\title{
ZWISCHENEVALUIERUNG DER aws-TECHNOLOGIEPROGRAMME 2007-2010
}

\author{
ENDBERICHT
}

\author{
HELMUT GASSLER, BRIGITTE ECKER \\ CHRISTIAN REINER, GERHARD STREICHER (JR) \\ SABINE MAYER, IRIS FISCHL, JÜRGEN STREICHER \\ (KMUFA)
}

APRIL 2011

\section{JOANNEUM RESEARCH Forschungsgesellschaft mbH}

POLICIES - Zentrum für Wirtschafts- und Innovationsforschung

Haus der Forschung, Sensengasse 1, 1090 Wien, Tel. +43-1-581 75 20, und

Elisabethstraße 17, $8010 \mathrm{Graz}$, Tel. +43-316-876 1488

policies@joanneum.at 


\section{JOANNEUM N1/N POLICIES \\ RESEARCH )) ZENTRUM FÜR WIRTSCHAFTS- UND \\ POLICIES IN), INNOVATIONSFORSCHUNG \\ KMU Forschung Austria \\ Austrian Institute for SME Research}

Diese Studie wurde im Auftrag des BMWFJ Bundesministerium für Wirtschaft, Familie und Jugend durchgeführt.

JOANNEUM RESEARCH Forschungsgesellschaft mbH

Helmut Gassler

Brigitte Ecker

Christian Reiner

Gerhard Streicher

KMU FORSCHUNG AUSTRIA

Sabine Mayer

Iris Fischl

Jürgen Streicher 


\section{Inhalt}

EINLEITUNG

1 MODUL 1: DARSTELLUNG DER EINZELNEN PROGRAMME UND DEREN

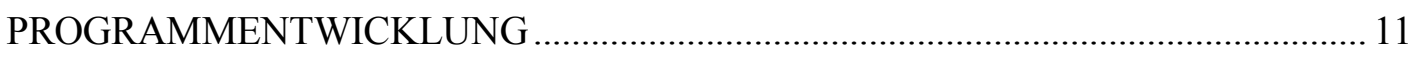

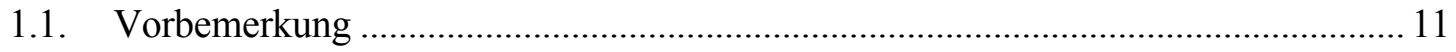

1.2. Programme zur Förderung von Gründung und Aufbau junger innovativer technologieorientierter Unternehmen.................................................................... 11

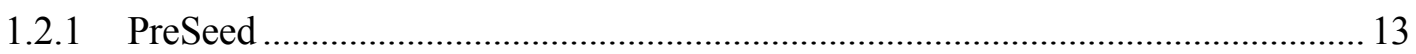

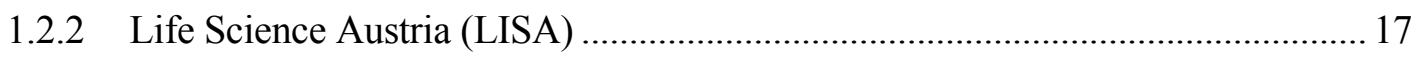

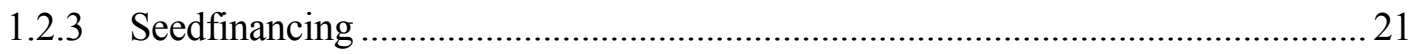

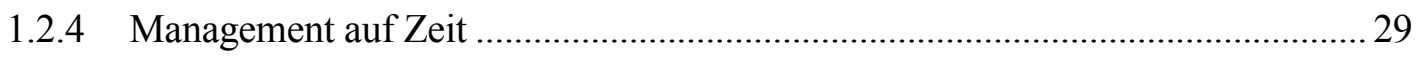

1.2.5 i2 - Die Börse für Business Angels .................................................................... 31

1.2.6 tecnet (Markt- und Technologierecherchen) ……................................................. 35

1.3. Programme zur Patentverwertung ........................................................................... 41

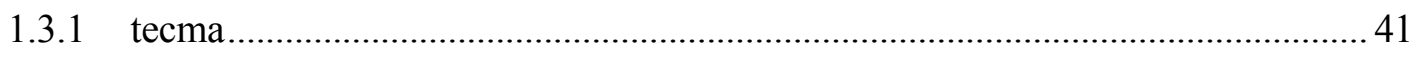

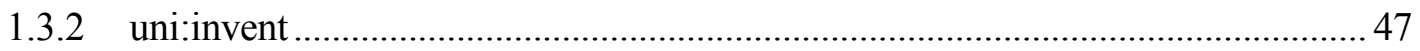

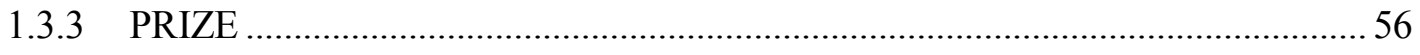

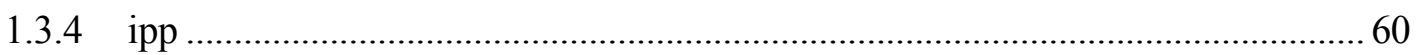

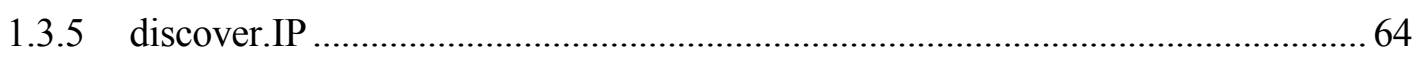

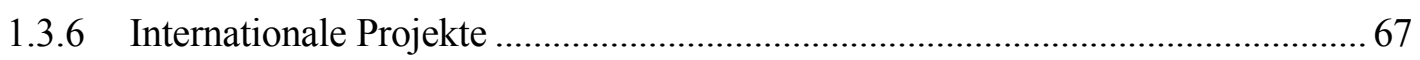

1.4. Programm zur Unterstützung der Kreativwirtschaft ................................................. 71

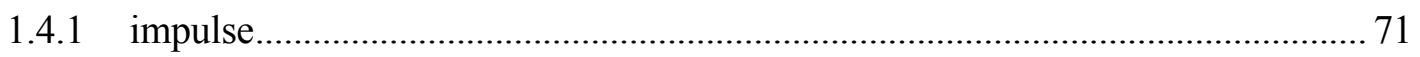

1.5. Programm zur Förderung von Produktfindungsstrategien ...................................... 85

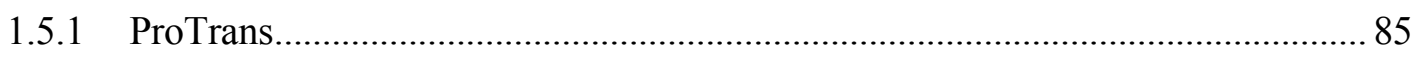

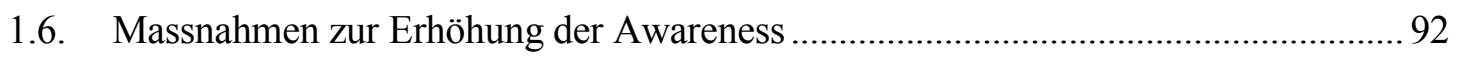

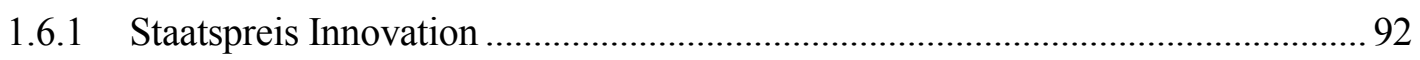

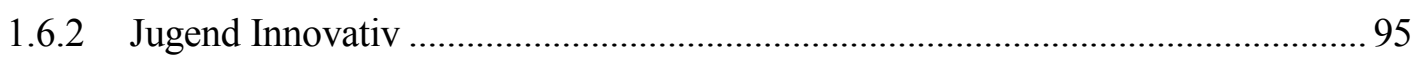

2 MODUL 2: PORTFOLIOBETRACHTUNG: SYNERGIEN UND ABSTIMMUNG . 102

2.1. Zielsystem und -konsistenz der Programmlinien.................................................... 102

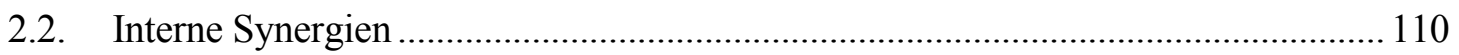

3 MODUL 3: FUNKTION UND POSITIONIERUNG IM INNOVATIONSSYSTEM . 116

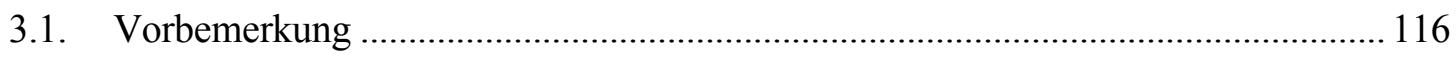

3.2. Charakteristika der Antragsteller in den Technologieprogrammen der aws............. 117

3.3. Zusammenspiel verschiedener Förderungen........................................................ 119

3.3.1 Nutzung durch die Unternehmen..................................................................... 119

3.3.2 Zusammenspiel aus Sicht der Agenturen und Stakeholder................................. 124

3.4. Kriterien für die Auswahl von Förderungsprogrammen aus der Sicht der Antragsteller bei den Technologieprogrammen der aws ....................................... 127

3.5. Strategien, wenn beantragte Förderungen nicht genehmigt werden ........................ 129

3.6. Stellenwert des FTI-Förderungsangebots für die Unternehmen............................... 130 
4 MODUL 4: AWARENESS UND AUßENDARSTELLUNG.

4.1. Vorbemerkung

4.2. Außendarstellung der aws-Technologieprogramme und Awareness seitens der Kunden

4.2.1 Awareness gegenüber Innovationen und Unterstützungsbedürfnisse 135

4.2.2 Bekanntheit der aws-Technologieprogramme.

5 MODUL 5: INTERNATIONALE BEISPIELE UND ERFAHRUNGEN.

5.1. Vergleichsstaaten im Überblick: Rahmenbedingungen und Trends .

5.1.1 Stellenwert und Entwicklung der Gründungspolitik in den FTI-Politiken der Vergleichsstaaten. 147

5.1.2 Innovationsökonomische Rahmenbedingungen der FTI-Politik 149

5.1.3 Venture Capital-Investitionen und Gründungsperformance. 151

5.1.4 Patentperformance und IPRs im Vergleich 155

5.2. Internationale Programme zur Förderung von Hochtechnologieunternehmensgründungen. 158

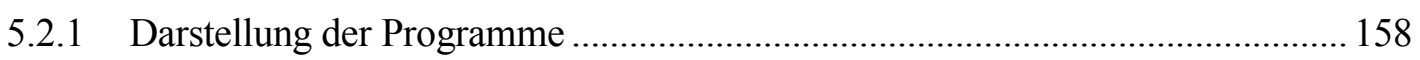

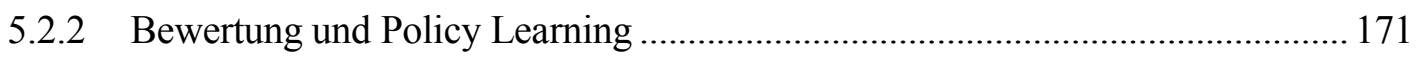

5.3. Internationale Programme zur Förderung von IPR-Aktivitäten ................................ 177

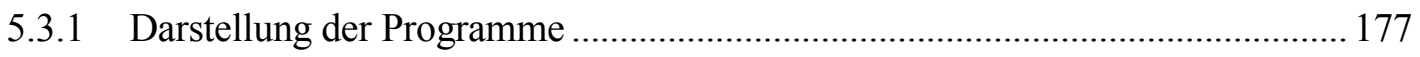

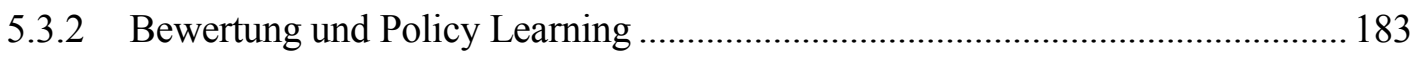

6 ALLGEMEINE SCHLUSSFOLGERUNGEN UND SPEZIFISCHE EMPFEHLUNGEN 185

7 LITERATUR. 199 


\section{Abbildungsverzeichnis}

Abbildung 1: aws-Technologieprogramme.....

Abbildung 2: Zahl der beschlossenen Projekte nach Themenschwerpunkten 2007 - 2010..... 15

Abbildung 3: Verteilung der PreSeed-Projekte nach Bundesländern 2007 - 2010 ................... 15

Abbildung 4: Kennzahlen zur Entwicklung von Seedfinancing seit 1995 .............................. 24

Abbildung 5: Monetäre Charakteristika der Seedfinancing-Projekte (IKT und Physical

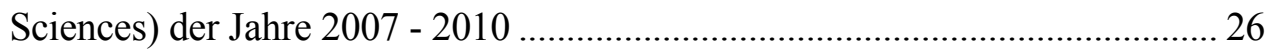

Abbildung 6: Entwicklung von Seedfinancing im Berichtszeitraum ...................................... 27

Abbildung 7: Beschäftigungsentwicklung der Portfoliounternehmen der Gründungsjahrgänge

2008 bis 2010 im Berichtszeitraum 28

Abbildung 8: Projektanfragen und versendete Projekte ............................................................. 33

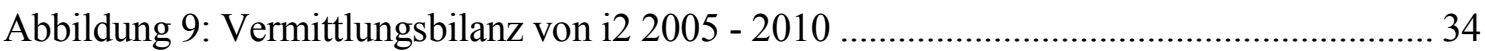

Abbildung 10: Auftragsrecherchen nach Technologiefeldern 2007 - 2010 ............................ 38

Abbildung 11: Auftragsrecherchen nach Kundentypen 2007 - 2010 ....................................... 39

Abbildung 12: Ergebnisse der Kundenzufriedenheitsanalyse (2007 - 2010$)$ )........................... 40

Abbildung 13: Zur Vermarktung übernommene Projekte 2007 - 2010 .................................... 44

Abbildung 14: Lizenzverträge nach Lizenznehmer (geographische Verteilung) 2007 - 201046

Abbildung 15: Anzahl der Erfindungsmeldungen (Datum der Meldung an die Universität).. 49

Abbildung 16: Erfindungsmeldungen nach Universitäten...................................................... 49

Abbildung 17: Erfindungsmeldungen nach Technologiefeldern ............................................. 50

Abbildung 18: Erfindungsmeldungen seit Programmbeginn.................................................... 51

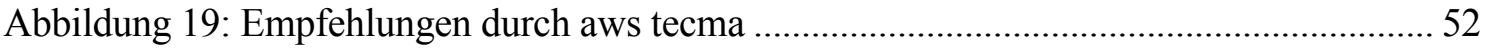

Abbildung 20: Erfindungsmeldungen im Jahr 2010 nach Technologiefeldern........................ 55

Abbildung 21: Geförderte Teilnehmer im Rahmen von ipp nach Bundesländern, 2007 - 201062

Abbildung 22: Eingereichte Projekte von discover.IP nach Bundesland, 2008-2010 65

Abbildung 23: Einreichungen und genehmigte Projekte in der Programmlinie impulse XS in den Jahren 2009 und 2010

Abbildung 24: Eingereichte und genehmigte Projekt- bzw. Fördervolumina (in EUR) in der Programmlinie impulse XS ....................................................................... 76

Abbildung 25: Typus des Antragsstellers in der Programmlinie impulse XS........................... 77

Abbildung 26: Einreichungen und genehmigte Projekte in der Programmlinie impulse XL in den Jahren 2009 und 2010

Abbildung 27: Eingereichte und genehmigte Projekt- bzw. Fördervolumina (in EUR) in der Programmlinie impulse XL 
Abbildung 28: Typus des Antragsstellers in der Programmlinie impulse XL

Abbildung 29: Geographische Verteilung der eingereichten und geförderten impulse XL

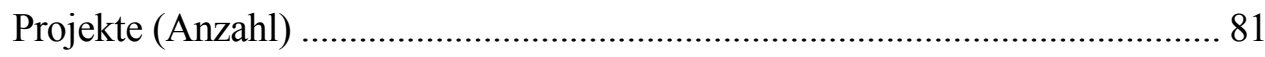

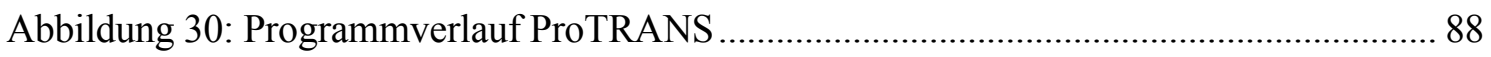

Abbildung 31: Geographische Verteilung der geförderten Projekte (Konzept- und Umsetzungsphase)...

Abbildung 32: Verteilung der geförderten Projekte (Konzept- und Umsetzungsphase) nach

Branchen 90

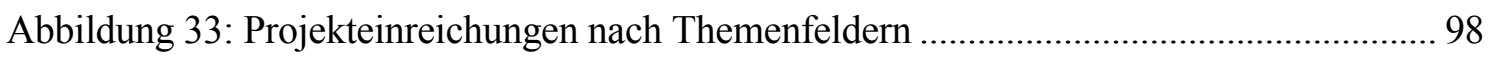

Abbildung 34: Projekteinreichungen nach Schultypen .............................................................. 99

Abbildung 35: Projekteinreichungen nach Bundesländern. 100

Abbildung 36: Überblick über die Zielebenen der aws-Technologieprogramme (Zahl der Einzelziele in Klammer) 105

Abbildung 37: Die Zielebenen der aws-Technologieprogramme und die jeweils zugeordneten Einzelprogramme 106

Abbildung 38: Beziehungen zwischen den aws-Programme untereinander 111

Abbildung 39: Inanspruchnahme von aws-Programmen durch Seedfinancing-Antragsteller113

Abbildung 40: Inanspruchnahme zusätzlicher aws Produkte (Programme) durch

Seedfinancing-Antragsteller (tatsächlich geförderte versus abgelehnte) 114

Abbildung 41: Anzahl der Unternehmen, die in den Technologieprogrammen der aws und in anderen (ausgewählten) Programmen im Zeitraum 2005-2008 mindestens einen Antrag eingereicht haben (Kombinationen) 122

Abbildung 42: Anzahl der Unternehmen, die mindestens einen Antrag bei den

Technologieprogrammen gestellt haben und bei weiteren

Förderungsprogrammen (2005-08).

Abbildung 43:Wirtschaftsnahe Förderungen in den Bundesländern - institutioneller Überblick126

Abbildung 44: Kriterien für die Auswahl von Förderprogrammen (ex-ante) 128

Abbildung 45: Strategien der Unternehmen bei Nicht-Bewilligung von FTI-Förderungen.. 130

Abbildung 46: Stellenwert des Angebots an FTI-Förderung 131

Abbildung 47: Bedeutung von Innovationen und Unterstützungsbedarf, befragte

Unternehmen in \%. 136

Abbildung 48: Bekanntheit der aws-Technologieprogramme, befragte Unternehmen in \%. 138

Abbildung 49: Kenntnis der aws-Technologieprogramme in Abhängigkeit von dem für das Unternehmen zutreffenden Unterstützungsbedarf in einzelnen Innovationsphasen*. 141

Abbildung 50: Militärausgaben in \% des BIP (2007) 145 
Abbildung 51: Netto-Migrationsraten im Vergleich (EinwanderInnen -

AuswanderInnen/1000) 146

Abbildung 52: Erwerbsquoten im Vergleich

Abbildung 53: Ausgaben des Unternehmenssektors für F\&E in \% des BIP 150

Abbildung 54: Humankapitalausstattung im Vergleich 151

Abbildung 55: Venture Capital-Investments in der Gründungsphase in \% des BIP 155

Abbildung 56: Venture Capital-Investments in der Expansionsphase in \% des BIP 155

Abbildung 57: Hochtechnologiepatentanmeldungen je Mio. EinwohnerInnen beim Europäischen Patentamt 156

Abbildung 58: Patentregime nach Gesetzeslage und ManagerInnen-Umfrage 157

Abbildung 59: Seedinvestitionen und Beteiligungen des HTGF 159

Abbildung 60: Die Position von Go-Bio im Biotechnologischen Innovationszyklus. 162

Abbildung 61: Die Finanzierungsfunktion der Fonds der Bayern Kapital im Unternehmens-

Lebenszyklus 163

Abbildung 62: Die Rolle des Coach im Programm Seedfonds Bayern. 164

Abbildung 63: Finanzierungsangebote von Vaekstfonden nach Unternehmenstypus. 168

Abbildung 64: F\&E-Förderprogramme der israelischen FTI-Politik 169

Abbildung 65: Technologische Spezialisierung der mit Juli 2010 durch das Incubator Program geförderten Unternehmen

Abbildung 66: Öffentliche und private Venture Capital-Investition in Hochtechnologiegründunge in Inkubatoren 171

Abbildung 67: Die Förderschienen des SIGNO-Programms 178 


\section{Tabellenverzeichnis}

Tabelle 1: Übersicht zu Zielen und Zielerreichung PreSeed................................................... 14

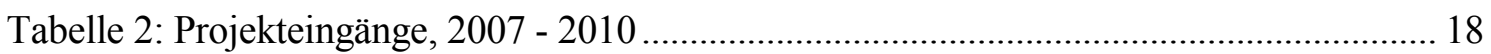

Tabelle 3: Anzahl der Projekte im Rahmen von PreSeed und Seedfinancing, 2007 - 2010 .... 18

Tabelle 4: Projekteinreichungen und geförderte Projekte beim Businessplan-Wettbewerb für Life Sciences (BOB), 2008 und 2010 ............................................................... 19

Tabelle 5: Übersicht der Entwicklung von tecnet anhand ausgewählter

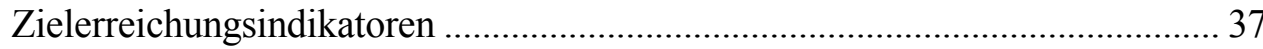

Tabelle 6: Verteilung der Einreichungen nach Bundesländern.................................................. 43

Tabelle 7: Verteilung der Einreichungen nach Technologiefeldern .......................................... 43

Tabelle 8: Zahl der Einreichungen nach Typus des Einreichers.............................................. 44

Tabelle 9: Erfindungsmeldungen nach Technologiefeldern, 2004 - 2009 (Datum des Eingangs bei der aws tecma) .......................................................................................... 50

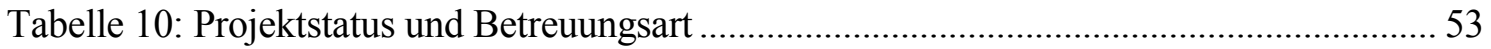

Tabelle 11: Frauenanteil an den ErsterfinderInnen sowie an allen Erfinderinnen, 2006 - 200954

Tabelle 12: Eingegangene Erfindungsmeldungen im Jahr 2010 nach Organisation.................. 55

Tabelle 13: Anzahl der PRIZE Einreichungen und davon positive Juryentscheidungen nach Organisation und Call-Jahr, 2008-2010 ......................................................... 57

Tabelle 14: Anzahl der geförderten PRIZE-Projekte nach Technologiefeld und Call-Jahr, 2008-2010

Tabelle 15: Anzahl der geförderten PRIZE-Projekte nach Technologiefeld und Call-Jahr .... 58

Tabelle 16: PRIZE-Fördersummen nach Organisation und Call-Jahr, 2008 - 2010 ................. 59

Tabelle 17: Durch PRIZE erzielte Vertragsabschlüsse nach Organisation seit 2006 .............. 59

Tabelle 18: Förderanträge und geförderte Projekte im Rahmen von ipp, 2007 - 2010............ 61

Tabelle 19: Geförderte Projekte im Rahmen von ipp nach Technologie, 2007 - 2010............ 62

Tabelle 20: Eingereichte Projekte von discover.IP nach Unternehmensgröße, 2008 - 2010 ... 64

Tabelle 21: Eingereichte Projekte von discover.IP nach Technologie, 2008 - 2010 ............... 65

Tabelle 22: aws Beteiligungen an EU-Programmen im Bereich IPR, 2005-2010 ................... 69

Tabelle 23: aws Beteiligungen am 6. und 7. RP und Strukturfonds, 2005 - 2010 ................... 70

Tabelle 24: Übersicht über die Förderungslinien von impulse .............................................. 73

Tabelle 25: Übersicht der Calls in der Programmlinie XS........................................................ 78

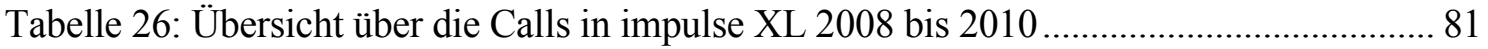

Tabelle 27: Kriterienkatalog und Gewichtung des Staatspreis Innovation, 2011 .................... 93

Tabelle 28: Einreichungen zum Staatspreis Innovation nach Bundesland, 2007 - 2010 ......... 93 
Tabelle 29: Zielvereinfachung für die Zielebene „Awareness“.....

Tabelle 30: Zielvereinfachung für die Zielebene „High-Tech Gründungen“.. 107

Tabelle 31: Zielvereinfachung für die Zielebene „High-Tech Risikokapital“ 108

Tabelle 32: Zielsystem für die Zielebene „Geistiges Eigentum“ (Anm.: keine Änderungen vorgenommen) 108

Tabelle 33: Zielvereinfachung für die Zielebene „Kreativwirtschaft“ 109

Tabelle 34: Zielvereinfachung für die Zielebene „Tech-Information“ 109

Tabelle 35: Zielvereinfachung für die Zielebene „Technologietransfer“. 110

Tabelle 36: Strukturdaten der untersuchten Unternehmen. 118

Tabelle 37: Anzahl der Unternehmen, die in den Technologieprogrammen der aws im Zeitraum 2005-2008 mindestens einen Antrag eingereicht haben

Tabelle 38: Anzahl der Unternehmen, die in den Technologieprogrammen der aws im Zeitraum 2005-2008 mindestens einen Antrag eingereicht haben (Kombinationen)

Tabelle 39: Zusammensetzung der Stichprobe nach Bundesländern, Verteilung der Unternehmen im Gewerbe und Handwerk in Österreich 134

Tabelle 40: Zusammensetzung der Stichprobe nach Sektoren, Verteilung der Unternehmen im Gewerbe und Handwerk in Österreich

Tabelle 41: Bedeutung von Innovationen und Unterstützungsbedarf, befragte Unternehmen ausgewählter Fachgruppen in Prozent.

Tabelle 42: Bekanntheit der aws-Technologieprogramme, befragte Unternehmen ausgewählter Fachgruppen in Prozent

Tabelle 43: Gründungsperformance und Venture Capital-Investment

Tabelle 44: Vergleichsprogramme zur Förderung von

Hochtechnologieunternehmensgründungen 158

Tabelle 45: Vergleichsprogramme Patentverwertung. 


\section{Einleitung}

\section{Hintergrund der Studie}

Das BMWFJ beauftragte die aws mit der Durchführung verschiedener Technologie- und Innovationsprogramme, die in ihren jeweiligen Zielsetzungen, ihrer Ausrichtung und in ihren Instrumenten ein breites Spektrum von innovations- und technologiepolitischen Fördermaßnahmen und -instrumenten abdecken:

Ein großer Pool an Maßnahmen und Instrumenten zielt auf die Gründung von Unternehmen im Hochtechnologiebereich (JITU) ab. Dazu zählen die innerhalb der neuen JITU-Richtlinien organisierten Programme PreSeed, Seedfinancing und Management auf Zeit sowie i2-Die Börse für Business Angels und tecnet - Markt- und Technologierecherche. Dieses Programmportfolio inkludiert Maßnahmen, die über die reine Finanzierung bzw. monetäre Unterstützung hinausgehen und beinhaltet auch immaterielle Fördermaßnahmen, etwa in Form von Beratungspakten bzw. Angeboten gezielter Informationsbereitstellung und -aufbereitung. Damit wird gleichsam die Verknüpfung zum zweiten Programmportfolio hergestellt, nämlich das Programmportfolio im Bereich Wissensmanagement, also der Patentverwertung und der geistigen Eigentumsrechte im Allgemeinen. Dazu zählen Programme wie das Ende 2009 ausgelaufene Programm uni:invent und PRIZE, weiters tecma - Innovationsvermarktung, ipp - Innovationsschutzprogramm, discover.IP sowie die Internationalisierungsmaßnahmen seitens der aws. Die Förderungslinien im Programm impulse zielen auf den Bereich der Kreativwirtschaft, ProTRANS auf die Förderung von Produktfindungsstrategien, und der Staatspreis Innovation und Jugend Innovativ setzen Akzente im Bereich der Awareness.

Diese Programme bilden im Zusammenspiel ein komplexes Instrumenten- und Förderportfolio und decken in ihren Zielrichtungen unterschiedliche funktionale bzw. thematische Bereiche ab (siehe Abbildung 1). Einige Programme zeigen dabei schon eine lange Laufzeit und entsprechen einer konstant eingerichteten Institution (wie beispielsweise das SeedfinancingProgamm), andere sind jüngeren Datums, mit einer kürzeren Laufzeit (wie zum Beispiel impulse).

Abbildung 1: aws-Technologieprogramme

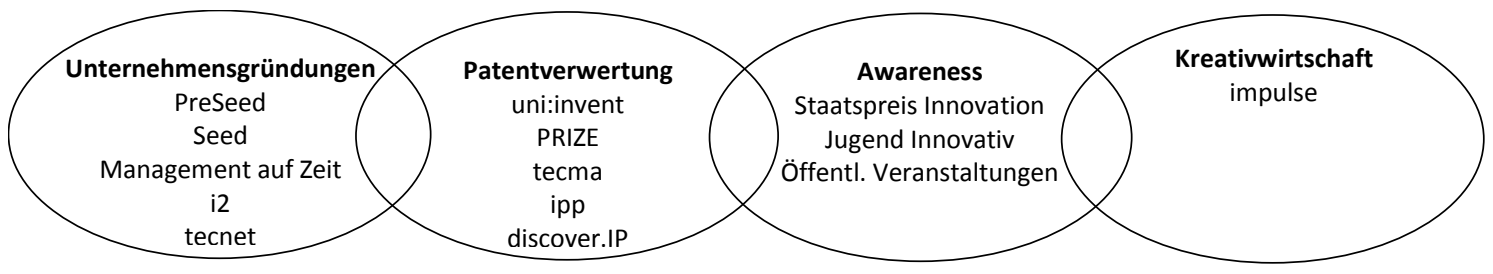

Die aws-Technologieprogramme wurden im Jahr 2006 (zwischen)evaluiert ${ }^{1}$. Wesentliche Empfehlungen dieser Zwischenevaluierung (durchgeführt von Technopolis gemeinsam mit JOANNEUM RESEARCH) waren:

- Stärkere Profilgebung des vorhandenen Instrumentenportfolio bei entsprechender Clusterung der Unterstützungsmaßnahmen

\footnotetext{
${ }^{1}$ Jörg et al. (2006)
} 
- Optimierung der Zusammenarbeit mit den relevanten Politikinitiativen der Bundesländer

- Weiterentwicklung der Programmsteuerung und -finanzierung.

Die Genese und Entwicklung der aws-Technologieprogramme zeigen exemplarische policy learning Prozesse. Neben der erwähnten Zwischenevaluierung aus dem Jahr 2006 wurde auch eine Reihe von Detailevaluierungen auf Programmebene durchgeführt und laufend Adaptierungen und Programmänderungen vorgenommen (z.B. die Etablierung der JITU-Richtlinie) oder die komplette organisatorische Eingliederung von impulse in den Förderungskanon der aws. Diese Ergebnisse sollen in der hier vorgeschlagenen Evaluierung mit berücksichtigt werden, gleichzeitig stellen sie auch einen wesentlichen Input an Informationen und Datengrundlagen dar.

Die vorliegende Zwischenevaluierung kann daher auf den Ergebnissen der ersten Zwischenevaluierung aus dem Jahr 2006 und den jeweiligen Detailevaluierungen aufsetzen und verfolgt diese wesentlichen Zielsetzungen:

- Darstellung des Programmverlaufs in den einzelnen Programmen und Bewertung des jeweiligen Programmerfolgs

- Bewertung des Zusammenspiels der Programme innerhalb der aws (interne Schnittstellenanalyse) und deren generelle Einbettung im österreichischen Technologiepolitiksystem bzw. Innovationssystem (externe Schnittstellenanalyse)

- Durch einen ,Blick nach außen' werden internationale Erfahrungen mit ähnlich gelagerten Programmen und Instrumenten für eine Positionierung und mögliche Weiterentwicklungen der aws-Technologieprogramme nutzbar gemacht.

\section{Berichtsstruktur}

Anfang Dezember 2010 sowie im Februar 2011 fanden Gesprächsrunden zwischen VertreterInnen der aws und dem Evaluierungsteam statt, bei denen die Strukturen und Entwicklungstendenzen in den einzelnen Programmen diskutiert wurden. Darüber hinaus wurde bei diesen Gesprächen auch der Datenaustausch (insbesondere Daten in Hinblick auf die definierten Zielerreichungsindikatoren) geregelt. Auf Basis dieser Daten wurde im Modul 1 die Programmentwicklung der einzelnen Technologieprogramme für die Jahre 2007 - 2010 nachgezeichnet.

In Modul 2 wird eine Diskussion des Zielkatalogs vorgenommen und ein Vorschlag für eine Strukturierung der Zielsysteme inklusive einer Vereinfachung/Kürzung der Einzelziele für die Programme gegeben. Um die internen Synergien zwischen den einzelnen Programmen bzw. Instrumenten der aws zu bewerten, wird in Form einer Netzwerkanalyse das Beziehungsgeflecht zwischen den Programmen analysiert.

Modul 3 diskutiert die Positionierung der aws-Technologieprogramme innerhalb des Gesamtsystems der technologiepolitischen Förderungslandschaft Österreichs, wobei auf die umfangreichen Daten hinsichtlich der Fördernutzung von österreichischen Unternehmen, die im Rahmen der Systemanalyse des österreichischen Innovationssystems aufgebaut wurden, zurückgegriffen werden konnte.

In Modul 4 werden die Wahrnehmung des Instrumentenportfolios der aws sowie die Awareness für innovative Ideen in Österreich von Seiten der Unternehmen diskutiert. Im Rahmen 
dieses Kapitels werden dabei auch Ergebnisse der Konjunkturbeobachtung der KMU Forschung Austria, die speziell für die Zwischenevaluierung der aws-Technologieprogramme um ausgewählte Fragen zur Nutzung/Kenntnis von Technologie-programmen sowie zur Awareness hinsichtlich der Bedeutung von Innovationen erweitert wurde, herangezogen.

Modul 5 widmet sich dem Vergleich ausgewählter aws-Programme (im Wesentlichen die gründungsorientierten Programme sowie IP-bezogenen Programme) mit einschlägigen Programmen bzw. Instrumenten in ausgewählten Ländern (Deutschland, Bayern, Dänemark, Israel, Frankreich). Die Auswahl der zu berücksichtigenden Programme und Länder wurde dabei in Abstimmung mit der aws getätigt.

In Kapitel 6 werden die wesentlichen Ergebnisse der Evaluierung zusammengefasst, Schlussfolgerungen abgeleitet und Handlungsempfehlungen gegeben. 


\section{Modul 1: Darstellung der einzelnen Programme und deren Programmentwicklung}

\subsection{VORBEMERKUNG}

Modul 1 behandelt die Technologieprogramme der aws im Einzelnen, es steht also das individuelle Programm, seine Ziele und seine generelle Performance im Fokus der Betrachtung. Im Kern geht es somit darum, die jeweiligen Ziele der Programme sowie die durchgeführten Aktivitäten und deren Beitrag zur Zielerreichung zu überprüfen. Ausgangspunkt hierbei sind die Ergebnisse der Zwischenevaluierung aus dem Jahr 2006. Folgende Fragen stehen dabei im Vordergrund:

- Wie hat sich das jeweilige Programm im Untersuchungszeitraum 2007 - 2010 qualitativ und quantitativ entwickelt?

- Inwieweit wurden die vereinbarten Ziele erreicht (Analyse der Performance) und welche Wirkungen wurden erzielt?

- Gibt es Zielabweichungen und wenn ja, welche Ursachen lassen sich dafür identifizieren?

- Werden die in den Programmzielen angesprochenen Zielgruppen erreicht?

- Inwieweit wurden die Empfehlungen der Zwischenevaluierung 2006 berücksichtigt und umgesetzt?

- Welche Perspektiven und ggf. Änderungsnotwendigkeiten ergeben sich für die $\mathrm{Zu}-$ kunft?

\subsection{PROGRAMME ZUR FÖRDERUNG VON GRÜNDUNG UND AUFBAU JUNGER INNOVATIVER TECHNOLOGIEORIENTIERTER UNTERNEHMEN}

Im Jahr 2009 wurden die Programme PreSeed, Seedfinancing und Management auf Zeit als aufeinander abgestimmte Module unter einem Dach als „Programm zur Förderung von Gründung und Aufbau junger innovativer technologieorientierter Unternehmen“ positioniert. Dabei wird den Erkenntnissen der Innovationsforschung Rechnung getragen, dass im Rahmen des Innovations-, Gründungs- und Aufbauprozesses i.w.S. jeweils unterschiedliche Problemlagen zu differenzierten Bedürfnissen hinsichtlich monetärer wie nicht-monetärer Förderung vorliegen.

Durch den modularen Aufbau des Programms und der vorhandenen Expertise in der aws können somit unterschiedliche Finanzierungsinstrumente sowie Beratungs- und Betreuungsleistungen auf die jeweiligen Bedürfnisse des Förderungswerbers „maßgeschneidert“ werden.

Die Ziele des Programms werden in der JITU-Richtlinie definiert und stellen gleichsam die „Oberziele“ für die unter dieser Richtlinie operierenden Einzelprogramme bzw. Module dar (JITU-Richtlinien, 2007, S. 3-4):

- Förderungen auf Grundlage dieser Richtlinien werden grundsätzlich im Rahmen von spezifischen Programmen vergeben, deren Ziele schriftlich in den Programmdokumenten festzulegen und zu veröffentlichen sind. 
- Die Ziele müssen in nachvollziehbarer Weise begründet sein, operationalisierbar und an Hand von qualitativen bzw. quantitativen Indikatoren überprüfbar sein.

- Die Förderung und Unterstützung von Gründung und Aufbau junger innovativer technologieorientierter Unternehmen stellt ein hohes Risiko dar, das den Einsatz öffentlicher Mittel im Interesse des Gesamtnutzens ambitionierter Forschungs- und Entwicklungsprojekte rechtfertigt, wenn die betreffenden Vorhaben sonst nicht oder nur in geringerem Umfang durchgeführt würden.

- Besonderes Augenmerk ist auf die Förderung von Projekten zu richten, welche eine Hebelwirkung in Richtung Forschung, Entwicklung und Innovation aufweisen. Es sollen Anstöße für innovative Prozesse, Produkte und Dienstleistungen bewirkt werden.

- Generelle Zielsetzung der gegenständlichen Richtlinien ist die Schaffung eines geeigneten Instrumentariums zur Unterstützung von potentiellen GründerInnen sowie zur Förderung junger kleiner innovativer technologieorientierter Unternehmen.

- Die Gründungsidee muss technologisch orientiert und innovativ sein sowie nachhaltige wirtschaftliche Erfolgsaussichten besitzen. Nicht gefördert werden Projekte, die dem Stand der Technik entsprechen bzw. lediglich eine graduelle Weiterentwicklung zum Ziel haben.

- Weiters sollen diejenigen jungen, kleinen, innovativen, technologieorientierten Unternehmen gefördert werden, welche zum Zeitpunkt der Gewährung der Beihilfe weniger als sechs Jahre bestanden haben, neue oder wesentlich verbesserte Produkte, Dienstleistungen oder Verfahren entwickeln, verglichen mit dem Stand in diesem Wirtschaftszweig in der Gemeinschaft, und die entweder das Risiko eines technischen oder wirtschaftlichen Misserfolges in sich tragen oder dessen FuE-Aufwendungen wenigstens $15 \%$ seiner gesamten Betriebsausgaben ausmachen.

- Das Gesamtziel aller dieser Förderungsprogramme ist die Unterstützung von technologieorientierten Unternehmensneugründungen mittels sonstiger Geldzuwendungen ohne Gewinnbeteiligung (nicht rückzahlbarer Zuschüsse) und Beratungsleistungen sowie die Förderung junger innovativer technologieorientierter Unternehmen innerhalb der ersten sechs Jahre seit deren Gründung mittels sonstiger Geldzuwendungen mit Gewinnbeteiligung (Zuschüsse, welche im Erfolgsfall zurückzuzahlen sind).

- Dieses Gesamtziel ist verknüpft mit wirtschaftspolitischen und gesellschaftspolitischen Zielen zu betrachten. Die geförderten Vorhaben sollen einen wesentlichen Beitrag zur Verbesserung der österreichischen Wirtschaftsstruktur, zur Schaffung dauerhafter hochqualitativer Arbeitsplätze sowie zur Stärkung der heimischen Leistungsbilanz leisten. Den umweltrelevanten, sozialen und gesellschaftlichen Auswirkungen kommt dabei eine wesentliche Bedeutung zu. 


\subsubsection{PreSeed}

\section{IKT und Physical Sciences}

PreSeed (IKT und Physical Sciences) ${ }^{2}$ wurde im Jahr 2005 vom BMWFJ initiiert und adressiert die Vorgründungsphase im Bereich der Hochtechnologie. Damit soll jene Lücke geschlossen werden, die aufgrund asymmetrischer Informationen am privaten Kapitalmarkt hinsichtlich der Finanzierung der Frühphase (Vorgründungsphase) von HochtechnologieGründungsprojekten besteht. Seit 2008 ist PreSeed als Programmmodul Teil des „Programms zur Förderung von Gründung und Aufbau junger, innovativer, technologieorientierter Unternehmen“ (JITU-Programm). Die Konzeption von PreSeed IKT und Physical Sciences basiert dabei auf den Erfahrungen des PreSeed-Moduls innerhalb von LISA, das bereits im Jahr 2003 etabliert wurde (vgl. Kapitel 1.2.2).

Programmziel ist die nachhaltige Steigerung der Zahl der österreichischen High-Tech Gründungen mit ausgeprägten Forschungs- und Entwicklungsaktivitäten und die Überleitung von Forschungsergebnissen in wettbewerbsfähige Produkte, Verfahren oder Dienstleistungen. Das Programmmodul PreSeed innerhalb des JITU-Daches erfüllt somit die Funktion, dem Programmmodul Seedfinancing entsprechend attraktive Gründungsprojekte zu liefern.

Im Kern geht es bei PreSeed darum, durch frühzeitige Bewertung (Überprüfung auf Machbarkeit und Kommerzialisierungspotential) und (monetäre) Unterstützung die Entwicklung eines Projekts hin zu einer marktfähigen Geschäftsidee zu begleiten und zu fördern.

Bis zum dritten Quartal 2009 betrug die maximale Fördersumme 100.000 EUR (im Rahmen der ,de-minimis“-Regelung der EU-Wettbewerbsregeln), seit dem 4. Quartal 2009 beträgt die maximale Fördersumme 200.000 EUR, wobei die Auszahlung der Fördermittel gemäß eines vertraglich fixierten Meilensteinkonzepts erfolgt.

Gemäß dem „Anbot für die Fortsetzung der Technologieprogramme für die Jahre 2007 bis 2009 sind die Indikatoren zur Überprüfung der Ziele des Förderprogramms wie folgt definiert:

- $\quad$ Forcierung und Etablierung Österreichs als Hochtechnologiestandort

- $\quad$ Steigerung der Gründungsbereitschaft im High-Tech Bereich

- Anzahl der PreSeed Anfragen

- Anzahl der geförderten PreSeed Projekte

- Projektkosten in EUR

- gegründete Unternehmen

- geplante/geschaffene Arbeitsplätze (m/w)

Die gegenständlichen Hauptindikatoren sind wie folgt zu detaillieren:

- $\quad$ nach Wirtschaftssektoren bzw. Technologiefelder

- nach Bundesländern

Die quantitative Programmentwicklung ist in Tabelle 1 dargestellt. Dabei ist zu berücksichtigen, dass durch die Umstellung auf die neuen JITU-Richtlinien die im Jahr 2007 beschlosse-

\footnotetext{
2 Das Schwerpunktprogramm LISA (Life Sciences Austria), das ebenfalls ein PreSeed-Element beinhaltet, wird in Kapitel 1.2 .2 behandelt.
} 
nen Projekte in die neuen JITU-Richtlinie übernommen wurden und „formal“ eigentlich erst im Jahr 2008 begonnen wurden.

Von den Projektanfragen führen ca. 27 \% zu Projekten („,beschlossene Projekte“). In Summe gab es somit im Zeitraum zwischen 2007 - 2010198 Projektanfragen, die zu 53 Projekten führten. Insgesamt betragen die (geplanten) Gesamtprojektkosten dieser Periode 8,6 Mio. EUR, wovon 7,1 Mio. EUR von der aws als Zuschüsse bewilligt wurden.

Von den Projekten der Jahre 2007 - 2010 führten bis dato 26 Projekte zu Unternehmensgründungen. Betrachtet man nur die Projekte zwischen 2007 und 2009, ergibt sich also eine Gründungsquote von ca. 64 \% (Anm.: Für die Projekte aus dem Jahr 2010 ist eine Berechnung einer Gründungsquote noch nicht sinnvoll, da diese Projekte noch im Laufen sind). Dies spricht zum einen für die hohe Qualität des Auswahlprozesses aus den Projektanfragen und zum anderen für die Effizienz des Betreuungsprozesses innerhalb der Projekte. Acht dieser Gründungen der Jahre 2007 bis 2009 wurden bislang vom „Anschlussmodul“ Seedfinancing übernommen (aus den Projekten des Jahres 2010 gibt es bereits eine erfolgte Unternehmensgründung, aber noch keine Übernahme in Seedfinancing).

Tabelle 1: Übersicht zu Zielen und Zielerreichung PreSeed

\begin{tabular}{|c|c|c|c|c|c|c|}
\hline Indikator & Zielwert & 2007 & 2008 & 2009 & 2010 & $\begin{array}{c}\text { Summe } \\
2007-2010\end{array}$ \\
\hline Anzahl der PreSeed Anfragen & & 46 & 36 & 51 & 65 & 198 \\
\hline $\begin{array}{l}\text { Anzahl der genehmigten PreSeed } \\
\text { Anträge }\end{array}$ & $15-20$ & $13^{*}$ & 11 & $15^{* *}$ & 14 & 53 \\
\hline $\begin{array}{l}\begin{array}{l}\text { Bewilligte } \\
\text { (Mio. EUR) }\end{array} \\
\text { aws }\end{array}$ & & 1,3 & 1,1 & 2,1 & 2,6 & 7,1 \\
\hline Gesamtprojektkosten (Mio. EUR) & & 1,5 & 1,2 & 2,7 & 3,2 & 8,6 \\
\hline $\begin{array}{l}\text { Anteil der aus PreSeed Projekten } \\
\text { gegründeten Unternehmen }\end{array}$ & $60-80 \%$ & $55 \%$ & $91 \%$ & $64 \%$ & $(7 \%) * * *$ & $64 \% * * * *$ \\
\hline
\end{tabular}

Anmerkungen: * davon 2 Abbrüche; ** davon 1 Abbruch; *** Projektlaufzeit noch zu kurz für eine aussagekräftige Gründungsquote; **** in diesem Wert ist das Jahr 2010 nicht enthalten

Quelle: aws

Eine wesentliche „Quelle“ für Projekte stellen die AplusB-Zentren an Universitäten dar, deren Aufgabe es ist, akademische Gründungsideen zu betreuen. Ein Gutteil der geförderten PreSeed-Projekte (2010: 43 \%; 2009: 80 \%; 2008: 73 \%, 2007: $92 \%$ ) wird auch im Rahmen eines AplusB-Zentrums betreut.

Die Verteilung über die beiden angesprochenen Themenschwerpunkte (IKT und Physical Sciences) ist relativ gleichmäßig und bleibt auch im Zeitablauf annähernd konstant (siehe Abbildung 2), d.h. die Aufteilung zwischen IKT und Physical Sciences beträgt ca. 50:50. 
Abbildung 2: Zahl der beschlossenen Projekte nach Themenschwerpunkten 2007 - 2010

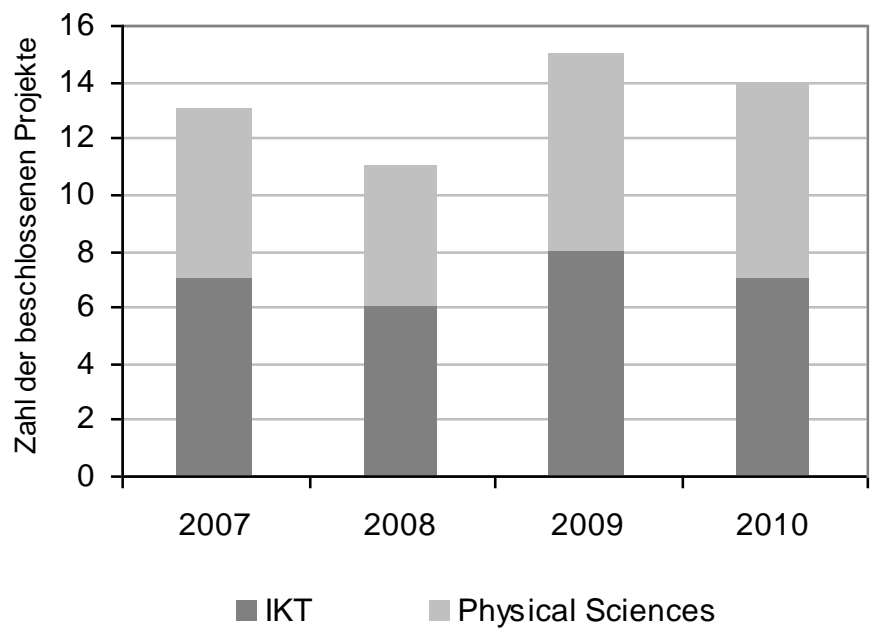

Quelle: aws

Hinsichtlich der geographischen Verteilung der PreSeed-Projekte gibt es einen eindeutigen Schwerpunkt auf Wien, gefolgt von Oberösterreich und der Steiermark (Abbildung 3). Lediglich im Jahr 2009 kam es zu einer deutlichen Veränderung, da auch Niederösterreich mehrere Projekte (mit vier Projekten sogar gleich viel wie Oberösterreich und um eines mehr als Wien) auf sich ziehen konnte. Von den kleineren Bundesländern (Vorarlberg, Kärnten und Burgenland fehlen durchgängig) sind lediglich Tirol und Salzburg präsent, dafür allerdings konstant mit jeweils ein bis zwei Projekten über den gesamten Evaluierungszeitraum.

$\mathrm{Zu}$ berücksichtigen bei der geographischen Betrachtung sind allerdings die doch kleinen Fallzahlen, so dass die Ergebnisse lediglich indikativ zu verstehen sind und keine allgemeinen Schlussfolgerungen erlauben.

Abbildung 3: Verteilung der PreSeed-Projekte nach Bundesländern 2007 - 2010

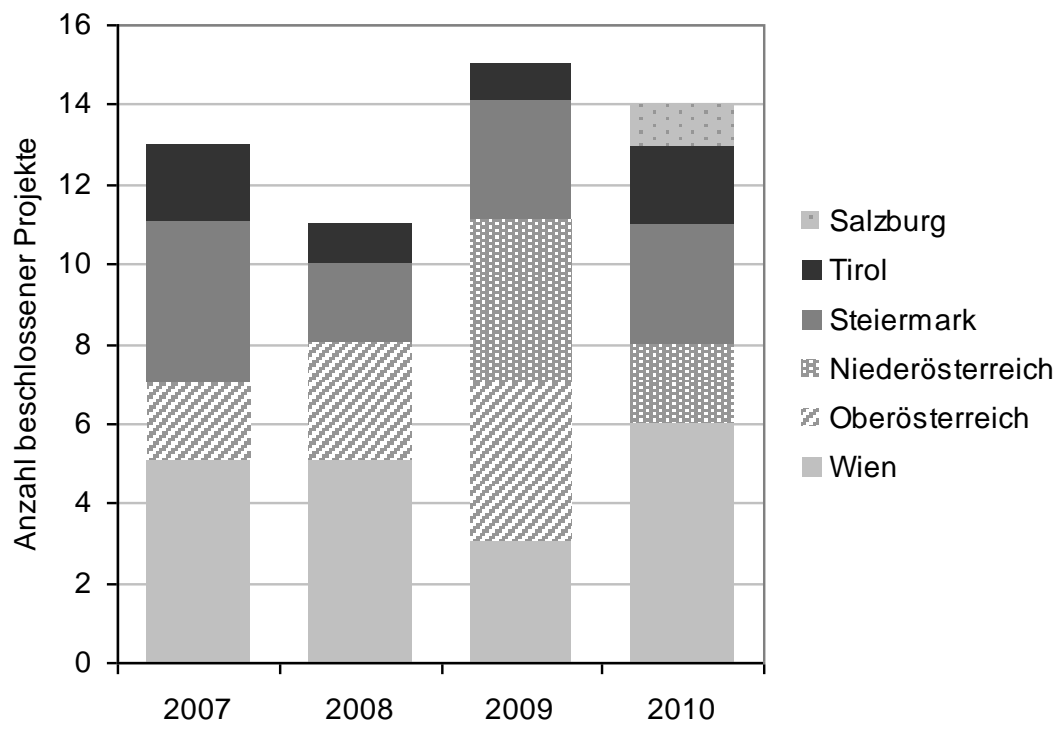

Quelle: aws 
Für die Projekte der Jahre 2007 bis 2009 können auch bereits Angaben zur Zahl der bislang geschaffenen Arbeitsplätze (Kopfzahl) getätigt werden. Insgesamt beträgt die Zahl der bislang geschaffenen Arbeitsplätze der Projekte aus dem Jahr 200746 (Kopfzahl inkl. GründerInnen selbst und TeilzeitmitarbeiterInnen bzw. freie MitarbeiterInnen), jener aus dem Jahr 200824 sowie $21 \mathrm{im}$ Jahr 2009. Der Frauenanteil bei diesen Arbeitsplätzen ist mit 11 \% (2007) bzw. $10 \%$ (2008) sowie $19 \%$ (2009) durchwegs gering.

Zusätzlich zu den angeführten Aktivitäten im Bereich der Themenfelder IKT und Physical Sciences erfolge im Jahr 2010 eine thematische Erweiterung durch ein Pilotprojekt in Richtung innovative Dienstleistungen. Dabei wurde nach den gleichen Richtlinien wie PreSeed ein Call für Frühphasen-Gründungsprojekte im Bereich innovativer Dienstleistungen ausgeschrieben. Dieser Call führte zu 91 Einreichungen, wovon eine international besetzte Jury 22 Projekte mit einem Gesamtförderungsvolumen von 3,1 Mio. EUR zur Förderung genehmigte.

\section{Bewertung durch das Evaluierungsteam}

Das Programmmodul PreSeed nimmt innerhalb der (technologieorientierten) Gründungsförderung Österreichs eine besondere Rolle ein, indem es bewusst auf die früheste Phase des Gründungsprozesses (Vorgründungsphase) abzielt und gleichzeitig ein hohes technologisches Anspruchsniveau an die Projekte stellt. Von der Konzeption erfüllt es eine Aktivierungsrolle für das zentrale JITU-Modul Seedfinancing, indem es eine Pipeline-Funktion für die Generierung von einschlägigen Gründungsprojekten übernimmt und somit einen entsprechenden Deal-Flow für Seedfinancing garantieren soll.

Eine Bewertung des Programmerfolgs hat diese hohen Selektionskriterien sowie die PipelineFunktion zu berücksichtigen. Zwar mag die Zahl der induzierten Gründungen prima facie als niedrig erscheinen, die Tatsache, dass diese Gründungen ohne dem Programm nicht erfolgt wären, lässt sich allerdings aus der Konstruktion des Programms (Konzentration auf Vorgründungsphase mit intensiver Betreuung und relativ hoher maximaler Förderung der Gesamtkosten - die monetäre Förderung deckt dabei oft bis zu 100 \% der kalkulierten Projektkosten ab) ableiten. Daher kann dem Programm eine hohe Additionalität bescheinigt werden (d.h. es werden Projekte / Gründungen initiiert, die ohne Förderungsprogramm mit hoher Wahrscheinlichkeit nicht entstanden wären). Mittel- und langfristig im Vordergrund steht aber die Pipeline-Funktion für Seedfinancing, indem qualitativ hochwerte Gründungen im Hoch- und Spitzentechnologiebereich für das Investmentportfolio von Seedfinancing generiert werden.

Die hohe „Gründungsquote“ (d.h. der Anteil von betreuten Projekten, die auch tatsächlich zu einer Unternehmensgründung führen) zeigt, dass das Projekt inhaltlich richtig aufgestellt ist. Weiters ist das PreSeed-Modul auch eine wichtige Anlaufstation für GründerInnen, die in AplusB-Zentren betreut werden und bei entsprechendem Projektfortschritt im Rahmen der PreSeed-Förderung in die entscheidende Gründungsphase eintreten können. Somit kommt dem PreSeed-Modul auch eine wichtige Rolle für den ambitionierten (d.h. besonders technologieintensiven bzw. High-Tech-orientierten) Wissens- und Technologietransfer zwischen Universitäten und Wirtschaft in Österreich in Form von akademischen Spin-off-Gründungen zu.

Trotz der vergleichsweise geringen Fördermittel und der geringen Ressourcenausstattung des
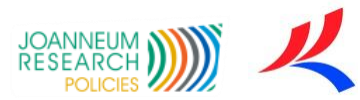
Programms (ca. jährlich ein bis zweieinhalb Mio. EUR Zuschüsse jeweils für IKT/Physical Sciences) kann somit das Programm als wichtiger Ankerpunkt der technologierorientierten Frühphasenförderung Österreichs eingestuft werden. Ein Programmerfolg von PreSeed wirkt sich somit indirekt auch positiv auf die ,nachgeordnete“ Modulstufe Seedfinancing aus (je mehr PreSeed-Projekte gefördert und je besser diese betreut werden können, desto mehr und bessere Projekte finden sich auch für Seedfinancing).

\subsubsection{Life Science Austria (LISA)}

Um den Life Science Standort Österreich zu stärken und die Forschungskapazitäten in der Wirtschaft durch Forcierung des Technologietransfers zu steigern, wurde das Programm Life Science Austria (LISA) als Nachfolgeprogramm des Impulsprogramms Biotechnologie (1999 bis 2002) im Auftrag des BMWFJ ins Leben gerufen. LISA versteht sich als zentrale Anlaufund Schnittstelle für Gründungen und nachhaltige Unternehmensförderung in den Bereichen Biotechnologie, Pharma, Medizintechnik und Verfahrenstechnik. Ziel ist „die Forcierung von Unternehmensgründungen und umfassende Unterstützung in der Wachstumsphase durch Erschließung neuer Finanzierungsinstrumente, Verknüpfung und Verstärkung bestehender Förderungsangebote und begleitender Beratung". Neben gezielten Betreuungs- und Finanzierungsmaßnahmen, Awareness- und Ausbildungsaktivitäten (BOB-Best of Biotech: Businessplan-Wettbewerb) rücken dabei Aufgaben wie die internationale Vermarktung (Dachmarke LISA) und das Clustermanagement immer mehr in den Mittelpunkt der Aktivitäten.

Die Indikatoren zur Überprüfung der Ziele des Förderprogramms LISA sind laut „Anbot für die Fortsetzung der Technologieprogramme für die Jahre 2007 bis 2009“" wie folgt definiert:

- Anzahl der Beratungsprojekte

- Anzahl der PreSeed- und Seedfinancing-Projekte

- Anzahl der weiterfinanzierten Seedfinancing-Projekte durch (inter-)nationales Venture Capital

- TeilnehmerInnenzahl und KundInnenzufriedenheit bei Ausbildungs- und Plattformveranstaltungen

- Anzahl der bei BOB eingereichten Projekte sowie daraus folgende Gründungen

- Anzahl der internationalen bei BOB eingereichten Projekte

\section{Begutachtungen/Beratungen}

Wie in Tabelle 2 dargestellt, hat die aws im Rahmen von LISA in den Jahren 2007 - 2010 insgesamt 255 Projektbegutachtungen, 121 allgemeine Beratungen und 47 Gründungsberatungen durch-geführt. Gegenüber den Vorjahren hat sich dabei im Jahr 2010 die Anzahl der Projektbegutachtungen sowie der allgemeinen Beratungen abermals erhöht. So wurden 2010 insgesamt 80 Projektbegutachtungen und 40 allgemeine Beratungen durchgeführt, darüber hinaus noch 14 Gründungsberatungen. Damit hat LISA - nach dem merklichen Rückgang im Jahr 2008 - wieder an die Zahlen des Jahres 2008 anknüpfen können. 
Tabelle 2: Projekteingänge, 2007 - 2010

\begin{tabular}{lrrrrc}
\hline Anzahl der Projekteingänge & $\mathbf{2 0 0 7}$ & $\mathbf{2 0 0 8}$ & $\mathbf{2 0 0 9}$ & $\mathbf{2 0 1 0}$ & gesamt \\
\hline Projektbegutachtungen & 89 & 74 & 12 & 80 & 255 \\
Beratungen & 21 & 31 & 29 & 40 & 121 \\
Gründungsberatungen & 12 & 8 & 13 & 14 & 47 \\
\hline Quelle: aws & & & & &
\end{tabular}

\section{Finanzierung}

Im Rahmen von LISA stehen zwei Finanzierungsinstrumente zur Verfügung: PreSeed und Seedfinancing. LISA-PreSeed ist eine Vorgründungsfinanzierung (dotiert mit max. 200.000 EUR) für alle Personen, die - ausgehend vomersten Erfolg versprechenden, wissenschaftlichen Daten - ein Unternehmen gründen möchten. In dieser ersten Phase geht es vor allem darum, die Erbringung eines wissenschaftlichen proof of principle zu fördern. Wie aus Tabelle 3 ersichtlich wurden im Zeitraum 2007 - 2010 insgesamt 17 PreSeed-Projekte für eine Förderung empfohlen. Seit 2007 wurden insgesamt 17 Projekte gefördert, wovon bereits fünf abgeschlossen sind. Bis auf acht Projekte haben alle zu Unternehmensgründungen geführt. Zwei Unternehmen wurden mit LISA-Seedfinancing und insgesamt drei mit Business Angels und Venture Kapital weiterfinanziert. $29 \%$ der PreSeed finanzierten Projekte sind universitären Ursprungs.

Mit dem Förderinstrument Seedfinancing können Hochtechnologieunternehmen mit max. 1 Mio. EUR in der Start-Up Phase unterstützt werden. Wie Tabelle 3 zeigt, bewegt sich auch die jährlich genehmigte Anzahl von Seedfinacing-Projekten um die vier bzw. fünf Projekte, wobei insgesamt 10,05 Mio. EUR in der Zeitspanne 2007 - 2010 zugesagt wurden. Von den 13 (in diesem Zeitraum) empfohlenen Unternehmen konnten 11 in der Folge Eigenkapital in der Höhe von 15 Mio. EUR einwerben.

Tabelle 3: Anzahl der Projekte im Rahmen von PreSeed und Seedfinancing, 2007 - 2010

\begin{tabular}{lrrrrr}
\hline Projektanzahl & $\mathbf{2 0 0 7}$ & $\mathbf{2 0 0 8}$ & $\mathbf{2 0 0 9}$ & $\mathbf{2 0 1 0}$ & gesamt \\
\hline PreSeed & 0 & 6 & 4 & 7 & 17 \\
Seedfinancing & 0 & 5 & 4 & 4 & 13 \\
\hline
\end{tabular}

Im Jahr 2007 wurden die Verhandlungen über die Richtlinien und den Auftragsvertrag nicht rechtzeitig abgeschlossen; aufgrund dessen gab es in diesem Jahr keine formal zugesagten Fördermittel, wobei dies für die Jahre davor und danach kompensiert wurde.

Quelle: aws

\section{Begleitmaßnahmen: Qualifizierung und Awareness}

Neben der direkten Begleitung und Beratung von Gründungs- und Aufbauprozessen ist LISA zusätzlich noch auf anderen Ebenen operativ tätig, so bietet LISA regelmäßig Qualifizierungsmaßnahmen im Bereich Life Science an. Diese Qualifizierungsmaßnahmen werden von Jahr zu Jahr fortgesetzt und umfassten im Jahr 2010 z.B. folgende Aktivitäten:

- Die Ausbildungsreihe Business Seminare für potentielle UnternehmensgründerInnen (durchgeführt von LISA VR), darunter: 
- „Crossing the Bridge from Preclinical to Clinical Studies Fast Track Approaches to Concept Testing \& Market Authorization" (11.2.2010)

- „Partnering-Messen - ein neues Format in der Medizintechnik“ (12.3.2010)

- „Partnering-Messen - Do's and Don'ts” (17. 9. 2010)

- Lehrveranstaltungsmodul „Business in Life Sciences: From Bench to Product“ an der Universität Wien, welches fünf Lehrveranstaltungen im Bachelor- und drei weitere im Master-Studium anbietet. Im Zuge dessen hat LISA VR auch die Betreuung von zwei PraktikantInnen übernommen.

- Vorlesung „Biotechnologie in Österreich“ im Studiengang „Bioengineering“ der Fachhochschule fh campus Wien. Ziel dieser Lehrveranstaltung ist es, einen Überblick über die Unternehmenslandschaft im Bereich Biotech und Pharma inklusive relevanter wirtschaftlicher Rahmenbedingungen zu geben. Auch UnternehmensvertreterInnen werden hierzu jährlich eingeladen.

- Eine Lehreinheit mit dem Titel „Förder- und Finanzierungsmöglichkeiten im Hochtechnologiesektor“ im Rahmen der Lehrveranstaltung „Grundlagen Biomedizinischer Ökonomie" an der Veterinärmedizinischen Universität Wien.

Hinzu kommen umfassende Awareness-Maßnahmen wie z.B. der Businessplan-Wettbewerb für Life Sciences (BOB), welcher 2010 im Auftrag des BMWFJ im Rahmen von LISA zum fünften Mal veranstaltet wurde. Ziel ist es, WissenschafterInnen, StudentInnen und IdeenträgerInnen aus Universitäten und außeruniversitären Einrichtungen zu motivieren, den Schritt ins Unternehmertum zu wagen und diese dabei professionell zu unterstützen. Sponsoren aus der Pharmaindustrie und Wirtschaft, die AplusB Zentren sowie internationale Partner unterstützen diesen Wettbewerb, in welchem - wie Tabelle 4 darstellt - in den letzten zwei Ausschreibungsrunden 2008 und 2010 insgesamt 94 Projekte eingereicht und davon 14 Projekte mit Preisgeldern von insgesamt 77.900.- EUR gefördert wurden. Verzeichnet die absolute Anzahl der Projekteinreichungen im Jahr 2010 ein Plus, so konnte auch beim Anteil an nicht-österreichischen Projekten ein Anstieg verzeichnet werden: War dieser Anteil 2008 mit $21 \%$ noch sehr gering, so konnte dieser 2010 auf immerhin $47 \%$ gesteigert werden. Was die Anzahl der Unternehmensgründungen zurückgehend auf den BOB betrifft, so sind seit seiner Gründung im Jahr 2000 bislang insgesamt 55 Unternehmen gegründet worden.

Tabelle 4: Projekteinreichungen und geförderte Projekte beim Businessplan-Wettbewerb für Life Sciences (BOB), 2008 und 2010

\begin{tabular}{lrrc}
\hline Anzahl & $\mathbf{2 0 0 8}$ & $\mathbf{2 0 1 0}$ & gesamt \\
\hline Projekteinreichungen & 45 & 49 & 94 \\
Geförderte/ ausgezeichnete Projekte & 8 & 6 & 14 \\
\hline
\end{tabular}

Quelle: aws

Um nun auch die Sichtbarkeit auf internationaler Ebene zu erhöhen und den Life Science Standort Österreich nachhaltig abzusichern, wurde auf Empfehlung des Rates für Forschung und Technologieentwicklung im Oktober 2007 „Life Science Austria (LISA)“ als Dachmar- 
$\boldsymbol{k} \boldsymbol{e}$ für alle österreichischen Life Science Aktivitäten im Außenauftritt geschaffen: „LISA fungiert dabei als Marketingplattform der noch stärkeren Zusammenarbeit der Regionen auf internationaler Ebene, um die Sichtbarkeit als attraktiven Standort und der Life Science Unternehmen in der internationalen Corporate und InvestorInnen Szene zu steigern sowie um den Life Science Standort Österreich nachhaltig abzusichern und auszubauen. " 3 Dabei erfährt LISA Unterstützung seitens wichtiger regionaler Cluster-Partner wie:

- ARGE LISA Vienna Region

- Tiroler Standortagentur/ des Life Science Clusters Tirol

- Human Technology Styria,

- Gesundheitscluster Oberösterreich sowie

- Technopolprogramm des Landes Niederösterreich/ ecoplus.

Gemeinsam mit den Cluster-Partnern steht LISA somit nicht nur als Ansprechpartner für Interessenten aus aller Welt zur Verfügung, sondern ermöglicht - unter Bündelung ihres KnowHows und ihrer Dienstleistungen - insbesondere jungen Unternehmen und Forschungsinstitutionen am internationalen Markt auftreten zu können. Seit ihrem Bestehen - somit seit beinahe drei Jahren - hat LISA auf diese Weise 135 Unternehmen, Start-Ups und Universitäts-Spin-offs durch Messeauftritte auf 27 internationalen Biotech- und Medizintechnikmessen präsentiert und 1.800 Kontaktanbahnungen zu potentiellen KundInnen, Kooperationspartner und Lieferanten ermöglicht. Allein im Jahr 2010 war LISA auf neun internationalen Messen im Bereich Biotechnologie und Medizintechnik vertreten, wovon acht mit einem Gemeinschafts-stand besucht wurden. Weiters wurde die Medienarbeit von LISA ausgebaut: 2010 gab es mehr als 60 von LSA initiierte Beiträge, was einer Verdoppelung von 2009 entspricht. Neben zahlreichen Schaltungen und redaktionellen Artikeln in ausgewählten Zeitschriften bzw. Magazinen gilt es hier vor allem auch die Medienkooperation mit dem Chemiereport zu nennen.

\section{Bewertung durch das Evaluierungsteam}

LISA wurde bereits in der Evaluierung von Technopolis und JOANNEUM RESEARCH im Jahr 2006 als best-practice hervorgehoben, was seine Gültigkeit bis heute hat. Nicht nur dass LISA - sehr erfolgreich - seinen Fokus auf die Förderung einer der innovativsten Technologiefelder, dem Life Science-Bereich, legt, auch hat LISA, insbesondere durch die Etablierung der Dachmarke zur Nachhaltigkeit beigetragen und auf internationaler Ebene den Unternehmensstandort Österreich merklich sichtbarer gemacht. Diesbezüglich hat gerade die jüngste Evaluierung der Dachmarke LISA (Radauer 2009) gezeigt, dass hier durchaus noch Verbesserungspotentiale auszuschöpfen sind. Hierzu zählen u.a., um der Heterogenität der zwei Bereiche Biotechnologie und Medizintechnik gerecht zu werden, weitere Regelungen zur Nutzung der Dachmarke als Corporate Identity anzustellen sowie eine klarere Rollenverteilung zwischen den Kooperationspartnern zu definieren und damit einhergehend auch die Entscheidungs- und Kommunikationsprozesse effizienter zu gestalten - nicht zuletzt um der Prämisse

\footnotetext{
${ }^{3}$ LISA Leistungsbericht (2010), S. 9.
} 
Folge zu leisten, dass sich ,alle Kooperationspartner zu einem gemeinsamen Zielsystem verpflichten“ und damit „auch zu gemeinsamen Vorgehensweisen“ (Radauer (2009), S. 22).

Eine erfolgreiche Weiterentwicklung ist die zweite große Awareness-Schiene von LISA, der BOB (Best Of Biotech: Businessplan-Wettbewerb). Ähnlich wie in den Jahren zuvor wurde der Wettbewerb in zwei Phasen organisiert, wobei man das 2007/ 2008 neu eingeführte Boot Camp durch die Coaching Session im Jahr 2010 ersetzte. Damit hat man nicht nur die Möglichkeit wahrgenommen, die zehn besten (und somit eine reduzierte Anzahl an) Teams in der Coaching Session zu schulen, sondern auch die Intervention wahrgenommen, dem hohen Kosten- und Organisationsaufwand der Jahre zuvor entgegenzusteuern. Des Weiteren hat man eine neue Website mit einem Online Einreich- und Begutachtungstool kreiert sowie die Kooperation mit den Technologietransferbüros an den Universitäten verstärkt. Damit konnte abermals - so-wohl auf nationaler als auch auf internationaler Ebene - die Anzahl der Einreichungen erhöht und des Weiteren die Sichtbarkeit von LISA gestärkt werden.

Dass man zudem versucht hat, mit der Ausschreibung eines zweiten Preises, des Medizintechnikpreises im Jahr 2010, zusätzliche Breitenwirkung zu erzielen, zeigt mit Erfolg die hohe Zahl an Einreichungen von Medizintechnikprojekten. Es stellt sich nun die Frage, inwieweit sich diese Heterogenität Medizintechnik versus Biotechnologie, der beim BOB bereits Rechnung getragen und die beim internationalen Marketing bereits andiskutiert wird, auch in den anderen Programmmaßnahmen (insbesondere in der PR/ Öffentlichkeitsarbeit) von LISA wiederfinden soll. Beantwortet man diese Frage nicht nur vor dem Hinter-grund der Programmkonsistenz, sondern auch hinsichtlich einer auf Zielgruppen abgestimmten Vermarktung, so wäre dies durchaus zu befürworten, auch weil durch eine differenzierte Vermarktung Investoren und Unternehmen gezielter angesprochen werden können. Gleiches gilt für die Universitäten, deren Technologietransferbüros auch in Zukunft ein wichtiger Kooperationspartner von LISA sein werden. Es gilt daher, diese Kooperation in Zukunft nicht nur fortzusetzen, sondern weiterhin zu verstärken und die Universitäten in die Öffentlichkeitsarbeit/ PRAktivitäten von LISA verstärkt einzubeziehen.

\subsubsection{Seedfinancing}

Mit der Etablierung im Jahr 1989 (damals im Rahmen des ITF-Fonds) weist das Förderungsprogramm Seedfinancing bereits eine außerordentlich lange Kontinuität auf. Seit 2004 wird es gemeinsam vom BMWFJ und dem BMVIT im Rahmen ihrer jeweiligen Schwerpunktfelder beauftragt und von der aws operativ abgewickelt. Als eines der Kernmodule innerhalb der „Richtlinien zur Förderung von Gründung und Aufbau junger, innovativer, technologieorientierter Unternehmen“ (JITU-Richtlinien) stellt es das zentrale Programm zur Dynamisierung der High-Tech-Gründungskultur in Österreich dar.

Im Kern geht es bei Seedfinancing darum, GründerInnen in der frühen Gründungsphase (,seed“) zu unterstützen, wobei diese Unterstützung im Wesentlichen durch Kapitalbereitstellung in Form von bedingt rückzahlbaren Zuschüssen erfolgt. Die Zurückzahlung ist an bestimmte, vertraglich fixierte Bedingungen wie z.B. Erreichung von Bilanzgewinnen oder erfolgreiche Veräußerung am privaten Kapitalmarkt, geknüpft. Es handelt sich bei Seedfinancing nicht um eine Projektförderung i.e.S., sondern um ein Programm zur Unternehmensfinanzierung. Gründungsprojekte bzw. Unternehmen im Seedfinancing-Portfolio soll zudem mittelfristig - in Anschluss an die öffentlich zur Verfügung gestellten bedingt rückzahlbaren 
Mittel - der Zugang zum privaten Venture Capital (VC)-Markt in Österreich erleichtert werden.

Die Einzelziele des Programms folgen der Motivation der JITU-Richtlinien und zielen ab auf:

- Verbesserung der österreichischen Wirtschaftsstruktur durch die Fokussierung auf dynamische, technologieorientierte Branchen sowie die Schaffung dauerhafter, hochqualitativer Arbeitsplätze

- Erhöhung der Anzahl von technologieorientierten Unternehmensneugründungen

- Nachhaltige Unterstützung des Aufbaus von Unternehmen zur wirtschaftlichen Nutzung innovativer und technologisch avancierter Produktideen, Verfahren oder Dienstleistungen mit überdurchschnittlichem Marktpotential und Wachstumschancen durch die Bereitstellung einer Seed-Finanzierung und einer projektbegleitenden Beratung

- Förderung von dynamischem und qualitativem Wachstum von innovativen Unternehmen

- Unterstützung bei der Ausweitung der Kapitalbasis (insbesondere für Risikokapital).

Konkret sind im Rahmen des Förderungsprogramms die Kosten aller Aktivitäten, die notwendig zum Aufbau des Unternehmens beitragen, förderbar, wie z.B. Personalkosten (v.a. Forscher und Techniker), Reise- und Ausbildungskosten, projektbezogene anteilige Sachkosten, wie z.B.: Laborgeräte, Prüfgeräte, Material für Prototypenbau, Kosten für Schutzrechte wie z.B. Patentkosten, Lizenzrechte, Konzept- und Studienkosten, Betriebsmittel und Markterschließungskosten.

Zusätzlich werden im Rahmen des Förderungsprogramms von Seiten der aws auch immaterielle Leistungen (in Form von maßgeschneiderten Beratungsleistungen) zur Verfügung gestellt.

Mit dem Inkrafttreten der JITU-Richtlinie (April 2008) wurde die maximal mögliche Förderungssumme von 500.000 EUR auf 1 Mio. EUR verdoppelt. Die Auszahlung des Betrags erfolgt in Tranchen, die mit der Erfüllung von vertraglich fixierten Projektmeilensteinen junktimiert ist.

Die Indikatoren zur Überprüfung der Ziele des Förderprogramms sind laut „Anbot für die Fortsetzung der Technologieprogramme für die Jahre 2007 bis 2009“ wie folgt definiert:

- $\quad$ Forcierung und Etablierung Österreichs als Hochtechnologiestandort

- Steigerung der Gründungsbereitschaft im High-Tech Bereich

- Anzahl der Seedfinancing Anfragen

- Anzahl der geförderten Seedfinancing Unternehmen

- Fördervolumen EUR

- $\quad$ erfolgreiche Weiterfinanzierung

- geplante/geschaffene Arbeitsplätze (m/w)

- Anzahl der erfolgreichen Rückführungen (High Flyer)

- Insolvenzen

- $\quad$ Rückflüsse

Die gegenständlichen Hauptindikatoren sind wie folgt zu detaillieren:

- $\quad$ nach Wirtschaftssektoren bzw. Technologiefelder

- $\quad$ nach Bundesländern 
Die Programmentwicklung in der Periode 2007 - 2010 lässt sich folgenderweise zusammenfassen: 2007 war durch die ausstehende Genehmigung der JITU-Richtlinien geprägt, allerdings konnten dennoch im Advisory Board vier neue Projekte interimistisch beschlossen werden (die endgültige Genehmigung dieser Projekte erfolgte dann im Jahr 2008). Zusätzlich zu den vier bereits 2007 vorläufig genehmigten Projekten wurden im Jahr 200812 weitere Projekte genehmigt. Insgesamt konnten im Jahr 2008 bereits nach den neuen JITU-Richtlinien 2,7 Mio. EUR ausbezahlt werden. Gleichzeitig kam es im Jahr 2008 zu einem Rückfluss von 3 Mio. EUR. Neben der monetären Förderung wurden im Jahr 2008 insgesamt elf pauschale Beratungspakete im Gesamtwert von 110.000 EUR zuerkannt. Im Jahr 2009 kam es bei 38 Anträgen zu einer Genehmigung von elf neuen Projekten mit einer Gesamtzusage von 7,4 Mio. EUR. Daneben wurden 30 pauschale Beratungspakete mit einem Gesamtwert von 300.000 EUR erteilt. Zu einem kurzfristigen Ressourcenproblem kam es im Jahr 2010, da durch Budgetrestriktionen das BMVIT die ursprünglich geplanten Mittel zurückhalten musste. Dadurch konnten die entsprechenden Projektbewertungen von zwei Jury-Sitzungen bislang noch nicht mit monetären Mitteln ausgestattet werden.

Die bisherigen Projektergebnisse der Gesamtperiode 1989-2010 von Seedfinancing kann wie folgt zusammengefasst werden (Stand 31.12. 2010):

- Seit Beginn wurden insgesamt 203 Unternehmen gefördert (Gesamtauszahlung 67,3 Mio. EUR)

- Mit Stichtag 31.12.2010 waren 93 Unternehmen mit einem Portfoliowert von 33,7 Mio. EUR (Investments minus Tilgungen) in Betreuung.

- 41 Unternehmen erreichten bislang Highflyer-Status.

- Bis Ende 2010 kam es zu 110 Exits, davon 55 erfolgreiche Abschichtungen.

- Seit 1989 wurden 47 Firmen insolvent mit 14,3 Mio. EUR Verlusten.

- Von Beginn bis 2010 wurden insgesamt knapp 2.342 Arbeitsplätze geschaffen.

Die langfristige Entwicklung (seit 1995) der Unternehmen im Seedfinancing-Portfolio ist in Abbildung 4 anhand von ausgewählten Kennzahlen dargestellt. Die Zahl der Unternehmen stieg annähernd kontinuierlich (die Abflachung im Jahr 2006 ist auf die Umstellung nach den neuen JITU-Richtlinien, die rechtlich erst im Jahr 2008 beendet wurde, zurückzuführen) auf mittlerweile $203^{4}$, was gegenüber 1995 mit damals ca. 70 Unternehmen beinahe eine Verdreifachung darstellt. Die Gesamtzahl der Beschäftigten in diesen Unternehmen hat sich noch positiver entwickelt. Sie beträgt derzeit 2342 und ist somit mehr zehnmal (!) so hoch wie im Jahr 1995. Auch die Umsätze haben sich entsprechend erhöht und zwar um den Faktor 13.

\footnotetext{
${ }^{4}$ Diese Zahl enthält auch jene Unternehmen, die bereits abgeschichtet wurden. Diese Unternehmen werden mit den entsprechenden Kennzahlen (Umsatz, Beschäftigte) zum Zeitpunkt ihres Exits in die Berechnung ein.
} 
Abbildung 4: Kennzahlen zur Entwicklung von Seedfinancing seit 1995

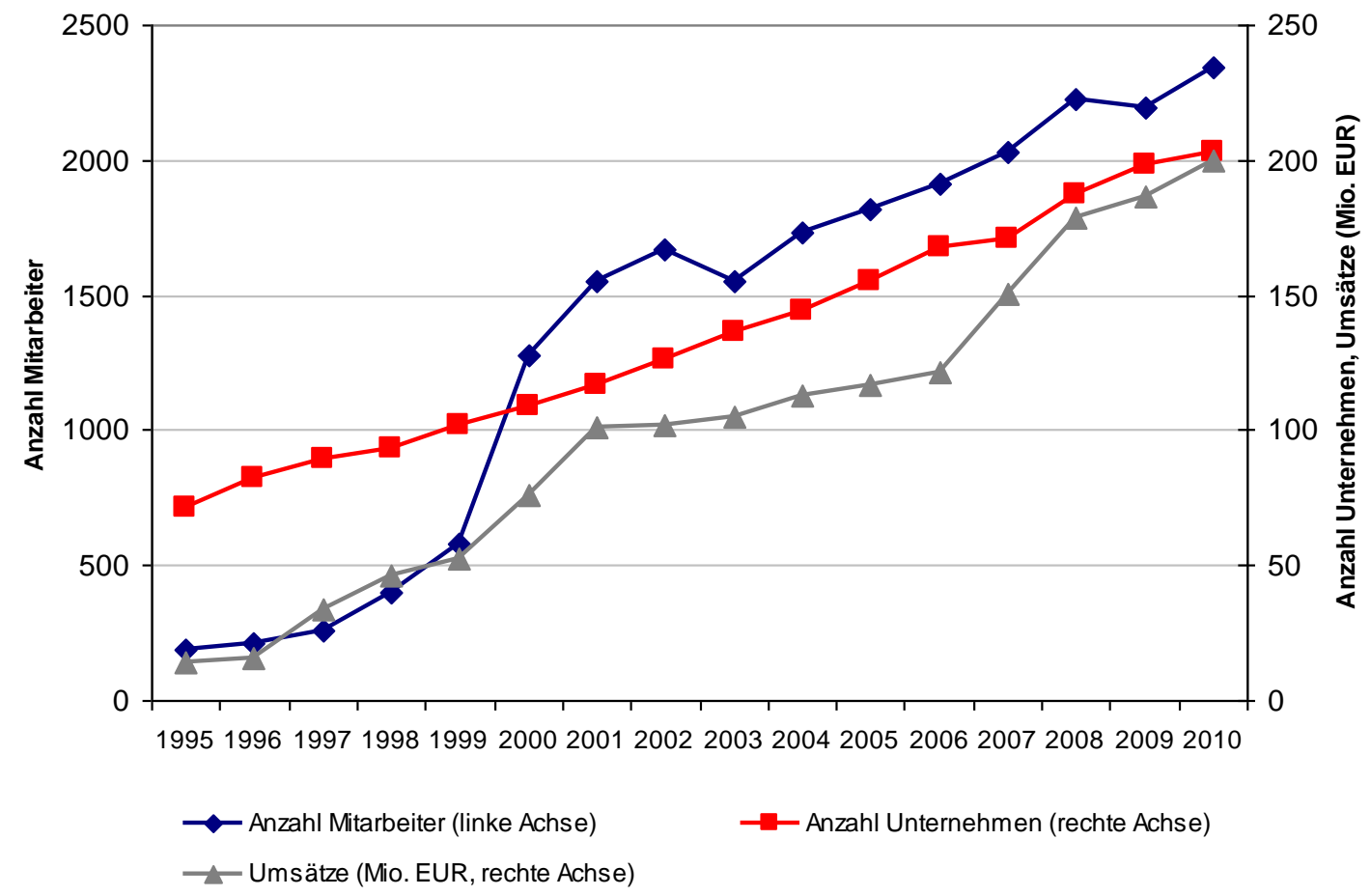

Quelle: aws

Diese Entwicklung deutet auf die Notwendigkeit hin, einschlägige Förderprogramme gerade im Gründungsbereich langfristig anzulegen. Die bewegten Summen und Effekte pro Jahr mögen zunächst als gering beurteilt werden, aber kumulativ zeigen sich bei längerer Betrachtung durchaus entsprechend eindrückliche Programmergebnisse. Dieser Befund wird auch durch eine Betrachtung der sogenannten „High Flyers“ bestätigt, worunter Unternehmen verstanden werden, die entweder innerhalb von fünf Jahren einen Mindestumsatz von 4 Mio. EUR erreichen oder eine Abschichtung mit maximaler Rendite ermöglichen. Derzeit (2010) konnten bereits 41 derartige High-Flyers identifiziert werden. Hingegen kam es während der Gesamtlaufzeit des Programms (1989 - 2010) zu 47 Insolvenzen (plus 8 Verluste durch sonstige Gründe), angesichts der Technologieorientierung sowie des hohen Innovationsgrades (und der damit implizierten erhöhten Unsicherheit) eine erstaunlich geringe Ausfallsquote.

Die Programmentwicklung von Seedfinancing in der hier relevanten Evaluierungsperiode (2007 - 2010 ) lässt sich nun im Detail folgenderweise charakterisieren, wobei die Entwicklung getrennt nach den Technologiefeldern IKT sowie Physical Sciences einerseits und Biotechnologie (als Teil der LISA-Dachmarke) andererseits nachgezeichnet wird (siehe Abbildung 5 und Abbildung 6 für eine graphische Zusammenfassung der Programmentwicklung).

\section{IKT und Physical Sciences}

- Im Jahr 2007 gab es 33 und im Jahr 200836 Anfragen, wovon dann im Jahr 2008 (unter den neuen JITU-Richtlinien) insgesamt zehn Projekte genehmigt wurden. Die Gesamtprojektkosten (als „Projektkosten“ werden dabei die gesamten Kosten des Unternehmens mit Ausnahme von Handelsgüter o.ä., also insbesondere die Personal- und 
F\&E-Kosten definiert) dieser zehn Projekte des Jahres 2008 werden dabei auf ca. 78 Mio. EUR geschätzt (über Projektlaufzeiten von drei bis fünf Jahren), die Förderungssumme für diese Projekte beträgt 6,4 Mio. EUR. Mit Stichtag 31.12.2008 hatten diese zehn im Jahr 2008 genehmigten Unternehmen 46 Beschäftigte (Vollzeitäquivalente-VZÄ) mit einem Frauenanteil von $15 \%$. Am Stichtag 31.12.2009 hat sich dann das Beschäftigungsvolumen (VZÄ) dieser Unternehmen bereits auf 89 (Frauenanteil $14 \%$ ) erhöht. Sieben Unternehmen sind thematisch dem Technologiefeld IKT zuzuordnen, zwei der „Physik“ und eines der Elektrotechnik. Insgesamt konnten bereits 4,71 Mio. EUR an privaten Mitteln für Zusatz- und Ausfinanzierung vertraglich festgemacht werden. Geographisch sind die Unternehmen dieser Kohorte in Wien (vier Unternehmen), in Oberösterreich (zwei Unternehmen), der Steiermark (zwei Unternehmen) sowie in Kärnten und in Burgenland (jeweils in Unternehmen) lokalisiert.

- Im Jahr 2009 kam es zu 38 Anfragen, wovon sieben Projekte für Seedfinancing genehmigt wurden. Die Gesamtprojektkosten dieser sieben Projekte werden auf ca. 77,3 Mio. EUR geschätzt, die genehmigte Förderungssumme beträgt 4,5 Mio. EUR. Zum Stichtag 31.12.2009 betrug das VZÄ dieser Unternehmen 22 Beschäftigte (Frauenanteil 14 \%). Vertraglich fixiert werden konnten bereits 1,85 Mio. EUR an privater Weiter- und Ausfinanzierung. Mit vier Unternehmen stellt Wien wiederum den wichtigsten Standort dar, zwei Unternehmen kommen aus Oberösterreich, eines aus der Steiermark. Sechs Unternehmen sind dem Technologiefeld IKT und ein Unternehmen der „Physik“ zuzuordnen.

- Im Jahr 2010 konnten bei 31 Anfragen lediglich drei Projekte mit einer Förderungssumme von 2,1 Mio. EUR genehmigt werden, wofür der kurzfristige Ausfall der Finanzierung durch das BMVIT verantwortlich ist. Die entsprechend qualifizierten Projektanträge mussten daher in das Jahr 2011 verschoben werden. Von den genehmigten Projekten sind zwei Unternehmen dem Technologiefeld Physik zugeordnet, das dritte der IKT.

- Für die gesamte Periode 2007 - 2010 ergeben sich somit in Summe 138 Anfragen, davon 20 genehmigte Projekte und eine gesamte Förderungssumme von 13 Mio. EUR. Die geschätzten Gesamtprojektkosten (bei drei bis fünf Jahren Laufzeit) dieser 20 Seedfinancing-Projekte werden mit 179 Mio. EUR veranschlagt. An privater Weiter- und Ausfinanzierung konnten für diese 20 Projekte bislang ca. 6,72 Mio. EUR vertraglich fixiert werden. 
Abbildung 5: Monetäre Charakteristika der Seedfinancing-Projekte (IKT und Physical Sciences) der Jahre 2007 - 2010

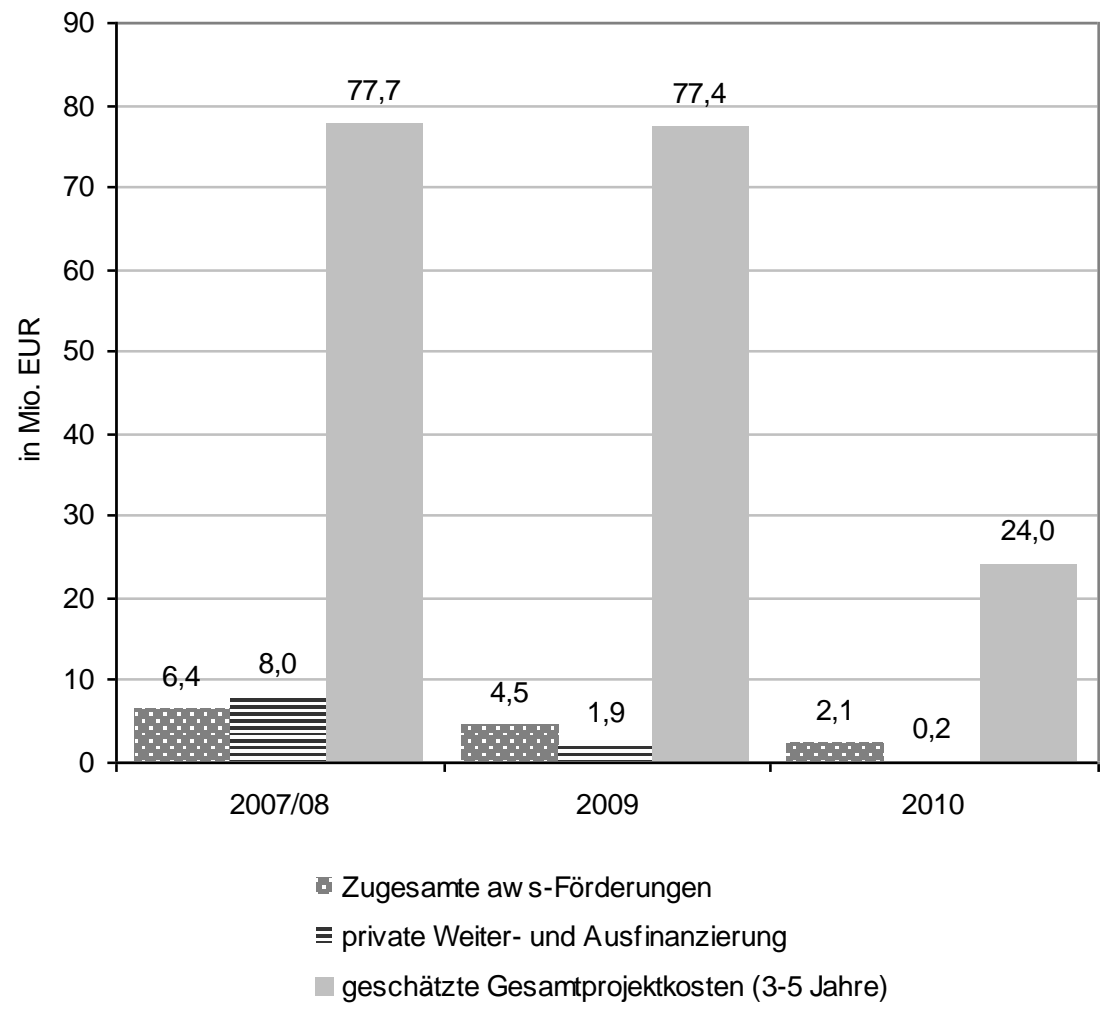

Quelle: aws

\section{Biotechnologie (LISA)}

- Im Technologiefeld Biotechnologie wurden in den Jahren 2008 bis 2010 jeweils 4 Projekte genehmigt (vgl. Abbildung 6), so dass insgesamt im einschlägigen Portfolio der Untersuchungsperiode 12 Biotechnologieprojekte enthalten sind.

- Die geschätzten Gesamtprojektkosten (über Projektlaufzeiten von drei bis fünf Jahren) belaufen sich für diese Projekte auf insgesamt knapp 110 Mio. EUR, dabei entfallen auf die Projekte des Jahres 2010 geschätzte 55,6 Mio. EUR (die Projekte der Jahre 2008 und 2009 werden auf jeweils 27 Mio. EUR geschätzt). Zwischen den einzelnen Projekten zeigen sich nennenswerte Größenunterschiede. Ein Projekt des Jahres 2010 wird allein auf ca. 30,1 Mio. EUR geschätzt, das kleinste Projekt kommt hingegen auf ein Volumen von knapp 2 Mio. EUR.

- Die zugesagten Fördersummen für die Projekte betrugen im Jahr 2008 3,6 Mio. EUR, 2,9 Mio. EUR im Jahr 2009 und schließlich 3,6 Mio. EUR im Jahr 2010, so dass sich insgesamt eine zugesagte Fördersumme für den Berichtszeitraum von 10,05 Mio. EUR ergibt.

- Die vertraglich zugesagten privaten Weiter- und Ausfinanzierungen betrugen im Jahr 2008 6,6 Mio. EUR, im Jahr 2009, 7,6 Mio. EUR und im Jahr 2010 0,9 Mio. EUR. Insgesamt wurde also bereits kurzfristig eine Summe von 15,1 Mio. EUR an zusätzlichen privaten Finanzierungsmitteln induziert.

- Betrachtet man die bisherigen Beschäftigungseffekte (jeweils in VZÄ) des Projektport- 
folios der Untersuchungsperiode, so ergeben sich 19 Beschäftigte im Startjahr 2008, 35 Beschäftigte im Jahr 2009 (bestehend aus den Beschäftigungswachstum auf 25 VZÄ der Unternehmenskohorte der 2008 geförderten Projekte plus 10 VZÄ in den vier neu geförderten Projekten) sowie $62 \mathrm{im}$ Jahr 2010 (32 VZÄ aus der 2008er Kohorte, 19 VZÄ aus der 2009er Kohorte plus 11 VZÄ aus den neuen 2010 geförderten Projekte). Die älteste Kohorte des Projektportfolios (bestehend aus vier Unternehmen, die 2008 gefördert wurden) ist also innerhalb von nur drei Jahren deutlich gewachsen, und zwar von 19 auf 32, was einer prozentuellen Zunahme von $63 \%$ entspricht.

- Die Förderhöhe pro geschaffenen VZÄ beträgt also in der kürzesten Frist (d.h. noch im gleichen Jahr der Förderung) zwischen 190.000 EUR (2008) und 330.000 EUR (2010). Betrachtet man die Förderkosten pro VZÄ der Kohorte aus dem Jahr 2008 gemessen an ihrem Beschäftigungseffekt innerhalb der ersten drei Jahre ergeben sich ca. 110.000 EUR (3,6 Mio. EUR Förderung resultieren in 32 VZÄ drei Jahre später).

- Im Jahr 2010 konnten aws Portfoliofirmen Lizenzverträge mit potentiellen Einnahmen (bei entsprechend erfolgreichem Durchlaufen der definierten Milestones) von ca. 1,5 Mrd. EUR abschließen.

Abbildung 6: Entwicklung von Seedfinancing im Berichtszeitraum
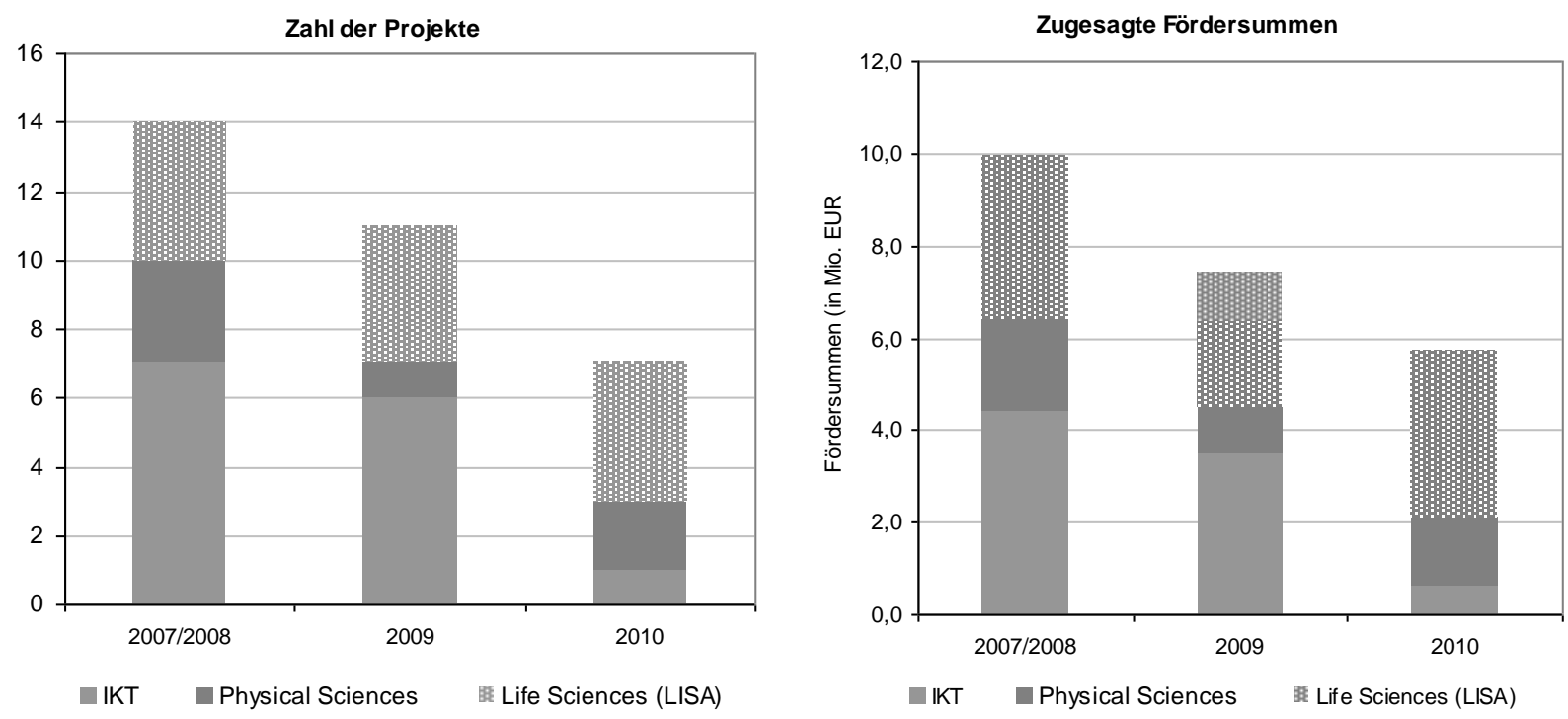

Anm.: Die (interimistisch) genehmigten Projekte des Jahres 2007 mussten aus administrativen Gründen in das Jahr 2008 verschoben werden

Quelle: aws

In Abbildung 7 ist die Entwicklung der Beschäftigung (in VZÄ) der im Berichtszeitraum gegründeten und betreuten Portfoliounternehmen dargestellt, wobei über die einzelnen thematischen Felder für diese Überblicksdarstellung aggregiert wurde. Deutlich ist ein „Verzögerungseffekt" zu erkennen, d.h. die Beschäftigung ist im Gründungsjahr noch vergleichsweise gering, steigt dann aber bereits im darauffolgenden Jahr (z.B. 2009 für die Gründungskohorte 2008 bzw. 2010 für die Gründungskohorte 2009) deutlich an. Dieser starke Anstieg bereits im zweiten Jahr nach der Gründung deutet darauf hin, dass es für Hochtechnologieunternehmen notwendig ist, möglichst rasch die mindestoptimale Unternehmensgröße anzustreben, z.B. um die Fixkosten zu reduzieren, erste Skalenvorteile zu erzielen etc. Dies macht die öffentliche 
Förderung umso kritischer, da dadurch diese Herausforderung des raschen Wachstums umso leichter gemeistert werden kann. Auch wenn die absoluten Zahlen (300 Beschäftigte in VZÄ in allen geförderten Projekten nach einer dreijährigen Berichtsperiode, d.h. mit drei unterschiedlichen Gründungskohorten) auf den ersten Blick gering erscheinen mag, sollte doch berücksichtigt werden, dass hinter dieser Beschäftigungswirkung nur 32 Projekte stehen, wovon sich sieben Projekte wiederum erst im Startjahr (mit der dementsprechend noch geringen Beschäftigungswirkung) befinden.

Abbildung 7: Beschäftigungsentwicklung der Portfoliounternehmen der Gründungsjahrgänge 2008 bis 2010 im Berichtszeitraum

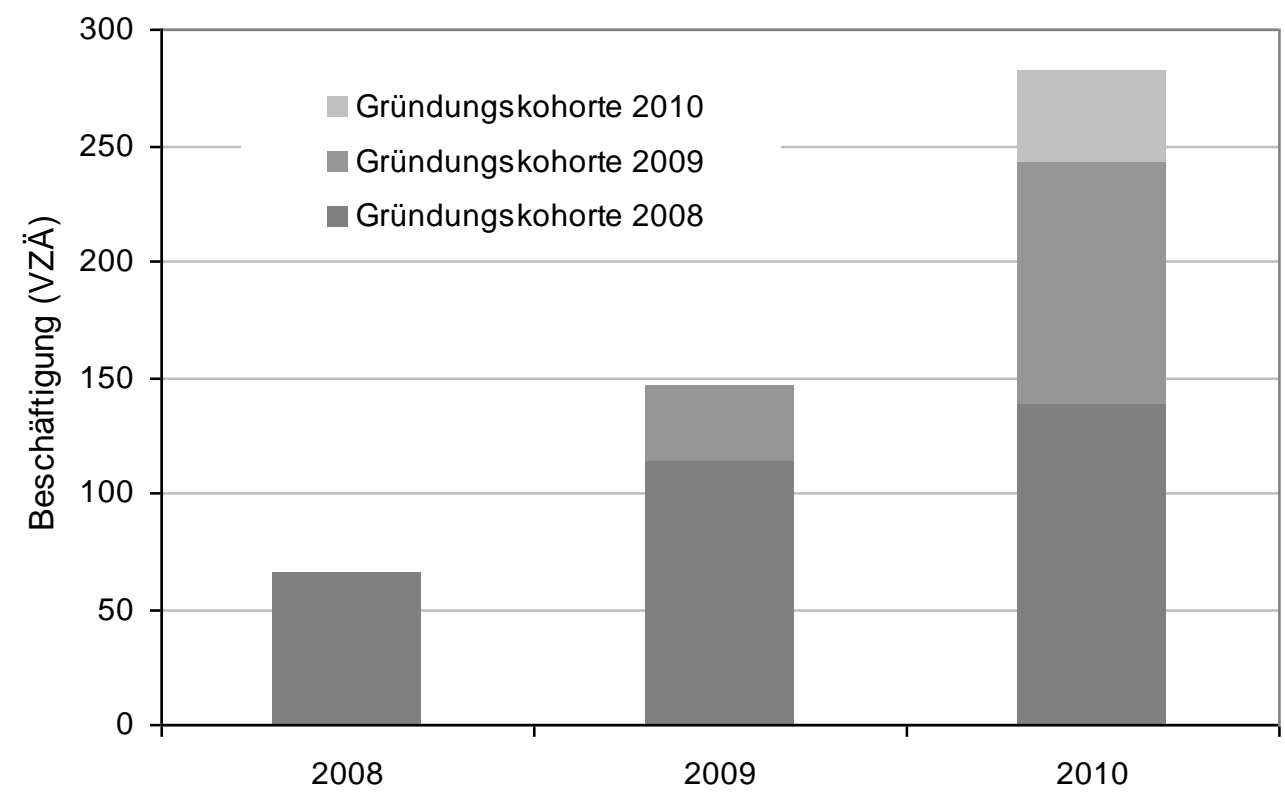

Quelle: aws

\section{Bewertung durch das Evaluierungsteam}

Seedfinancing ist das zentrale Programmmodul innerhalb des JITU-Daches und nimmt somit eine herausragende Stellung für die (hoch-)technologieorientierte Gründungsförderung in Österreich ein. Eine Besonderheit des Programms stellt seine lange bisherige Programmlaufzeit (seit 1989!) dar, was es zu einem der traditionsreichsten Hochtechnologieförderprogramme Österreichs macht. Die bisherige Bilanz von Seedfinancing zeigt dabei deutlich, dass sich - gerade in der Gründungsförderung im Bereich der Hoch- und Spitzentechnologie - dieser „lange Atem“ lohnt. Bislang wurden ca. 200 High-Tech-Gründungen unterstützt, bis Ende 2009 kam es zu etwas mehr als 100 exits, wovon 52 erfolgreiche Abschichtungen waren.

Das Programm Seedfinancing ist bewusst hochselektiv konzipiert. Die in Summe 138 Anfragen im Technologiesegment IKT\&Physical Sciences der Programmlaufzeit 2007 - 2010 führten (bislang) zu 20 genehmigten Projekten. Das Programmmanagement von Seedfinancing fokussiert dabei auf jene kleine Gruppe von Gründungsprojekten im Hoch- und Spitzentechnologiesegment, der besonderes Potential für eine spätere Beteiligung am privaten VC-Markt eingeräumt wird. 
Auch das Life Sciences Segment hat sich im Berichtszeitraum weiterhin bewährt. Insgesamt kamen 12 neue Unternehmen in das aws Portfolio hinzu, wobei mit einem öffentlichen Förderungsvolumen von in Summe 10,05 Mio. EUR ein mittelfristiges Gesamtprojektvolumen (d.h. auf Sicht von drei bis fünf Jahren) von geschätzten 109,6 Mio. EUR angestoßen werden konnte und eine zusätzliche private Weiter- und Ausfinanzierung von ca. 15,1 Mio. EUR bereits vertraglich fixiert wurde. $\mathrm{Zu}$ bemerken ist, dass aws Portfoliounternehmen gerade im Jahr 2010 entsprechende Verwertungsverträge mit Pharmakonzernen mit hohen potentiellen zukünftigen Einnahmen abwickeln konnten. Diese Erfolge sprechen für die hohe Kompetenz bei der Auswahl und Betreuung der Projekte.

Diese Konzeption von Seedfinancing, nämlich die geförderten Unternehmen für den privaten VC-Markt „fit“ zu machen, erhöht einerseits die „Hebelwirkung“5 des Programms, indem die öffentlichen Mittel bei erfolgreicher Investorenakquisition mit privaten VC-Kapital u.U. vervielfacht werden, macht das Programm aber andererseits auch abhängig von der Entwicklung des privaten VC-Marktes in Österreich. Durch die strenge Auswahl von Projekten stellt das Programm aber auch qualitativ interessante, potentielle Investmentziele für den privaten VCMarkt zur Verfügung, der tendenziell aufgrund unterschiedlicher Marktversagensmomente die Frühphase generell vernachlässigt. Das Seedfinancing Programm erfüllt somit auch eine wichtige Pipeline-Funktion für den privaten VC-Markt, indem es die Finanzierungslücke in der Frühphase überbrückt und die Portfoliounternehmen in der Anfangsphase (wo sie für den privaten Kapitalmarkt aufgrund von Unsicherheiten und hohem Risiko noch von entsprechend geringer Attraktivität sind) erfolgreich betreut und sie so für den privaten VC-Markt „fit" macht.

Die Zwischenevaluierung der aws-Technologieprogramme aus dem Jahr 2006 (Jörg et al., 2006) hatte als eine der wesentlichen Empfehlungen die Clusterung von Einzelprogrammen vorgeschlagen. Mit dem Inkrafttreten der JITU-Richtlinien und seinen Modulen PreSeed, Seedfinancing und Management auf Zeit wurde im Bereich der Hochtechnologiegründerförderung eine derartige Clusterung erfolgreich vorgenommen und die entsprechenden Programmmodule synergetisch aufeinander abgestimmt. Die drei Module werden mittlerweile von Seiten der aws auch gemeinsam als Gesamtpaket vermarktet.

\subsubsection{Management auf Zeit}

Das Programmmodul MaZ zielt darauf ab, die „Überlebenschance der geförderten Unternehmen durch aktive Hilfestellung und schnelle Problemlösung mittels externer Experten bei sich abzeichnenden Krisen (z.B. Verfehlung von Meilensteinen, Liquiditätsengpässen etc.) zu erhöhen, dabei die Qualifizierung der MitarbeiterInnen des geförderten Unternehmens durch den Know-How-Transfer während des Experteneinsatzes zu verbessern und somit den unternehmerischen Erfolg von innovativen High-Tech-Unternehmen nachhaltig zu steigern. " Management auf Zeit (MaZ) stellt fokussierte, zeitlich befristete Management-Unterstützung zur Verfügung mit dem Ziel, die Erfolgschancen (bzw. Überlebenschancen) von im Seedfinan-

\footnotetext{
${ }^{5}$ Derzeit wird intern von Seiten der aws versucht, diesen Hebel empirisch zu erfassen, wobei die entsprechenden privaten Investitionszusagen der bestehenden Portfoliounternehmen erhoben werden. Für die nach den JITU-Richtlinien geförderten Unternehmen ist diese Datenerfassung bereits „,automatisiert“. Allerdings lässt sich aufgrund der bis dahin geringen Laufzeit der JTU-Richtlinien noch kein endgültiger Hebel errechnen, da VC-Investitionen vielfach erst Jahre nach der Förderung getätigt werden bzw. u.U. auch in mehreren - zeitlich versetzten - Runden. Die aws-internen Berechnungen hinsichtlich der Hebelwirkung von öffentlichem Seedfinancing-Kapital und privatem VC-Kapital werden voraussichtlich im Sommer 2011 vorliegen.
} 
cing-Programm (= Modul 2 des JITU-Programms) geförderten Unternehmen zu erhöhen. Zielgruppe sind also alle High-Tech-Unternehmen, die bereits im Rahmen von Seedfinancing eine Förderung erhalten haben. Somit stellt MaZ eine unterstützende Begleitmaßnahme dar, welche die Effektivität und Performance des Seedfinancing-Programms insgesamt erhöhen bzw. optimieren soll.

Die konkrete Leistung besteht in Form eines nicht-rückzahlbaren Zuschusses bis zu $50 \%$ der Gesamtkosten eines externen Beratungsprojekts (maximal 50.000 EUR Zuschuss). Üblicherweise liegen die Laufzeiten zwischen sechs und neun Monaten, wobei Karenzvertretungen jedoch auch bis zu einem Jahr gefördert werden.

Die Grundidee besteht darin, dass gerade in der Gründungs- und Aufbauphase eines Unternehmens die Managementkapazitäten üblicherweise begrenzt sind. Dies trifft insbesondere im Rahmen einer Hochtechnologiegründung zu, wo der GründerInnen meist auch der technologische Ideengeber und -treiber ist. Außerdem haben gerade High-Tech-GründerInnen mit technisch-naturwissenschaftlichem Kompetenzhintergrund u.U. eine mangelnde betriebswirtschaftliche Ausbildung. Eine eventuell entstehende Kapazitätslücke kann und soll daher gegebenenfalls kurzfristig (und zeitlich begrenzt) durch den Einsatz externer ExpertInnen geschlossen werden.

Die Indikatoren zur Überprüfung der MaZ-Ziele sind laut „Anbot für die Fortsetzung der Technologieprogramme für die Jahre 2007 bis 2009“" wie folgt definiert:

- Die Auswertung soll nach Outputindikatoren wie die Anzahl der geförderten Firmen erfolgen, Kosten des Experteneinsatzes bezogen auf Sicherung des Investments der öffentlichen Hand und der Zielerreichung des Einsatzes. Ein jährlicher Workshop mit GründerInnen/BeraterInnen soll zeitnah die Lernerfahrungen sichern und sinnvolle Weiterentwicklungsoptionen aufzeigen.

Das Programm wurde zwar bereits im Jahr 2008 etabliert, hatte seinen operativen Programmstart aber erst im Dezember 2008, so dass erst das Jahr 2009 das erste Jahr mit ,durchlaufendem“ operativem Betrieb war ${ }^{6}$. Im gleichen Jahr wurden auch alle bereits bestehenden Portfolio-Unternehmen innerhalb des Seedfinancing Programms über das neue Angebot im Rahmen von MaZ informiert sowie das MaZ-Modul innerhalb des JITU-Programms bei einschlägigen Marketingaktivitäten adressiert.

In Summe gab es im Jahr 200913 Anfragen, wovon zehn als MaZ-Projekt für die Förderung empfohlen wurde. Die geförderten Gesamtprojektkosten beliefen sich 2009 auf knapp 700.000 EUR bzw. auf eine Zuschusshöhe von 338.000 EUR (= ca. 48 \%, was annähernd der maximal vorgesehen Zuschusshöhe von $50 \%$ entspricht).

Die Programmentwicklung für das Jahr 2010 stellt sich wie folgt dar: Insgesamt wurden im Jahr 2010 drei Gremiumsitzungen abgehalten, bei denen in Summe sechs Projekte dem BMWFJ vorgeschlagen bzw. empfohlen wurden, wovon bislang drei genehmigt wurden (ein vorgeschlagenes/empfohlenes Projekt aus der Gremiumsitzung von 10. Februar 2010 wurde abgelehnt). Zwei vorgeschlagene/empfohlene Projekte der Gremiumsitzung vom 5. Oktober 2010 warten noch auf eine Entscheidung.

\section{Bewertung durch das Evaluationsteam}

\footnotetext{
${ }^{6}$ Am 9. Dezember 2008 wurde das erste MaZ-Projekt dem Bewertungsgremium zum Beschluss vorgelegt und bewilligt.
} 
Grundsätzlich wird in der einschlägigen Literatur betont, dass Gründungen (bzw. junge Unternehmen) im Hochtechnologiebereich neben Finanzierungsengpässen auch Defizite im Bereich von Managementressourcen aufweisen, die durch geringe personellen Ressourcen und dem damit verbundenen geringen Grad an interner Arbeitsteilung (der/die GründerIn bzw. das Gründungsteam ist für „,alles“ verantwortlich bzw. muss sich um ,alles“ selbst kümmern) gekennzeichnet sind. Gerade in kritischen Phasen (z.B. Verzögerungen in der Projektentwicklung; gescheiterte Lizenzverhandlungen, kurzfristiger Ausfall von SchlüsselmitarbeiterInnen etc.) können derartige Ressourcenknappheiten für ein neugegründetes Unternehmen - trotz prinzipiell vorhandener Marktchancen bzw. ersten Markterfolgen - eine existentielle Bedrohung darstellen.

Das Programm Management auf Zeit adressiert diese Problematik direkt und bietet damit die Möglichkeit, kurzfristig und für einen definierten Zeitraum Engpässe zu überwinden. Die Anknüpfung an Seedfinancing (sowohl für die bereits bestehenden Portfolio-Unternehmen als auch für neue Förderungswerber) erscheint aus zwei Überlegungen heraus besonders sinnvoll:

(a) Einerseits wird dadurch die Zielgruppe klar definiert und abgegrenzt - was insbesondere in der Aufbauphase des Programms von großem Vorteil ist - auf eine „enge“ Gruppe von avancierten Hochtechnologieunternehmen (was ja gleichsam automatisch durch den Auswahlprozess mit den entsprechend hohen Anforderungen des Seedfinancing-Programms garantiert wird).

(b) Anderseits wird durch das ergänzende Modul MaZ das Programmportfolio für die JITU-Förderung zusätzlich attraktiviert und optimiert. Dem SeedfinancingProgramm wird also mit MaZ eine Ergänzung gegeben, die speziell auf die Probleme der Seedfinancing-Zielgruppe ,maßgeschneidert“ ist und gegebenenfalls in Anspruch genommen werden kann.

Die bisherigen Erfahrungen und Daten (Anzahl der Projekte etc.) lassen auf einen reibungslosen und erfolgreichen Programmstart hindeuten. Aufgrund der geringen Laufzeit (knapp zwei Jahre) und den dadurch erst sehr begrenzt vorliegenden Erfahrungswerten kann im Rahmen dieser Zwischenevaluierung aber keine abschließende Einschätzung gegeben werden.

\subsection{5 i2 - Die Börse für Business Angels}

Das Programm i2 - die Börse für Business Angels - geht zurück bis ins Jahr 1997, als in der Innovationsagentur im Auftrag des damaligen BMWA mit dem Aufbau eines Business Angel Networks begonnen wurde. Österreich war mit dieser Initiative eines der führenden europäischen Länder. I2 ist Mitglied der EBAN (European Trade Association for Business Angels, Seed Funds, and other Early Stage Market Players).

Die Grundidee von i2 besteht darin, private Investoren (Business Angels) an technologieorientierte Unternehmen (insbesondere in deren Frühphase) mit entsprechendem Kapitalbedarf für den Unternehmensaufbau bzw. -ausbau zu vermitteln. Im Wesentlichen geht es also um den Abbau von Informationsasymmetrien, die den privaten (Risiko-)Kapitalmarkt notorisch prägen. Die Ziele sind somit

- dem Marktversagen in der Frühphasenfinanzierung entgegenzuwirken

- die Vermittlung von privaten Risikokapital von Investoren (Business Angels) an Technologieunternehmen zur Schließung ihrer Eigenkapitallücke (equity gap) 
- Angebot einer offenen und effizienten Plattform für alle Beteiligten (Unternehmen mit Kapital- und Unterstützungsbedarf, Investoren, Netzwerkpartner)

- Ansprechen von möglichst vielen Playern durch unterschiedliche Awareness- und Vermarktungsmaßnahmen.

Die Indikatoren zur Prüfung der Zielerreichung sind laut „Anbot für die Fortsetzung der Technologieprogramme für die Jahre 2007 bis 2009“ wie folgt definiert:

- Anzahl von Investoren unter Vertrag

- Anzahl von Unternehmen, die über i2 Kapital suchen

- Anzahl von vermittelten Beteiligungsprojekten (dv. mit Vertragsabschluss)

- Einnahmen (Teilnahmegebühren, Vermittlungsprovisionen)

Im Rahmen von i2 werden also Matching-Dienstleistungen zwischen kapitalsuchenden Unternehmen einerseits und potentiell geeigneten Investoren andererseits zur Verfügung gestellt, wobei die aws als neutraler "Broker" auftritt, der die kapitalsuchenden Unternehmen entsprechend bewertet und aus dem Pool der Investoren jene versucht heraus zu selektieren, die für das entsprechende Unternehmen / Projekt inhaltlich geeignet erscheinen. Die Investoren (Business Angels) stellen bei erfolgreicher Vermittlung dem Unternehmen dann neben Kapital auch einschlägiges Know-How sowie entsprechende Kontakte zur Verfügung.

Das Programm steht grundsätzlich Projekten / Unternehmen aus allen Branchen / Technologiefeldern offen, schwerpunktmäßig sollen jedoch Unternehmen in frühen Phasen adressiert werden. Kriterien für die Aufnahme sind das Vorhandensein einer neuartigen Geschäftsidee mit großem Marktpotential und hohe Wachstumschancen, klare Alleinstellungsmerkmal, ein erkennbarer Wettbewerbsvorsprung sowie ein qualifiziertes Managementteam im Unternehmen. Die Kosten für das Vermittlungsservice betragen für das Unternehmen 390 EUR pro sechs Monate, wobei für eine tatsächlich erfolgreiche Vermittlung zusätzlich eine Vermittlungsprovision in Hohe von 1 bis $2 \%$ der Transaktionssumme verrechnet wird.

Für die interessierten Investoren betragen die Kosten der Aufnahme in das Business Angels Network 90 EUR pro Jahr, strategischen und institutionellen Investoren werden 390 EUR pro Jahr verrechnet.

Die Entwicklung der Projektanfragen (= Unternehmen, die um Vermittlung anfragen) sowie der dann tatsächlich versendeten Projekte (= Vermittlungsversuche nach Vorabrecherche bezüglich inhaltlicher Tauglichkeit etc.) ist in Abbildung 8 dargestellt. Die Anzahl der einlangenden Anfragen (= Dealflow) zeigt sich dabei über den Berichtszeitraum im Wesentlichen stabil und liegt bei jährlich zwischen 300 und knapp 400 Anfragen. Stark zurückgegangen ist hingegen die Anzahl der an die Investoren versendeten Projekte, und zwar von über 50 (in den Jahren 2007 und 2008) auf nunmehr $30 \mathrm{im}$ Jahr 2010. Dies ist allerdings eine von Seiten des i2-Programmmanagements bewusst verfolgte Strategie, um die Qualität der ausgewählten Projekte zu verbessern und somit die Vermittlungswahrscheinlichkeit zu erhöhen. D.h. die Prüfung der potentiellen Tauglichkeit für den Einstieg eines Business Angels wurde während des Berichtszeitraums nach immer strengeren Kriterien getätigt. Insgesamt wurden im Berichtszeitraum (2007 - 2010) 1.407 Vermittlungsanfragen bearbeitetet und bewertet, wovon dann in Summe $177(=12,6 \%)$ an potentielle Investoren (Business Angels) weitergeleitet wurden. 
Abbildung 8: Projektanfragen und versendete Projekte

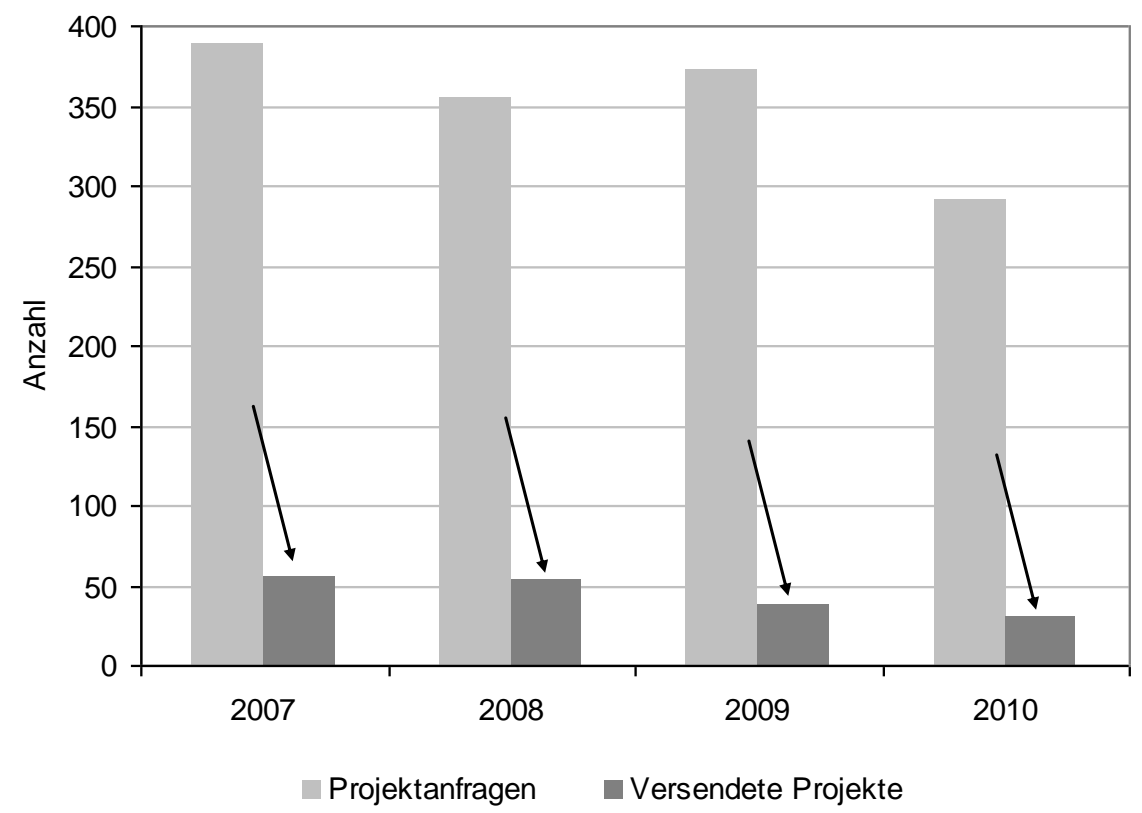

Quelle: aws; eigene Darstellung

Die Vermittlungsbilanz, d.h. die tatsächlich zustande gekommenen Kapitalbeteiligungen und die damit verbundenen Kapitalsummen, ist in Abbildung 9 dargestellt7. Von den 177 an potentielle Investoren weiter geleiteten Projekten führten insgesamt $18 \mathrm{zu}$ tatsächlich erfolgten Beteiligungen, die zusammen ein Investitionsvolumen von 2,04 Mio. EUR ausmachen. Dies entspricht einer Vermittlungsquote von 10,2 \%. Damit ist im gegenwärtigen Berichtszeitraum die Vermittlungsquote gegenüber dem Zeitraum 2002 - 2006 (7,3 \%) merkbar angestiegen. Die Evaluierung aus dem Jahr 2006 ( Jörg et al. 2006) gibt für den damaligen Berichtszeitraum (2002-2005) eine Vermittlungsquote von 6,5\% an. Dieser Wert wurde mit den Zahlen von 2006 ergänzt, so dass sich für den Zeitraum 2002 - 2006 eine damalige Vermittlungsquote von 7,3 \% ergibt. In der ersten Berichtsperiode (1997 - 2001) lag die Vermittlungsquote bei ca. $4 \%$. Somit hat sich also die nunmehrige Vermittlungsquote gegenüber den Anfangsjahren von i2 mehr als verdoppelt! Dies ist umso bemerkenswerter, als die globale Finanz- und Wirtschaftskrise ab 2007/08 zu einem drastischen Einbruch der VC-Märkte geführt ${ }^{8}$ und sich somit das Umfeld für erfolgreiche Vermittlung deutlich erschwert hat.

Deutlich zurückgegangen ist vor allem das Volumen der Vermittlungen. Noch in den Jahren 2005 und 2006 wurden ca. 1 Mio. EUR bzw. 1,6 Mio. EUR Investitionsvolumen vermittelt. Dagegen schwankt das Volumen der vermittelten Beteiligungen in der Berichtsperiode 2007 2010 zwischen 332.000 EUR und 600.000 EUR. Die Höhe des durchschnittlichen Investitionsvolumens pro vermittelter Beteiligung lag im Berichtszeitraum 2007 - 2010 bei 113.000 EUR gegenüber durchschnittlich 224.000 in den Jahren 2002 - 2006.

\footnotetext{
${ }^{7}$ Der in der Abbildung 9 dargestellt Zeitraum erfasst auch die Jahre 2005 und 2006, um das Volumen vor Einsetzen der globalen Finanz- und Wirtschaftskrise aufzuzeigen.

8 Ein ähnliches Einbrechen der VC-Märkte war auch in den Jahren 2001/2002 nach dem Platzen der New Economy Blase zu beobachten.
} 
Nach Angaben der aws erreichen die erfolgreichen Vermittlungen von i2 in den letzten Jahren einen Anteil von immerhin 10-20 \% aller Frühphasenbeteiligungen in Österreich. ${ }^{9}$

Abbildung 9: Vermittlungsbilanz von i2 2005 - 2010

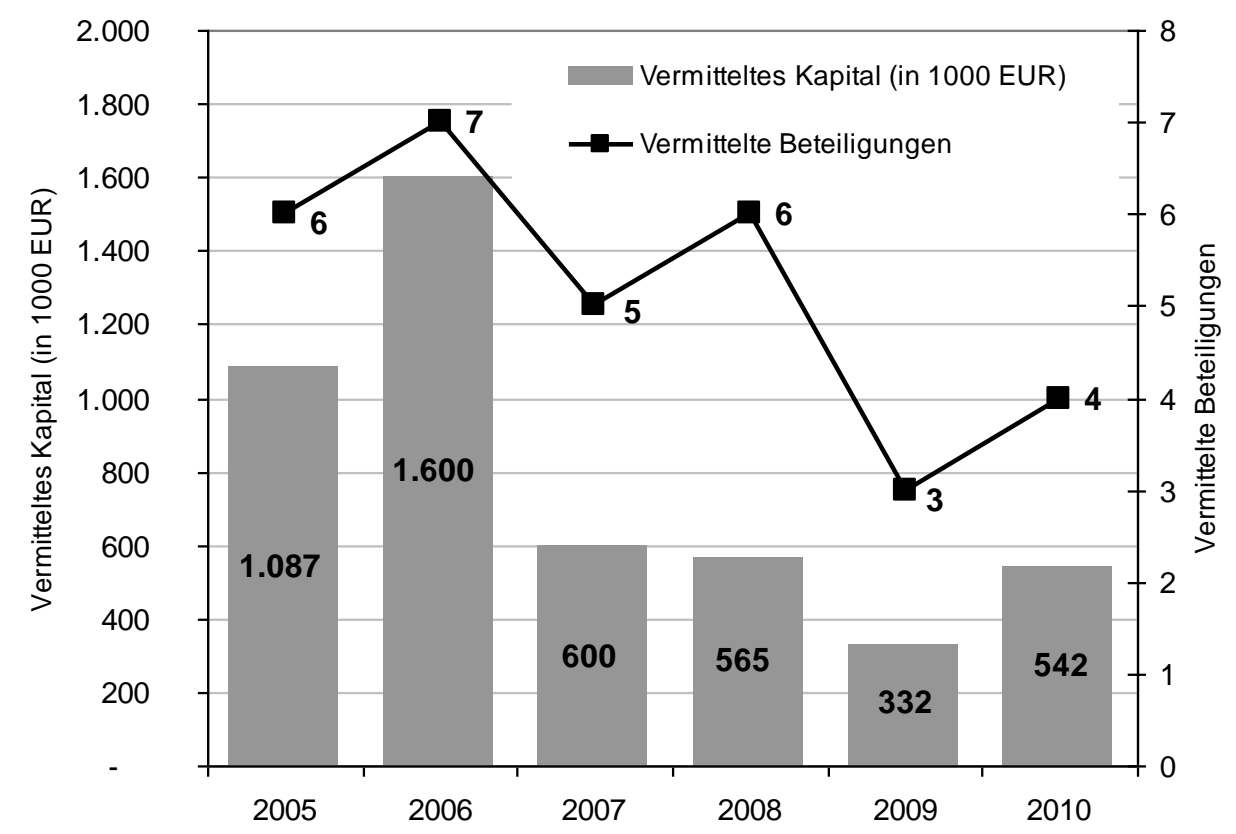

Anm.: Die Zeitachse beginnt bei 2005, um den Effekt der Finanz- und Wirtschaftskrise demonstrieren zu können

Quelle: aws; eigene Darstellung

\section{Bewertung durch das Evaluationsteam}

Das Programm adressiert ein eindeutiges Marktversagen, nämlich die Informationsasymmetrien am privaten Risikokapitalmarkt, die ein ,effizientes“ Zueinanderfinden von Kapitalsuchenden und potentiell interessierten Investoren behindern. Als „,neutraler“ Vermittler (Broker) trägt i2 mit dazu bei, einen „Marktplatz“ für kapitalsuchende Unternehmen einerseits und potentiellen Business Angels andererseits zu schaffen. Neben der reinen Vermittlungstätigkeit (d.h. dem „Matchen“ von Projekten zu kapitalgebenden Business Angels) verbessert i2 diesen Marktplatz zusätzlich durch Bewertung und Vorselektion der Projekte. Als neutrale Instanz mit einschlägiger wirtschaftlicher und technologischer Kompetenz stellen diese Aktivitäten der aws einen beträchtlichen Mehrwert für alle Beteiligten dar, da einerseits die kapitalsuchenden Unternehmen Rückmeldung über die Qualität (im Sinne von VC-Markttauglichkeit) ihrer Projekte erhalten und andererseits die potentiellen Investoren mit einer qualifizierten Auswahl von Projekten konfrontiert werden, die ihre Suchkosten erheblich verringern kann (gegenüber singulärem, selbständigen Suchen nach interessanten Projekten durch die Business Angels). Gerade für Österreich als Land mit unterdurchschnittlicher VC-Marktreife (die sich nicht zuletzt in einem extrem geringen Anteil von VC am BIP niederschlägt) ist ein Programm wie i2 von besonderer Bedeutung. Diese Bedeutung sollte nicht allein an der Zahl erfolgreicher Vermittlungen gemessen werden, sondern zusätzlich auch in seinem Beitrag zur Entwicklung des VC-Marktes allgemein. Der durchaus bemerkenswerte Dealflow des Pro-

\footnotetext{
${ }^{9}$ Unter Heranziehung von Marktdaten der EVCA (European Private Equity and Venture Capital Association) über das VC-Aufkommen in Österreich.
} 
gramms (immerhin zwischen 300 und 400 Projektanfragen pro Jahr) zeigt, dass die Nachfrage nach Venture Capital in Österreich durch Unternehmen mit entsprechendem Kapitalbedarf durchaus gegeben ist. Allein durch die Tatsache der Existenz des Programms erhöht sich somit nicht nur die Awareness für VC in Österreich, sondern es werden auch laufend potentielle Projekte generiert. Gleichzeitig nimmt auch die Zahl der im Business-Angel-Pool vorhandenen Investoren laufend zu und betrug im Jahr 2010 ca. $120^{10}$.

$\mathrm{Zu}$ berücksichtigen ist weiters, dass im Lauf der Berichtsperiode durch die globale Finanzund Wirtschaftskrise der VC-Markt weltweit eingebrochen ist und sich die Risikoakzeptanz von Investoren erheblich verringert hat. Insofern ist es durchaus bemerkenswert, dass - selbst im kritischen Krisenjahr 2009 - immerhin drei erfolgreiche Vermittlungsprojekte abgeschlossen werden konnte. Generell deutet die beachtliche Erhöhung der Vermittlungsquote (gegenüber der Startperiode von i2 mehr als Verdopplung, plus $40 \%$ gegenüber der Vorperiode 2001-2006) auf eine erfolgreiche Weiterentwicklung von i2 und ein professionelles Ausfüllen der Brokerfunktion hin. Auch die Entscheidung, die einlangenden Projekteinreichungen intensiver zu prüfen bzw. stärker zu selektieren hat sicherlich zu dieser gestiegenen Vermittlungsquote beigetragen. Damit steigt zwar vordergründig der Aufwand aufgrund der restriktiveren Bewertung und Auswahl der Projekte (die notwendigerweise zu einem vermehrten zeitlichen Ressourcenbedarf pro eingereichtem und selektierten Projekt führt), letztlich führt dies aber zu einer erhöhten Effizienz des Gesamtnetzwerks (im Sinne, dass Investoren „,besser passende" Projekte vorgestellt bekommen und sich die Wahrscheinlichkeit einer erfolgreichen Vermittlung erhöht), die für beide Seiten (kapitalsuchende Unternehmen einerseits und Business Angels andererseits) Vorteile bringt.

Insgesamt ist also die Programmentwicklung von i2 als positiv zu bewerten, wobei gleichzeitig anzumerken ist, dass die Erwartungen an ein derartiges Programm nicht zu hoch gestellt werden sollten. ${ }^{11}$ Vor dem Hintergrund der tristen Lage des VC-Marktes in Österreich ist jedoch ein derartiges Programm - auch wenn seine direkten Wirkungen minimal sind - eine wichtige Säule.

\subsection{6 tecnet (Markt- und Technologierecherchen)}

tecnet (Markt- und Technologierecherchen) wurde bereits im Jahr 1998 etabliert und ursprünglich (bis zur Fusion 2003) von der damaligen Innovationsagentur operativ abgewickelt. Es handelt sich dabei um ein Programm, das Gründungen im High-Tech Bereich sowie technologieorientierten KMU generell Marktrecherchen, und -analysen sowie Technologie- und Unternehmensrecherchen zur Unterstützung von wichtigen Unternehmensentscheidungen (z.B. Finanzierungs-, Expansion oder Produktentwicklungsentscheidungen) liefern soll. Hintergrund des Programms ist es somit, jungen/ kleinen technologieorientierten Unternehmen (bzw. auch einschlägigen GründerInnen) den Informationszugang so zu erleichtern, dass die generell bestehenden Informationsdefizite gegenüber Großunternehmen zumindest in der Tendenz abgebaut werden. Neben der eigentlichen Zielgruppe (GründerInnen, junge kleine Unternehmen) steht das Rechercheangebot auch mittleren und großen Unternehmen sowie

\footnotetext{
${ }^{10}$ Im Jänner 2010 lag die Zahl bei 118 und im Juli 2010 bei 121, derzeit (April 2011) beträgt sie 127.

${ }^{11}$ Diese Einschätzung wurde bereits von Jörg et al. (2006) - und zwar den gegenüber den Erwartungen an das Potential der europäischen Landschaft von Businss Angel Netzwerken generell - vorgenommen.
} 
Investoren bzw. potentiellen Kapitalgebern offen, wobei hier marktgerechte Preise verrechnet werden (siehe Ausführungen weiter unten).

Die konkreten Zieldefinitionen des Programms lauten:

- Vermittlung von Markt- und Technologieinformation zur Untermauerung von Businessplänen und Vermeidung von Fehlinvestitionen

- Erleichterung von Finanzierungsentscheidungen von Förderungsgebern um Fehlinvestitionen zu vermeiden

- Schaffung von Zugang zu (Patent-)Datenbanken und Bereitstellung von RechercheKnow-How und -kapazitäten (Verringerung des Informationsdefizits von KMUs gegenüber Großunternehmen)

- Unterstützung strategischer Entscheidungen bei der Produktentwicklung und -vermarktung von neuen Technologien

- Markt- und Technologieinformationen so verfügbar zu machen, dass sie von Unternehmen und Kapitalgebern sinnvoll genutzt werden können und Unternehmen wichtige Ressourcen (Zeit, Geld und Personal) sparen.

Im Evaluierungszeitraum kam es zu einer signifikanten Änderung der Preisgestaltung für die Rechercheprojekte dergestalt, dass ein differenziertes System der in Rechnung gestellten Stundensätze eingeführt wurde. GründerInnen (bzw. Kleinunternehmen bis zu einem Alter von sechs Jahren) zahlen seit 2008 einen reduzierten Stundensatz von 45 EUR, Kleinunternehmen mit einem Alter von über sechs Jahren zahlen 90 EUR und alle anderen Anfrager (Großunternehmen, Kapitalgeber/Investoren) zahlen 130 EUR. Die wesentliche Motivation für die Einführung dieses differenzierten Preissystem war die weitere Attraktivierung des Angebots für die Kernzielgruppe (GründerInnen, junge Kleinunternehmen), die nun zu einem stark subventionierten Preis von deutlich reduzierten Projektkosten der Rechercheaufträge (übliche Rechercheaufträge umfassen ca. 20 - 40 Stunden) profitieren. Gleichzeitig wird durch die marktgerechte Preisgestaltung für Großunternehmen bzw. Kapitalgeber/Investoren eine ungerechtfertigte Marktverzerrung hintangestellt. Ein derart differenziertes Preissystem wurde bereits in der Zwischenevaluierung der aws-Technologieprogramme (Jörg et al. 2006) angeregt und somit in der darauffolgenden Programmperiode auch erfolgreich umgesetzt.

tecnet war ursprünglich durch geringe personelle Ressourcen geprägt (eine TechnologieExpertin und ein halbe Bürokraft zur organisatorischen Unterstützung) und wurde dann aber ab 2005 sukzessive ausgebaut. Derzeit sind fünf TechnologieexpertInnen als tecnetProjektbetreuerIn tätig, wobei sich die ExpertInnen jeweils auf unterschiedliche Technologiebzw. Branchenthemen (Maschinenbau, Elektrotechnik/Elektronik, Chemie/Umwelt/Energie, IKT, Life Sciences/Medizintechnik) spezialisieren.

Die Indikatoren zur Prüfung der Zielerreichung sind laut „Anbot für die Fortsetzung der Technologieprogramme für die Jahre 2007 bis 2009“ wie folgt definiert: 
Anzahl der Förderansuchen

- Anzahl der geförderten Projekte (=Rechercheaufträge) aufgeteilt nach Bundesländer und Technolgiefelder

- Einnahmen aus geförderten Projekten

- Nutzung der Rechercheergebnisse bzw. -berichte durch die Förderwerber

- Anzahl der Dienstleistungen innerhalb der aws

- Kundenzufriedenheit

Die Entwicklung des Programms entlang dieser Zielerreichungsindikatoren ist für den Evaluierungszeitraum (2007 - 2010) in Tabelle 5 dargestellt. Die Anzahl der Projektanfragen liegt zwischen 110 und 150 (bzw. 170 hochgerechnet für das Gesamtjahr 2010) und liegt somit etwas über jenem der Jahre 2004/2005 und deutlich über den Vergleichszahlen der Jahre 2000 bis 2003 (damals gab es zwischen 80 und 100 Projektanfragen jährlich).

Deutlich gestiegen ist hingegen die Zahl der durchgeführten Recherchen (interne plus externe), was letztlich auch mit der personellen Aufstockung in Zusammenhang zu bringen ist. Die Zahl der Rechercheprojekte schwankte in den Jahren 2000 bis 2005 zwischen ca. 40 und knapp unter 60 Projekte pro Jahr. Im hier betrachteten Evaluierungszeitraum schwankt die Zahl der Rechercheprojekte um ca. 80 pro Jahr. Die Aufträge innerhalb der aws (z.B. Rechercheaufträge im Zusammenhang von PreSeed-Projekten etc.) sind im Zeitablauf mit ca. 30 Recherchen pro Jahr konstant und letztlich von der jeweiligen Entwicklung dieser anderen aws-Programme abhängig.

Tabelle 5: Übersicht der Entwicklung von tecnet anhand ausgewählter Zielerreichungsindikatoren

\begin{tabular}{lrrrr}
\hline & $\mathbf{2 0 0 7}$ & $\mathbf{2 0 0 8}$ & $\mathbf{2 0 0 9}$ & $\mathbf{2 0 1 0 *}$ \\
\hline $\begin{array}{l}\text { Anzahl der Förderansuchen } \\
\text { Anzahl der geförderten Projekte }\end{array}$ & 113 & 150 & 130 & 170 \\
(= ext. Rechercheaufträge) & 48 & 51 & 48 & 50 \\
Anzahl der Dienstleistungen innerhalb der aws & 29 & 28 & 29 & 30 \\
$\begin{array}{l}\text { Einnahmen aus geförderten Projekten } \\
\text { (= ext. Rechercheaufträge) in EUR }\end{array}$ & 48.307 & 50.210 & 58.682 & 60.000 \\
\hline * Zahlen für 2010 Hochrechnung auf Basis der Entwicklung bis zum 3. Quartal & & \\
Quelle: aws & & &
\end{tabular}

Gestiegen ist auch die Höhe der Einnahmen und zwar insbesondere ab 2009 (im Vergleich zu den Jahren 2007 und 2008), wobei diesbezüglich zu berücksichtigen ist, dass mit dem im Jahr 2008 eingeführten differenzierten Preisgestaltungssystem die Stundensätze für die Hauptzielgruppe (die auch den mit Abstand größten Anteil an allen Rechercheaufträgen stellt) halbiert wurden.

Hinsichtlich der Technologie- / Branchenthemen zeigt sich in der Evaluierungsperiode eine interessante Entwicklung (siehe Abbildung 10). Zwar entfällt auf die fünf vordefinierten Kernthemen aus dem Hoch- bzw. Spitzentechnologiebereich noch immer die Mehrheit aller durchgeführten Auftragsrecherchen, die „Restkategorie“ Sonstiges hat aber deutlich an Anteil gewonnen (und zwar von unter 20\% in den Jahren 2007 und 2008 auf knapp ein Drittel bis ein Viertel). Unter diese heterogene Restkategorie entfallen aber meist ebenfalls durchaus 
technologieintensive Themenfelder. In jüngster Zeit deutlich zugenommen haben z.B. Recherchen im Bereich energieeffizienter Gebäudetechnologien. Daneben finden sich jüngst vermehrt auch Recherchen im Themenbereich Kreativwirtschaft.

Hinsichtlich der Verteilung über die vordefinierten Kernthemen aus der Spitzen- und Hochtechnologie zeigt sich ein einigermaßen stabiles Bild. Lediglich die tendenzielle Anteilsabnahme der Informations- und Kommunikationstechnologie ist auffällig. Die Schwankungen bezüglich der anderen Technologiefelder sind im Wesentlichen zufällig und zeigen keine besonderen Entwicklungstrends bzw. Muster.

Abbildung 10: Auftragsrecherchen nach Technologiefeldern 2007 - 2010

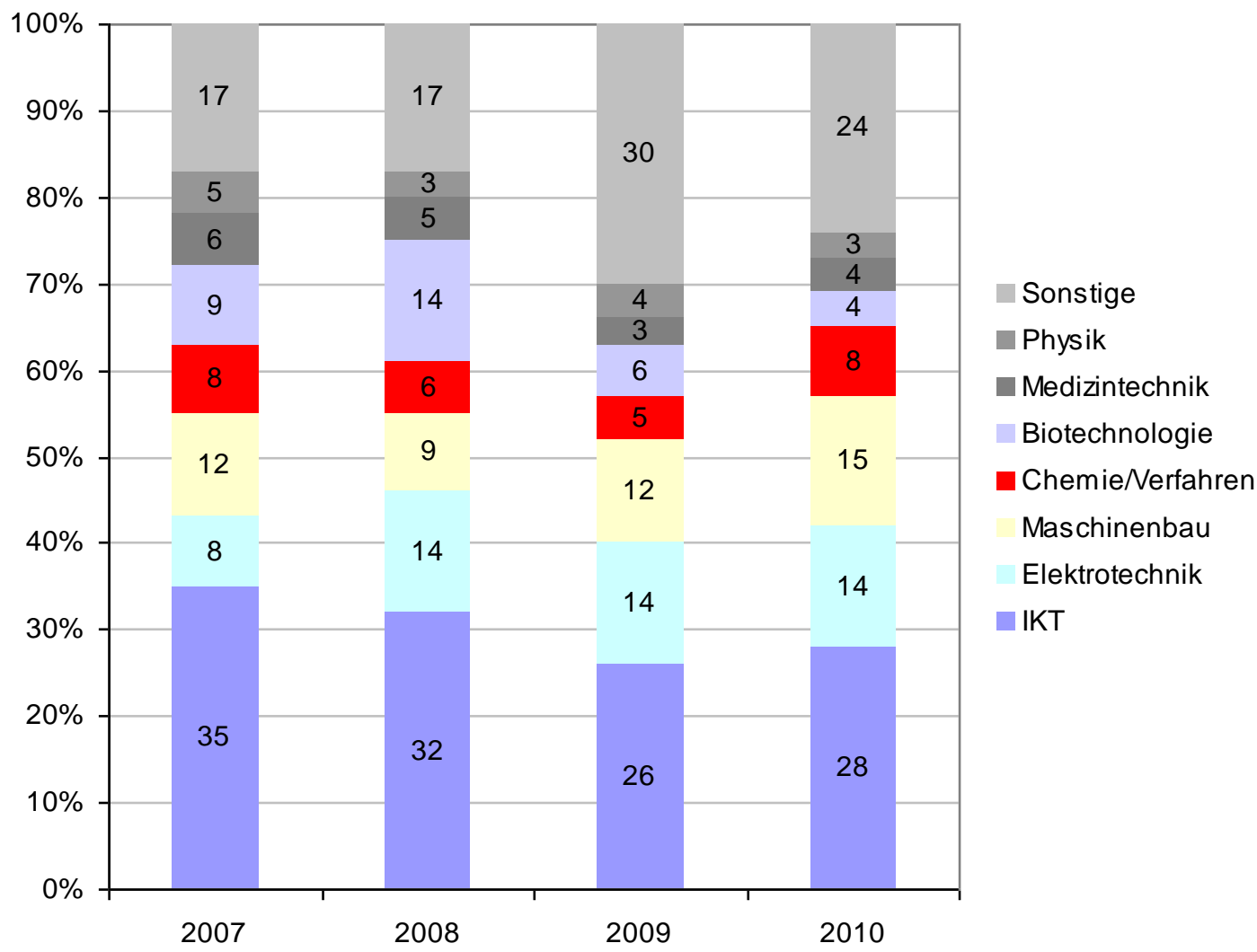

Anmerkung: Der starke Zuwachs der Kategorie „, Sonstiges “ im Jahr 2009/10 gegenüber den Vorjahren ist vor allem den Segmenten Baustoffe/Gebäudetechnologie sowie Kreativwirtschaft/Design geschuldet.

Quelle: aws

Die Verteilung der (externen) Auftragsrecherchen nach Kundentyp zeigt Abbildung 11. Die vorrangige Zielgruppe von tecnet, nämlich Kleinunternehmen (mit einem Alter von unter sechs Jahren) sowie GründerInnen sind auch die mit Abstand wichtigsten Nachfrager nach den Rechercheleistungen des Programms. Auf diese beiden Kundentypen entfallen zusammen etwa 80 bis $90 \%$ aller Anfragen, wobei es im Zeitablauf zu einem deutlichen Anstieg des Anteils von GründerInnen gekommen ist (von $24 \%$ im Jahr 2007 auf 70 \% im Jahr 2010), wobei sich spiegelbildlich dazu der Anteil von etablierten KMUs - von einem hohen Niveau ausgehend - stark verringert hat. Großunternehmen und Investoren/Kapitalgeber spielen nur eine untergeordnete Rolle. Ebenfalls an Gewicht verloren haben Forschungszentren und Universitäten. 
Abbildung 11: Auftragsrecherchen nach Kundentypen 2007 - 2010

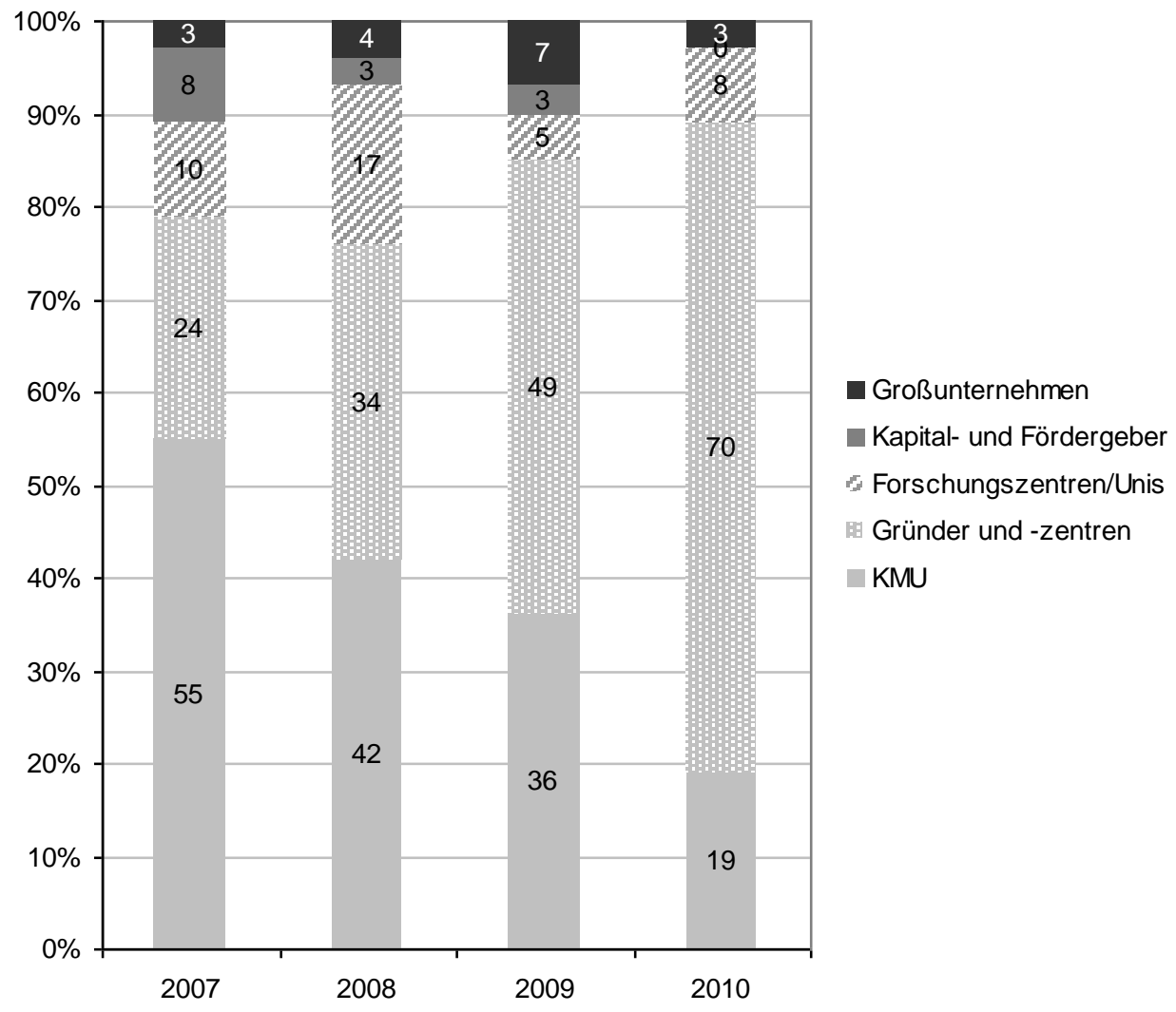

Quelle: aws

Die aws führt hinsichtlich der externen Rechercheaufträge regelmäßig eine Kundenzufriedenheitsanalyse mittels eines online-Fragebogens durch. Die wesentlichen Ergebnisse dieser Kundenbefragung sind in Abbildung 12 zusammengefasst. Die Beurteilung durch die (externen) Nutzer von tecnet ist dabei überwiegend sehr gut und gut, wobei diese positive Einschätzung sowohl hinsichtlich der Bewertung des Nutzens der Marktrecherche für die entsprechende Geschäftstätigkeit (bzw. Gründungsabsicht) als auch für die Qualität der Recherche bzw. der Rechercheergebnisse gilt. Im Zeitablauf hat vor allem die positive Beurteilung des Nutzens der tecnet-Recherchen noch weiter zugenommen. „Schlechte“ Bewertungen (genügend oder gar nicht genügend) finden sich ab 2009 was die Einschätzung des Nutzens betrifft überhaupt nicht mehr. Dies ist einerseits ein Hinweis auf die Wertschätzung der Arbeit der tecnet-ExpertInnen, andererseits deutet dies aber auch darauf hin, dass kleine Unternehmen (bzw. GründerInnen) Kenntnisse bezüglich ihres Marktumfeldes als (zunehmend) wichtig einschätzen. 
Abbildung 12: Ergebnisse der Kundenzufriedenheitsanalyse (2007 - 2010)
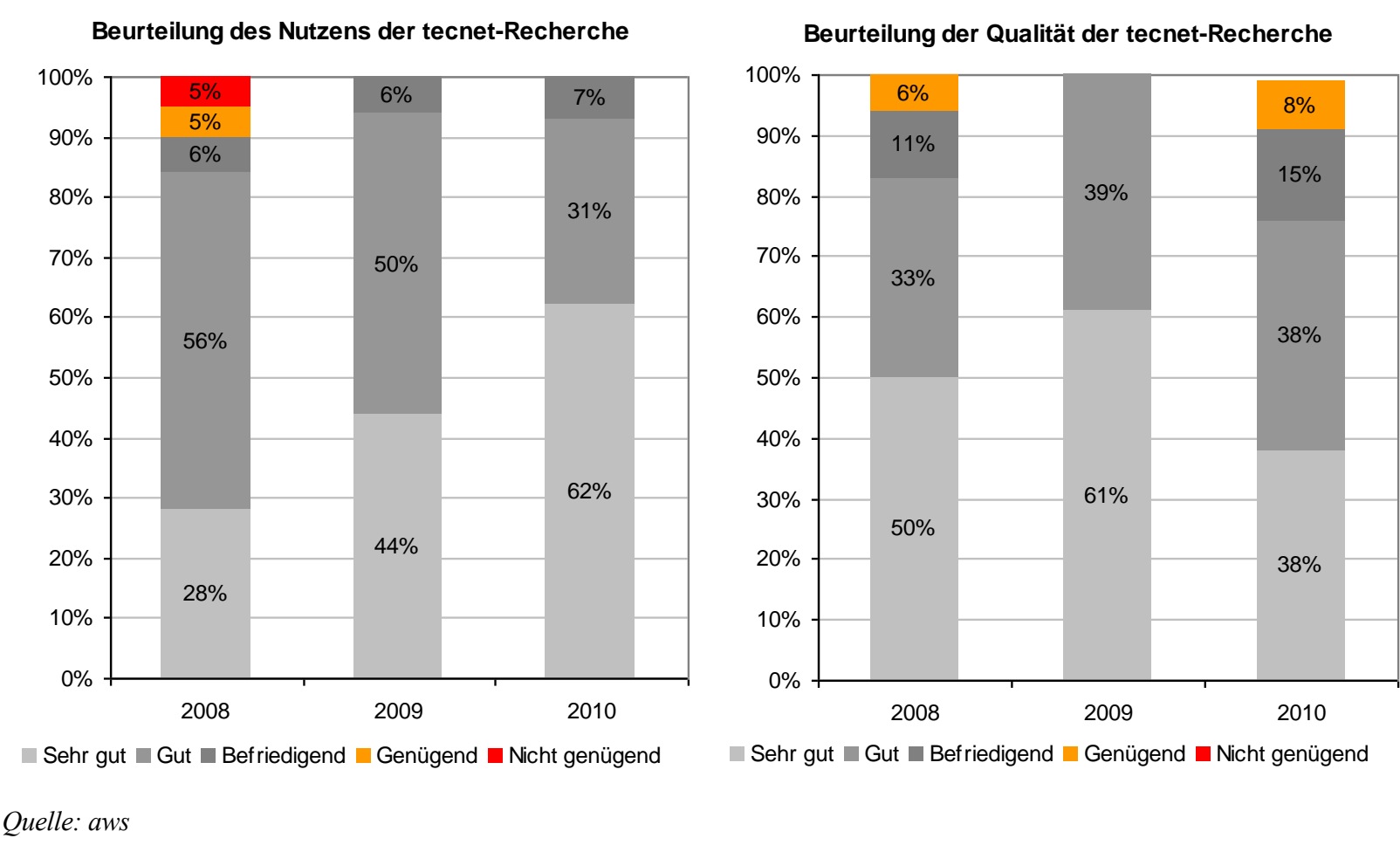

\section{Bewertung durch das Evaluierungsteam}

tecnet adressiert die Barrieren, mit denen Gründungen (bzw. KMUs generell) in Bezug auf die Informationsbeschaffung konfrontiert werden, und versucht das Informationsdefizit dieser Zielgruppe gegenüber Großunternehmen abzubauen. Diese Barrieren für GründerInnen / Kleinunternehmen bestehen sowohl in Bezug auf Ressourcenknappheit (Kosten der Nutzung von einschlägigen Datenbanken, kostenpflichtige Marktstudien etc.) als auch im Informations- und Wissensdefizit bezüglich der Nutzung dieser Informationsquellen. Beide Problembereiche bzw. Barrieren werden von tecnet gezielt adressiert.

Durch tecnet wird GründerInnen bzw. kleinen, jungen Unternehmen ein günstiger (subventionierter) Zugang zu Datenbanken mit entsprechenden markt- und technologierelevanten Informationen (z.B. Marktstudien, Marktdaten etc.) geboten, wobei die Information durch die tecnet-ExpertInnen gemäß den spezifizierten Bedürfnissen der Anfrager aufbereitet wird. GründerInnen profitieren dabei einerseits durch eine empirisch abgestützte Marktpotentialabschätzung ihrer Geschäftsidee und andererseits wird durch die empirische Absicherung auch die Qualität ihres Business Plans erhöht, was wiederum ein wichtiges „Signalling“ in Richtung potentieller Investoren bzw. Kreditgeber darstellt.

Betrachtet man die Verteilung der (Auftrags-)Recherchen nach den entsprechenden Anfragertypen (GründerInnen/KMUs, Großunternehmen, Investoren) lässt sich beobachten, dass die vorrangige Zielgruppe (nämlich Gründungen und junge Kleinunternehmen) eindeutig dominiert und anteilsmäßig im Zeitablauf auch weiter zugenommen hat. Das Programm erreicht somit eindeutig seine intendierte Zielgruppe. Die Bewertungen der Nutzer durch aws eigene Kundenzufriedenheitsanalysen zeigen durchwegs gute bis sehr gute Ergebnisse, was die Professionalität des tecnet-ExpertInnenteams unterstreicht. 
Insgesamt zeigen also die unterschiedlichen Indikatoren über die letzten vier Jahre, dass sich das Programm gemäß den Zielsetzungen entwickelt hat und sich keine unerwünschten Abweichungen feststellen lassen.

Der weitere personelle Ausbau von tecnet hat sich bewährt, insbesondere die Spezialisierung der tecnet-TechnologieexpertInnen auf jeweils bestimmte Technologie- / Branchenthemen garantiert eine professionelle Bearbeitung der Anfragen bzw. Recherchen. Die Zahl der Recherchen (und auch der Einnahmen - trotz neuem, differenzierten Preisgestaltungssystem) ist gegenüber der Programmperiode der ersten Zwischenevaluierung (2006) deutlich gestiegen. Gleichzeitig zeigt aber die zunehmende thematische Heterogenität der Anfragen / Recherchen (z.B. in Richtung Umwelttechnologie i.w.S., aber auch in Richtung Kreativwirtschaft) die Notwendigkeit für (thematische) Flexibilität. Sollten sich - neben den bestehenden fünf vordefinierten Themenbereichen - weitere Themenfelder (die derzeit unter der „Restkategorie“ Sonstiges gesammelt sind) verdichten und sich eine weiter zunehmende Nachfrage nach Recherchen in der Zielgruppe abzeichnen, wäre also eine entsprechende Ressourcenaufstockung anzudenken.

\subsection{PROGRAMME ZUR PATENTVERWERTUNG}

Die Programme zur Patentverwertung umfassen derzeit einen Cluster unterschiedlicher Programme, die einerseits v.a. das IP-Bewusstsein bzw. das Verständnis für das Patentsystem bei kleinen und mittleren Unternehmen adressieren, Internationalisierungsaktivitäten österreichischer KMU durch IP-spezifische Beratungen unterstützen sowie die Vermarktung von Erfindungen / Patenten in Form von Lizenzen von unterschiedlichen Organisationstypen (KMUs, Universitäten etc.) vorantreiben sollen.

Die Indikatoren zur Überprüfung der Ziele des Förderprogramms sind laut Anbot für die Fortsetzung der Technologieprogramme für die Jahre 2007 bis 2009 wie folgt definiert:

- $\quad$ Steigende Patentanmeldungen, wobei besonderer Wert auf starke Patentpositionen gelegt werden sollte (z.B. Triadenpatente, also Patente die in den wesentlichen Wirtschaftsräumen USA, Europa, Asien relevanten Schutz bieten)

- Stärkung des Innovationsstandortes Österreich

- Anzahl der angefragten Projekte

- Anzahl der betreuten Projekte

- Aufteilung sowohl angefragter als auch betreuter Projekte nach Bundesland

- Branchenzugehörigkeit bzw. Technologiefelder

- Stärkung des Bewusstseins für die Notwendigkeit einer Patentierungsstrategie

- $\quad$ Platzierung im Innovation-Scoreboard

\subsection{1 tecma}

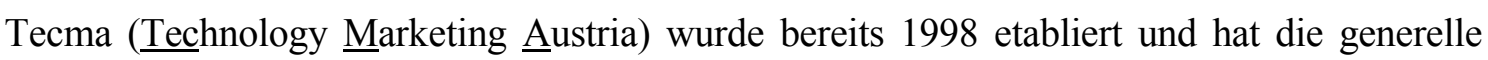
Zielsetzung, österreichischen ErfinderInnn, Unternehmen und WissenschaftlerInnn hinsichtlich der Vermarktung von erfolgversprechenden Innovationen zu unterstützen.

Das Leistungsspektrum von tecma sind zielt im Wesentlichen auf folgende zwei Ebenen ab:

- Begleitende Awareness-Maßnahmen hinsichtlich des Patentsystems im Allgemeinen bzw. zur Nutzung geistiger Eigentumsrechte im Speziellen sowie 
- Unterstützungsleistungen bei der kommerziellen Verwertung von bzw. Vermarktung von österreichischen Erfindungen, wobei die Suche nach Umsetzungspartnern (z.B. LizenznehmerInnen) für Erfindungen von KMUs oder Forschungseinrichtungen (einschließlich Universitäten) im Vordergrund steht.

Tecma bietet dabei ein breites Unterstützungsangebot entlang folgender Ebenen ${ }^{12}$ :

- Potential- und Marktanalysen über nationale und internationale Verwertungsmöglichkeiten für Technologien, Erfindungen, Patente und Nutzungsrechte

- Anmeldung von Patenten und Schutzrechten und Einschaltung von Patentanwälten (die Finanzierung kann über tecma erfolgen)

- Unterstützung bei der optimalen Gestaltung von Technologieangeboten

- Suche nach geeigneten Verwertungspartnern

- Aktive Betreuung des Evaluierungsprozesses bei potentiellen Verwertungspartnern

- Führung der Vertragsverhandlungen im Rahmen des Verwertungsprozesses im Auftrag der Projektnehmer

- Monitoring der Lizenzverträge.

Die Leistungen von tecma werden in zwei Kategorien angeboten, nämlich

(1) exklusive Lizenzvermittlung: Die aws und der Patentinhaber schließen einen Alleinvermittlungsvertrag und die aws sucht aktiv nach einem Lizenznehmer, begleitet und unterstützt die Lizenzverhandlungen und übernimmt gegebenenfalls das Monitoring der Lizenzgebühren. Dadurch erhält der Lizenzgeber einen optimalen Verwertungspartner und wird bei der Suche entlastet.

(2) Lizenzvermittlung mit Patentierung: Hier übernimmt die aws die nationale und internationale Patentanmeldung für die Erfindung und betreut den gesamten Prozess der Lizenzvermittlung und -finanzierung. Die Leistungen und das Risiko im Rahmen der Innovationsvermarktung werden durch eine Erfolgsbeteiligung abgegolten. Die Anteile bestimmen sich nach Leistungsumfang der aws.

Die Zahl der eingegangen Projekte schwankte im Evaluierungszeitraum zwischen 89 (2010) und 114 (jeweils 2007 bzw. 2009). Im Jahr 2008 lag die Zahl der Einreichungen zwischen diesen Extremwerten bei 94. Insgesamt bedeutet dies, dass es in den vier Jahren 2007 - 2010 in Summe 411 Projekteinreichungen gegeben hat.

Geographisch stammen die meisten Einreichung aus Wien, das insgesamt (über die Gesamtperiode) einen Anteil von $37 \%$ aller Projekteinreichungen für sich verbucht. Es folgen Niederösterreich (knapp $17 \%$ ) und Oberösterreich (ca. $11 \%$ ), alle anderen Bundesländer weisen nur noch Anteile von unter $10 \%$ auf (siehe Tabelle 6).

\footnotetext{
${ }^{12}$ aws: Patentverwertung tecma im Detail, http://www.awsg.at/portal/media/174.pdf
} 
Tabelle 6: Verteilung der Einreichungen nach Bundesländern

\begin{tabular}{lrrrrrr}
\hline & $\mathbf{2 0 0 7}$ & $\mathbf{2 0 0 8}$ & $\mathbf{2 0 0 9}$ & $\mathbf{2 0 1 0}$ & $\mathbf{2 0 0 7} \mathbf{- 2 0 1 0}$ & $\begin{array}{c}\text { Anteil 2007 } \\
\mathbf{- 2 0 1 0}\end{array}$ \\
\hline Burgenland & 1 & 2 & 0 & 1 & 4 & 1,0 \\
Kärnten & 12 & 4 & 8 & 8 & 32 & 7,8 \\
Niederösterreich & 28 & 14 & 15 & 12 & 69 & 16,8 \\
Oberösterreich & 15 & 15 & 9 & 5 & 44 & 10,7 \\
Salzburg & 4 & 8 & 13 & 6 & 31 & 7,5 \\
Steiermark & 6 & 9 & 4 & 15 & 34 & 8,3 \\
Tirol & 5 & 7 & 7 & 3 & 22 & 5,4 \\
Vorarlberg & 2 & 0 & 1 & 1 & 4 & 1,0 \\
Wien & 35 & 27 & 55 & 34 & 151 & 36,7 \\
Deutschland & 5 & 4 & 0 & 2 & 11 & 2,7 \\
Sonstiges Ausland & 1 & 4 & 2 & 2 & 9 & 2,2 \\
\hline Gesamtergebnis & 114 & 94 & 114 & 89 & 411 & 100,0 \\
\hline Quelle: aws & & & & & &
\end{tabular}

Am meisten Einreichungen finden sich mit in Summe $237 \mathrm{im}$ Technologiefeld Maschinenbau (Anteil 57,7\%), gefolgt von der Elektrotechnik mit 53 Einreichungen (12,9\%), der Medizintechnik mit 40 Einreichungen (9,7\%) und der Biotechnologie mit 36 Einreichungen $(8,8 \%)$. Der hohe Anteil des Technologiefelds Maschinenbau ist einerseits Ausdruck der branchenmäßigen Konzentration der österreichischen Industrie (bzw. der KMUs) und weist andererseits auch auf die hohe Affinität des Technologiefelds Maschinenbau in Bezug auf die Nutzung patentrechtlicher Schutzmaßnahmen hin (siehe Tabelle 7).

Tabelle 7: Verteilung der Einreichungen nach Technologiefeldern

\begin{tabular}{lrrrrrr}
\hline & $\mathbf{2 0 0 7}$ & $\mathbf{2 0 0 8}$ & $\mathbf{2 0 0 9}$ & $\mathbf{2 0 1 0}$ & $\mathbf{2 0 0 7} \mathbf{- 2 0 1 0}$ & $\begin{array}{c}\text { Anteil } \\
\mathbf{2 0 0 7} \mathbf{- 2 0 1 0}\end{array}$ \\
\hline Biotechnologie & 13 & 8 & 9 & 6 & 36 & 8,8 \\
Chemie Verfahrenstechnik & 5 & 4 & 4 & 7 & 20 & 4,9 \\
Computerprogramm & 0 & 1 & 1 & 0 & 2 & 0,5 \\
Elektrotechnik & 17 & 10 & 14 & 12 & 53 & 12,9 \\
Informationstechnologie & 4 & 3 & 2 & 3 & 12 & 2,9 \\
keine Technologie & 0 & 0 & 2 & 4 & 6 & 1,5 \\
Maschinenbau & 65 & 60 & 64 & 48 & 237 & 57,7 \\
Medizintechnik & 8 & 7 & 16 & 9 & 40 & 9,7 \\
Physik & 2 & 1 & 2 & 0 & 5 & 1,2 \\
\hline Gesamtergebnis & 114 & 94 & 114 & 89 & 411 & 100,0 \\
\hline
\end{tabular}

Quelle: aws

Die meisten Einreichungen kamen mit insgesamt 218 von PrivaterfinderInnn (53\%), gefolgt von Kleinen und Mittleren Unternehmen (KMU) mit insgesamt 153 (bzw. 37,2 \%). Außeruniversitäre Forschungseinrichtungen und Universitäten spielen mit Anteilen an den Einreichungen von ca. $6 \%$ bzw. $3 \%$ nur eine sehr untergeordnete Rolle. Von Fachhochschulen gab es im Berichtszeitraum nur eine einzige Einreichung (siehe Tabelle 8). 
Tabelle 8: Zahl der Einreichungen nach Typus des Einreichers

\begin{tabular}{lrrrrrrr}
\hline & $\mathbf{2 0 0 7}$ & $\mathbf{2 0 0 8}$ & $\mathbf{2 0 0 9}$ & $\mathbf{2 0 1 0}$ & $\mathbf{2 0 0 7} \mathbf{- 2 0 1 0}$ & $\begin{array}{c}\text { Anteil } \\
\mathbf{2 0 0 7} \mathbf{- 2 0 1 0}\end{array}$ \\
\hline KMU & 38 & 35 & 48 & 32 & 153 & 37,2 \\
Privaterfinder & 69 & 54 & 51 & 44 & 218 & 53,0 \\
Universität & 3 & 0 & 4 & 6 & 13 & 3,2 \\
außeruniv. F\&E-Einrichtg. & 4 & 5 & 11 & 6 & 26 & 6,3 \\
Fachhochschule & 0 & 0 & 0 & 1 & 1 & 0,2 \\
\hline Gesamtergebnis & 114 & 94 & 114 & 89 & 411 & 100,0 \\
\hline
\end{tabular}

Quelle: aws

Die Anzahl der tatsächlich zur Betreuung bzw. Vermarktungsbestrebungen übernommenen Projekte zeigt Abbildung 13, wobei jeweils nach dem Kundentypus unterschieden wird. Insgesamt wurden im Berichtszeitraum 28 Projekte zur Vermarktung übernommen, wovon 11 Projekte von EinzelerfinderInnn, 10 von außeruniversitären Einrichtungen und 7 Projekte von KMUs sind; Projekte von Fachhochschulen wurden im Berichtszeitraum nicht in Betreuung übernommen.

Abbildung 13: Zur Vermarktung übernommene Projekte 2007 - 2010

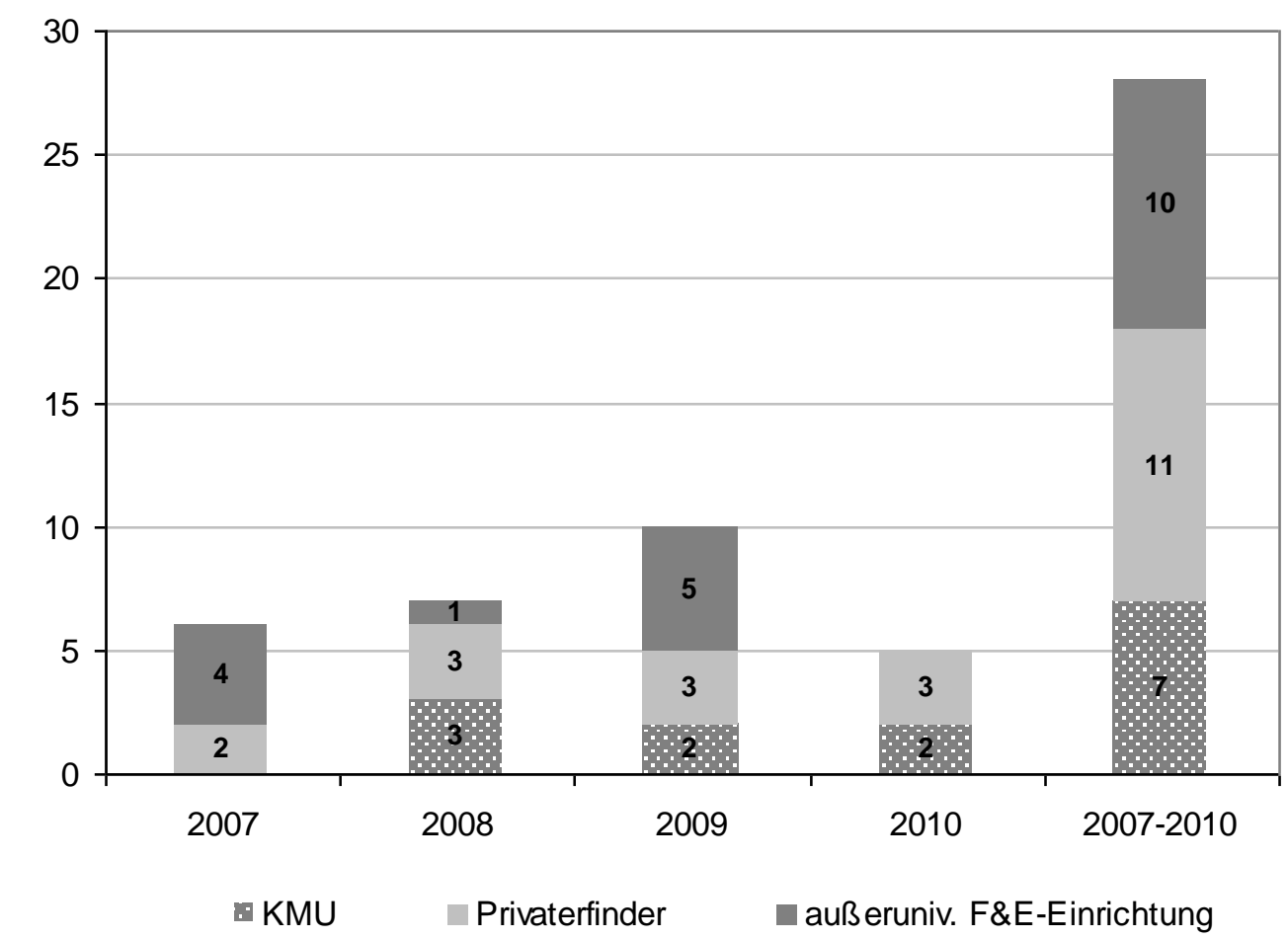

\begin{tabular}{lrrrrrr}
\hline & 2007 & $\mathbf{2 0 0 8}$ & $\mathbf{2 0 0 9}$ & $\mathbf{2 0 1 0}$ & $\mathbf{2 0 0 7 - 2 0 1 0}$ & $\begin{array}{c}\text { Anteil } \\
\mathbf{2 0 0 7 - 2 0 1 0}\end{array}$ \\
\hline KMU & 0 & 3 & 2 & 2 & 7 & 25,0 \\
PrivaterfinderInnen & 2 & 3 & 3 & 3 & 11 & 39,3 \\
außeruniv. F\&E-Einrichtung & 4 & 1 & 5 & 0 & 10 & 35,7 \\
\hline Gesamtergebnis & 6 & 7 & 10 & 5 & 28 & 100,0 \\
\hline
\end{tabular}

Quelle: aws

Beim Vergleich der Projekteinreichungen mit der Zahl der übernommenen Projekte ist zu beachten, dass der Zeitraum zwischen Einreichung und Übernahme durchaus relativ lang 
(mehrere Monate bzw. in Einzelfälle auch länger) sein kann. Eine Berechnung jährlicher „Übernahmequote“ ist daher nicht angebracht (eingereichte Projekte des Jahres 2008 können sich beispielsweise im Jahr 2009 als übernommene Projekte wiederfinden). Für den gesamten Zeitraum 2007 - 2010 ergibt sich somit eine „Übernahmequote“ (bzw. Betreuungsquote) von knapp 7 \% (28 übernommene Projekte von in Summe 411 Projekteinreichungen). ${ }^{13}$ Gegenüber dem Zeitraum 1998 - 2005, in dem eine durchschnittliche Betreuungsquote von ca. 17 \% vorlag (Jörg et al. 2006) bedeutet das einen klaren Rückgang. Laut Angaben der aws ist dieser Rückgang auf eine deutliche Verminderung der Qualität der Projekteinreichungen zurückzuführen. Betrachtet man die Betreuungsquote nach Kundentypen zeigt sich, dass die außeruniversitären Forschungseinrichtungen mit durchschnittlich ca. 39\% (über alle Jahre 2007 2010) die höchste Quote aufweisen. Die Betreuungsquoten bei PrivaterfinderInnen sowie bei KMUs liegen jeweils bei $5 \%$. Eine Aufgliederung nach einzelnen Jahren ist wenig aussagekräftig, da es aufgrund der geringen Fallzahlen zu teils erheblichen Schwankungen (Spannweite von 0 bis $100 \%$ ) kommt.

Im Berichtszeitraum kam es insgesamt zu 48 erfolgreichen Lizenzvergaben. Abbildung 14 stellt die geographische Verteilung der 48 Lizenznehmer dar. Dabei zeigt sich der internationale Charakter der Vermarktungserfolge, der Anteil von Lizenznahmen aus dem Ausland beträgt immerhin ca. $44 \% .{ }^{14}$ Mit 7 Lizenznahmen ist Deutschland dabei der wichtigste Auslandsmarkt für die Patentverwertung, gefolgt von der Schweiz (4 Lizenznahmen) sowie Großbritannien (3) und die USA (3). Auch wenn in Summe mit 27 Fällen der Großteil (56 \%) der Lizenznahmen national erfolgt, verdeutlichen diese Werte dennoch die Notwendigkeit einer internationalen Orientierung der Bemühungen zur Patentverwertung.

\footnotetext{
${ }^{13}$ Auch hier ist zu beachten, dass theoretisch bei der Zahl der übernommenen Projekte auch Einreichungen aus dem Jahr 2006 und davor dabei sein könnten. Es wird aber davon ausgegangen, dass bei Betrachtung über vier Jahre hinweg (2007 bis 2010) dieser Effekt nur noch sehr gering ist.

${ }^{14}$ In den Jahren 1998 bis 2005 betrug der Anteil ausländischer Lizenznehmer 58 \% (Jörg et al. 2006).
} 


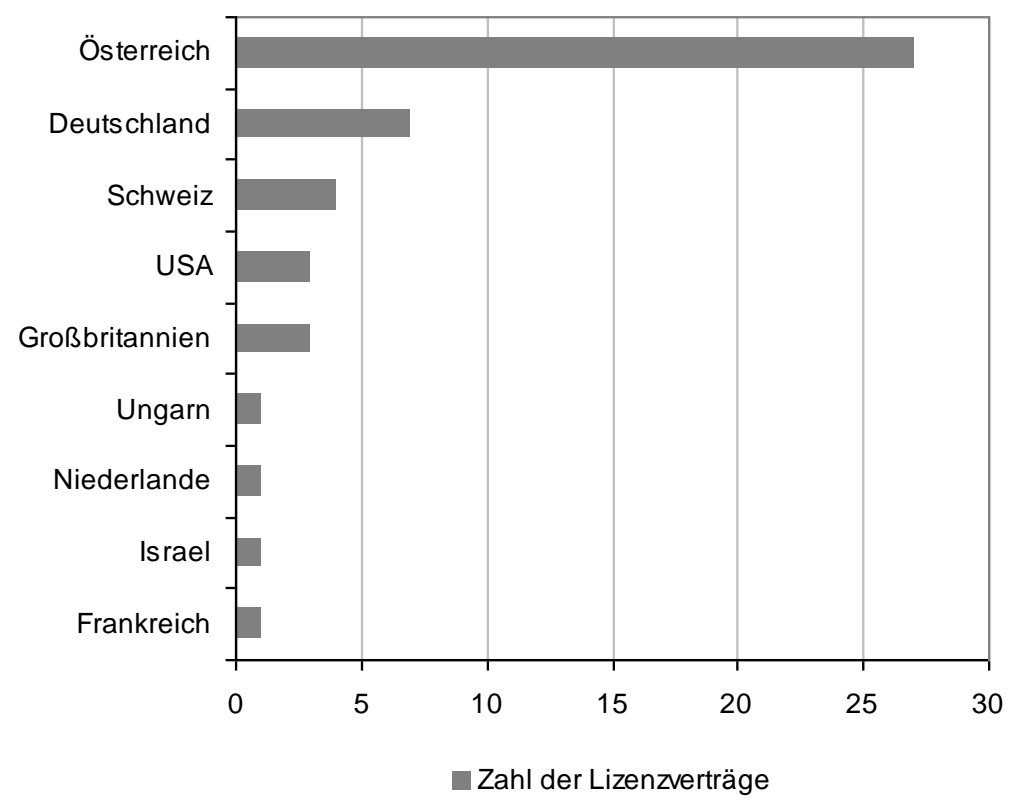

Quelle: aws

\section{Bewertung durch das Evaluationsteam}

Das Programm tecma adressiert die Problematik, der sich vor allem Kleine und Mittlere Unternehmen sowie EinzelerfinderInnen, bezüglich der ökonomischen Verwertung ihrer Erfindungen gegenüberstehen. Die hohen Such- und Transaktionskosten bezüglich der Vermarktung von Erfindungsrechten wirken vielfach prohibitiv, so dass KMUs und EinzelerfinderInnen ihre Patente nicht ökonomisch verwerten können ${ }^{15}$. Hier setzt tecma direkt an, indem es ein professionelles Vermarktungsinstrument, das auch eine internationale Reichweite garantieren kann, anbietet. Tecma kann aufgrund des vorhandenen Know-How, des beständigen Dealflow dynamische Skaleneffekte (Lerneffekte / Professionalisierung) erzielen, die einem einzelnen KMU bzw. einem/r EinzelerfinderIn nicht zur Verfügung stehen. Das „Poolen“ und Abarbeiten der Verwertungsbestrebungen einer Vielzahl von Einzelakteuren über ein zentrales Programm führt also letztlich zu Effizienzgewinnen gegenüber dem Fall, wo jeder Akteur für sich allein Vermarktungsaktivitäten setzt.

Bei der Bewertung des quantitativen Ausmaß der betreuten Projekte des Programms ist zu beachten, dass in den vergangenen Jahren ein Schwerpunkt der Aktivitäten der MitarbeiterInnen von tecma auf der Betreuung der universitären Erfindungen im Rahmen von uni:invent lag. Dementsprechend waren die freien Ressourcen für KMUs und EinzelerfinderInnen im Vergleich zur Vorgängerperiode geringer, was sich auch in einem geringeren Dealflow niederschlug. Durch das Auslaufen von uni:invent kann daher für die nächste Zeit wieder eine Verschiebung in Richtung Betreuung von KMUs/ EinzelerfinderInnen erwartet werden bzw. sollte aktiv angestrebt werden, wobei allerdings die aws weiterhin Bewertungen (sowie in Einzelfällen die entsprechende Vermarktung) von universitären Erfindungsmeldungen (auf bilateraler Vertragsbasis mit den jeweiligen Universitäten) durch.

\footnotetext{
${ }^{15}$ Anzumerken ist hier, dass natürlich die ErfinderInnen nicht jede Erfindung bzw. jedes Patent aktiv via Lizenzvergaben verwerten wollen. Es kann davon ausgegangen werden, dass vielfach die Verwertung ,in-house“ erfolgt bzw. ,passiv“ insofern, als der Patentschutz Dritten den Einstieg in die geschützte Technologie verwehrt.
} 
Insgesamt stellt das Programm tecma aber ein adäquates Instrument zur Nutzung und Verwertung der innovativen Ressourcen von KMUs dar, das durch seine Intervention ein Marktsagen adressiert und die der Zielgruppe inhärente Ressourcen- und Informationsschwäche im Umgang mit dem Patentsystem i.w.S. abzubauen hilft.

\subsection{2 uni:invent}

Ein ungenügend intensives Ausmaß des Technologie- und Wissenstransfers zwischen dem Hochschul- und dem Wirtschaftssektor war eine der zentralen Problemdiagnosen für das österreichische Innovationssystem in den 1990er Jahren. Konzeptionell können einschlägige Interaktionen zwischen Universitäten und Unternehmen als Aktivitäten auf einem spezifischen „Wissensmarkt“" verstanden werden. Wie auf allen Märkten ist auf diesem „Wissensmarkt" für effiziente Transaktionen Klarheit über die bestehenden Eigentumsrechte (im Falle des Wissenstransfers also jene der geistigen Eigentumsrechte, etwa in Form von Patenten/Lizenzen) eine notwendige Bedingung. Ausgehend von den USA und deren Erfahrung mit dem Bayh-Dole-Act (1980) haben in den vergangenen Jahren mehr und mehr OECDLänder die entsprechenden regulatorisch-legistischen Rahmenbedingungen für eine Klärung der geistigen Eigentumsrechte, resultierend aus Forschungsaktivitäten von öffentlichen Forschungseinrichtungen bzw. Universitäten, etabliert.

Im diesem Zusammenhang ist das Programm uni:invent zu sehen, das auf der Basis des UG 2002 zur Unterstützung österreichischer Universitäten bei der Bewertung, Patentierung und Verwertung von Erfindungen im Jahr 2003 vom Rat für Forschung und Technologieentwicklung (RFT) empfohlen und 2004 etabliert wurde (die erste Programmlaufzeit - uni:invent Phase I - war von 2004 bis 2006; die zweite Programmlaufzeit - uni:invent Phase II - schloss 2007 daran an und endete 2009).

Gemäß der Empfehlung des FTE-Rates ,... Forschungsergebnisse aus den Universitäten in größerem Umfang einer wirtschaftlichen Verwertung zuzuführen ... (um dadurch) die kommerzielle Nutzung möglichst vieler guter Erfindungen zu forcieren" lag die Zielsetzung von uni:invent bei folgenden Säulen:

- Aufbau von Know-How an den Universitäten und außeruniversitären Einrichtungen

- Aufbau von Verwertungsinfrastrukturen in Partnerschaft zwischen Universitäten, Fachhochschulen und außeruniversitären Forschungseinrichtungen

- Nutzung von professionellen Verwertungsagenturen für die Vermarktung von Patenten.

Die Indikatoren zur Prüfung der Zielerreichung von uni:invent sind laut „Anbot für die Fortsetzung der Technologieprogramme für die Jahre 2007 bis 2009 “ wie folgt definiert:

- Anzahl der Erfindungsmeldungen aufgeteilt nach Universitäten

- Anzahl der positiven Empfehlungen der aws

- Steigende Patentanmeldungen von Universitäten aufgeteilt nach Bundesländern und

- Forschungsfeldern

- $\quad$ Steigende Patenterteilungen

- Umsetzung der Patente mit PartnerInnen im In- und Ausland

- Stärkung universitärer Transfer-Eigenaktivitäten

Im Programm uni:invent soll das Patentierungs-/Lizenzierungspotential für österreichische Universitäten gezielt aktiviert und möglichst effizient einer wirtschaftlichen Nutzung, zum 
Vorteil für die Forschung und in Folge für die österreichische Volkswirtschaft, zugeführt werden. Über einen Zeitraum von 3 Jahren werden 9 Mio. EUR zur Verfügung gestellt, um eine Verwertungskultur an den Universitäten in Gang zu setzen. Wesentliche Bestandteile sind dabei, Anlaufstellen an den Universitäten, professionelle Unterstützung im IP Management und bei der Vermarktung, Schulung der AkteurInnen sowie die Finanzierung von Patenten.

Programmträger sind das BMWA und das BMWF. Die administrative und operative Abwicklung des Programmes erfolgt durch aws tecma, die folgende Aufgaben übernimmt: Schulungen für die ErfinderberaterInnen, Begutachtung der Erfindungsmeldungen, Verwaltung der Patentkonten und Verwertungsaktivitäten.

Teilnahmeberichtigt sind alle österreichischen Universitäten, die Erfindungs- und Patentberatungsstellen bzw. Innovationsscouts an ihrer Einrichtung etablieren wollen. Entsprechend haben die teilnahmeinteressierten Universitäten zu Programmbeginn ein individuelles Umsetzungskonzept erstellt. An den Einrichtungen sind verschiedene Modelle zur Etablierung der Innovationsscout(s) gewählt worden.

Das Programm ist modular aufgebaut und umfasst (i) die Unterstützung von ErfinderberaterInnen und PatentberaterInnen (sog. Innovationsscouts), (ii) die Erfindungsprüfung, (iii) die Patentverwertung und (iv) Patentfonds, ein virtueller Fonds, der mit Mitteln des BMWF dotiert und für die Deckung entstehender Patentkosten genutzt wird.

Die Förderung umfasst Zuschüsse und Services für die im Rahmen der Erfindungsverwertung an den Universitäten nötigen Aktivitäten. Für die teilnehmenden Universitäten selbst entstehen keine zusätzlichen Kosten. Die Einreichungen erfolgen direkt bei der aws. Das Ausmaß der Leistungen für die einzelnen Universitäten variiert mit der Zahl der Erfindungs- und Patentmeldungen.

Die vorliegende Analyse der Programmergebnisse beruht auf dem von der aws tecma am 28.10.2010 übermittelten Datenbankauszug. Er enthält 1.552 Einträge der Erfindungsmeldungen der an dem Programm beteiligten Universitäten, welche im Rahmen des Vertrages zwischen der jeweiligen Universität und der aws tecma gemeldet wurden. Bis 2006 zeigt sich ein Anstieg der Erfindungsmeldungen auf 330, in den beiden Folgejahren waren es 275 Meldungen pro Jahr. Im letzten Programmjahr 2009 zeigt sich wiederum eine Zunahme, auf 343 Meldungen. Die deutliche Zunahme zwischen 2004 und 2005 lässt darauf schließen, dass sich das Programm gut etablieren konnte (siehe Abbildung 15). 
Abbildung 15: Anzahl der Erfindungsmeldungen (Datum der Meldung an die Universität)

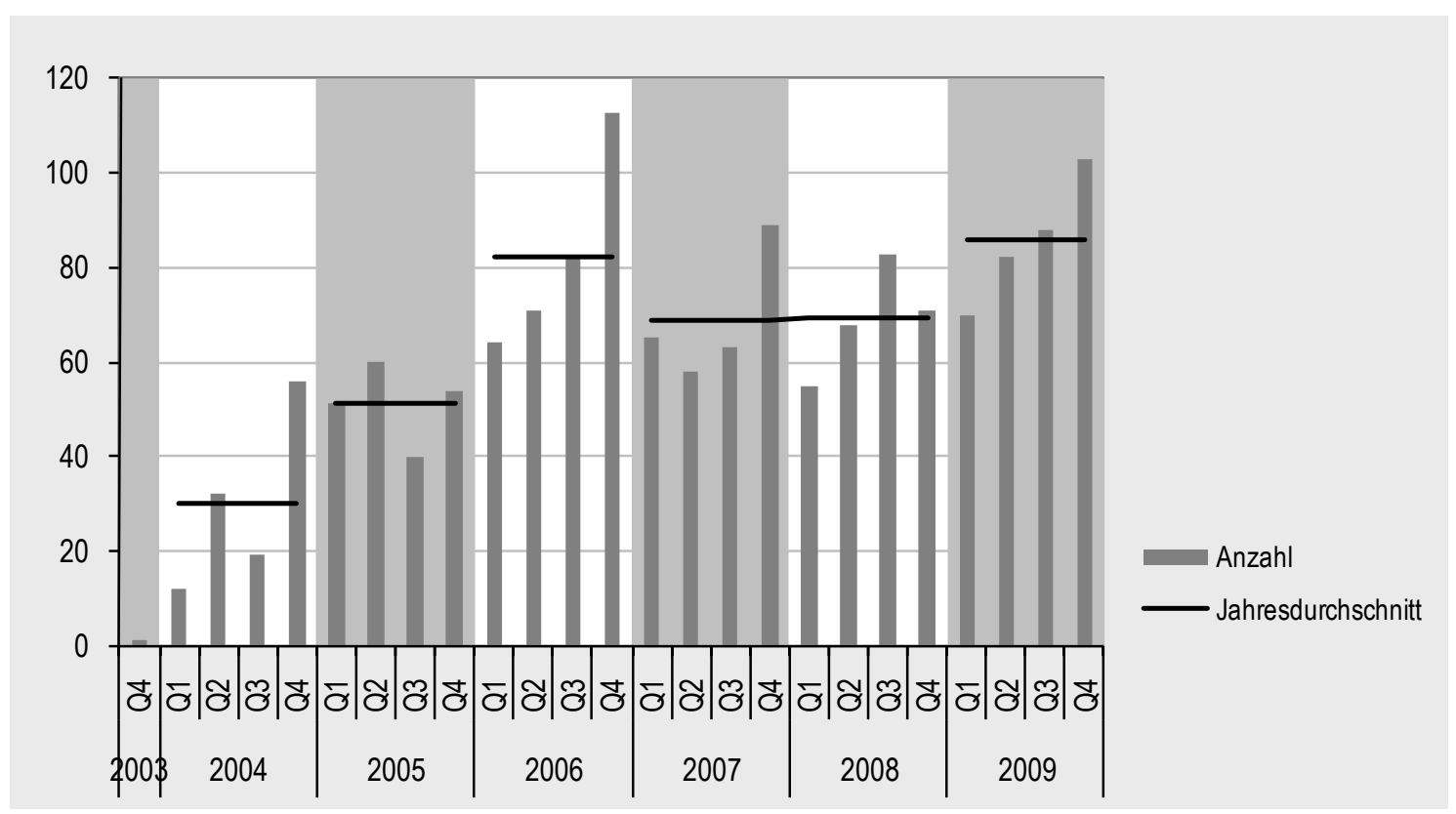

Quelle: aws tecma; eigene Berechnungen

Diese 1552 Erfindungsmeldungen wurden von 801 (Erst-)ErfinderInnen eingereicht; im Durchschnitt entfallen also mehr als 1,9 Erfindungsmeldungen auf eine/n ErfinderIn, das Maximum beträgt 18 Erfindungsmeldungen. Abbildung 16 gibt einen Überblick über die Erfindungsmeldungen für die 16 beteiligten Universitäten.

Abbildung 16: Erfindungsmeldungen nach Universitäten

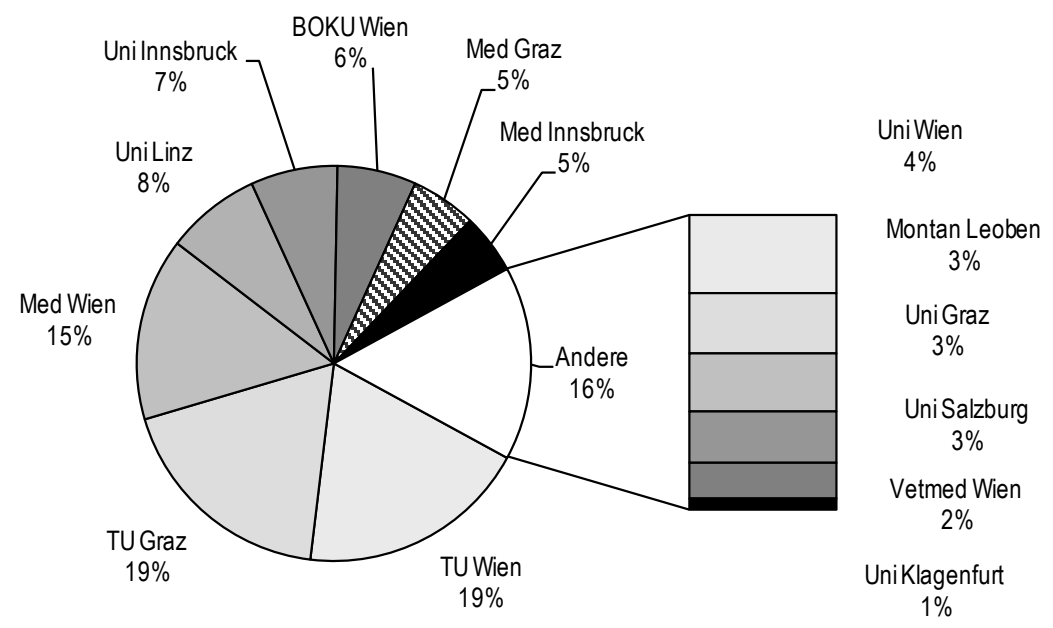

Quelle: aws tecma; eigene Berechnungen

Mit einem Anteil von jeweils $19 \%$ sind die TU Wien und die TU Graz die aktivsten Universitäten, gefolgt von der Medizinischen Universität Wien (15\%) und der Johannes Kepler Universität Linz (8\%). Mit fast zwei Drittel der Erfindungsmeldungen stellen also - wenig überraschend - die medizinischen und technischen Universitäten das Gros der Erfindungsmeldungen (inklusive BOKU Wien und Montanuniversität Leoben beträgt dieser Anteil $72 \%$ ). Im Zeitablauf sind zwar Schwankungen dieses Anteils zu beobachten, diese sind aller- 
dings durch die - speziell im ersten Programmjahr - relativ geringen Fallzahlen zumindest mit verursacht.

Die Bedeutung der einzelnen Technologiefelder insgesamt (d.h. über alle Universitäten hinweg) ist in Abbildung 17 dargestellt. Ein Drittel der Erfindungsmeldungen entfallen auf die Biotechnologie, gefolgt von Chemie/Verfahrenstechnik (16\%) sowie Elektronik und Maschinenbau (mit jeweils $13 \%$ ). Der hohe Anteil der Biotechnologie an den Erfindungsmeldungen zeigt zum einen den hohen Stellenwert, den der Schutz geistigen Eigentums durch Patente in diesem Technologiewert einnimmt, und zum andern lässt der hohe Anteil auf eine intensive Forschungsarbeit schließen. Die österreichischen Universitäten spielen zudem auch eine bedeutsame Rolle innerhalb der „Clusterpolitik“ im Bereich Biotechnologie (Kooperationen mit einschlägigen Unternehmen mit verschiedensten institutionellen Ausprägungen). Die jährlichen Erfindungsmeldungen nach Technologiefeldern (in absoluten als auch in relativen Anteilen) werden zudem in Tabelle 9 angeführt.

\section{Abbildung 17: Erfindungsmeldungen nach Technologiefeldern}

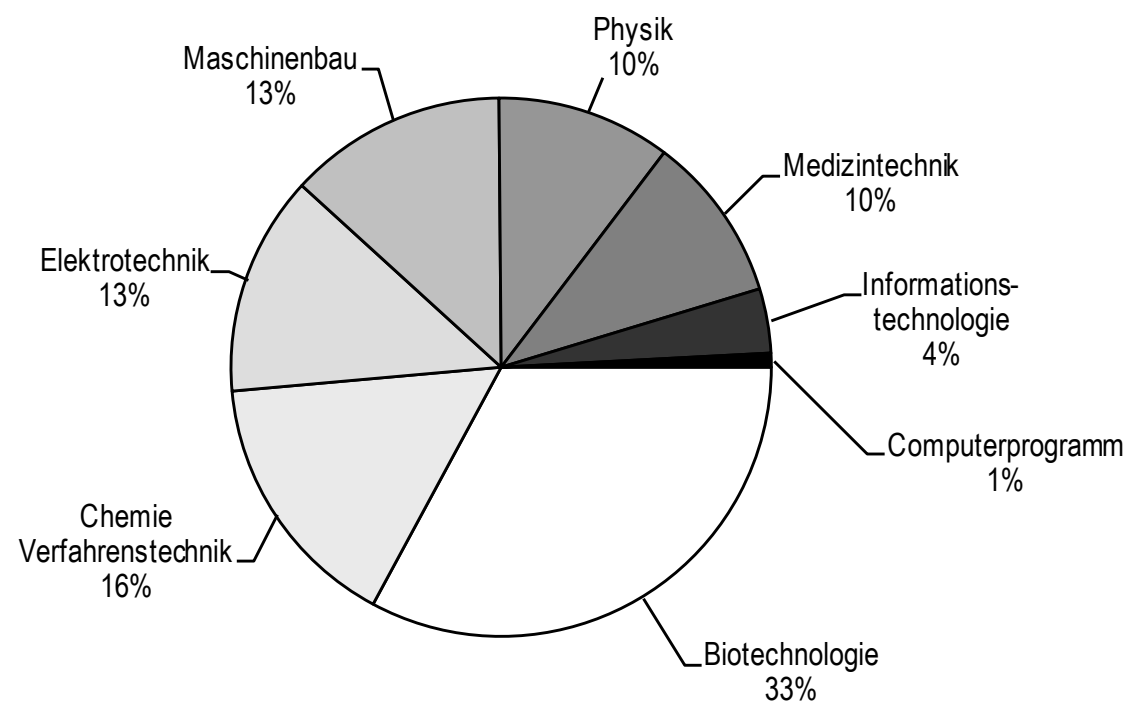

Quelle: aws tecma; eigene Berechnungen

Tabelle 9: Erfindungsmeldungen nach Technologiefeldern, 2004 - 2009 (Datum des Eingangs bei der aws tecma)

\begin{tabular}{|c|c|c|c|c|c|c|c|c|c|c|c|c|c|c|}
\hline \multirow[b]{2}{*}{ Institution } & \multicolumn{6}{|c|}{ \# Meldungen im Jahr } & \multirow[b]{2}{*}{ 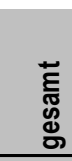 } & \multicolumn{7}{|c|}{ Anteile } \\
\hline & ণัे & 옹 & ণั & 옹 & 옹 & 옹 & & ర్ & 옹 & ণั & స్ํ․ & 。ั & ㅇํㄹ & $\begin{array}{l}\overrightarrow{\mathrm{E}} \\
\mathbb{J} \\
\stackrel{\mathrm{g}}{\sigma}\end{array}$ \\
\hline \# Meldungen & 110 & 199 & 341 & 253 & 288 & 361 & 1552 & $100 \%$ & $100 \%$ & $100 \%$ & $100 \%$ & $100 \%$ & $100 \%$ & $100 \%$ \\
\hline Biotechnologie & 37 & 70 & 98 & 94 & 94 & 117 & 510 & $34 \%$ & $35 \%$ & $29 \%$ & $37 \%$ & $33 \%$ & $32 \%$ & $33 \%$ \\
\hline Chemie Verfahrenstechnik & 10 & 39 & 55 & 37 & 50 & 53 & 244 & $9 \%$ & $20 \%$ & $16 \%$ & $15 \%$ & $17 \%$ & $15 \%$ & $16 \%$ \\
\hline Elektrotechnik & 13 & 7 & 43 & 23 & 42 & 77 & 205 & $12 \%$ & $4 \%$ & $13 \%$ & $9 \%$ & $15 \%$ & $21 \%$ & $13 \%$ \\
\hline Maschinenbau & 12 & 25 & 43 & 29 & 41 & 53 & 203 & $11 \%$ & $13 \%$ & $13 \%$ & $11 \%$ & $14 \%$ & $15 \%$ & $13 \%$ \\
\hline Physik & 10 & 20 & 57 & 36 & 22 & 17 & 162 & $9 \%$ & $10 \%$ & $17 \%$ & $14 \%$ & $8 \%$ & $5 \%$ & $10 \%$ \\
\hline Medizintechnik & 16 & 29 & 32 & 26 & 16 & 36 & 155 & $15 \%$ & $15 \%$ & $9 \%$ & $10 \%$ & $6 \%$ & $10 \%$ & $10 \%$ \\
\hline Informationstechnologie & 10 & 8 & 10 & 5 & 20 & 7 & 60 & $9 \%$ & $4 \%$ & $3 \%$ & $2 \%$ & $7 \%$ & $2 \%$ & $4 \%$ \\
\hline Computerprogramm & 2 & 1 & 3 & 3 & 3 & 1 & 13 & $2 \%$ & $1 \%$ & $1 \%$ & $1 \%$ & $1 \%$ & $0 \%$ & $1 \%$ \\
\hline
\end{tabular}

Quelle: aws tecma; eigene Berechnungen 
Die Programmentwicklung mit den entsprechenden „Betreuungsschritten“ von Seiten der tecma ist in Abbildung 18 kumulativ dargestellt. Die ersten Erfindungsmeldungen - sowohl an die Universitäten als auch an die aws tecma - konnten (abgesehen von zwei „Frühmeldungen“ aus dem Jahre 2003) im März 2004 registriert werden, die ersten Empfehlungen durch die aws tecma im April. Im vierten Quartal 2004 zeigt sich eine deutliche Zunahme der monatlichen Erfindungsmeldungen; seitdem liegt - von einigen Ausreißern abgesehen - die Zahl der Erfindungsmeldungen pro Quartal zwischen etwa 50 und 100. Der stärkste Ausreißer, im 4.Quartal 2006, markiert sicherlich auch das Auslaufen der ersten Programmperiode (dies ist durchaus als für uni:invent positiv zu beurteilen). Eine Zunahme - allerdings nicht so sprunghaft wie im 4. Quartal 2006 - zeigt sich auch gegen Programmende.

Die durchschnittliche Dauer zwischen dem Eingang bei der Universität und der Weiterleitung an die aws tecma beträgt im Gesamtdurchschnitt etwa 22 Tage. Im Verlauf des ersten uni:invent-Jahres ist dieser Zeitraum merkbar gesunken, seit 2005 liegt er recht konstant bei etwa 2-4 Wochen (mit einer merklichen Abnahme seit 2008). Im Wirkungsbereich der aws tecma blieb während der gesamten Programmlaufzeit der Bearbeitungszeitraum (der Zeitraum von der allfälligen Prüfungsübernahme bis zur Empfehlung) recht konstant bei etwa 30 Tagen (und damit deutlich unter der vorgegebenen 6-Wochen-Frist).

Abbildung 18: Erfindungsmeldungen seit Programmbeginn

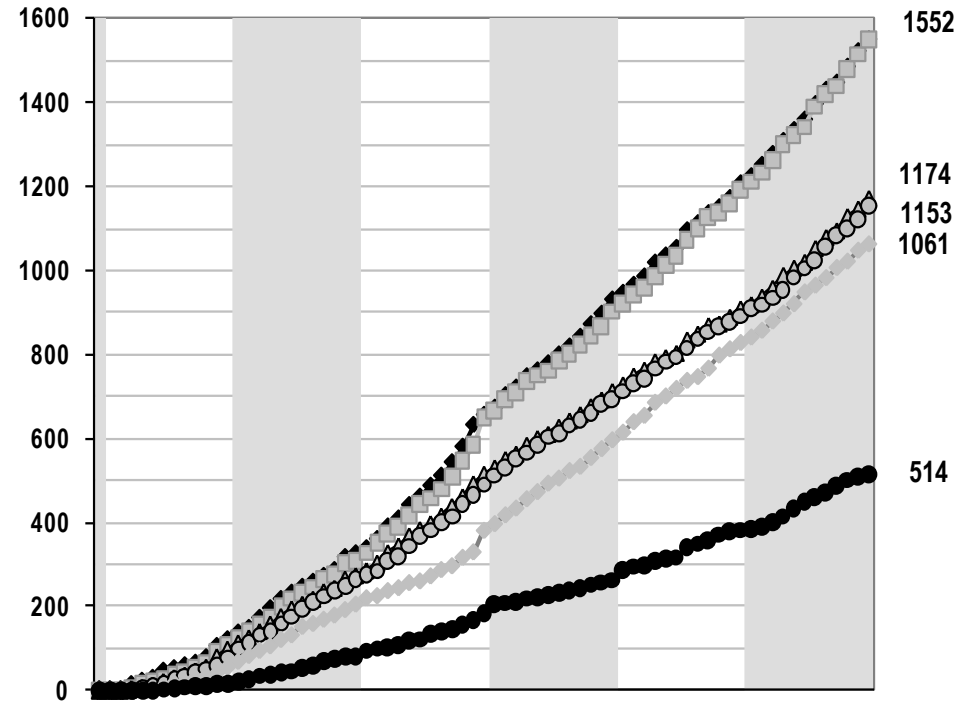

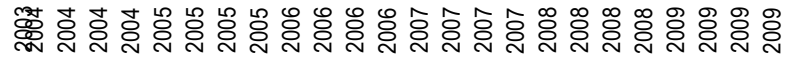
i. kumulierte Erfindungsmeldungen (Stand Programmende 2009):

Quelle: aws tecma; eigene Berechnungen

Von den 1.552 Erfindungsmeldungen wurden 1.182 zur Prüfung übernommen; die 370 nicht übernommenen sind in der Datenbank als ,,beendet“ eingetragen. Als Hauptgrund für die Beendigung ist „Drittmittelprojekt“" genannt (in 367 Fällen).

Aus der Gesamtheit der Erfindungsmeldungen wurden für $40 \%$ positive Empfehlungen abgegeben (Empfehlung „Erfindungsmeldung aufgreifen“); für $36 \%$ wurde eine negative Empfehlung erteilt, wobei „,fehlende Neuheit“, „ungenügende Erfindungshöhe“, „ungenügender Markt" die wichtigsten Gründe für den negativen Bescheid darstellen.

Der aws tecma wird von Seiten der Programmkonzeption eine 6-Wochen-Frist als maximaler 
Zeitraum bis zur Abgabe einer Empfehlung eingeräumt. Bei den Projekten, die in eine Empfehlung mündeten, wird diese sehr gut eingehalten: Lediglich bei 14 der 1.552 Erfindungsmeldungen (bzw. der 1.182 zur Prüfung übernommenen Meldungen) wird diese Frist überschritten.

In 624 Fällen (bzw. $40 \%$ ) wurde von der aws tecma der Aufgriff der Erfindungsmeldung empfohlen (vgl. Abbildung 19). Von den 558 negativen Empfehlungen wurde fast ein Drittel mit „fehlender Neuheit“ begründet, ein weiteres Viertel mit „ungenügender Erfindungshöhe“.

Abbildung 19: Empfehlungen durch aws tecma

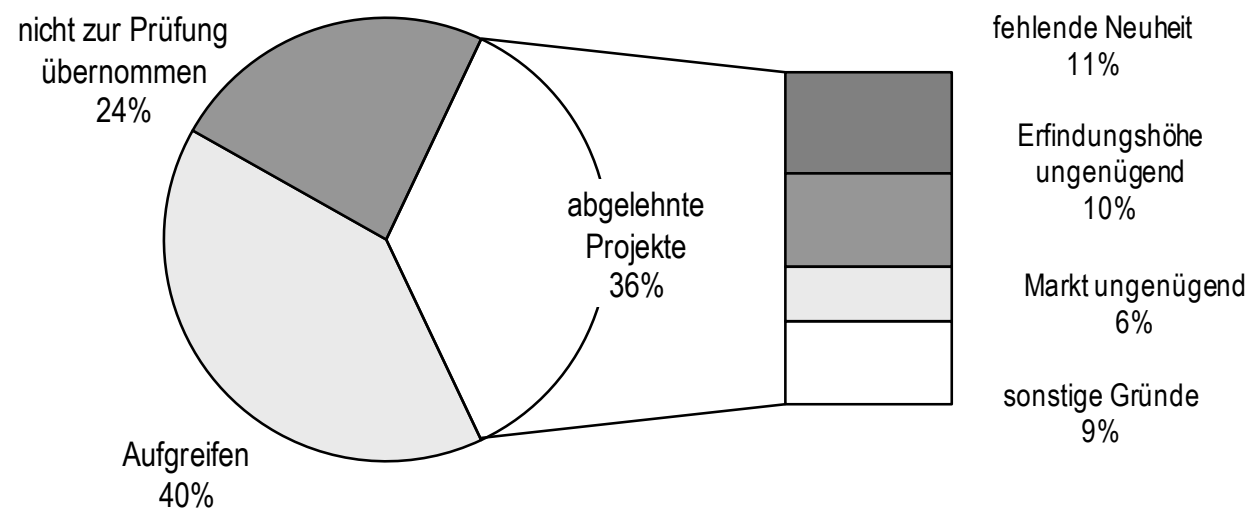

Quelle: aws tecma; eigene Berechnungen

In den weitaus meisten Fällen schließt sich die Universität der Empfehlung der aws tecma an. Nur in 79 Fällen (von 1552) ist eine abweichende Stellungnahme verzeichnet (die meisten davon betreffen negative Empfehlungen, begründet mit „ungenügendem Markt“ bzw. „fehlender Neuheit" oder „mangelnder Erfindungshöhe“).

Für 520 Erfindungsmeldungen (34\%) ist eine Betreuungsart ausgewiesen; in 417 Fällen (27\%) besteht diese in einer Finanzierung, in weiteren 91 Fällen (6\%) zusätzlich in der Vermarktung (Tabelle 10). Nur in 11 Fällen ist nur die Betreuungsart „Vermarktung“ verzeichnet. 1032 Erfindungsmeldungen wurden nicht zur Betreuung übernommen; der Hauptgrund für die vorzeitige Beendigung ist mit $35 \%$ der Vermerk „Drittmittelprojekt“ (d.h., dass die Rechte an der Erfindung außerhalb der Universität liegen), gefolgt von „Freigabe / kein Aufgriff“ (29\%). Der Tatsache entsprechend, dass das Programmende bereits 2009 war, ist nur für $3 \%$ der beendeten Projekte kein Beendigungsgrund eingetragen (diese befinden sich im Status „Empfehlung“ bzw. „Förderbeschluss“; hier wurde also noch keine Entscheidung über die Betreuungsart getroffen). 
Tabelle 10: Projektstatus und Betreuungsart

\begin{tabular}{|c|c|c|c|c|c|}
\hline BetreuungsArt & Anzahl & Anteil & Status & Anzahl & Anteil \\
\hline Finanzierung & 417 & $27 \%$ & Eingang & 1 & $0 \%$ \\
\hline Finanzierung+Vermarktung & 91 & $6 \%$ & Prüfung & 0 & $0 \%$ \\
\hline Vermarktung & 11 & $1 \%$ & Empfehlung & 9 & $1 \%$ \\
\hline Finanzierung+Durchsezung & 1 & $0 \%$ & Förderbeschluss & 4 & $0 \%$ \\
\hline \multirow[t]{3}{*}{ ohne Angabe } & 1032 & $66 \%$ & Betreuung & 28 & $2 \%$ \\
\hline & & & Verwertung & 11 & $1 \%$ \\
\hline & & & Ende & 1499 & $97 \%$ \\
\hline Gesamtergebnis & 1552 & $100 \%$ & Gesamtergebnis & 1552 & $100 \%$ \\
\hline
\end{tabular}

Quelle: aws tecma; eigene Berechnungen

Auch im Projektstatus zeigt sich, dass uni:invent als Programm bereits vor einiger Zeit abgeschlossen wurde: Nur noch 9 der 1.552 Erfindungsmeldungen werden als im Stadium der „Empfehlung“ geführt (d.h. dass bislang weder ein Förderbeschluss des Rektorats über den Aufgriff noch ein Vertragsdatum über Finanzierung und/oder Verwertung oder anderweitige Beendigung durch die aws tecma verzeichnet sind). 1499 Erfindungsmeldungen (97\%) sind als „,beendet“ aus dem uni:invent-Programm ausgeschieden.

\section{Erfindungsmeldungen und Erfinderinnen}

Der Anteil von Ersterfinderinnen beträgt im Schnitt der gesamten Programmlaufzeit 6,9 \% (107 von 1552 Erfindungsmeldungen, siehe Tabelle 11). Dieser Wert ist über die Zeit relativ konstant, er betrug in den einzelnen Jahren zwischen 4 und $9 \%$ - seine Schwankungen sind wahrscheinlich eher durch die relativ geringe Fallzahl erklärbar als durch tatsächliche Verschiebungen. Dies ist allerdings nur der Frauenanteil an den ErsterfinderInnen: für jede Erfindungsmeldung ist eine Person als Hauptansprechperson definiert. Der Frauenanteil bei der Gesamtheit der ErfinderInnen ist deutlich höher: er liegt im Schnitt bei etwa 14\% (und ist über die Zeit recht konstant). Bei der genauen Analyse ergibt sich allerdings das Problem, dass nicht für alle Erfindungsmeldungen alle ErfinderInnen in der Datenbank vermerkt sind: zum einen wurde diese Information erst während der Programmlaufzeit in die Datenbankstruktur aufgenommen (was bedeutet, dass gemäß aws tecma erst ab etwa der zweiten Hälfte 2006 diese Information wirklich vollständig in der Datenbank enthalten ist ${ }^{16}$ ), zum anderen werden bei Drittmittelprojekten, die von den Universitäten nur gemeldet werden, keine vollständigen ErfinderInnenlisten erfasst. In der Tabelle ist daher der Anteil der Frauen an den ErsterfinderInnen und an allen ErfinderInnen nur für die Jahre 2006-2009 ausgewiesen. Nach Technologiefeldern ist der Anteil weiblicher Ersterfinderinnen mit $14 \%$ (bzw. $27 \%$ an allen ErfinderInnen) in der Biotechnologie am höchsten.

\footnotetext{
${ }^{16}$ Davor wurde diese Information zwar erfasst, aber nicht elektronisch zugänglich gemacht.
} 
Tabelle 11: Frauenanteil an den ErsterfinderInnen sowie an allen Erfinderinnen, 2006 - 2009

\begin{tabular}{|c|c|c|c|}
\hline Organisation & 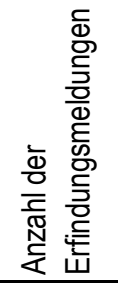 & 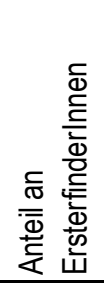 & 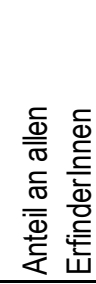 \\
\hline BOKU Wien & 85 & $15 \%$ & $22 \%$ \\
\hline Kunst Linz & 2 & $0 \%$ & $20 \%$ \\
\hline Med Graz & 57 & $16 \%$ & $24 \%$ \\
\hline Med Innsbruck & 60 & $5 \%$ & $17 \%$ \\
\hline Med Wien & 170 & $11 \%$ & $26 \%$ \\
\hline Montan Leoben & 39 & $5 \%$ & $8 \%$ \\
\hline Musik Graz & 6 & $17 \%$ & $7 \%$ \\
\hline TU Graz & 244 & $5 \%$ & $11 \%$ \\
\hline TU Wien & 233 & $2 \%$ & $8 \%$ \\
\hline Uni Graz & 42 & $10 \%$ & $22 \%$ \\
\hline Uni Innsbruck & 78 & $4 \%$ & $19 \%$ \\
\hline Uni Klagenfurt & 9 & $0 \%$ & $0 \%$ \\
\hline Uni Linz & 109 & $4 \%$ & $6 \%$ \\
\hline Uni Salzburg & 34 & $15 \%$ & $31 \%$ \\
\hline Uni Wien & 54 & $6 \%$ & $20 \%$ \\
\hline Vetmed Wien & 21 & $24 \%$ & $25 \%$ \\
\hline Gesamt & 1243 & $1 \%$ & \\
\hline
\end{tabular}

\begin{tabular}{|c|c|c|c|}
\hline Technologiefeld & 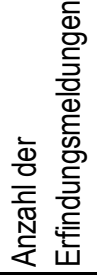 & 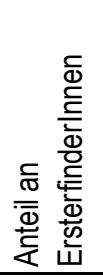 & 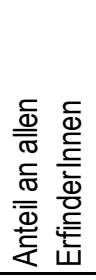 \\
\hline Biotechnologie & 403 & $14 \%$ & $27 \%$ \\
\hline Chemie Verfahrenstechr & 195 & $5 \%$ & $18 \%$ \\
\hline Computerprogramm & 10 & $10 \%$ & $10 \%$ \\
\hline Elektrotechnik & 185 & $2 \%$ & $2 \%$ \\
\hline Informationstechnologie & 42 & $2 \%$ & $5 \%$ \\
\hline Maschinenbau & 166 & $4 \%$ & $5 \%$ \\
\hline Medizintechnik & 110 & $6 \%$ & $11 \%$ \\
\hline Physik & 132 & $4 \%$ & $7 \%$ \\
\hline
\end{tabular}

Quelle: aws tecma; eigene Berechnungen

\section{Aktivitäten im Jahr 2010}

Obgleich das Programm uni:invent plangemäß mit dem Jahr 2009 ausgelaufen ist, wurden von Seiten der aws - in Vereinbarung mit dem BMWFJ - die IP-Aktivitäten der österreichischen Universitäten weiter betreut. Insgesamt gingen (bis Anfang Dezember 2010) 208 Erfindungsmeldungen bei aws tecma ein, wovon 70 Erfindungsmeldungen (=33,7\%) als positiv empfohlen wurden. Die rege Erfindungsmeldungsaktivität deutet darauf hin, dass - nicht zuletzt ausgelöst durch uni:invent - ein Bewusstsein für intellektuelles Eigentum an den österreichischen Universitäten verankert ist.

Die Verteilung der Erfindungsmeldungen nach Technologiefeldern war auch im Jahr 2010 wieder von der Biotechnologie mit 78 Meldungen (37,5\%) geprägt (vgl. Abbildung 20), gefolgt von der Elektrotechnik (36 Meldungen bzw. 17,3\%), der Chemie/Verfahrenstechnik (25 Meldungen bzw. $12 \%$ ), dem Maschinenbau (24 Meldungen bzw. 11,5\%) und der Medizintechnik (21 Meldungen bzw. 10,1\%). 


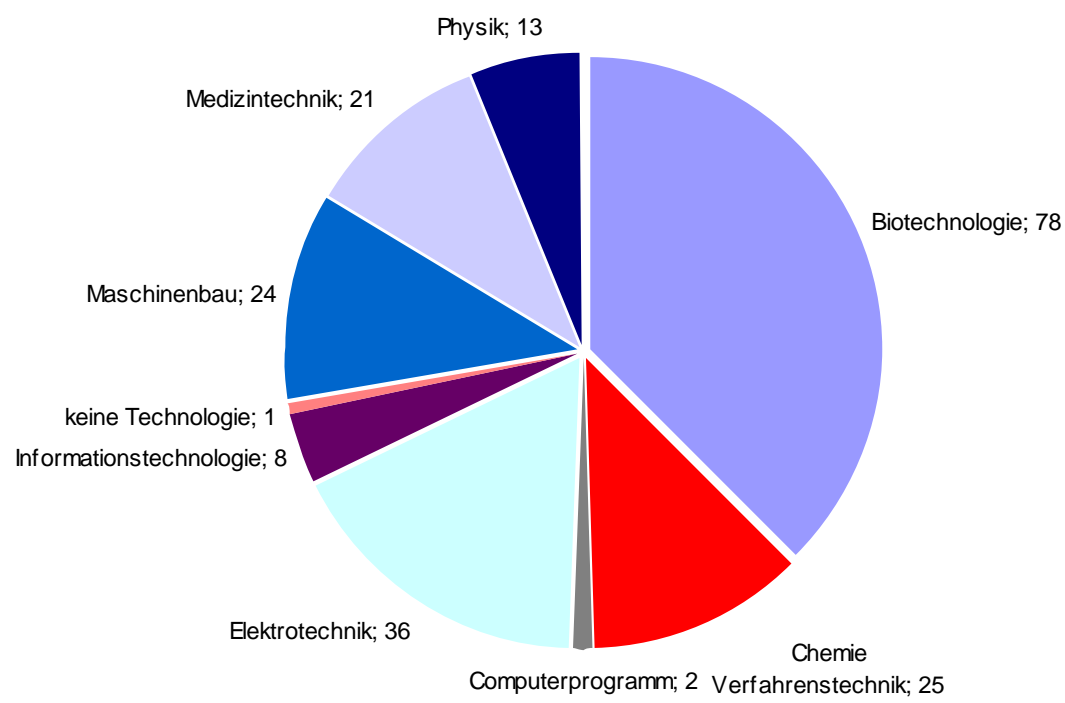

Quelle: aws

Die meisten Erfindungsmeldungen des Jahres 2010 stellte die Technische Universität Graz mit 55 Meldungen (Anteil von 26,4\%), gefolgt von der Technischen Universität Wien (41 Meldungen bzw. 19,7 \%), der Medizinischen Universität Wien (36 Meldungen bzw. 17,3 \%) und der Leopold-Franzens-Universität Innsbruck (20 Meldungen bzw. 9,6 \%) (siehe Tabelle 12). Alle anderen Universitäten meldeten hingegen weniger als zehn Erfindungen.

Tabelle 12: Eingegangene Erfindungsmeldungen im Jahr 2010 nach Organisation

\begin{tabular}{lrr}
\hline Organisation & Anzahl & Anteil \\
\hline Alpen-Adria-Universität Klagenfurt & 1 & 0,5 \\
IFA Tulln & 1 & 0,5 \\
Johannes Kepler Universität Linz & 8 & 3,8 \\
Karl-Franzens-Universität Graz & 7 & 3,4 \\
Leopold-Franzens-Universität Innsbruck & 20 & 9,6 \\
Medizinische Universität Graz & 10 & 4,8 \\
Medizinische Universität Innsbruck & 7 & 3,4 \\
Medizinische Universität Wien & 36 & 17,3 \\
Paris-Lodron-Universität Salzburg & 2 & 1,0 \\
Private & 1 & 0,5 \\
Technische Universität Graz & 55 & 26,4 \\
Technische Universität Wien & 41 & 19,7 \\
Universität für Angewandte Kunst Wien & 1 & 0,5 \\
Universität für Bodenkultur Wien & 7 & 3,4 \\
Universität Wien & 5 & 2,4 \\
Veterinärmedizinische Universität Wien & 6 & 2,9 \\
\hline Gesamtergebnis & 208 & 100,0 \\
\hline Otle: & &
\end{tabular}

Quelle: aws 


\section{Bewertung durch das Evaluierungsteam}

Der Programmverlauf von uni:invent kann abschließend durchwegs als positiv eingeschätzt werden. Im Zuge der Programmlaufzeit ist es gelungen, den Stellenwert von geistigen Eigentumsrechten an den Universitäten zu verankern, einen entsprechenden Bewusstseinswandel zu initialisieren und das IPR-Management zu professionalisieren. Immerhin wurden während der Programmperiode über 1500 Erfindungen gemeldet (wovon $40 \%$ zum Aufgreifen empfohlen wurden). Mit dem Auslaufen des Programms im Jahr 2009 stellt sich nun die Frage nach der weiteren Perspektive universitärer IPR-Strategien. Dass selbst nach Beendigung des Programms einschlägige Aktivitäten (wenn auch in etwas geringerem Umfang) in Form entsprechender Erfindungsmeldungen von Seiten der österreichischen Universitäten weiterliefen, zeigt, dass das Programm durchaus nachhaltige Wirkungen erzielen konnte. IP-orientierte Nachfolgemaßnahmen wären daher - um den Impetus nicht zu verlieren - durchaus angebracht. Dementsprechend wird in den Leistungsvereinbarungen mit den Universitäten von Seiten des BMWF darauf gepocht, dass diese bis spätestens 2012 entsprechende IPRStrategien vorlegen müssen. Für die Zukunft werden Überlegungen in Richtung der Etablierung von Wissenstransferzentren an Universitäten, die von externen Fachjurys einschlägig geprüft und gegebenenfalls zertifiziert werden, angestellt. Für eine Nachhaltigkeit der eingeschlagenen positiven und durch das Programm uni:invent in den vergangenen Jahren erfolgreich initiierten Entwicklungspfade im Bereich des IPR-Managements ist eine rasche Umsetzung derartiger strategischer Überlegungen mit ausreichender Ressourcenausstattung notwendig.

\subsubsection{PRIZE}

PRIZE hat sich aus dem Programm uni:invent entwickelt (Start im Jahr 2006 als Pilotversuch, anschließend die Regelung in der Sonderrichtlinie uni:invent II 2007 bis 2009) und verfolgt das Ziel, Erfindungen/ Innovationen von Universitäten potentiellen Entwicklungspartnern und/ oder Lizenznehmern vorzustellen. Es adressiert somit den Themenkreis Weiterentwicklung einer Erfindung in Richtung marktfähiges Produkt. Organisiert ist PRIZE als Prototypenwettbewerb, wo unter operativer Abwicklung der aws prinzipiell patentfähige Konzepte von einer unabhängigen Fachjury ausgewählt, ausgezeichnet und bei der Erarbeitung von Prototypen (proof of concept) finanziell unterstützt werden. Bislang erfolgte die Ausschreibung zur Projekteinreichung jährlich. Voraussetzung auf Seiten des Fördernehmers dabei ist, dass es sich 1) um eine universitäre Institution handelt und 2) dass diese auch am Programm uni:invent teilgenommen hat.

Gemäß den Richtlinien des Förderprogramms uni:invent II 2007-2009 können je nach Art und Umfang des beantragten Projekts bis zu 100 \% der förderbaren Kosten (Material-, Geräte- und Personalkosten, die direkt dem Projekt zugeordnet werden können sowie Kosten für Machbarkeitsstudien und Eigenschaftsblätter) von der aws getragen werden, maximal jedoch 150.000.- EUR pro Projekt. Der Zeitraum für die Förderung ist auf maximal 1,5 Jahre begrenzt.

Die Indikatoren zur Prüfung der PRIZE-Zielerreichung sind laut „Anbot für die Fortsetzung der Technologieprogramme für die Jahre 2007 bis 2009“ wie folgt definiert: 
- Anzahl der eingereichten Projekte

- Anzahl der geförderten Projekte aufgeteilt nach Bundesland, Universität und Technologiefeld

- Anzahl erfolgreicher Kooperationen/Lizenzeinnahmen auf Basis der entwickelten Prototypen

Nach einer Pilotphase im Jahr 2006 sind in den darauf folgenden Call-Jahren 2008-2010 insgesamt 77 Projekte zur Prototypenförderung PRIZE eingereicht worden; das letzte Jahr zeigte sich dabei am stärksten, was die Anzahl der Einreichungen anbelangt - 2010 wurden insgesamt 34 Projekte eingereicht, beinahe doppelt so viele wie ein Jahr zuvor (siehe Tabelle 13. Bemerkenswert ist, dass von diesen im Jahr 2010 insgesamt 34 eingereichten Projekten, lediglich acht Einreichungen eine positive Juryentscheidung erfahren durften; dies entspricht einer Bewilligungsquote von unter $25 \%, 2009$ betrug die Bewilligungsquote immerhin noch $39 \%$ und 2008 beachtliche $48 \%$. Dass die Bewilligungsquote sich innerhalb von nur drei Jahren in etwa halbierte, ist vor allem auf die abnehmende Qualität der Projekteinreichungen zurückzuführen. Auch war zu beobachten, dass Universitäten versuchten, für ein und dasselbe Projekt mehrere Förderschienen bzw. Drittmittelstellen zwecks Förderung zu adressieren.

Tabelle 13: Anzahl der PRIZE Einreichungen und davon positive Juryentscheidungen nach

Organisation und Call-Jahr, 2008-2010

\begin{tabular}{lrr|rr|rr}
\hline \multicolumn{1}{c}{ Organisation } & $\mathbf{2 0 1 0}$ & $\begin{array}{r}\text { davon } \\
\text { positiv }\end{array}$ & $\mathbf{2 0 0 9}$ & $\begin{array}{r}\text { davon } \\
\text { positiv }\end{array}$ & $\mathbf{2 0 0 8}$ & $\begin{array}{r}\text { davon } \\
\text { positiv }\end{array}$ \\
\hline Johannes Kepler Universität Linz & 4 & 0 & 1 & 1 & 3 & 2 \\
Karl-Franzens-Universiät Graz & 1 & 0 & & & & \\
Leopold-Franzens Universität Innsbruck & 2 & 1 & 1 & 1 & 2 & 1 \\
Medizinische Universität Graz & 1 & 0 & 1 & 0 & & \\
Medizinische Universität Innsbruck & 1 & 1 & 1 & 1 & 1 & 0 \\
Medizinische Universität Wien & 5 & 2 & 2 & 0 & 4 & 4 \\
Montanuniversität Leoben & & & 1 & 1 & 1 & 1 \\
Paris Lodron Universität Salzburg & & & & & 1 & 0 \\
Technische Universität Graz & 6 & 0 & 3 & 1 & 8 & 2 \\
Technische Universität Wien & 9 & 3 & 7 & 2 & 1 & 0 \\
Universität für Bodenkultur Wien & 1 & 0 & & & 2 & 1 \\
Universität für künstlerische und industrielle & & & & & 1 & 1 \\
Gestaltung Linz & 3 & 1 & 1 & 0 & & \\
Universität Wien & 1 & 0 & & & 1 & 0 \\
Veterinärmedizinische Universität Wien & 34 & 8 & 18 & 7 & 25 & 12 \\
\hline Gesamtergebnis & & & & & \\
\hline
\end{tabular}

Anmerkung: Leere Zellen bedeuten, dass die betreffende Organisation in dem jeweiligen Jahr nicht eingereicht hat. Quelle: aws

Tabelle 13 führt darüber hinaus die Zahl der Einreichungen sowie die Zahl der positiven Juryentscheidungen nach teilnehmenden Organisationen über die Jahre der Programmlaufzeit an. Mit jeweils in Summe 17 Einreichungen waren die beiden Technischen Universitäten Wien und Graz am aktivsten, gefolgt von der Medizinischen Universität Wien (11) und der Johannes Kepler Universität Linz (8). Demzufolge sind auch diese drei genannten Universitä- 
ten führend bei der Zahl der positiven Juryentscheidungen, wenn auch hier die Medizinische Universität Wien mit insgesamt 6 Bewilligungen den ersten Rang einnimmt, gefolgt von der Technischen Universität Wien (5) und der Technischen Universität Graz (3).

Insgesamt sind somit in den Jahren 2008-2010 insgesamt 27 Projekte im Rahmen von PRIZE gefördert worden. Die Zahl der geförderten Projekte war dabei im ersten Programmjahr am höchsten (12) und hat sich in den beiden letzten Jahren auf einem Niveau von 7 (2009) bzw. 8 (2010) Projekten eingependelt. Dabei stellt das Technologiefeld Biotechnologie eindeutig einen Schwerpunkt dar. In den Jahren 2008-2010 wurde insgesamt ein Drittel der Projekte (9) im Bereich der Biotechnologie unterstützt, gefolgt von 5 Projekten in der Medizintechnik und jeweils 4 Projekten in der Elektrotechnik und Physik (siehe Tabelle 14).

Tabelle 14: Anzahl der geförderten PRIZE-Projekte nach Technologiefeld und Call-Jahr, 2008-2010

\begin{tabular}{|c|c|c|c|c|}
\hline Projekte & 2010 & 2009 & 2008 & 2008-2010 \\
\hline Biotechnologie & 3 & 1 & 5 & 9 \\
\hline Chemie Verfahrenstechnik & 1 & & & 1 \\
\hline Elektrotechnik & & 1 & 3 & 4 \\
\hline Informationstechnologie & & & 1 & 1 \\
\hline Maschinenbau & & 2 & 1 & 3 \\
\hline Medizintechnik & 3 & 1 & 1 & 5 \\
\hline Physik & 1 & 2 & 1 & 4 \\
\hline Gesamtergebnis & 8 & 7 & 12 & 27 \\
\hline
\end{tabular}

Dementsprechend haben Projekte aus dem Bereich Biotechnologie über die Jahre hinweg die höchsten Fördersummen im Rahmen von PRIZE akquirieren können - insgesamt immerhin über $30 \%$ des gesamten Fördervolumens (772.063.- EUR). Auch durchaus erfolgreich waren die Bereiche Medizintechnik und Physik mit $19 \%$ bzw. $14 \%$ des gesamten Fördervolumens sowie die Elektrotechnik, welche in den Jahren 2008 und 2009 insgesamt $16 \%$ akquirieren konnte. Insgesamt betrug das Fördervolumen von PRIZE in den Jahren 2008-2010 über 2,5 Mio. EUR.

Tabelle 15: Anzahl der geförderten PRIZE-Projekte nach Technologiefeld und Call-Jahr

\begin{tabular}{|c|c|c|c|c|c|}
\hline Projekte & 2010 & 2009 & 2008 & 2008-2010 & $\%$ gesamt \\
\hline Biotechnologie & $328.852,23$ & $89.974,00$ & $353.237,00$ & $772.063,23$ & $30 \%$ \\
\hline Chemie Verfahrenstechnik & $147.140,00$ & 0,00 & 0,00 & $147.140,00$ & $6 \%$ \\
\hline Elektrotechnik & 0,00 & $149.500,00$ & $247.960,00$ & $397.460,00$ & $16 \%$ \\
\hline Informationstechnologie & & & $89.000,00$ & $89.000,00$ & $4 \%$ \\
\hline Maschinenbau & 0,00 & $192.603,00$ & $95.567,00$ & $288.170,00$ & $11 \%$ \\
\hline Medizintechnik & $265.062,50$ & $142.698,00$ & $76.359,00$ & $484.119,50$ & $19 \%$ \\
\hline Physik & $77.750,00$ & $227.084,00$ & $53.752,00$ & $358.586,00$ & $14 \%$ \\
\hline Gesamtergebnis & $818.804,73$ & $801.859,00$ & $915.875,00$ & $2.536 .538,73$ & $100 \%$ \\
\hline
\end{tabular}

Anmerkung: Leere Zellen bedeuten, dass die betreffende Organisation in dem jeweiligen Jahr nicht eingereicht hat. Quelle: aws 
Spricht eine Prototypenförderung per definitionem technische, innovative Wissenschaften an, so stellt sich dementsprechend auch das Bild der PRIZE-Förderung unter den Universitäten dar (siehe Tabelle 16). In den Jahren 2008-2010 konnte dabei die Technische Universität Wien 20 \% (über 0,5 Mio. EUR) der Gesamtfördersumme von PRIZE einwerben, gefolgt von der Medizinischen Universität Wien (18\%), der Leopold-Franzens Universität Innsbruck (15\%) und der Johannes Kepler Universität Linz (13\%).

Tabelle 16: PRIZE-Fördersummen nach Organisation und Call-Jahr, 2008 - 2010

\begin{tabular}{|c|c|c|c|c|c|}
\hline Organisation & 2010 & 2009 & 2008 & $\begin{array}{l}\text { Gesamt- } \\
\text { ergebnis }\end{array}$ & Gesamt \% \\
\hline Johannes Kepler Universität Linz & 0,00 & $149.500,00$ & $189.212,00$ & $338.712,00$ & $13 \%$ \\
\hline Karl-Franzens-Universität Graz & 0,00 & & & 0,00 & $0 \%$ \\
\hline Leopold-Franzens Universität Innsbruck & $144.668,63$ & $106.028,00$ & $133.346,00$ & $384.042,63$ & $15 \%$ \\
\hline Medizinische Universität Graz & 0,00 & 0,00 & & 0,00 & $0 \%$ \\
\hline Medizinische Universität Innsbruck & $77.750,00$ & $142,698,00$ & 0,00 & $220.448,00$ & $9 \%$ \\
\hline Medizinische Universität Wien & $207.752,00$ & 0,00 & $239.742,00$ & $447.494,00$ & $18 \%$ \\
\hline Montanuniversität Leoben & & $86.575,00$ & $95.567,00$ & $182.142,00$ & $7 \%$ \\
\hline Paris Lodron Universität Salzburg & & & 0,00 & 0,00 & $0 \%$ \\
\hline Technische Universität Graz & 0,00 & $113.000,00$ & $112.500,00$ & $225.500,00$ & $9 \%$ \\
\hline Technische Universität Wien & $300.162,10$ & $204.058,00$ & 0,00 & $504.220,10$ & $20 \%$ \\
\hline Universität für Bodenkultur Wien & 0,00 & & $56.508,00$ & $56.508,00$ & $2 \%$ \\
\hline $\begin{array}{l}\text { Universität für künstlerische und industri- } \\
\text { elle Gestaltung Linz }\end{array}$ & & & $89.000,00$ & $89.000,00$ & $4 \%$ \\
\hline Universität Wien & $88.472,00$ & 0,00 & & $88.472,00$ & $3 \%$ \\
\hline Veterinärmedizinische Universität Wien & 0,00 & & 0,00 & 0,00 & $0 \%$ \\
\hline Gesamtergebnis & $818.804,73$ & $801.859,00$ & $915.875,00$ & $2.536 .538,73$ & $100 \%$ \\
\hline
\end{tabular}

Anmerkung: Leere Zellen bedeuten, dass die betreffende Organisation in dem jeweiligen Jahr nicht eingereicht hat. Quelle: aws

Tabelle 17: Durch PRIZE erzielte Vertragsabschlüsse nach Organisation seit 2006

\begin{tabular}{ll}
\hline Organisation & Vertragsabschluss \\
\hline Johannes Kepler Universität Linz & 1 Kooperationsvertrag \\
Leopold Franzens Universität Innsbruck & 1 Gründung \\
Medizinische Universität Wien & 1 Gründung \\
& 1 Lizenzabkommen mit CD Laborfinanzierung \\
Montanuniversität Leoben & 3 Kooperationsverträge \\
Technische Universität Graz & 1 Forschungsvertrag \\
Technische Universität Wien & 1 Optionsvertrag mit Patentkostenübernahme \\
Universität für künstlerische und industrielle & 1 Lizenzvertrag \\
Gestaltung Linz & Gewinner zahlreicher Preise \\
\hline
\end{tabular}

Quelle: aws 
Was den Erfolg der im Rahmen von PRIZE geförderten Projekte anbelangt so zeigt der Überblick in Tabelle 17 , dass von insgesamt 34 bis dato geförderten Projekten zwölf Erfolge hervorgingen: Zwei Gründungen, je eine abstammend von der Medizinischen Universität Wien und der Leopold Franzens Universität Innsbruck, ein Lizenzvertrag, ein Lizenzabkommen, fünf Kooperationsverträge, ein Forschungsvertrag sowie zwei Optionsverträge; d.h. mehr als ein Drittel aller durch PRIZE geförderten Projekte konnten erfolgreiche Verträge abschließen. Lizenzeinnahmen konnten lediglich in zwei Fällen erzielt werden. Ist letzteres eher als ernüchternd zu bewerten, so ist es umso erstaunlicher, dass die Prototypenförderung durch PRIZE zu zwei Unternehmensgründungen geführt hat.

\section{Bewertung durch das Evaluierungsteam}

Wie bereits die Zwischenevaluierung von uni:invent II im Jahr 2009 zeigt, hat sich die Idee, ein Förderprogramm zwecks Unterstützung der Entwicklung von Prototypen bzw. der Finanzierung von Machbarkeitsstudien im universitären Bereich, durchaus bewährt. Die steigende Anzahl der Projekteinreichungen spiegelt das Interesse an einem solchen Programm wider, zumal es über ein "Alleinstellungsmerkmal" verfügt; stehen für die Unternehmen (vor allem seitens der FFG) zahlreiche Fördermöglichkeiten für die Herstellung von Prototypen und Finanzierung von Machbarkeitsstudien zur Verfügung, so ist PRIZE das einzige Programm im österreichischen Förderportfolio, welches eine Förderung im Bereich der Prototypenentwicklung für universitäre Institutionen anbietet.

Angesichts dessen dass uni:invent II ausgelaufen ist, steht man nun vor der Herausforderung, PRIZE als eigenständiges Programm für die Universitäten zu etablieren und damit im Förderportfolio zu positionieren. Der Mehrwert von PRIZE steht dabei außer Frage. Die Prototypenförderung hat durchaus dazu geführt, dass Kooperations-, Options- und Forschungsverträge abgeschlossen wurden. Als weniger gut ist hingegen der Erfolg bei den Lizenzeinnahmen zu bewerten; hier ist festzuhalten, dass nur in zwei von 34 geförderten Fällen solche erzielt werden konnten. Zumal PRIZE ein noch sehr junges Programm ist, soll dieses in Zukunft durchaus weiterentwickelt werden - unter der Prämisse, universitäre Innovationen nicht nur zugänglich, sondern insbesondere auch für Klein- und Mittelbetriebe rascher verwertbar zu machen.

\subsection{4 ipp}

Im Jahr 2006 wurde - im Rahmen eines umfassenden Pakets für kleine und mittlere Unternehmen - das Innovationsschutzprogramm ipp entwickelt, wobei das Bundesministerium für Wirtschaft, Familie und Jugend die aws mit der Durchführung dieses Programms beauftragte. Die Programmmotivation des ipp liegt darin, „österreichische, primär Klein- und Mittelunternehmen, bei der Schaffung, Durchsetzung, Verwertung und Überwachung ihrer geistigen Eigentumsrechte zur Stärkung und Festigung ihres Wachstums-, Innovations- und Internationalisierungspotentials effizient zu unterstützen. ${ }^{117}$ Bis dato zielt das Programm insbesondere auf den Schutz geistiger Eigentumsrechte in Ländern mit erheblichen Marktpotentialen wie China, Indien und Russland ab. Vor allem China und Indien weisen dabei eine zunehmend

${ }^{17}$ Siehe hierzu Innovationsschutz und Innovationsvermarktung, Programmdokument gemäß Punkt 1.3 der Richtlinie „Jungunternehmer- und Innovationsförderung für KMU - Prämienförderung“ vom 19. Mai 2009. 
große, wissenschaftlich-technologische Aufholdynamik auf, die jedoch auch durch komplexe legistisch-institutionelle Rahmenbedingungen charakterisiert ist. Gerade diese Rahmenbedingungen sind es auch, die für Klein- und Mittelunternehmen allein nur schwer zu meistern sind bzw. erhebliche (und für viele Klein- und Mittelunternehmen unter Umständen prohibitive) Kosten verursachen. Angesichts einer Gefahr wie z.B. Produktpiraterie ist ein nachhaltig erfolgreicher Marktauftritt (bzw. die Nutzung potentieller Wissens- bzw. Technologieressourcen vor Ort) ohne ausreichende Absicherung der geistigen Eigentumsrechte kaum mehr denkbar.

Gemäß „Anbot für die Fortsetzung der Technologieprogramme für die Jahre 2007 bis 2009 “ lautet der Auftrag des Innovationsschutzprogramms ipp wie folgt:

Ziel des 2006 neu gestarteten und getrennt beauftragten Innovationsschutzprogramms ipp ist es, österreichischen Unternehmen und Forschungseinrichtungen bei der Erlangung und Durchsetzung von Schutzrechten zu unterstützen. In diesem Zusammenhang soll besonders versucht werden, die Position österreichischer Unternehmen im europäischen Kontext und durch entsprechende Kooperationen zu stärken.

Unterstützung dabei bietet die aws explizit den in die genannten Schwellenländer expandierenden (Klein- und Mittel-)Unternehmen an, sei es in Form von Serviceleistungen wie z.B. Aufklärung im Rahmen von Informationsveranstaltungen, themenspezifisch aufbereiteten Materialien, Beratung bei der Entwicklung von individuellen Patentstrategien oder in Form einer monetären Förderung - Zuschüsse, die nicht rückzahlbar sind und eine maximale Höhe von 250.000.- EUR betragen können.

Die Einreichung von Anträgen im Rahmen von ipp ist laufend möglich und erfolgt direkt bei der aws. Die Laufzeit des derzeitigen Programms ist vom 1. Juli 2008 bis 31. Dezember 2010 anberaumt; gefördert werden kleine und mittlere Unternehmen im Sinne der jeweils aktuellen Bestimmungen des EU-Wettbewerbsrechts, welche über einen Sitz oder eine Betriebsstätte in Österreich verfügen und deren Projekte dazu beitragen, geistige Eigentumsrechte national und international zu schützen, zu vermarkten und zu verteidigen.

In der Förderperiode 2007 - 2010 wurden im Rahmen des Innovationsschutzprogramms ipp insgesamt 156 eingegangene Förderanträge von der aws bearbeitet; ein Spitzenwert wurde dabei im Jahr 2008 mit insgesamt 48 eingegangenen Förderanträgen erreicht. In der Regel werden in etwa die Hälfte oder sogar etwas mehr der eingegangenen Förderanträge zur Förderung frei gegeben. So konnten zwischen 2007 und 2010 insgesamt 95 geförderte Projekte gezählt werden; die Zahl der Patentförderungen beläuft sich insgesamt auf 278, wobei auch hier das Jahr 2008 mit insgesamt 125 Patentförderungen herausragend ist.

Tabelle 18: Förderanträge und geförderte Projekte im Rahmen von ipp, 2007 - 2010

\begin{tabular}{lccccc}
\hline \multicolumn{1}{c}{ Projekte } & $\mathbf{2 0 1 0}$ & $\mathbf{2 0 0 9}$ & $\mathbf{2 0 0 8}$ & $\mathbf{2 0 0 7}$ & $\mathbf{2 0 0 7} \mathbf{- 2 0 1 0}$ \\
\hline Eingegangene Förderanträge & 29 & 39 & 48 & 40 & 156 \\
Geförderte Projekte (Gesamt) & 16 & 29 & 29 & 21 & 95 \\
Patentförderungen & 37 & 64 & 125 & 52 & 278 \\
\hline Quelle: aws & & & &
\end{tabular}


Die Förderleistungen des Programms ipp orientieren sich vor allem an den Bedürfnissen der österreichischen Klein- und Mittelunternehmen, wobei auch durchaus Großunternehmen die Expertise und Serviceleistungen der aws - in diesem Fall im Bereich der Schutzrechtsanmeldungen und Rechtsdurchsetzung zu marktkonformen Bedingungen ${ }^{18}$ - in Anspruch nehmen können. Erwartungsgemäß waren daher in den Jahren 2007 - 201083 von insgesamt 95 Fördernehmern (rund $87 \%$ ) Klein- und Mittelunternehmen, gefolgt von insgesamt zehn Privatpersonen und lediglich zwei Großunternehmen, welche die Leistungen der aws im Rahmen des ipp in Anspruch genommen haben. Bei den zwei Großunternehmen ging es dabei typischerweise um die Entwicklung einer effizienten Patentstrategie bzw. um die Weitervermittlung von Netzwerkpartnern. Obwohl das Innovationsschutzprogramm durchaus auch für die Universitäten zugänglich ist, hat in der Periode 2007 - 2010 bemerkenswerterweise keine einzige Universität die Leistungen des Programms in Anspruch genommen.

Im Allgemeinen kommen die Fördernehmer - wie Abbildung 21 darstellt - vorwiegend aus den Bundesländern Wien (28 von insgesamt 95 Fördernehmern in der Periode 2007 - 2010 ), Niederösterreich (22), Steiermark (16) und Oberösterreich (12).

Abbildung 21: Geförderte Teilnehmer im Rahmen von ipp nach Bundesländern, 2007 - 2010

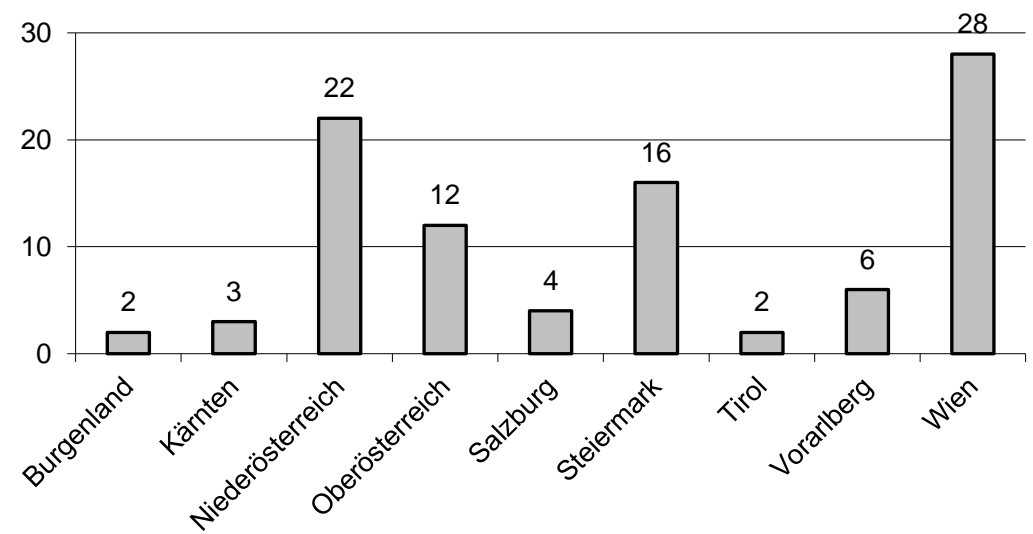

Quelle: aws

Tabelle 19: Geförderte Projekte im Rahmen von ipp nach Technologie, 2007 - 2010

\begin{tabular}{lrrrrr}
\hline \multicolumn{1}{c}{ Projekte } & $\mathbf{2 0 1 0}$ & $\mathbf{2 0 0 9}$ & $\mathbf{2 0 0 8}$ & $\mathbf{2 0 0 7}$ & $\mathbf{2 0 0 7} \mathbf{- 2 0 1 0}$ \\
\hline Biotechnologie & 5 & 5 & 2 & 1 & 13 \\
Chemie Verfahrenstechnik & 2 & 1 & 4 & 1 & 8 \\
Elektrotechnik & 1 & 2 & 4 & 4 & 11 \\
Informationstechnologie & 1 & & 1 & & 2 \\
keine Technologie & 5 & 5 & & 12 & 10 \\
Maschinenbau & 2 & 9 & 17 & 4 & 10 \\
Medizintechnik & & 2 & 4 & 22 & 94 \\
\hline Gesamtergebnis & 16 & 24 & 32 & &
\end{tabular}

\footnotetext{
${ }^{18}$ Anmerkung: "zu marktkonformen Bedingungen" bedeutet in diesem Fall gegen vollem Kostenersatz.
} 
Im Rahmen des Innovationsschutzprogramms ipp wurden die meisten Projekte (in Summe 40 von 94 Projekten) im Bereich Maschinenbau gefördert; andere Technologiebereiche wie die Biotechnologie (mit insgesamt 13 geförderten Projekten), die Elektrotechnik (11), die Medizintechnik (10) und die Chemie Verfahrenstechnik (8) sind zwar durchaus vertreten, jedoch eindeutig weniger häufig. Bemerkenswert ist, dass gerade der Bereich der Biotechnologie in den letzten Jahren eine zunehmende Tendenz in den geförderten Projekten verzeichnen konnte; so wurde 2007 lediglich ein Projekt in diesem Bereich gefördert, während 2009 und 2010 die Zahl der geförderten Projekte auf immerhin jeweils 5 anstieg; ein gegenläufiger Trend ist unterdessen im Bereich Elektrotechnik und Maschinenbau zu verzeichnen.

Geht es um die Wirkungssteuerung des Programms ipp insgesamt, so stellten Gassler et al. (2010, S. 78) hierzu fest, dass ,...das Innovationsschutzprogramm in der ersten Förderperiode durchaus dazu beigetragen hat, das IP-Bewusstsein österreichischer Unternehmen zu erhöhen und den Schutz geistigen Eigentums in Zielländern wie etwa China zu verbessern." Darüber hinaus hat eine Umfrage unter ausgewählten Fördernehmern gezeigt, dass diese angesichts der Erfahrungen mit der aws und deren Leistungsangebot - durchaus motiviert sind, ihre unternehmensinterne Strategie zwecks Innovationsschutzes in Zukunft auszubauen.

\section{Bewertung durch das Evaluierungsteam}

Mit dem Innovationsschutzprogramm ipp hat die aws einen weiteren Schritt gesetzt, geistiges Eigentum von Klein- und Mittelunternehmen durch Vermarktung und Durchsetzung von Schutzrechten zu stärken. Gerade die operative Hilfestellung durch aws ExpertInnen hat dazu beigetragen, aws intern länderspezifisches Know-How und weitreichende Kompetenzen aufzubauen. Ergänzt wird dies durch das vielschichtige Netzwerk von externen Kooperationspartnern/ Dienstleistern, welche- fallabhängig - zur Unterstützung beigezogen werden.

Sind bis dato all die Serviceleistungen der aws für Klein- und Mittelunternehmen unentgeltlich so stellt sich die Frage, ob und inwieweit Unternehmen einen (finanziellen) Beitrag für ihre in Anspruch genommenen Leistungen in Zukunft leisten können. Auch ist anzustreben, dass die Vermarktung und Durchsetzung von Schutzrechten ohne regionale Einschränkung seitens der aws angeboten wird, wobei bei der Durchsetzung von Schutzrechten bereits ab Mitte 2011 der Fokus auf alle Wachstumsmärkte ohne regionale Einschränkung ausgedehnt wird. Die aws - als österreichisches Kompetenzzentrum für die Vermarktung und Durchsetzung von Schutzrechten - hat hierzu bereits umfassende Expertise aufgebaut und soll diese weiterhin vor allem dem Unternehmenssektor zur Verfügung stellen. Neben der Prämisse, soweit wie möglich unbürokratisch zu sein, sollen auch die Entscheidungskriterien für eine Förderung im IPR-Bereich transparent sein. Richtungsweisend sollen hierbei (1) das zu erwartende wirtschaftliche Potential sowie (2) die Qualität des Entwicklungs- und Nutzungsplans des zu unterstützenden Unternehmens sein.

Was die Universitäten als Fördernehmer anbelangt so hat bislang keine einzige Universität das Unterstützungsangebot der aws im Rahmen von ipp genutzt; demnach gilt es auch zu überlegen, inwieweit ein solches Programm auch in Zukunft für Forschungseinrichtungen offen (bzw. in abgeschwächter Form unentgeltlich) sein soll. Angesichts der Programmentwicklung liegt der Betreuungsfokus und dessen Erfolg im Rahmen des Innovationsschutzpro-
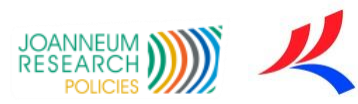
gramms eindeutig auf dem Unternehmenssektor.

\subsection{5 discover.IP}

Das Österreichische Patentamt und die aws starteten im Oktober 2008 - mit Unterstützung des Europäischen Patentamts - die Initiative discover.IP. Bei der Initiative discover.IP werden technologieorientierte Klein- und Mittelunternehmen in Österreich beraten, wie sie geistige Eigentumsrechte optimal nutzen können bzw. diese werden hinsichtlich ihres Rechtsschutzes informiert. Adressiert werden vor allem Klein- und Mittelunternehmen, die wenig bis gar keine Erfahrung im Bereich Schutzrechtsanmeldungen besitzen.

Das Projekt wird vom Österreichischen Patentamt in Kooperation mit der aws durchgeführt. Aufgabe des Österreichischen Patentamts dabei ist es, Expertise in den Bereichen gewerbliche Schutzrechte, Patentrecherche, Geheimhaltung und Dokumentation einzubringen; die aws ist demgegenüber angehalten, Expertise, die das wirtschaftliche Know-How umfasst (wie z.B. Finanzierung, Patentverwertung, Lizenzierung) bereitzustellen.

Im Konkreten besuchen ExpertInnen des Österreichischen Patentamts und der aws das (interessierte) Unternehmen und erheben direkt vor Ort die jeweiligen Schutzmöglichkeiten; dabei werden die Schutzrechte vor dem Hintergrund der spezifischen Situation und Strategie des Unternehmens sowie im Weiteren im Kontext mit dem jeweiligen Kooperations- und Lieferantennetzwerk erhoben. Basierend auf diesen Ermittlungen wird von den ExpertInnen eine Analyse erstellt, welche in der Regel in einem Bericht zusammengefasst und gegebenenfalls auch präsentiert werden kann. Ziel ist es, die wichtigsten Handlungsoptionen mit dem jeweiligen Unternehmen zu besprechen und Fragen im Bereich IPR zu beantworten. All diese (Service-)Leistungen werden sowohl seitens des Österreichischen Patentamts als auch seitens der aws unentgeltlich angeboten.

In der Periode 2008-2010 sind insgesamt 118 Projekte eingereicht worden, wobei sich die Zahl der eingereichten Projekte - wie Tabelle 21 darstellt - im ersten Jahr auf 12 belief; diese Zahl hat sich in den folgenden Jahren beträchtlich gesteigert, sogar mehr als vervierfacht, auf 51 Projektanträge im Jahr 2009 bzw. 55 Projektanträge im Jahr 2010. Die teilnehmenden Unternehmen sind erwartungsgemäß - nicht zuletzt auf Grund der Programmintention von discover.IP - fast ausschließlich Klein- und Mittelunternehmen: Von insgesamt 118 Fördernehmern sind 114 (97\%) als Klein- und Mittelunternehmen zu bezeichnen; demgegenüber ist die Zahl der eingereichten Projekte von Seiten der Großunternehmen und Privaten verschwindend gering.

Tabelle 20: Eingereichte Projekte von discover.IP nach Unternehmensgröße, 2008 - 2010

\begin{tabular}{lrrrr}
\hline Projekte & $\mathbf{2 0 1 0}$ & $\mathbf{2 0 0 9}$ & $\mathbf{2 0 0 8}$ & $\mathbf{2 0 0 8 - 2 0 1 0}$ \\
\hline Großunternehmen & & 1 & & 1 \\
KMU & 55 & 49 & 10 & 114 \\
Privat & & 1 & 2 & 3 \\
\hline Gesamt & 55 & 51 & 12 & 118 \\
\hline Quelle: aws & & & &
\end{tabular}


Etwa die Hälfte der Antragsteller im Rahmen von discover.IP kommt - die Förderperiode 2008-2010 betrachtend (siehe Abbildung 22) - aus Wien, gefolgt von Niederösterreich (13\%), Oberösterreich (11\%) und der Steiermark (10\%).

Abbildung 22: Eingereichte Projekte von discover.IP nach Bundesland, 2008-2010

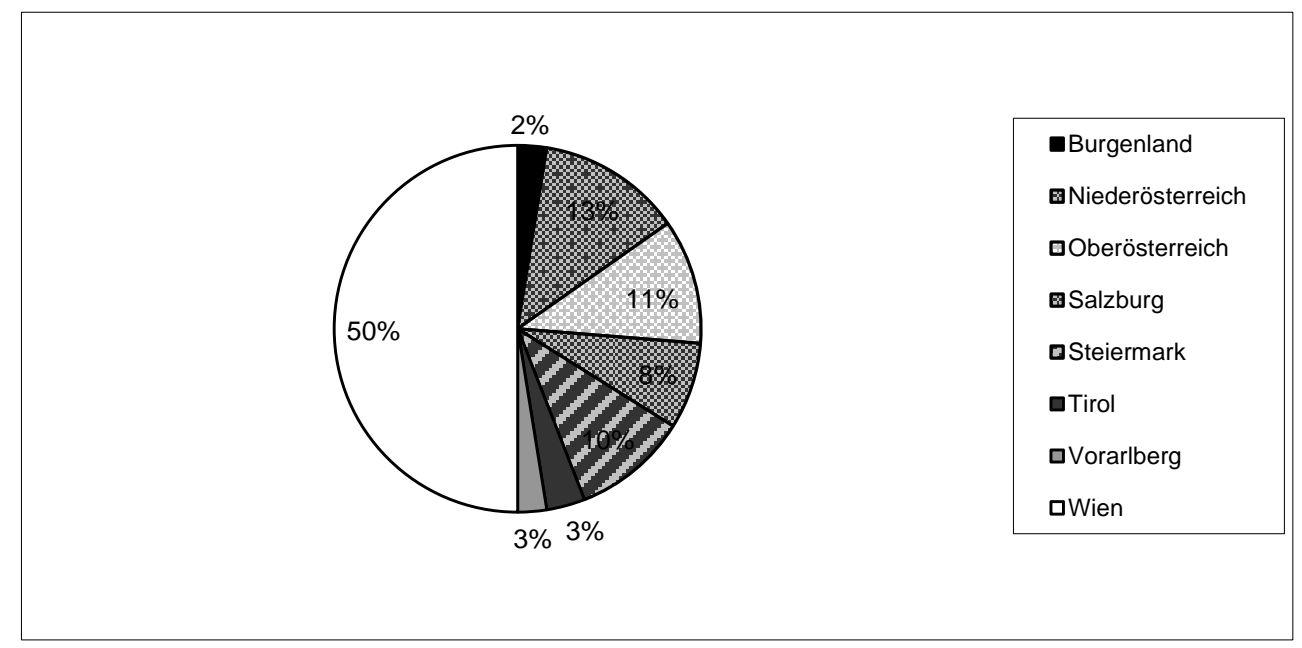

Quelle: aws

Was die Aktivitäten der Initiative discover.IP in den einzelnen Technologiebereichen betrifft (siehe Tabelle 21), so ist über die gesamte Förderperiode hinweg der Bereich Maschinenbau mit insgesamt 36 eingereichten Projekten der stärkste Technologiezweig, gefolgt von der Kreativwirtschaft mit insgesamt 21, der Elektrotechnik mit insgesamt 20 und der Informationstechnologie mit insgesamt 17 Projekten. Auffällig dabei ist, dass in jüngster Zeit - ins besonders Unternehmen aus der Kreativwirtschaft verstärktes Interesse an den unentgeltlichen Leistungen der Initiative discover.IP gezeigt haben. Ansonsten liegt der Schwerpunkt der eingereichten Projekte durchgängig im technischen, mittel-innovativen Bereich.

Tabelle 21: Eingereichte Projekte von discover.IP nach Technologie, 2008 - 2010

\begin{tabular}{lrrrr}
\hline Projekte & $\mathbf{2 0 1 0}$ & $\mathbf{2 0 0 9}$ & $\mathbf{2 0 0 8}$ & $\mathbf{2 0 0 8 - 2 0 1 0}$ \\
\hline Biotechnologie & & 1 & 1 & 2 \\
Chemie Verfahrenstechnik & 2 & 5 & 1 & 8 \\
Elektrotechnik & 11 & 6 & 3 & 20 \\
Informationstechnologie & 5 & 10 & 2 & 17 \\
keine Technologie & 1 & 7 & 1 & 9 \\
Kreativwirtschaft & 17 & 2 & 2 & 21 \\
Maschinenbau & 18 & 16 & 2 & 36 \\
Medizintechnik & 1 & 2 & & 3 \\
Physik & & 1 & & 1 \\
Verkehr und Logistik & & 1 & & 1 \\
\hline Gesamtergebnis & 55 & 51 & 12 & 118 \\
\hline Quelle: aws & & & &
\end{tabular}


Die Initiative discover.IP wurde ausgehend vom Europäischen Patent implementiert. Sie wird nicht nur in Österreich, sondern auch in einer Vielzahl anderer europäischer Länder umgesetzt und zeichnet sich dadurch aus, dass nicht nur die Beratungskomponente, sondern auch die wirtschaftliche Verwertung abgedeckt wird. Die anfallenden Kosten werden dabei auf die auf den IPR spezialisierten Organisationen aufgeteilt: So beteiligt sich u.a. das Europäische Patentamt an den Kosten (25\%), während die anderen $75 \%$ der Kosten vom Österreichischen Patentamt gemeinsam mit der aws - mit Zustimmung des BMWFJ - getragen werden. Möchte man die Leistungen pro Projekt in etwa beziffern, so belaufen sich die Beratungskosten im Rahmen von discover.IP auf rund 1.500.- EUR.

Die beiden Kooperationspartner aws und das Österreichische Patentamt teilen sich jedoch nicht nur die Kosten der auf Klein- und Mittelunternehmen fokussierten IPR-Initiative. Wie bereits zuvor erwähnt ist es ein großes Asset von discover.IP, dass die bereits in der aws vorhandenen Kompetenzen durch das fachliche Detailwissen der Patentamt-KollegInnen ergänzt werden. Damit werden nicht nur Synergieeffekte genutzt, sondern es steht den betreuten Unternehmen ein Team an ExpertInnen zur Verfügung, welches sowohl betriebliche Notwendigkeiten als auch schutzrechtliche Erfordernisse für den jeweiligen Fall individuell berücksichtigen kann.

Auch eine Evaluierung der erbrachten Leistungen findet im Rahmen von discover.IP bereits statt. So werden nach Auskunft des Österreichischen Patentamts die erbrachten Leistungen regelmäßig in Form einer Kundenzufriedenheitsanalyse unterzogen. Erste Auswertungen zeigen, dass das Feedback der teilnehmenden Unternehmen überdurchschnittlich gut ist und weiterhin Bedarf an einer solchen umfassenden Dienstleistung (im Sinne einer kundenindividuellen IPR-Beratung) besteht. Wichtig dabei ist, dass nicht nur der Eigentumsschutz per se in Abstimmung mit der Unternehmensstrategie analysiert wird, sondern dass ein ganzheitlicher Ansatz verfolgt wird, d.h. dass alle Optionen des Eigentumsschutzes (Geheimhaltungsstrategien bzw. -vereinbarungen, Patente, Marken, Urheberrechte, Kooperationsverträge etc.) dargelegt werden.

\section{Bewertung durch das Evaluierungsteam}

Überaus positiv zu bewerten ist das Faktum, dass die Initiative discover.IP einen ganzheitlichen Ansatz verfolgt, d.h. dass die Bewertung von Innovationen bzw. Erfindungen umfassend vor dem Hintergrund des unternehmerischen Kontextes stattfindet. Die Analyse wird unter Berücksichtigung der Unternehmensziele, der Kapazitäten, des Technologiefeldes, der Einbettung in das jeweilige Umfeld inklusive Lieferanten und Kooperationspartnern sowie der Finanzmittel erstellt und darauf basierend individuelle Schutzrechtsstrategien erarbeitet. Erste Evaluierungsergebnisse bestärken die Intention von discover.IP, dass gerade Klein- und Mittelunternehmen beim Aufbau von Schutzrechten auf externe Expertise und finanzielle Unterstützung angewiesen sind.

Weiters bestärkt werden die Ziele von discover.IP, dass solche Initiativen angesichts der Relevanz des geistigen Eigentumsschutzes auch bereits in anderen europäischen Ländern gestartet wurden. Das Europäische Patentamt nimmt dabei durchaus die Rolle des Multiplikators wahr. Vor diesen Hintergrund gilt es nun auch discover.IP weiterzuentwickeln. Dabei sollte nicht nur der Bedarf österreichischer Klein- und Mittelunternehmen an einer von ExpertInnen auszuarbeitenden Schutzrechtsstrategie wegweisend sein, auch Erfahrungen anderer

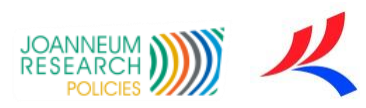


Länder in die Weiterentwicklung sollten miteinfließen; letzteres, um bspw. abzuklären, inwieweit Unternehmen in Zukunft auch einen finanziellen Beitrag zu discover.IP leisten könnten.

\subsubsection{Internationale Projekte}

Um bestehende Förderprodukte weiterzuentwickeln und stets zu verbessern sowie um zusätzlich einen Zugang zu Finanzierungsmitteln zwecks Förderung des Wissens- und Technologietransfers zwischen Wissenschaft und Wirtschaft auch auf europäischer Ebene für österreichische Unternehmen zu ermöglichen, kooperiert die aws aktiv in supra-nationalen Netzwerken mit Playern wie Europäischen Förderbanken und Finanzinstituten.

Die Kooperation und Beteiligung an solchen auf europäischer Ebene institutionalisierten Netzwerken haben dabei zum Ziel:

a. Erfahrungen und Know-How im Finanz- und Förderwesen auszutauschen sowie

b. die Zusammenarbeit mit (Finanz-)Institutionen der Europäischen Union zu vertiefen.

Für die aws von strategischer Bedeutung sind dabei vor allem zwei Mitgliedschaften, zum einen die Mitgliedschaft an der Association Européenne du Cautionnement Mutuel und zum anderen die Mitgliedschaft am Network of European Financial Institutions for SMEs. Beide solchen auf europäischer Ebene institutionalisierten Netzwerke sollen in Folge kurz dargestellt werden:

\section{Association Européenne du Cautionnement Mutuel (AECM)}

AECM ist die Vereinigung der europäischen Bürgschaftsbanken. Im Jahr 1992 gegründet vertritt AECM heute 34 Gesellschaften und Verbände, die als Netzwerk von Bürgschaftsorganisationen in 18 Ländern des Europäischen Wirtschaftsraums kooperieren bzw. zusammenarbeiten. Dabei strebt die Vereinigung folgende Ziele an:

- Vertretung der gemeinsamen Interessen der Mitglieder,

- Stärkung und Weiterentwicklung der Rolle von Bürgschaften als ein besonderes Mittel zur Förderung von KMUs

- Werbung für die Harmonisierung der rechtlichen Rahmenbedingungen und Verbesserung der finanziellen Bedingungen der Unternehmen,

- Förderung des Informationsaustausches zwischen den Mitgliedern sowie

- Einbringen von Ideen und Vorschlägen gegenüber den verschiedenen Beteiligten in der Wirtschaftspolitik einschließlich der Europäischen Union.

\section{Network of European Financial Institutions for SMEs (NEFI)}

Das Netzwerk Europäischer KMU-Finanzierungsinstitute NEFI wurde 1999 gegründet und besteht heute aus 13 Finanzierungsinstituten aus 13 Mitgliedstaaten der Europäischen Union. Als europäisches Netzwerk der KMU-Finanzierungsinstitute hat NEFI Ziele wie folgt:

- Einen langfristigen und konstruktiven Dialog zum Thema KMU-Finanzierung mit den Institutionen der Europäischen Union oder mit anderen europäischen Verbänden und Vereinigungen, die sich mit KMU-Themen beschäftigen, aufrecht zu erhalten.

- Der EU und ihren Finanzierungsinstitutionen bei der Planung und Durchführung von EU- 
weiten Förderungs- und Finanzierungsplänen Know-How und Beratung anzubieten.

- Den Zugang für KMUs sowohl zu europäischen als auch nationalen Finanzierungsinstrumenten zu erleichtern.

Zählt es zu einen der prioritären Zielen der Europäischen Union - gerade im Vergleich zu globalen Innovationsführern wie USA oder Japan - strukturelle Schwächen im Technologieund Wissenstransfer auszugleichen, so hat die Europäische Kommission selbst bereits zahlreiche Schwerpunkte zum Thema Stärkung des Technologie- und Wissenstransfersystems gesetzt. Um hier anzuknüpfen, hat die aws im Jahr 2005 - in Abstimmung mit den Auftraggebern/ Ministerien - begonnen, die Teilnahme an Europäischen Projekten strategisch zur Verbesserung der eigenen Handlungsfelder im Bereich Patent- und Lizenzmanagement (PLM) zu nutzen. Folgende vier Zielsetzungen wurden dabei festgehalten:

○ Verstärkte Nutzung des europäischen Marktes für die transnationale Vermarktung österreichischer Forschungsergebnisse und Technologien

○ Erhöhung des Wirkungspotentials der PLM-Kernprogramme (tecma, uni:invent, ipp etc.)

- Mitgestaltung der Rahmenbedingungen für Wissens- und Technologietransfer (u.a. Förderschwerpunkte, Qualitätsstandards, Kompetenzentwicklung)

○ Bildung strategischer Partnerschaften und Austausch von Good Practices

In den Jahren 2005 bis 2010 hat sich die aws, im Speziellen der Bereich PLM, somit an insgesamt 19 Einreichungen beteiligt, wobei neun Projekte erfolgreich evaluiert und mit Mitteln europäischer Programme kofinanziert wurden. Vier Projekte wurden in diesem Zeitraum bereits abgeschlossen (siehe in Tabelle 22 die Projekte 1-4), weitere fünf Projekte wurden gestartet. Bei vier Projekten übernahm die aws die Rolle des Koordinators, ebenso oft war bzw. ist die aws Partner. 
Tabelle 22: aws Beteiligungen an EU-Programmen im Bereich IPR, 2005-2010

\begin{tabular}{|c|c|c|c|c|}
\hline & Projekt & Ziel/Inhalt des Projekts & Rolle der aws & Motivation der aws \\
\hline 1 & $\begin{array}{l}\text { IRC Stakeholder- } \\
\text { Tool }\end{array}$ & $\begin{array}{l}\text { Entwicklung einer Softwareplattform } \\
\text { zur Implementierung von TT* Profi- } \\
\text { len auf Webseiten }\end{array}$ & Koordinator & $\begin{array}{l}\text { Zugang zu web- } \\
\text { basierten TT- } \\
\text { Plattformen; Zusam- } \\
\text { menarbeit mit der FFG }\end{array}$ \\
\hline 2 & Trans-Bio & $\begin{array}{l}\text { Förderung des TT Potentials zwi- } \\
\text { schen Europa und Nordamerika }\end{array}$ & Partner & $\begin{array}{l}\text { Abschluss von Lizenz- } \\
\text { und Options- } \\
\text { vereinbarungen für } \\
\text { österr. Biotech- } \\
\text { Technologien }\end{array}$ \\
\hline 3 & CERT-TTT-M & $\begin{array}{l}\text { Ausbildungsrahmen zur Zertifizie- } \\
\text { rung von TT Managern in Europa }\end{array}$ & Koordinator & $\begin{array}{l}\text { Standardisierter und } \\
\text { europaweit anerkannter } \\
\text { Trainingsstandard für } \\
\text { aws MitarbeiterInnen } \\
\text { im Bereich TT }\end{array}$ \\
\hline 4 & $\begin{array}{l}\text { Transatlantic IPR } \\
\text { Collaboration }\end{array}$ & $\begin{array}{l}\text { Abstimmung der IPR-Strategie zwi- } \\
\text { schen EU und U.S. bezüglich Dritt- } \\
\text { staaten }\end{array}$ & Koordinator & $\begin{array}{l}\text { Bekanntmachung von } \\
\text { ipp und Einholen von } \\
\text { Best Practise- } \\
\text { Erfahrungen }\end{array}$ \\
\hline 5 & $\begin{array}{l}\text { CIP/ EEN Net- } \\
\text { work }\end{array}$ & $\begin{array}{l}\text { Service-Netzwerk für KMUs, Unter- } \\
\text { stützung bei Internationalisierungsak- } \\
\text { tivitäten }\end{array}$ & Subcontractor & $\begin{array}{l}\text { Abschlüsse von Ko- } \\
\text { operationen und IPR } \\
\text { Services für das EEN } \\
\text { Netzwerk }\end{array}$ \\
\hline 6 & Centrope_TT & $\begin{array}{l}\text { Förderung von Wissens- und Techno- } \\
\text { logietransferkapazitäten in der Regi- } \\
\text { on Centrope }\end{array}$ & Partner & $\begin{array}{l}\text { Entwicklung von TT } \\
\text { Trainingsmodulen; } \\
\text { Schulung eigener Mit- } \\
\text { arbeiterInnen }\end{array}$ \\
\hline 7 & $\begin{array}{l}\text { SEE-IFA Net- } \\
\text { work }\end{array}$ & $\begin{array}{l}\text { Aufbau eines Netzwerks von Innova- } \\
\text { tionsfinanzierungsagenturen }\end{array}$ & Koordinator & $\begin{array}{l}\text { u.a. Prüfung der } \\
\text { Machbarkeit eines } \\
\text { Patentfonds }\end{array}$ \\
\hline 8 & IPRforSEE & $\begin{array}{l}\text { Analyse und Implementierung von } \\
\text { IPR Services für KMU in der Südost- } \\
\text { europäischen Region }\end{array}$ & Partner & $\begin{array}{l}\text { Benchmarking und } \\
\text { Weiterentwicklung der } \\
\text { aws IP-Services insb. } \\
\text { von discover.IP }\end{array}$ \\
\hline 9 & $\begin{array}{l}\text { EuKTS - Europe- } \\
\text { an Knowledge } \\
\text { Transfer Society }\end{array}$ & $\begin{array}{l}\text { Machbarkeitsstudie zur Gründung } \\
\text { einer Europäischen Gesellschaft zur } \\
\text { Förderung des Berufsbilds eines } \\
\text { Knowledge Transfer Professional }\end{array}$ & Partner & $\begin{array}{l}\text { Fortsetzung von } \\
\text { CERT-TTT-M in } \\
\text { Richtung eines institu- } \\
\text { tionellen Rahmens für } \\
\text { die Zertifizierung von } \\
\text { TT- Managern }\end{array}$ \\
\hline
\end{tabular}

*Technologietransfer

Quelle: aws

Wie Tabelle 23 darstellt hat sich die aws von 2005-2010 an Projekten mit einem Gesamtvolumen von mehr als 10 Mio. EUR beteiligt, wobei der aws Budgetanteil bei etwa einem Fünftel (2 Mio. EUR) liegt. Die Finanzierungsquoten der EU liegen zwischen 75 und $100 \%$, allerdings beziehen sich diese lediglich auf die direkten Projektkosten. Thema-tisch sind drei Projekte dem 6. Rahmenprogramm, ein Projekt dem 7. Forschungsrahmenprogramm sowie 
drei Projekte den territorialen Strukturfondsprogrammen für South East Europe und Central Europe zuzuordnen. Dabei steuerte die aws - den zeitlichen Ablauf betrachtend - in den ersten Jahren vorrangig die Rahmenprogramme an, während mit Beginn der aktuellen Förderperiode (2007-2013) der Fokus auf Programme des Strukturfonds gelegt wurde.

Tabelle 23: aws Beteiligungen am 6. und 7. RP und Strukturfonds, 2005 - 2010

\begin{tabular}{|c|c|c|c|c|}
\hline & $\begin{array}{l}\text { Programm/ EU-Ko- } \\
\text { Finanzierungsanteil }\end{array}$ & Projekt & Eckdaten des Projekts & aws Budget \\
\hline 1 & $\begin{array}{l}\text { 6. RP } \\
75 \%\end{array}$ & $\begin{array}{l}\text { IRC Stakeholder- } \\
\text { Tool }\end{array}$ & $\begin{array}{l}\text { Partner } \\
\text { Laufzeit: 2006-2008 } \\
\text { Gesamtvolumen: 500.000.- EUR }\end{array}$ & 230.000.- EUR \\
\hline 2 & 6. RP $75 \%$ & Trans-Bio & $\begin{array}{l}8 \text { Partner } \\
\text { Laufzeit: } 2006-2008 \\
\text { Gesamtvolumen: } 1,2 \text { Mio. EUR }\end{array}$ & 144.000.- EUR \\
\hline 3 & $\begin{array}{l}\text { 6. RP } \\
100 \%\end{array}$ & CERT-TTT-M & $\begin{array}{l}11 \text { Partner } \\
\text { Laufzeit: 2007-2009 } \\
\text { Gesamtvolumen: 1,3 Mio. EUR }\end{array}$ & 342.000.- EUR \\
\hline 4 & $\begin{array}{l}\text { DG Relex } \\
90 \%\end{array}$ & $\begin{array}{l}\text { Transatlantic IPR } \\
\text { Collaboration }\end{array}$ & $\begin{array}{l}5 \text { Partner } \\
\text { Laufzeit: 2008-2009 } \\
\text { Gesamtvolumen: } 480.000 .- \text { EUR }\end{array}$ & 102.000.- EUR \\
\hline 5 & $\begin{array}{l}\text { Competitiveness \& } \\
\text { Innovation Programme } \\
\text { (CIP) } \\
100 \%\end{array}$ & CIP/ EEN Network & $\begin{array}{l}23 \text { Partner } \\
\text { Laufzeit: 2008-2011 (plus } 2 \text { Jahre Option) } \\
\text { Gesamtvolumen: } 3 \text {,6 Mrd. EUR }\end{array}$ & 73.600.- EUR \\
\hline 6 & $\begin{array}{l}\text { Strukturfonds } \\
\text { Central Europe Pro- } \\
\text { gramme } \\
75 \%\end{array}$ & Centrope_TT & $\begin{array}{l}15 \text { Partner } \\
\text { Laufzeit: 2009-2012 } \\
\text { Gesamtvolumen: 1,9 Mio. EUR }\end{array}$ & 143.000.- EUR \\
\hline 7 & $\begin{array}{l}\text { Strukturfonds } \\
\text { South East Europe } \\
\text { Programme } \\
85 \%\end{array}$ & SEE-IFA Network & $\begin{array}{l}11 \text { Partner } \\
\text { Laufzeit: 2009-2012 } \\
\text { Gesamtvolumen: 2,9 Mio. EUR }\end{array}$ & $\begin{array}{l}\text { 900.000.- EUR } \\
\text { (davon } \\
\text { 200.000.- EUR } \\
\text { für Patentfonds) }\end{array}$ \\
\hline 8 & $\begin{array}{l}\text { Strukturfonds } \\
\text { South East Europe } \\
\text { Programme } \\
85 \%\end{array}$ & IPRforSEE & $\begin{array}{l}11 \text { Partner } \\
\text { Laufzeit: 2009-2012 } \\
\text { Gesamtvolumen: 1,4 Mio. EUR }\end{array}$ & 200.000.- EUR \\
\hline 9 & 7. RP100\% & $\begin{array}{l}\text { EuKTS - European } \\
\text { Knowledge Trans- } \\
\text { fer Society }\end{array}$ & $\begin{array}{l}15 \text { Partner } \\
\text { Laufzeit: 2010-2012 } \\
\text { Gesamtvolumen: 0,9 Mio. EUR }\end{array}$ & 54.000.- EUR \\
\hline
\end{tabular}

Anmerkung: Der EU-Ko-Finanzierungsanteil bezieht sich lediglich auf die direkten Kosten. Quelle: aws

Die erfolgreiche Beteiligung an zahlreichen EU-Projekten zeigt, dass die zuvor erwähnten Zielsetzungen seitens der aws durchaus erreicht werden konnten. Tatsächlich war es im Zuge dieser neun EU-Projekte möglich, Technologiekooperationen abzuschließen sowie Marktkenntnisse, Kompetenzen und Programmlinien weiterzuentwickeln. Eine fast $50 \%$ Erfolgsquote bei der Einreichung ist dabei nicht nur Ausdruck für die Kompetenz in der aws, wissensintensive, länderübergreifende Konsortien aufzubauen, sondern zeigt auch, dass die 
aws - auf operativer Ebene - den oft zeitkritischen Einreichprozess in hoher Qualität organisieren kann.

Die aws hat mehrfach die Rolle des Koordinators übernommen und weist darüber hinaus einen überdurchschnittlich hohen Projektanteil am Gesamtvolumen von $20 \%$ aus. Letzteres ist auch der Ausweis dafür, dass die aws MitarbeiterInnen mit hohem Engagement die Rolle in solchen internationalen Projekten wahrnehmen. Dies wird auch dadurch bestätigt, dass die aws heute immer wieder ein angefragter Partner für EU-Projektkonsortien ist.

Was die nicht-zeitunkritischen, administrativen, internen Prozesse (wie z.B. das Verfassen von Financial Reportings) betrifft, so hat auch die aws selbst, im Besonderen der Bereich PLM, Lernprozesse durchmachen müssen. Nach anfänglichen Schwierigkeiten hat die aws gelernt, sich den administrativen Herausforderungen internationaler Projekte zu stellen, und dementsprechend sind sowohl die Prüfung von zwei Projekten durch den Rechnungshof als auch eine Systemprüfung durch einen von der EU beauftragten Wirtschaftsprüfer positiv verlaufen.

Ernüchternd ist hingegen die Situation hinsichtlich der Abdeckung der aws Overheads. Werden die direkten Kosten i.d.R. zumindest bis zu $75 \%$ von der EU finanziert, so erfolgt die Refundierung von indirekten Kosten nur in einem sehr geringen Ausmaß. Im Ergebnis sinkt die EU-Finanzierungsquote der aws Vollkosten somit je nach Programm bis zu 50 \%.

\section{Bewertung durch das Evaluierungsteam}

Im aktuellen, dem BMWFJ vorliegenden Anbot der aws zur Durchführung der Technologieförderprogramme 2011-2013, ist die Teilnahme an EU-Projekten - definiert als Begleitmaßnahme - weiterhin vorgesehen. Infolgedessen hat sich die aws im 1. Quartal des Jahres 2011 bereits an drei weiteren Projekteinreichungen beteiligt, deren Fokus nach wie vor auf den Bereichen IPR sowie Technologie- und Wissenstransfer liegt. Für die Zukunft überlegenswert wäre, dieses thematische Spannungsfeld weiter aufzumachen und dementsprechend auch die Expertise im Bereich PLM auszubauen. Als potentielle Themenfelder sind hierbei zu nennen: Creative Industries, Early Stage-Finanzierung und Open Innovation.

Was die Beteiligung an internationalen/ EU-Projekten betrifft, so ist festzuhalten, dass diese als Begleitmaßnahme der aws zu sehen ist. Damit geht einher, dass eine Entscheidung über die Teilnahme an solchen Projekten immer nach Maßgabe der unterstützenden Wirkung für die aws-Förderprogramme und Beratungsleistungen sowie der zur Verfügung stehenden Ressourcen zu treffen ist. EU-Projekte dienen vor allem dazu, die Kompetenz der aws MitarbeiterInnen zu erweitern, aber auch die Reputation der aws als Institution auf supra-nationaler als auch auf nationaler Ebene zu stärken. Vor diesem Hintergrund lässt sich auch die Abdeckung eines Minus bei den Vollkosten durch aws Mittel aus dem Auftragsvertrag rechtfertigen.

\subsection{PROGRAMM ZUR UNTERSTÜTZUNG DER KREATIVWIRTSCHAFT}

\subsection{1 impulse}

Das Programm impulse wurde im Jahr 2004 etabliert und zunächst aus den Mitteln der Nationalstiftung FTE finanziert. Im Rahmen der Vertiefung der innovationspolitischen Förde- 
rungsmaßnahmen für die Kreativwirtschaft ${ }^{19}$ wurde von Seiten des BMWFJ im Jahr 2008 die „Dachmarke“ evolve für die Zielgruppe Kreativwirtschaft initiiert, woran das Programm impulse angeknüpft wurde und nunmehr als unabhängiges Programm von Seiten der aws abgewickelt wird. Die „Dachmarke“ evolve hat die allgemeine Mission, das Innovationspotential des österreichischen Kreativsektors auszuschöpfen und weiter auszubauen und gleichzeitig dieses Innovationspotential auch für die Gesamtwirtschaft Österreich zu nutzen (im Sinne einer Vernetzung der Kreativwirtschaft mit Branchen außerhalb des eigentlichen Sektors).

Die Ziele von impulse unterscheiden sich in Primär- und Sekundärzielen und definieren sich im Einzelnen wie folgt:

Primäre Ziele

- Etablierung der Kreativwirtschaft als Impulsgeber für Innovationen und maßgeblichen Wertschöpfungsfaktor

- Stärkung der wirtschaftlichen Leistungsfähigkeit von Kreativwirtschaftsunternehmen

- Stärkung des Innovationspotentials und der Wettbewerbsfähigkeit von KMUs aller Branchen durch die Integration kreativer Leistungen in unternehmerische Vorhaben

- Optimale Betreuung potentieller GründerInnen und Unternehmen im Kreativwirtschaftsbereich

- Erhöhung der Anzahl von Kreativwirtschaftsunternehmen in Österreich, insbesondere durch Neugründungen

- Verbesserung des betriebswirtschaftlichen Know-Hows in den Kreativbranchen

- Stimulation von Investitionen - materiell und immateriell

- Etablierung Österreichs als international anerkannten Kreativstandort.

Sekundäre Ziele

- Anregung von Kooperationen sowohl zwischen Kreativwirtschaftsunternehmen als auch mit der „traditionellen“ Wirtschaft

- Erhöhung der Bedeutung kreativer Leistungen in der Wirtschaft.

Innerhalb des Programms bestehen drei unterschiedliche Förderungslinien, die entsprechend dem jeweiligen Reifegrad bzw. der Ausrichtung der Projekte jeweils angepasste Förderungsinstrumente zur Verfügung stellen und deren wesentlichen Charakteristika in Tabelle 24 dargestellt sind.

\footnotetext{
${ }^{19}$ Im Kontext von evolve/impulse wird die Kreativwirtschaft im engeren Sinne mit den Segmenten Design, Architektur, Multimedia/Spiele, Mode, Musikwirtschaft/Musikverwertung, Audiovision und Film/Filmverwertung, Medien- und Verlagswesen, Grafik, Werbewirtschaft und Kunstmarkt definiert.
} 
Tabelle 24: Übersicht über die Förderungslinien von impulse

\begin{tabular}{|c|c|c|c|}
\hline & impulse XS & impulse XL & impulse LEAD \\
\hline Programmstart & 2009 & $\begin{array}{l}2008 \text { (2. Call mit neuen } \\
\text { Richtlinien) }\end{array}$ & Erster Call 2010 \\
\hline Projektphasen & $\begin{array}{l}\text { Aktivitäten der experimen- } \\
\text { tellen Entwicklung - aus- } \\
\text { schließlich Maßnahmen zur } \\
\text { Prüfung der inhaltlichen } \\
\text { und wirtschaftlichen Mach- } \\
\text { barkeit }\end{array}$ & $\begin{array}{l}\text { Aktivitäten der experimen- } \\
\text { tellen Entwicklung - alle } \\
\text { Maßnahmen der Entwick- } \\
\text { lung und gegebenenfalls } \\
\text { auch der ersten Anwen- } \\
\text { dung und/oder Marktüber- } \\
\text { leitung. }\end{array}$ & $\begin{array}{l}\text { Aktivitäten der experimentel- } \\
\text { len Entwicklung - alle Maß- } \\
\text { nahmen der Entwicklung und } \\
\text { gegebenenfalls auch der } \\
\text { ersten Anwendung und/oder } \\
\text { Marktüberleitung }\end{array}$ \\
\hline Einreicher & $\begin{array}{l}\text { Kleinstunternehmen - aller } \\
\text { Branchen (bestehend / in } \\
\text { Gründung) / Natürliche } \\
\text { Personen }\end{array}$ & $\begin{array}{l}\text { KMU - aller Branchen } \\
\text { (bestehend / in Gründung) }\end{array}$ & $\begin{array}{l}\text { Leistungsverbünde unter } \\
\text { Mitwirkung von KMUs }\end{array}$ \\
\hline Projektinhalt & $\begin{array}{l}\text { innovative unternehmeri- } \\
\text { sche Projekte im Kontext } \\
\text { der Kreativwirtschaft }\end{array}$ & $\begin{array}{l}\text { innovative unternehmeri- } \\
\text { sche Projekte im Kontext } \\
\text { der Kreativwirtschaft }\end{array}$ & $\begin{array}{l}\text { zukunftsweisende Projekte } \\
\text { mit Modellcharakter, die auf } \\
\text { Vernetzung, Professionalisie- } \\
\text { rung und Sichtbarmachung } \\
\text { der Kreativwirtschaft abzie- } \\
\text { len }\end{array}$ \\
\hline Fördersumme & $\begin{array}{l}70 \% \text { der Projektkosten } \\
\text { bzw. max. } 45.000 \text { EUR }\end{array}$ & $\begin{array}{l}50 \% \text { der Projektkosten } \\
\text { bzw. max. } 200.000 \text { EUR }\end{array}$ & $\begin{array}{l}80 \% \text { der Projektkosten bzw. } \\
\text { max. } 300.000 \text { EUR }\end{array}$ \\
\hline Projektlaufzeit & $1 \mathrm{Jahr}$ & 3 Jahre & 1 bis 3 Jahre \\
\hline
\end{tabular}

Neben den eigentlichen monetären Förderschienen (XS, XL, LEAD) finden sich zusätzlich noch eine Reihe von begleitenden Maßnahmen und Aktivitäten, die das Ziel verfolgen einerseits die Awareness (impulse awareness) für die Leistungen der österreichischen Kreativwirtschaft zu heben und anderseits mit einschlägigen Aus- und Weiterbildungsangeboten fachspezifisches Know-How zu vermitteln und allgemein die wirtschaftliche bzw. unternehmerische Kompetenz in den Kreativbranchen anzuheben (impulse training).

Laut „Anbot für die Fortsetzung der Technologieprogramme für die Jahre 2007 bis 2009“ sind die Indikatoren zur Überprüfung der Ziele des Förderprogramms Kreativwirtschaft wie folgt definiert: 
Gesamtziele des Programms:

- Erhöhung der Bedeutung kreativer Leistungen in der Wirtschaft / Erhöhung der Awareness für die Thematik

- Entwicklung regionaler Maßnahmen für die Creative Industries

- Akzeptanz der Ausbildungsmaßnahmen (u.a. we - workshops for entrepreneurs)

- Einzelziele der Förderungslinien:

- Anzahl der Einreichungen / Anzahl der geförderten Projekte

- Projektvolumen der geförderten Projekte (Projektvolumen versus Fördervolumen)

- geplante /geschaffene Arbeitsplätze

- Steigerung der Neugründungen

- Aufteilung der Projektanträge nach Bundesländer und Branchen

Im Rahmen dieser Zwischenevaluierung wird der Zeitraum während impulse aus Mitteln der Nationalstiftung dotiert wurde, nicht weiter berücksichtigt. $\mathrm{Zu}$ beachten ist, dass das Programm durch Calls in den jeweiligen Programmlinien (XS, XL und LEAD) abgewickelt wird, wobei jährlich zwei Calls (im Frühsommer bzw. im Herbst) stattfinden ${ }^{20}$. Im Jahr 2008 wurde der erste Call noch aus den Mitteln der Nationalstiftung für FTE finanziert und erst der zweite Call basiert auf den neuen Richtlinien. Für den Aufbau einer stringenten Zeitreihe eignet sich somit erst das Jahr 2009.

\section{Programmlinie XS}

Die Programmlinie wurde im Jahr 2009 gestartet und adressiert Projekte in der frühen Phase der Ideengenerierung bzw. -bewertung. Bereits im ersten Jahr wurden insgesamt 170 Projekte eingereicht (siehe Abbildung 23), wovon 28 für eine Förderung genehmigt wurden (=Quote von 16,5 \%). Im Jahr 2010 wurden im „Call 1“ (Sommer 2010) 100 Projekte eingereicht, die bislang zu 16 genehmigten Projekten geführt haben, sowie im „Call 2“ (Oktober 2010) 59 Projekteinreichungen bei 10 genehmigten Projekten (vgl. Tabelle 25 für eine Darstellung ausgewählter Programmkennzahlen im Zeitablauf auf Ebene der einzelnen Calls). Somit ist die Genehmigungsquote wiederum annähernd auf dem Niveau von 2009. Zu berücksichtigen ist sicherlich, dass die Zielgruppe vielfach wenig bis gar keine Erfahrung mit technologiepolitischen Förderinstrumenten und -verfahren aufweist, so dass anzunehmen ist, dass teilweise Projekteinreichungen einfach nicht den formalen Kriterien entsprechen. Zusätzlich ist auch die Nachweis der Existenz von experimenteller Entwicklung für zumindest einen Teil der Projekte unmöglich bzw. sehr schwierig. Dennoch sind die Genehmigungsquoten als sehr niedrig zu bewerten und es stellt sich die Frage, inwieweit durch diese niedrigen Genehmigungsquoten künftig potentielle Antragsteller ,abgeschreckt“ werden könnten, da das Verhältnis des Aufwands zu Nutzen einer Antragstellung unter dieser niedrigen Genehmigungsquote leidet.

\footnotetext{
${ }^{20}$ Die Einreichung von Projekten ist laufend möglich, allerdings erfolgt die Bewertung im Rahmen von Juryrunden zweimal jährlich, so dass hier von „Calls“ gesprochen wird. Auch die Datenaufbereitung im Rahmen des impulse Monitoring folgt dieser Struktur.
} 
Abbildung 23: Einreichungen und genehmigte Projekte in der Programmlinie impulse XS in den Jahren 2009 und 2010

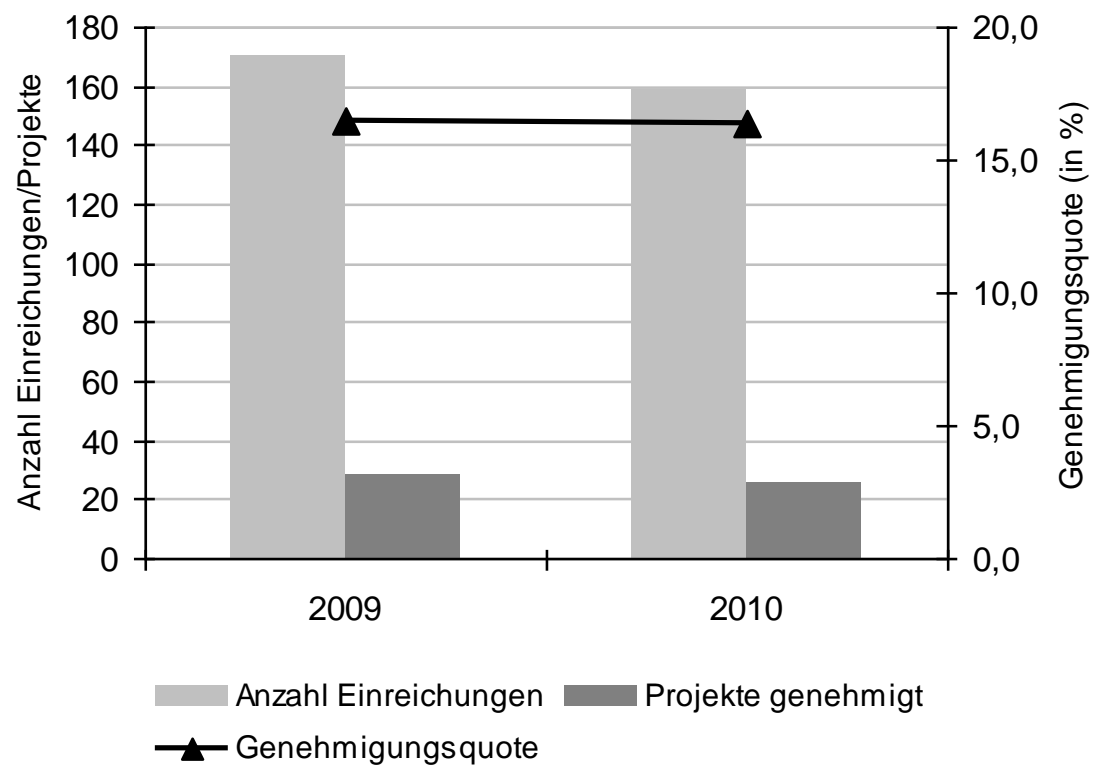

Quelle: aws, Programmmonitoring impulse

Die hohe Zahl an Projekteinreichungen - bereits in den ersten beiden Jahren - lässt darauf schließen, dass die Programmlinie XS die Zielgruppe gut erreicht und ein hoher Unterstützungsbedarf von Seiten der Kreativszene besteht.

Abbildung 24 zeigt die Volumina der eingereichten sowie genehmigten Projekte bzw. Fördersummen. Im Jahr 2009 wurden Projekte mit einem Gesamtvolumen von knapp 12 Mio. EUR eingereicht und damit eine Fördersumme von etwas über 6 Mio. EUR beantragt. Davon wurden Projekte mit einem Gesamtvolumen von knapp 1,8 Mio. EUR tatsächlich genehmigt und eine Fördersumme von knapp über 1 Mio. EUR bewilligt. Der Anteil der bewilligten Förderung an den Projektkosten liegt bei knapp $60 \%$ und somit etwas unter der in der Programmlinie vorgesehen maximalen Förderquote von $70 \%$ der Projektkosten. Die durchschnittliche Förderhöhe pro Projekt liegt bei ca. 37.500 EUR im Jahr 2009 bzw. ca. 40.000 EUR im Jahr 2010 und damit ebenfalls leicht unter der maximalen Zuschusshöhe von 45.000 EUR. 
Abbildung 24: Eingereichte und genehmigte Projekt-bzw. Fördervolumina (in EUR) in der Programmlinie impulse XS

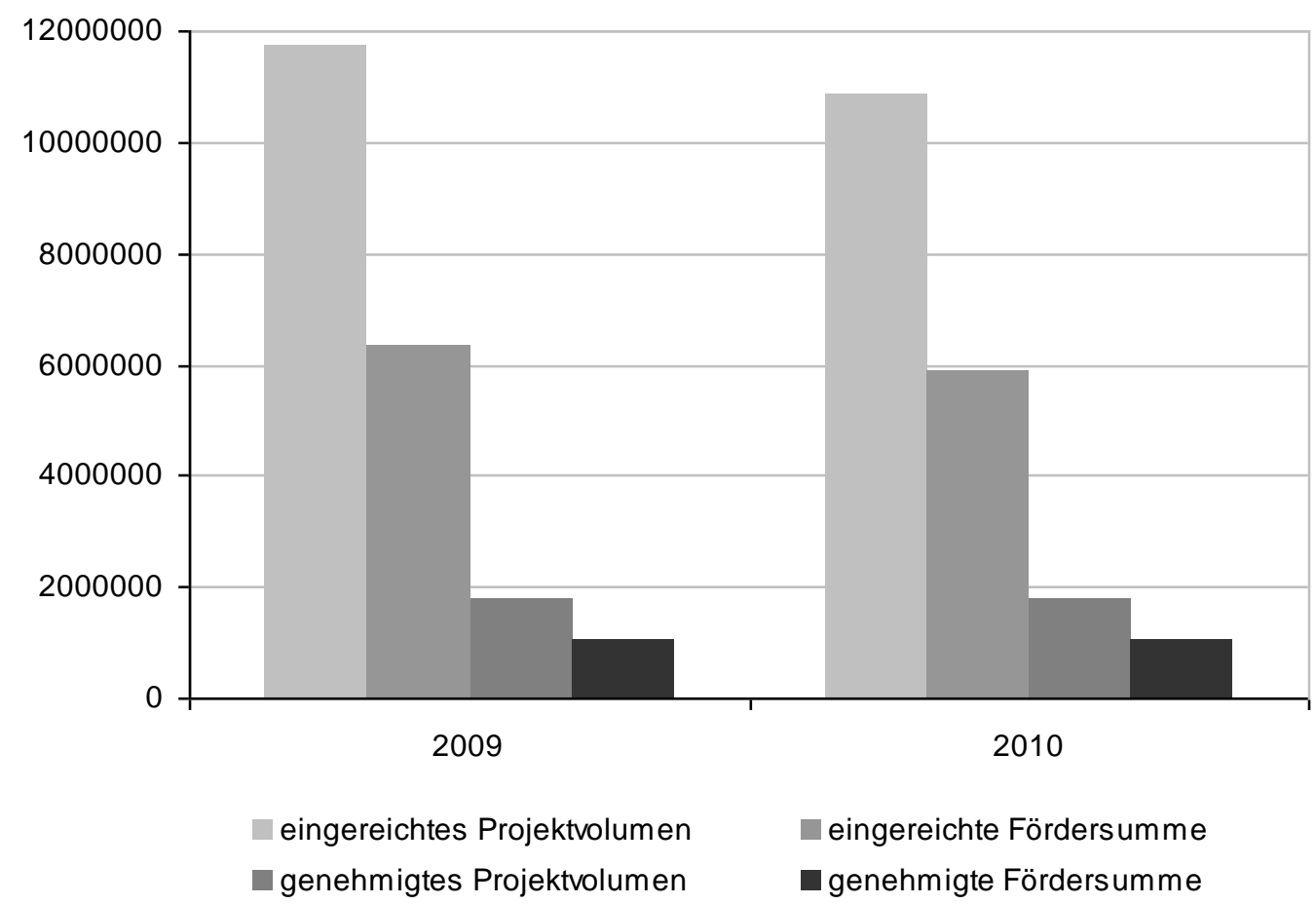

Quelle: aws, Programmmonitoring impulse

Thematisch verteilen sich die Projekte relativ gleichmäßig auf die beiden großen Themenbereiche Design und Innovative Medien mit 84 bzw. 86 eingereichten Projekten im Jahr 2009 und 75 bzw. 84 eingereichten Projekten im Jahr 2010. Betrachtet man die Themenfelder im Einzelnen dominieren im Bereich Design Projekte aus dem „Designbereich i.e.S.“ vor dem „Modebereich“, während es im Bereich Innovative Medien die Projekte aus dem Themenfeld „Multimedia/Spiele“ vor dem Themenfeld „Medien und Verlagswesen“ sind, auf die die größte Anzahl entfällt. Die Verteilung über diese Themenfelder ist dabei im bisherigen Zeitablauf sehr stabil, d.h. die anteilsmäßige Bedeutung der einzelnen Themenfelder ist zwischen 2009 und 2010 sehr ähnlich.

Hinsichtlich der geographischen Verteilung der Projekte zeigt sich ein eindeutiger Schwerpunkt auf Wien als hauptsächliche Herkunftsregion sowohl der eingereichten als auch der genehmigten Projekte. Der Anteil Wiens an den eingereichten Projekten beträgt $50 \%$ (2009) bzw. 66 \% (2010), bei den geförderten Projekten beträgt er $71 \%$ (2009) bzw. $62 \%$ (2010). Der hohe Anteil Wiens spiegelt sicherlich die überragende Bedeutung des Standorts Wien für die österreichische Kreativwirtschaft wider; inwieweit die geographische Koinzidenz mit der Förderungsagentur ebenfalls für diesen hohen Anteil von Einfluss ist, kann aufgrund der vorliegenden Datenlage nicht abgeschätzt werden. Die einzelne Verteilung auf die anderen Bundesländer ist - v.a. was die genehmigten Projekte betrifft - aufgrund der geringen Fallzahl arbiträr und lässt kaum entsprechende Rückschlüsse zu. Auffällig ist jedoch, dass Tirol - sowohl bei den Einreichungen als auch bei den genehmigten Projekten - durchgängig stark vertreten ist und jeweils mehr Aktivität aufweist als die größeren Flächenbundesländer.

Gemäß der Programmphilosophie richtet sich impulse XS an kreativwirtschaftliche Projekte 
in der Ideen- bzw. Frühphase, wo eine explizite Marktorientierung oft noch nicht gegeben ist bzw. eventuelle Marktpotentiale oft erst einer Überprüfung im Laufe des Projekts zu unterziehen sind. Daher kann erwartet werden, dass Projekte vielfach von natürlichen Personen (Angehörige der „Kreativszene“ inklusive einschlägige StudentInnen bzw. AbsolventInnen) sowie von (Mikro-)Unternehmen in Gründung getragen bzw. eingereicht werden.

Die Verteilung über drei potentielle Einreichertypen (bestehende Unternehmen, natürliche Personen, Unternehmen in Gründung) ist in Abbildung 25 dargestellt. Tatsächlich weisen Projekte von natürlichen Personen bzw. GründerInnen zusammen einen Anteil von 59 \% (2009) bzw. $61 \%$ (2010) auf. Bei den einreichenden bestehenden Unternehmen handelt es sich darüber hinaus zum weitaus überwiegenden Teil um Kleinstunternehmen. Es werden somit eindeutig die von der Programmkonzeption definierten Zielgruppen bedient. Die Tatsache, dass die Einreicher zu 29 \% (2009) bzw. zu 35 \% (2010) sich in Gründung befindliche Unternehmen sind, deutet darauf hin, dass die Programmlinie XS eine „Pipeline-Rolle“ für Unternehmensgründungen in der Kreativwirtschaft einnehmen kann. Inwieweit der beobachtete Anstieg des GründerInnenanteils an den einreichenden Projekten im Jahr 2010 ein einmaliger „Ausreißer“ ist oder den Beginn einer nachhaltigen Entwickelt darstellt, kann derzeit noch nicht abgeschätzt werden.

Abbildung 25: Typus des Antragsstellers in der Programmlinie impulse XS

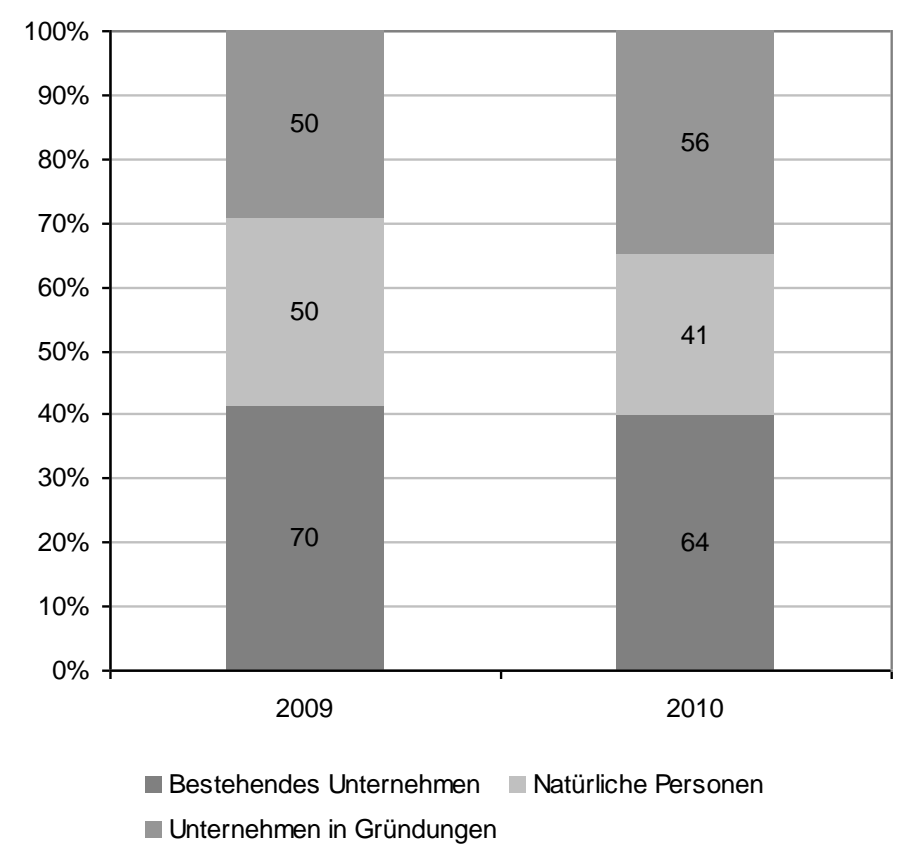

Quelle: aws, Programmmonitoring impulse

Betrachtet man den Gender-Aspekt innerhalb der Programmlinie XS so zeigt sich bei den Einreichungen ein Frauenanteil (projektbeteiligte weibliche Personen an allen projektbeteiligten Personen) von 31 \% im Jahr 2009 und 32 \% im Jahr 2010. 
Tabelle 25: Übersicht der Calls in der Programmlinie XS

\begin{tabular}{lcccc}
\hline & Call 2009/1 & Call 2009/2 & Call 2010/1 & Call 2010/2 \\
\hline Einreichungen & 109 & 61 & 100 & 59 \\
durchschnittliche Projektkosten & 65.000 & 75.000 & 69.000 & 68.000 \\
geförderte Projekte & 11 & 17 & 16 & 10 \\
durchschnittliche Fördersumme & 35.000 & 39.000 & 40.000 & 39.000 \\
Gesamtprojektvolumen (genehmigte Projekte) & 0,67 Mio. $€$ & 1,1 Mio. $€$ & 1,1 Mio. $€$ & 0,6 Mio. $€$. \\
Förderung gesamt & 0,38 Mio. $€$ & 0,67 Mio. $€$ & 0,65 Mio. $€$ & 0,39 Mio. $€$ \\
\hline
\end{tabular}

Quelle: aws, Programmmonitoring impulse

\section{Programmlinie XL}

Das erste volle Jahr auf Basis der neuen Richtlinien ist für die Programmlinie impulse XL ebenfalls 2009. Trotzdem sollen hier für die Programmlinie XL - als Vergleichswerte - einige Ergebnisse aus dem Jahr 2008 angeführt werden: Zusammen (Call 1 aus Mitteln der Nationalstiftung und Call 2 unter der neuen Richtlinie) gab es im Jahr 2008120 Projekteinreichungen $(71+49)$, wovon 20 (11 plus 9) Projekte auch tatsächlich gefördert wurden, d.h. die Genehmigungsquote lag bei 16,7\% (bzw. 15,7\% und 18,4\%). Die Gesamtprojektkosten beliefen sich für diese Projekte auf 5,8 Mio. EUR (3,6 Mio. + 2,2 Mio.), wovon 2,6 Mio. EUR (1,6 + 1,0 Mio.) durch impulse XL gefördert wurde (vgl. Tabelle 26 für eine Darstellung ausgewählter Programmkennzahlen über den Zeitablauf auf Ebene der einzelnen Calls).

Im Jahr 2009 wurden insgesamt 107 Projekte eingereicht, wovon 20 zur Förderung bewilligt wurden (was einer Genehmigungsquote von 19 \% entspricht). Im Jahr 2010 wurden 75 Projekte eingereicht, wovon 21 zur Förderung genehmigt wurden. Die Genehmigungsquote im 2010 lag bei $28 \%$ (Abbildung 26) und somit deutlich höher als im Jahr 2009. Trotzdem ist auch in der Programmlinie XL die Genehmigungsquote als relativ niedrig zu betrachten, was auf eine Unterdotierung des Programms angesichts der gegebenen Förderungsnachfrage der Zielgruppe schließen lässt. 
Abbildung 26: Einreichungen und genehmigte Projekte in der Programmlinie impulse XL in den Jahren 2009 und 2010

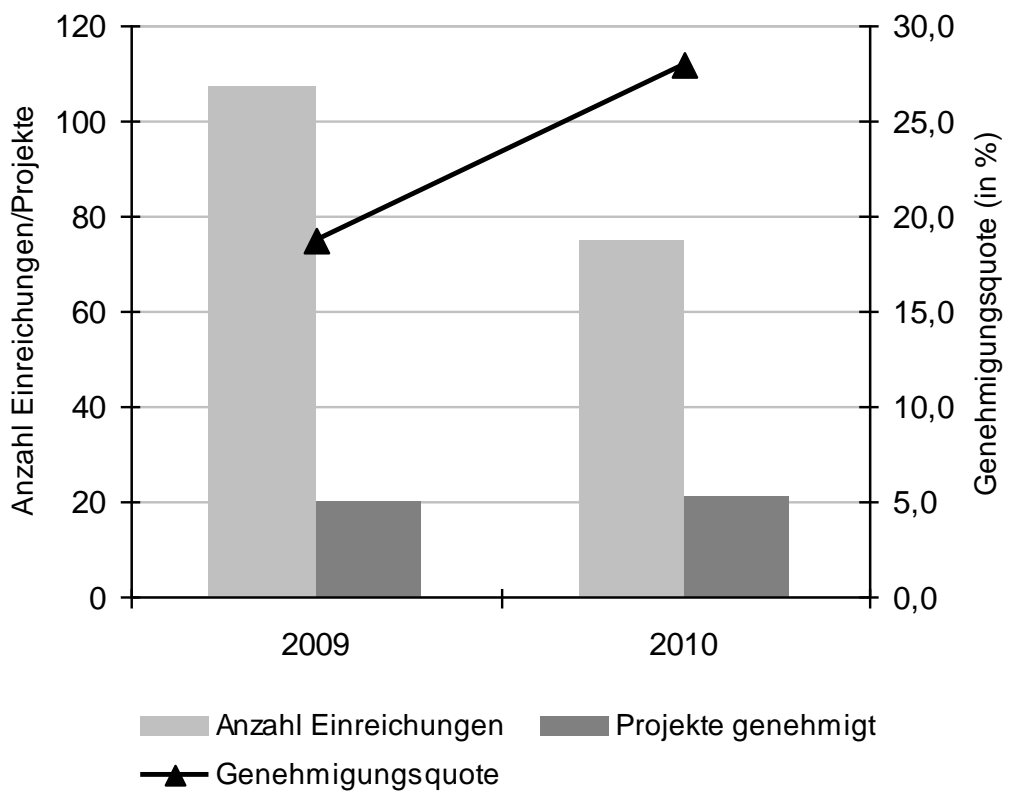

Quelle: aws, Programmmonitoring impulse

Die Volumina der eingereichten und genehmigten Projekte (bzw. der tatsächlich geflossenen Förderungen) sind in Abbildung 27 dargestellt. Die 107 eingereichten Projekte des Jahres 2009 wiesen ein gesamtes Projektvolumen von 37,8 Mio. EUR auf, die einer Förderungssumme (bei einer angenommen Förderquote von $100 \%$ ) von 13,9 Mio. EUR entsprochen hätten. Letztlich wurden Projekte mit einem Gesamtvolumen von 5,8 Mio. EUR zur Förderung genehmigt. Diese Projekte erhielten dann eine tatsächliche Förderung von insgesamt 2,6 Mio. EUR. Im Durchschnitt wurde also im Jahr 2009 ein Projekt mit einem Zuschuss von 132.000 EUR gefördert (d.h. der durchschnittliche Zuschussanteil in einem Projekt lag bei $45 \%$, wobei die Programmrichtlinien einen maximalen Förderanteil von $50 \%$ vorsehen). Die 75 eingereichten Projekte des Jahres 2010 wiesen ein Projektvolumen von 24,7 Mio. EUR auf, die einer Förderungssumme (wiederum unter der Annahme einer 100-prozentigen Förderquote) von 10,7 Mio. EUR entsprächen. Tatsächlich genehmigt wurden davon die erwähnten 21 Projekte mit einem Gesamtvolumen von 5,7 Mio. EUR, die mit insgesamt 2,8 Mio. EUR gefördert wurden, was einer durchschnittlichen Fördersumme pro gefördertem Projekt von 133.000 EUR entspricht. 
Abbildung 27: Eingereichte und genehmigte Projekt- bzw. Fördervolumina (in EUR) in der Programmlinie impulse XL

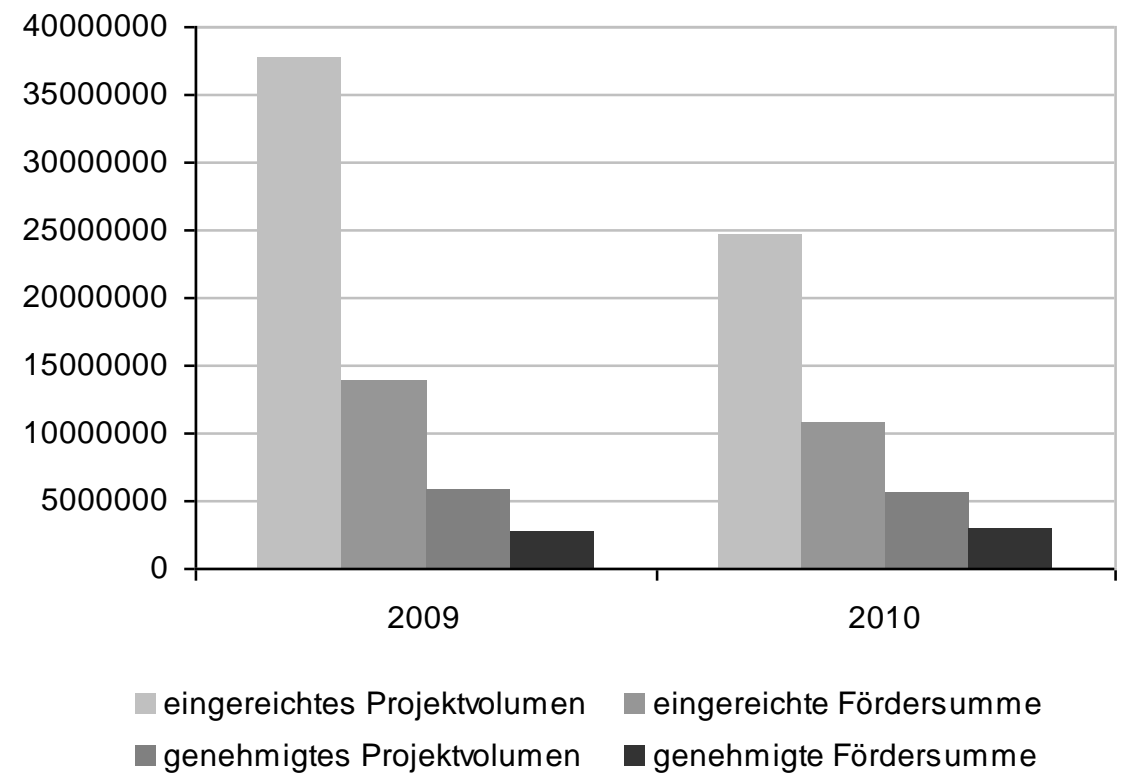

Quelle: aws, Programmmonitoring impulse

Die Verteilung der Antragssteller auf die zwei als Zielgruppen vorgesehenen „Einreichertypen“ zeigt Abbildung 28. Der Anteil bestehender Unternehmen liegt in beiden Jahren bei ca. $75 \%$. Der Anteil von in Gründung befindlichen Unternehmen von etwa einem Viertel deutet immerhin darauf hin, dass auch von der Programmlinie XL noch deutliche impulse in Richtung Neugründungen ausgehen, wie es auch den entsprechenden (Teil-)Zielen des Gesamtprogramms entspricht.

Abbildung 28: Typus des Antragsstellers in der Programmlinie impulse XL

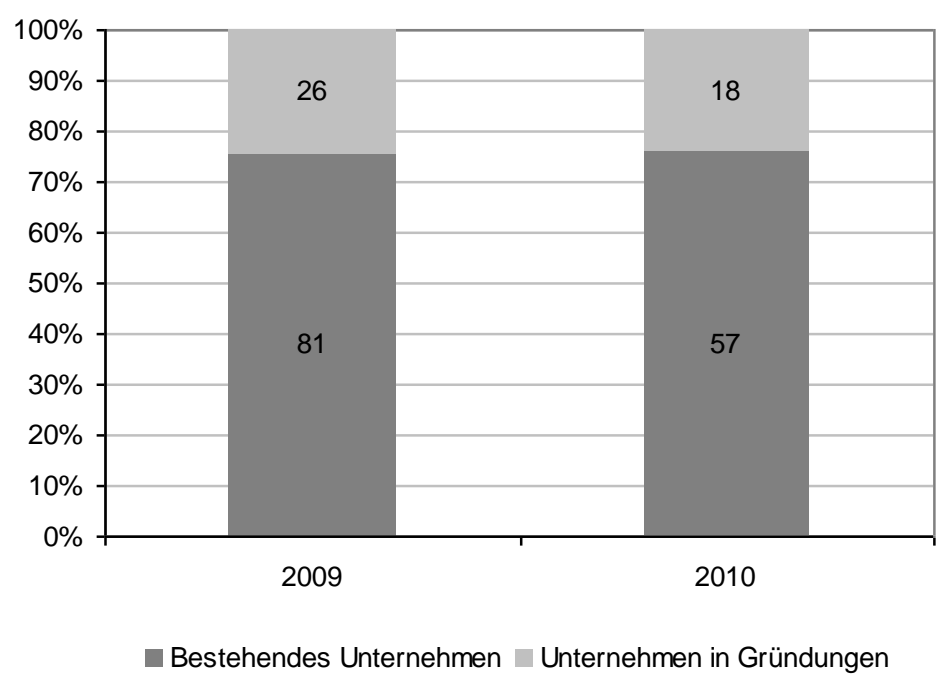

Quelle: aws, Programmmonitoring impulse

Die überwiegende Mehrzahl (2009: 61 \%, 2010: 69 \%) der eingereichten Projekte findet sich im Themenkomplex Innovative Medien und hier wiederum v.a. im Themenfeld Multime$\mathrm{dia} /$ Spiele. 
Der Frauenanteil bei den Einreichungen beträgt $19 \%$ (im Durchschnitt der beiden Calls des Jahres 2009) bzw. knapp 28 \% im Jahr 2010.

Hinsichtlich der geographischen Verteilung der Einreichungen und der Projekte zeigt sich ein starker Fokus auf Wien, wobei dieser besonders im Jahr 2009 stark ausgeprägt war (siehe Abbildung 29). Der Anteil Wiens machte im Jahr 2009 bei den Einreichungen ca. 64 \% sowie bei den geförderten Projekten ca. $70 \%$ aus. Diese starke Konzentration auf Wien ging dann im Jahr 2010 etwas zurück (Anteil Wiens $57 \%$ bei den Einreichungen und $51 \%$ bei den geförderten Projekten), wobei jedoch noch nicht abgeschätzt werden kann, inwieweit dieser Rückgang ein durch entsprechende Marketingmaßnahmen der aws (Aktivitäten in den Bundesländern etc.) bedingter Trend ist oder ob es sich um eine einmalige Verschiebung handelt.

Abbildung 29: Geographische Verteilung der eingereichten und geförderten impulse XL Projekte (Anzahl)

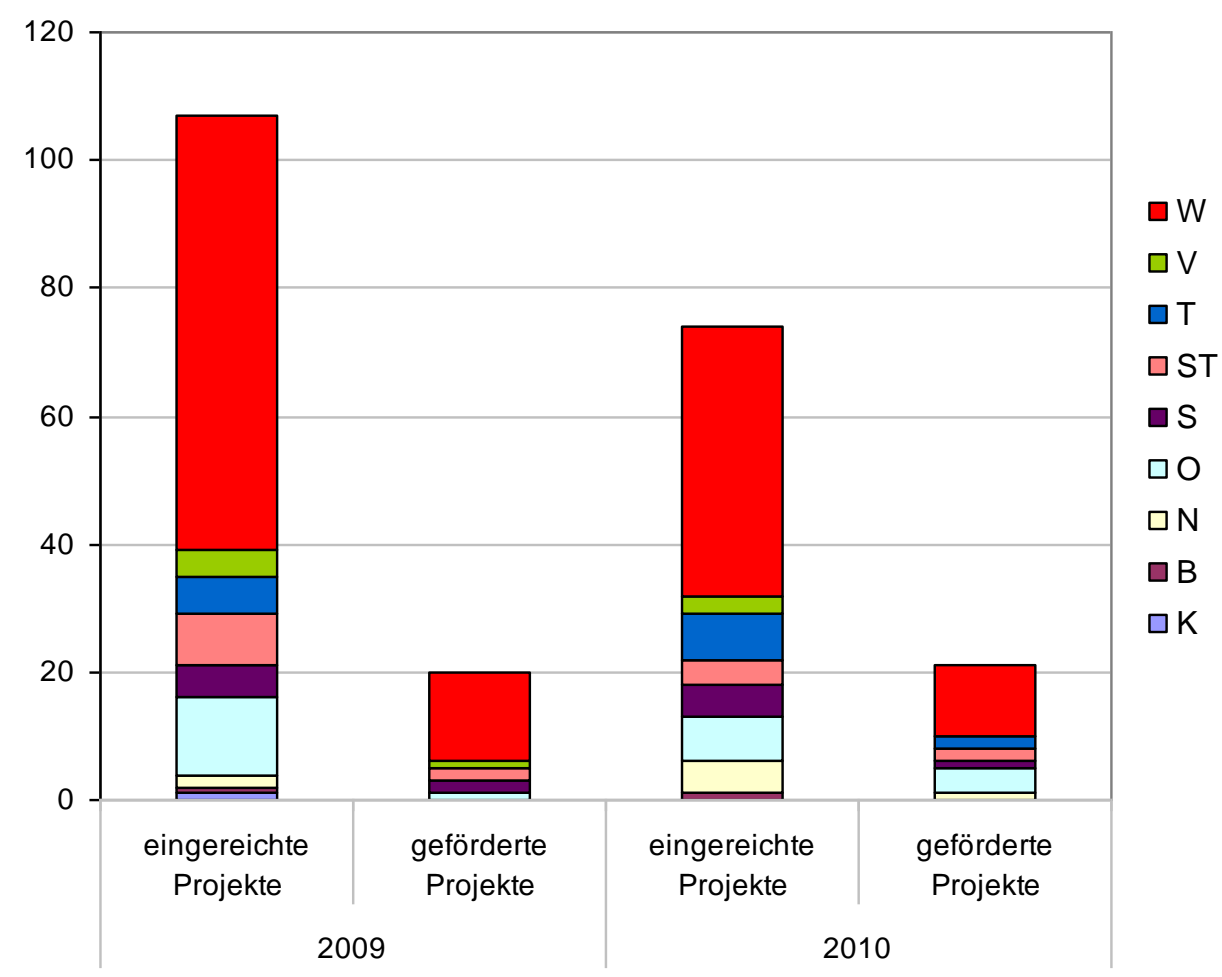

Quelle: aws

Tabelle 26: Übersicht über die Calls in impulse XL 2008 bis 2010

\begin{tabular}{lcccccc}
\hline & $\begin{array}{c}\text { Call } \\
2008 / 1^{*}\end{array}$ & $\begin{array}{c}\text { Call } \\
2008 / 2\end{array}$ & $\begin{array}{c}\text { Call } \\
2009 / 1\end{array}$ & $\begin{array}{c}\text { Call } \\
2009 / 2\end{array}$ & $\begin{array}{c}\text { Call } \\
2010 / 1\end{array}$ & $\begin{array}{c}\text { Call } \\
2010 / 2\end{array}$ \\
\hline $\begin{array}{l}\text { Einreichungen } \\
\text { durchschnittliche Projektkosten } \\
\text { (Einreichungen) }\end{array}$ & 71 & 49 & 54 & 53 & 41 & 34 \\
$\begin{array}{l}\text { geförderte Projekte } \\
\text { durchschnittliche Fördersumme }\end{array}$ & 11 & 9 & 9 & 11 & 10 & 11 \\
$\begin{array}{l}\text { Gesamtprojektvol. (gen. Projekte) } \\
\text { Förderung gesamt }\end{array}$ & 3,6 Mio. & 2,3 Mio. & 2,2 Mio. & 3,5 Mio. & 2,6 Mio. & 3,0 Mio. \\
\hline
\end{tabular}

* Call 2008/1 wurde noch mit Mitteln der Nationalstiftung finanziert Quelle: aws, Programmmonitoring impulse 


\section{Programmlinie LEAD}

Die Programmlinie impulse LEAD wurde ebenfalls im Jahr 2009 (wobei der erste Call allerdings erst im Jahr 2010 abgeschlossen wurde) als Ergänzung zu den beiden anderen Instrumenten gestartet und zielt auf die Förderung und Etablierung von Leistungsverbünden im Bereich der Kreativwirtschaft ab. Dadurch soll der Aufbau von kritischen Massen initiiert und unterstützt werden, was letztlich der Professionalisierung entlang der Wertschöpfungskette innerhalb der Kreativwirtschaft, der Vernetzung mit „herkömmlichen“ Wirtschaftsbereichen sowie generell der Sichtbarkeit kreativwirtschaftlicher Leistungen dienlich sein soll. Im Call 2010/1 wurden insgesamt 29 Projekte (eingereichtes Projektvolumen: 11,2 Mio. EUR) eingereicht, wobei drei Projekte genehmigt wurden (Projektvolumen der bewilligten Projekte: 1,1 Mio. EUR) und letztlich 0,83 Mio. EUR an Förderungszuschüssen ausbezahlt wurden. Typischerweise wurden die Projekte von Konsortien bestehend aus zwei oder mehr Partnern eingereicht (ein Projekt wurde von einer ARGE und vier Projekte von Vereinen eingereicht). Auch in der Programmlinie LEAD stammt die überwiegende Mehrheit (ca. zwei Drittel) der Einreicher (wie auch der Projektpartner insgesamt) aus Wien. Ca. $92 \%$ der Einreicher sind bestehende Unternehmen, der Rest sind in Gründung befindliche.

Aufgrund der geringen Fallzahl und der Tatsache, dass Informationen erst zu einem einzigen Call vorliegen, können für LEAD noch keine evaluierungsrelevanten Aussagen getroffen werden. Einzig die Tatsache, dass auch in dieser Programmlinie die Genehmigungsquote sehr niedrig ist (mit lediglich $10 \%$ sogar noch deutlich niedriger als in den anderen Förderungslinien), ist hervorzuheben und deutet auch hier auf einen erheblichen Überhang an förderungsfähigen Projekten gegenüber den zur Verfügung stehenden Mitteln hin.

\section{Begleitendes Maßnahmenbündel Awareness (impulse awareness)}

Mit diesem Maßnahmenbündel werden diverse Aktivitäten gesetzt, um allgemein die Relevanz der österreichischen Kreativwirtschaft und die Potentiale der vielfältigen kreativwirtschaftlichen Leistungen aufzuzeigen. Hierfür werden eine Reihe vielfältiger Initiativen und Projekte durchgeführt, vielfach auch in Kooperation mit Partnern in den Bundesländern. Die Aktivitäten reichen dabei von einer eigenen Veranstaltungsreihe in den Bundesländern (impulse infohours) bis hin zu allgemeinen Public Relations Aktivitäten (z.B. Sponsoring von entsprechenden Veranstaltungen wie Festivals, Messen etc.) und einschlägiger Medienarbeit. Jüngst werden vermehrt auch international orientierte Aktivitäten (u.a. in Kooperation / Schnittstelle mit einschlägigen kultur-/kunstorientierten Tourismusmarketing-Maßnahmen) gesetzt. Zu bemerken ist, dass aufgrund des Sponsorings nicht nur die Bekanntheit/Awareness des eigentlichen Programms impulse und der durch impulse geförderten Projekte erhöht wird, sondern positiver Nutzen für den Kreativbereich insgesamt erzielt werden kann, da die gesponserten Veranstaltungen ohne diese Unterstützung u.U. nicht im gleichen Umfang stattfinden könnten.

\section{Begleitendes Maßnahmenbündel Aus- und Weiterbildung (impulse training)}

Im Zentrum dieses Maßnahmenbündels steht das im Jahr 2006 ursprünglich in Kooperation mit departure (einem Kreativwirtschafts-Förderungsprogramm der Wirtschaftsagentur Wien) entwickelten Aus- und Weiterbildungsprogramm „we-workshops for entrepreneurs“. Mit 
diesem Programm werden GründerInnen und JungunternehmerInnen angesprochen, wobei in Form von Workshops (5-tägig aufgeteilt an jeweils zwei Wochenenden), wirtschaftsrelevantes Basiswissen (Erstellen von Business Plänen, Finanz- und Vertriebs Know-How etc.) vermittelt werden. Zusätzlich berichten einschlägige (auch internationale) UnternehmerInnen aus der Kreativwirtschaft (Mode, Musik, Design, Multimedia, Architektur) über ihre Erfahrungen und geben somit Einblick in die unternehmerische Praxis. Integrierter Bestandteil dieser Workshops ist die Vorstellung der monetären Förderungsschienen des impulse-Programms, so dass diese Workshops ebenfalls ein wichtiges Bindeglied zur angesprochenen Zielgruppe des Förderungsprogramms darstellen. Gerade aufgrund der Tatsache, dass die mit impulse angesprochene Zielgruppe geringere Erfahrungen mit betriebswirtschaftlichen Inhalten hat sowie mit den in der Wirtschaftsförderung üblichen Verfahren und Abläufen vergleichsweise wenig vertraut ist (viele kreativwirtschaftliche AkteurInnen definieren sich aus künstlerischer Sicht heraus), kommt diesen begleitenden Aus- und Weiterbildungsaktivitäten eine besondere Rolle zu.

Ab 2009 wurden diese Maßnahmen - jeweils in enger Kooperation mit einschlägigen Institutionen der Bundesländer (Tirol, Salzburg, Vorarlberg), auch in Innsbruck (unter dem Titel „we-west") durchgeführt. Typischerweise liegt die Teilnehmerzahl an diesen Workshops um die 50 bis $60^{21}$, wobei sowohl originäre GründerInnen angesprochen werden als auch MitarbeiterInnen von bestehenden Unternehmen (ca. die Hälfte der Teilnehmer sind GründerInnen).

Neben diesen we-workshops bestehen seit 2009 zusätzlich auch noch die sogenannten welectures, die in Kooperation mit design austria durchgeführt werden. Diese we-lectures sind als Vertiefung der we-workshops gedacht, wobei branchenspezifisch ein spezielles Themengebiet präsentiert wird. Zielgruppe sind v.a. AbsolventInnen von Designausbildungseinrichtungen, wobei diese auch den Standort dieser lectures darstellen. Der thematische Schwerpunkt orientiert sich hauptsächlich auf für die Gründung (bzw. das Selbstständigmachen) und den damit verbundenen Aspekten (z.B. rechtliche Situation, Urheberrecht, Kalkulation, Vermarktung etc.).

\section{Bewertung durch das Evaluierungsteam}

Das Programm impulse mit seinen unterschiedlichen Programmlinien hat sich bereits innerhalb der wenigen Jahre seines Bestehens (die Linien XS und LEAD sind im Jahr 2009 bzw. 2010 gestartet, XL wird erst seit Herbst 2008 nach den neuen Richtlinien zur Gänze von der aws abgewickelt) als exklusive Anlaufstelle für die „Kreativszene“ in Österreich etabliert. Durch die unterschiedliche Ausgestaltung der einzelnen Programmlinien ist es gelungen nicht nur die Kreativwirtschaft i.e.S. (verstanden als marktwirtschaftlich orientierte kreativwirtschaftliche Unternehmen), sondern darüber hinaus auch die Kreativszene i.w.S. (z.B. StudentInnen/AbsolventInnen von einschlägigen Studienrichtungen, Freiberufler, potentielle GründerInnen etc.) anzusprechen.

Dabei weisen die Programmlinien von impulse (insbesondere impulse XS und impulse LEAD) aufgrund ihrer erst kurzen Laufzeit und ihrer heterogenen Zielgruppe (die potentiellen FördernehmerInnen besitzen vielfach wenig bis keine Erfahrung mit wirtschaftsbezogenen

\footnotetext{
${ }^{21}$ Diese TeilnehmerInnenanzahl bezieht sich auf die Wiener Workshops.
} 
Förderungsmaßnahmen und -agenturen) noch durchaus Pilotcharakter auf. Insofern sind die bisherigen Ergebnisse so kurz nach dem Programmstart als durchgängig bemerkenswert einzustufen.

impulse XS erzielte bereits im ersten Jahr 170 Einreichungen. Diese hohe Zahl von Einreichungen hat letztlich aufgrund der begrenzten für das Programm zur Verfügung stehenden Ressourcen auch zu einer niedrigen Genehmigungsquote von ca. $16 \%$ geführt). Immer wieder geäußerte Befürchtungen hinsichtlich eventueller förderungstechnischer Schwierigkeiten, Projektförderung im Dienstleistungssektor (bzw. im konkreten Fall der Kreativwirtschaft) durchzuführen, haben sich nicht bewahrheitet. Die Tatsache, dass über $60 \%$ der Antragsteller natürliche Personen oder in Gründung befindliche Unternehmen sind, zeigt, dass eine wesentliche Zielgruppe des Programms sehr gut erreicht werden konnte und eines der zentralen (Teil-)Ziele, nämlich die Aktivierung des Gründungspotentials in der Kreativwirtschaft, realisiert werden konnte. Eine erhebliche Aufstockung der Mittel gerade im „niedrigschwelligen“ Bereich von impulse XS wäre daher für die nächsten Jahre anzudenken.

Die Programmlinie XL stellt den zentralen Baustein von impulse dar und richtet sich vorrangig an bereits bestehende Unternehmen der Kreativwirtschaft. Dennoch findet sich auch in dieser Programmlinie ein gewisser Anteil von in Gründung befindlichen Unternehmen, was die Zielorientierung des Gesamtprogramms in Richtung Forcierung der kreativwirtschaftlichen Gründungslandschaft noch einmal unterstreicht. Die geringe Genehmigungsquote (ca. 20 bis $25 \%$ ) der eingereichten Projekte deutet auch für diese Programmlinie auf eine Unterdotierung des Programms hin. Von den in den Jahren 2009 und 2010 bislang eingereichten Gesamtprojektvolumen von ca. 50 Mio. EUR konnte lediglich ein Projektvolumen von 8,4 Mio. EUR mit einer Zuschusshöhe von 3,9 Mio. EUR bedacht werden. Geht man davon aus, dass zumindest ein Teil der abgelehnten Projekte den eigentlichen Qualitätskriterien von impulse genügen würde, kann also angenommen werden, dass ein durchaus beträchtliches Volumen aufgrund von Unterdotierung nicht aktiviert werden kann ${ }^{22}$.

Die Programmlinie LEAD startete mit einem ersten Call erst im Jahr 2010, so dass derzeit noch keine robusten Aussagen möglich sind. Allerdings zeigte sich auch hier bereits im ersten Call, dass die Genehmigungsquote aufgrund der verfügbaren Ressourcen sehr niedrig (10\%) ist. Dies ist insofern bemerkenswert, als das Programm eine durchaus anspruchsvolle Zielsetzung hat, indem es keine Einzelanträge anspricht, sondern Leistungsverbünde von kreativwirtschaftlichen Unternehmen. Erste Ergebnisse des diesjährigen Kreativwirtschaftsberichts weisen auf die positiven Effekte von Kooperationsverbünden innerhalb der Kreativwirtschaft hin. Durch das Programm werden derartige Kooperationen generell attraktiviert und die Kooperationsbarrieren (z.B. Transaktionskosten der Kooperation, Fehlen von trust) abgebaut. Die rege Beteiligung zeigt, dass mit der Programmlinie tatsächlich ein bestehender Bedarf adressiert wird. Es kann somit erwartet werden, dass die Programmlinie einen wichtigen Beitrag zur spezifischen Zielsetzung, nämlich der Etablierung kritischer Massen und der synergetischen Nutzung von Kooperationen innerhalb der Kreativwirtschaft beitragen kann.

Abgerundet werden diese monetären Förderungsschienen von einschlägigen AwarenessMaßnahmen (Public Relations, Sponsoring von Veranstaltungen) sowie von einer speziell auf

\footnotetext{
${ }^{22}$ Hier wird dabei zusätzlich eine hohe Additionalität der Förderung unterstellt (d.h. abgelehnte Projekte kommen ohne Förderung nicht oder nur in wesentlich reduziertem Volumen zustande). Aufgrund des hohen durchschnittlichen Förderanteils in genehmigten Projekten (ca. 45 \%) erscheint diese Annahme hier durchaus gerechtfertigt.
} 
die Zielgruppe zugeschnittenen Ausbildungsschiene. Letztere bietet neben der Vermittlung von einschlägigem branchenrelevanten Theorie- und Praxiswissens zusätzlich die Möglichkeit, die Kenntnisse hinsichtlich der monetären Förderungsschienen von impulse zu erhöhen.

Insgesamt stellt sich das Programm impulse somit als ein bereits gut etabliertes, die verschiedenen Zielgruppen erreichendes Programm dar, das eine zentrale Funktion in der österreichischen Förderungslandschaft für die Kreativwirtschaft einnimmt. Die einzelnen Programmlinien fügen sich modulartig zu einem „Ganzen“ zusammen und adressieren jeweils unterschiedliche Problemkreise. So spricht die Programmlinie impulse XS die „Vorgründungsphase“ bzw. Frühphasen in der Projektentwicklung an, während impulse XL konkrete Projektentwicklungen mit bereits ausgeprägter Marktorientierung adressiert. Dies wird ergänzt durch die explizit netzwerkbildende bzw. kooperationsfördernde Programmlinie impulse LEAD. Gemeinsam mit den nicht-monetären Schulungsleistungen wird in Summe ein breites Spektrum an „Förderungsleistungen“ für die Kreativwirtschaft abgedeckt, welches durch einschlägige Marketing- und Awareness-Maßnahmen flankiert wird und die Sichtbarkeit der Leistungen der österreichischen Kreativwirtschaft insgesamt erhöht.

Der bisherige Erfolg (im Sinne der „Akzeptanz“ durch die adressierten Förderungsnehmer, was sich in der großen Zahl an Förderansuchen ausdrückt) der Programmlinien hat allerdings auch dazu geführt, dass die Ablehnungsquoten sehr hoch sind. Im Sinne einer „Nachhaltigkeit" des Programms wäre eine Reduzierung dieser Unterdotierung angebracht, da hohe Ablehnungsquoten mittelfristig u.U. zu einem geringeren Interesse von Seiten der potentiellen Förderungswerber führen können.

\subsection{PROGRAMM ZUR FÖRDERUNG VON PRODUKTFINDUNGSSTRATEGIEN}

\subsubsection{ProTrans}

Das Programm ProTRANS zielt auf die Steigerung der Innovationsfähigkeit v.a. von KMUs durch bessere und breitere Umsetzung von Wissen in Innovation ab und hat als organisatorischen Vorläufer das Technologietransfer-Programm protec 2002+ (protec-TRANS und protec-INNO), wobei entsprechende Evaluierungsergebnisse dieser beiden Programme aufgegriffen wurden und zu einem Programm zusammengefasst wurden. Der operative Programmbeginn war ursprünglich mit 1. Jänner 2008 geplant, wobei jedoch in Folge eines verzögerten Programmstarts im Jahr 2008 noch keine Förderzusagen getroffen werden konnten.

ProTrans soll KMU dabei unterstützen, ihr Innovationspotential optimal auszuschöpfen, wobei hierfür die strategische Produktfindung durch moderne Methoden des Innovations- und Wissensmanagement im Unternehmen systematisiert und professionalisiert werden soll. Dafür sollen konkrete Forschungs- und Entwicklungs- und Technologietransferprojekten von Unternehmen in Kooperation mit externen Dienstleistern unterstützt werden.

Die Ziele des Programms sind folgende:

Allgemeine Ziele:

- Stärkung der Innovationskraft und Hebung der F\&E-Leistung der Unternehmen der mittelständischen Wirtschaft durch Stimulierung von Technologietransfer

- Optimierung von Unternehmensstrategien mittelständischer Unternehmen zur Forcierung deren FEI-Potentials im Hinblick auf die Verbesserung der Produktportfolios 
durch die Implementierung der strategischen Produktfindung mit geeigneten Methoden und der Erschließung neuer Märkte

- Verbesserung der Innovations- und Kooperationskultur österreichischer KMU und ihres Zugangs zu den Methoden und dem Know-How von Forschungsreinrichtungen.

Aus diesen allgemeinen Zielen leiten sich folgende Subziele ab:

- Verbesserung der Nutzung externer Ressourcen für KMU, im Hinblick auf die Implementierung geeigneter Methoden der strategischen Produktfindung insbesondere die Optimierung des Zuganges zur Expertise von Forschungseinrichtungen (u.a. Universitäten, Fachhochschulen, außeruniversitäre Forschungsreinrichtungen, kooperative Forschungseinrichtungen) und Unternehmen (inklusive spezialisierte Beratungsunternehmen)

- Verbesserung des unternehmensinternen Innovations- und Wissensmanagement

- Stimulierung von strategischen Partnerschaften für die Weiterentwicklung und Markteinführung neuer Produkte, Prozesse und Dienstleistungen.

Laut dem „Anbot für die Fortsetzung der Technologieprogramme für die Jahre 2007 bis 2009“ sind die Indikatoren zur Prüfung der ProTRANS -Zielerreichung wie folgt definiert:

- $\quad$ Erhöhung des FEI-Outputs

- Steigerung des Anteils der F\&E MitarbeiterInnen

- Produkt- und Prozessinnovationen aus dem Projekt

- Entwicklung der organisatorischen Grundlagen im Unternehmen

- Zahl der Patente, Gebrauchsmuster etc.

- Entwicklung der FEI-Ausgaben der Unternehmen im Vergleich zum Umsatz

- Entwicklung Umsatzanteil innovativer Produkte, Verfahren und Dienstleistungen

- Optimierung des Produkt- bzw. Dienstleistungsportfolios

- Implementierung strategischer Produktfindungsprozesse

- Erhöhung der Kooperationsbereitschaft von Wirtschaft und Wissenschaft bzw. von Wirtschaft/Wirtschaft anhand konkreter Projekte bzw. Anzahl der Projekte über das Projekt hinaus

- Nutzung zusätzlicher Innovationsinformations- und -managementsysteme

Grundsätzlich besteht das Förderungsangebot ProTRANS in Zuschüssen (in Form von Barzuschüssen) bis zu einer Höhe von EUR 300.000. Gefördert wird die Stärkung der Innovationsleistung durch Kooperationen mit universitären /außeruniversitären Forschungseinrichtungen sowie mit anderen Unternehmen auf Basis konkreter F\&E- bzw. Technologietransferprojekte. Als Zielgruppe spricht das Förderungsangebot kleine und mittlere Unternehmen an.

Das Programm ProTRANS fördert Kooperationsprojekte auf der Basis spezifischer F\&EVorhaben, die folgende Eigenschaften aufweisen:

- Entwicklung bzw. Verbesserung von Unternehmensstrategien in Hinblick auf die Optimierung der Produktportfolios zur Nutzung technologischer Potentiale

- Implementierung bzw. Etablierung von effektiven Innovations-Management-Methoden, welche die Innovationsleistung des Fördernehmers nachhaltig stärken

- Strategischen Produktfindung, die substanzielle Produkt- und Verfahrensinnovationen 
auf Basis von Forschungs-, Entwicklungs- und Innovationsprojekte mit stark ausgeprägter Kooperationskomponente ermöglicht.

Als förderbare Kosten gelten Personal- und Beratungskosten, Dienstleistungskosten Dritter, technische Durchführbarkeitsstudien, Verbrauchsmaterialien für F\&E-Aktivitäten sowie Reisekosten, die unmittelbar im Zusammenhang mit dem Projekt stehen.

Durch ProTRANS geförderte Projekte werden in zwei Phasen abgewickelt. In der Definitionsphase beträgt die maximale Förderung 50.000 EUR (bzw. maximal $50 \%$ der förderbaren Kosten) und in der darauffolgenden Umsetzungsphase beträgt die maximale Förderung 250.000 EUR (bzw. maximal 35 \% der förderbaren Kosten). Die maximale Gesamtförderung für ein Pro-TRANS-Gesamtprojekt (Definitions- und Umsetzungsphase) beträgt somit 300.000 EUR bei einer Laufzeit von maximal 24 Monaten.

Der Programmverlauf von ProTRANS für die beiden ersten operativen Jahre (2009 und 2010) ist in Abbildung 30 dargestellt. Im Jahr 2009 konnten 31 Projekte in der Konzeptphase gefördert werden (Fördersumme = 1,2 Mio. EUR). Davon konnten noch im gleichen Jahr 9 Projekte in die Umsetzungsphase übergeleitet und mit zusätzlichen 1,3 Mio. EUR gefördert wer$\operatorname{den}^{23}$. Von den verbleibenden 22 Konzeptprojekten wurden dann 14 weitere im Jahr 2010 in die Umsetzungsphase übergeleitet.

Im Jahr 2010 kam es dann zu 21 neuen Förderprojekten in der Konzeptphase (Fördersumme Konzeptphase = 0,9 Mio. EUR), wovon wiederum ein Teil der Projekte (nämlich 5) noch im gleichen Jahr in die Umsetzungsphase übergeleitet wurden. Dazu kamen dann die erwähnten 14 Umsetzungsprojekte der bereits im Jahr 2009 geförderten Konzeptphasenprojekte. Insgesamt ergibt das für das Jahr 201020 Förderprojekte in der Umsetzungsphase (14 plus 5 plus ein Projekt, das wiederum sofort mit der Umsetzungsphase startete ${ }^{24}$ ). Die Fördersumme für Projekte in der Umsetzungsphase betrug im Jahr 2010 2,4 Mio. EUR.

\footnotetext{
${ }^{23}$ Das sofort in der Umsetzungsperiode aufgenommene Projekt wurde mit ca. 60.000 EUR gefördert. Dieses Unternehmen konnte deswegen gleich in der Umsetzungsphase starten, da bereits nach dem Ende der Vorgänger-Förderaktion Protec-Trans mit den Arbeiten zum Konzept begonnen wurde und durch die Verzögerungen im Zusammenhang mit dem Programmanlauf von ProTrans hier sonst ungerechtfertigter Weise Verzögerungen entstanden wären.

${ }^{24}$ Dabei handelt es sich um einen Wechsel des Begünstigten, nachdem das ursprüngliche Projekt der Konzeptphase von einem anderen Unternehmen durchgeführt wurde, das mittlerweile aufgrund seiner Insolvenz ausgeschieden ist. Aufgrund der Insolvenz des Technologiegebers wurde durch den Wechsel des Begünstigten der Erhalt des erarbeiteten Know-hows gesichert.
} 
Abbildung 30: Programmverlauf ProTRANS

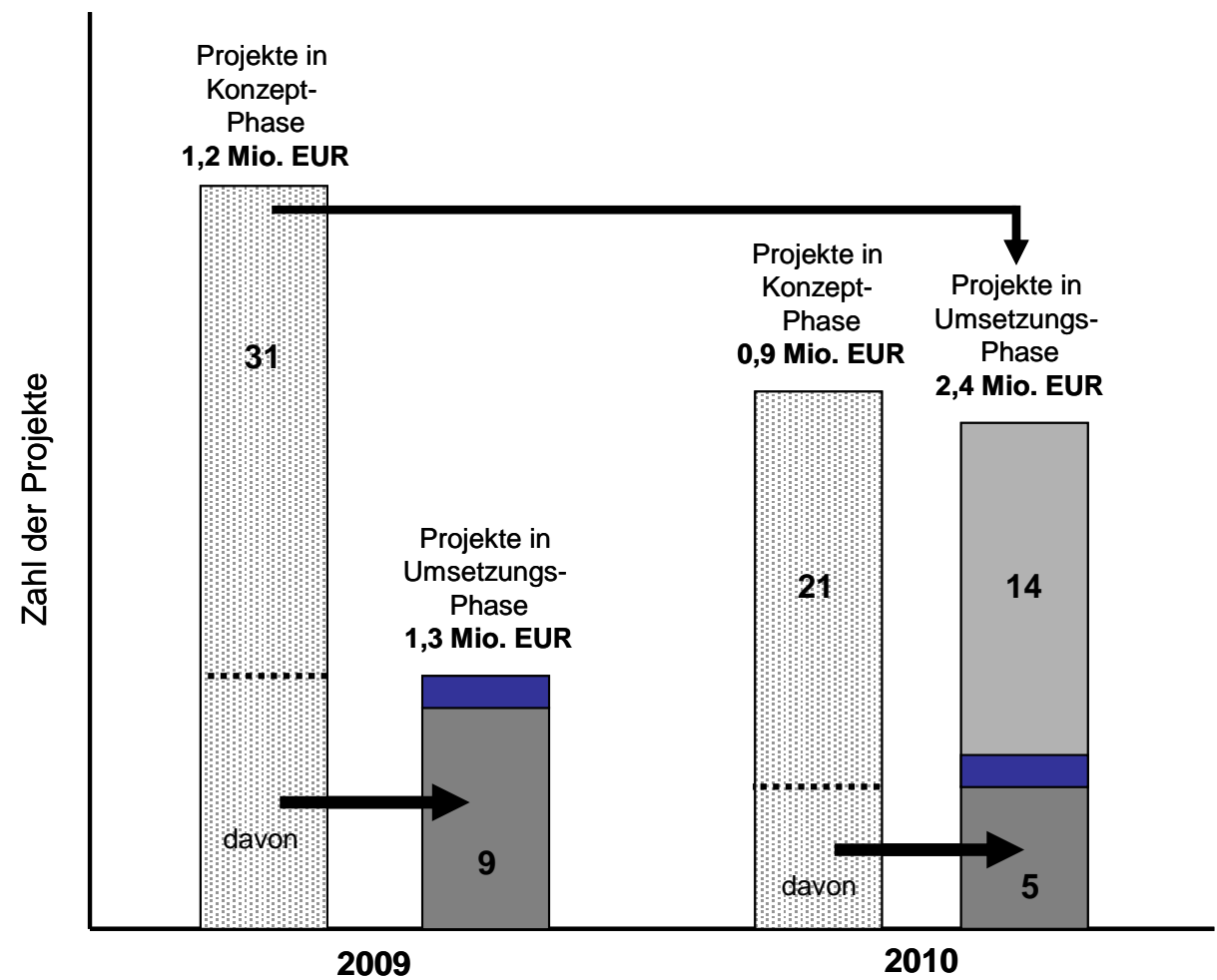

Anm.: Jeweils ein Projekt wurde in beiden Jahren sogleich in der Umsetzungsphase gefördert (blau dargestellt) Quelle: aws, eigene Darstellung

Hinsichtlich der geographischen Verteilung der geförderten Projekte sticht besonders Oberösterreich hervor, das für beide Programmphasen die meisten Projekte stellt. Erst an zweiter Stelle folgt Wien. Auffällig ist, dass die Steiermark - trotz ihres theoretisch großen Potentials als traditionsreiches Industrieland - mit nur wenigen Projekten vertreten ist. Des Weiteren zeigt sich eine Lücke im äußersten Westen mit dem Bundesland Vorarlberg, das derzeit mit lediglich einem einzigen Projekt in der Konzeptphase im Programm vertreten ist. 
Abbildung 31: Geographische Verteilung der geförderten Projekte (Konzept- und Umsetzungsphase)

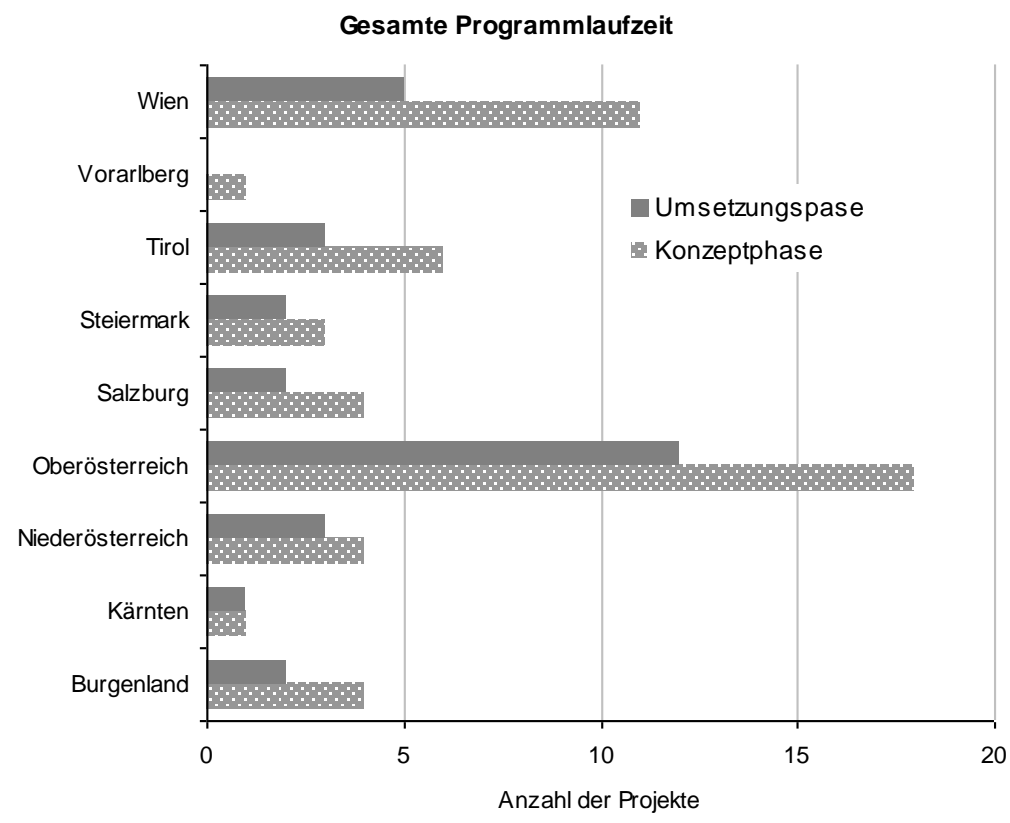

Quelle: aws

Die geförderten Projekte verteilen sich über eine Vielzahl von unterschiedlichen Branchen, wobei nicht ausschließlich die „klassischen“ High-Tech-Branchen (wie z.B. Elektronik, Pharma) vertreten sind (siehe Abbildung 32). Die meisten Projekte verbucht der Maschinenbau (inklusive Metallverarbeitung) auf sich, gefolgt vom Wirtschaftszweig „Forschung und Entwicklung“ (hier zusammengefasst mit den „Technischen Büros“). Auffällig ist auch, dass der Dienstleistungssektor ${ }^{25}$ gut vertreten ist, was aufzeigt, dass auch Klein- und Mittlere Unternehmen dieses Sektors Innovationspotential aufweisen, das mit den einschlägigen Instrumenten der Innovationspolitik unterstützt und gefördert werden kann.

\footnotetext{
${ }^{25}$ Bei diesen Projekten handelt es sich hauptsächlich um Projekte aus dem Einzel- und Großhandel sowie aus der Marktforschung.
} 
Abbildung 32: Verteilung der geförderten Projekte (Konzept- und Umsetzungsphase) nach Branchen

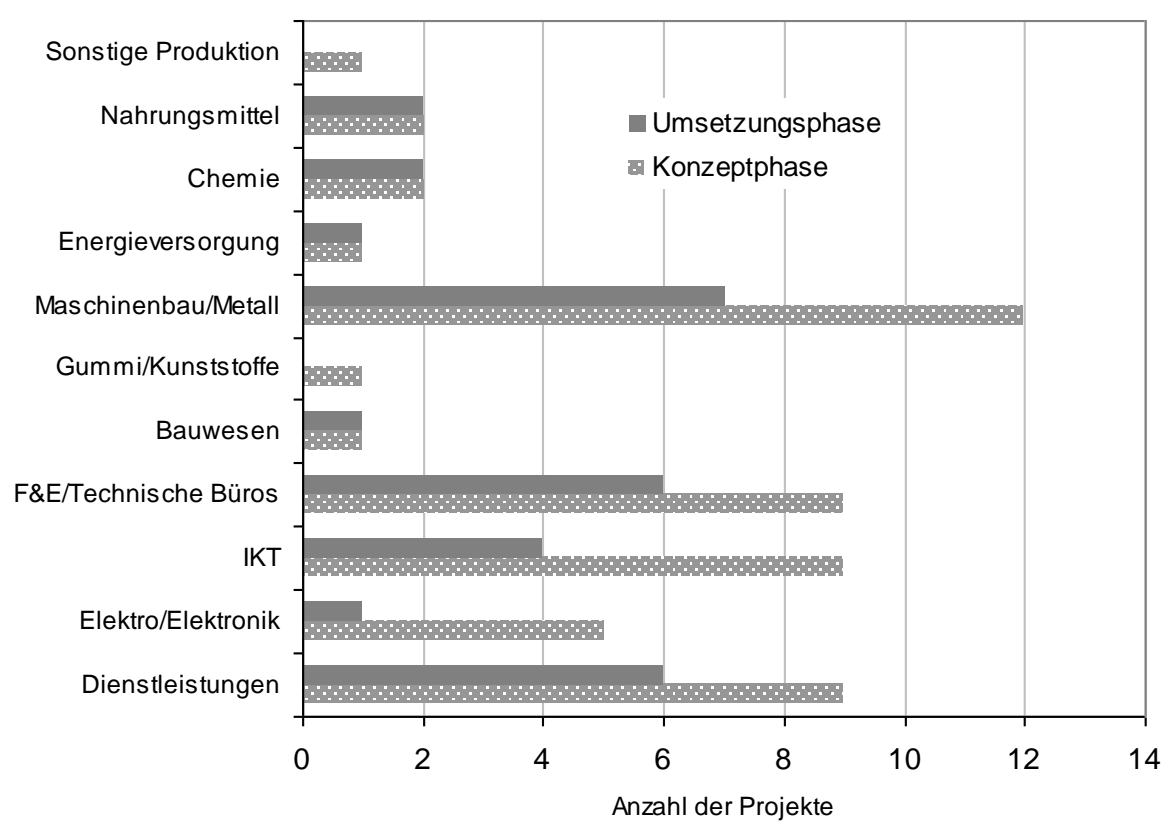

Quelle: aws

Im Jahr 2010 wurde von Seiten der aws eine interne Analyse des Programms ProTRANS durchgeführt, deren zentralen Ergebnisse im Folgenden kurz zusammengefasst werden:

- Die Zielgruppe von PROTRANS bilden primär Klein- und Mikrounternehmen, wobei bezogen auf die geringe Unternehmensgröße der von ProTRANS geförderten Unternehmen die Projekte durch vergleichsweise hohe Volumina geprägt sind.

- Ca. ein Drittel der ProTRANS-Nutzer wird/wurde auch von anderen aws-Programmen gefördert

- Die Einreichkriterien werden von den Programmnutzern als adäquat betrachtet und es lassen sich keine Hinweise auf „Projektkosmetik“ feststellen.

- Die Förderungen wird als attraktiv eingeschätzt und von den Nutzern werden keine (bzw. kaum) Förderungsalternativen (d.h. Programme, die ähnliche Förderungsintention verfolgen) wahrgenommen.

- Es stehen Methoden des Informations- und Wissensmanagement im Vordergrund und es lässt sich ein hoher Anteil von Projekten in Richtung Produktfindung beobachten.

- Ca. je $40 \%$ der ProTRANS-Nutzer wurde auf das Programm durch eigene Recherchen, Hinweise von (späteren) Kooperationspartnern bzw. durch Beratungs- und Informationsaktivitäten der aws aufmerksam.

- Nach Auskunft der ProTRANS-Nutzer handelt es sich bei den geförderten Projekten um intensiv geplante „Schlüsselprojekte“ mit überdurchschnittlich hohem Ressourceneinsatz und nicht vernachlässigbarem Fehlschlagrisiko.

- Zudem besteht starker Zugriff auf externes Wissen (Transferaspekt) \& Lernen von Methoden. Insgesamt sind die Projekte bedeutsam für die Umsetzung von Unternehmensstrategien zur Diversifizierung des Portfolios.

- Eine Kundenzufriedenheitsanalyse ergab eine generell hohe Zufriedenheit von Seiten 
der geförderten Unternehmen mit der Abwicklung/Betreuung durch die aws während der Projektlaufzeit.

- Dem Programm kann eine hohe Additionalität bescheinigt werden, d.h. ohne Förderung wären die Projekte nicht zustande gekommen bzw. nur in einem deutlich reduzierten Ausmaß.

- Die unmittelbaren Konsequenzen der Projektumsetzung gehen prinzipiell konform mit den Programmzielen, d.h. die Programmteilnahme ermöglicht Diversifizierung des Produktportfolios und gleichzeitig wurde die Bereitschaft, auf externes Wissen für den eigenen Innovationsprozess zuzugreifen, erhöht.

- Generell hat die Programmteilnahme zu Folge, dass Wissen, wie man kooperiert, geschaffen wurde.

- Die Teilnahme am Programm lässt substanzielle Beiträge zur Unternehmensentwicklung insgesamt und zur Erweiterung des Portfolios um innovative Produkte zu erwarten.

- Häufig wird eine Erweiterung des Produktportfolios um echte Marktneuheiten sowie das Erlangen der Technologieführerschaft in einem Marktsegment angestrebt.

\section{Bewertung durch das Evaluationsteam}

Forschungs- und Technologiekooperationen (und im Speziellen kooperative Forschungsprojekte zwischen Unternehmen und Universitäten / Forschungseinrichtungen) haben seit den 1990er Jahren an Aufmerksamkeit gewonnen. Es wird davon ausgegangen, dass die wissenschaftliche Problemlösungskompetenz an Forschungseinrichtungen von Unternehmen für ihre Innovationsprozesse gewinn- und nutzenbringend verwertet werden kann. Gleichzeitig bestehen jedoch - und dies in besonderem Maße für Kleine und Mittlere Unternehmen - eine Reihe von Hürden und Barrieren einer erfolgreichen Kooperation entgegen. Diese Barrieren liegen nicht zuletzt in den begrenzten Ressourcen innerhalb von KMUs sowie den hohen Suchund Transaktionskosten (unterschiedliche Kultur und „Sprachen“ zwischen Wirtschaft und Wissenschaft), die mit einer Kooperation einhergehen ${ }^{26}$. Diese Hürden spricht das Programm ProTRANS an, indem es durch Barzuschüsse die Kooperationskosten der Unternehmen senkt.

Aufgrund der vorliegenden Daten sowie der aws eigenen Bewertungs- und Kundenzufriedenheitsanalyse kann dem Programm ein seinen Zielen entsprechender Erfolg zugesprochen werden. Insgesamt ergibt sich also derzeit kein Handlungsbedarf in Bezug auf die Programmgestaltung, zumal auch zu berücksichtigen ist, dass das Programm in seiner jetzigen Ausgestaltung erst zwei operative Jahre hinter sich hat. Anzumerken ist jedoch, dass die geographische Verteilung der geförderten Projekte nach Bundesländern Lücken im Süden (v.a. Steiermark) und im äußersten Westen (Vorarlberg) aufweist. Hier wären entsprechende verstärkte Informationsaktivitäten, etwa in Zusammenarbeit mit den regionalen Wirtschaftsförderagenturen, anzudenken.

\footnotetext{
${ }^{26}$ Vgl. für eine empirische Analyse der Situation in Österreich Schartinger et al. (2001).
} 


\subsection{MASSNAHMEN ZUR ERHÖHUNG DER AWARENESS}

\subsubsection{Staatspreis Innovation}

Seit 1979 wird der Staatspreis jedes Jahr an das innovativste Unternehmen Österreichs verliehen. Es ist die höchste Auszeichnung für besonders innovative Leistungen, welche im Rahmen einer feierlichen Verleihung verliehen wird. Diese Verleihung wird im Auftrag des BMWFJ von der aws organisiert und abgewickelt und wird u.a. auch als eine „Drehscheibe der Innovation“, als Wissens- und Informationsplattform zwischen wichtigen Playern aus Wissenschaft, Wirtschaft und Politik, angesehen.

Die Konzeption des Staatspreis Innovation ruht dabei auf drei Säulen:

(1) Durch Branding des Wettbewerbs wird eine Auszeichnung für hochwertige innovative Leistungen von österreichischen Unternehmen geschaffen.

(2) Das Image des Wettbewerbs soll so überragend sein, dass Unternehmen allein wegen der Auszeichnung und der guten Reputation, die sie dadurch erhalten, zur Teilnahme motiviert werden.

(3) Das Image des Wettbewerbes soll durch eine exzellente Organisation, von der Einreichung bis zur Auszeichnung, einem professionellen, einheitlichen Auftritt sowie von imagestärkenden Persönlichkeiten und Partnern getragen werden.

Laut „Anbot für die Fortsetzung der Technologieprogramme für die Jahre 2007 bis 2009“ sind im Rahmen des Wettbewerbs und der begleitenden PR-Maßnahmen des Staatspreises für Innovation die Ziele wie folgt zu erreichen:

- Sensibilisierung österreichischer UnternehmerInnen für das Thema Innovation und Technologie und Motivation zur praktischen Umsetzung

- Bewusstseinsbildung in der interessierten Öffentlichkeit für das Thema Innovation und Technologie (,Was leisten österreichische Unternehmen auf diesem Sektor?“)

- Wissensplattform und Infoplattform bilden, um Innovation nachhaltig zu forcieren und Chancen zu erhöhen (Vernetzung der wichtigsten Player aus Wissenschaft, Wirtschaft und Politik, AnbieterInnen und NachfragerInnen zusammenbringen)

- Positionierung/ Imagetransfer des BMWFJ in der Öffentlichkeit

Der Staatspreis Innovation wird in einem zweistufigen Verfahren in Kooperation mit den Bundesländern abgewickelt; nur Unternehmen, die sich in ihrem Landesinnovationswettbewerb qualifizieren, können auch beim Staatspreis für Innovation einreichen. In Summe können bis zu 27 innovative Unternehmen mit Firmen- oder Gewerbesitz in Österreich einreichen, je ein Bundeslandsieger und zwei weitere Projekte aus unterschiedlichen Technologiefeldern.

Alle jeweiligen Bundesland gemeldeten Unternehmen erhalten einen sogenannten „Jurybogen", dessen Daten und Informationen schließlich Entscheidungsgrundlage für die Jury sind. Die Jury arbeitet ehrenamtlich und bestand im Jahr 2010 aus acht Mitgliedern, davon sind sechs stimmberechtigt: je ein/e VertreterIn der Österreichischen Forschungsförderungsgesellschaft, des Österreichischen Patentamts, der Wirtschaftskammer Österreich, der Vereinigung 
österreichischer Industrieller sowie der Universitäten (2010 der Montanuniversität Leoben und der Universität Wien). Nicht stimmberechtigt sind hingegen der/ die VertreterIn des BMWFJ und der aws (in der Rolle des Veranstalters bzw. Organisators).

Aus dem Pool von maximal 27 Einreichungen ist es Aufgabe der Jury, maximal sechs Projekte zum Staatspreis zu nominieren. Danach wird aus den Nominierungen ein Siegerprojekt für den Staatspreis ausgewählt, wobei der Jury die Entscheidung vorenthalten bleibt, auch kein Unternehmen mit dem Staatspreis auszuzeichnen bzw. den Staatspreis im Ausnahmefall sogar an zwei Unternehmen ex aequo zu verleihen. Zur Beurteilung wird dabei der Kriterienkatalog sowie dessen Gewichtung - wie Tabelle 26 dargestellt - herangezogen.

Tabelle 27: Kriterienkatalog und Gewichtung des Staatspreis Innovation, 2011

\begin{tabular}{cl}
\hline Gewichtung & Kriterium \\
\hline $25 \%$ & Neuheit (innovatorische Leistung) \\
$20 \%$ & Schwierigkeit (unternehmerische Leistung) \\
$25 \%$ & Nutzen (Wirkung der Innovation) \\
$15 \%$ & Markt (Chancen und Marketingkonzept) \\
$5 \%$ & Volkswirtschaftliche Effekte inkl. IPR \\
$10 \%$ & Kooperationsaspekte (universitär/ außeruniversitär, Unternehmen) \\
\hline Quelle: aws &
\end{tabular}

Insgesamt haben in der Periode 2007 - 2010 über 2.300 Unternehmen Projekte zum Staatspreis Innovation eingereicht (siehe Tabelle 28); in den letzten beiden Jahren wurde dabei jeweils die 600er-Marke überschritten; im Jahr 2010 zählte man insgesamt 616 Einreichungen, ein Jahr zuvor sogar 639. Führend auf Bundesländerebene sind dabei stets die industrielastigen Länder wie Steiermark mit insgesamt $26 \%$ aller Einreichungen, gefolgt von Wien (18\%), Oberösterreich (15\%) und Niederösterreich (12\%).

Tabelle 28: Einreichungen zum Staatspreis Innovation nach Bundesland, 2007 - 2010

\begin{tabular}{|c|c|c|c|c|c|c|}
\hline Bundesland & 2007 & 2008 & 2009 & 2010 & gesamt & $\%$ \\
\hline Burgenland & 19 & 0 & 15 & 14 & 48 & $2 \%$ \\
\hline Kärnten & 45 & 28 & 39 & 52 & 164 & $7 \%$ \\
\hline Niederösterreich & 36 & 52 & 90 & 100 & 278 & $12 \%$ \\
\hline Oberösterreich & 76 & 78 & 100 & 100 & 354 & $15 \%$ \\
\hline Salzburg & 67 & 49 & 50 & 44 & 210 & $9 \%$ \\
\hline Steiermark & 223 & 128 & 119 & 118 & 588 & $26 \%$ \\
\hline Tirol & 63 & 63 & 65 & 0 & 191 & $8 \%$ \\
\hline Vorarlberg & 0 & 24 & 10 & 28 & 62 & $3 \%$ \\
\hline Wien & 0 & 100 & 151 & 160 & 411 & $18 \%$ \\
\hline Gesamt & 529 & 522 & 639 & 616 & 2.306 & $100 \%$ \\
\hline
\end{tabular}


Dementsprechend zeigt sich auch das Bild der Staatspreissieger: So kamen in der Periode 2007-2011 $1^{27}$ je zwei Sieger aus dem Bundesland Steiermark (2009/2011) und Oberösterreich (2008/ 2011) und ein Sieger aus dem Burgenland (2007).

Gleichsam mit dem Staatspreis für Innovation wird seit dem Jahr 2003 auch der Sonderpreis für ein innovatives kleines oder mittleres Unternehmen, der „Econovius“, verliehen. Ein innovatives KMU kann sich im Rahmen des jeweiligen Landesinnovationspreises für den Sonderpreis „Econovius“ bewerben, jährlich können bis zu neun KMUs am Sonderpreis „Econovius“ teilnehmen. Die Beurteilung der Projekte erfolgt nach den gleichen Kriterien wie beim Staatspreis Innovation. In den Jahren 2007 - 2010 waren die Sieger auf Bundesländerebene wie folgt: Je zwei KMUs aus Oberösterreich (2007/ 2011), je zwei KMUs aus der Steiermark (2008/ 2009) und ein KMU aus Vorarlberg (2007). ${ }^{28}$

Wurden der Staatspreis Innovation und der Sonderpreis „Econovius“ von der aws nur organisatorisch abgewickelt, so hat man sich seit 2008 intensiv bemüht, den Staatspreis Innovation auch mit weiteren Inhalten zu bereichern und breiter aufzustellen. Diese Bemühungen hatten zur Folge, dass Unternehmen - mit dem Start der Ausschreibungen in den Bundesländern ab dem Jahr 2011 - nun auch mit einem Sonderpreis „VERENA“ ausgezeichnet werden können. Der Sonderpreis „VERENA powered by VERBUND“ zeichnet Unternehmen aus, die in Kooperation mit Universitäten, Fachhochschulen oder außeruniversitären Forschungseinrichtungen innovative Projekte in den Bereichen Energieeffizienz/-management, erneuerbare Energien, E-Mobilität und Energiesysteme umgesetzt haben. Auch für diesen neu geschaffenen Sonderpreis gelten die gleichen Beurteilungskriterien wie für den Staatspreis Innovation; entsprechende Projekte - mit Einreichung bei den Landesorganisationen ab dem Jahr 2011 - im Rahmen der Verleihung des Staatspreis Innovation im Frühjahr 2012 ausgezeichnet werden können. Damit soll pro Bundesland zusätzlich zu den Projekten für den Staatspreis Innovation und den Sonderpreis ECONOVIUS jeweils ein Unternehmen für die Auszeichnung mit „VERENA powered by VERBUND“ entsandt werden. Die Vergabe des Sonderpreises VERENA ist vorerst befristet, mit Option auf Verlängerung soll dieser Preis zunächst in den Jahren 2012, 2013 und 2014 verliehen werden.

\section{Bewertung durch das Evaluierungsteam}

Wurden angesichts der Erweiterung der Kategorien die Statuten des Staatspeises Innovation entsprechend angepasst sowie das Einvernehmen auf Bundesländerebene mit den Organisatoren des Landesinnovationswettbewerbs hergestellt, so gilt es nunmehr für die aws, sich der Herausforderung zu stellen, diese neue Kategorie in den kommenden Jahren nachhaltig zu etablieren. Ein Blick in die vergangenen Jahre zeigt, dass die aws solchen Anforderungen durchaus gewachsen ist, hat sie doch seit der Evaluierung im Jahr 2006 eine gewisse „Neuorientierung" des Staatspreis nicht nur mitgetragen, sondern die Abwicklung und Organisation auf ein Niveau gehoben, welches das Attribut ,exzellent“ verdient. Neben dem Perfektionismus, dem erstklassigen Eventmanagement, über welches die aws MitarbeiterInnen heute verfügen, waren es vor allem vier Punkte aus der Evaluierung 2006, deren Abänderung bzw.

\footnotetext{
${ }^{27} 2011$ wurde der Jahreskreislauf durchbrochen; d.h. ab dem Jahr 2011 wurde das Namensschema umgestellt und somit der Staatspreis des aktuellen Jahres verliehen und nicht, wie in den Jahren zuvor, der des Vorjahres. Unabhängig davon wurde im Jahr 2011 der Staatspreis an zwei Sieger ex aequo vergeben.

${ }^{28} \mathrm{Im}$ Jahr 2007 wurde der Sonderpreis „Econovius“ ex aequo an zwei Unternehmen vergeben.
} 
Weiterentwicklung zu diesem Erfolg führten: (1) Erweiterung des Teilnehmerkreises auf Bundesländerebene, (2) stärkere Gewichtung der Auswahlkriterien auf Innovativität, (3) die Zusammensetzung der Jury aus unabhängigen FachexpertInnen, sowie (4) die verbesserte PR bzw. Öffentlichkeitsarbeit.

Was letzteren Punkt, die Medienarbeit, anbelangt, so ist es der aws gelungen, die Reichweite zu erhöhen, indem u.a. bisherige Medienpartner stärker genutzt, aber auch zusätzlich neue Medien aktiv bearbeitet wurden. Damit war es auch möglich, die mediale Präsenz des Staatspreis Innovation erheblich zu erhöhen, von insgesamt 94 Meldungen in Hörfunk, Online-, Printmedien und TV im Jahr 2007 auf 115 im Jahr 2008 und im Jahr 2009 auf weitere beachtliche 217 Meldungen; die Medienpräsenz hat sich somit im Zeitraum 2007 - 2010 mehr als verdoppelt.

Was die zukünftige Ausrichtung des Staatspreis Innovation anbelangt, so stellen zumal die Erweiterung der Kategorien und damit verbunden die weitere Verbreiterung (u.a. auf Bundesländerebene) die prioritären Entwicklungsziele dar. Darüber hinaus wäre anzudenken, die bis dato aufgebauten Erfahrungen und Kompetenzen der aws zu nutzen, um künftig auch andere, weitere Staatspreise in der aws abzuwickeln. Die aws würde somit in ihrem AwarenessPortfolio ein Kompetenzzentrum für Staatspreise beinhalten, dessen Aufgabe sowohl die inhaltliche Gesamtkonzeption als auch die Abwicklung mehrerer Staatspreise umfasst. Damit würde der Auftraggeber nicht nur das Potential an Erfahrung und Know-How der aws optimal ausschöpfen, auch könnten durch eine solche konzentrierte Abwicklung von Staatspreisen Synergieeffekte genutzt werden und daraus Effizienzsteigerungen - sowohl auf Seiten der aws als auch auf Seiten des Auftraggebers - erzielt werden. Letztlich ginge es auch um eine Option der Kosteneinsparung auf Auftraggeberseite, stellt man die bislang gehandhabte, vielschichtige, an zahlreiche Agenturen vergebene Abwicklung von Staatspreisen einer konzentrierten Abwicklung in der aws gegenüber.

Über all diese inhaltlichen (Zukunfts-)Themen steht jedenfalls die Erfordernis der rechtzeitigen Beauftragung und Budgetbereitstellung an die aws; d.h. dass für all die zukünftigen Ausbauschritte entsprechende finanzielle Rahmenbedingungen gewährt werden müssen. Im Konkreten bedeutet dies, dass - um die inhaltlichen Anforderungen des Auftraggebers erfüllen zu können - die damit verbundenen Budgets zum Start der Umsetzung verbindlich zur Verfügung gestellt werden müssen, im Falle der Abwicklung des Staatspreis 2012 und somit dessen Vergabe im März 2012 müsste die Beauftragung und der Budgetrahmen zumindest sechs Monate im Voraus, im Oktober 2011, von Seiten des Auftraggebers erfolgen bzw. bekannt gegeben werden.

\subsubsection{Jugend Innovativ}

Jugend Innovativ ist Österreichs größter Schulwettbewerb für innovative und kreative Ideen aus den Bereichen Business, Design, Engineering, Science sowie aus den Themenfeldern IKT $^{29}$ und Klimaschutz ${ }^{30}$ und wird bereits seit dem Jahr 1987 durchgeführt $^{31}$. Das Programm

\footnotetext{
${ }^{29}$ Zusammenarbeit mit T-Systems im Rahmen des „T-Systems Young Innovation-Award - die Sonderpreiskategorie für Informations- und Kommunikationstechnik“.

${ }^{30}$ Zusammenarbeit mit Raiffeisen im Rahmen des „Raiffeisen Klimaschutz Award - die Sonderpreiskategorie für Klimaschutz".
} 
wird gemeinsam vom bm:ukk und dem bmwfj getragen. Die allgemeinen Ziele des Programms Jugend Innovativ sind laut Programmdokumenten folgenderweise definiert:

- Bildungsauftrag „Innovation ist Zukunft“ - nachhaltige Verankerung der Bedeutung des Themas vor allem bei Jugendlichen (von guten Ideen/Innovation lebt die Wirtschaft)

- Jugendliche - insbesondere Mädchen - für Technologie und Innovation begeistern und Interesse an naturwissenschaftlichen und technischen Studienrichtungen wecken

- Vermittlung von Spaß und Motivation beim Experimentieren und Ausarbeiten von innovativen Lösungsansätzen

- Bewusstseinsbildung in der breiten Öffentlichkeit für das Thema „Jugend und Innovation.

Auf der operationellen Ebene finden sich folgende Ziele (Wettbewerbsziele):

- Junge Talente zur Umsetzung ihrer Ideen motivieren

- SchülerInnen mit ihren Projekten eine optimale „Bühne“ bieten und außergewöhnliche Leistungen auszeichnen

- Sensibilisierung österreichischer LehrerInnen für das Thema Innovation und Motivation zur praktischen Projektumsetzung (,Teaching Innovation“)

- Das Kreativitäts- und Innovationspotential an Österreichs Schulen vorstellen.

Die Indikatoren für Jugend Innovativ sind laut Anbot für die Fortsetzung der Technologieprogramme für die Jahre 2007 bis 2009 wie folgt definiert:

- Anzahl Projekteinreichungen (nach Schularten, nach Bundesländern, nach Geschlecht, nach Kategorien)

- Anzahl Projektförderungen (nach Schularten, nach Bundesländern, nach Geschlecht, nach Kategorien)

- Anzahl Patent-, Marken- und Musteranmeldungen

- Erfolge/Gewinne bei internationalen Wettbewerben (z.B.: EU-Contest for Young Scientists)

- Anzahl TeilnehmerInnen bei Jugend Innovativ-Veranstaltungen (Kick Off/Info-Events, LehrerInnen-Fortbildungs-Seminare, Ausstellung Finale, Gäste Preisverleihung)

- Medienpräsenz, -resonanz

Das Programm setzt Anreize für die Teilnahme bzw. für entsprechende Projekteinreichungen durch Geldpreise sowie zusätzlich durch Reisepreise an internationalen Veranstaltungen ${ }^{32}$.

Jugend Innovativ gliedert sich thematisch in vier Kategorien, Business, Engineering, Science und Design. Dazu kommen noch thematische Sonderpreise, nämlich seit 2007/08 der IKT Sonderpreis (in Zusammenarbeit mit T-Systems) sowie seit 2008/09 der Klimaschutz Sonderpreis (in Zusammenarbeit mit Raiffeisen). Die besten Projekte aus den Einreichungen werden in einem 2-stufigen Verfahren ermittelt und auf Bundesland-Ebene vorgestellt und prämiert.

\footnotetext{
${ }^{31}$ SchülerInnenwettbewerbe haben auch international eine lange Tradition. Das Programm ,jugend forscht“ in Deutschland besteht bereits seit dem Jahr 1965 und führt derzeit pro Jahr ca. 100 verschiedene Wettbewerbe durch. Seit der Gründung haben sich rund 190.000 junge Menschen an diesen Wettbewerben beteiligt.

${ }^{32}$ Reisepreise stehen beispielsweise für 2010 und 2011 für folgende internationale Veranstaltungen zur Verfügung: „International Wildlife Research Week“ (CH); „22. European Union Contest for Young Scientists“ (PT); „London International Youth Science Forum 2010“(UK), IENA - Nürnberger Erfindermesse 2010” (DE); "INTEL ISEF - Int. Science and Engineering Fair 2011" (US); ISWEEP - Int. Sustainable World” (US) sowie dem "25. China Adolescents Science \& Technology Innovation Contest $(\mathrm{CH})$ ” zur Verfügung.
} 
Davon steigen die jeweils 3-5 besten Projekte pro Kategorie ins Bundes-Finale auf ${ }^{33}$. Für die jeweiligen thematischen Kategorien werden im Rahmen des Wettbewerbs für die Siegerteams (bzw. für die ersten drei Plätze) Geldpreise (in Höhe von 2000 EUR für Platz 1, 1500 EUR für Platz 2 und 1000 EUR für Platz 3) vergeben. Zusätzlich gibt es noch Anerkennungspreise, die mit 500 EUR je Projekt dotiert sind. Im Jahr 2009/10 wurden so für die 29 Finalprojekte in Summe 32.500 EUR zur Verfügung gestellt.

Die Entwicklung der Zahl der Projekteinreichungen nach den thematischen Kategorien zeigt Abbildung 33. Die Summe der Einreichungen in den vier „Standardkategorien“ bewegt sich dabei annähernd konstant in der Spannweite zwischen 300 und 370. Die Einführung der Sonderpreise ab dem Jahr 2007/08 hat zu zusätzlichen Einreichungen geführt, so dass ab dem Jahr 2008/09 (mit dann bereits zwei thematischen Sonderpreisen) die Gesamtzahl der Einreichungen auf ca. 450 gestiegen ist. Die Verbreiterung der thematischen Kategorien hat offensichtlich eine positive Anreizwirkung für die Beteiligung gezeitigt. Dies ist insofern von besonderer Relevanz, da sicherlich etliche Projekte der Sonderpreiskategorien auch thematischinhaltlich unter einer anderen Kategorie eingereicht hätten werden können. Die zusätzlichen Kategorien erzielten jedoch einen echten Stimulus für die Zahl der Einreichungen. Einen weiteren Sprung bezüglich der Zahl der Projekteinreichungen gab es mit der letzten Runde von Jugend Innovation (2010/11), in der die Gesamtzahl auf 549 stieg (dabei kam es auch nochmals zu einer deutlichen Steigerung der Gesamtzahl in den „herkömmlichen“ Kategorien, nämlich auf ca. 440).

Bezüglich der Verteilung der Einreichungen nach thematischen Kategorien zeigt sich traditionell eine Konzentration auf die Kategorie „Engineering“, deren Anteil im Berichtszeitraum zwischen $44 \%$ und 53 \% lag. Durch die Verbreiterung der Kategorien im Zuge der Etablierung der Sonderpreise IKT und Klimaschutz nahm diese hohe Konzentration auf Engineering zwar etwas ab, Engineering bleibt aber unbestritten die Kernkategorie von Jugend Innovativ. Bemerkenswert ist der rasche Erfolg der neuen Kategorien in Form von Sonderpreisen (die jeweils in Zusammenarbeit mit Sponsoren aus der Wirtschaft vergeben werden). Beide Kategorien erreichen - nur drei (IKT) bzw. zwei Jahre (Klimaschutz) nach ihrer Etablierung bereits Anteile an allen Einreichungen von jeweils ca. $12 \%$ (Klima) bzw. $17 \%$ (IKT). Die beiden - bereits länger bestehenden - Kategorien Science und Design schwanken mit ihren Anteilen zwischen $5 \%$ und $10 \%$. Auffällig ist der - auch in absoluten Zahlen feststellbare Rückgang der Kategorie Business, der von einem Hoch im Jahr 2007/08 mit einem Anteil von mehr als $20 \%$ auf etwas über $10 \%$ im Jahr 2009/10 geprägt war.

\footnotetext{
${ }^{33}$ Die regionale Staffelung durch ein zweistufiges Verfahren (Bundesland - Bund) wurde in der letzten Zwischenevaluierung der aws-Technologieprogramme (Jörg et al. 2006) vorgeschlagen.
} 
Abbildung 33: Projekteinreichungen nach Themenfeldern

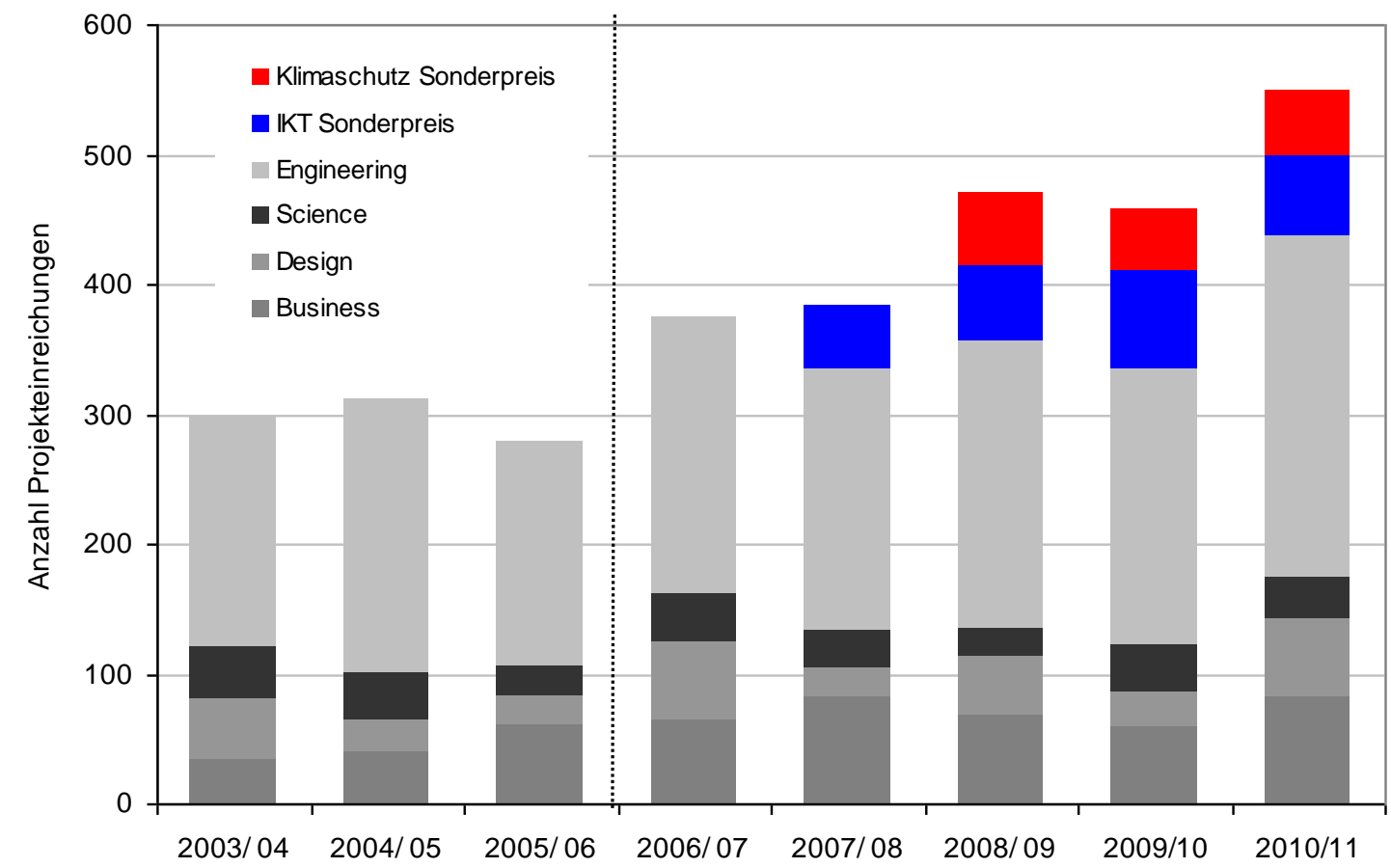

Anmerkung: Die Sonderpreise für Klimaschutz bzw. IKT wurden von privaten Sponsoren finanziert Quelle: aws

Hinsichtlich der Projekteinreichungen nach Schultypen zeigt sich weiterhin eine hohe Konzentration auf HTLs, auf die jeweils zwischen $70 \%$ und $80 \%$ aller Projekteinreichungen entfallen (Abbildung 34), dahinter folgen HAK/HAS mit jeweils ca. $12 \%$ der Projekte. Auf die anderen Schultypen entfallen dementsprechend nur sehr geringe Anteile, wobei wiederum die sehr geringen Anteile (lediglich um die vier bis fünf Prozent) der AHS bemerkenswert sind, deren Anteile in den „Urzeiten“ von Jugend Innovativ Anfang der 1990er Jahre noch um die $40 \%$ bis $50 \%$ lagen. Die hohe Konzentration auf HTLs findet sich auch bei den beiden neuen Sonderpreis-Kategorien mit über $90 \%$ beim Sonderpreis IKT und um die $70 \%$ beim Sonderpreis Klima.

Wie bereits bei Jörg et al. (2006) erwähnt sind derartige Konzentrationsprozesse bei als Preise gestalteten Wettbewerben nicht untypisch, da die Wettbewerbssituation mit dem spezifischen Teilnahmeanreizen oft eine Eigendynamik innerhalb einer Zielgruppe initiieren kann. Dies führt dann zur Situation, wo die einzelnen HTLs miteinander konkurrieren und andere Schultypen gleichsam ,an den Rand“ gedrängt werden und nur noch einen geringen Anreiz haben am Wettbewerb teilzunehmen. Gerade im Fall von Jugend Innovativ, wo der Wettbewerb aufgrund seiner Technik- und Businessorientierung den HTLs in gewissen Sinn einen generischen Wettbewerbsvorteil gewährt, ist somit der zu beobachtende Konzentrationsprozess wenig überraschend. 
Abbildung 34: Projekteinreichungen nach Schultypen

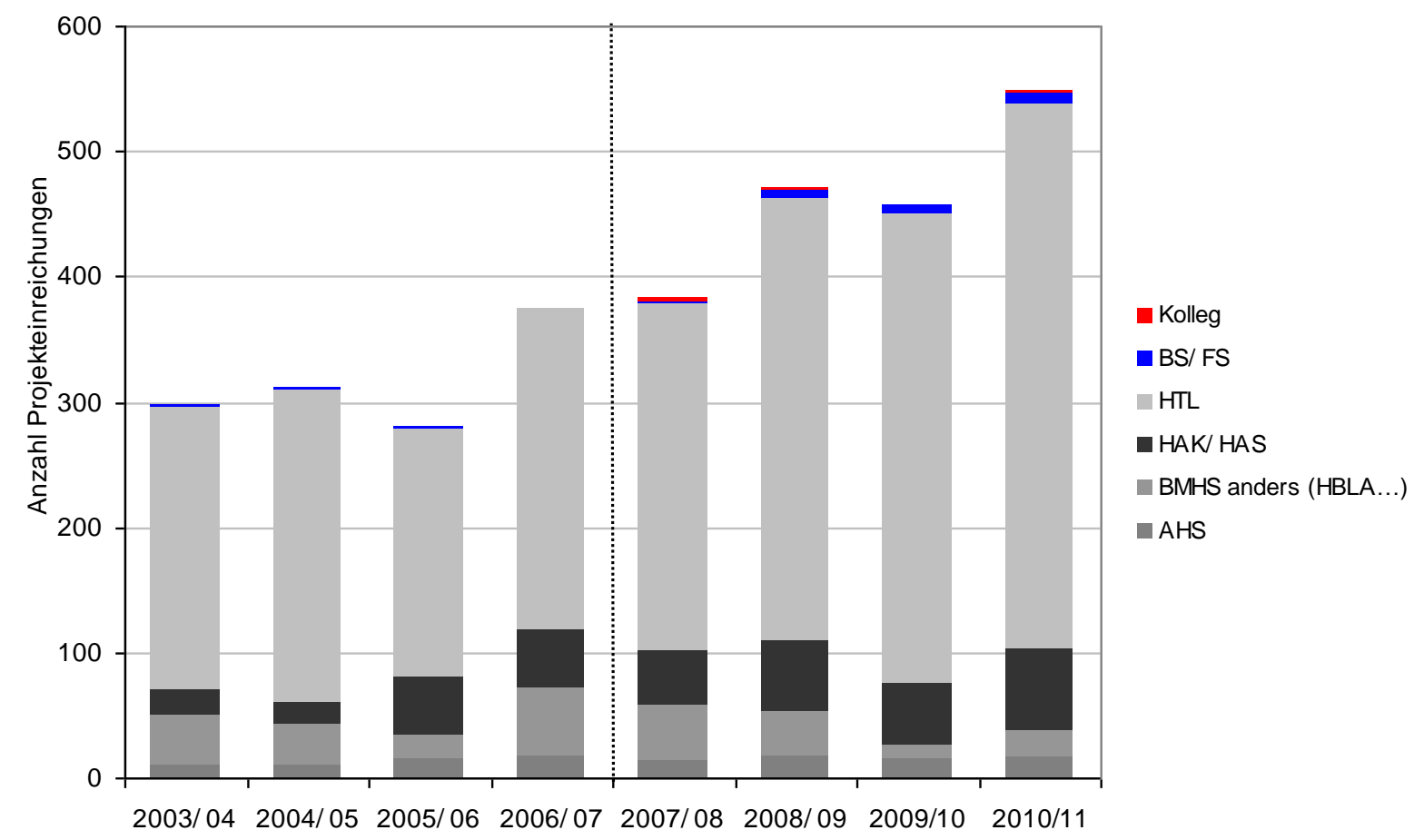

Quelle: aws

Bei der geographischen Verteilung der Projekteinreichungen nach Bundesländern liegt in der Berichtsperiode Oberösterreich recht deutlich voran, gefolgt von Wien, Niederösterreich und Kärnten (siehe Abbildung 35). In Oberösterreich und in Wien gab es mit einer Verdreifachung bzw. Verdoppelung gegenüber 2006/07 auch den stärksten Anstieg bezüglich der Zahl der Projekteinreichungen. Tatsächlich sind diese beiden Bundesländer also die wesentlichen Treiber der starken Zunahme der Beteiligung an Jugend Innovativ. In den anderen Bundesländern gibt es z.T. starke Schwankungen, mit Ausnahme von Kärnten, das eine überraschend konstante TeilnehmerInnenanzahl aufweist. 
Abbildung 35: Projekteinreichungen nach Bundesländern

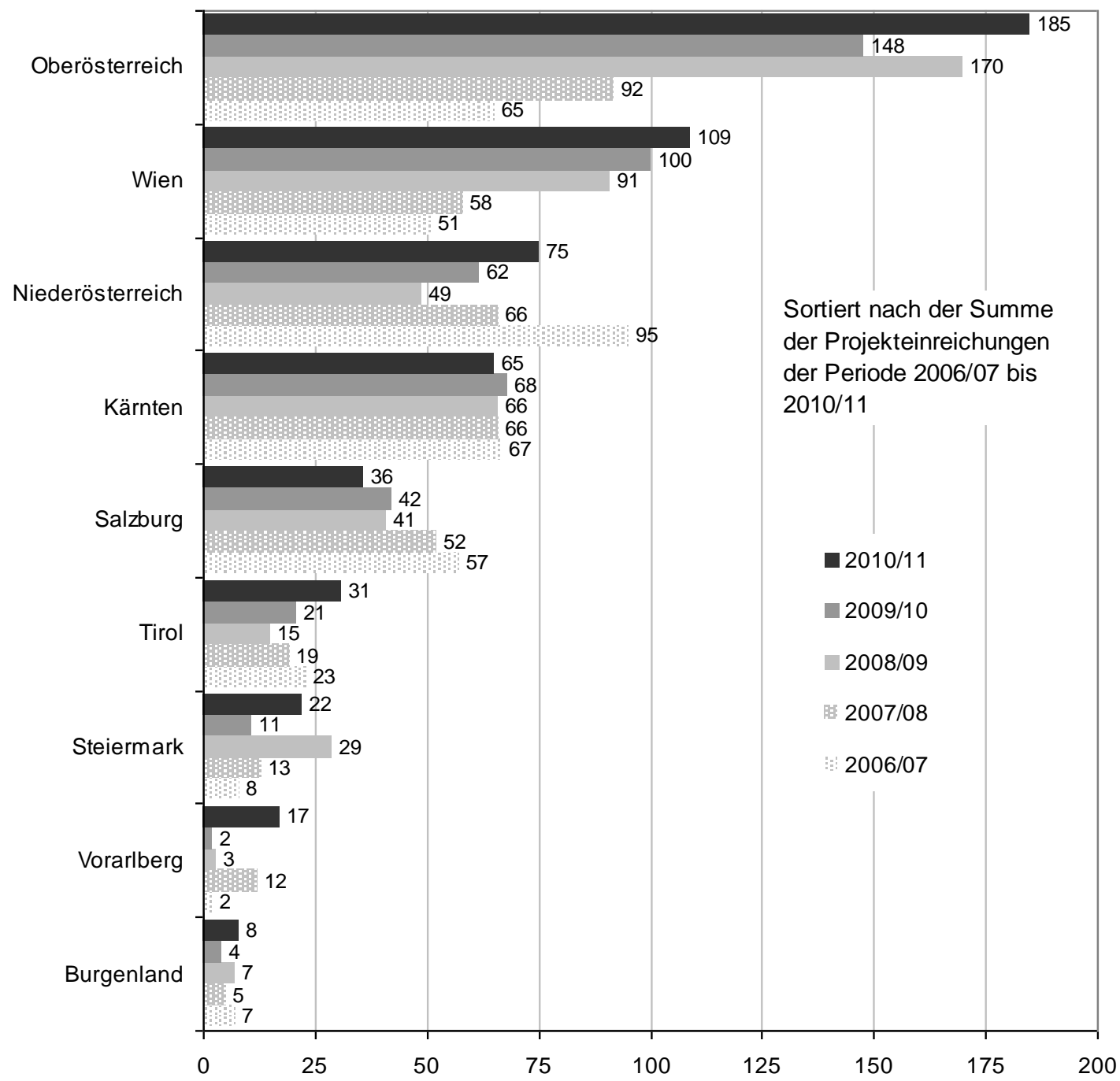

Quelle: aws

\section{Bewertung durch das Evaluationsteam}

Die grundsätzlich positive Entwicklung der Reichweite des Wettbewerbs Jugend Innovativ (gemessen an der Zahl der Projekteinreichungen), die schon in der Evaluierung der awsTechnologieprogramme aus dem Jahr 2006 (Jörg et al. 2006) festgestellt wurde, bestätigt sich auch mit den einschlägigen Zahlen der Jahre 2007 - 2010 . Die wesentlichen Programmziele (Motivierung und Aktivierung der Jugend in Richtung Befassung mit und Umsetzung von innovativen Ideen) können als erfüllt betrachtet werden. Dem Programm Jugend Innovativ kommt somit auch eine wichtiger Rolle in Hinblick auf das übergeordnete Ziel zu, den Stellenwert von Technik, Innovation und Wissenschaft in der österreichischen Gesellschaft generell zu verbessern. Nicht zuletzt kann das Programm auch dazu beitragen, die Bereitschaft der jugendlichen SchulabgängerInnen ingenieur- und naturwissenschaftlich ausgerichtete Studiengänge zu wählen, zu erhöhen. Frühe einschlägige Erfolgserlebnisse - eben durch eine Teilnahme an einem derartigen Wettbewerb - können letztlich nachhaltig den weiteren beruflichen Werdegang bzw. die Karrierevorstellungen der Jugendlichen prägen.

Gute Erfahrungen wurden auch mit den beiden neuen thematischen Kategorien (in Form von 
Sonderpreisen unter Zusammenarbeit mit Sponsoren aus der Wirtschaft) gemacht. Die Zahlen für die Projekteinreichungen belegen, dass dadurch eine echte Ausweitung gelungen ist und nicht nur Verschiebungen zwischen den thematischen Feldern. Immerhin zwischen 20 und $30 \%$ der Projekteinreichungen entfallen mittlerweile auf diese Sonderpreiskategorien.

Aufgrund dieser positiven Erfahrungen mit den Sonderpreisen wäre eine weitere thematische Ausweitung (im Idealfall wiederum mit Beteiligung bzw. unter Zusammenarbeit mit privaten Sponsoren aus der Wirtschaft) überlegenswert. Bei einer gezielten Auswahl von neuen Kategorien könnte es durchaus wieder gelingen, die Reichweite von Jugend Innovativ neuerlich anzuheben $^{34}$. Denkbare thematische Kategorien wären etwa übergreifende Themenfelder wie z.B. „Wasser“, „Lebensmittel“ oder „Stadt der Zukunft“.

Weiterhin augenfällig bleibt die große Dominanz des Schultyps HTL. Zwar weist dieser Schultyp unzweifelhaft generische Vorteile in einem technikorientierten Wettbewerb wie Jugend Innovativ auf, trotzdem sollte eine stärkere Einbindung anderer Schultypen angestrebt werden. Insbesondere der nunmehr sehr geringen Teilnahme von AHS, die noch zu Beginn der 1990er Jahre die Mehrzahl der Teilnehmer gestellt hatten, sollte gegengesteuert werden. Eine Möglichkeit hierfür bietet wiederum die Definition zusätzlicher Kategorien. Wird die Themengestaltung dieser zusätzlichen Kategorien eher an generischen Wissensgebieten (statt spezifischer Technologiesegmente wie z.B. IKT) ausgerichtet, hat dies eventuell einen für die AHS (deren Fächerkanon sich tendenziell eher an die traditionell akademische Gliederung der Wissenschaftsdisziplinen orientiert) motivierenden Effekt.

\footnotetext{
${ }^{34}$ Theoretisch ,passen“ in die bereits bestehenden Kategorien nahezu alle denkbaren Technologie- bzw. Wissenschaftsthemen. Allerdings zeigt sich, dass durch zusätzliche spezifische Kategorien (wie im Berichtszeitraum IKT und Klima) offensichtlich zusätzliche Jugendliche für eine Teilnahme motiviert werden können.
} 


\section{Modul 2: Portfoliobetrachtung: Synergien und Ab- stimmung}

In Modul 2 erfolgt eine Analyse und Bewertung des Programmportfolios der aws in Hinblick auf das konzeptionelle und faktische Zusammenspiel der einzelnen Programmlinien innerhalb der aws. Konkret geht werden dabei folgende Ebenen analysiert:

- Prüfung inwieweit die Programmlinien im Verhältnis zueinander konsistent sind, d.h. es wird untersucht, ob eventuell Zielkonflikte zwischen den einzelnen Programmen existieren.

- Analyse internen Abstimmungsprozesse, wobei der Frage nachgegangen wird, inwieweit gegenseitige Lerneffekte zwischen den Programmen bestehen, ein regelmäßiger Informationsaustausch erfolgt etc.

- Analyse des Zusammenspiels zwischen Programmlinien und den Instrumenten. Lassen sich Synergien zwischen dem Beratungsinstrumentarium und den monetären Förderungsangeboten identifizieren? Wie erfolgt die Abstimmung zwischen den Instrumenten und den jeweiligen Programmen (z.B. Double Equity in den jeweiligen gründungsorientierten Programmen) etc.

\subsection{ZIELSYSTEM UND -KONSISTENZ DER PROGRAMMLINIEN}

Übersicht 1 listet alle einzelnen Ziele der aws-Technologieprogramme auf, wobei die Reihenfolge der Auflistung keine Hierarchie impliziert (sie folgt lediglich der Behandlung der einzelnen Programme in Modul 1). In Summe ergeben sich für die aws-Technologieprogramme 52 unterschiedliche Ziele, wobei die Zielebenen sehr heterogen sind. Sie reichen von eher allgemeinen Zielen (,,Verbesserung der österreichischen Wirtschaftsstruktur ...; „Etablierung Österreichs als international anerkannter Kreativstandort“; ...) bis hin zu konkreten Einzelzielen (z.B. ,Vermittlung von Markt- und Technologieinformation zur Untermauerung von Businessplänen und Vermeidung von Fehlinvestitionen“).

Grundsätzlich stellt sich die Frage, inwieweit eine derart lange Zielliste angesichts der notwendigerweise begrenzten Ressourcen, die für die Technologieprogramme insgesamt zur Verfügung stehen, nicht eine Überfrachtung darstellt. Auch wenn sich keine offensichtlichen Zielinkonsistenzen feststellen lassen, ist eine derartige Zielüberfrachtung als wenig zweckdienlich anzusehen. Ein Durchforsten des Zielkataloges wäre daher empfehlenswert.

Tatsächlich ließe sich beispielsweise eine Hierarchisierung von Zielebenen dergestalt vorstellen, dass die einzelne Programmfamilien (JITU, Wissens- und IP-Programme, Kreativwirtschaft, Awarenessprogramme) jeweils mit einer allgemeinen Mission als übergeordnete Zielebene ausgestattet werden und dann daraus für die jeweiligen Einzelprogramme zwei bis drei konkrete Programmziele abgeleitet werden.

Eine derartige Trennung der Zielebenen zwischen Programmfamilie und Einzelprogrammen würde auch eine konsistentere Hierarchisierung der Ziele ermöglichen: die allgemeinen Ziele („Missionen“) würden auf der Ebene der Programmfamilie aufgespannt werden, während die konkreteren Einzelziele jeweils den Programmen zugeordnet wären. Insbesondere sollte eine 
innovationspolitische „Überladung“ der Programmziele mit makroökonomischen Aggregatzielen (z.B. „Verbesserung der Position Österreichs“ im EIS etc.) in Zukunft vermieden werden. Derartige Aggregatziele haben eine Vielzahl an Einflussfaktoren (von denen viele die öffentliche Hand nicht direkt beeinflussen kann) und gehen weit über den Einflusshorizont von einzelnen (oft mit relativ begrenzten monetären Ressourcen ausgestatteten) Programmen hinaus. Für die Bewertung des Programmerfolgs sind derartige Aggregatziele letztlich wertlos und nachgerade kontraproduktiv.

Da zwischen den einzelnen Zielen vielfach erhebliche Redundanzen bestehen, führt eine Bereinigung des Zielkatalog nicht zu einem qualitativen Verlust an Zieladressierungen (im Sinne, dass bestimmte Problemkreise nicht mehr adressiert würden), hätte aber ein deutlich gestiegenes Ausmaß an Übersichtlichkeit und Konsistenz zur Folge.

\section{Übersicht 1: Zielkatalog aller aws-Technologieprogramme}

1. Programmziel ist die nachhaltige Steigerung der Zahl der österreichischen High-Tech -Gründungen mit ausgeprägten Forschungs- und Entwicklungsaktivitäten und die Überleitung von Forschungsergebnissen in wettbewerbsfähige Produkte, Verfahren oder Dienstleistungen

2. Verbesserung der österreichischen Wirtschaftsstruktur durch die Fokussierung auf dynamische, technologieorientierte Branchen sowie die Schaffung dauerhafter, hochqualitativer Arbeitsplätze

3. Erhöhung der Anzahl von technologieorientierten Unternehmensneugründungen

4. Nachhaltige Unterstützung des Aufbaus von Unternehmen zur wirtschaftlichen Nutzung innovativer und technologisch avancierter Produktideen, Verfahren oder Dienstleistungen mit überdurchschnittlichem Marktpotential und Wachstumschancen durch die Bereitstellung einer Seed-Finanzierung und einer projektbegleitenden Beratung.

5. Förderung von dynamischem und qualitativem Wachstum von innovativen Unternehmen

6. Unterstützung bei der Ausweitung der Kapitalbasis (insbesondere für Risikokapital).

7. Überlebenschance der geförderten Unternehmen durch aktive Hilfestellung und schnelle Problemlösung mittels externer Experten bei sich abzeichnenden Krisen (z.B. Verfehlung von Meilensteinen, Liquiditätsengpässen etc.) zu erhöhen, dabei die Qualifizierung der MitarbeiterInnen des geförderten Unternehmens durch den Know-How-Transfer während des Experteneinsatzes zu verbessern und somit den unternehmerischen Erfolg von innovativen High-Tech-Unternehmen nachhaltig zu steigern.

8. Dem Marktversagen in der Frühphasenfinanzierung entgegenzuwirken

9. Die Vermittlung von privaten Risikokapital von Investoren (Business Angels) an Technologieunternehmen zur Schließung ihrer Eigenkapitallücke (equity gap)

10. Angebot einer offenen und effizienten Plattform für alle Beteiligten (Unternehmen mit Kapital- und Unterstützungsbedarf, Investoren, Netzwerkpartner)

11. Ansprechen von möglichst vielen Player durch unterschiedliche Awareness- und Vermarktungsmaßnahmen

12. Vermittlung von Markt- und Technologieinformation zur Untermauerung von Businessplänen und Vermeidung von Fehlinvestitionen;

13. Erleichterung von Finanzierungsentscheidungen von Förderungsgebern um Fehlinvestitionen zu vermeiden;

14. Schaffung von Zugang zu (Patent-)Datenbanken und Bereitstellung von Recherche-Know-How und -kapazitäten (Verringerung des Informationsdefizits von KMUs gegenüber Großunternehmen);

15. Unterstützung strategischer Entscheidungen bei der Produktentwicklung und -vermarktung von neuen Technologien

16. Markt- und Technologieinformationen so verfügbar zu machen, dass sie von Unternehmen und Kapitalgebern sinnvoll genutzt werden können und Unternehmen wichtige Ressourcen (Zeit, Geld und Personal) sparen.

17. österreichischen Erfindern, Unternehmen und Wissenschaftlern hinsichtlich der Vermarktung von erfolgversprechenden Innovationen zu unterstützen

18. Aufbau von Know-How an den Universitäten und außeruniversitären Einrichtungen

19. Aufbau von Verwertungsinfrastrukturen in Partnerschaft zwischen Universitäten, Fachhochschulen und au- 
Beruniversitären Forschungseinrichtungen

20. Nutzung von professionellen Verwertungsagenturen für die Vermarktung von Patenten

21. Grundlagen-Innovationen potentiellen Entwicklungspartner vorzustellen, um den Schritt von der Weiterentwicklung hin zu einem marktfähigen Produkt zu ermöglichen bzw. zu erleichtern

22. österreichische, primär Klein- und Mittelunternehmen, bei der Schaffung, Durchsetzung, Verwertung und Überwachung ihrer geistigen Eigentumsrechte zur Stärkung und Festigung ihres Wachstums-, Innovationsund Internationalisierungspotentials effizient zu unterstützen

23. die Bewusstseinsbildung der KMUs für ihre eigenen IPs zu stärken, das Erkennen von Gefahren fremden IPs zu sensibilisieren, sowie die Etablierung eines strategischen IP-Management-Konzepts voranzutreiben

24. Schaffen, Stärken und Erweitern der internationalen Netzwerke

25. Etablierung der Kreativwirtschaft als Impulsgeber für Innovationen und maßgeblichen Wertschöpfungsfaktor

26. Stärkung der wirtschaftlichen Leistungsfähigkeit von Kreativwirtschaftsunternehmen

27. Stärkung des Innovationspotentials und der Wettbewerbsfähigkeit von KMUs aller Branchen durch die Integration kreativer Leistungen in unternehmerische Vorhaben

28. Optimale Betreuung potentieller GründerInnen und Unternehmen im Kreativwirtschaftsbereich

29. Erhöhung der Anzahl von Kreativwirtschaftsunternehmen in Österreich, insbesondere durch Neugründungen

30. Verbesserung des betriebswirtschaftlichen Know-Hows in den Kreativbranchen

31. Stimulation von Investitionen - materiell und immateriell

32. Etablierung Österreichs als international anerkannten Kreativstandort

33. Anregung von Kooperationen sowohl zwischen Kreativwirtschaftsunternehmen als auch mit der ,traditionellen" Wirtschaft

34. Erhöhung der Bedeutung kreativer Leistungen in der Wirtschaft

35. „Stärkung der Innovationskraft und Hebung der F\&E-Leistung der Unternehmen der mittelständischen Wirtschaft durch Stimulierung von Technologietransfer.

36. Optimierung von Unternehmensstrategien mittelständischer Unternehmen zur Forcierung deren FEI-Potentials im Hinblick auf die Verbesserung der Produktportfolios durch die Implementierung der strategischen Produktfindung mit geeigneten Methoden und der Erschließung neuer Märkte.

37. Verbesserung der Innovations- und Kooperationskultur österreichischer KMU und ihres Zugangs zu den Methoden und dem Know-How von Forschungsreinrichtungen.“

38. Verbesserung der Nutzung externer Ressourcen für KMU, im Hinblick auf die Implementierung geeigneter Methoden der strategischen Produktfindung insbesondere die Optimierung des Zuganges zur Expertise von Forschungseinrichtungen (u.a. Universitäten, Fachhochschulen, außeruniversitäre Forschungsreinrichtungen, kooperative Forschungseinrichtungen) und Unternehmen (inklusive spezialisierte Beratungsunternehmen).

39. Verbesserung des unternehmensinternen Innovations- und Wissensmanagement

40. Stimulierung von strategischen Partnerschaften für die Weiterentwicklung und Markteinführung neuer Produkte, Prozesse und Dienstleistungen.

41. Sensibilisierung österreichischer UnternehmerInnen für das Thema „Innovation und Technologie“ und Motivation zur praktischen Umsetzung

42. Bewusstseinsbildung in der interessierten Öffentlichkeit für das Thema Innovation und Technologie (,Was leisten österreichische Unternehmen auf diesem Sektor?“")

43. Wissensplattform und Infoplattform bilden, um Innovation nachhaltig zu forcieren und Chancen zu erhöhen (Vernetzung der wichtigsten Player aus Wissenschaft, Wirtschaft und Politik: AnbieterInnen und NachfragerInnen zusammenbringen);

44. Positionierung/Imagetransfer des BMWFJ in der Öffentlichkeit

45. Bildungsauftrag „Innovation ist Zukunft“ - nachhaltige Verankerung der Bedeutung des Themas vor allem bei Jugendlichen (von guten Ideen/Innovation lebt die Wirtschaft)

46. Jugendliche - insbesondere Mädchen - für Technologie und Innovation begeistern und Interesse an naturwissenschaftlichen und technischen Studienrichtungen wecken

47. Vermittlung von Spaß und Motivation beim Experimentieren und Ausarbeiten von innovativen Lösungsansätzen 
48. Bewusstseinsbildung in der breiten Öffentlichkeit für das Thema „Jugend und Innovation“

49. Junge Talente zur Umsetzung ihrer Ideen motivieren

50. Schüler mit ihren Projekten eine optimale „Bühne“ bieten und außergewöhnliche Leistungen auszeichnen

51. Sensibilisierung österreichischer Lehrer für das Thema Innovation und Motivation zur praktischen Projektumsetzung (,Teaching Innovation“)

52. Das Kreativitäts- und Innovationspotential an Österreichs Schulen vorstellen.

Quelle: Diverse Programmdokumente der aws-Technologieprogramme, aws Jahresberichte

Um diese umfangreiche Zielliste der einzelnen Programme zu strukturieren wurden zunächst die einzelnen Ziele gruppiert und zu entsprechenden Gruppen von „Oberzielen“ zusammengefasst. Dabei wurde in einem ersten Schritt bewusst auf die Zuordnung der Einzelziele zu den jeweiligen Programmen ausgeblendet. Das Ergebnis ist graphisch in Abbildung 36 dargestellt. Es zeigt sich, dass sich die Einzelziele durchaus trennscharf einigen Oberzielen zuzuordnen sind. Insgesamt zu den Einzelzielen sieben „Oberziele“ (Zielcluster) verdichtet. Davon entfallen drei Oberziele (High-Tech-Gründungen, Risikokapital und Kreativwirtschaft) auf den Förderungsfokus Gründungen, ebenfalls drei auf den Förderfokus Wissens- und IPManagement (Technologieinformation, Intellektuelle Eigentumsrechte sowie Technologietransfer). Gleichsam als „Unterbau“ fungiert der Zielcluster „Awareness“, der letztlich ,indirekt" (durch die Erhöhung des Stellenwerts der Thematik Technologie und Innovation im öffentlichen Diskurs) für alle anderen Zielcluster von Relevanz ist.

Abbildung 36: Überblick über die Zielebenen der aws-Technologieprogramme (Zahl der Einzelziele in Klammer)

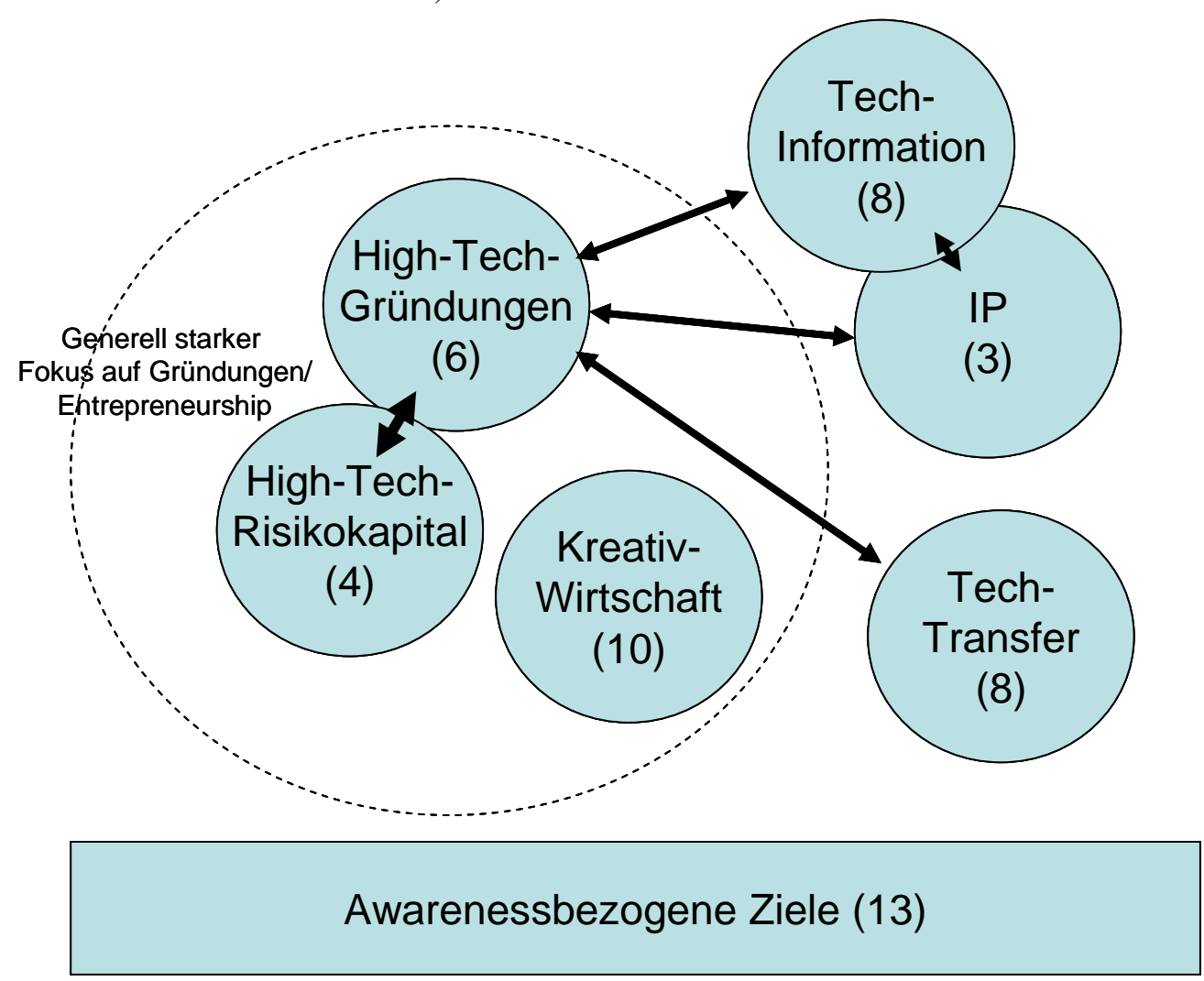

Quelle: diverse Programmdokumente der aws-Technologieprogramme

In einem nächsten Schritt wurden den Zielebenen die einzelnen Technologieprogramme zugeordnet (vgl. Abbildung 37). Dabei zeigt sich, dass die Zuordnung - mit zwei Ausnahmen - 
trennscharf ist, d.h. die Programme finden sich in jeweils einer Zielebene. Eine Ausnahme stellt das Programm Seedfinancing dar, dessen Programmziele sich sowohl auf die Zielebene der High-Tech-Gründungen als auch auf die Ebene der Risikokapitalbereitstellung beziehen. Anzumerken ist dabei, dass sich diese Verschränkung der Zielebenen für das Programm Seedfinancing als sinnvoll darstellt, da dieses Programm von seiner Konstruktionsweise her die Förderung von technologieintensiven Neugründungen mit Hilfe von risikokapitalähnlichen Instrumenten (bedingt rückzahlbare öffentliche Zuschüsse) adressiert. Die zweite Ausnahme ist das Programm ProTRANS, das neben den transferorientierten Zielen auch ein Ziel in Bezug auf Wissensmanagement/Information beinhaltet.

Insgesamt zeigt sich also ein konsistentes System der Zielebenen, das die Schwerpunkte der aws-Technologieprogramme, nämlich High-Tech -orientierte Gründungen, Kreativwirtschaft, Wissensmanagement \& Technologietransfer sowie allgemeine Awareness für FTI, gut abbildet. Lediglich die Anzahl der Einzelziele bleibt somit als Kritikpunkt der Zielanalyse übrig, wobei im Folgenden gezeigt werden soll, dass durch Reduktion (bzw. Zusammenfassen) von redundanten Einzelzielen der umfangreiche Zielkatalog vereinfacht werden kann, ohne dass dies wichtige Zielbereiche ausklammern würde.

Abbildung 37: Die Zielebenen der aws-Technologieprogramme und die jeweils zugeordneten

\section{Einzelprogramme}

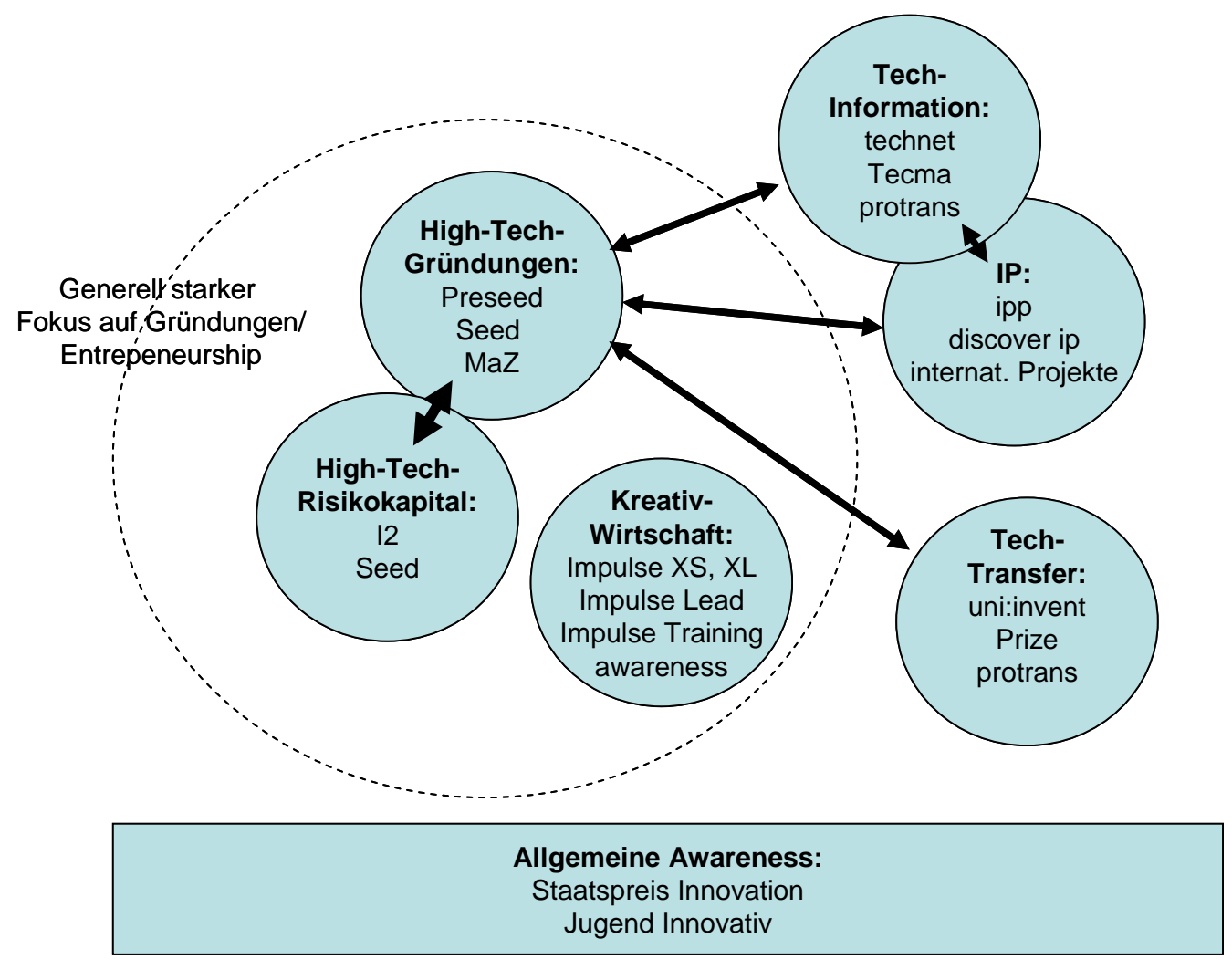

Quelle: diverse Programmdokumente der aws-Technologieprogramme

In den folgenden Tabellen (Tabelle 29 bis

Tabelle 35) wird eine Vereinfachung der Einzelziele in den sieben unterschiedlichen Zielebenen vorgenommen. Dabei werden folgende Kürzungen bzw. Vereinfachungen vorgeschlagen:

- Streichung von zu unspezifischen (d.h. zu allgemein gehaltenen) Zielen (in den Tabel- 
len durch „durchgestrichenen Text“ gekennzeichnet),

- Streichung eines Einzelziels aufgrund hoher Redundanz zu einem anderen Einzelziel innerhalb der Zielebene (in den Tabellen durch „Farb " gekennzeichnet),

- Verknüpfung eines Einzelziels mit einem anderen Ziel innerhalb der Zielebene (in den Tabellen durch "Far b " gekennzeichnet).

Tabelle 29: Zielvereinfachung für die Zielebene „Awareness“

\begin{tabular}{|c|c|c|c|c|}
\hline Zieltyp & Nr. & Ziel & Programm & Bemerkung \\
\hline Awareness & A1 & $\begin{array}{l}\text { Ansprechen von möglichst vielen Player durch unterschiedliche Awareness- und } \\
\text { Vermarktungsmaßnahmen }\end{array}$ & $\mathrm{I} 2$ & $\begin{array}{l}\text { Streichen, da zu } \\
\text { unspezifisch }\end{array}$ \\
\hline Awareness & A2 & $\begin{array}{l}\text { Sensibilisierung österreichischer UnternehmerInnen für das Thema „Innovation } \\
\text { und Technologie“ und Motivation zur praktischen Umsetzung }\end{array}$ & $\begin{array}{l}\text { Staatspreis } \\
\text { Innovation }\end{array}$ & \\
\hline Awareness & A3 & $\begin{array}{l}\text { Bewusstseinsbildung in der interessierten Öffentlichkeit für das Thema Innovati- } \\
\text { on und Technologie (,Was leisten österreichische Unternehmen auf diesem } \\
\text { Sektor?“) }\end{array}$ & $\begin{array}{l}\text { Staatspreis } \\
\text { Innovation }\end{array}$ & \\
\hline Awareness & A4 & $\begin{array}{l}\text { Wissensplattform und Infoplattform bilden, um Innovation nachhaltig zu forcie- } \\
\text { ren und Chancen zu erhöhen (Vernetzung der wichtigsten Player aus Wissen- } \\
\text { schaft, Wirtschaft und Politik: AnbieterInnen und NachfragerInnen zusammen- } \\
\text { bringen); }\end{array}$ & $\begin{array}{l}\text { Staatspreis } \\
\text { Innovation }\end{array}$ & $\begin{array}{l}\text { Steichen, da } \\
\text { redundant } \mathrm{zu} \mathrm{A} 2 \\
\text { und A3 }\end{array}$ \\
\hline Awareness & A5 & Positionierung/Imagetransfer des BMWFJ in der Öffentlichkeit & $\begin{array}{l}\text { Staatspreis } \\
\text { Innovation }\end{array}$ & \\
\hline Awareness & A6 & $\begin{array}{l}\text { Bildungsauftrag „Innovation ist Zukunft“ - nachhaltige Verankerung der Bedeu- } \\
\text { tung des Themas vor allem bei Jugendlichen (von guten Ideen/Innovation lebt } \\
\text { die Wirtschaft) }\end{array}$ & $\begin{array}{l}\text { Jugend } \\
\text { Innovativ }\end{array}$ & \\
\hline Awareness & A7 & $\begin{array}{l}\text { Jugendliche - insbesondere Mädchen - für Technologie und Innovation begeis- } \\
\text { tern und Interesse an naturwissenschaftlichen und technischen Studienrichtungen } \\
\text { wecken }\end{array}$ & $\begin{array}{l}\text { Jugend } \\
\text { Innovativ }\end{array}$ & \\
\hline Awareness & A8 & $\begin{array}{l}\text { Vermittlung von Spaß und Motivation beim Experimentieren und Ausarbeiten } \\
\text { von innovativen Lösungsansätzen }\end{array}$ & $\begin{array}{l}\text { Jugend } \\
\text { Innovativ }\end{array}$ & \\
\hline Awareness & A9 & $\begin{array}{l}\text { Bewusstseinsbildung in der breiten Öffentlichkeit für das Thema „Jugend und } \\
\text { Innovation“ }\end{array}$ & $\begin{array}{l}\text { Jugend } \\
\text { Innovativ }\end{array}$ & $\begin{array}{l}\text { Streichen, da } \\
\text { redundant } \mathrm{zu} \mathrm{A6}\end{array}$ \\
\hline Awareness & A10 & Junge Talente zur Umsetzung ihrer Ideen motivieren & $\begin{array}{l}\text { Jugend } \\
\text { Innovativ }\end{array}$ & \\
\hline Awareness & A11 & $\begin{array}{l}\text { Schüler mit ihren Projekten eine optimale „Bühne“ bieten und außergewöhnliche } \\
\text { Leistungen auszeichnen }\end{array}$ & $\begin{array}{l}\text { Jugend } \\
\text { Innovativ }\end{array}$ & $\begin{array}{l}\text { verknüpfen mit } \\
\text { A10 }\end{array}$ \\
\hline Awareness & A12 & $\begin{array}{l}\text { Sensibilisierung österreichischer Lehrer für das Thema Innovation und Motivati- } \\
\text { on zur praktischen Projektumsetzung (,Teaching Innovation“) }\end{array}$ & $\begin{array}{l}\text { Jugend } \\
\text { Innovativ }\end{array}$ & \\
\hline Awareness & A13 & Das Kreativitäts- und Innovationspotential an Österreichs Schulen vorstellen. & $\begin{array}{l}\text { Jugend } \\
\text { Innovativ }\end{array}$ & $\begin{array}{l}\text { Streichen, da } \\
\text { redundant } \mathrm{zu} \\
\mathrm{A} 10 \text { und } \mathrm{A} 11\end{array}$ \\
\hline
\end{tabular}

Tabelle 30: Zielvereinfachung für die Zielebene „High-Tech Gründungen“

\begin{tabular}{|l|l|l|l|l|}
\hline Zieltyp & Nr. & Ziel & Programm & Bemerkung \\
\hline HTG & H1 & $\begin{array}{l}\text { Programmziel ist die nachhaltige Steigerung der Zahl der österreichischen High- } \\
\text { und die Überleitung von Forschungsergebnissen in wettbewerbsfähige Produkte, } \\
\text { Verfahren oder Dienstleistungen }\end{array}$ & PreSeed \\
\hline HTG & H2 & $\begin{array}{l}\text { Verbesserung der österreichischen Wirtschaftsstruktur durch die Fokussierung } \\
\text { auf dynamische, technologieorientierte Branchen sowie die Schaffung dauerhaf- } \\
\text { ter, hochqualitativer Arbeitsplätze }\end{array}$ & Seedfinancing \\
\hline
\end{tabular}




\begin{tabular}{|l|l|l|l|l|} 
HTG & H3 & Erhöhung der Anzahl von technologieorientierten Unternehmensneugründungen & Seedfinancing & $\begin{array}{l}\text { redundant zu H1, } \\
\text { aber anderes } \\
\text { Programm }\end{array}$ \\
\hline HTG & H4 & $\begin{array}{l}\text { Nachhaltige Unterstützung des Aufbaus von Unternehmen zur wirtschaftlichen } \\
\text { Nutzung innovativer und technologisch avancierter Produktideen, Verfahren } \\
\text { oder Dienstleistungen mit überdurchschnittlichem Marktpotential und Wachs- } \\
\text { tumschancen durch die Bereitstellung einer Seed-Finanzierung und einer pro- } \\
\text { jektbegleitenden Beratung. }\end{array}$ & Seedfinancing \\
\hline HTG & H5 $\begin{array}{l}\text { Förderung von dynamischem und qualitativem Wachstum von innovativen } \\
\text { Unternehmen }\end{array}$ & Seedfinancing & $\begin{array}{l}\text { streichen, da } \\
\text { redundant zu H4 }\end{array}$ \\
\hline HTG & H6 $\begin{array}{l}\text { Überlebenschance der geförderten Unternehmen durch aktive Hilfestellung und } \\
\text { schnelle Problemlösung mittels externer Experten bei sich abzeichnenden Krisen } \\
\text { (z.B. Verfehlung von Meilensteinen, Liquiditätsengpässen etc.) zu erhöhen, } \\
\text { dabei die Qualifizierung der MitarbeiterInnen des geförderten Unternehmens } \\
\text { durch den Know-How-Transfer während des Experteneinsatzes zu verbessern } \\
\text { und somit den unternehmerischen Erfolg von innovativen High-Tech- } \\
\text { Unternehmen nachhaltig zu steigern. }\end{array}$ & \begin{tabular}{l} 
MaZ \\
\hline
\end{tabular} \\
\hline
\end{tabular}

Tabelle 31: Zielvereinfachung für die Zielebene „High-Tech Risikokapital“

\begin{tabular}{|l|l|l|l|l|}
\hline Zieltyp & Nr. & Ziel & Programm & Bemerkung \\
\hline HTG-RK & R1 & $\begin{array}{l}\text { Unterstützung bei der Ausweitung der Kapitalbasis (insbesondere für Risikokapi- } \\
\text { tal). }\end{array}$ & Seedfinancing & \\
\hline HTG-RK & R2 & Dem Marktversagen in der Frühphasenfinanzierung entgegenzuwirken & I2 & \\
\hline HTG-RK & R3 & $\begin{array}{l}\text { Die Vermittlung von privaten Risikokapital von Investoren (Business Angels) an } \\
\text { Technologieunternehmen zur Schließung ihrer Eigenkapitallücke (equity gap) }\end{array}$ & I2 & verknüpfen mit R3 \\
\hline HTG-RK & R4 & $\begin{array}{l}\text { Angebot einer offenen und effizienten Plattform für alle Beteiligten (Unterneh- } \\
\text { men mit Kapital- und Unterstützungsbedarf, Investoren, Netzwerkpartner) }\end{array}$ & I2 & \\
\hline
\end{tabular}

Tabelle 32: Zielsystem für die Zielebene ,, Geistiges Eigentum“ (Anm.: keine Änderungen vorgenommen)

\begin{tabular}{|l|l|l|l|l|}
\hline Zieltyp & Nr. & Ziel & Programm & Bemerkung \\
\hline IP & I1 & $\begin{array}{l}\text { österreichischen Unternehmen, primär KMU, bei der Schaffung, Durchsetzung, } \\
\text { Ferwertung und Überwachung ihrer geistigen Eigentumsrechte zur Stärkung und } \\
\text { effizient zu unterstützen }\end{array}$ & ipp \\
\hline IP & I2 & $\begin{array}{l}\text { Iie Bewusstseinsbildung der KMUs für ihre eigenen IPs zu stärken, das Erken- } \\
\text { nen von Gefahren fremden IPs zu sensibilisieren, sowie die Etablierung eines } \\
\text { strategischen IP-Management-Konzepts voranzutreiben }\end{array}$ & discover IP & \\
\hline IP & I3 & Schaffen, Stärken und Erweitern der internationalen Netzwerke & $\begin{array}{l}\text { internationale } \\
\text { Projekte }\end{array}$ & \\
\hline
\end{tabular}


Tabelle 33: Zielvereinfachung für die Zielebene „,Kreativwirtschaft"

\begin{tabular}{|c|c|c|c|c|}
\hline Zieltyp & Nr. & Ziel & Programm & Bemerkung \\
\hline Kreativ & K1 & $\begin{array}{l}\text { Etablierung der Kreativwirtschaft als Impulsgeber für Innovationen und maßgeb- } \\
\text { lichen Wertschöpfungsfaktor }\end{array}$ & impulse & \\
\hline Kreativ & $\mathrm{K} 2$ & $\begin{array}{l}\text { Stärkung der wirtschaftlichen Leistungsfähigkeit von Kreativwirtschaftsunter- } \\
\text { nehmen }\end{array}$ & impulse & \\
\hline Kreativ & K3 & $\begin{array}{l}\text { Stärkung des Innovationspotentials und der Wettbewerbsfähigkeit von KMUs } \\
\text { aller Branchen durch die Integration kreativer Leistungen in unternehmerische } \\
\text { Vorhaben }\end{array}$ & impulse & \\
\hline Kreativ & K4 & $\begin{array}{l}\text { Optimale Betreuung potentieller GründerInnen und Unternehmen im Kreativ- } \\
\text { wirtschaftsbereich }\end{array}$ & impulse & \\
\hline Kreativ & K5 & $\begin{array}{l}\text { Erhöhung der Anzahl von Kreativwirtschaftsunternehmen in Österreich, insbe- } \\
\text { sondere durch Neugründungen }\end{array}$ & impulse & verknüpfen mit $\mathrm{K} 4$ \\
\hline Kreativ & K6 & Verbesserung des betriebswirtschaftlichen Know-Hows in den Kreativbranchen & impulse & verknüpfen mit K2 \\
\hline Kreativ & K7 & Stimulation von Investitionen materiell und immateriell & impulse & $\begin{array}{l}\text { streichen, da zu } \\
\text { unspezifisch }\end{array}$ \\
\hline Kreativ & K8 & Etablierung Österreichs als international anerkannten Kreativstandort & impulse & \\
\hline Kreativ & K9 & $\begin{array}{l}\text { Anregung von Kooperationen sowohl zwischen Kreativwirtschaftsunternehmen } \\
\text { als auch mit der „traditionellen“ Wirtschaft }\end{array}$ & impulse & \\
\hline Kreativ & K10 & Erhöhung der Bedeutung kreativer Leistungen in der Wirtschaft & impulse & redundant zu K1 \\
\hline
\end{tabular}

Tabelle 34: Zielvereinfachung für die Zielebene „Tech-Information“

\begin{tabular}{|c|c|c|c|c|}
\hline Zieltyp & Nr. & Ziel & Programm & Bemerkung \\
\hline TecInfo & $\mathrm{T} 1$ & $\begin{array}{l}\text { Vermittlung von Markt- und Technologieinformation zur Untermauerung von } \\
\text { Businessplänen und Vermeidung von Fehlinvestitionen; }\end{array}$ & tecnet & \\
\hline TecInfo & $\mathrm{T} 2$ & $\begin{array}{l}\text { Erleichterung von Finanzierungsentscheidungen von Förderungsgebern um } \\
\text { Fehlinvestitionen zu vermeiden; }\end{array}$ & tecnet & $\begin{array}{l}\text { Anm.: Kapitalge- } \\
\text { ber aus T5 hier mit } \\
\text { berücksichtigen }\end{array}$ \\
\hline TecInfo & $\mathrm{T} 3$ & $\begin{array}{l}\text { Schaffung von Zugang zu (Patent-)Datenbanken und Bereitstellung von Recher- } \\
\text { che-Know-How und -kapazitäten (Verringerung des Informationsdefizits von } \\
\text { KMUs gegenüber Großunternehmen); }\end{array}$ & tecnet & \\
\hline TecInfo & $\mathrm{T} 4$ & $\begin{array}{l}\text { Unterstützung strategischer Entscheidungen bei der Produktentwicklung und - } \\
\text { vermarktung von neuen Technologien; }\end{array}$ & tecnet & verknüpfen mit $\mathrm{T} 1$ \\
\hline TecInfo & T5 & $\begin{array}{l}\text { Markt- und Technologieinformationen so verfügbar } \mathrm{zu} \text { machen, dass sie von } \\
\text { Unternehmen und Kapitalgebern sinnvoll genutzt werden können und Unter- } \\
\text { nehmen wichtige Ressourcen (Zeit, Geld und Personal) sparen. }\end{array}$ & tecnet & $\begin{array}{l}\text { redundant zu T3 } \\
\text { (siehe Bemerkung } \\
\text { zu T2) }\end{array}$ \\
\hline TecInfo & T6 & $\begin{array}{l}\text { österreichischen Erfindern, Unternehmen und Wissenschaftlern hinsichtlich der } \\
\text { Vermarktung von erfolgversprechenden Innovationen zu unterstützen }\end{array}$ & tecma & \\
\hline
\end{tabular}




\begin{tabular}{|l|l|l|l|l|} 
TecInfo & T7 & $\begin{array}{l}\text { Optimierung von Unternehmensstrategien mittelständischer Unternehmen zur } \\
\text { Forcierung deren FEI-Potentials im Hinblick auf die Verbesserung der Produkt- } \\
\text { portfolios durch die Implementierung der strategischen Produktfindung mit } \\
\text { geeigneten Methoden und der Erschließung neuer Märkte. }\end{array}$ & ProTRANS & $\begin{array}{l}\text { streichen, da } \\
\text { bereits in T8 } \\
\text { beinhaltet }\end{array}$ \\
\hline TecInfo & T8 & Verbesserung des unternehmensinternen Innovations- und Wissensmanagement & ProTRANS & \\
\hline
\end{tabular}

Tabelle 35: Zielvereinfachung für die Zielebene ,,Technologietransfer“

\begin{tabular}{|c|c|c|c|c|}
\hline Zieltyp & Nr. & Ziel & Programm & Bemerkung \\
\hline Transfer & TT1 & $\begin{array}{l}\text { Aufbau von Know-How an den Universitäten und außeruniversitären Einrich- } \\
\text { tungen }\end{array}$ & uniinvent & $\begin{array}{l}\text { streichen, da zu } \\
\text { unspezifisch }\end{array}$ \\
\hline Transfer & TT2 & $\begin{array}{l}\text { Aufbau von Verwertungsinfrastrukturen in Partnerschaft zwischen Universitäten, } \\
\text { Fachhochschulen und außeruniversitären Forschungseinrichtungen }\end{array}$ & uniinvent & \\
\hline Transfer & TT3 & $\begin{array}{l}\text { Nutzung von professionellen Verwertungsagenturen für die Vermarktung von } \\
\text { Patenten }\end{array}$ & uniinvent & redundant $\mathrm{zu}$ TT2 \\
\hline Transfer & TT4 & $\begin{array}{l}\text { Grundlagen-Innovationen potentiellen Entwicklungspartner vorzustellen, um den } \\
\text { Schritt von der Weiterentwicklung hin zu einem marktfähigen Produkt zu ermög- } \\
\text { lichen bzw. zu erleichtern }\end{array}$ & prize & \\
\hline Transfer & TT5 & $\begin{array}{l}\text { Stärkung der Innovationskraft und Hebung der F\&E-Leistung der Unternehmen } \\
\text { der mittelständischen Wirtschaft durch Stimulierung von Technologietransfer. }\end{array}$ & ProTRANS & \\
\hline Transfer & TT6 & $\begin{array}{l}\text { Verbesserung der Innovations- und Kooperationskultur österreichischer KMU } \\
\text { und ihres Zugangs zu den Methoden und dem Know-How von Forschungsrein- } \\
\text { richtungen." }\end{array}$ & ProTRANS & \\
\hline Transfer & TT7 & $\begin{array}{l}\text { Verbesserung der Nutzung externer Ressourcen für KMU, im Hinblick auf die } \\
\text { Implementierung geeigneter Methoden der strategischen Produktfindung insbe- } \\
\text { sondere die Optimierung des Zuganges zur Expertise von Forschungseinrichtun- } \\
\text { gen (u.a. Universitäten, Fachhochschulen, außeruniversitäre Forschungsreinrich- } \\
\text { tungen, kooperative Forschungseinrichtungen) und Unternehmen (inklusive } \\
\text { spezialisierte Beratungsunternehmen). }\end{array}$ & ProTRANS & redundant zu TT6 \\
\hline Transfer & TT8 & $\begin{array}{l}\text { Stimulierung von strategischen Partnerschaften für die Weiterentwicklung und } \\
\text { Markteinführung neuer Produkte, Prozesse und Dienstleistungen. }\end{array}$ & ProTRANS & redundant zu TT6 \\
\hline
\end{tabular}

Der hier gegebene Vorschlag zur Ziel-Reformulierung zeigt also, dass der etwas ,überfrachtete“ Zielkatalog der Technologieprogramme allein durch Reduktion der Redundanz zwischen ähnlichen Zielen der jeweils gleichen Zielebene sowie durch Streichen einiger zu unspezifisch gehaltener Ziele vereinfacht werden kann.

\subsection{INTERNE SYNERGIEN}

Die internen Schnittstellen und Synergien nehmen eine wichtige Rolle für die Gesamtbewertung eines Programmportfolios ein. Zunächst sollen die „Verknüpfungen“ der Programme zueinander dergestalt analysiert werden, dass jene Programme als in Verknüpfung befindlich definiert werden, die von einem Unternehmen jeweils als Förderungsinstrument beansprucht wurden (unabhängig vom Zeitpunkt der Inanspruchnahme, d.h. die Förderungsinstrumente müssen nicht unbedingt gleichzeitig in Anspruch genommen werden). Die Verbindung (und 
damit die potentiellen Synergieeffekte) zwischen zwei Programmen ist demnach umso stärker, je mehr Unternehmen diese beiden Programme in Anspruch nehmen. Als Datenbasis dient hierfür die im Rahmen der Systemevaluierung ermittelte Inanspruchnahme der Förderungsinstrumente durch Unternehmen. In Abbildung 38 ist das so verstandene Beziehungsgefüge der aws-Technologieprogramme (plus einiger zusätzlich ausgewählter awsFörderungsprogramme) in Form einer Netzwerkanalyse dargestellt. Das Programm Seedfinancing findet sich dabei im Zentrum des Netzwerks ${ }^{35}$ mit einer Reihe von engen Verknüpfungen zu anderen Förderungsinstrumenten der aws, wie vor allem Double Equity und der KMU - Innovationsförderung Unternehmensdynamik. Diese Programme sind von ihrer Zielsetzung und Programmkonzeption her komplementär. Die nun empirisch bestätigte enge Verknüpfung dieser Programme via Förderfälle ist ein weiterer Hinweis auf synergetische Beziehungen zwischen den Programmen / Instrumenten innerhalb der aws. Zu beachten ist, dass die Förderungsmaßnahmen nur eine der Finanzierungsquellen darstellen und zusätzliche private Finanzierungen (wie sie z.B. beim Seedfinancing-Programm in signifikanter Höhe zu finden sind) nicht in die Analyse mit aufgenommen werden konnten.

\section{Abbildung 38: Beziehungen zwischen den aws-Programme untereinander}

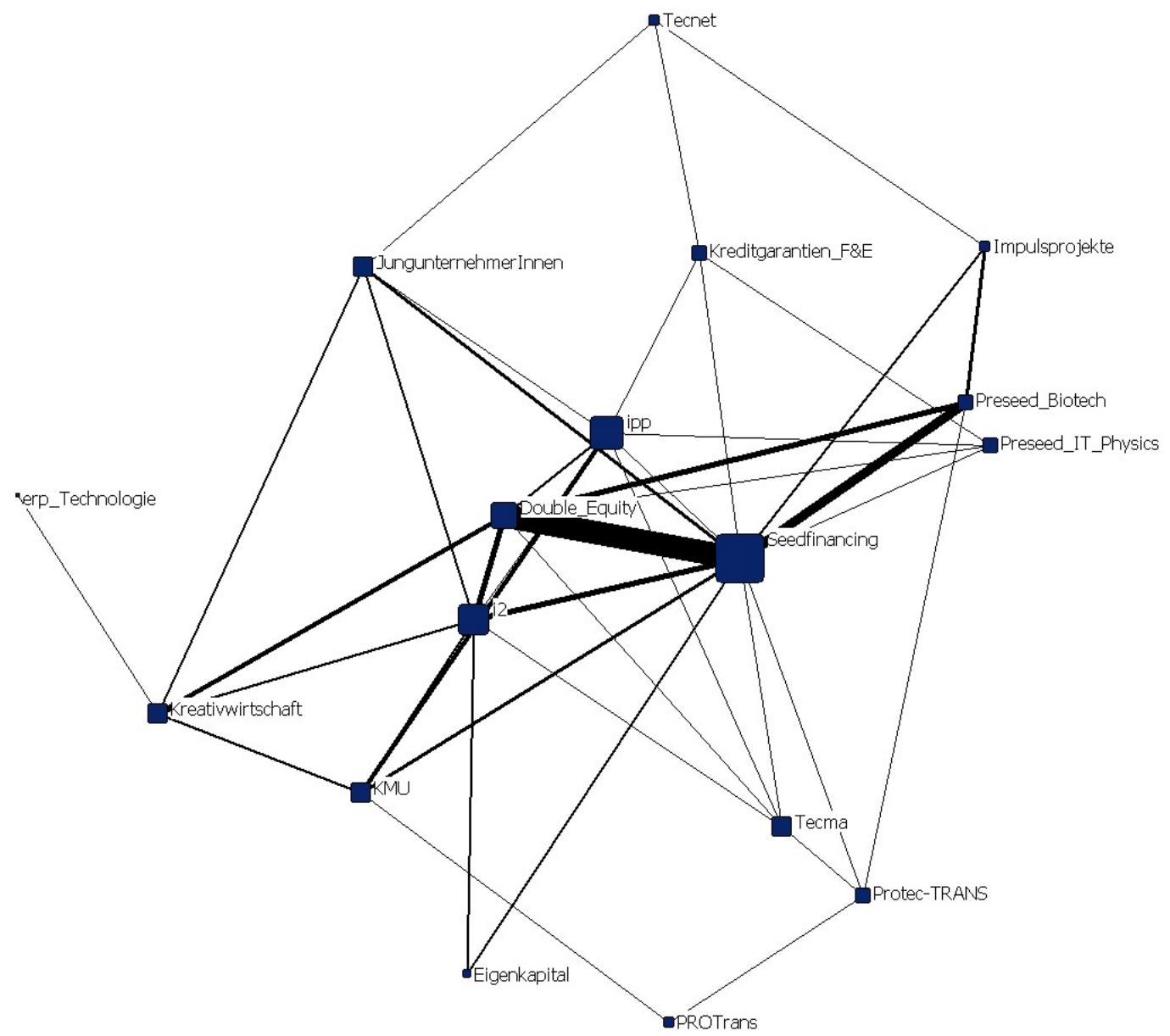

Anmerkungen: Größe des Knoten basiert auf dem Degree (Anzahl der Verbindungen mit anderen aws Programmen unabhängig von der Stärke der Verbindung); Linienstärke basiert auf der Anzahl der Verbindungen;

Layout: Multidimensional Scaling (angepasst zur besseren Übersicht)

\footnotetext{
${ }^{35}$ Je näher am Zentrum der graphischen Netzwerkdarstellung ein Programm liegt, desto mehr ist es auch mit allen anderen Programmen verknüpft, während ein Programm, das z.B. nur mit einem einzigen anderen Programm verknüpft ist, eher an den Rand der graphischen Darstellung ,gedrängt“ wird.
} 
Quelle: WIFO/KMFA (2008/2010): Unternehmensbefragung im Rahmen der Systemevaluierung, KMFA-Berechnungen; Darstellung JOANNEUM RESEARCH

Betrachtet man nur die aws-Technologieprogramme, so ist insbesondere PreSeed-Biotech und i2-Business Angel Börse eng mit dem Seedfinancing-Programm verflochten. Die geringe Anzahl an Verknüpfungen mit PreSeed-IT/Physik dürfte eher in der Struktur der Datenbasis begründet sein, von der angenommen werden kann, dass sie bei sehr jungen und noch tendenziell sehr kleinen Unternehmen eine überdurchschnittlich hohe Untererfassung aufweist.

Mit dem ipp-Innovationsschutzprogrammbefindet sich noch ein Programm aus dem Bereich Wissenskapital/IP im Zentrum des Netzwerkes. Die anderen Programme der awsTechnologieprogramme finden sich hingegen tendenziell an der Peripherie. Da dieser Befund auf Basis der hier herangezogenen Systemevaluierungsdaten teilweise Auswertungen von Seiten der aws auf Basis aws interner Programmdaten widerspricht, sollten diese Ergebnisse aber lediglich mit Vorsicht interpretiert werden. Ein Grund der hier angedeuteten geringen Vernetzung könnte in der Untererfassung von bestimmten Unternehmenstypen (neu gegründete, kleine Unternehmen bzw. überhaupt erst in Gründung befindliche Unternehmen) von Seiten der Systemevaluierungsdatenbank liegen. Da einige der aws-Technologieprogramme gerade die Frühphase der Unternehmensgründung adressieren und auch bei anderen Technologieprogrammen explizit ganz junge, kleine Unternehmen (bzw. sich überhaupt erst in Gründung befindliche Projekte) ansprechen, könnte es somit zu systematischen Verzerrungen kommen.

Zusätzlich zu der oben beschriebenen Datenquelle wurde für die Analyse der internen Synergien im Februar 2011 von der aws eine Spezialauswertung aller Seedfinancing-Anträge durchgeführt, wobei für Antragsteller (in Summe 293) erhoben wurde, inwieweit - neben der (beabsichtigten) Nutzung von Seedfinancing - auch andere Förderungsinstrumente der aws (sowohl aws-Technologieprogramme als auch die nicht technologieorientierten Instrumente der aws) in Anspruch genommen wurden ${ }^{36}$. Diese Analyse wird dabei sowohl für die tatsächlich in das Seedfinancing Programm aufgenommenen Anträge durchgeführt als auch für jene Fälle, wo der Antrag zum Seedfinancing zwar abgelehnt wurde, die Antragsteller aber durch andere Programme der aws betreut wurden. Abbildung 39 zeigt, inwieweit die Antragsteller an Seedfinancing auch andere aws-Programmen in Anspruch nehmen, wobei zwischen erfolgreichen Antragstellern (= ins Seedfinancing-Programm aufgenommene) und abgelehnten Fällen unterschieden wird.

\footnotetext{
36 Aus organisatorischen Gründen war es nicht möglich, diese Analyse für alle Antragsteller an awsTechnologieprogramme durchzuführen. Ein Problem besteht auch darin, dass z.B. Anträge bei gewissen Programmen in der Vorgründungsphase noch durch natürliche Personen erfolgen. Kommt dann eine Unternehmensgründung zustande und werden auch andere Technologieprogramme in Anspruch genommen, lässt sich dieses Unternehmen dann nicht mehr auf die ursprüngliche natürliche Person zurückführen.
} 
Abbildung 39: Inanspruchnahme von aws-Programmen durch Seedfinancing-Antragsteller

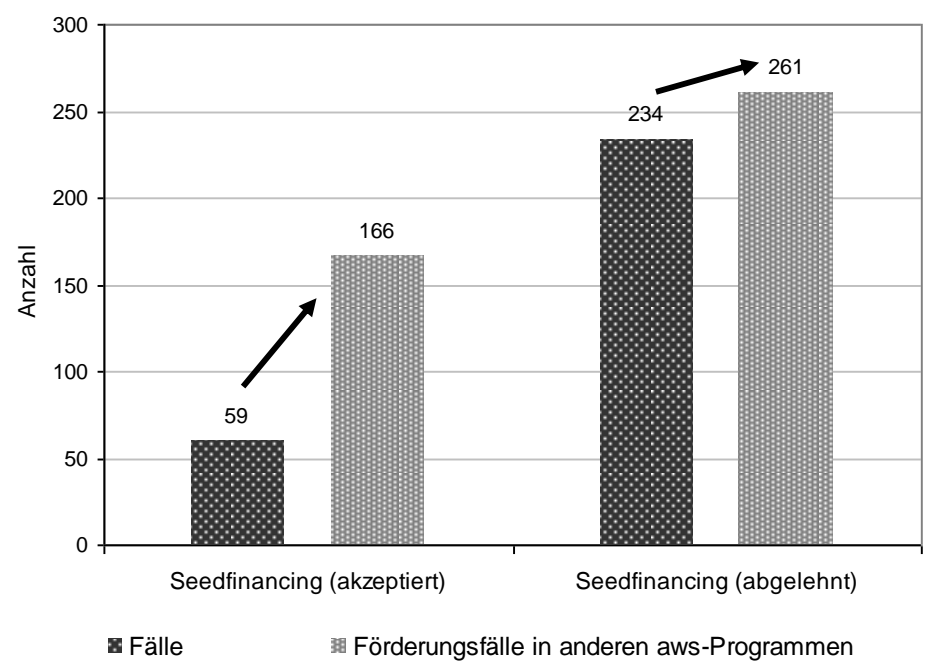

Quelle: aws

Insgesamt wurden 293 Anträge identifiziert und weiter analysiert, wovon 59 Anträge tatsächlich für das Seedfinancing-Programm genehmigt und 234 abgelehnt wurden. Wie Abbildung 39 zeigt, nehmen die 59 durch Seedfinancing geförderten Fälle in Summe 166 zusätzliche Förderungen in anderen aws-Programmen in Anspruch. Dies ist ein deutlicher Hinweis auf synergetische Beziehungen der einzelnen Förderinstrumente (und zwar nicht nur der awsTechnologieprogramme) innerhalb der aws. Auch die letztlich nicht durch Seedfinancing geförderten Fälle (= Ablehnung des Seedfinancing-Antrags) weisen trotzdem Förderkontakte zu anderen aws-Programmen auf. Allerdings ist diesbezüglich der „Vernetzungsgrad“ deutlich geringer, die 234 abgelehnten Fälle finden sich in Summe in nur 261 andere Förderfälle in anderen aws-Programmen. Letztlich ist dies ein weiterer Hinweis darauf, dass durch die hohe Selektion des Seedfinancing-Programms die Seedfinancing-Projekte eine besondere Attraktivität (z.B. Wachstumspotential, spezifischer Beratungsbedarf etc.) für Förderer haben und dadurch auch bei anderen Programmen entsprechend Förderung finden.

Welche aws-Programme nun tatsächlich zusätzlich durch Seedfinancing-Antragsteller genutzt werden, zeigt Abbildung 40, wobei wiederum zwischen erfolgreichen SeedfinancingAntragsteller und jenen, die vom Seedfinancing-Programm abgelehnt wurden, differenziert wird. 
Abbildung 40: Inanspruchnahme zusätzlicher aws Produkte (Programme) durch Seedfinancing-Antragsteller (tatsächlich geförderte versus abgelehnte)

Anteil der Unternehmen/Gründer, die zusätzlich das jeweilige awsProgramm in Anspruch nehmen

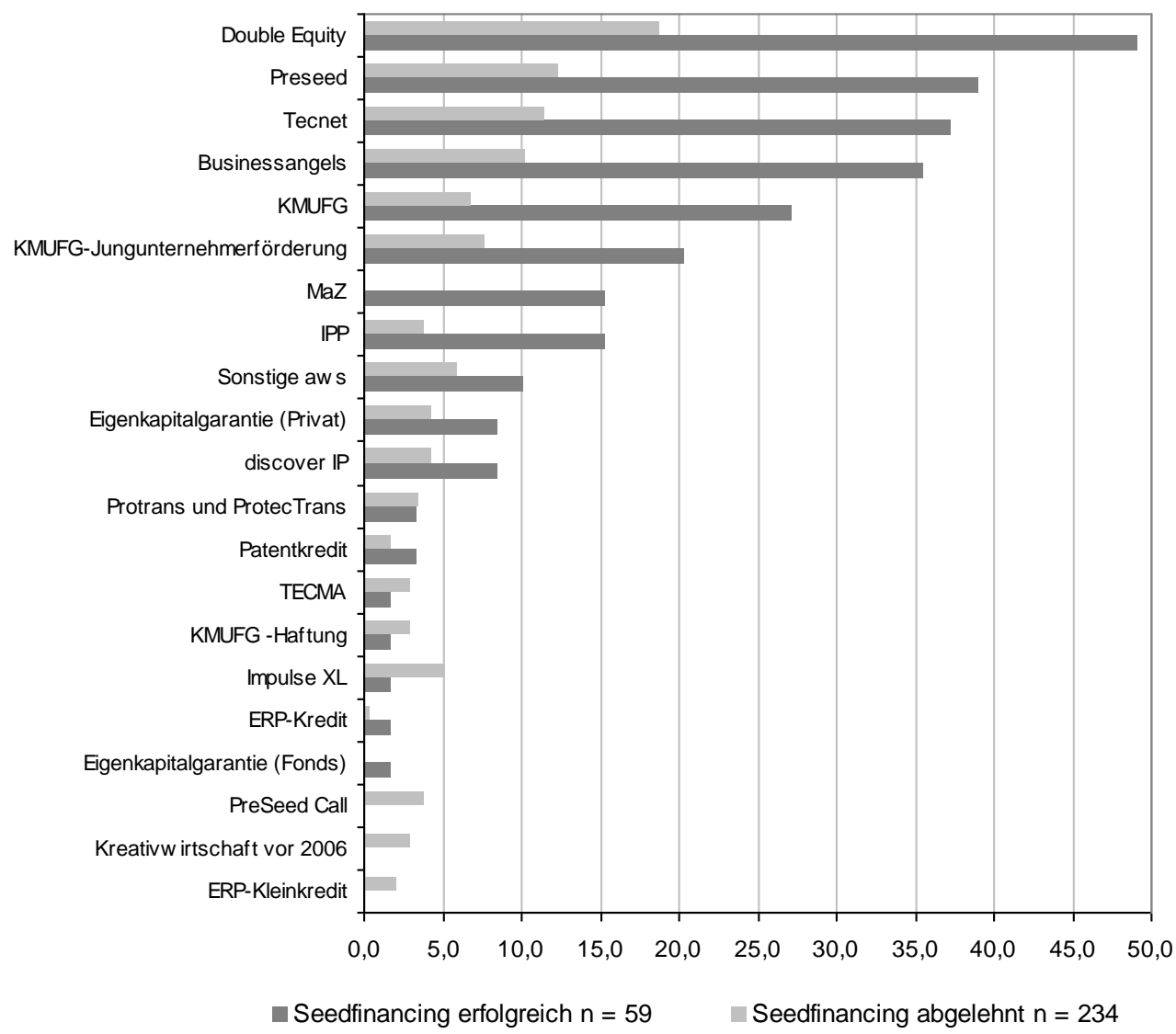

Quelle: aws

Die Ergebnisse zeigen deutlich die starke Verflechtung des Seedfinancing Programms mit anderen aws Instrumenten. Dabei sind es gerade Instrumente zur Wachstumsförderung bzw. zur Bereitstellung von Risikokapital die von vielen Seedfinancing-geförderten Unternehmen zusätzlich genutzt werden. So erhalten knapp 50 \% der durch Seedfinancing geförderten Projekte zusätzlich auch Mittel aus dem Double Equity Programm der aws $;{ }^{37}$ dagegen erhalten nur knapp 20 \% der bei Seedfinancing abgelehnten Projekte eine Förderung via Double Equity. Die starke „Verknüpfung“ zu PreSeed ist aufgrund der Programmkonzeption beider Programme nicht überraschend, bestätigt aber, dass durch PreSeed tatsächlich eine laufende „Projektpipeline“ für darauffolgende Seedfinancing-Förderungsprojekte generiert wird (immerhin knapp $40 \%$ der hier analysierten Seedfinancing-Projekte wurden via PreSeed generiert). Auffällig ist auch, dass das Programm tecnet ebenfalls intensiv von Seedfinancinggeförderten Unternehmen genutzt wird. Das zeigt, dass High-Tech Neugründungen neben der

\footnotetext{
${ }^{37}$ Double Equity ermöglicht die Übernahme einer Haftung. Adressiert wird damit die Verbesserung der Finanzstruktur von kleinen und mittleren Unternehmen (Ausnahme: Tourismus- und Freizeitwirtschaft), die für die Finanzierung bereits privates Eigenkapital erhalten haben. Konkret besteht die Förderung in einer Garantieübernahme von bis zu $80 \%$ für einen Kredit in Höhe von bis zu 2.500.000 EUR. Der im Double Equity Garantiefonds verbürgte Kredit kann zur Finanzierung aller betrieblichen Ausgaben (z.B. Investitionen) im Rahmen einer Expansionsstrategie verwendet werden.
} 
Finanzierungslücke auch einen erheblichen Informationsbedarf hinsichtlich Markt- und Technologiesituation bezüglich ihrer Geschäftsideen aufweisen. 


\section{Modul 3: Funktion und Positionierung im Innovations-} system

\subsection{VORBEMERKUNG}

Ziel von Modul 3 ist die Analyse der Positionierung der aws-Technologieprogramme im gesamten österreichischen Innovationssystem. Dabei stehen folgende Fragen im Vordergrund:

Die wesentlichen Leitfragen des Moduls 3 sind:

- Wie sind die aws-Technologieprogramme im gesamten österreichischen Innovationssystem positioniert?

- Wie sind die aws-Technologieprogramme in die Förderungsaktivitäten im Bereich der Innovationspolitik auf Ebene der Bundesländer eingebettet? Wie fügen Sie sich in die jeweils regionalen Förderstrukturen ein?

- Wie grenzen sich die aws-Technologieprogramme gegenüber dem Förderungsportfolio der FFG ab? Welche Wechselwirkungen gibt es zwischen den awsTechnologieprogrammen und den FTI-Programmen der FFG?

In Modul 2wurden die Zielbündel der verschiedenen Programme der Gruppe „Technologieprogramme“ der aws analysiert. Alle diese Zielbündel adressieren für Österreich und international wesentliche Motivbereiche für politisches Handeln. Ihre Relevanz muss wohl hier nicht einzeln begründet werden sondern ist vielfach belegt, exemplarisch sei z. B. auf das WIFO Weißbuch, auf verschiedene Empfehlungen des Rates für Forschung und Technologieentwicklung, auf dessen Strategie 2020, auf das Grünbuch der Europäischen Kommission zur „Erschließung des Potenzials der Kultur- und Kreativindustrien“ usw. verwiesen.

Schnittstellen auf Bundesebene gibt es in Bezug auf die Technologieprogramme einerseits aws intern zu anderen Förderungen (sieh dazu auch Modul 2), aber auch z.B. in Bezug auf die Förderungen der FFG oder der Bundesländer. Diese Zusammenhänge werden aus der Perspektive der Nutzer dargestellt, weiters wird die Perspektive von Stakeholdern/ ExpertInnen auf Basis von Interviews berücksichtigt und auf die Abstimmung zwischen den Förderungsorganisationen eingegangen, dies auch im Verhältnis mit den Bundesländern.

Methodisch beruht dieses Kapitel neben der Dokumentenanalyse und Interviews mit Stakeholdern und Kunden der aws auch auf der Auswertung der Befragungsergebnisse aus der Systemevaluierung der österreichischen Forschungsförderung und -finanzierung, die 2008-2009 im Auftrag des BMWFJ und des BMVIT durch ein Konsortium aus WIFO, KMU FORSCHUNG AUSTRIA, Prognos Berlin und convelop durchgeführt wurde ${ }^{38}$. Befragt wurden 5.308 Unternehmen aus FEI-affinen Sektoren, der Rücklauf betrug 28\%. Die Adressen der befragten Unternehmen stammen von Herold, aws und FFG.

Für den vorliegenden Bericht werden Auswertungen dieser Daten herangezogen. In der Befragung wurde erhoben, in welchen der als für die Systemevaluierung als relevant ausgewählten 77 Förderungsprogrammen die befragten Unternehmen Anträge eingereicht haben und

\footnotetext{
${ }^{38}$ Die Nutzung dieser Daten wurde durch die Auftraggeber für die vorliegende Evaluierung freigegeben. Die Ergebnisse der spezifischen Datenauswertung werden den Auftraggebern der Systemevaluierung zur Verfügung gestellt.
} 
Projekte genehmigt wurden (bezogen auf den Zeitraum 2002 bis 2008). Von den gegenständlichen Technologieprogrammen der aws sind folgende Programme in dieser Befragung erfasst:

- i2- Die Börse für Business-Angels

- ipp - Innovationsschutzprogramm

- PROtrans

- tecma

- tecnet

- PreSeed Biotech

- PreSeed IT \& Physical Sciences

- Seedfinancing

- Impulsprogramm Kreativwirtschaft

Die vorliegenden Auswertungen der Befragung im Rahmen der Systemevaluierung fokussieren auf die Perspektive der Nutzung der Förderungsprogramme, d.h. darauf, in welchen Programmen Anträge gestellt wurden. Als ,Systemnutzer“" wurden dabei, ebenso wie in der Systemevaluierung, diejenigen Unternehmen bezeichnet, die mindestens einen Antrag für direkte FTI-Förderung in den für die Systemevaluierung relevanten Programmen zwischen 2005 und 2008 gestellt haben und/oder indirekte Forschungsförderung (Forschungsfreibetrag, Forschungsprämie) zw. 2002 und 2006 in Anspruch genommen haben.

\subsection{CHARAKTERISTIKA DER ANTRAGSTELLER IN DEN TECHNOLOGIEPROGRAMMEN DER aws}

Um die Nutzer der Technologieprogramme (62 Unternehmen, die mindestens einen Antrag in einem der gegenständlichen Programme eingereicht haben) zu charakterisieren, wurden Strukturdaten der Unternehmen, die bei den Technologieprogrammen der aws mindestens einen Antrag eingereicht haben, der Gesamtgruppe der Systemnutzer aus der Befragung im Zuge der Systemevaluierung gegenübergestellt. ${ }^{39}$ Klar ersichtlich ist der hohe Anteil an Mikro- bzw. Kleinunternehmen ${ }^{40}$ im Vergleich zur Gruppe der Systemnutzer insgesamt. Außerdem sind die Unternehmen, die bei den aws-Technologieprogrammen eingereicht haben, deutlich jünger und mehrheitlich in High-Tech-Industrien sowie im Bereich wissensintensive Dienstleistungen (inkl. Spitzentechnologie nutzende wissensintensive Dienstleistungen) tätig. Von jenen 33 Unternehmen (53\%), die Antragsteller bei den aws-Technologieprogrammen waren und den wissensintensiven Dienstleistungen zugeordnet werden, entfallen die meisten Unternehmen auf die Branchen "Forschung und Entwicklung" (10 Unternehmen), "Erbringung von Dienstleistungen der Informationstechnologie" (9 Unternehmen) und "Architekturund Ingenieurbüros; technische, physikalische und chemische Untersuchung" (6 Unternehmen).

\footnotetext{
${ }^{39}$ Bei der Auswertung über genehmigte Projekte anstelle der eingereichten Anträge wurden für die konkreten Fragestellungen der vorliegenden Evaluierung keine signifikanten Unterschiede in den Ergebnissen festgestellt; daher und vor allem aufgrund einer zu geringen Stichprobengröße für die Unternehmen mit genehmigten Anträgen wurde in Bezug auf die Antragstellung ausgewertet.

${ }^{40}$ Die Differenzierung erfolgte ausschließlich anhand der Anzahl der MitarbeiterInnen.
} 
Tabelle 36: Strukturdaten der untersuchten Unternehmen

\begin{tabular}{lcc}
\hline & $\begin{array}{c}\text { Antragsteller aws Tech- } \\
\text { Programme (in \%) } \\
\mathrm{n}=62\end{array}$ & $\begin{array}{c}\text { Systemnutzer Syseval } \\
\text { (in \%) } \\
\mathrm{n}=1.410\end{array}$ \\
\hline Unternehmensgröße (nach Anzahl der MitarbeiterInnen) & & 33,3 \\
Mikrounternehmen (<10 Beschäftigte) & 65,5 & 28,6 \\
Kleinunternehmen (10-49) & 22,4 & 21,7 \\
Mittlere Unternehmen (50-249) & 8,6 & 8,1 \\
Große Unternehmen (250-499) & 0,0 & 8,2 \\
Sehr große Unternehmen (+500) & 3,4 & \\
Gründungsjahr & & 55,0 \\
Bis 1994 & 17,5 & 33,4 \\
1995 bis 2004 & 36,8 & 11,6 \\
Seit 2005 & 45,6 & \\
Sektorale Klassifikation (OECD) & & 6,6 \\
low tech Industrien & 3,2 & 9,5 \\
medium-low tech Industrien & 4,8 & 23,0 \\
medium-high tech Industrien & 11,3 & 9,6 \\
high-tech Industrien & 16,10 & 15,2 \\
Nicht-wissensintensive Dienstleistungen & 11,3 & 35,6 \\
Wissensintensive Dienstleistungen (inkl. Spitzen- & 53,3 & \\
technologie nutzende wissensintensive DL) & & \\
\hline
\end{tabular}

Anmerkung: Die sektorale Klassifikation wurde im Rahmen der Befragung von den befragten Unternehmen selbst vorgenommen. Dabei bezog sich die Klassifikation auf das gesamte Unternehmen (z.B. im Bereich Life Sciences: Messleistungen und Diagnostik) und nicht auf ein spezielles Projekt.

Quelle: WIFO/KMFA (2008/2010): Unternehmensbefragung im Rahmen der Systemevaluierung, KMFA-Berechnungen

Die Selektion auf Seiten der Antragsteller zeigt also ein deutliches Übergewicht der Mikrounternehmen; auch jüngere Unternehmen fühlen sich eher angesprochen. Dienstleistungsunternehmen sind stärker vertreten unter den Antragstellern der Technologieprogramme der aws als unter den Systemnutzern insgesamt, vor allem gilt dies für wissensintensive Dienstleistungen. Dieses Bild entspricht im Grunde der Förderungslogik der gegenständlichen Programme, die ja stärker den Innovationsbereich der Unternehmen (strategische Unternehmensentwicklung) fokussieren, als beispielsweise klassische, projektbezogene F\&E. Auch eine aktuelle Studie über Dienstleistungsunternehmen ${ }^{41}$ zeigt, dass Dienstleistungsunternehmen und insbesondere Unternehmen in den wissensintensiven Dienstleistungen weniger abgegrenzte, sondern vielmehr das gesamte Unternehmen betreffende Aktivitäten (z.B.. Organisationsentwicklung) verfolgen.

\footnotetext{
${ }^{41}$ Ruhland et.al. (2010): Dienstleistungslandschaft in Österreich, Studie im Auftrag der Österreichischen Forschungsförderungsgesellschaft FFG und des Bundesministeriums für Wirtschaft, Familie und Jugend BMWFJ, Wien
} 


\subsection{ZUSAMMENSPIEL VERSCHIEDENER FÖRDERUNGEN}

\subsubsection{Nutzung durch die Unternehmen}

Im Folgenden wird auf Basis der Befragung im Rahmen der Systemevaluierung dargestellt und diskutiert, wie viele Unternehmen im Zuge der Befragung angaben, mindestens einen Antrag im Zeitraum 2005-08 in einem der Technologieprogramme der aws eingereicht zu haben.

Basierend auf den erhobenen Daten ist festzustellen, dass die Programme PROTrans, Tecma und tecnet im fraglichen Zeitraum offenkundig nur von einer kleinen Zahl an Unternehmen (jeweils drei) beantragt wurden. Hier muss jedoch anmerkt werden, dass die Befragung im Rahmen der Systemevaluierung nicht dezidiert auf Antragsteller dieser Programme abgestellt hat.

Unter den betrachteten Programmen weisen diejenigen der Frühphasenfinanzierung insgesamt die größte Nachfrage (Anzahl der antragstellenden Unternehmen) auf, gefolgt von impulse (Impulsprogramm für die Kreativwirtschaft) und I2. In der zweiten Spalte ist dargestellt, wie viele der Unternehmen, die mindestens einen Antrag in einem bestimmten Programm der Technologieprogramme eingereicht haben, nur in diesem Programm eingereicht haben, d.h. wie selektiv die einzelnen Technologieprogramme genutzt werden. Dabei zeigt sich deutlich, dass das Impulsprogramm Kreativwirtschaft von seiner Zielgruppe sehr selektiv genutzt wird; ähnlich PreSeed IT \& Physical Sciences; interessanterweise gilt das nicht für PreSeed Biotech, was möglicherweise auf die weiterführenden Begleitmaßnahmen (Stichwort LISA) und den Anschluss Seedfinancing zurückzuführen sein kann. Die Kombination mit Seedfinancing ist bei Nutzern von PreSeed Biotech deutlich häufiger als bei Nutzern von PreSeed IT \& Physical Sciences (vgl. Modul 2). Die Börse für Business Angels I2 wird auch vorrangig in Kombination mit anderen Förderungen genutzt, vor allem ist dies innerhalb der Technologieprogramme wieder Seedfinancing. Das Programm Seedfinancing wird - außerhalb der Technologieprogramme - am häufigsten mit dem Programm Double Equity kombiniert. Die Programme PROtrans und tecnet wurden von einer geringen Zahl der befragten Unternehmen, von diesen aber sehr selektiv genutzt. 
Tabelle 37: Anzahl der Unternehmen, die in den Technologieprogrammen der aws im Zeitraum 2005-2008 mindestens einen Antrag eingereicht haben

\begin{tabular}{lccc}
\hline & $\begin{array}{c}\text { Anzahl der Antrag- } \\
\text { stellerInnen (Unter- } \\
\text { nehmen) }\end{array}$ & $\begin{array}{c}\text { Innerhalb der aws } \\
\text { Technologie- } \\
\text { programme nur bei } \\
\text { Programm ... Bean- } \\
\text { tragt * }\end{array}$ & Anteil in \% \\
\hline i2-Die Börse für Business Angels & 10 & 3 & 30,0 \\
Innovationsschutzprogramm ipp & 9 & 6 & 66,6 \\
PROTrans & 3 & 3 & 100,0 \\
tecma & 3 & 1 & 33,3 \\
tecnet & 3 & 3 & 100,0 \\
PreSeed Biotech & 9 & 2 & 22,2 \\
PreSeedIT \& Physical Sciences & 8 & 6 & 75,0 \\
Seedfinancing & 24 & 11 & 45,8 \\
Impulsprogramm Kreativwirtschaft & 12 & 10 & 83,3 \\
\hline
\end{tabular}

Anmerkung: *z.B.: 3 Unternehmen, die bei einem der 9 ausgewählten Programme eingereicht haben, haben nur bei i2 (Die Börse für Business Angels) eingereicht. Einreichungen bei anderen Programmen (z.B. Basisförderung) wurden hier nicht berücksichtigt.

Quelle: WIFO/KMFA (2008/2010): Unternehmensbefragung im Rahmen der Systemevaluierung, KMFA-Berechnungen

In weiterer Folge wurde analysiert, bei welchen anderen Förderungen die Antragsteller bei den Technologieprogrammen ebenfalls Förderungsanträge eingereicht haben.

Die folgende Matrix zeigt, in welchen Programmen durch die Unternehmen ,kombiniert“ Anträge im Zeitraum 2005-2008 eingereicht wurden. Im ersten Schritt erfolgte hier eine Auswertung für Kombinationen innerhalb der Technologieprogramme. Zusätzlich wurden die am häufigsten in Kombination mit den Technologieprogrammen genutzten Programme auBerhalb der aws mit aufgenommen. 
Tabelle 38: Anzahl der Unternehmen, die in den Technologieprogrammen der aws im Zeitraum 2005-2008 mindestens einen Antrag eingereicht haben (Kombinationen)

\begin{tabular}{|c|c|c|c|c|c|c|c|c|c|}
\hline & 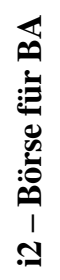 &. & 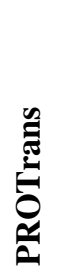 & 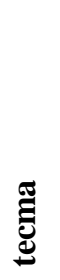 & $\begin{array}{l}\stackrel{\mathscr{E}}{\mathscr{E}} \\
\text { }\end{array}$ & 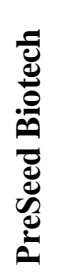 & 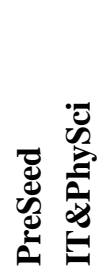 & 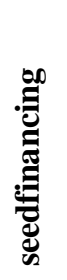 & 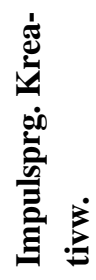 \\
\hline i2 - Börse für BA & 10 & & & & & & & & \\
\hline ipp & 1 & 9 & & & & & & & \\
\hline PROTrans & & & 3 & & & & & & \\
\hline tecma & 1 & 1 & & 3 & & & & & \\
\hline tecnet & & & & & 3 & & & & \\
\hline PreSeed Biotech & & & & & & 9 & & & \\
\hline PreSeedIT\&PhySci & 1 & & & & & & 8 & & \\
\hline Seedfinancing & 5 & 1 & & 1 & & 7 & 1 & 24 & \\
\hline Impulsprg. Kreativw. & 2 & & & & & & & & 12 \\
\hline Basisförderung (FFG-BP) & 5 & 8 & & 1 & 3 & 7 & 2 & 17 & 6 \\
\hline Bridge (FFG-BP) & 3 & 1 & & & & 4 & 1 & 12 & 3 \\
\hline
\end{tabular}

Innerhalb der Technologieprogramme der aws wurde am häufigsten mit seedfinancing kombiniert, an zweiter Stelle liegt I2. Die Unternehmen, die z. B. beim Impulsprogramm für die Kreativwirtschaft eingereicht haben, haben darüber hinaus innerhalb der Technologieprogramme der aws nur bei 12 eingereicht. Von den anderen Förderungen der aws wurde von den Nutzern der Seed-Förderung vor allem Double Equity beantragt.

Häufig wurden von den Antragstellern der aws-Technologieprogramme auch Anträge bei der Basisförderung der FFG eingereicht, aber auch Förderungsanträge bei Bridge eingereicht (beides vorrangig von den Nutzern von seedfinancing). Zwischen den (Pre)Seed Programmen und der direkten Förderung von Unternehmen in AplusB Zentren ist ebenfalls ein Zusammenhang sichtbar. Die häufigste Kombination besteht jedoch zwischen Seedfinancing und der Basisförderung in den Basisprogrammen der FFG. Diese werden auch häufig kombiniert mit ipp, und PreSeed Biotech, gefolgt von impulse. Ein deutlicher Zusammenhang in der Nutzung besteht auch zwischen Seedfinancing und Bridge. 
Abbildung 41: Anzahl der Unternehmen, die in den Technologieprogrammen der aws und in anderen (ausgewählten) Programmen im Zeitraum 2005-2008 mindestens einen Antrag eingereicht haben (Kombinationen)

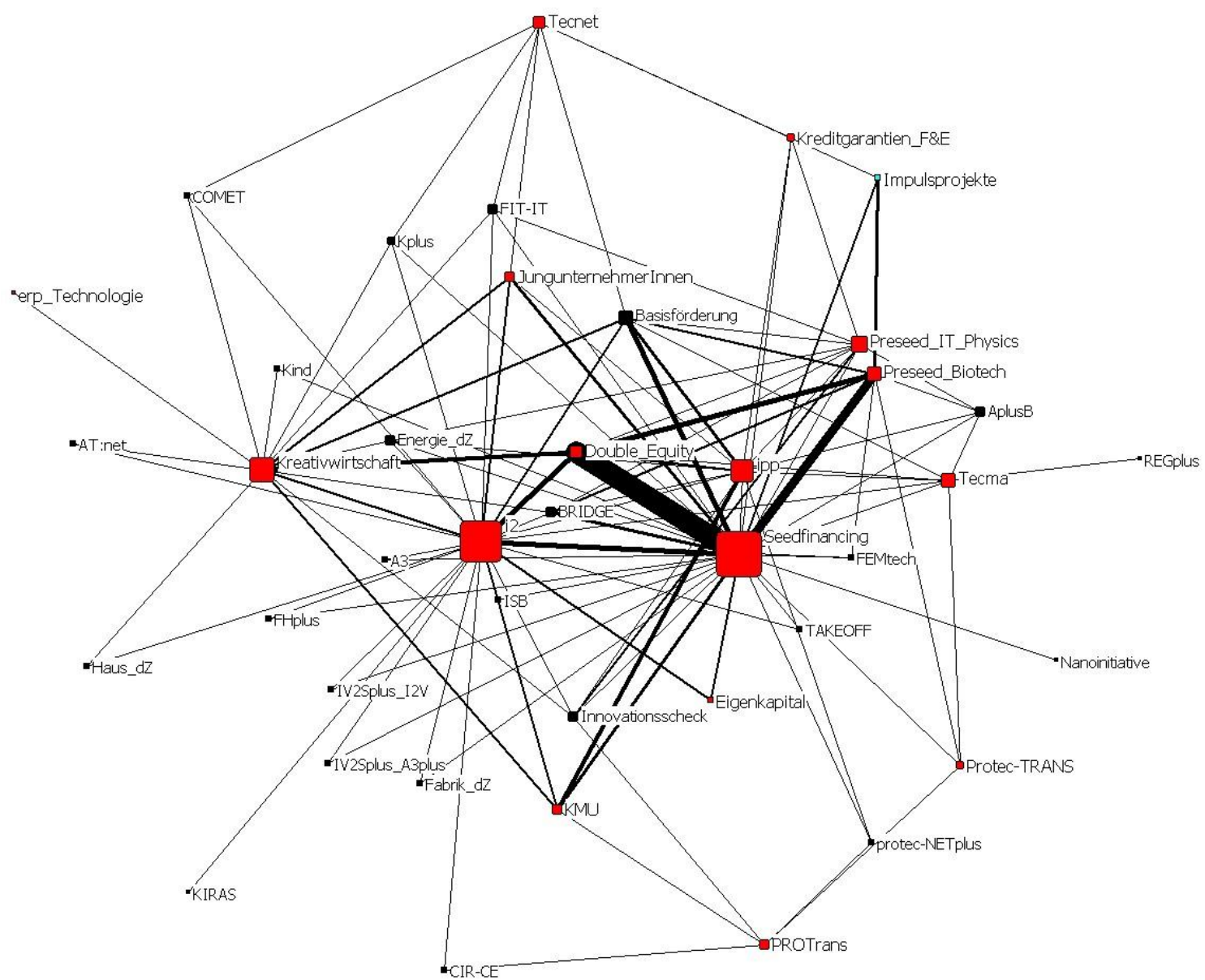

Anmerkungen: AWS: rot; blau: FWF; schwarz: FFG Programm; KMU=Unternehmensdynamikförderung Größe des Knoten basiert auf dem Degree (Anzahl der Verbindungen mit anderen Programmen unabhängig von der Stärke der Verbindung); Linienstärke basiert auf der Anzahl der Verbindungen;

Layout: Multidimensional Scaling

Quelle: WIFO/KMFA (2008/2010): Unternehmensbefragung im Rahmen der Systemevaluierung, KMFA-Berechnungen; Darstellung JOANNEUM RESEARCH

In der vorhergehenden sowie in der folgenden Abbildung 42 ist dargestellt, in welchen Programmen die Gruppe der Unternehmen, die Anträge bei den aws-Technologieprogrammen eingereicht haben, insgesamt jeweils mindestens einen Antrag eingereicht haben (mind. 3 Unternehmen). Die Technologieprogramme der aws sind demgemäß Teil dieser Darstellung. 37 der 62 Unternehmen, die bei den Technologieprogrammen der aws mindestens einen Antrag gestellt haben, haben dies auch bei der Basisförderung der FFG getan, 25 Unternehmen haben bei der aws Double Equity eingereicht. 
Abbildung 42: Anzahl der Unternehmen, die mindestens einen Antrag bei den Technologieprogrammen gestellt haben und bei weiteren Förderungsprogrammen (2005-08)

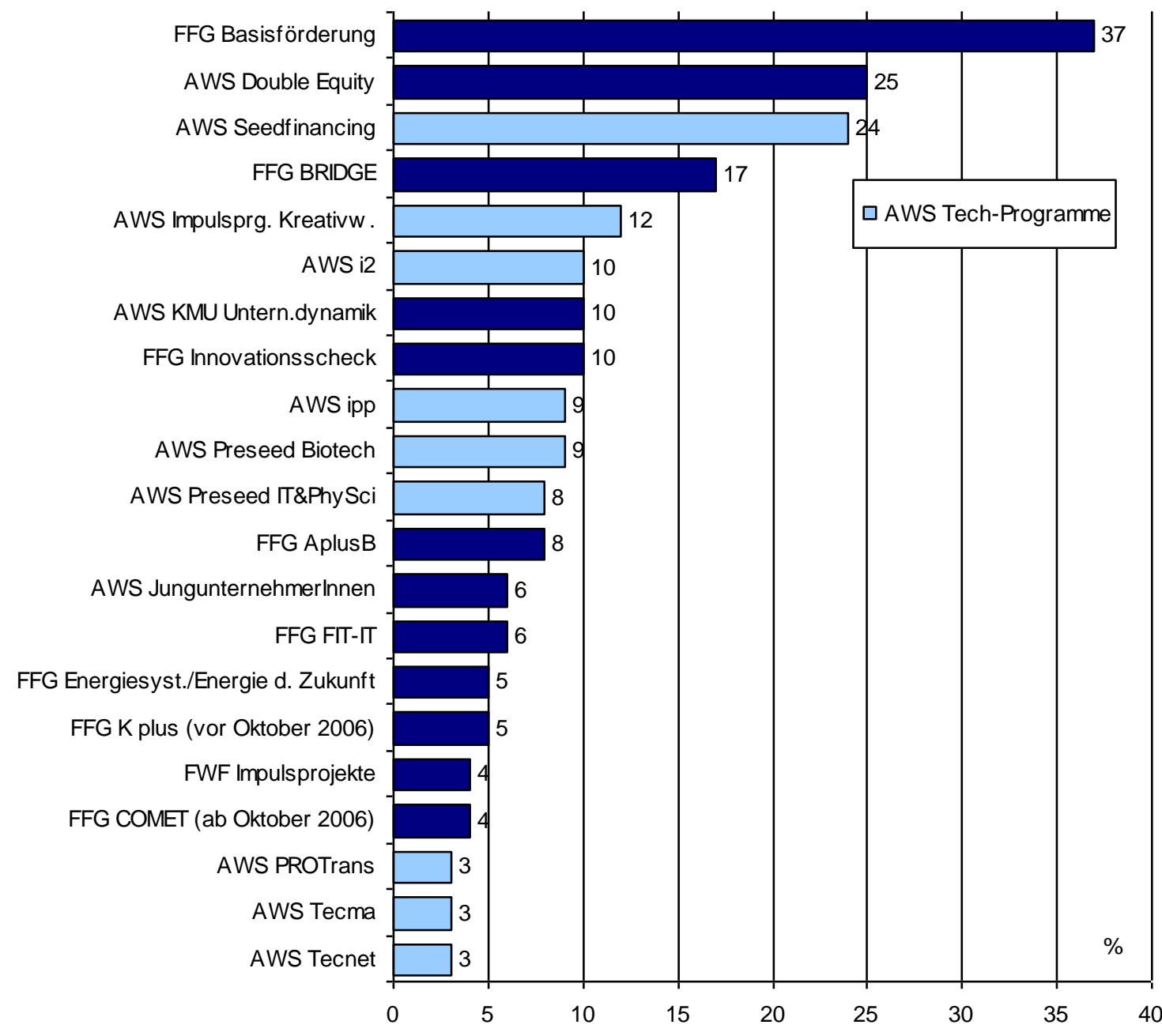

Anmerkung: $n=62$

Quelle: WIFO/KMFA (2008/2010): Unternehmensbefragung im Rahmen der Systemevaluierung, KMFA-Berechnungen

Bei AplusB haben nur 8 Unternehmen angegeben, einen Förderungsantrag gestellt zu haben (dabei ist jedoch zu berücksichtigen, dass die AplusB Förderung der FFG die Zentren fördert und nicht direkt die GründerInnen), diese vor allem in Kombination mit den (Pre)Seedprogrammen der aws-Technologieprogramme. Auch die Kombination mit Bridge Anträgen kommt vor allem mit den (Pre)Seedprogrammen vor (in 3 Fällen auch mit dem Impulsprogramm Kreativwirtschaft).

Jene Unternehmen, die bei den ausgewählten AWS Technologieprogrammen zumindest einen Antrag eingereicht haben, haben im Vergleich zu den Systemnutzern der FTI Förderung insgesamt etwas mehr FTI-Förderungen der österreichischen Bundesländer in Anspruch genommen (56 \%), dafür etwas weniger Förderungen der Europäischen Union (20 \%). Die Unterschiede belaufen sich nur auf 6 bzw. 4\%-Punkte. Einreichungen bei EURahmenprogrammförderungen bedeuten idR - verglichen zu nationalen/regionalen Förderungen - einen enormen Ressourcenaufwand (Antragstellung, Abwicklung, Aufwand für Kooperationen etc.), den insbesondere die intendierte Zielgruppe der aws-Technologieprogramme (kleine, junge Unternehmen) weniger auf sich nimmt, als andere (größere, ältere) Unternehmen. 
Auch in den Interviews mit Unternehmen, die aws-Programme in Anspruch genommen haben, wurden Zusammenhänge zwischen den Programmen innerhalb des Clusters „Technologieprogramme“ der aws, aber auch darüber hinaus, deutlich. Diejenigen der interviewten Unternehmen, die das Programm PreSeed bzw. auch Seed in Anspruch genommen haben, kamen über AplusB-Zentren zur aws, wobei die Zentren PreSeed aktiv als Anschlussförderung empfahlen und die Unternehmen auch bei der Kontaktaufnahme zur aws unterstützten.

In den Interviews wird diesbezüglich auch angegeben, dass die Basisprogramme einerseits von der aws empfohlen worden sind (z.B. im Anschluss an PreSeed oder Seed) und dass sie besonders naheliegend für die Nutzung sind, da die Basisprogramme thematisch offen konzipiert sind. Ein Unternehmen hat die Basisprogramme vor dem Technologieprogramm ipp genutzt. Diese qualitativen Ergebnisse decken sich demnach mit den oben dargestellten.

\subsubsection{Zusammenspiel aus Sicht der Agenturen und Stakeholder}

Die Zusammenarbeit der Förderungseinrichtungen auf Bundesebene (aws und FFG) erfolgt gerade an der Schnittstelle Basisprogramme - aws aus der Sicht der Förderungseinrichtungen gut. Es wurde dargestellt, dass die jeweiligen Sachbearbeiter das Portfolio der gegebenenfalls „anschließenden“ Programme kennen und dementsprechend Unternehmen beraten, weiterverweisen und sich abstimmen. Im Bereich Life Sciences wurde ein regelmäßiger jour fix angegeben. In Fällen, in denen z B. ein potenzielles Unternehmen aus der Denklogik eines geplanten Projektes einen Antrag bei den Basisprogrammen der FFG stellt, aber die Gründung noch nicht erfolgreich ist, kann dieser Antrag mit einem Genehmigungsvorbehalt bzgl. der Gründung genehmigt werden, und für die Gründung die Unterstützung der aws vermittelt werden. Dem Unternehmen nützt die in der FFG erfolgte Projektprüfung im Sinne eines „Technology Rating“. Ebenso wurde auf die Zusammenarbeit mit dem Bereich der Schutzrechte z. B. im Zusammenhang mit den Ergebnissen aus Basisprogramm-Projekten der FFG verwiesen.

Durch die Zusammenarbeit zwischen den Agenturen auf Sachbearbeiterebene soll auch sichergestellt werden, dass es bei paralleler Förderung z. B. mit PreSeed oder Seed und einen Basisprogrammprojekt nicht zu Doppelförderungen kommt.

Die Abstimmung und Zusammenarbeit in Bezug auf die AplusB Zentren ist differenziert zu betrachten. Das Programm AplusB der FFG fördert und unterstützt österreichweit in acht AplusB-Zentren akademische Unternehmensgründungen und akademische Spin-offs, wobei die FFG die Zentren an sich fördert, die an Universitäten eingerichtet sind.

Die Zusammenarbeit in Bezug auf die konkrete Unterstützung der GründerInnen erfolgt demgemäß nicht vorrangig mit dem Programmmanagement der FFG sondern mit den AplusB Zentren selbst. Diese sind tlw. - abhängig unter anderem auch von der jeweiligen Ausrichtung der Universitäten und den regionalen Gegebenheiten - recht unterschiedlich aufgestellt und kooperieren in unterschiedlicher Art und Intensität z. B. mit der aws. Dies dürfte sich auch in den Fallzahlen z. B. von PreSeed niederschlagen. Manche dieser Zentren verstehen sich aber auch als regionale Knotenpunkte und Ansprechstellen auch für Förderungen auf Bundesebene. Das CAST hat beispielsweise ein ,aws Büro“ eingerichtet. Eine zentrale Aufgabe der AplusB Betreuung ist die Beratung der GründerInnen und deren Unterstützung auch in Bezug auf Förderungsmöglichkeiten, wodurch sich automatisch ein enger Zusammenhang mit der aws ergibt. Dadurch fungieren die AplusB-Zentren vielfach auch als Multiplikatoren 
für aws-Programme (wie z.B. tecnet) mit entsprechend erheblichen Synergien.

In Bezug auf die Positionierung der aws-Technologieprogramme im Verhältnis zu den Bundesländern ergibt sich ein vielfältiges Bild. Es zeigt sich, dass die Förderungslandschaft auf Landesebene heterogen und ebenfalls ausdifferenziert ist (Abbildung 43).

Die Interviewpartner gaben sämtlich an, dass bei der Konzeption und Umsetzung der Landesförderungen darauf geachtet wird, keine Bundesaktion zu duplizieren. Allerdings zeigt sich bei der Recherche der verschiedenen Förderungsmöglichkeiten in den Bundesländern, dass es dort z. B. auch Frühphasen-Angebote, Beratung zu Schutzrechts-Fragen, Business-Angel Initiativen gibt. Ganz grundsätzlich wurde angeführt, dass auf der Ebene der Länder diejenigen Aktivitäten sinnvoll sind, bei denen es um die bessere Kenntnis des Unternehmens- und Gründerspektrums im Land geht und bei denen das Vertrauen und der Kontakt auf persönlicher Basis relevant sind, bzw. dass Formate, die denjenigen auf Bundesebene ähneln, nach ebendiesen Gesichtspunkten und unter besonderer Berücksichtigung der regionalen wirtschaftlichen Gegebenheiten aufgesetzt sind und es daher nicht zu Doppelungen komme. Die Zusammenarbeit mit der Bundesebene wurde für aws und FFG gleichermaßen positiv beschrieben. In einigen Fällen wurden auch institutionalisierte Kooperationen angesprochen. Formalisiert ist die Zusammenarbeit z. B. beim Staatspreis Innovation. Hilfreich z. B. für die Förderung durch die aws wäre aus der Sicht mancher Interviewpartner eine stärkere systematische regionale Präsenz der aws, wobei sich die aws insgesamt in enger Zusammenarbeit der regionalen Partner bedienen könnte. „Die Wirkung der aws nimmt mit jedem km ab“. 
Abbildung 43:Wirtschaftsnahe Förderungen in den Bundesländern - institutioneller Überblick
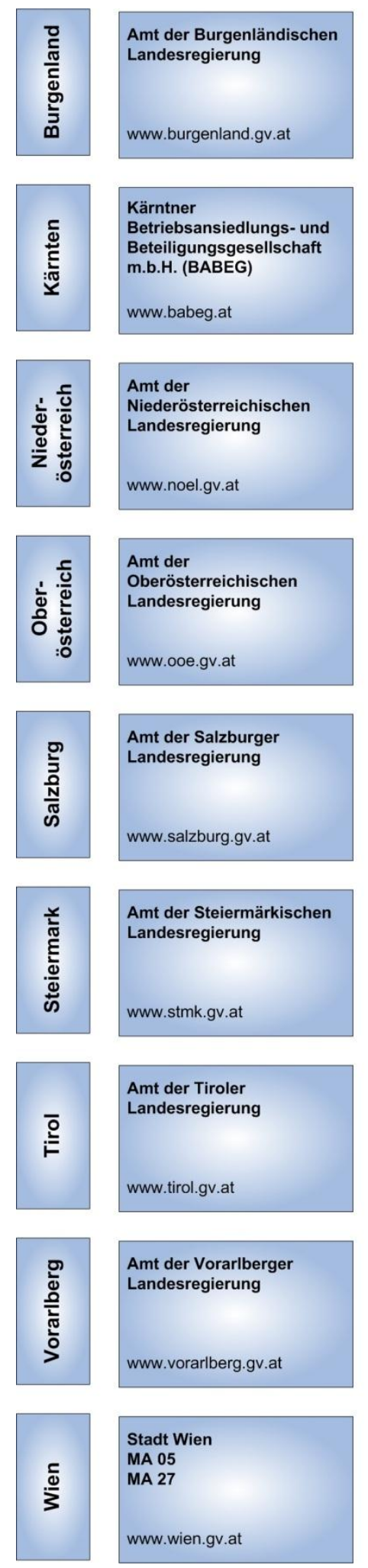

Quelle: KMFA, eigene Darstellung, 2011
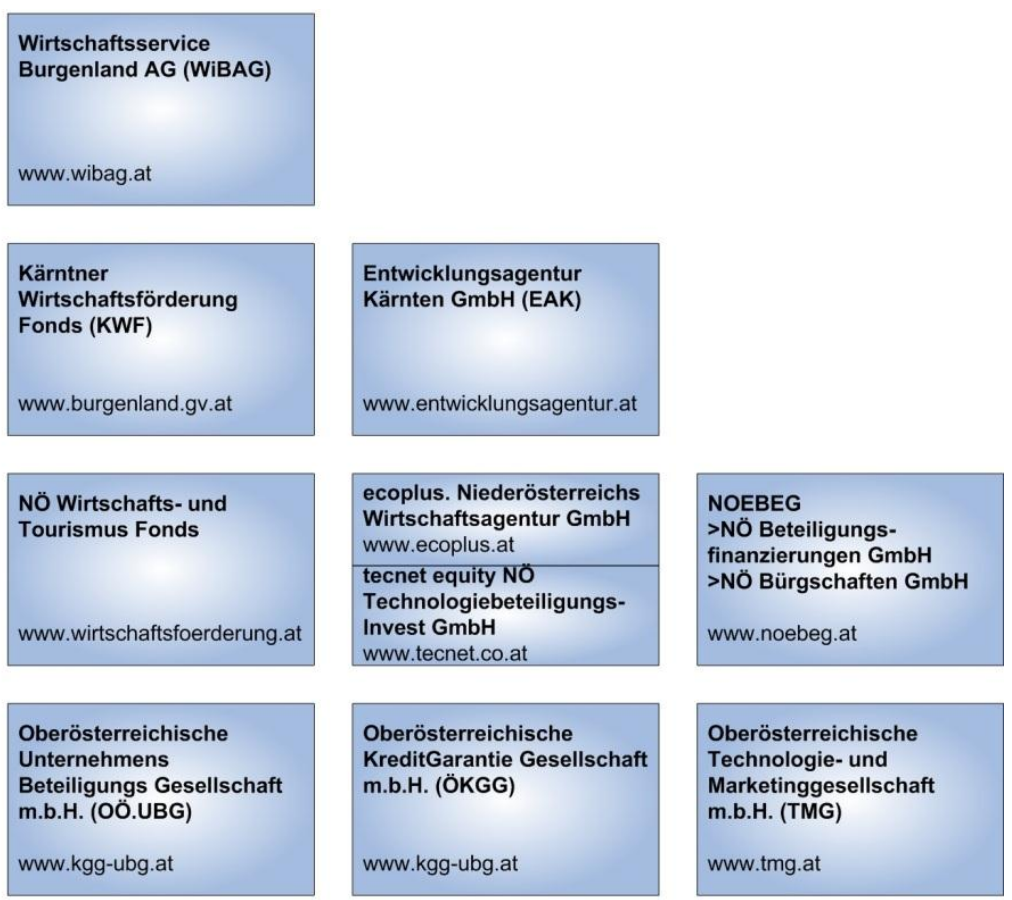

SUBG Salzburger

Unternehmensbeteiligungs

gesellschaft m.b.H.

www.subg-skgg.at
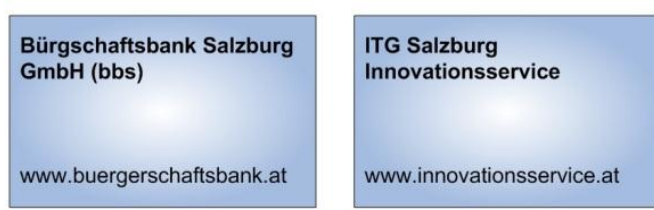

Steirische

Wirtschaftsförderungsgese

Ilschaft m.b.H. (SFG)

www.sfg.at
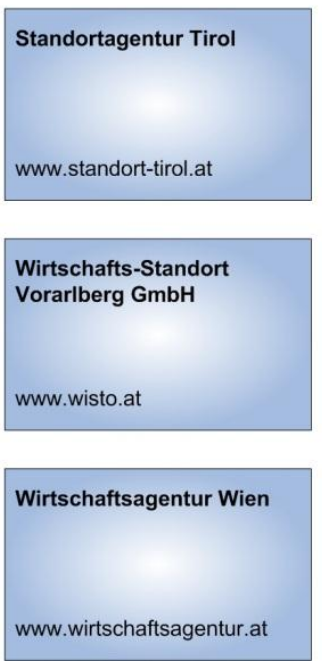
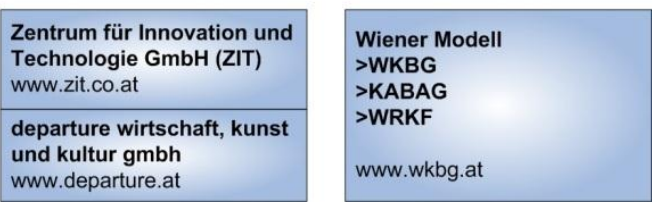

Aktionen auf Landesebene beziehen sich manchmal auf zu kleine abgegrenzte Zielgruppen, sodass z. B. Risikostreuung für Investoren nicht möglich ist oder kein Wettbewerb zwischen Anträgen machbar ist. Angebote, bei denen hochspezialisiertes Know-How nötig ist, sollten eher auf Bundesebene angeboten werden (z.B. ipp). Die allgemeine Einschätzung in 
den Interviews war dahingehend, dass die subsidiäre Aufteilung der Agenden im Grunde passend umgesetzt ist. Was kritisch diskutiert wurde, ist die Vielzahl an verschiedenen Programmen auf Bundesebene, die sehr spezifisch konstruiert sind und gleichzeitig oft unterkritisch mit Förderungsmitteln dotiert, sodass sich die Förderungschancen gerade in Bundesländern, deren Wirtschaftsstruktur im Ländervergleich insgesamt weniger im Sine der Zielgruppen der Technologieprogramme ausgerichtet ist, als sehr gering darstellen.

Die Zusammenarbeit zwischen den verschiedenen Landesförderungen und der aws erfolgt idR über die jeweiligen Themenexperten anhand konkreter Projekte / Unternehmen / Gründungsvorhaben, ähnlich wie zwischen aws und FFG geschildert. Die Bundesländer versuchen, durch spezifische Angebote, die auf die jeweilige Wirtschaftssituation ihres Bundeslandes abgestimmt sind, oftmals „Anschlussfähigkeit“ ihrer Zielgruppen zu Bundesförderungen herzustellen (exemplarisch sei hier das kreative Handwerk Tirol angeführt), oder einen regionalen Fokus (ein besonderes Stärkefeld) in den Vordergrund zu stellen und für ein spezialisiertes Angebot zu nutzen (z. B. Business Angels in der Steiermark) oder mit ihrer genauen Kenntnis der Unternehmen in der Region pro-aktiv auf diese zuzugehen um Awareness für bestimmte als relevant erachtete Themen zu schaffen (als Beispiel sei hier die Steiermark zum Thema Schutzrechte angeführt). Die regionalen Impulszentren wurden insgesamt als Portfolio eher kritisch beurteilt - für qualitative gute Beratung bedarf es einer gewissen kritischen Masse an BeraterInnen und an Nachfrage, dies sei bei der Vielzahl der existierenden Zentren in jedem einzelnen Zentrum nicht umsetzbar.

Schließlich sind auf der Ebene der Länder die Angebote der jeweiligen Landeswirtschaftskammern anzuführen (z. B. Patentberatungstage). Mit diesen gibt es institutionalisierte Abstimmungsrunden mit der aws.

\subsection{KRITERIEN FÜR DIE AUSWAHL VON FÖRDERUNGSPROGRAMMEN AUS DER SICHT DER ANTRAGSTELLER BEI DEN TECHNOLOGIEPROGRAMMEN DER AWS}

Hinsichtlich der Kriterien, nach denen Unternehmen ex-ante die FTI-Programme auswählen, bei denen sie Anträge einreichen, sind auf Basis der Daten aus der Unternehmensbefragung im Rahmen der Systemevaluierung keine wesentlichen Unterschiede der Nutzer der Technologieprogramme im Vergleich zu den Ergebnissen der Systemevaluierung insgesamt festzustellen. Dort scheint insgesamt der ,thematische Zuschnitt“ zwar erst an dritter Stelle auf, jedoch sind die Unterschiede in Prozentpunkten zu vernachlässigen. 
Abbildung 44: Kriterien für die Auswahl von Förderprogrammen (ex-ante)

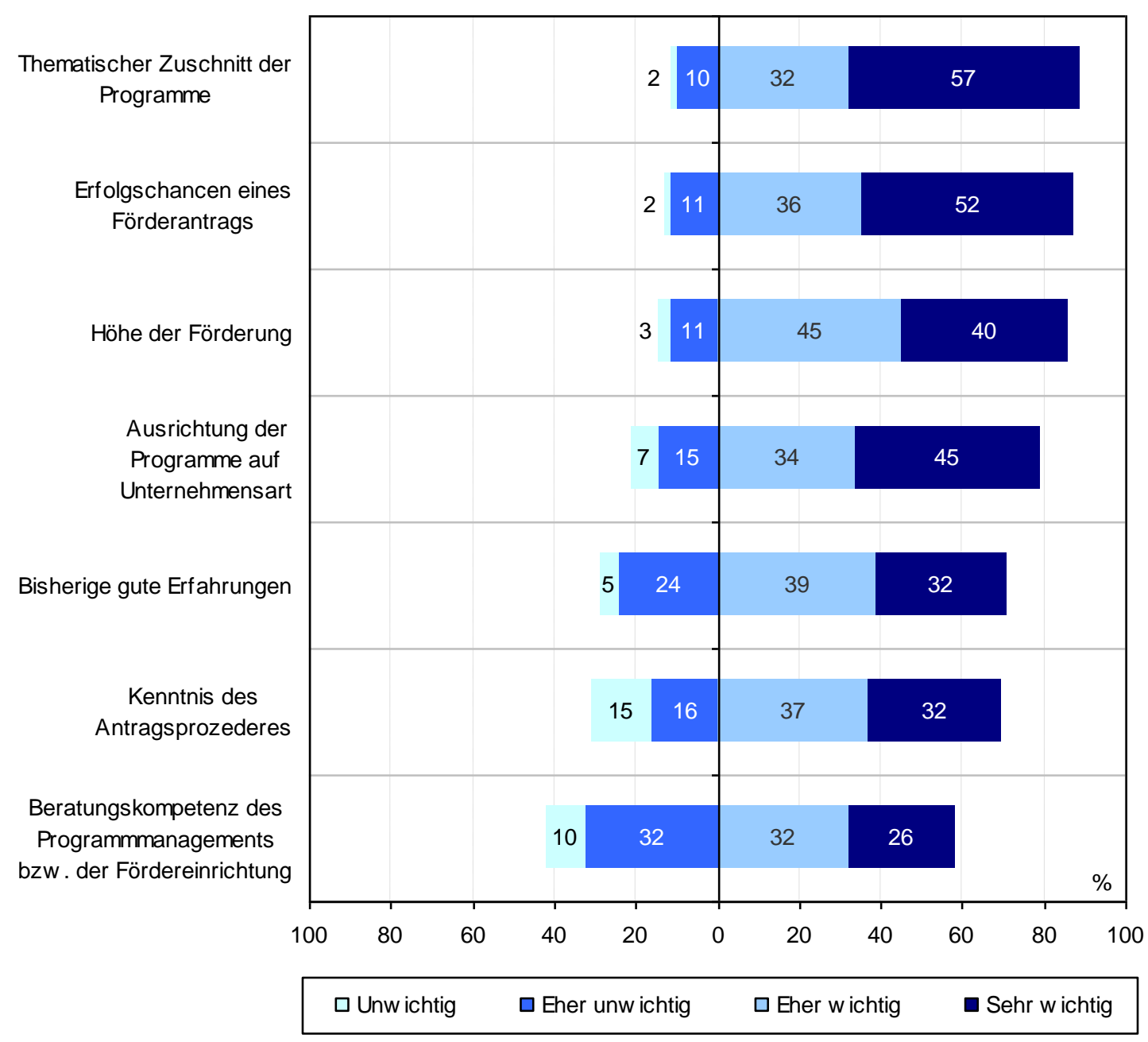

Anmerkung: $n=62$

Quelle: WIFO/KMFA (2008/2010): Unternehmensbefragung im Rahmen der Systemevaluierung, KMFA-Berechnungen

Dabei ist zu berücksichtigen, dass die Unternehmen bei der Befragung im Rahmen der Systemevaluierung vorrangig monetäre Förderung im Fokus hatten; die gestellte Frage bezog sich daher auf die Förderungsberatung zu Programmen der monetären Förderung. Die Ergebnisse betreffen daher nicht die grundsätzliche „Einstiegsberatung“ durch die aws vor der Auswahl bestimmter Förderungsschienen oder „Beratungsprogramme“. Bei letzteren wäre jedenfalls zu erwarten, dass die Beratungskompetenz höhere Relevanz hat. In der Evaluierung des Haftungsinstrumentariums der aws auf Basis des KMU Förderungsgesetzes, die die KMFA im Jahr 2010 im Auftrag des BMWFJ durchgeführt hat, hat sich gezeigt, dass diejenigen Unternehmen, die in diesem Programmen Beratung der aws in Anspruch genommen haben, diese auch sehr gut bewerten.

Zudem wurde in den durchgeführten Interviews mit Unternehmen, die awsTechnologieprogramme genutzt haben, deutlich, dass die Unternehmen mit der Beratung seitens der aws überaus zufrieden sind. Dies gilt für die Phase der Antragstellung, aber auch generell - z.B. bei eher allgemeinen Auskünften - sowie während der gesamten Projektlaufzeit. Nötige Informationen sind offenbar einfach abzurufen (Internet, häufig auch direkt via Telefon). Die Ansprechpartner in der aws sind, nach Meinung der interviewten Unternehmen, 
idR gut erreichbar, erteilen kundengerechte Auskünfte und agieren - im Vergleich zu anderen Förderungsagenturen - ,pragmatischer“.

\subsection{STRATEGIEN, WENN BEANTRAGTE FÖRDERUNGEN NICHT GENEHMIGT WERDEN}

Die Frage nach den Folgen, die sich ergeben, wenn beantragte Förderungen nicht genehmigt werden, wird von den Nutzern der aws-Technologieprogramme in einigen Punkten deutlich anders beantwortet als von den Systemnutzern der FTI-Förderung insgesamt. Die Nutzer der aws-Technologieprogramme geben zu einem deutlich geringeren Anteil an, die Projekte in der Regel trotzdem ohne Einschränkung durchzuführen und zu einem höheren Anteil, die Projekte in der Regel ohne die Förderung nicht umzusetzen. Die Abhängigkeit der Projektumsetzung von der Förderung scheint in dieser Gruppe also höher zu sein, was als Indiz gewertet werden kann, dass die aws-Technologieprogramme mit ihren auf kleine, junge Unternehmen zugeschnittenen Maßnahmen, auch die intendierte Zielgruppe ansprechen; in der Gruppe der Systemnutzer insgesamt sind auch große Unternehmen vertreten. Allerdings gibt ein großer Anteil der Antragsteller der aws-Technologieprogramme an, die Projekte anderswo einzureichen - die Spezifika einzelner Programme scheinen also weniger relevant zu sein. 
Abbildung 45: Strategien der Unternehmen bei Nicht-Bewilligung von FTI-Förderungen

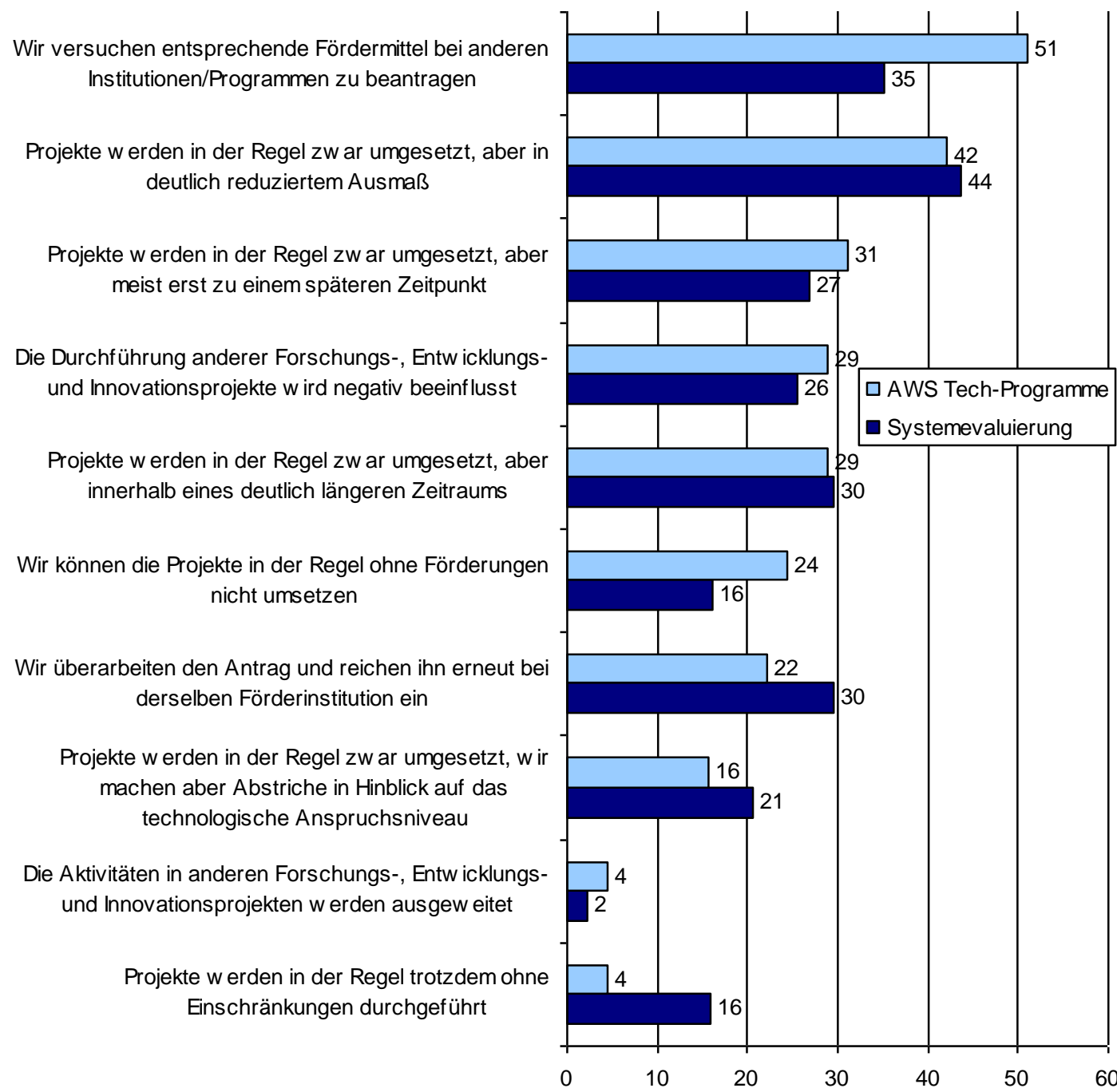

Anmerkung: $n=45$ bzw. 516 (Syseval)

Quelle: WIFO/KMFA (2008/2010): Unternehmensbefragung im Rahmen der Systemevaluierung, KMFA-Berechnungen

Eine differenzierte Betrachtung dieser Strategien zeigt Unterschiede zwischen den Gruppen der Antragsteller bei verschiedenen Programmen der Technologieprogramme der aws.

Die Unternehmen, die Anträge bei Seed oder PreSeed eingereicht haben, versuchen im Fall der Ablehnung des Förderungsantrags vor allem (14 von 24; 58\%), anderswo Förderung zu bekommen, reduzieren das Ausmaß des „Projekts“ oder führen es später durch.

Unternehmen, die Anträge beim Impulsprogramm Kreativwirtschaft eingereicht haben, geben mehrheitlich (6 von 10;60\%) an, dass sie das Projekt ohne die Förderung nicht umsetzen können. Immerhin 50\% versuchen, anderswo Förderung zu bekommen.

\subsection{STELLENWERT DES FTI-FÖRDERUNGSANGEBOTS FÜR DIE UNTERNEHMEN}

Die Unternehmen, die Anträge bei den Technologieprogrammen der aws eingereicht haben, planen in größeren Umfang als im gesamten Sample der Systemevaluierung das Förderungsangebot in ihre FTI-Tätigkeiten ein (Abbildung 46). Dies kann wie schon vorne als Indiz gewertet werden, dass bei der Zielgruppe der Technologieprogramme eine größere 
Abhängigkeit von der Förderung und/oder eine bessere Planbarkeit der Förderung zu verzeichnen sein kann. Dabei ist darauf hinzuweisen, dass sich diese Antworten auf das FTIFörderungsangebot der insgesamt beziehen. Die Differenzierung erfolgt anhand der Nutzung der verschiedenen Förderprogramme (Nutzer von aws-Technologieprogrammen versus Nutzer von systemevaluierungsrelevanten Förderprogrammen).

\section{Abbildung 46: Stellenwert des Angebots an FTI-Förderung}

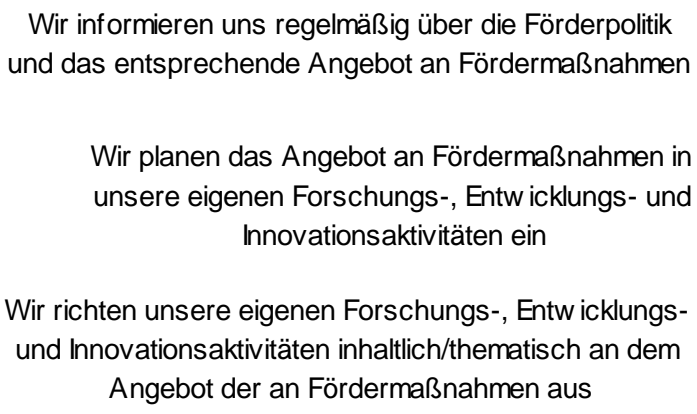

Wir informieren uns regelmäßig über die Förderpolitik und das entsprechende Angebot an Fördermaßnahmen

Wir planen das Angebot an Fördermaßnahmen in unsere eigenen Forschungs-, Entw icklungs- und Innovationsaktivitäten ein

Wir richten unsere eigenen Forschungs-, Entw icklungsund Innovationsaktivitäten inhaltlich/thematisch an dem Angebot der an Fördermaßnahmen aus

Keine dieser Möglichkeiten trifft zu

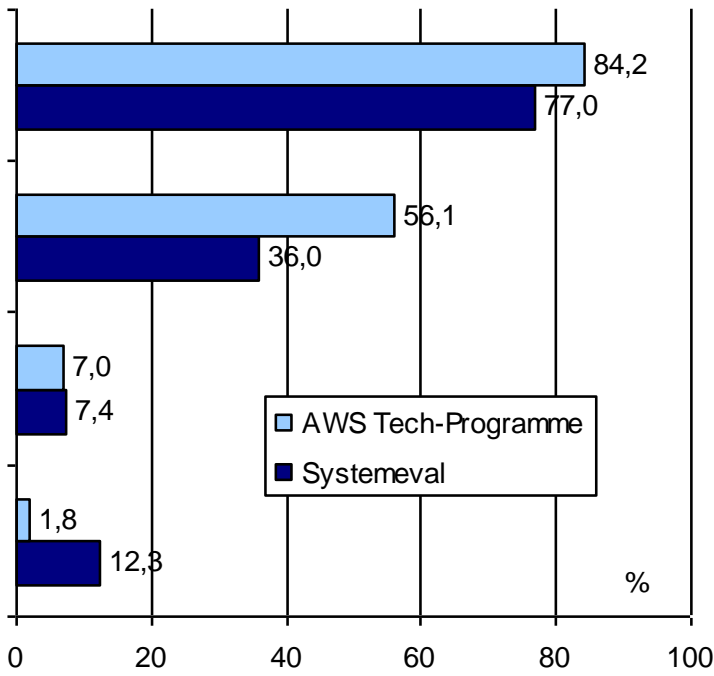

Anmerkung: $n=57$ bzw. 901 (Syseval)

Quelle: WIFO/KMFA (2008/2010): Unternehmensbefragung im Rahmen der Systemevaluierung, KMFA-Berechnungen

Unternehmen, die zumindest einmal für ein Seedfinancing bzw. bei den PreSeedprogrammen eingereiht haben, beziehen Ihre Informationen über die Möglichkeiten für FTI-Förderungen insgesamt vorwiegend über Recherche im Internet (91 \%, 29 Betriebe) bzw. direkt durch die Fördereinrichtungen (85 \%, 27 Betriebe). Die hauseigene Bank wurde lediglich von 5 Betrieben als Informationsquelle genannt.

Die folgenden Auswertungen differenziert nach Programmen können nur indikative Ergebnisse liefern, da die Anzahl der Unternehmen, die diese Fragen beantwortet haben und Anträge bei den genannten Programmen eingereicht haben, sehr gering ist. Die Analyse der Antworten auf die Frage, wie wichtig die Unterstützung für das Unternehmen in den einzelnen Phasen des FTI-Prozesses wahrgenommen wird und wie das Angebot beurteilt wird, zeigt für die Unternehmen, die bei Seed und PreSeed Anträge eingereicht haben im Vergleich zum Gesamtdurchschnitt (Systemnutzer), dass die Wichtigkeit der Unterstützung von Ideengenerierung bzw. Konzepterarbeitung bei diesen Unternehmen (noch) geringer eingeschätzt wird. Unterstützungsmaßnahmen für den Bereich Entwicklung wird im Vergleich zu den Systemnutzern insgesamt als wichtiger erachtet. Ansonsten sind die Ergebnisse ähnlich. Bei den Unternehmen, die Anträge im Impulsprogramm Kreativwirtschaft eingereicht haben, fällt im Vergleich zum Gesamtdurchschnitt (Systemnutzer) auf, dass Unterstützung für die Ideengenerierung, Konzepterarbeitung und Forschung bei diesen Unternehmen als weniger wichtig eingeschätzt werden. Unterstützung für die Produktion, Markteinführung und -durchdringung erscheint wichtiger. Das Angebot an Unterstützung für den Bereich Ideengewinnung schätzen diese Unternehmen zudem als deutlich schlechter ein als der Durchschnitt. Die Unternehmen, die Anträge bei ipp und/oder tecma eingereicht haben, geben im Vergleich zum Gesamt- 
durchschnitt (Systemnutzer) häufiger an, dass Unterstützung für den Bereich Forschung, aber auch für die Produktion, Markteinführung und -durchdringung als wichtig eingeschätzt wird. Wenig überraschend ist festzustellen, das die Relevanz von Unterstützungsmaßnahmen im Bereich Schutzrechte von diesen Unternehmen als sehr hoch angesehen wird - bei der Beurteilung des entsprechenden Angebots zeigen sich jedoch keine gravierenden Unterschiede zum Gesamtdurchschnitt. Diese Auswertungen differenziert nach Programmen kann jedoch nur indikative Ergebnisse liefern, da die Anzahl der Unternehmen, die diese Fragen beantwortet haben und Anträge bei den genannten Programmen eingereicht haben, sehr gering ist.

\section{Fazit}

Die Nutzer der aws-Technologieprogramme können im Vergleich zu Nutzern anderer Förderungsprogramme im österreichischen Forschungsförderungssystem (basierend auf Auswertungen der Unternehmensbefragung, die im Rahmen der Systemevaluierung durchgeführt wurde) als deutlich jüngere Unternehmen bzw. auch häufiger Mikrounternehmen aus den High-Tech-Industrien und insbesondere aus dem Bereich der wissensintensiven Dienstleistungen charakterisiert werden. Dies entspricht im Grunde der Förderungslogik der awsTechnologieprogramme.

Bezüglich des Zusammenspiels unterschiedlicher Förderungsprogramme (innerhalb der aws und auch nach außen) zeigt sich, dass diejenigen der Frühphasenfinanzierung insgesamt die größte Nachfrage (Anzahl der antragstellenden Unternehmen) aufweisen, gefolgt von impulse und i2. Bei impulse zeigt sich am deutlichsten, dass dieses Programm von den Unternehmen am selektivsten genutzt wird. Innerhalb der Technologieprogramme der aws wurde am häufigsten mit Seedfinancing kombiniert, an zweiter Stelle liegt i2. Die häufigste Kombination mit Seedfinancing ist PreSeed Biotech, was der Förderungslogik entspricht. Die höhere Anzahl im Bereich Biotech ist möglicherweise auf weiterführende Begleitmaßnahmen im Rahmen von LISA zurückzuführen. Das Programm Seedfinancing wird außerhalb der Technologieprogramme am häufigsten mit Double Equity kombiniert. Außerhalb der aws wurde von den Unternehmen, die bei aws-Technologieprogrammen eingereicht haben, auch häufig bei der Basisförderung (FFG) eingereicht, ebenso bei Bridge (beides vorrangig von Nutzern von Seedfinancing). Auch zwischen den PreSeedprogrammen und AplusB ist ein Zusammenhang ersichtlich.

Nutzer von aws-Technologieprogrammen nehmen tendenziell auch eher mehr Bundesländerförderungen und weniger EU-Förderungen in Anspruch.

Die Zusammenarbeit der Förderungseinrichtungen auf Bundesebene (aws, FFG) dürfte gut funktionieren, die jeweiligen FörderungsberaterInnen kennen die ,anschließenden“ Programme und beraten die Unternehmen dahingehend bzw. verweisen sie entsprechend weiter. Die Zusammenarbeit hat ebenfalls die Funktion der Vermeidung von Doppelförderungen. Bei der Abstimmung in Bezug auf AplusB muss beachtet werden, dass die Abstimmung weniger mit der FFG sondern vielmehr mit den unterschiedlich aufgestellten Zentren selbst erfolgt. In Bezug auf die Wirksamkeit der aws als Bundesagentur wurde seitens der Interviewpartner auf Landesebene auf deren Abnehmen mit zunehmender Distanz verwiesen und auf die Relevanz einer Präsenz vor Ort. 


\section{Modul 4: Awareness und Außendarstellung}

\subsection{VORBEMERKUNG}

Dieses Modul analysiert die Öffentlichkeitsarbeit und die Außendarstellung der awsTechnologieprogramme. Dabei werden zum einen die Auswirkungen von Maßnahmen zur Außendarstellung auf die Wahrnehmung des Instrumentenportfolios der aws sowie die Awareness für innovative Ideen in Österreich erfasst.

Dabei stehen folgende Leitfragen im Vordergrund:

- In welchem Ausmaß konnten die aws-Technologieprogramme sowie Außendarstellungsmaßnahmen der aws insgesamt einen Beitrag zur Stärkung von Awareness für innovative Ideen in Österreich leisten? Lassen sich dabei Verbesserungspotenziale erzielen?

- Sind die derzeitigen Maßnahmen der aws zur Präsentation der awsTechnologieprogramme ausreichend?

- Welche konkreten Erfahrungen wurden mit der verstärkt angestrebten Clusterung der Programme auf Seiten des BMWFJ und auf Seiten der aws gemacht? Hatten die Maßnahmen Auswirkungen auf die Wahrnehmung des Instrumentenportfolios auf Seiten der Kunden?

- Wie bewerten die Kunden und andere Stakeholder die Förderungsmaßnahmen des BMWFJ in den aws-Technologieprogrammen?

Um Hinweise über die Wahrnehmung bzw. Awareness der aws-Technologieprogramme auf der Kundenseite zu bekommen, wurden im Rahmen der vierteljährlich von der KMU FORSCHUNG AUSTRIA durchgeführten Konjunkturbeobachtung im Gewerbe und Handwerk zwei Zusatzfragen platziert. ${ }^{42}$ Darüber hinaus wurden Interviews mit Kunden der awsTechnologieprogramme sowie mit Stakeholdern auf Bundes- und Landesebene durchgeführt.

In der Befragung wurden die Unternehmen zum einen gefragt, wie wichtig Innovationen generell für die Unternehmen selbst sind bzw. in welcher Entwicklungsphase (von der Ideengenerierung bis zur Verwertung) sie vorrangig Unterstützung benötigen. Darüber hinaus wurden die befragten Unternehmen aufgefordert, Angaben zur Bekanntheit der einzelnen awsTechnologieprogramme zu tätigen. Dabei wurde unterschieden in ,nicht bekannt“, ,nicht in Anspruch genommen, aber Bezeichnung bekannt“, „nicht in Anspruch genommen, aber Inhalt bekannt“" und „bereits in Anspruch genommen“ unterschieden.

Die Stichprobe setzt sich mit Blick auf die Bundesländer wie folgt zusammen: niederösterreichische (19\%) und oberösterreichische (18\%) Unternehmen, gefolgt von steirischen Unternehmen (14\%), sind anteilsmäßig am höchsten in der Befragung repräsentiert. Unternehmen aus Kärnten, Tirol und Vorarlberg, sind mit jeweils 9\%, Unternehmen aus Salzburg und

\footnotetext{
${ }^{42}$ Die Konjunkturbeobachtung im Gewerbe und Handwerk wird im Auftrag der Wirtschaftskammer Österreich, Bundessparte und Landessparten Gewerbe und Handwerk durchgeführt. Die Stichprobe der Konjunkturerhebung der KMU FORSCHUNG AUSTRIA setzt sich aus 5.402 österreichischen Gewerbe- und Handwerksbetrieben zusammen (4. Quartal 2010). Zwar wird dadurch nicht die gesamte Zielgruppe der aws-Technologieprogramme abgebildet, da jedoch knapp $30 \%$ aller österreichischen KMU der Sparte Gewerbe und Handwerk zuzuordnen sind, ist es möglich mit den im Rahmen der Befragung gestellten Zusatzfragen einen guten Hinweis auf Bekanntheit der Programme zu bekommen.
} 
Wien mit jeweils $8 \%$ und Unternehmen aus dem Burgenland mit $5 \%$ in der Stichprobe vertreten.

Tabelle 39: Zusammensetzung der Stichprobe nach Bundesländern, Verteilung der Unternehmen im Gewerbe und Handwerk in Österreich

\begin{tabular}{lcc}
\hline & Stichprobe* & Gesamt Ö** \\
\hline Niederösterreich & $19 \%$ & $18 \%$ \\
Oberösterreich & $18 \%$ & $16 \%$ \\
Steiermark & $14 \%$ & $14 \%$ \\
Kärnten & $9 \%$ & $7 \%$ \\
Tirol & $9 \%$ & $10 \%$ \\
Vorarlberg & $9 \%$ & $5 \%$ \\
Salzburg & $8 \%$ & $8 \%$ \\
Wien & $8 \%$ & $19 \%$ \\
Burgenland & $5 \%$ & $4 \%$ \\
\hline
\end{tabular}

Quelle 1: KMU FORSCHUNG AUSTRIA, Konjunkturerhebung im Gewerbe und Handwerk (4. Quartal 2010); $N=$ 5.402; * Rauchfangkehrer, Bestattung und Allgemeiner Fachverband des Gewerbes wurden nicht befragt

Quelle 2: Wirtschaftskammer Österreich, Beschäftigtenstatistik 2009. ** Anteil der Unternehmen im Bundesland an der Sparte Gewerbe und Handwerk Österreich gesamt (ohne die Branche: Erzeugung kunstgewerblicher Gegenstände).

Die folgende Abbildung zeigt die Zusammensetzung der erzielten Stichprobe nach Sektoren im Vergleich zur Verteilung von Gewerbe- und Handwerksbetrieben in Österreich gesamt. Der Sektor Bauinstallationen/Ausbaugewerbe ist mit $45 \%$ in der Stichprobe vertreten (44 \% gesamt). Diesem Sektor werden auch die Fachgruppen Metalltechnik/Oberflächentechnik/Guss, Elektro- und Alarmanlagentechnik/Kommunikationselektronik, Chemisches Gewerbe zugerechnet, die zu den Zielgruppen von aws-Technologieprogrammen gezählt werden können. Weitere Fachgruppen, die zur Zielgruppen der aws-Technologieprogramme gezählt werden können sind Kunststoffverarbeiter (im Sektor Holz/Kunststoff subsumiert) und Mechatroniker (im Sektor KfZ/Mechatronik enthalten). Weiters relevant für die awsTechnologieprogramme ist der gesamte Sektor Kreativ/Design als Zielgruppe für impulse. In den weiteren Analysen wurden die Auswertungen immer auch mit Bezug zu den genannten Fachgruppen, die Relevanz für die aws-Technologieprogramme haben, vorgenommen.

Tabelle 40: Zusammensetzung der Stichprobe nach Sektoren, Verteilung der Unternehmen im Gewerbe und Handwerk in Österreich

\begin{tabular}{lcc}
\hline & Stichprobe* & Gesamt Ö** \\
\hline Bauinstallation/Ausbaugewerbe & $45 \%$ & $44 \%$ \\
KFZ/Mechatronik & $11 \%$ & $9 \%$ \\
Gesundheit/Wellness & $8 \%$ & $16 \%$ \\
Baugewerbe & $9 \%$ & $10 \%$ \\
Holz/Kunststoff & $9 \%$ & $10 \%$ \\
Kreativ/Design & $9 \%$ & $6 \%$ \\
Lebensmittel & $10 \%$ & $5 \%$ \\
\hline
\end{tabular}

Quelle 1: KMU FORSCHUNG AUSTRIA, Konjunkturerhebung im Gewerbe und Handwerk (4. Quartal 2010)$N=5.402$, * ohne Rauchfangkehrer, Bestattung und Allgemeiner Fachverband des Gewerbes wurden nicht befragt Quelle 2: Wirtschaftskammer Österreich, Beschäftigtenstatistik 2009. ** Anteil der Unternehmen nach Sektor an der Sparte Gewerbe und Handwerk Österreich gesamt (ohne die Branche: Erzeugung kunstgewerblicher Gegenstände, ohne Rauchfangkehrer, Bestattung und Allgemeiner Fachverband des Gewerbes). 
Mit Blick auf die Unternehmensgröße wird deutlich, dass in der Stichprobe Kleinstunternehmen (0 bis 9 MitarbeiterInnen) mit $64 \%$ den höchsten Anteil ausmachen. Weitere $31 \%$ sind Unternehmen mit 10 bis 49 MitarbeiterInnen. Die restlichen $5 \%$ sind Unternehmen mit mehr als 50 MitarbeiterInnen.

\subsection{AUßENDARSTELLUNG DER AWS-TECHNOLOGIEPROGRAMME UND AWARENESS SEITENS DER KUNDEN}

In diesem Kapitel werden zunächst die Ergebnisse aus den Interviews mit Unternehmen, die aws-Technologieprogramme genutzt haben sowie die Ergebnisse aus den Interviews mit Stakeholdern dargestellt. Darauf folgend widmen sich die Kapitel 5.2.1 und 5.2.2 den Ergebnissen aus der Konjunkturerhebung im Gewerbe und Handwerk der KMU FORSCHUNG AUSTRIA, in deren Rahmen im 4. Quartal 2010 zwei Zusatzfragen bezüglich Unterstützungsbedürfnissen und Bekanntheit der aws-Technologieprogramme gestellt wurden.

Den Außenauftritt der aws-Technologieprogramme kennen die Unternehmen idR weniger, sie nehmen eher den Gesamtauftritt der aws (Internet, Newsletter, Veranstaltungen) wahr. Generell dürfte der Außenauftritt für die Unternehmen wenig bedeutsam sein, da die aws Ansprechpartner gute und ,maßgeschneiderte“ Beratungen durchführen. Die Unternehmen dürften auch diejenigen Ansprechpartner, mit denen sie bereits Kontakt hatten, bei neuem Interesse direkt kontaktieren bzw. dürfte auch die aws die Unternehmen ,im Auge behalten“.

Die interviewten Unternehmen kennen idR auch die anderen Technologieprogramme - jedoch meist nur der Bezeichnung nach, viel weniger auch dem Inhalt nach. Da die Unternehmen seitens der aws für passende Förderungen stark beraten werden, befassen sie sich nicht selbstständig mit anderen Programmen. Die Unternehmen assoziieren als Zielgruppe der awsTechnologieprogramme primär KMU, aber auch High Tech-KMU (jene, die Gründungsprogramme in Anspruch genommen haben). Der Blick auf die aws-Technologieprogramme dürfte seitens der Unternehmen sehr fokussiert sein auf jene Förderungsprogramme, die sie auch in Anspruch genommen haben.

\subsubsection{Awareness gegenüber Innovationen und Unterstützungsbedürfnisse}

Innovation ist nach Angaben von über $50 \%$ der befragten Unternehmen von sehr großer bzw. eher großer Bedeutung. Wird nach dem Unterstützungsbedarf entlang einzelner Phasen bzw. Bereiche des Innovationsprozesses gefragt, so sehen die Unternehmen den größten Bedarf an Unterstützung in der Frühphasenfinanzierung (27\%) und auch in der Phase davor, bei der Ideengenerierung bzw. Produktfindung (22\%). In späteren Phasen, insbesondere bei der Verwertung/IPR wird seitens der befragten Unternehmen hingegen weniger Bedarf an Unterstützung angegeben; Ein Ergebnis, das vermutlich aus der Zusammensetzung des Samples (Gewerbe- und Handwerksbetriebe) resultiert. 
Abbildung 47: Bedeutung von Innovationen und Unterstützungsbedarf, befragte Unternehmen in $\%$

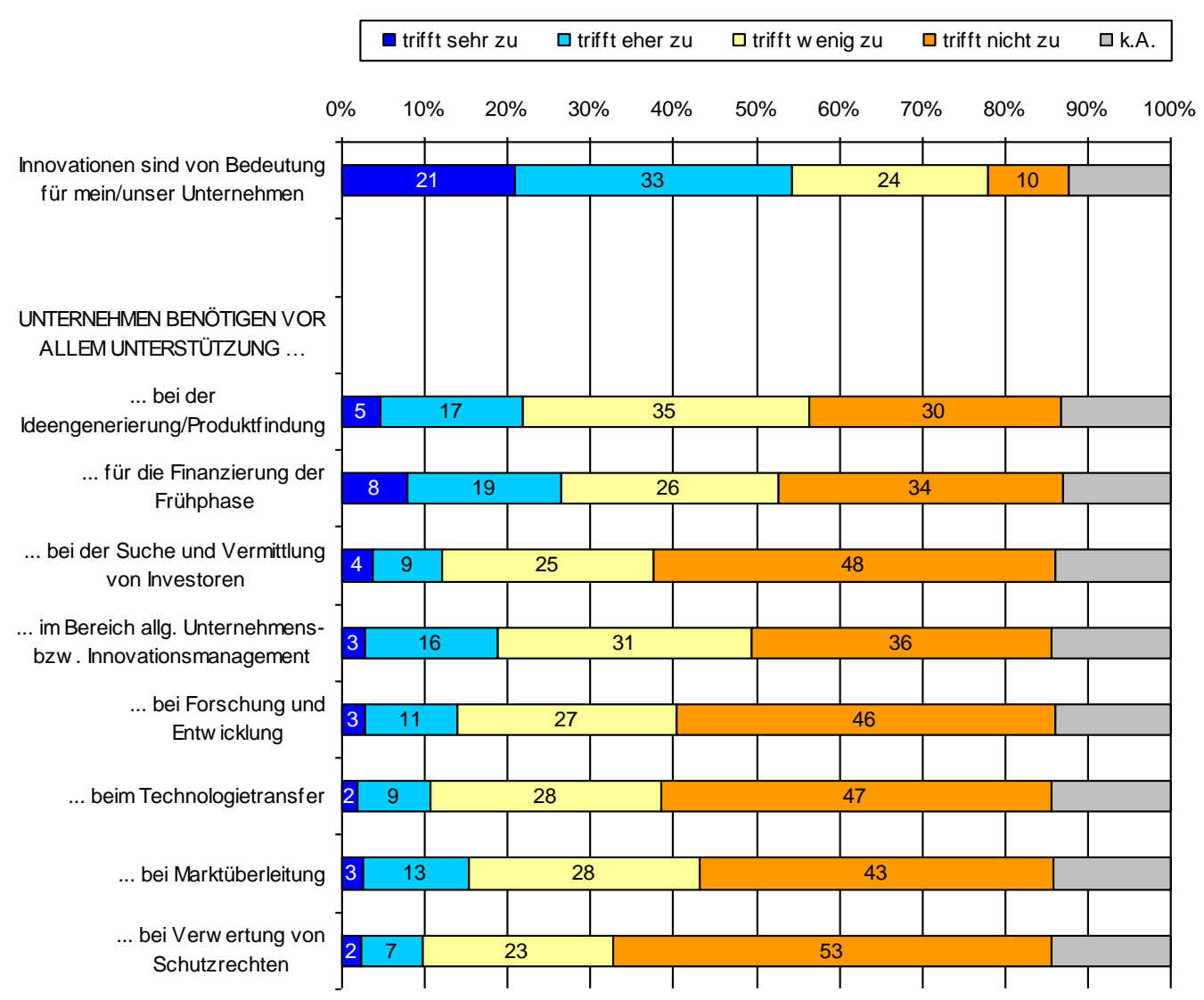

Quelle: KMU FORSCHUNG AUSTRIA, Konjunkturerhebung im Gewerbe und Handwerk (4. Quartal 2010) $N=5.402$

Die Ergebnisse bzgl. Unterstützungsbedarf für Ideengenerierung/Produktfindung und Frühphasenfinanzierung unterstützen gemeinsam die Relevanz der Frühphasenfinanzierung: Ideengenerierung und Produktfindung kann vielfach in der Vorgründungsphase relevant sein.

Differenziert nach ausgewählten Fachgruppen (siehe weiter oben), die zu den Zielgruppen der aws-Technologieprogramme gezählt werden können, ergibt sich folgendes Bild: Insbesondere Unternehmen aus den Fachgruppen Kunststoffverarbeiter und Mechatroniker messen Innovationen generell mehr Bedeutung für ihr Unternehmen zu, als andere Handwerks- und Gewerbebetriebe (siehe Tabelle 41). 
Tabelle 41: Bedeutung von Innovationen und Unterstützungsbedarf, befragte Unternehmen ausgewählter Fachgruppen in Prozent

\begin{tabular}{|c|c|c|c|c|c|c|c|c|}
\hline & \begin{tabular}{|l} 
ALLE \\
SEKTOREN
\end{tabular} & \multicolumn{3}{|c|}{ Sektor: Bauinstallation/Ausbaugewerbe } & \multirow{2}{*}{\begin{tabular}{|l|}
$\begin{array}{l}\text { Sektor:Holz/Kuns } \\
\text { tstoff }\end{array}$ \\
\\
\\
Kunststoff- \\
verarbeiter
\end{tabular}} & \multirow{2}{*}{\begin{tabular}{|l|}
$\begin{array}{l}\text { Sektor: Kfz/ } \\
\text { Mechatronik }\end{array}$ \\
Mechatroniker
\end{tabular}} & \multirow{2}{*}{\begin{tabular}{|l}
$\begin{array}{l}\text { Sektor: } \\
\text { Kreativ/Design }\end{array}$ \\
\\
(alle FG d. \\
Sektors) \\
\end{tabular}} \\
\hline & & ALLE FG & $\begin{array}{l}\text { Metalldesign, } \\
\text { Oberflächen- } \\
\text { technik und Guss }\end{array}$ & \begin{tabular}{|l} 
Elektro- und \\
Alarmanlagen- \\
technik/ Kom- \\
munikations- \\
elektronik \\
\end{tabular} & \begin{tabular}{|l|} 
Chemisches \\
Gewerbe und \\
Denkmal-, \\
Fassaden- und \\
Gebäude-reiniger \\
\end{tabular} & & & \\
\hline & & $n=5.402$ & $\mathrm{n}=44$ & $\mathrm{n}=211$ & $\mathrm{n}=71$ & $\mathrm{n}=86$ & $\mathrm{n}=212$ & $\mathrm{n}=463$ \\
\hline \multirow{5}{*}{\begin{tabular}{|l|} 
Innovationen \\
sind von \\
Bedeutung für \\
mein/unser \\
Unternehmen \\
\end{tabular}} & trifft sehr zu & 21 & 30 & 27 & 25 & 36 & 40 & 18 \\
\hline & trifft eher zu & 33 & 32 & 32 & 35 & 30 & 29 & 32 \\
\hline & trifft wenig zu & 24 & 27 & 15 & 20 & 14 & 14 & 24 \\
\hline & trifft nicht zu & 10 & 5 & 10 & 13 & 6 & 9 & 13 \\
\hline & k.A. & 12 & 7 & 16 & 7 & 14 & 8 & 14 \\
\hline \multicolumn{9}{|c|}{. BENÖTIGEN VOR ALLEM UNTERSTÜTZUNG ... } \\
\hline \multirow{5}{*}{$\begin{array}{l}\ldots \text { bei der } \\
\text { Ideengenerierung } \\
\text { /Produktfindung }\end{array}$} & trifft sehr zu & 5 & 2 & 6 & 3 & 7 & 8 & 6 \\
\hline & trifft eher zu & 17 & 11 & 20 & 14 & 24 & 24 & 13 \\
\hline & trifft wenig zu & 35 & 50 & 30 & 32 & 26 & 34 & 30 \\
\hline & trifft nicht zu & 30 & 32 & 27 & 38 & 22 & 26 & 34 \\
\hline & k.A. & 13 & 5 & 17 & 13 & 21 & 8 & 17 \\
\hline \multirow{5}{*}{\begin{tabular}{|l|}
... für die \\
Finanzierung der \\
Frühphase \\
\end{tabular}} & trifft sehr zu & 8 & 9 & 7 & 15 & 10 & 14 & 8 \\
\hline & trifft eher zu & 19 & 23 & 18 & 15 & 15 & 24 & 15 \\
\hline & trifft wenig zu & 26 & 25 & 26 & 18 & 20 & 26 & 21 \\
\hline & trifft nicht zu & 34 & 39 & 33 & 37 & 33 & 30 & 39 \\
\hline & k.A. & 13 & 5 & 17 & 14 & 22 & 7 & 17 \\
\hline \multirow{5}{*}{$\begin{array}{l}\text {... bei der Suche } \\
\text { und Vermittlung } \\
\text { von Investoren }\end{array}$} & trifft sehr zu & 4 & 2 & 5 & 10 & 7 & 6 & 4 \\
\hline & trifft eher zu & 9 & 14 & 9 & 10 & 8 & 10 & 8 \\
\hline & trifft wenig zu & 25 & 20 & 27 & 24 & 22 & 32 & 18 \\
\hline & trifft nicht zu & 48 & 57 & 42 & 45 & 40 & 44 & 52 \\
\hline & k.A. & 14 & 7 & 16 & 11 & 23 & 8 & 18 \\
\hline \multirow{5}{*}{$\begin{array}{l}\ldots \text { im Bereich } \\
\text { allg. } \\
\text { Unternehmens- / } \\
\text { Innovations- } \\
\text { management } \\
\end{array}$} & triifft sehr zu & 3 & 2 & 5 & 4 & 2 & 4 & 3 \\
\hline & trifft eher zu & 16 & 14 & 14 & 8 & 26 & 21 & 14 \\
\hline & trifft wenig zu & 31 & 41 & 31 & 35 & 27 & 35 & 24 \\
\hline & trifft nicht zu & 36 & 36 & 33 & 35 & 21 & 29 & 41 \\
\hline & k.A. & 14 & 7 & 18 & 17 & 24 & 10 & 17 \\
\hline \multirow{5}{*}{$\begin{array}{l}\text {... bei Forschung } \\
\text { und Entwicklung }\end{array}$} & trifft sehr zu & 3 & 0 & 3 & 7 & 7 & 10 & 1 \\
\hline & trifft eher zu & 11 & 27 & 12 & 15 & 29 & 25 & 5 \\
\hline & trifft wenig zu & 27 & 32 & 26 & 21 & 23 & 25 & 22 \\
\hline & trifft nicht zu & 46 & 34 & 42 & 37 & 19 & 31 & 54 \\
\hline & k.A. & 14 & 7 & 17 & 20 & 22 & 8 & 18 \\
\hline \multirow{5}{*}{\begin{tabular}{|l}
... beim \\
Technologie- \\
transfer
\end{tabular}} & trifft sehr zu & 2 & 0 & 2 & 0 & 5 & 7 & 1 \\
\hline & trifft eher zu & 9 & 23 & 12 & 14 & 22 & 20 & 5 \\
\hline & trifft wenig zu & 28 & 39 & 27 & 27 & 27 & 31 & 20 \\
\hline & trifft nicht zu & 47 & 32 & 42 & 42 & 22 & 33 & 54 \\
\hline & k.A. & 14 & 7 & 18 & 17 & 24 & 9 & 19 \\
\hline \multirow{5}{*}{$\begin{array}{l}\text {... bei Markt- } \\
\text { überleitung }\end{array}$} & trifft sehr zu & 3 & 5 & 2 & 6 & 10 & 7 & 2 \\
\hline & trifft eher zu & 13 & 25 & 13 & 17 & 19 & 23 & 10 \\
\hline & trifft wenig zu & 28 & 25 & 27 & 23 & 26 & 27 & 21 \\
\hline & trifft nicht zu & 43 & 39 & 41 & 41 & 22 & 34 & 48 \\
\hline & k.A. & 14 & 7 & 17 & 14 & 23 & 9 & 18 \\
\hline \multirow{5}{*}{$\begin{array}{l}\text {... bei Verwertung } \\
\text { von } \\
\text { Schutzrechten }\end{array}$} & trifft sehr zu & 2 & 5 & 1 & 4 & 9 & 7 & 5 \\
\hline & trifft eher zu & 7 & 9 & 8 & 7 & 15 & 17 & 10 \\
\hline & trifft wenig zu & 23 & 27 & 18 & 30 & 26 & 24 & 17 \\
\hline & trifft nicht zu & 53 & 48 & 55 & 44 & 28 & 43 & 50 \\
\hline & k.A. & 14 & 11 & 18 & 15 & 22 & 9 & 18 \\
\hline
\end{tabular}

Quelle: KMU FORSCHUNG AUSTRIA, Konjunkturerhebung im Gewerbe und Handwerk (4. Quartal 2010) $N=5.402$

Auch haben Unternehmen aus diesen Fachgruppen deutlich mehr Unterstützungsbedarf in den meisten Innovationsphasen (Ausnahme die Frühphase bei den Kunststoffverarbeitern und die Vermittlung von Investoren bei beiden Fachgruppen). Unternehmen der Fachgruppe chemisches Gewerbe zeigen, im Vergleich zur in der Stichprobe enthaltenen Gesamtheit, mehr Bedarf an Unterstützung für die Frühphase und bei der Vermittlung von Investoren (Tabelle 41). Unternehmen der Fachgruppe Metalldesign/Oberflächentechnik und Guss benötigen vor allem bei der Marktüberleitung und Verwertung sowie in der Frühphase und bei der Vermittlung von Investoren Unterstützung.

\subsubsection{Bekanntheit der aws-Technologieprogramme}

Werden die Angaben von im Rahmen der Konjunkturerhebung befragten Unternehmen zur 
Bekanntheit unterschiedlicher aws-Technologieprogramme betrachtet, so fällt zunächst auf, dass äußerst geringe Anteile der Unternehmen angeben, die entsprechenden Programme zu kennen. Die vergleichsweise bekanntesten Programme sind „Management auf Zeit“ (15\% ist die Bezeichnung bekannt und $3 \%$ auch der Inhalt) sowie impulse (12\% und $3 \%$ ). $\mathrm{Zu}$ berücksichtigen ist hier die Zusammensetzung des befragten Samples. ${ }^{43}$

Abbildung 48: Bekanntheit der aws-Technologieprogramme, befragte Unternehmen in \%

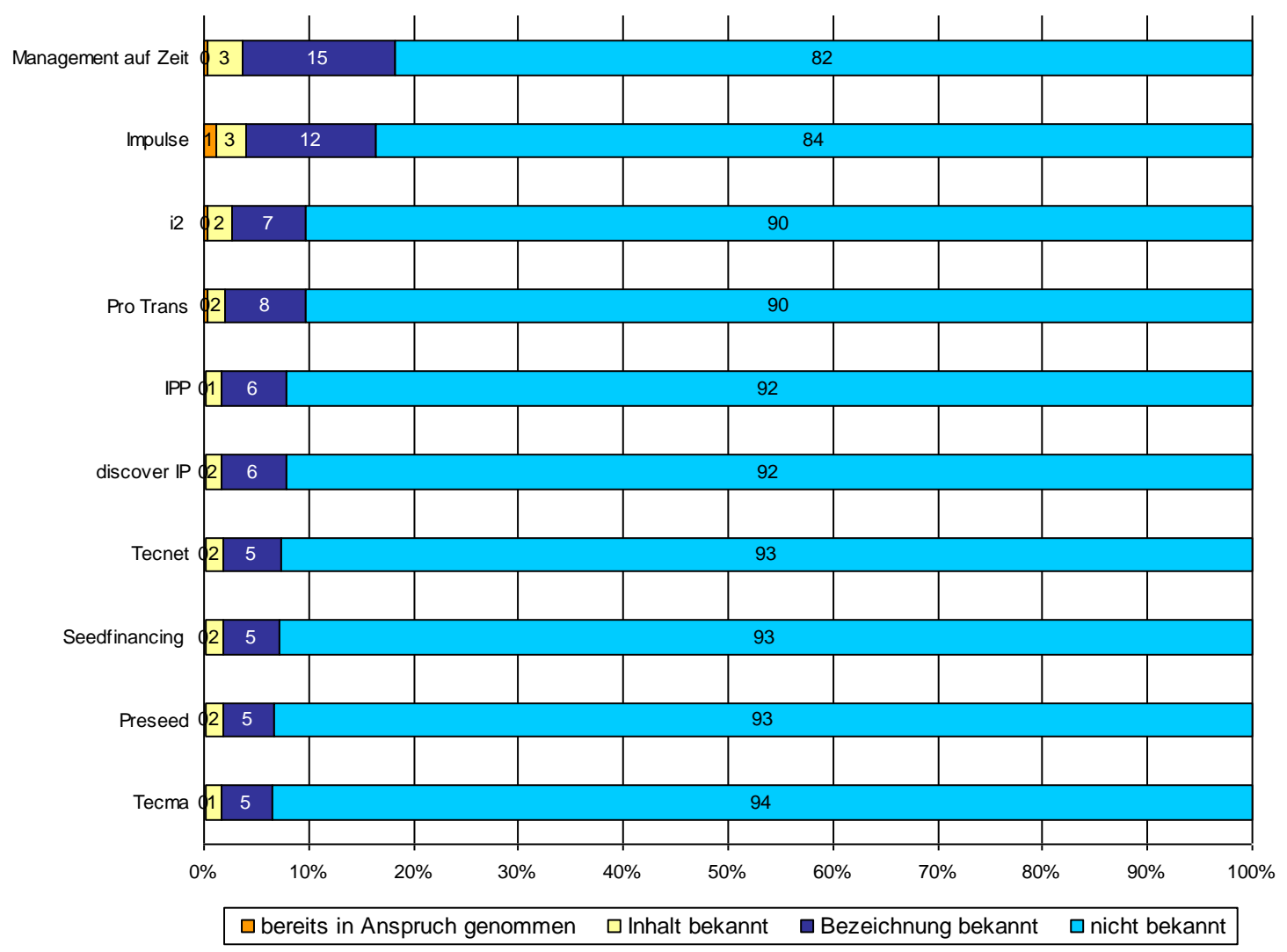

Quelle: KMU FORSCHUNG AUSTRIA, Konjunkturerhebung im Gewerbe und Handwerk (4. Quartal 2010) $n=4.756-4.888$, Mehrfachantworten (je abgefragtem Programm) waren möglich

Mit Bezug auf die ausgewählten Fachgruppen (siehe weiter oben), die zu den Zielgruppen der aws-Technologieprogramme gezählt werden können, wird deutlich, dass Unternehmen der Fachgruppe Chemisches Gewerbe die Programme (insbesondere tecma, PreSeed, Seedfinancing, tecnet, ipp, impulse) öfter kennen, als die Gesamtheit der in der Stichprobe enthaltenen Unternehmen. Auch haben diese Unternehmen die Programme schon (öfter) in Anspruch genommen. Kunststoffverarbeiter kennen insbesondere tecnet, impulse und Management auf Zeit öfter, Unternehmen der Fachgruppe Metalldesign, Oberflächentechnik und Guss kennen das Programm Management auf Zeit der Bezeichnung nach deutlich öfter. Unternehmen aus der Fachgruppe Mechatroniker kennen ebenfalls die meisten Programme, zumindest der Bezeichnung nach, öfter; die deutlichsten Abweichungen sind dabei bei den Programmen Management auf Zeit, i2, tecnet, Seedfinancing und PreSeed feststellbar. Interessant ist auch,

\footnotetext{
${ }^{43}$ Der hohe Wert beim Programm Management auf Zeit (MaZ) dürfte auf den selbsterklärenden Programmnamen zurückzuführen sein. Das Programm selbst ist sehr spezifisch und zudem auf Seed-Projekte beschränkt. Die antwortenden Unternehmen dürften somit eher das Konzept als solches, denn das konkrete Programm gemeint haben.
} 
dass Unternehmen aus dem Sektor Kreativ/Design das Programm Impulse etwas weniger oft kennen, als die Gesamtheit der Unternehmen in der Stichprobe.

Tabelle 42: Bekanntheit der aws-Technologieprogramme, befragte Unternehmen ausgewählter Fachgruppen in Prozent

\begin{tabular}{|c|c|c|c|c|c|c|c|c|}
\hline & \begin{tabular}{|l|} 
ALLE \\
SEKTOREN \\
\end{tabular} & \multicolumn{3}{|c|}{ Sektor: Bauinstallation/Ausbaugewerbe } & \multirow{2}{*}{\begin{tabular}{|l}
$\begin{array}{l}\text { Sektor:Holz/ } \\
\text { Kunststoff }\end{array}$ \\
\\
\\
$\begin{array}{l}\text { Kunststoff- } \\
\text { verarbeiter }\end{array}$ \\
\end{tabular}} & \multirow{2}{*}{\begin{tabular}{|l|}
$\begin{array}{l}\text { Sektor: Kfz/ } \\
\text { Mechatronik }\end{array}$ \\
\\
Mechatroniker
\end{tabular}} & \multirow{2}{*}{\begin{tabular}{|l|}
$\begin{array}{l}\text { Sektor: } \\
\text { Kreativ/Design }\end{array}$ \\
\\
(alle FG d. \\
Sektors)
\end{tabular}} \\
\hline & & ALLE FG & $\begin{array}{l}\text { Metalldesign, } \\
\text { Oberflächen- } \\
\text { technik und Guss }\end{array}$ & $\begin{array}{l}\text { Elektro- und } \\
\text { Alarm-anlagen- } \\
\text { technik/ Kom- } \\
\text { munikations- } \\
\text { elektronik } \\
\end{array}$ & \begin{tabular}{|l} 
Chemisches \\
Gewerbe und \\
Denkmal-, \\
Fassaden- und \\
Gebäude-reiniger
\end{tabular} & & & \\
\hline & & $n=4756-4888$ & $\mathrm{n}=42$ & $\mathrm{n}=189-191$ & $\mathrm{n}=61-64$ & $\mathrm{n}=71-73$ & $\mathrm{n}=194-200$ & $\mathrm{n}=408-415$ \\
\hline \multirow{4}{*}{ Tecma } & nicht bekannt & 94 & 95 & 94 & 89 & 92 & 88 & 93 \\
\hline & \begin{tabular}{|l|} 
Bezeichnung bekannt \\
\end{tabular} & 5 & 2 & 4 & 3 & 5 & 9 & 4 \\
\hline & \begin{tabular}{|l|l} 
Inhalt bekannt \\
\end{tabular} & 1 & 2 & 2 & 6 & 3 & 2 & 2 \\
\hline & bereits in Anspruch genommen & 0 & 0 & 0 & 2 & 0 & 1 & 0 \\
\hline \multirow{4}{*}{ Preseed } & nicht bekannt & 93 & 93 & 94 & 83 & 96 & 86 & 93 \\
\hline & Bezeichnung bekannt & 5 & 5 & 5 & 9 & 1 & 12 & 4 \\
\hline & Inhalt bekannt & 2 & 2 & 2 & 6 & 3 & 3 & 4 \\
\hline & bereits in Anspruch genommen & 0 & 0 & 0 & 2 & 0 & 1 & 0 \\
\hline \multirow{4}{*}{$\begin{array}{l}\text { Seed- } \\
\text { financing }\end{array}$} & Jicht bekannt & 93 & 95 & 93 & 86 & 93 & 86 & 92 \\
\hline & Bezeichnung bekannt & 5 & 2 & 5 & 8 & 6 & 11 & 5 \\
\hline & \begin{tabular}{|l|} 
Inhalt bekannt \\
\end{tabular} & 2 & 2 & 2 & 5 & 1 & 2 & 3 \\
\hline & bereits in Anspruch genommen & 0 & 0 & 0 & 2 & 0 & 1 & 0 \\
\hline \multirow{4}{*}{ Tecnet } & nicht bekannt & 93 & 95 & 93 & 87 & 85 & 87 & 92 \\
\hline & Bezeichnung bekannt & 5 & 2 & 5 & 6 & 10 & 11 & 5 \\
\hline & Inhalt bekannt & 2 & 2 & 2 & 5 & 5 & 2 & 3 \\
\hline & bereits in Anspruch genommen & 0 & 0 & 0 & 2 & 0 & 1 & 0 \\
\hline \multirow{4}{*}{ discover IP } & nicht bekannt & 92 & 90 & 95 & 89 & 92 & 91 & 90 \\
\hline & Bezeichnung bekannt & 6 & 7 & 4 & 6 & 5 & 7 & 6 \\
\hline & Inhalt bekannt & 2 & 2 & 1 & 3 & 3 & 2 & 4 \\
\hline & bereits in Anspruch genommen & 0 & 0 & 0 & 2 & 0 & 1 & 0 \\
\hline \multirow{4}{*}{ IPP } & nicht bekannt & 92 & 98 & 95 & 87 & 93 & 89 & 91 \\
\hline & Bezeichnung bekannt & 6 & 0 & 3 & 8 & 3 & 9 & 5 \\
\hline & Inhalt bekannt & 1 & 2 & 2 & 3 & 4 & 2 & 4 \\
\hline & bereits in Anspruch genommen & 0 & 0 & 0 & 2 & 0 & 1 & 0 \\
\hline \multirow{4}{*}{ Pro Trans } & nicht bekannt & 90 & 93 & 92 & 85 & 92 & 85 & 90 \\
\hline & Bezeichnung bekannt & 8 & 5 & 5 & 8 & 5 & 12 & 6 \\
\hline & Inhalt bekannt & 2 & 2 & 3 & 3 & 3 & 3 & 3 \\
\hline & bereits in Anspruch genommen & 0 & 0 & 0 & 3 & 0 & 1 & 0 \\
\hline \multirow{4}{*}{ i2 } & Jicht bekannt & 90 & 90 & 93 & 86 & 89 & 81 & 89 \\
\hline & Bezeichnung bekannt & 7 & 7 & 4 & 10 & 7 & 14 & 7 \\
\hline & Inhalt bekannt & 2 & 2 & 3 & 3 & 4 & 4 & 4 \\
\hline & bereits in Anspruch genommen & 0 & 0 & 0 & 2 & 0 & 1 & 0 \\
\hline \multirow{4}{*}{ Impulse } & nicht bekannt & 84 & 82 & 86 & 79 & 83 & 76 & 84 \\
\hline & Bezeichnung bekannt & 12 & 13 & 12 & 16 & 11 & 16 & 9 \\
\hline & Inhalt bekannt & 3 & 0 & 2 & 3 & 6 & 4 & 5 \\
\hline & bereits in Anspruch genommen & 1 & 5 & 0 & 2 & 0 & 3 & 1 \\
\hline \multirow{4}{*}{$\begin{array}{l}\text { Management } \\
\text { auf Zeit }\end{array}$} & nicht bekannt & 82 & 76 & 79 & 77 & 73 & 74 & 84 \\
\hline & Bezeichnung bekannt & 15 & 21 & 16 & 16 & 16 & 21 & 10 \\
\hline & \begin{tabular}{|l|} 
Inhalt bekannt \\
\end{tabular} & 3 & 2 & 4 & 6 & 10 & 5 & 6 \\
\hline & bereits in Anspruch genommen & 0 & 0 & 0 & 2 & 1 & 1 & 0 \\
\hline
\end{tabular}

Quelle: KMU FORSCHUNG AUSTRIA, Konjunkturerhebung im Gewerbe und Handwerk (4. Quartal 2010) $n=4.756-4.888$, Mehrfachantworten (je abgefragtem Programm) waren möglich

249 der 5.402 befragten Unternehmen gaben an, bereits andere Förderungen (außerhalb der aws-Technologieprogramme) in Anspruch genommen zu haben. Unter jenen Unternehmen haben $50 \%$ andere aws-Förderungen (Jungunternehmerförderung, ERP, Unternehmensdynamik und andere) in Anspruch genommen, $5 \%$ FFG-Förderungen und $45 \%$ weder aws noch FFG-Förderungen, sondern beispielsweise Förderungen der Bundesländer in Anspruch genommen.

Bei den Angaben der befragten Unternehmen zur Bekanntheit der einzelnen Programme kann weiters festgestellt werden, dass die Unternehmen, die Unterstützungsbedarf in den einzelnen Phasen äußern, die aws-Technologieprogramme auch kennen. Somit gibt es keine deutlichen Hinweise auf Defizite in der Kenntnis der Programme bei gleichzeitigem Unterstützungsbe- 
darf.

Die Einschätzung der Bekanntheit der Technologieprogramme durch die Interviewpartner lässt sich anhand der folgenden Argumente zusammenfassen:

- Die Logik der Abgrenzung der Technologieprogramme ist weniger eine der Interventionslogik als eine organisatorische - die Technologieprogramme bedienen sich keiner Bankeninstrumente. Das führt zum Teil dazu, dass diese Instrumente den Unternehmen nicht oder weniger bekannt sind, da der Vertriebs- und Informationskanal über die Kommerzbanken wegfällt.

- Umgekehrt wird argumentiert - und die Interviews mit Kunden stützen dieses Argument - dass gerade technologieorientierte Gründer mit der ersten Anfrage bzgl. Beratung oder Förderung in ein relativ engmaschiges Netz an Beratungs- und Förderungsinstitutionen eintreten, sodass die Informationskosten ab diesem Zeitpunkt nicht mehr allzu hoch sein dürften und Beratung oft auch pro-aktiv an die UnternehmerInnen oder GründerInnen herangetragen wird.

Die Problematik der Abgrenzung mit der Bezeichnung „Technologieprogramme“ zeigte sich auch in den Interviews mit Stakeholdern und ExpertInnen- dabei wurde wiederholt Bezug auch auf andere aws-Förderungen genommen, wie etwa Jungunternehmerförderung, Unternehmensdynamik etc. Umgekehrt wurde z B impulse kaum den Technologieprogrammen zugeordnet. Ebenso wurden die awareness Maßnahmen (Staatspreis, Jugend Innovativ) idR nicht von selbst angesprochen und als Teil des Clusters gesehen. 
Abbildung 49: Kenntnis der aws-Technologieprogramme in Abhängigkeit von dem für das Unternehmen zutreffenden Unterstützungsbedarf in einzelnen Innovationsphasen*

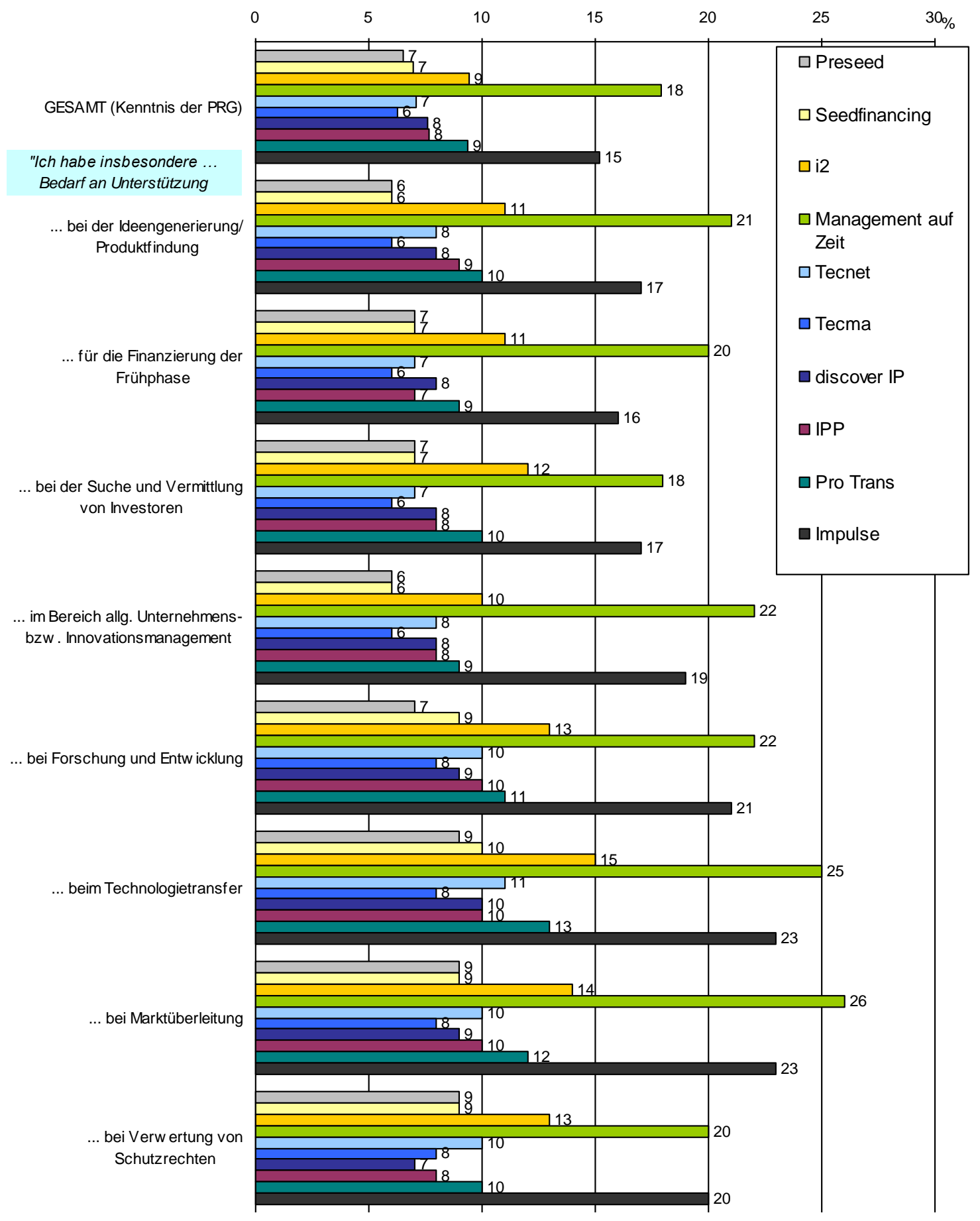

$n=4.756$ bis 4.888 bzw. 1.108 bis 1.134; 1.344 bis 1.382; 616 bis 633; 966 bis 989; 700 bis 720 ; 547 bis 565; 779 bis 803; 499 bis 512, Mehrfachantworten (je abgefragtem Programm) waren möglich.

* Angaben Programm bekannt (Bezeichnung und /oder Inhalt), wenn in einzelnen Phasen Angaben „trifft sehr zu bzw. trifft eher $z u$ ".

Quelle: KMU FORSCHUNG AUSTRIA, Konjunkturerhebung im Gewerbe und Handwerk (4. Quartal 2010) 
In Abbildung 49 fällt auf, dass jene Unternehmen, die Unterstützungsbedarf in den Phasen F\&E, Technologietransfer, Marktüberleitung (und mit Abstrichen auch in der Schutzrechtsverwertung) bekunden, in der Regel auch öfter die aws-Technologieprogramme kennen als Unternehmen, die in den davor gelagerten Phasen mehr Unterstützung benötigen. Am deutlichsten wird dies bei dem Programm impulse. Beim Programm Management auf Zeit kennen die Unternehmen, die im Bereich Technologietransfer und Marktüberleitung Unterstützungsbedarf bekunden, das Programm tendenziell öfter als Unternehmen, die in den vorgelagerten Phasen Bedarf an Unterstützung angeben. Unternehmen, die bei der Verwertung von Schutzrechten Unterstützungsbedarf angeben, kennen nicht notwendigerweise die beiden Innovationsschutzprogramme discover ip und ipp öfter als Unternehmen, die in anderen Phasen solchen Bedarf sehen.

\section{Fazit}

Für eine Einschätzung der Wahrnehmung und Bekanntheit der aws-Technologieprogramme und der Awareness für Innovationen seitens potenzieller Kunden wurde im Rahmen der Evaluierung auf Ergebnisse der vierteljährlichen Konjunkturerhebung der KMU FORSCHUNG AUSTRIA zurückgegriffen. Bei dieser Erhebung wurden Zusatzfragen zum Stellenwert von Innovationen und zur Bekanntheit der aws-Technologieprogramme platziert. Bei der Auswertung wurden auch in Abstimmung mit der aws ausgewählte Fachgruppen berücksichtigt, die für die aws relevante Zielgruppen darstellen. Zusätzlich wurden Interviews mit Nutzern der aws-Technologieprogramme und mit weiteren Stakeholdern auf Bundes- und Landesebene durchgeführt.

Die Unternehmen geben im Rahmen der Konjunkturerhebung an, dass sie insbesondere in der Frühphase und auch in der Phase davor (Ideengenerierung/Produktfindung) Unterstützung benötigen. Die Ergebnisse unterstützen also die Relevanz der Frühphasenfinanzierung bzw. der Unterstützung in dieser Phase: Ideengenerierung und Produktfindung kann vielfach in der Vorgründungsphase relevant sein.

Generell dürften die aws-Technologieprogramme von den Unternehmen eher wenig wahrgenommen werden. Die Logik der Abgrenzung der aws-Technologieprogramme als „Dachmar$\mathrm{ke}^{\prime}$ ist eher eine organisatorische als eine der Interventionslogik. Der Cluster der „Technologieprogramme“" ist dementsprechend eher als Label mit Signal für die policy-Ebene geeignet, aber nicht unbedingt in Richtung Zielgruppen, da aus deren Perspektive unterschiedlichste Motivbündel (siehe Modul 2) aufgegriffen werden. Zudem fehlt in der „Vermarktung“ der Informationskanal über die Kommerzbanken. Die Unternehmen kommen idR eher aus einer bestimmten Problemlage zu den einzelnen Programmen, die den in Modul 2 dargestellten Zielbündeln entsprechen. Die neue Homepage hat die Kommunikation und Außendarstellung bereits eher an diese Motivbündel angepasst, ähnlich die neuen Folder. Andererseits wird argumentiert, dass gerade technologieorientierte Gründer mit der ersten Anfrage bzgl. Beratung oder Förderung in ein relativ engmaschiges Netz an Beratungs- und Förderungsinstitutionen eintreten, sodass die Informationskosten ab diesem Zeitpunkt nicht mehr allzu hoch sein dürften und Beratung oft auch pro-aktiv an die UnternehmerInnen oder GründerInnen herangetragen wird. 


\section{Modul 5: Internationale Beispiele und Erfahrungen}

Die Entwicklung der FTI-Politik in den OECD-Staaten innerhalb der letzten Jahrzehnte lässt sich grob als eine konvergente Entwicklung charakterisieren. Die Ziele und Instrumente nationaler FTI-Politiken wurden zunehmend ähnlicher, wobei sowohl politische als auch ökonomische Faktoren dafür verantwortlich sind (Rammer et. al. 2004). Trotzdem bestehen nach wie vor relevante Unterschiede zwischen den Innovationssystemen, die vor allem vor dem Hintergrund von dauerhaften Performanceunterschieden von politischer Relevanz sind. In diesem Sinne besteht die Aufgabe von Modul 5 darin, die Technologieprogramme der aws mit internationalen Good-Practice Beispielen ähnlich strukturierter Programme zu vergleichen. Darüber hinaus werden auch Programme vorgestellt, die keine unmittelbare Entsprechung im aws-Programmportfolio haben, aber dennoch interessante impulse für eine Reflexion bzw. Weiterentwicklung technologiepolitischer Instrumente im Bereich Entrepreneurship und IPR-Aktivitäten geben können.

Die internationalen Beispiele lassen sich im Rahmen des aws-TechnologieprogrammPortfolio den Bereichen Unternehmensgründungen und Patentverwertung zuordnen (siehe Abbildung 1). Kap. 5.1 gibt zunächst einen Überblick über die für den Vergleich relevanten Merkmale der Innovationssysteme der ausgewählten Länder bzw. Regionen. Danach werden in Kap. 5.2 (bzw. 5.3) internationale Programme zur Förderung von Hochtechnologiegründungen (zur Patentverwertung) dargestellt und mit den aws-Programmen verglichen. ${ }^{44}$

\subsection{VERGLEICHSSTAATEN IM ÜBERBLICK: RAHMENBEDINGUNGEN UND TRENDS}

In Absprache mit dem BMWFJ und der aws wurden Technologieprogramme folgender Staaten bzw. Regionen zum Vergleich herangezogen: Deutschland, Bayern, Dänemark, Frankreich und Israel. Die Auswahl der Vergleichsländer hat zwei Bedingungen zu erfüllen: Erstens sollten diese Länder im Bereich von Hochtechnologiegründungen erfolgreich sein. Zweitens ist eine hinreichende Ähnlichkeit mit dem österreichischen Innovationssystem notwendig um einen Lernprozess von internationalen Instrumenten oder Programmen zu ermöglichen. Israel ist insofern als Sonderfall zu betrachten, als hier singuläre Entwicklungen einen Vergleich mit Österreich erschweren. Trotzdem wurde Israel aufgrund des zunehmenden internationalen Interesses an dem erfolgreichen Strukturwandel zugunsten von Hochtechnologiebranchen in den letzten zwanzig Jahren in dieses Modul 5 aufgenommen (siehe Kasten 1). Frankreich wurde weniger wegen einer besonderen Gründerdynamik ausgewählt, als wegen des Interesses an dem kürzlich gegründeten Patentfonds „France Brevets“.

Ein Vergleich hat die jeweils unterschiedlichen institutionellen und ökonomischen Voraussetzungen in diesen Ländern zu berücksichtigen. Daher sollen nachstehend die für den Vergleich relevanten Merkmale der Innovationssysteme erörtert werden. Zunächst werden die Grün-

\footnotetext{
${ }^{44}$ Die Informationen zu den Programmen stammen aus vier Quellen: Evaluationsberichte, Programmdokumente, EmailBefragungen und Telefoninterviews. Leider existieren nicht zu allen hier verglichenen Programmen Evaluationen oder sind zum Teil, falls sie existieren, nicht freigegeben. Die Befragungen und Interviews wurden im März 2011 mit den jeweils programmverantwortlichen Personen geführt. Die Programmdokumente wurden von den Homepages der Programme bezogen bzw. durch die Interviewpartner zur Verfügung gestellt.
} 
dungspolitiken dieser Staaten skizziert, danach zentrale FTI-Performance-Indikatoren verglichen und anschließend die beiden Bereiche Hochtechnologieunternehmensgründungen und Patentverwertung vergleichend dargestellt.

\section{Kasten 1: Israels Entwicklung zum Hochtechnologiestandort - Sonderentwicklung oder Rol- lenmodell?}

Die ökonomische und technologische Entwicklung Israels ist aktuell ein viel beachtetes Thema in innovations- und gründungspolitischen Debatten. Tatsächlich ist der Erfolg Israels beeindruckend, wie nachstehende ausgewählte Beispiele belegen ${ }^{45}$ : Abbildung 50 und Abbildung 51 zeigen für Israel Spitzenwerte bei F\&Eund AkademikerInnenquote; Israel gilt als das Land mit der höchsten Dichte an Hochtechnologiegründungen; Venture Capital-Investition in \% des BIP sind die höchsten weltweit, wobei Israel 2008 in absoluten Zahlen mehr Venture Capital-Investitionen attrahieren konnte als Frankreich und Deutschland zusammen; die Anzahl an der NASDAQ gelisteter israelischer Technologieunternehmen übersteigt jene von indischen und chinesischen Unternehmen zusammen; der Anteil von IKT an Wertschöpfung im Industriesektor (Dienstleistungssektor) ist mit ca. $21 \%$ (19\%) der zweithöchste (höchste) in der OECD. Zum Vergleich: Der Anteil der finnischen IKT-Industrie an der industriellen Wertschöpfung liegt bei etwa $20 \%$, während jener der IKT-Dienstleistungen mit ca. $12 \%$ weit unter dem Wert von Israel liegt.

Welche Faktoren können diese Entwicklung erklären? Bedenkt man, dass Israel noch vor zwei Jahrzehnten keineswegs zu den technologisch dynamischsten Volkswirtschaften zählte, sondern sich erst seit etwa 1990 laut Economist (29.12.2010) von einem „semi-socialist backwater“ zu einer „high-tech superpower“ entwickelte, so erscheint diese Frage umso interessanter. Neben diesem zeitlichen Entwicklungsverlauf ist insbesondere auch die Unterscheidung in tendenziell singuläre oder potentiell replizierbare Faktoren in der innovationspolitischen Diskussion zu beachten. Greift man diese Unterscheidung auf, so können, aufbauend auf der einschlägigen Literatur, vereinfacht folgende Erfolgsfaktoren genannt werden:

\section{Potentiell replizierbare Faktoren}

- Hohes Niveau an Bildungsinvestitionen und Humankapital

- Naturwissenschaftlich-technische Schwerpunktsetzungen der Forschungspolitik

- Offenheit für Immigration

\section{Singuläre Faktoren}

- Hohes Niveau an Militärausgaben und militärischer Forschung

- Historisch bedingte enge ökonomische, soziale und politische Verbindungen zu den USA

- Positiver Angebotsschock an hochqualifiziertem Humankapital nach 1989 (s.u.)

Während die genannten potentiell replizierbaren Faktoren mehr oder weniger den in einschlägigen österreichischen FTI-Studien genannten kritischen Punkten entsprechen (z.B. Janger 2009), soll nachstehend kurz auf die Bedeutung der singulären Faktoren eingegangen werden.

Die hohe Bedeutung des Militärs (Israel Defense Forces) für die israelische Ökonomie kann angenähert werden anhand der Ausgaben für militärische Zwecke am BIP (Abbildung 50). Konkret wirkt der militärische Komplex über vier Kanäle auf innovationssystem-relevante Variablen:

1. Der hohe Anteil an Militärdienstleistenden in technischen Eliteeinheiten (bei einem mindestens zwei Jahre langen Militärdienst) ist eine hochwertige Humankapitalinvestition.

2. Der Militärdienst stellt sich auch als eine bedeutende Sozialkapitalinvestition dar; viele Unternehmensgründungen stellen Projekte zwischen GründerInnen dar, deren soziale Beziehung auf den gemeinsamen

${ }^{45}$ Die zitierten Zahlen sind folgenden Quellen entnommen: OECD Factbook 2010, Chorev/ Anderson 2006, Saxenian 2006, The Economist, 29. Oktober 2009, The Economist, 29. Dezember 2010. 
Militärdienst zurückgeht. Auch das Einüben von Führungskompetenzen und Improvisationstalent im Rahmen des Militärdienstes dürften positive Effekte auf unternehmerische Qualifikationen haben.

3. Das Militär ist ein wichtiger Nachfrager nach technologieintensiven Produkten privater Unternehmen. Neben dem positiven Einfluss durch eine technologisch anspruchsvolle Nachfrage zeigt sich z.B. auch, dass Kontrakte mit dem Militär eine Signalling-Funktion für Venture-Capital-Gesellschaften haben, da diese als Hinweis auf gute Performance interpretiert werden. Konktrakte mit dem Militär ,may assist entrepreneurial new high-tech firms to gain support of potential financing sources, even in cases where they have not yet completed products" (Honig et.al. 2006, S.433).

4. Das Militär tritt selbst als Technologieproduzent auf und schafft damit die Grundlagen für zivile Folgenutzungen von ursprünglich militärischen Technologien. In diesem Zusammenhang ist insbesondere das 1948 gegründete Science Corps zu erwähnen, welches im Rahmen der israelischen Streitkräfte für die Entwicklung neuer Kriegsgeräte zuständig ist.

Abbildung 50: Militärausgaben in \% des BIP (2007)

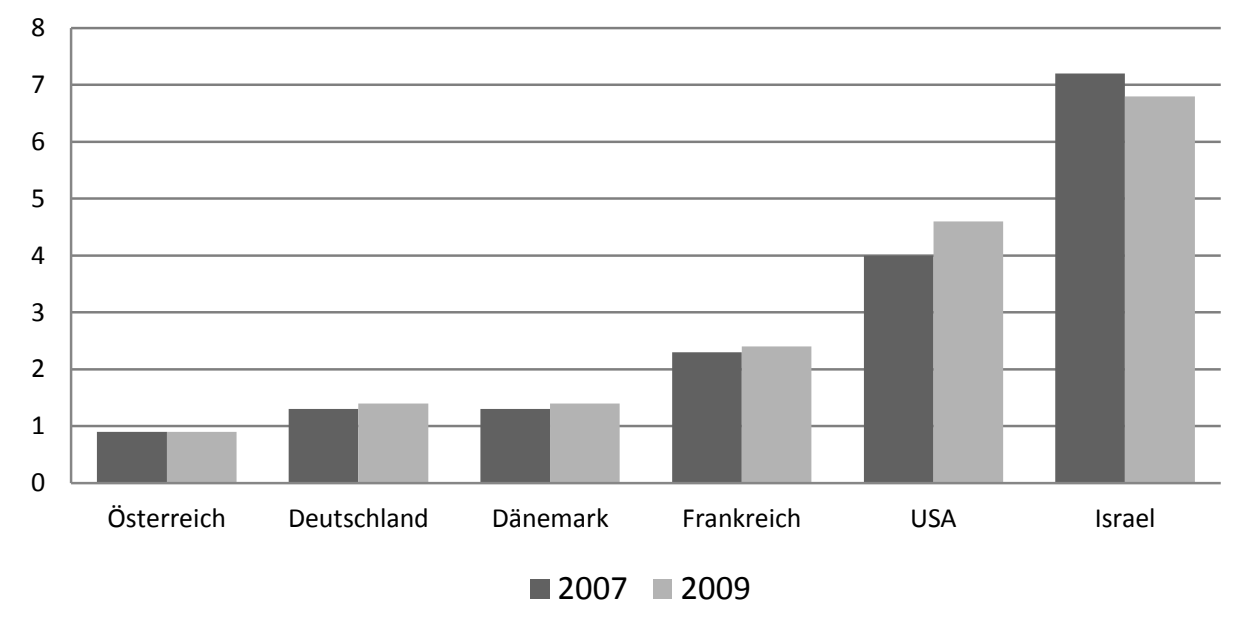

Quelle: Weltbank, eigene Darstellung

Neben dem Militär ist die enge politische und ökonomische Verbindung zwischen Israel und den USA zu berücksichtigen. Es leben etwa 500.000 israelische Staatsbürger in den USA. Darüber hinaus unterstützen auch viele jüdische US-Staatsbürger den Staat Israel. Ein kleiner aber qualitativ bedeutender Teil dieser Population ist auf einen Brain Drain israelischen Humankapitals in die USA in den 1970er und 1980er Jahren zurückzuführen ${ }^{46}$ Ein geteiltes Commitment zur Unterstützung des israelischen Staates sowie die erwähnten sozialen Netzwerke aus dem Militärdienst führten zur Entstehung eines dichten Netzwerks an Kontakten zwischen der israelischen Diaspora in amerikanischen Hochtechnologieregionen und Israel. In weiterer Folge wandelte sich dieser anfängliche Brain Drain in einen Brain Gain: Einerseits folgten multinationale Unternehmen wie IBM oder Intel ihren israelischen Ingenieuren bei ihrer Rückkehr nach Israel, um diese nicht zu verlieren, und gründeten Forschungsabteilungen in Israel („Jobs Follow People“). Freilich waren dabei auch das allgemein hohe Bildungsniveau sowie die relativ niedrigen Lohnkosten weitere wichtige Standortfaktoren. Andererseits transferierten mobile, israelische Ingenieure und Manager aus Silicon Valley Marktwissen, Management-Fähigkeiten und Entrepreneurship-Know-How aus der erfolgreichsten Technologieregion der Welt nach Israel. Insbesondere kam es zu Unternehmensgründungen durch Entrepreneure, die zuvor in Silicon Valley Erfahrungen sammeln konnten, und zu Investments amerikanischer Venture Capital-Gesellschaften in Israel sowie, mit Unterstützung der Politik, zum Aufbau einer eigenständigen

\footnotetext{
${ }^{46}$ Unter einem Brain Drain versteht man einen Abfluss von Humankapital ohne kompensatorische Zuflüsse. Bei etwa gleich großen Strömen spricht man von einem Brain Exchange und bei höheren Zuflüssen als Abflüssen von einem Brain Gain.
} 
israelischen Venture Capital-Industrie. Die enge Bindung an die USA wird nicht zuletzt dadurch deutlich, dass israelische Hochtechnologiegründungen ihren IPO eher an der NASDAQ als an der Tel Aviv Stock Exchange vornehmen (Saxenian 2006).

Als weiterer singulärer Faktor ist der positive Angebotsschock an Humankapital am israelischen Arbeitsmarkt in der Folge des Zusammenbruchs der osteuropäischen Regime nach 1989 zu nennen. Abbildung 51 zeigt diese Sonderentwicklung mit dem dramatischen Anstieg der Netto-Migrationsrate unmittelbar um 1990 und das danach noch länger anhaltend sehr hohen Einwanderungsvolumen.

Abbildung 51: Netto-Migrationsraten im Vergleich (EinwanderInnen - AuswanderInnen/1000)

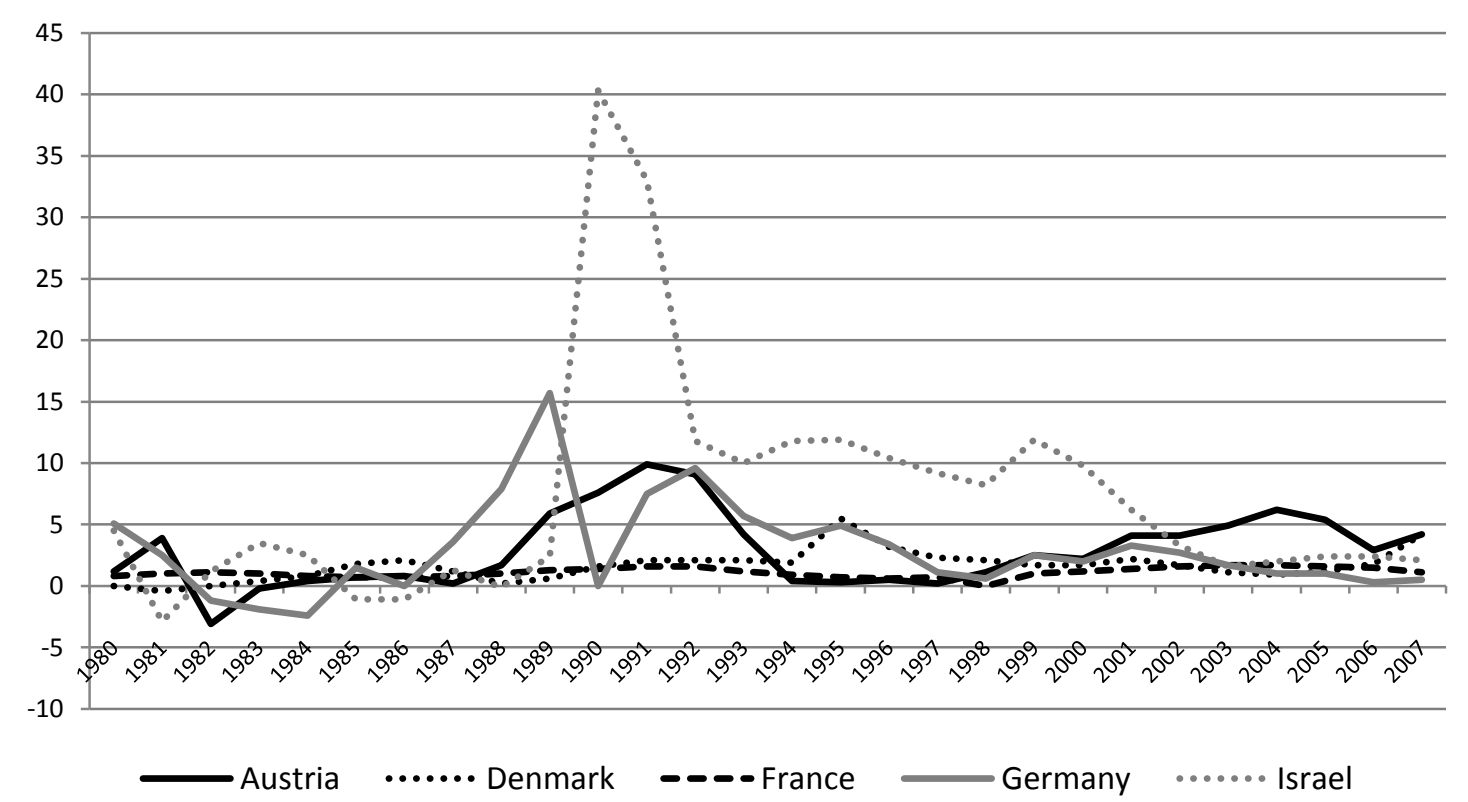

Quelle: OECD, eigene Darstellung

In absoluten Zahlen kam es zwischen 1990 und 2000 zur Einwanderung von ca. 800.000 Personen aus der ehemaligen Sowjetunion (Senor/ Singer 2009). Die Angaben bezüglich des Anteils an hochqualifizierten ImmigrantInnen an dieser Einwanderungswelle sind in der Literatur divergierend. Einstimmig wird jedoch betont, dass es sich generell um eine deutlich überdurchschnittlich gebildete Bevölkerungsgruppe mit vorwiegend ingenieurwissenschaftlicher und naturwissenschaftlicher Ausbildung handelte. Durch den positiven Angebotsschock kam es kurzfristig zu einem Lohndifferenzial von $30 \%$ im Vergleich zu den USA, jedoch zeichnete sich durch den IT-Boom der Jahre vor 2000 bereits Ende der 1990er Jahre eine Knappheit am Arbeitsmarkt ab. Es sei an dieser Stelle angemerkt, dass der tatsächliche Beitrag dieser EinwanderInnen differenziert beurteilt werden muss, denn neben der hohen formalen Qualifikation fehlte regelhaft unternehmerisches Know-How, das in der planwirtschaftlichen Sozialisierung kaum erworben werden konnte; auch sprachliche Barrieren waren zu überwinden.

Um den Aufschwung des israelischen Hochtechnologiesektors in den 1990er Jahren zu verstehen, sind mindestens zwei Faktoren in ihrer zeitlichen Koinzidenz entscheidend: Einerseits das zunehmende Engagement der israelischen Diaspora sowie multinationaler Technologieunternehmen in Israel, andererseits die Einwanderung einer deutlich überdurchschnittlich qualifizierten Population von WissenschafterInnen und IngenieurInnen. Alle weiteren Faktoren, wie das Militär oder die hohen Bildungsinvestitionen ermöglichten aber erst diese Entwicklung, wenngleich sie deren zeitlichen Verlauf nicht erklären können.

Blickt man nicht nur auf die „Strengths“ sondern auch auf die „Weaknesses“ des israelischen Modells, so sind unter anderem zwei wachstumshemmende Faktoren zu nennen: Erstens hat Israel zwar viele Hochtechnologiegründungen, aber kaum ein Unternehmen schafft es zum Großunternehmen (Cohen 2002, The Eco- 
nomist, 29.12.2010). Bislang gibt es keinen israelischen Global Player wie etwa Google oder Cisco. Zweitens ist die sehr niedrige Erwerbsbeteiligung von arabischen und orthodoxen Juden, die sich auf eine insgesamt niedrige Erwerbsquote auswirkt (Abbildung 52), zu nennen. Dieser Befund relativiert die gemeinhin sehr positiv beurteilte israelische Wettbewerbsfähigkeit. Bei männlichen orthodoxen Juden liegt die Erwerbsquote etwa bei $39 \%$, bei arabisch-jüdischen Frauen bei $25 \%$. Bedenkt man, dass diese beiden Bevölkerungsgruppen 2025 zusammen etwa ein Drittel der Bevölkerung ausmachen werden, droht hier möglicherweise demnächst ein Flaschenhals für eine weitere ökonomische Expansion (The Economist, 29.12.2010).

Abbildung 52: Erwerbsquoten im Vergleich

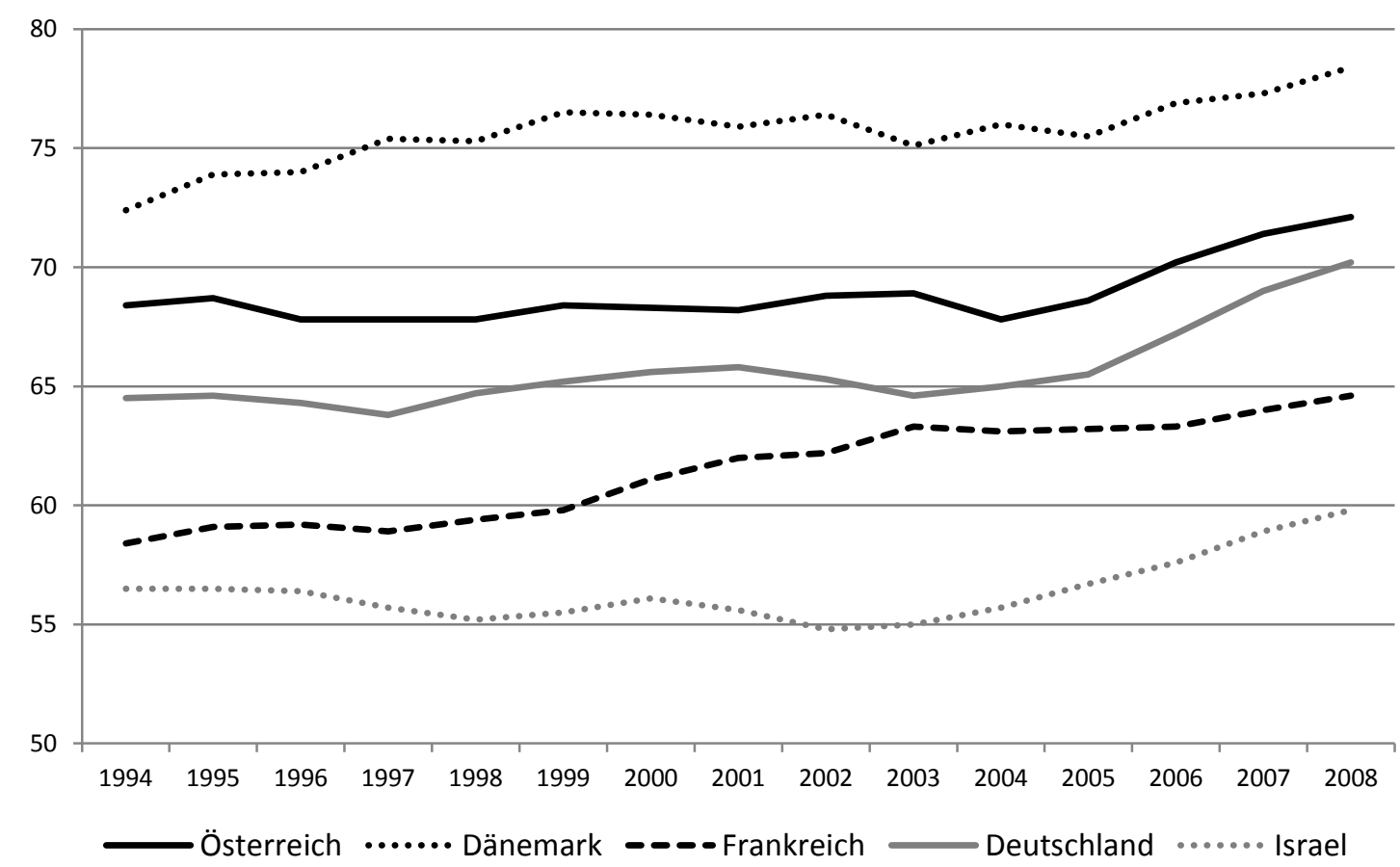

Quelle: OECD, eigene Darstellung

Zusammengenommen kann festgehalten werden, dass die Entwicklung Israels in den letzten 20 Jahren im Wesentlichen auf Faktoren beruht, die jedenfalls für Österreich kaum replizierbar sind, sofern das z.B. in Bezug auf die Bedeutung des militärischen Apparats überhaupt wünschenswert erscheint. Wichtige Punkte, die aber trotzdem durchaus interessant sein könnten, betreffen die Bedeutung der Diaspora sowie die soziale Offenheit einer Gesellschaft für Einwanderung. Auch Österreich hat im Bereich Wissenschaft durchaus eine bedeutende Diaspora in den USA und anderen Staaten. Überlegungen hinsichtlich Möglichkeiten der Nutzung des Know-Hows dieser Diaspora auch für Hochtechnologieunternehmensgründungen in Österreich wären möglicherweise eine interessante Ergänzung zum bestehenden FTI-Instrumentenportfolio. Oberösterreich hat eine diesbezügliche Netzwerk-Initiative (Oberösterreich International) im Jahr 2007 gegründet. Der zweite Punkt betrifft die Offenheit einer Gesellschaft für Einwanderung. Senor und Singer (2009, S.132) beschreiben bildhaft die Einstellung in Israel zur Immigration: „If Israelis hear on the radio at the end of the year that immigration was down, this is received as bad news, like reports there was not enough rainfall that year." An diesem Beispiel lassen sich auch Anregungen für die österreichische Politik gewinnen.

\subsubsection{Stellenwert und Entwicklung der Gründungspolitik in den FTI-Politiken der Vergleichsstaaten}

Die Förderung innovativer Unternehmensgründungen und KMUs sowie die Mobilisierung von Venture Capital sind regelhafte Elemente der FTI-Politik von OECD-Staaten. Auf Instrumentenebene gibt es typischerweise einen Mix aus Transfers in Kind, Transfers in Cash 
und regulatorischen Maßnahmen. Der Fokus der FTI-Politik auf Hochtechnologiegründungen ist im Wesentlichen ein Ergebnis der 1990er Jahre, wobei insbesondere angelsächsische Staaten eine Vorreiterrolle einnahmen. So wurde beispielsweise in den USA bereits 1958 mit dem Small Business Investment Company (SBIC)-Programm ein wichtiges Instrument zum Aufbau der Infrastruktur der heute existierenden Venture Capital-Industrie eingeführt (Lerner 2009). Ein quantitativer Vergleich der Politiken hinsichtlich der Ausgaben für Hochtechnologiegründungen ist nur sehr eingeschränkt möglich. Daher wird im Folgenden ein kurzer qualitativer Überblick zur Entwicklung und Bedeutung der politischen Förderungen von Hochtechnologiegründungen in den Vergleichsstaaten gegeben.

Politikmaßnahmen zur Förderung von Hochtechnologiegründungen in Österreich sind eng mit der Gründung des Förderprogramms Seedfinancing im Jahr 1989 verbunden. Ein weiterer wichtiger Meilenstein, um die als mäßig wahrgenommene Gründungsdynamik in Österreich zu steigern, war der Start des AplusB Programms im Jahr 2001. Die jüngst veröffentlichte FTI-Strategie der österreichischen Bundesregierung betont die Rolle von HochtechnologieGründungen. Es wird ein explizites quantitatives Ziel definiert, wonach die jährlichen wissens- und forschungsintensiven Neugründungen um durchschnittlich $3 \%$ p.a. bis 2020 ansteigen sollen (Republik Österreich 2011).

Dänemark ist eine kleine offene Volkswirtschaft mit einem dominanten KMU-Sektor. Seit dem Ende der 1990er Jahre wurden zahlreiche Initiativen zur Förderung von Entrepreneurship gesetzt. Diese umfassten Finanzierungsförderungen, Ausbau von Entrepreneurship-Education, Beratungsleistungen, Technologietransfer und Internationalisierung. Die Förderung der Finanzierungsbedingungen in Form von Venture Capital wurde durch den 1993 gegründeten öffentlichen Fonds „Vakstfonden“ bereits frühzeitig institutionalisiert. Hervorzuheben ist ein regelmäßiges Monitoring der Gründungsaktivitäten in Dänemark, welches den Informationsgrad der Politik systematisch verbessert und damit die Wahrscheinlichkeit rationaler Politikgestaltung erhöht. In der 2006 veröffentlichten „Globalisation Strategy“ ist eines unter insgesamt 14 Zielen die Förderung von rasch wachsenden Unternehmen. Dahinter steht die Wahrnehmung einer im internationalen Vergleich hohen Gründungsintensität bei gleichzeitig niedrigem Anteil an rasch wachsenden Gründungen. In dem Strategiedokument wird das Ziel definiert, Dänemark bis 2015 in der Spitzengruppe der Staaten mit rasch wachswenden Unternehmen zu positionieren. Letztere wird typischerweise von den USA dominiert (Veugelers 2009). Damit verfolgt die dänische Regierung ein deutlich fokussiertes Ziel in der Gründungspolitik, das an die operativen Förderinstitutionen den Anspruch einer erfolgreichen Selektion von Unternehmen mit hohem Wachstumspotential stellt.

In Deutschland besitzt die Förderung von Hochtechnologiegründungen hohe politische Aufmerksamkeit. Der jährlich publizierte Bericht des ZEW in Mannheim zu Hochtechnologiegründungen (Mannheimer Unternehmenspanel) ist ein einschlägiges MonitoringInstrument. Wichtige Meilensteine in der deutschen FTI-Politik zur Gründungsförderung waren der Start von EXIST als Programm für Existenzgründungen aus der Wissenschaft sowie im Jahr 1998 die Etablierung des High-Tech Gründerfonds 2005. 2010 wurden weitere Initiativen zum bereits bestehenden, umfangreichen Förderportfolio gestartet. Die vom deutschen Wirtschaftsministerium gemeinsam mit Wirtschaftsverbänden getragene Initiative „Gründerland Deutschland“ zielt unter anderem auf eine weitere Verbesserung der Finanzierungsmöglichkeiten für innovative und wachstumsintensive Gründungen durch Auflage eines 
„High-Tech Gründerfonds II“ sowie auf die Attraktivierung des Marktumfelds für Business Angels (BMWi 2010) ab.

Auch Frankreich mit einer FTI-politischen Tradition in der Förderung nationaler Champions („High-Tech Colbertism“) zeigt eine Reorientierung in Richtung Unterstützung von innovativen KMUs. Während einige einschlägige Maßnahmen bereits seit längerem bestanden, markiert das 1999 beschlossene Gesetz zur Innovations- und Forschungsförderung mit Maßnahmen zur Förderung von Seedkapital, dem Aufbau von Inkubatoren für innovationsintensive Gründungen sowie der Auflage von GründerInnen-Wettbewerben einen wichtigen Meilenstein in der Schwerpunktsetzung der französischen FTI-Politik auf die Förderung von Hochtechnologie-gründungen.

Wie auch in anderen Staaten ist die Implementierung von Instrumenten zur Förderung von Hochtechnologiegründungen in Israel eine Entwicklung der 1990er Jahre. Allerdings gab es mit BIRD (Binational Industrial Research and Development Foundation) bereits seit 1976 ein wichtiges Instrument, welches u.a. zur Finanzierung von jungen Hochtechnologieunternehmen diente. Dieser Fonds verfolgte den Zweck, US-amerikanische und israelische JointVentures zu finanzieren. Die Bedeutung dieses Fonds liegt vor allem darin, dass sich israelische Unternehmer durch die enge Zusammenarbeit mit amerikanischen Unternehmen Markt-, Management- und Technologiewissen aneignen konnten. 1992 waren $60 \%$ der an der New York Stock Exchange gelisteten israelischen Unternehmen von BIRD unterstützt worden; der entsprechende Wert für die NASDAQ liegt sogar bei 75 \%. In den frühen 1990er Jahren kamen dann die bis heute am häufigsten genannten innovationspolitischen Instrumente hinzu: Der öffentliche Venture Capital-Fonds Yozma (Gründung 1993) und das Inkubator Programm (Gründung 1991) zur Förderung von Hochtechnologiegründungen durch hochqualifizierte jüdische Einwanderer aus dem ehemaligen Ostblock.

\subsubsection{Innovationsökonomische Rahmenbedingungen der FTI-Politik}

Abbildung 53 gibt einen Überblick über die Innovationsintensität des Unternehmenssektors in den Vergleichsstaaten. Zunächst einmal zeigt sich für Österreich ein rasanter Wachstumsprozess in der F\&E-Quote des Unternehmenssektors (UF\&E-Quote) von knapp über 0,5 \% im Jahr 1990 auf beinahe $2 \%$ im Jahr 2009. 2002 war die österreichische UF\&E-Quote erstmals höher als die französische und erreichte um 2005 das deutsche Niveau. Interessanterweise ist die Entwicklung Dänemarks sehr ähnlich dem österreichischen Catching-up-Prozess; die beiden Kurven verlaufen annähernd parallel. Die beiden Ausreißer mit deutlich überdurchschnittlicher UF\&E-Quote in Abbildung 53 sind Bayern und Israel. Israel zeigt dabei ein seit der ersten Hälfte der 1990er Jahre einsetzendes dramatisches Wachstum, was letztlich dazu führte, dass Israel nunmehr die höchste F\&E- und die höchste UF\&E-Quote innerhalb der OECD aufweist; noch in der ersten Hälfte der 1990er Jahre lag die UF\&E-Quote niedriger als in Deutschland und Frankreich. Darüber hinaus ist auch der Anteil der unternehmerischen F\&E-Ausgaben an den gesamten israelischen F\&E-Aufwendungen mit 81 \% (2008) einer der höchsten in der OECD. Für Österreich beträgt der entsprechende Wert für 2008 46 \%; rechnet man auch noch die F\&E-Ausgaben ausländischer Unternehmen in Österreich hinzu, kommt man auf etwa $60 \%$. Bayern liegt als das neben Baden-Württemberg zweitinnovativste deutsche Bundesland deutlich unter dem Niveau von Israel, aber klar über dem von Österreich, Dänemark, Frankreich und Deutschland. Für Bayern zeigt sich ähnlich wie für Frankreich und 
Deutschland eine Stagnation im Wachstum der UF\&E-Quote seit den 1990er Jahren.

Abbildung 53: Ausgaben des Unternehmenssektors für F\&E in \% des BIP

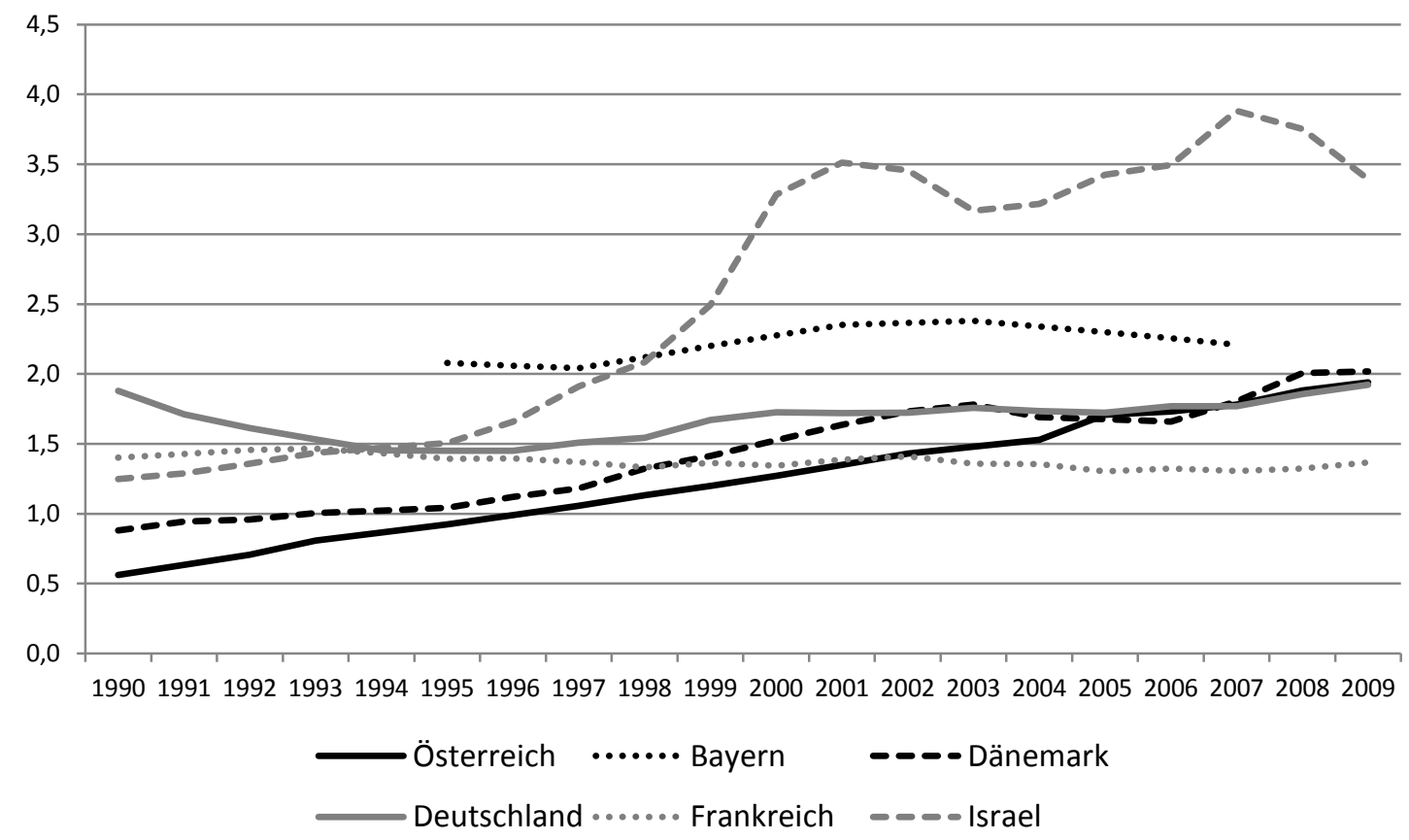

Quelle: OECD (MSTI), RWI 2009, eigene Darstellung

Das Niveau von F\&E-Aktivitäten des Unternehmenssektors kann neben einer allgemeinen Einschätzung des technologischen Niveaus einer Volkswirtschaft auch wichtige Hinweise auf das Potential an hochtechnologieorientiertem Entrepreneurship geben. Gemäß der „Knowledge Spillover Theory of Entrepreneurship“ (Acs et al. 2009) ist Entrepreneurship (u.a.) auf Wissen angewiesen, das durch bereits etablierte Firmen zwar produziert, aber dort nicht verwertet wird. Ceteris paribus erhöht ein F\&E-intensiver Unternehmenssektor auch die Wahrscheinlichkeit von Hochtechnologiegründungen.

Neben F\&E-Quoten kommt humankapitalbezogenen Variablen eine überragende Bedeutung im Innovationsprozess zu (OECD 2010, Leo et. al. 2006). Mit der Höherqualifizierung der Bevölkerung nimmt auch das Gründungspotential zu: AkademikerInnen weisen die höchste Gründungsneigung unter allen Bildungsgruppen auf, PflichtschulabsolventInnen die niedrigste (De 2005).

Abbildung 54 zeigt Österreich und die Vergleichsländer anhand zweier Humankapitalindikatoren: Bevölkerung (25-64) mit Tertiärabschluss in \% dieser Alterskohorte (Tertiärquote) sowie den Anteil natur- und ingenieurwissenschaftlicher Doktorate in \% aller Doktorate. Die Länder sind geordnet nach aufsteigender Tertiärquote. Hierbei zeigt sich das für Mitteleuropa typische elitäre Universitätsmodell anhand der niedrigen Tertiärquoten in Österreich, Bayern und im gesamten Deutschland. Israel liegt mit $44 \%$ an der Spitze, gefolgt von Dänemark mit einem Abstand von immerhin 10 Prozentpunkten. Interessant ist, dass Bayern als überdurchschnittlich innovationsintensives Bundesland keine höhere AkademikerInnenquote als Deutschland aufweist. ${ }^{47}$

\footnotetext{
47 Tatsächlich sind die Zahlen für die Vergleichsstaaten von 2008, jene für Bayern von 2007. Die Tertiärquote 2007 war in Bayern um einen Prozentpunk höher als jene vom gesamten Deutschland (RWI 2009).
} 
Für Hochtechnologiegründungen relevant sind insbesondere natur- und ingenieurwissenschaftliche Studienrichtungen und AbsolventInnen. Die rechten, hellen Säulen in Abbildung 54 zeigen den höchsten Anteil einschlägiger Doktorate für Frankreich, gefolgt von Israel und dann etwa mit gleichem Anteil Österreich und Dänemark. ${ }^{48}$ Für Bayern liegen keine vergleichbaren Zahlen vor, jedoch zeigen nationale Statistiken im Vergleich zu Deutschland einen überproportionalen Anteil an AbsolventInnen in den Studienrichtungen Biowissenschaften, Physik und Agrarwissenschaften sowie einen unterproportionalen Anteil in den Studienrichtungen Mathematik und Informatik sowie Ingenieurwissenschaften, Fertigung und Bauwesen. Baden-Württemberg weist bei allen 3 Studiengruppen überdurchschnittliche Werte auf - ein Hinweis auf deren innovationapolitische und ökonomische Relevanz. Zusammengenommen weist Österreich in der Innovationsintensität des Unternehmenssektors eine mittlere Position, beim Humankapital eine eher unterdurchschnittliche Performance auf.

Abbildung 54: Humankapitalausstattung im Vergleich

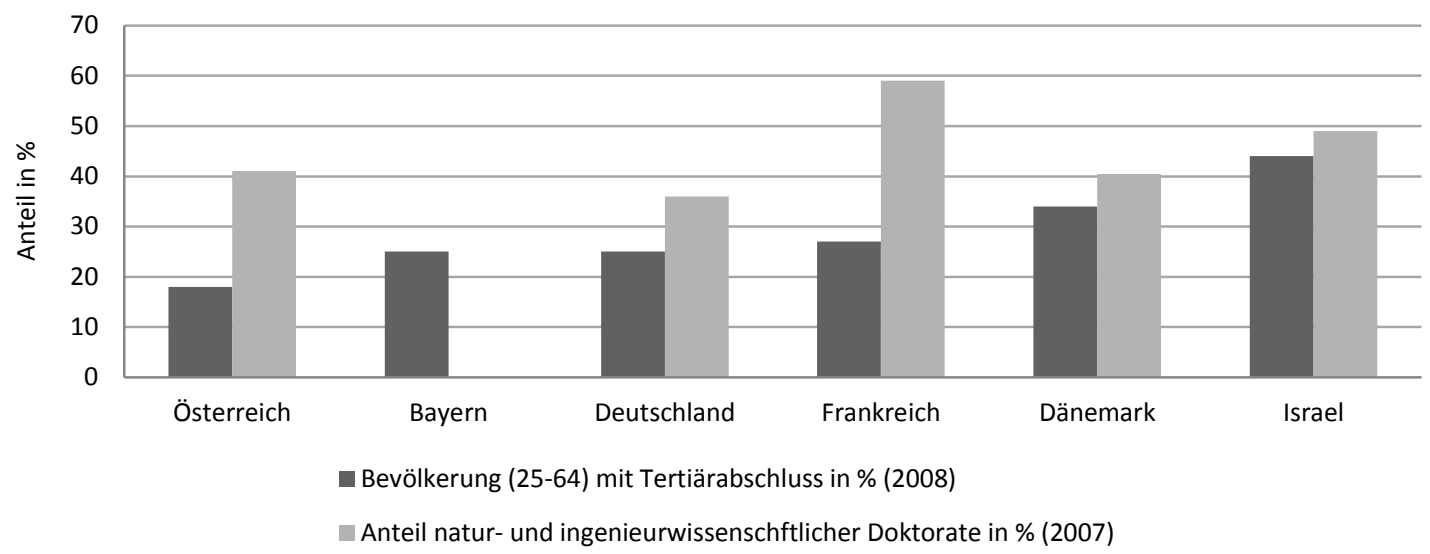

Quelle: OECD 2010, RWI 2009, eigene Darstellung

\subsubsection{Venture Capital-Investitionen und Gründungsperformance}

In diesem Unterkapitel soll die Gründungsdynamik skizziert sowie Niveau und Entwicklung von Venture Capital-Investitionen in den Vergleichsstaaten dargestellt werden.

Generell ist die Datenlage für einen Vergleich von Gründungsaktivitäten eher schlecht. Vertrauenswürdige vergleichbare Datensätze zu Hochtechnologiegründungen sind so gut wie nicht verfügbar. Hölzl (2006, S.14) referiert in einer Teilstudie zum WIFO-Weißbuch eine Reihe von Studien und Datenquellen und kommt zu folgendem, differenzierten Ergebnis: „Die verfügbare Evidenz legt insgesamt nahe, dass Österreich bei Gründungsaktivitäten ungefähr auf dem gleichen Niveau vergleichbarer Länder liegt, jedoch allenfalls im Mittelfeld. Diese Struktur der Gründungen legt allerdings nahe, dass viele der Gründungen eher traditionelle denn innovative Gründungen sind." Anhand von Tabelle 43 wurde trotzdem der Versuch unternommen, Österreich mit den Vergleichsstaaten anhand einiger traditioneller, aber auch neuerer Indikatoren hinsichtlich Gründerperformance bzw. deren politisch gestaltete Umwelt darzustellen. Da keine vergleichbaren Daten für Bayern verfügbar sind, wird die bayrische Gründungsperformance separat diskutiert.

\footnotetext{
${ }^{48}$ Nach anderen Indikatoren zur Messung der Bedeutung natur- und ingenieurwissenschaftlichen Humankapitals liegt Österreich signifikant schlechter als Dänemark oder auch Deutschland (Österreichischer Forschungs- und Technologiebericht 2010, Leo et.al. 2006).
} 
Tabelle 43 zeigt Dänemark als eines der attraktivsten Länder für Gründungen. Dänemark hat unter den Vergleichsstaaten die niedrigsten Barrieren für unternehmerische Tätigkeit, den Rang eins beim GEDI-Index (Kasten 2) sowie den höchsten Anteil an Venture CapitalFinanzierung am BIP. 2008 hatte Dänemark den höchsten Anteil an Venture Capital am BIP unter allen OECD-Staaten (OECD 2009). Allerdings ist für den Vergleich der fehlende Wert für Israel zu beachten (siehe Kasten 1). Damit ist Dänemark ein Beispiel dafür, wie auch in einem bankbasiertem Kapitalmarktsystem hohe Venture Capital-Investitionen möglich sind, jedoch ist auch der öffentliche Anteil überdurchschnittlich hoch. Die Beurteilung der Gründungsintensität Dänemarks variiert je nach Indikator. Während der Global Entrepreneurship Monitor auf eine eher mittelmäßige Gründungsperformance hinweist, zeigen andere Indikatoren Dänemark als eines der gründungsaktivsten Länder der OECD. Generell ist aber eine isolierte Betrachtung der Gründungsintensität unabhängig vom innovationssystemischen und makro-ökonomischen Kontext problematisch. So weist etwa Dänemark durch niedrige Arbeitslosenquoten und hohe Lohnersatzraten im Fall der Arbeitslosigkeit strukturell auf niedrige Gründungen aus ökonomischer Not heraus hin; das Gegenteil gilt für Deutschland.

Österreich liegt bei den Indikatoren in Tabelle 43 relativ weit zurück: Bei den beiden Weltbankindikatoren ist es insbesondere beim Indikator „Starting a Business“ abgeschlagen, was auf relativ hohe Gründungskosten verweist; beim GEDI liegt Österreich am letzten Platz unter den Vergleichsstaaten und auch der Venture Capital-Anteil ist hinter Deutschland der niedrigste. Betrachtet man den unmittelbar das Gründungsniveau betreffenden TAE-Index, so liegt Österreich jedenfalls für den Wert von 2005 durchaus gleichauf mit den anderen Staaten. Der starke Abfall für 2007 deutet auf ein erratisches Verhalten dieser Variable hin. Erstaunlicherweise ist Israel bei keinem der Indikatoren, abgesehen vom TAE-Index, in den Spitzenrängen platziert.

Tabelle 43: Gründungsperformance und Venture Capital-Investment

\begin{tabular}{lccccc}
\hline & Österreich & Dänemark & Deutschland & Frankreich & Israel \\
\hline $\begin{array}{l}\text { Ease of doing business (2010), Rang } \\
\text { Starting a Business (2010), Rang }\end{array}$ & 32 & 6 & 22 & 26 & 29 \\
& 125 & 27 & 88 & 21 & 36 \\
$\begin{array}{l}\text { Early Stage Entrepreneurial Activity (TAE) } \\
\text { in } \%^{2}\end{array}$ & $2,4(2007)$ & $3,6(2009)$ & $4,1(2009)$ & $4,3(2009)$ & $(2009)$ \\
Global Entrepreneurship Index (GEDI), Rang & $5,3(2005)$ & $5,4(2007)$ & $4,2(2006)$ & $3,2(2007)$ & 5,4 \\
$(2010)$ & & & & $16007)$ & 21 \\
$\begin{array}{l}\text { Venture Capital Investment in \% des BIP } \\
(2008)^{3}\end{array}$ & 0,036 & 0,298 & 0,048 & 0,091 & k.A. \\
\hline
\end{tabular}

Quelle: Worldbank, Global Entrepreneurship Monitor verschieden er Jahrgänge, OECD 2009, Acs/ Szerb 2010

Anmerkungen. 1: Der von der Weltbank erstellte, auf Umfragen basierende Ease of Doing Business-Index reiht 183 Staaten gemäß ihrer Performance in 9 Teilbereichen. Einer davon ist „Starting a Business“. Es gilt: Je niedriger der Rank desto besser die Position in der Rangliste. 2: Der TEA-Index ist das weit verbreitetste Maß des Global Entrepreneurship Monitors. Es misst das Niveau an früher unternehmerischer Aktivität anhand des Anteils der „Nascent Entrepreneurs“ (Gründungsaktivitäten vor der Unternehmensgründung) und der „Owner Manager of new Businesses“ (new bedeutet jünger als 3,5 Jahre) an der Bevölkerung zwischen 18-64. Die Verwendung unterschiedlicher Jahreswerte ergibt sich aus der Tatsache, dass nicht jedes Land jedes Jahr an der Umfrage des Global Entrepreneurship Monitors teilnimmt. 3: Beim GEDI werden 71 Staaten nach ihrem Rang gereiht. Für weitere Informationen siehe Kasten 2. 


\section{Kasten 2: Der Global Entrepreneurship and Development Index (GEDI) als neues Maß zur} Erfassung der Qualität von Entrepreneurship

Die Mehrzahl der traditionell verwendeten Indikatoren, wie z.B. der TEA-Index, geben vor allem quantitative Informationen zum Gründergeschehen. Diese Indikatoren geben damit aber gerade für eine an der Erhöhung der Qualität oder Technologieintensität der Gründungen interessierte FTI-Politik wenig hilfreiche Informationen. Typischerweise weisen Staaten wie Griechenland, Spanien oder Italien sehr hohe Werte bei quantitativen Gründerindikatoren auf. Jedoch ist davon auszugehen, dass diese Gründungen eine geringere durchschnittliche Qualität haben als etwa jene in Bayern oder Dänemark. Dies als Ausgangspunkt nehmend, konstruierte Zoltan Acs, Leiter des „Center for Entrepreneurship and Public Policy“ der George Mason Universität, USA, gemeinsam mit KollegInnen einen Index, der versucht, qualitative Aspekte der Gründungsdynamik zu berücksichtigen.

Der daraus entstandene GEDI-Index wurde 2010 erstmals publiziert. Er setzt sich aus drei Subindizes, die wiederum aus insgesamt 31 Variablen bestehen, zusammen und berücksichtigt mögliche Flaschenhalseffekte im Gründergeschehen durch Algorithmen, die einen Abschlag für schwache Performance in Teilbereichen der Indizes vorsehen. Die drei Teilindizes sind folgende:

\section{Entrepreneurial Attitude}

Dieser Subindex misst die Einstellung zu Entrepreneurship anhand von Variablen wie „Opportunity Perception“, Startup Skills“, „No Fear of Failure“ oder „Networking“.

\section{Entrepreneurial Activity}

Hier geht es um die Erfassung der tatsächlichen Aktivitäten. Um die Qualität der Aktivitäten zu berücksichtigen, werden insbesondere die Zugehörigkeit der Gründung zu technologieintensiven Sektoren und das Ausbildungsniveau der GründerInnen erfasst. Weiterhin werden hier auch institutionelle Variablen, etwa aus den „Doing Business“-Berichten der Weltbank berücksichtigt.

\section{Entrepreneurial Aspiration}

Fragen nach der unternehmerischen Strategie und den Ambitionen stehen hier im Zentrum des Interesses. Neben der Neuheit des Produkts und der Technologie gehen auch Internationalisierungsstrategien oder Kapitalverfügbarkeit als Variablen in die Berechnung des Index ein.

Zusammenfassend ist festzustellen, dass dieser neue Index eine interessante Weiterentwicklung in der Entrepreneurship-Forschung darstellt und der Politik mögliche Hinweise zur strategischen Ausrichtung geben kann. Trotzdem ist auch hier Vorsicht geboten: So schneidet Österreich etwa beim Indikator „Venture Capital“ im GEDI 2010 besser als Israel ab. (Vgl. dazu Acs/ Szerb 2010, sowie die Homepage des Projekts GEDI unter http://www.thegedi.org/.)

Wie stellt sich die Gründungsperformance in Bayern dar? Generell kann festgestellt werden, dass insbesondere die Region München sowie zunehmend auch der Raum Nürnberg eine dominante Position im deutschen Hochtechnologie-Gründergeschehen einnehmen. Im Gegensatz dazu fällt das - gemessen an der Forschungsquote - technologieintensivste Bundesland Baden-Württemberg bzw. die Region Stuttgart zunehmend zurück. München gilt als das Zentrum der deutschen Venture Capital-Szene. Der bei Venture Capital deutlich ausgeprägte „Home-Bias“ sowie eine hohe Nachfrage seitens innovativer GründerInnen führen dazu, dass München vor allem auch bei Early-Stage Investitionen die führende deutsche Region ist 
(Geyer/ Heimer 2010). Zwischen 2005 und 2007 hatte Bayern die höchste Dichte an Hochtechnologiegründungen unter den deutschen Bundesländern.

Abbildung 55 und Abbildung 56 zeigen die zeitliche Entwicklung des Anteils der Venture Capital-Finanzierungen am BIP von 1997-2009, differenziert nach Gründungsphase und Expansionsphase. Diese beiden Phasen zusammen machen nur einen kleinen Anteil am gesamten Venture Capital-Investitionsvolumen aus, sind jedoch, gerade was die Legitimation politischer Intervention aufgrund eines durch asymmetrische Information verursachten Marktversagens am Kapitalmarkt für innovative Unternehmensgründungen betrifft, von besonderer Bedeutung. Generell zeigt diese Abbildung das bekannte Bild einer niedrigen Venture Capital-Intensität für Österreich. Auch das Wachstum derselben ist gerade in der Gründungsphasenfinanzierung für die letzten 12 Jahre beinahe Null. Weiterhin zeigt sich, dass in der Gründungsphase eine größere Lücke relativ zu den anderen Staaten besteht als in der Expansionsphase. Für letztere ist auch ein Wachstumstrend erkennbar. Interessant ist auch, dass Dänemark bis 1999/2000 keineswegs eine bessere Performance als Österreich aufzuweisen hatte, dann jedoch deutlich stärkere Zuwächse erzielen konnte. Während Dänemark bei der Gründungsphase die beste Performance aufweist, ist Frankreich bei der Finanzierung der Expansionsphase erfolgreicher. Die gute Venture Capital-Performance Frankreichs ist unter anderem eine Folge der in Europa umfangreichsten steuerlichen Begünstigungen für Venture CapitalFinanzierungen. Damit wird aber auch deutlich, dass die Aussagekraft der undifferenzierten Venture Capital-Quote (Tabelle 43) nur ein unzureichendes Bild über die innovationspolitisch relevanten Frühphasen der Unternehmensgründung gibt: Während Dänemark für die Gesamtquote einen dreimal höheren Wert als Frankreich aufweist, hat letzteres bei der Expansionsphase höhere Venture Capital-Investitionen als Dänemark. In der Gründungsphase hat Dänemark eine bessere Performance als Frankreich; in dieser Phase ist in Dänemark auch der Anteil öffentlichen Kapitals am Risikokapitalmarkt besonders hoch. Zusammenfassend zeigen die Abbildungen 6 und 7 vor allem in der frühen Gründungsphase ein deutliches Defizit Österreichs, auch gegenüber Deutschland. In der Expansionsphase ist der Venture Capital-Anteil annähernd gleich hoch wie in Deutschland. 
Abbildung 55: Venture Capital-Investments in der Gründungsphase in \% des BIP

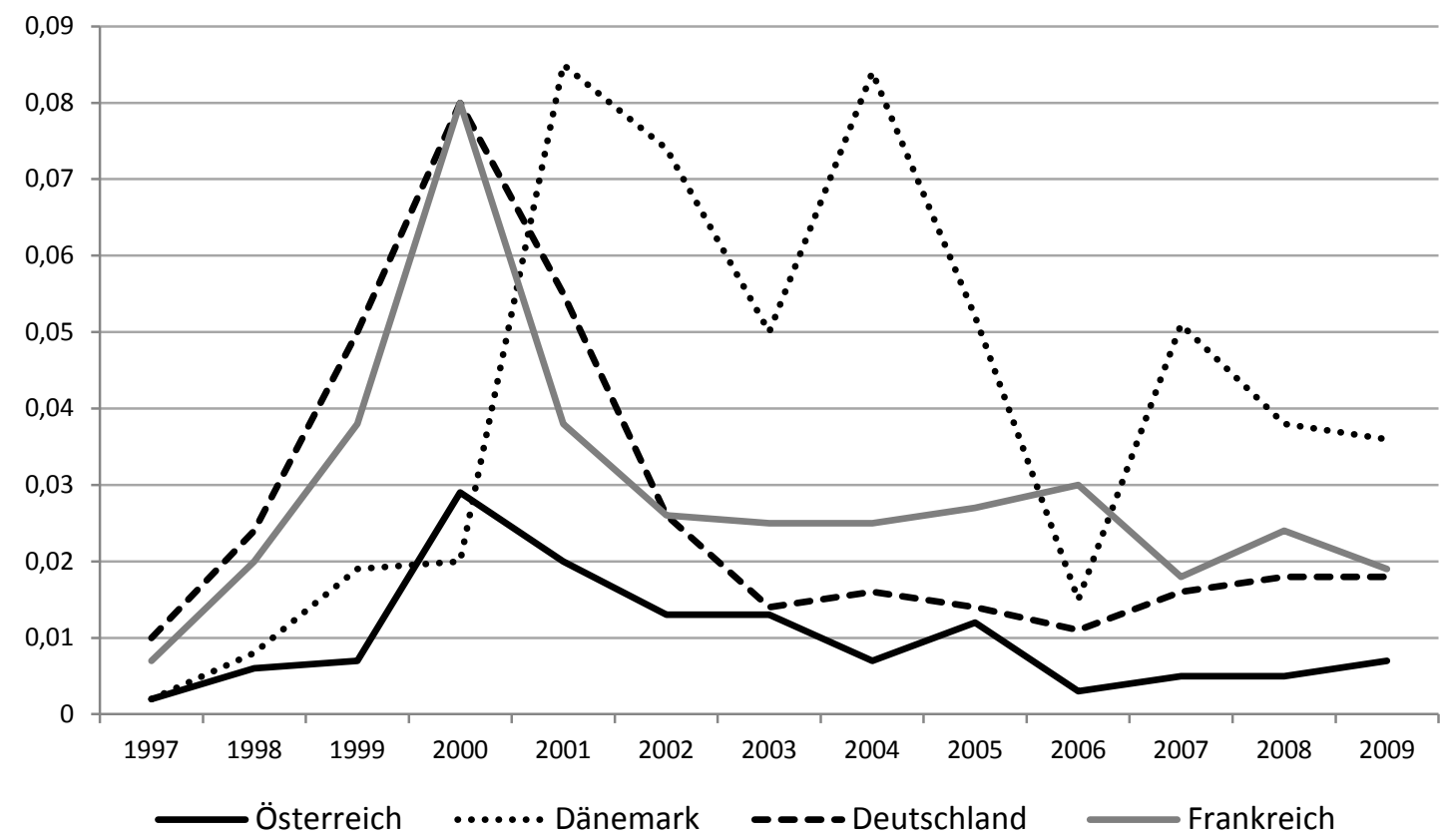

Quelle: Eurostat, eigene Darstellung

Abbildung 56: Venture Capital-Investments in der Expansionsphase in \% des BIP

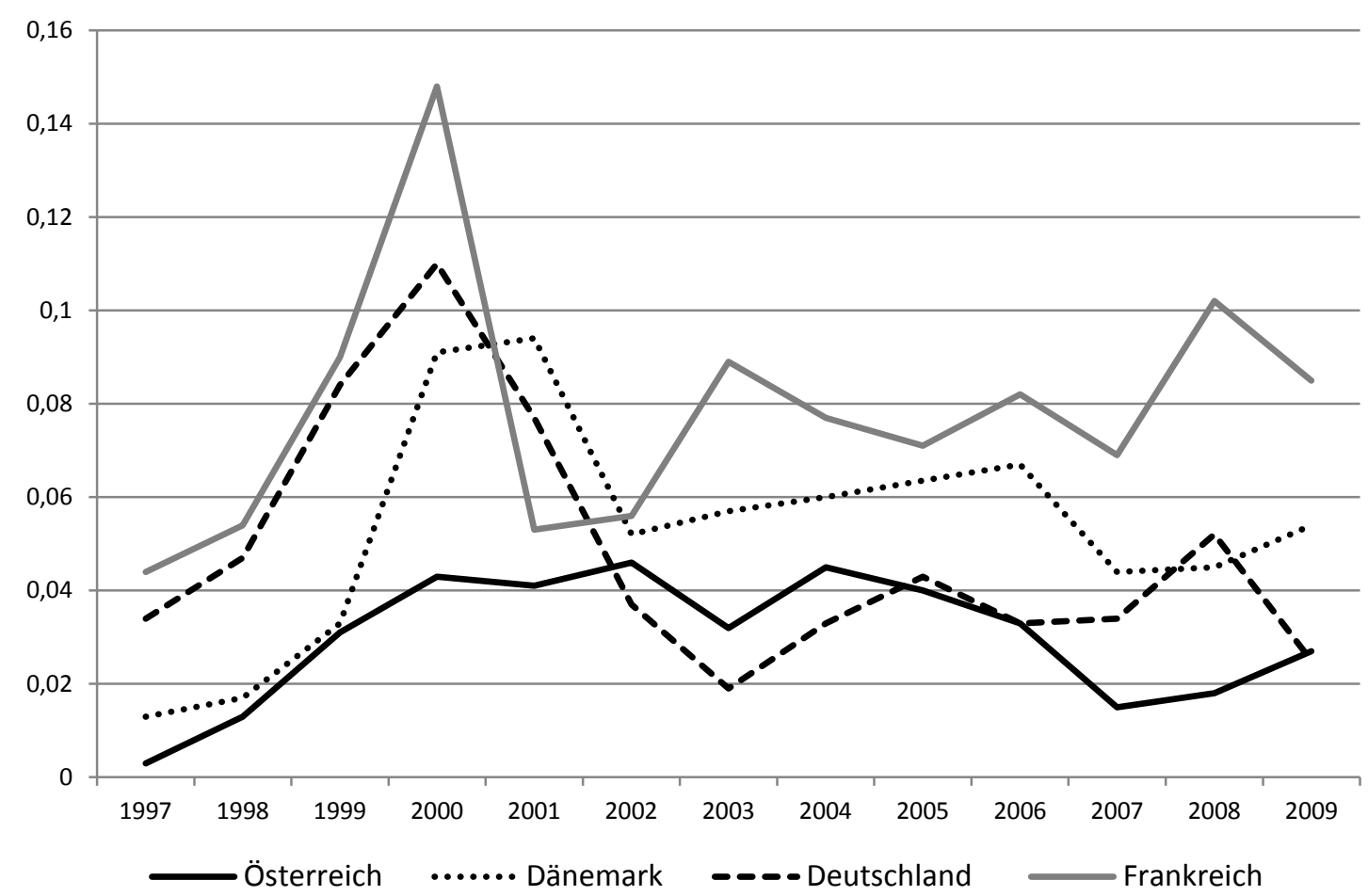

Quelle: Eurostat, eigene Darstellung

\subsubsection{Patentperformance und IPRs im Vergleich}

Neben den Programmen zur Förderung von Hochtechnologiegründungen werden in Modul 5 auch aws-Programme zur Nutzung des IPR-Systems mit internationalen Programmen vergli- 
chen. Wie alle Technologieprogramme des aws haben auch diese Programme KMUs als explizite Zielgruppe. Diese Orientierung ist nicht zuletzt auch deshalb von Bedeutung, weil KMUs im Gegensatz zu Großunternehmen signifikant eingeschränkte Möglichkeiten zum Schutz ihrer Innovation abseits des Patents haben, während letztere durch Produktionsanlagen, Markenname und Distributionsnetzwerk einen de facto-Schutz besitzen: „By protecting new entrants against incumbents [Herv.d.Verf.], patents can in such cases enable entry on innovative markets and thus boost dynamic competition" (OECD 2010, S.147). In diesem Kapitel sollen nachstehend die empirischen und regulatorischen Rahmenbedingungen skizziert werden.

Abbildung 57 stellt die Patentperformance der Vergleichsstaaten an Hand des Indikators Hochtechnologie-Patentanmeldungen beim Europäischen Patentamt dar. Wie zu erwarten hat Bayern über den gesamten Datenzeitraum die höchste Anzahl an Patentmeldungen. Die Uförmige Kurve dürfte primär durch die Dotcom-Blase um 2000 und den damit verbundenen Anstieg und Abfall an IKT-Patentanmeldungen erklärbar sein. Österreich liegt im Vergleich zu diesen Staaten an letzter Stelle. Dies könnte einerseits eine Folge des überwiegend mittleren Technologieniveaus der österreichischen Branchenstruktur und andererseits durch eine geringere Patentier-Neigung österreichischer Unternehmen verursacht sein. Allerdings zeigt sich, dass Österreich in den letzten zehn Jahren einen kontinuierlichen Wachstumsprozess durchlief, so dass im Jahr 2006 die Differenziale zu Dänemark und Deutschland egalisiert werden konnten.

Abbildung 57: Hochtechnologiepatentanmeldungen je Mio. EinwohnerInnen beim Europäischen Patentamt

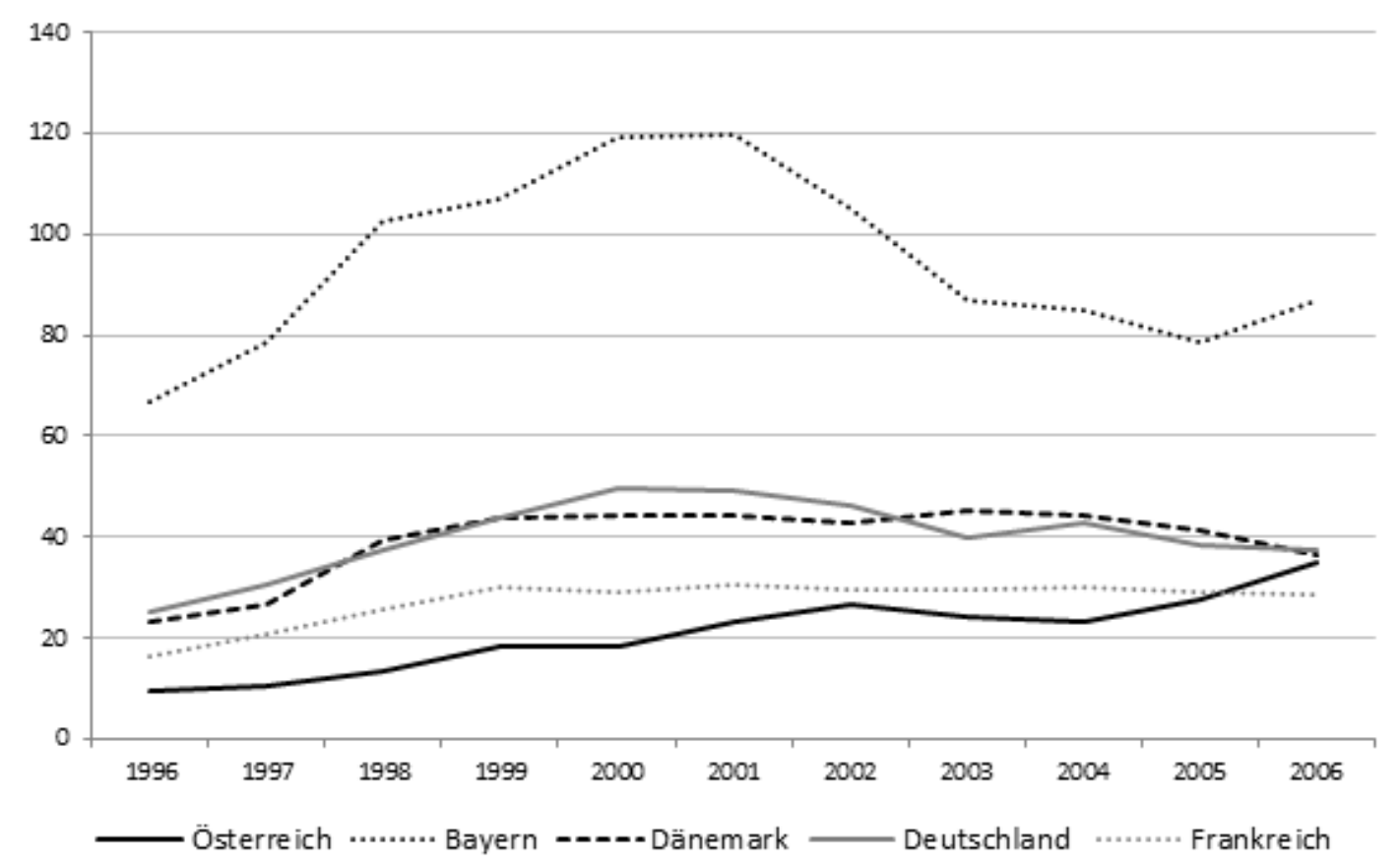

Daten: Eurostat, eigene Darstellung 
Die Entwicklung nationaler und internationaler Patentrechte ist in den letzten beiden Dekaden durch eine ständige Ausweitung und Verschärfung gekennzeichnet. Die Position Österreichs in Bezug auf patentrechtliche Rahmenbedingungen relativ zu den Vergleichsstaaten zeigt Abbildung 58 (siehe auch Janger 2009). Der Index der Patentrechte basiert auf Analysen einschlägiger nationaler Rechtsbestände und misst das durch Patente gewährte Schutzniveau, wobei 5 als Maximalwert das höchste Schutzniveau abbildet. Österreich weist hier unter den Vergleichsländern das höchste Schutzniveau auf, wobei die Unterschiede hier zwischen den Staaten eher gering sind. Der rechte, hellgraue Balken stammt aus Manager-Befragungen des World Competitiveness Report 2010-2011 und gibt die Antwort von Managern auf einer 7stufigen Skala auf die Frage: „How would you rate intellectual porperty protection, including anti-counterfeiting measures in your country? [1=very weak, 7=very strong] ", wieder. Auch hier ist die Position Österreichs ähnlich mit jener der Vergleichsstaaten; einzig Israel fällt hier deutlich zurück.

\section{Abbildung 58: Patentregime nach Gesetzeslage und ManagerInnen-Umfrage}

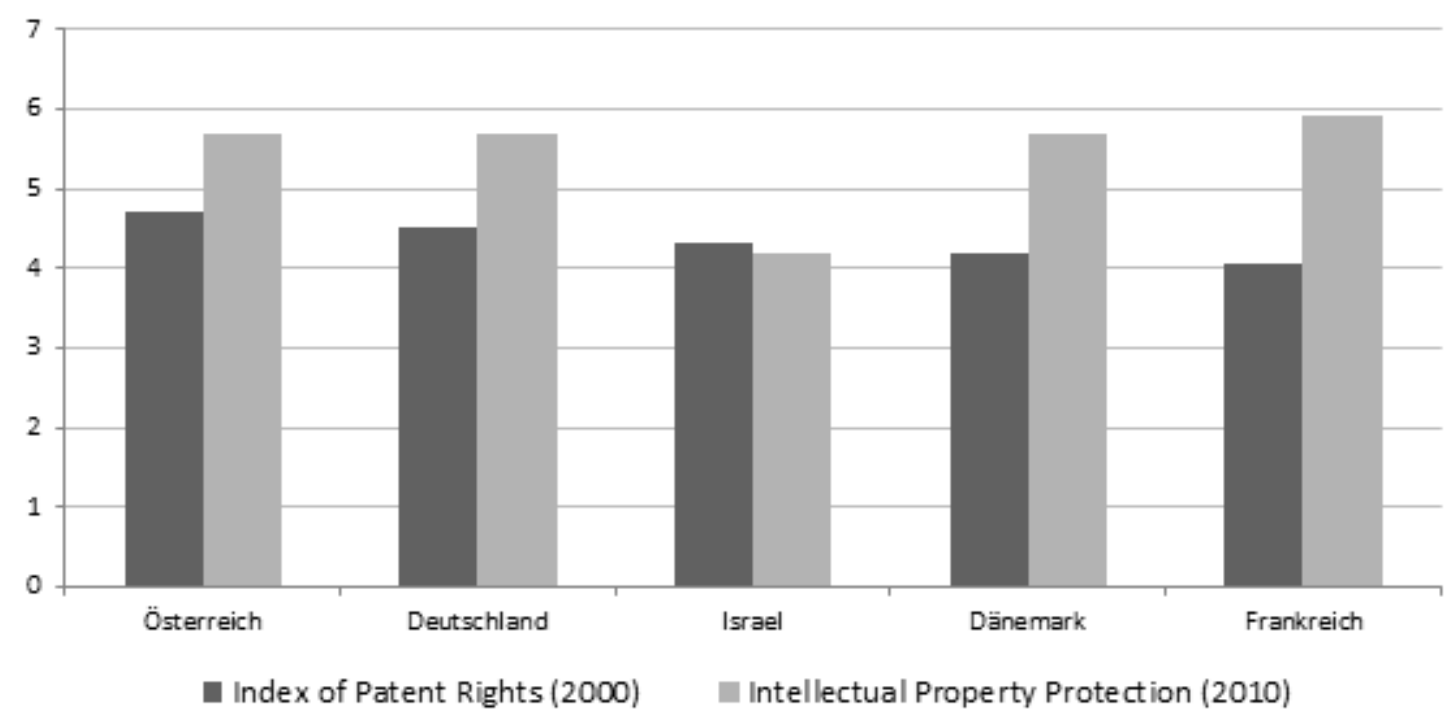

Quelle: Park/Wagh 2002, Global Competitiveness Report 2010-2011, eigene Darstellung

Zusammengefasst ergeben sich bei Patentperformance und Patentregime weit weniger deutliche Unterschiede zwischen Österreich und den Vergleichsländern als im Bereich Gründungsperformance und Venture Capital-Investitionsvolumen. Wesentliche Verbesserungen im Patentregime im Sinne einer Kostenreduktion der Patentierung werden vorrangig von der Einführung eines Europäischen Patents erwartet (Pottelsberghe 2010). Solange aber die Patentkosten in Europa signifikant über jenen der USA liegen, scheint eine politische Unterstützung von Patentangelegenheiten von KMUs nicht nur als wettbewerbspolitische Kompensation gegenüber Großunternehmen relevant, sondern auch als Reduktion des Kostennachteils relativ zu den USA. Die jüngste Entscheidung des EuGH (März 2011) hat jedoch die Europarechtswidrigkeit eines eigenständigen, neuen Europäischen Patentgerichtshofes festgestellt. Das dürfte die Einrichtung eines Europäischen Patents weiter erschweren. 


\subsection{INTERNATIONALE PROGRAMME ZUR FÖRDERUNG VON HOCHTECHNOLOGIEUNTERNEHMENSGRÜNDUNGEN}

Kapitel 1.2 diskutiert die in Tabelle 44 aufgelisteten innovationspolitischen Programme, vergleicht diese mit bestehenden aws-Programmen und versucht mögliche Elemente, die als Grundlage eines Policy Learning fungieren könnten, zu identifizieren. Dabei sollen die in Kapitel 1.1 dargestellten Rahmenbedingungen explizit berücksichtigt werden.

Tabelle 44: Vergleichsprogramme zur Förderung von Hochtechnologieunternehmensgründungen

\begin{tabular}{|c|c|c|}
\hline Staat/ Region & Institution & Programm/ Instrument \\
\hline \multirow{2}{*}{ Deutschland } & $\begin{array}{l}\text { Bundesministerium für Wirtschaft } \\
\text { und Technologie }\end{array}$ & High-Tech Gründerfonds \\
\hline & $\begin{array}{l}\text { Bundesministerium für Bildung } \\
\text { und Forschung }\end{array}$ & $\begin{array}{l}\text { Gründungsoffensive Biotechnolo- } \\
\text { gie (GO-Bio) }\end{array}$ \\
\hline \multirow{6}{*}{ Bayern } & \multirow{4}{*}{ Bayern Kapital } & Seedfonds \\
\hline & & Clusterfonds Start-Up! \\
\hline & & Clusterfonds Innovation \\
\hline & & Clusterfonds EFRE Bayern \\
\hline & \multirow{2}{*}{$\begin{array}{l}\text { Bayrisches Staatsministerium für } \\
\text { Wirtschaft, Infrastruktur, Verkehr } \\
\text { und Technologie }\end{array}$} & $\begin{array}{l}\text { Technologieorientierte Unterneh- } \\
\text { mensgründung (BayTOU) }\end{array}$ \\
\hline & & $\begin{array}{l}\text { Münchner Business Plan Wettbe- } \\
\text { werb }\end{array}$ \\
\hline Dänemark & $\begin{array}{l}\text { Ministry of Economic and Busi- } \\
\text { ness Affairs }\end{array}$ & $\begin{array}{l}\text { Vaekstfonden (The Danish In- } \\
\text { vestment Fund) }\end{array}$ \\
\hline \multirow{3}{*}{ Israel } & $\begin{array}{l}\text { Ministry of Industry, Trade and } \\
\text { Labor (Office of the Chief Scien- } \\
\text { tist) }\end{array}$ & $\begin{array}{l}\text { Technological Incubators Tnufa } \\
\text { Nofar }\end{array}$ \\
\hline & Ministry of Immigrant Absorption & 2B In Israel \\
\hline & $\begin{array}{l}\text { Israel Small and Medium Enter- } \\
\text { prise Authority }\end{array}$ & $\begin{array}{l}\text { Encourages immigrant entrepre- } \\
\text { neurship }\end{array}$ \\
\hline
\end{tabular}

\subsubsection{Darstellung der Programme}

\section{Deutschland}

\section{High-Tech Gründerfonds}

Der High-Tech Gründerfonds (HTGF) wurde 2005 vom Bundesministerium für Wirtschaft und Technologie unter Beteiligung der KfW-Bankengruppe sowie einiger Großunternehmen gegründet. Insgesamt wurde der Fonds mit 272 Mio. EUR dotiert, wobei sich der Unternehmensanteil auf 32 Mio. EUR beläuft. Hintergrund der Auflage dieses Fonds ist die Prozyklizität im Investitionsverhalten privater Venture Capital-Gesellschaften, die nach der DotcomBlase zum Einbruch des Venture Capital-Marktes geführt hat. Diesen Markt durch staatliches Beteiligungskapital wieder zu beleben war der wesentliche motivierende Faktor bei der Gründung des Fonds. Abbildung 59 zeigt die Entwicklung der Beteiligungen des HTGF und der gesamten Seedinvestitionsprojekte in Deutschland. Hier wird der Einbruch in der Phase nach 2000 ebenso deutlich, wie die bedeutsame Rolle des HTGF als Investor am Markt für Hoch- 
technologiegründungen. So wurden etwa $76 \%$ von den Seedinvestitionen im Jahr 2006 mit HTGF-Beteiligung getätigt.

Abbildung 59: Seedinvestitionen und Beteiligungen des HTGF

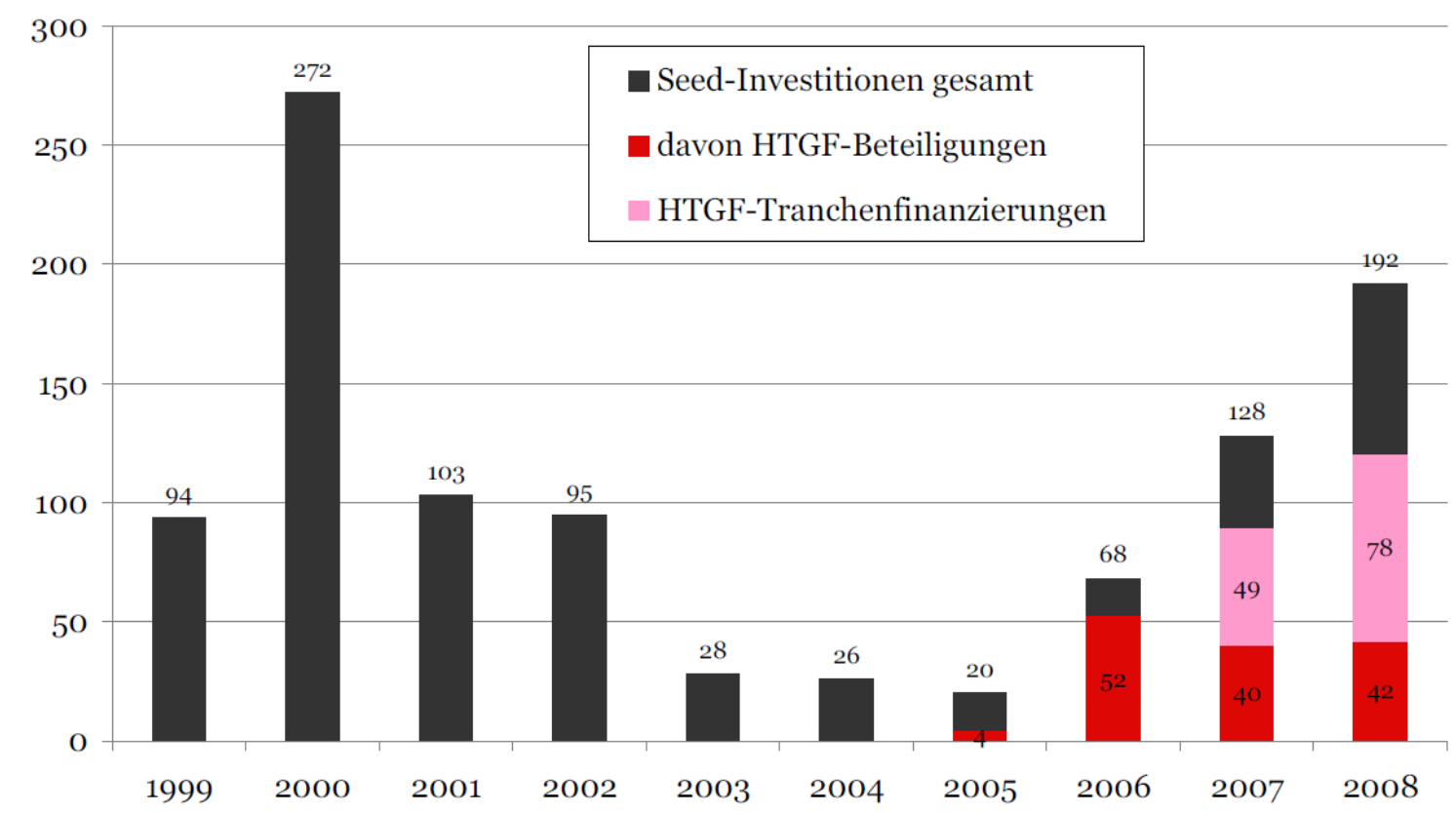

Quelle: Geyer/ Heimer 2010

Ziel des HTGF ist die Schließung der durch Marktversagen entstehenden Finanzierungslücke in der Seedphase im Hochtechnologiebereich, um damit GründerInnen die Finanzierung bis zum proof of concept zu ermöglichen. Die Zielgruppe umfasst junge und technologieintensive KMUs. Investitionsobjekte müssen dabei folgenden Kriterien entsprechen:

- Antragstellende Unternehmen dürfen nicht älter als 12 Monate sein.

- Die Gründung muss weniger als 50 MitarbeiterInnen oder weniger als 10 Mio. EUR Jahresumsatz bzw. Jahresbilanzsumme aufweisen.

- Unternehmensstandort ist Deutschland.

- Das Unternehmen weist eine hohe Technologieintensität mit einer innovativen Geschäftsidee auf.

- Die Spezialisierung der Antragsteller auf ein bestimmtes Technologiefeld ist kein Selektionskriterium. Eher im Gegenteil scheint das Programm bewusst offen gestaltet zu sein. Dass lässt sich jedenfalls aus der Liste der Coaches schließen, die den Unternehmen in der Antragsphase und Gründungsphase zur Verfügung stehen. Diese Liste beinhaltet Coaches für Biotechnologie bis Umwelttechnologie inklusive einer Kategorie „Sonstiges“. Aufgrund des angebotenen limitierten Finanzierungsvolumen ergibt sich jedoch ein de factoSelektionsmechanismen, der eine positive Diskriminierung von IKT-Gründungsprojekten und eine negative Diskriminierung von Gründungen in den Bereichen Energie-, Materialund Umwelttechnologie bewirkt. Die Erwartungen bezüglich Gründungszahlen in diesen letztgenannten Branchen sind beim Start des Fonds signifikant über den realisierten Ergebnissen gelegen. Es hat sich jedoch herausgestellt, dass das limitierte Finanzierungsvolumen für Gründungen in diesen Branchen zu gering, während es etwa für IKT-Projekte hinreichend ist. Durch die Substitutionsmöglichkeit zwischen Innovations- und Techno- 
logiegrad ist der High-Tech Gründerfonds aber gerade auch für Softwareunternehmen ein möglicher Investor.

Der Auswahlprozess umfasst ein mehrstufiges Verfahren; von der Gründung des Fonds bis 31.3.2009 führten 1.859 Anfragen zur Einleitung von 272 due diligence-Prüfungen und schließlich zu 181 Beteiligungsverträgen.

Die Förderleistungen setzen sich aus Beteiligungskapital und Beratungsleistungen durch einen Pool von ExpertInnen (Coachs) zusammen. Die Beteiligungsbedingungen erfolgen zu standardisierten Konditionen für Erst- und Folgefinanzierungen. Die Erstfinanzierung beinhaltet eine Beteiligung des HTGF bis zu 500 Tsd. EUR, wobei sich dieser Betrag aus einer Kombination von offener Beteiligung und Darlehen zusammensetzt. Die Laufzeit des endfälligen Darlehens beträgt sieben Jahre mit Stundung der Zinsen für die ersten Jahre und Fälligkeit im fünften Jahr. Um diese Finanzierung zu erhalten, muss das Unternehmen einen Eigenmittelanteil von $20 \%$ aufbringen, wobei die Hälfte davon durch private Seedinvestoren eingebracht werden kann. Eine Beteiligung des in der Gründungsphase beratenden Coachs wird begrüßt. Side-Investments sind sowohl durch öffentliche als auch private Seedinvestoren unter bestimmten Bedingungen möglich. Bei privaten Investoren bestehen Obergrenzen hinsichtlich Investitionsvolumen (max. 200 Tsd. EUR) und Anteil relativ zum Investitionsvolumen des HTGF (max. 50 \%). Folgefinanzierungen sind nach Erreichen von Milestones bis zu einer Gesamtsumme von 1 Mio. EUR inkl. Erstfinanzierung möglich. Diese Finanzierung setzt sich aus einer offenen Beteiligung und einem Nachrang-Darlehen mit Wandlungsoption in eine offene Beteiligung zwecks eines Schutzes vor Verwässerung des öffentlichen Investments zusammen.

Eine besondere Rolle kommt den Netzwerkpartnern des Programms zu. Diese umfassen Coachs, potentielle Manager und PersonalberaterInnen, Business Angels, Family Offices, nationale und internationale Venture Capital-Gesellschaften, Industrieunternehmen sowie ein Netzwerk der Portfoliounternehmen. Die regional agierenden Coachs haben dabei die wichtigste Rolle. Einerseits sollen sie dazu dienen, förderungswürdige Projekte zu identifizieren und die Awareness des HTGF zu heben, andererseits besteht ihre Rolle in der umfassenden Unterstützung der GründerInnen von der Antragsphase bis hin zur möglichen Beteiligung am Unternehmen. Die GründerInnen können sich dabei einen Coach aus einer Datenbank auswählen und müssen diesen auch finanzieren.

Eine Evaluierung durch Technopolis von 2010 kommt zu folgenden ausgewählten Ergebnissen (Geyer/ Heimer 2010): Insgesamt ist die Arbeit des Fonds erfolgreich und positiv zu beurteilen. Es gibt keine Hinweise auf Crowding-out von privatem Kapital. Die Rolle des LeadInvestors ermöglicht eine relativ konjunkturunabhängige Verfügbarkeit von Venture Capital in der Frühphase von Unternehmensgründungen. Der sektorale Schwerpunkt der Gründungen ist dominiert von IKT-Gründungen. Gründungen im Sachgüterbereich benötigen in der Regel wesentlich mehr Kapital als es der HTGF zur Verfügung stellt. Überlegt werden sollte nach Ansicht der EvaluatorInnen, eine Ausweitung der Folgefinanzierung auf 2 bis 3 Mio. EUR anzuheben, um auch komplexere Gründungen weiter entwickeln zu können. Weiterhin wird empfohlen, die Beschränkung der Höhe privater Side-Investments auf 200 Tsd. EUR aufzuheben. 


\section{Gründungsoffensive Biotechnologie (GO-Bio)}

Das Programm Go-Bio (GB) ist eine gründungsorientierte Fördermaßnahme im Bereich Life Sciences des Bundesministeriums für Bildung und Forschung. Für die operative Tätigkeit wurde der Projektträger Jülich beauftragt. Die Initiative ist als Wettbewerb konzipiert und wurde 2005 zum ersten Mal ausgeschrieben. Das Auswahlverfahren des Wettbewerbs basiert auf einem zweistufigem Verfahren, das etwa ein Jahr in Anspruch nimmt und daher neben einem hohen Aufwand zur Antragserstellung auch eine längere Phase der Unsicherheit für die Antragsteller mit sich bringt. Geplant sind insgesamt 5 Bewerbungsrunden, drei $(2005,2006$, 2008) wurden bislang realisiert, die vierte Runde wurde 2010 gestartet und dauert aktuell noch an. Für diese fünf Runden stehen max. 150 Mio. EUR bis zum Jahr 2015 zur Verfügung. In den ersten drei Runden wurden 62 Mio. EUR Fördermittel vergeben. Bei insgesamt 28 Förderfällen beläuft sich die Fördersumme pro Projekt damit auf ca. 2,2 Mio. EUR. Der Selektionsgrad der ersten drei Runden ergibt sich aus dem Verhältnis von 315 eingereichten Projektskizzen, daraus folgenden 62 Anträgen und schließlich 28 geförderten Projekten. D.h., von den ursprünglich eingereichten Skizzen erlangten ca. $9 \%$ eine Förderung. Von diesen $9 \%$ sind mehr als die Hälfte der Projekte im Bereich roter (medizinischer) Biotechnologie angesiedelt.

Interessanterweise nahm die Anzahl der eingereichten Skizzen von 176 (1. Runde) auf 54 (3. Runde) und die geförderten Projekte von 12 (1. Runde) auf 6 (3. Runde) ab. In der 4. Runde zeichnet sich jedoch ein neuerlicher Anstieg ab. Mit Stand Februar 2011 bestehen 14 Gründungen aus dem GB-Programm.

Das Ziel von GB besteht darin, Forschungsergebnisse derart weiterzuentwickeln, so dass sie im Anschluss daran ökonomisch verwertet und Basis einer Unternehmensgründung werden können. Durch diese Validisierungsförderung soll der Reifegrad der Forschung erhöht sowie die Marktfähigkeit gesteigert werden, um die Lücke zwischen Wissenschaft und Verwertung zu schließen. Zielgruppe sind in- und ausländische ForscherInnen- bzw. GründerInnenteams, die kommerziell Erfolg versprechende neue Forschungsansätze in den Lebenswissenschaften mit einer eigenständigen Arbeitsgruppe in Deutschland bearbeiten und einer kommerziellen Anwendung zuführen wollen. Diese Anwendung geschieht im Idealfall durch eine Unternehmensgründung. Antragsberechtigt sind Universitäten, Fachhochschulen und außeruniversitäre Forschungseinrichtungen.

In Ergänzung zu anderen Förderprogrammen zu Hochtechnologiegründungen adressiert GB die spezifischen Problemlagen in den Lebenswissenschaften (lange Entwicklungszeit, hoher Kapitalbedarf, hohes Risiko). Abbildung 60 zeigt die Einbettung von GB in den Innovationszyklus eines biotechnologischen Unternehmens. 
Abbildung 60: Die Position von Go-Bio im Biotechnologischen Innovationszyklus

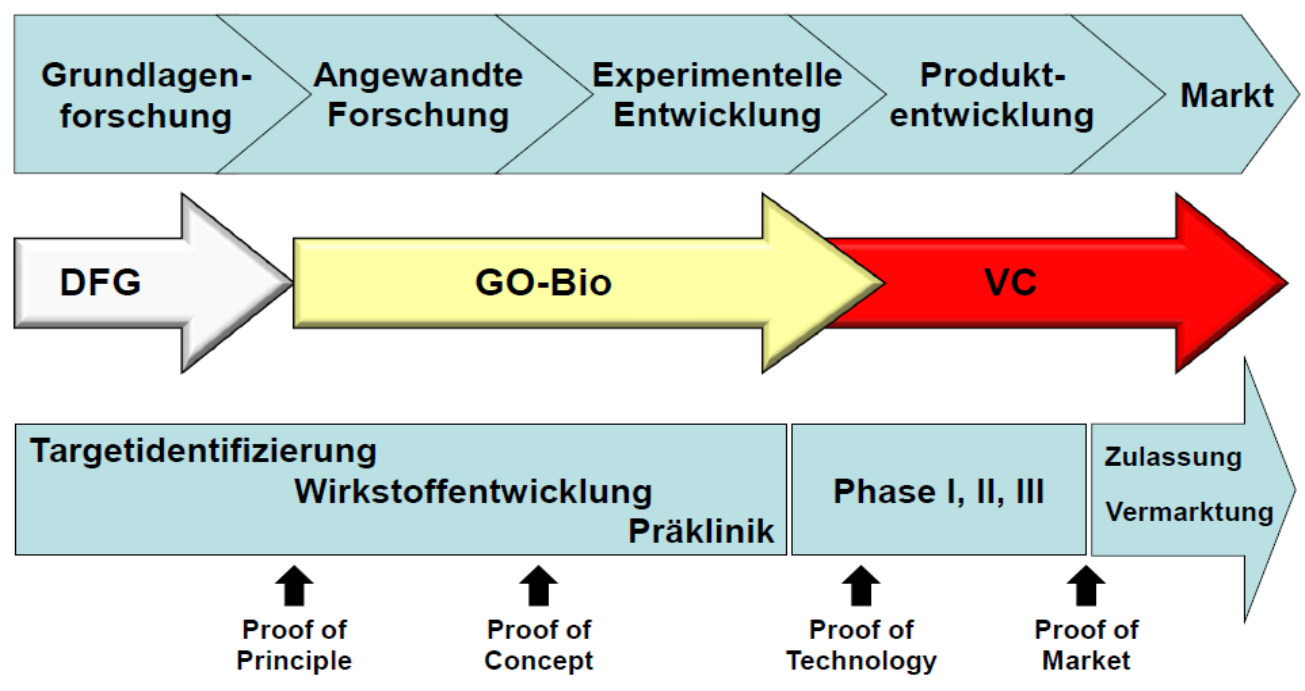

Quelle: http://www.go-bio.de/lw resource/datapool/GO-Bio Praesentation aktualisiert.pdf [15.03.2011]

Die Förderung erfolgt in zwei Phasen von jeweils maximal drei Jahren. In der ersten Phase wird vom GründerInnenteam ein proof of concept erarbeitet, wobei begleitend Kommerzialisierungsstrategien entwickelt werden sollen. Dies betrifft vor allem die Ausarbeitung eines Businessplanes und die Aufbringung des notwendigen Eigenanteils für eine mögliche zweite Förderphase. In dieser ersten Phase wird auch die Einbeziehung von Personen mit Wirtschaftserfahrung gefördert. Der Eigenanteil für die zweite Phase beläuft sich dabei auf mindestens $50 \%$ der entstehenden zuwendungsfähigen Kosten (BMBF 2010). Basis für die Erlangung einer Förderung im Rahmen der zweiten Förderphase sind erfolgreich absolvierte Zwischen- und Abschlussevaluierungen. Ziel der zweiten Förderphase ist der proof of technology sowie der proof of market. Insbesondere soll dadurch die notwendige Folgefinanzierung sichergestellt werden. Bislang wurde dieses Programm keiner externen Evaluierung unterzogen.

\section{Bayern}

\section{Bayern Kapital}

Die Beteiligungsgesellschaft Bayern Kapital (BK) wurde 1995 als 100 \%ige Tochtergesellschaft der LfA Förderbank Bayern, der Förderbank des Freistaates Bayern, gegründet. Die unmittelbare politische Motivation war durch die Initiative der Bayrischen Staatsregierung „Offensive Zukunft Bayern“ gegeben. Die Intention der Gründung war es, jungen technologieorientierten Unternehmen den Zugang zu Venture Capital zu erleichtern. BK betreut insgesamt sieben Beteiligungsfonds mit einem Gesamtvolumen von rd. 200 Mio. EUR, von denen bislang ca. 162 Mio. EUR in 195 Unternehmen investiert wurden. Durch die Kooperation mit anderen öffentlichen (insbes. High-Tech -Gründerfonds) und privaten Fonds wurden weitere 330 Mio. EUR an Lead- und Co-Investment-Finanzierung mobilisiert. Generell erfolgt das Investment von BK in Kooperation mit (Lead-)Investoren, die sich mindestens in gleicher Höhe wie BK beteiligen. Zu Beginn des Fonds war BK als reiner Co-Investor aktiv, allerdings kam es nach $2000 \mathrm{zu}$ einem Mangel an potentiellen Leadinvestoren. Als Reaktion darauf wurde das Coach-Konzept entwickelt (Abbildung 13); dabei beteiligt sich die BK in Koopera- 
tion mit einem Coach, der insbesondere betriebswirtschaftliches Wissen in das Unternehmen einbringt und dessen Investment explizit erwünscht ist (Abbildung 62).

Von den insgesamt sieben Fonds sind vier im Neugeschäft aktiv: (1) Seedfonds Bayern, (2) Clusterfonds Start-Up!, (3) Clusterfonds Innovation und (4) Clusterfonds EFRE. Wie Abbildung 61 zeigt, ergänzen sich diese Fonds entlang der Frühphasenentwicklung junger Technologieunternehmen. Im Folgenden werden diese Fonds kurz charakterisiert. Nach Auskunft von BK entsprachen 2005 die akkumulierten Rückflüsse aus den Beteiligungen in etwa dem Investitionsvolumen aus dem ersten Geschäftsjahr (40 Mio. EUR) zuzüglich der Managementkosten. Keiner der vier Fonds ist mit einem Selektionskriterium hinsichtlich der technologischen oder branchenspezifischen Orientierung der Antragsteller versehen. Ausgeschlossen sind lediglich Klein- und Kleinstunternehmen aus den Sektoren Landwirtschaft, Fischerei, Fischzucht, Kohle, Bergbau, Schiffbau und Stahl. Bemerkenswert ist der explizite Ausschluss von Vorhaben, welche heimische Erzeugnisse zu Lasten von Importerzeugnissen begünstigen. Damit wird die Effizienz des wichtigen Technologietransfer-Kanals via Importe von Gütern und Faktoren befördert.

Abbildung 61: Die Finanzierungsfunktion der Fonds der Bayern Kapital im UnternehmensLebenszyklus

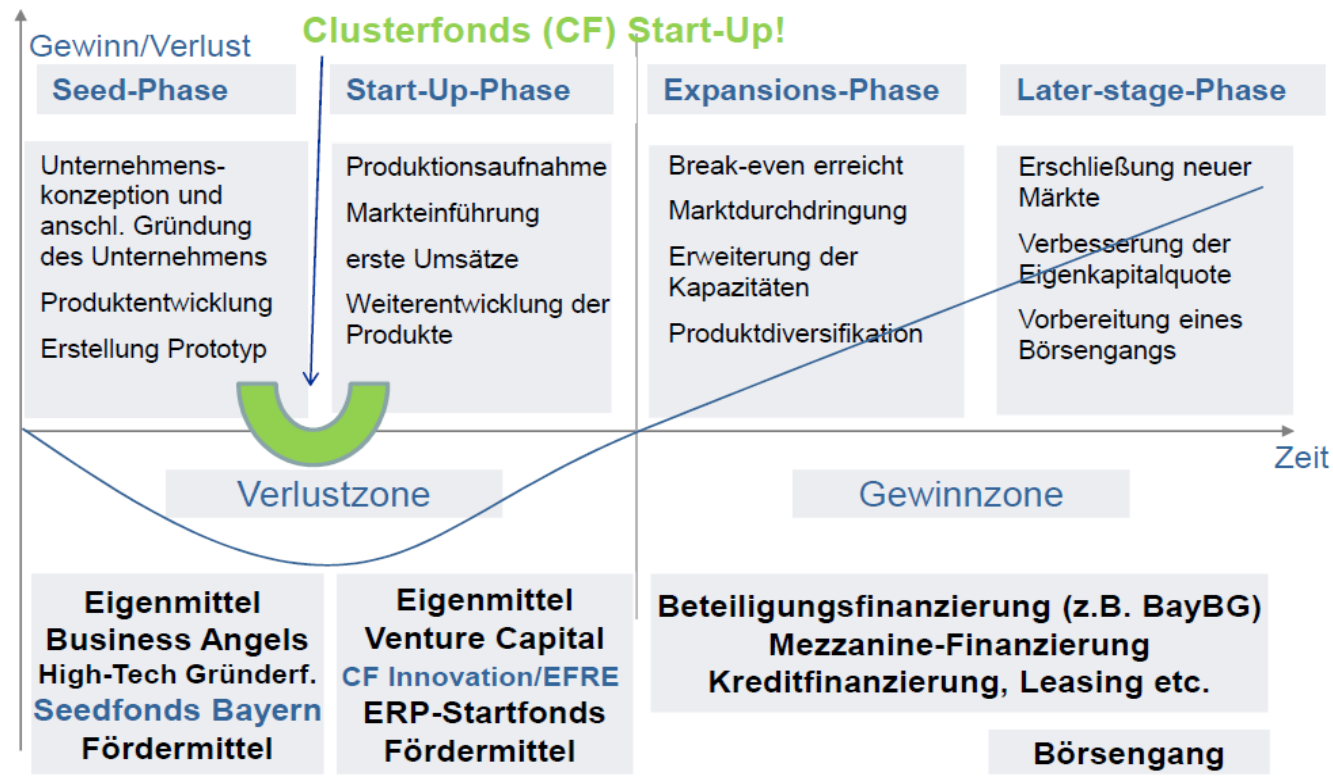

Quelle: http://www.bayernkapital.de/user/eesy.de/bayernkapital.de/dwn/1 Pr sentation k Jan 2011.pdf [16.03.2010

Der Seedfonds Bayern, auch als Clusterfonds Seed bezeichnet, finanziert junge, technologieorientierte Unternehmen grundsätzlich aller Branchen mit dem Ziel, diesen ein F\&EVorhaben bis zum proof of concept zu ermöglichen. Dies soll bei den Unternehmen die Voraussetzung für weitere Finanzierungsrunden schaffen. Der Seedfonds bleibt Minderheitsgesellschafter und strebt keine Beteiligung am Management an. Die Beteiligung erfolgt in Kooperation mit einem Coach (Abbildung 62).

BK strebt mit dem Seedfonds grundsätzlich eine gemeinsame Finanzierung mit dem HighTech Gründerfonds an, wobei auch eine Alleinfinanzierung möglich ist. Im ersten Fall investiert der Seedfonds max. 200.000 EUR als Kombination aus offener Beteiligung und Nachrangdarlehen, wobei der Gründergesellschafter einen Eigenmittelbetrag von 80.000 EUR leisten muss, der bis zu $50 \%$ von Seedinvestoren oder Coach finanziert werden darf. Der High- 
Tech Gründerfonds investiert max. 400 Tsd. EUR, womit sich das Gesamtvolumen an verfügbarer öffentlicher Venture Capital-Finanzierung auf 600 Tsd. EUR beläuft. Im relativ seltenen, zweiten Fall investiert der Seedfonds max. 250.000 EUR in Form einer typisch stillen Beteiligung und der GründerInnen muss einen Eigenbetrag von min. 25.000 EUR aufbringen.

Abbildung 62: Die Rolle des Coach im Programm Seedfonds Bayern

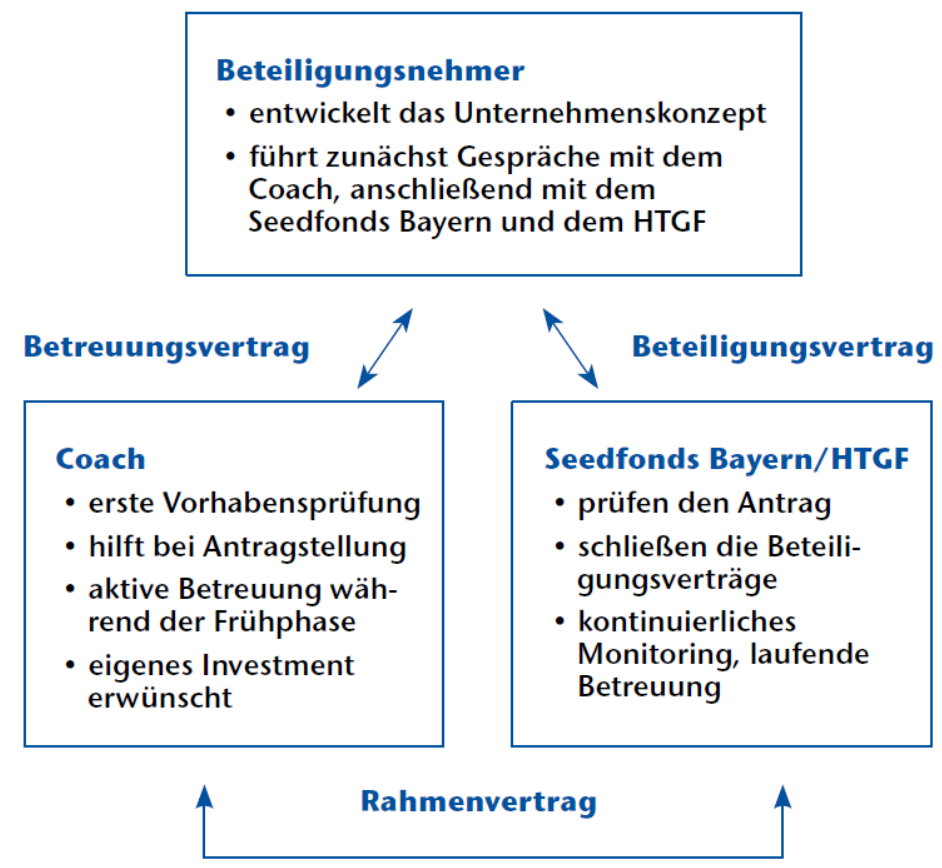

Quelle: http://www.bayernkapital.de/user/eesy.de/bayernkapital.de/dwn/BayernKapital_Seedfonds.pdf [3.3.2011]

Das Ziel des Clusterfonds Start-Up ist die Schließung der Lücke zwischen Seed- und Startup-Phase. Generell ist dieser Fonds als eine residuale Option für jene Unternehmen gedacht, die erfolgreich die Seed-Phase absolviert und den proof of technics erreicht haben, aber durch unerwartete Umstände keine private Folgefinanzierung erreichen konnten. Mit dem Clusterfonds Start-Up können diese Unternehmen eine Finanzierung zur Erreichung des proof of markets erlangen. Finanziert wird dabei der Aufbau des Unternehmens, Aufwendungen für Verfahrens- und Produktentwicklung inkl. Patentanmeldungen, Aufwendungen zum Markteintritt sowie Gebühren des Coachs. Ebenso wie beim Seedfonds strebt der Clusterfonds Start-Up die Position eines Minderheitsgesellschafters ohne Managementbeteiligung an, wobei die Beteiligung in Kooperation mit einem Coach erfolgt. Die max. 500.000 EUR betragende Beteiligung erfolgt vorrangig in Form einer offenen Beteiligung in Kombination mit einem Nachrangdarlehen. Dabei muss ein Finanzierungsanteil von 100.000 EUR durch GründerInnen und andere privaten Seedkapitalgeber gegeben sein.

Der Clusterfonds Innovation dient ebenso wie der Clusterfonds EFRE ${ }^{49}$ der Kofinanzierung von jungen innovativen KMUS grundsätzlich aller Branchen, die neue Produkte und/oder Produktionsverfahren einführen wollen. Der Clusterfonds Innovation beteiligt sich nur in Kooperation mit einem privaten Leadinvestor, der min. $50 \%$ der Beteiligung übernehmen muss. Die Beteiligung erfolgt regelhaft in Form einer offenen und/oder typisch stillen Beteiligung. Die Höhe der Beteiligung ist auf 2,0 Mio. EUR begrenzt, kann jedoch unter be-

\footnotetext{
${ }^{49}$ EFRE ist das Akronym für „Europäischer Fonds für Regionale Entwicklung““.
} 
stimmten Umständen auf bis zu 2,5 Mio. EUR ausgedehnt werden. Für die Beteiligungsformen werden marktübliche Konditionen verrechnet. Der wesentliche Unterschied zwischen dem Clusterfonds Innovation und dem Clusterfonds EFRE liegt in der Ausrichtung des EFRE-Fonds auf ländliche Gebiete. In sogenannten C-Fördergebieten (strukturschwachen Regionen) müssen private Leadinvestoren, die gemeinsam mit der BK investieren, anstatt $50 \%$ lediglich $30 \%$ Finanzierungsanteil übernehmen.

Es liegen keine öffentlich zugänglichen Evaluationsergebnisse vor.

\section{Bay-TOU - Technologieorientierte Unternehmensgründungen}

Das vom Bayrischen Staatsministerium für Wirtschaft, Infrastruktur, Verkehr und Technologie aufgelegte Programm Bay-TOU (BT) wurde 1995 begründet um den Strukturwandel Bayerns durch Unternehmensgründungen hin zum Hochtechnologiestandort zu unterstützen. Zielgruppe des Programms sind UnternehmensgründerInnen und junge Technologieunternehmen, die an einem innovativen, technologieintensiven Unternehmenskonzept, Produkt oder Verfahren arbeiten. Die Förderung kann in zwei Phasen des Unternehmenszyklus erfolgen: Während der Arbeit am technologischen Konzept einer Unternehmensgründung oder während der Durchführung eines Entwicklungsvorhabens. Bestehende Unternehmen dürfen nach den aktuellen Programmrichtlinien von 2009 nicht älter als 6 Jahre sein und max. 10 MitarbeiterInnen beschäftigen. Die Förderung erfolgt als Anteil-Finanzierung im Rahmen einer Projektfinanzierung. Der Fördersatz beträgt max. $30 \%$ der zuwendungsfähigen Kosten in der Konzept-Phase und max. $40 \%$ für Entwicklungsvorhaben. Hierbei kam es zu einer Veränderung der Programmrichtlinien von 2001, die folgende Grenzen vorgesehen hatten: Beantragende Unternehmen dürfen nicht älter als 3 Jahre sein und max. 5 MitarbeiterInnen beschäftigen sowie der EU-Definition von KMUs entsprechen. Damit wurde der Kreis der potentiellen Anspruchsberechtigten deutlich ausgeweitet.

Für technologieorientierte Aktivitäten in der Konzeptphase beträgt der Zuschuss max. 26.000 EUR, bei Softwareentwicklung max. 150.000 EUR. BT ist auf ein Zusammenwirken mit anderen Förderinstrumenten durch öffentliche Venture Capital-Geber sowie mit privaten Venture Capital-Gesellschaften hin ausgerichtet.

Von 1996 bis Ende 2009 wurden im Rahmen von BT 278 Projekte mit Zuschüssen in der Höhe von insgesamt 25 Mio. EUR gefördert. Damit beträgt der durchschnittliche jährliche Mitteleinsatz für diese Periode ca. 1,9 Mio. EUR; für 2011 sind 2,4 Mio. EUR veranschlagt. Nach Auskunft des Programmverantwortlichen im Ministerium ist BT das erfolgreichste Förderprogramm Bayerns in Bezug auf die Kennzahl aufgewendete Fördermittel pro mittelfristig geschaffenen Arbeitsplatz. Mittelfristig ist hier definiert als drei Jahre nach Vorhabensende. Bei mittlerer bis hoher Technologie beläuft sich dieses Verhältnis auf 17.000 EUR/Arbeitsplatz und im Bereich Hoch- und Spitzentechnologie auf 25.000 EUR/ Arbeitsplatz. Eine Evaluierung von BT wurde durchgeführt, ist jedoch nicht freigegeben.

\section{Münchner Business Plan Wettbewerb}

Der jährlich stattfindende Münchner Business Plan Wettbewerb (MBPW) wurde 1996 gegründet. Dabei wurde explizit an den ersten Businesswettbewerben am Massachusetts Institute of Technology (MIT) in den USA in den 1980er Jahren angeknüpft. 1996 wurde die Idee 
des BP-Wettbewerbs durch die Unternehmensberatung McKinsey in Deutschland popularisiert und zusammen mit drei Münchner Hochschulen, Unternehmen, Förderinstitutionen und weiteren Akteuren der MBPW initiiert.

Aufgrund der relativ langen Programmdauer konnte sich der MBPW mittlerweile als eine wichtige Plattformfunktion für die Gründung und Finanzierung innovativer Unternehmen in München bzw. Südbayern etabliert. Der MBPW ist eine Initiative von Wirtschaft, Hochschulen und Forschungseinrichtungen und wird durch das Bayrische Staatsministerium für Wirtschaft, Infrastruktur, Verkehr und Technologie gefördert. Die Finanzierung erfolgt durch öffentliche und private Akteure.

Die Zielgruppe umfasst sowohl Personen mit der Intention, ein Unternehmen zu gründen, als auch Unternehmen, die jünger als 5 Jahre sind. Der MBPW bietet neben dem mehrstufigen Wettbewerb auch Coaching sowie Unterstützung bei Investorensuche an. Das MBPW offeriert den TeilnehmerInnen die Möglichkeit, in einem dreistufigen Verfahren einen vollständigen Business- und Finanzierungsplan zu entwickeln. Die Stufen sind aufeinander aufbauend und das Anspruchsniveau nimmt von Stufe zu Stufe zu. Parallel dazu werden Seminare und Workshops angeboten, die das notwendige Wissen vermitteln.

Die Businesspläne werden auf jeder Stufe von min. zwei Unternehmer-JurorInnen begutachtet, wobei diese nicht nur Punkte vergeben sondern durch Kommentare den WettbewerbsteilnehmerInnen qualifizierte Rückmeldungen zum Projekt liefern. Die Businesspläne der Stufen 2 und 3 werden zusätzlich von Investoren beurteilt. Diesen wird die Frage gestellt, ob ein Kapitalgeber in dieses Unternehmen investieren würde oder nicht. Die Preisgelder steigen stufenweise von der ersten Runde (10 x 500 EUR) bis zum ersten Preis in der dritten Runde an (15.000 EUR). Generell ist der mögliche Austausch zwischen Gründerteams, Coachs und Juroren eine wichtige Motivation für die Teilnahme am Wettbewerb.

Pro Jahr nehmen etwa 250 Unternehmen an dem Wettbewerb teil, wobei ca. $50 \%$ aus dem IT-Sektor kommen, $25 \%$ dem Maschinenbau und der Elektrotechnik sowie weitere $10 \%$ den Life-Sciences zuzurechnen sind. Laut Homepage des MBPW wurden bislang ca. 600 Unternehmen mit Unterstützung des MBPW gegründet und finanziert. In diesen Unternehmen sind ca. 45.880 neue Arbeitsplätze entstanden.

\section{Dänemark}

\section{Vaekstfonden (The Danish Investment Fund) - Vaekstkapital}

Im Gegensatz zu anderen Venture Capital-Märkten Europas ist der dänische Markt durch einen hohen Anteil öffentlichen Kapitals, investiert durch Vaekstfonden (VF), und von Pensionsfonds gekennzeichnet. Da beide Akteure eine eher langfristige Investmentstrategie verfolgen, zeigt der dänische Markt relativ geringere Volatilitäten (Lindgaard et.al. 2008). Neben VF, der dem Ministry of Economic and Business Affairs zugeordnet ist, hat die dänische FTIPolitik mit den Innovation Incubators des Ministry of Science, Technology and Innovation eine zweite Förderinstitution zur Förderung des Venture Capital-Markts eingerichtet.

Vaekstfonden ist ein 1992 gegründeter staatlicher Investmentfonds mit einer Kapitalbasis von rund 300 Mio. EUR. Seit 1992 hat VF in Kooperation mit privaten Co-Investoren mehr als 4200 Unternehmen mit ca. 8,1 Mrd. DKK finanziert, was umgerechnet zu aktuellem Kurs (März 2011) etwa 1,1 Mrd. EUR entspricht. Im Wesentlichen besteht die Tätigkeit 
von VF im Angebot von Information, Eigenkapital, Darlehen und Garantien in Kooperation mit öffentlichen und privaten Institutionen. Diese Angebote sollen die Finanzierungskonditionen von „New Growth Companies“ verbessern.

Übergeordnetes Ziel von VF ist die Etablierung des attraktivsten Venture Capital-Marktes für Innovationsfinanzierung in Europa. Dazu werden drei Strategien verfolgt: Erstens soll VF durch Investitionen als Katalysator am Venture-Capital-Markt fungieren; zweitens soll durch dieses Engagement ein Signalling-Effekt erzielt werden, der potentiellen Markteilnehmern wie z.B. Pensionsfonds die Attraktivität von Venture-Capital-Investitionen demonstriert; drittens soll VF den Informationsstand der Marktteilnehmer erhöhen.

Ursprünglich finanzierte VF in Form von Soft Loans und Garantien. Das Kapital dazu stammte aus Erträgen aus der in Anleihen investierten Kapitalbasis. 2001 kam es zu einem radikalen Strategiewechsel hin zu eigenkapitalbasierten Finanzierungsmodellen. Wie Abbildung 14 zeigt umfasst die aktuelle Tätigkeit von VF eine Vielzahl von Venture CapitalFinanzierungsmodellen. Je nach Unternehmensmodell und Wachstumsperspektive kommen unterschiedliche Finanzierungsinstrumente zum Einsatz.

Die Finanzierung des Markteintritts von technologieintensiven, von Beginn an global orientierten Unternehmen („Global Companies“) benötigt hohe Kapitalsummen. VF beteiligt sich an diesen Unternehmen durch Funds in Funds-Investments sowie einen Public-Private Fonds (Sunstone Capital), der zu Marktkonditionen Kapital bereitstellt. „Internationale Companies“ (Abbildung 63) werden durch „Growth Capital“ unterstützt. Mit „Growth Capital“ investiert VF direkt in Kooperation mit anderen Investoren in Unternehmen. Dabei werden Eigenkapital oder Darlehn oder eine Mischung dieser Finanzierungsarten angeboten. Die Investments liegen zwischen 5 und 25 Mio. DKK, was zu aktuellem Referenzwechselkurs etwa 675.Tsd. und EUR 3,4 Mio. EUR entspricht. Für regional und lokal orientierte Unternehmen bestehen mit „Partner Capital“ und Kreditgarantien weitere, spezifische Finanzierungsangebote (siehe hierzu auch OECD 2008). 
Abbildung 63: Finanzierungsangebote von Vaekstfonden nach Unternehmenstypus

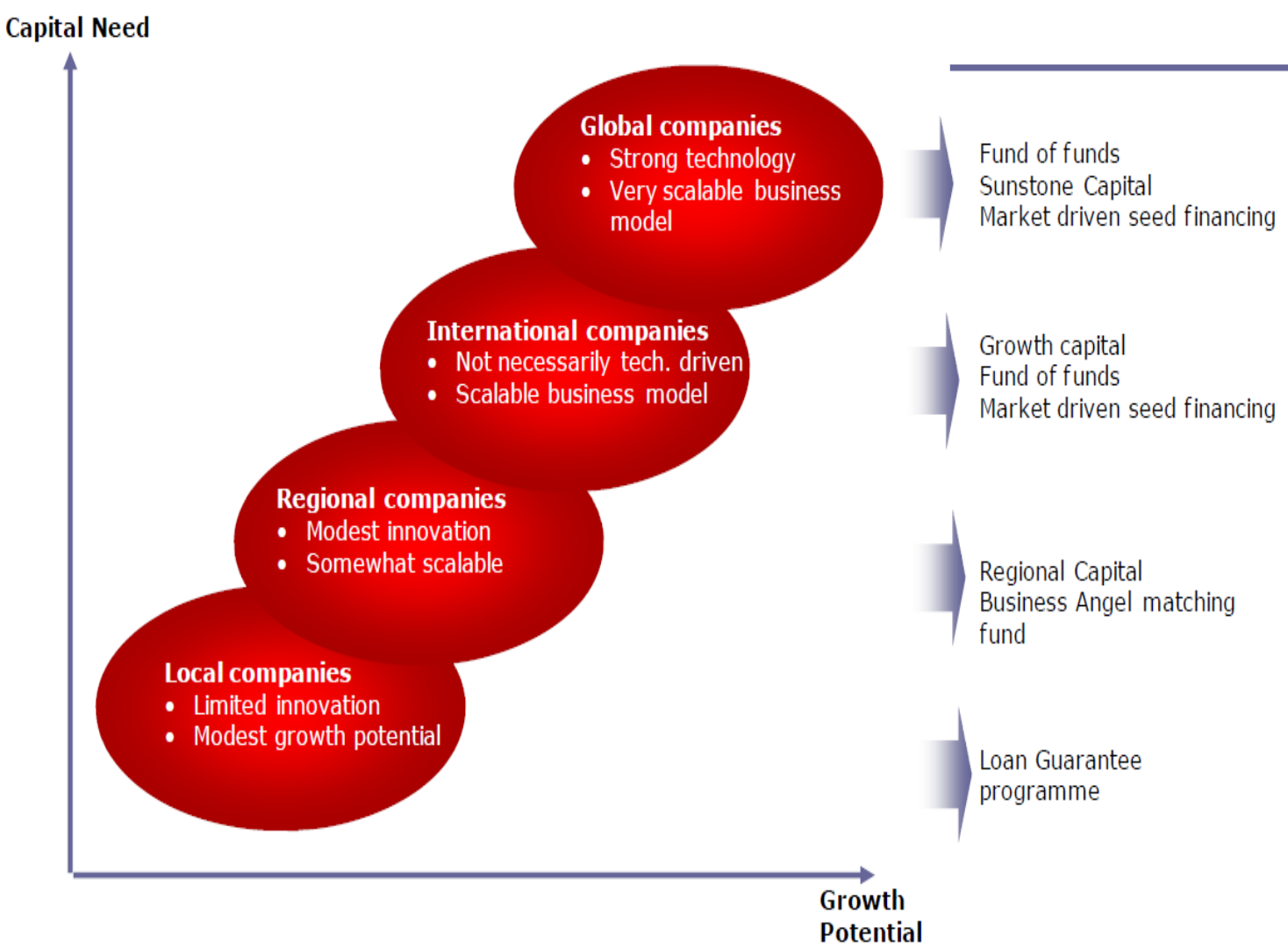

Quelle: OECD 2008

Die drei wichtigsten Programme zur operativen Umsetzung dieser Finanzierungsmodelle umfassen Vaekstkapital, Fondskapital und Vaekstauktion. Vaekstkapital ist eine Investmentgruppe von Vaekstfonden, die Eigenkapital in junge Unternehmen mit hohem Wachstumspotential investiert. Die Zielbranchen von Vaekstkapital sind IT, Medizintechnik, Umwelttechnik und Werkstofftechnik. Aber es wird auch ausdrücklich auf die Offenheit gegenüber anderen Branchen hingewiesen; „But we are open to investing in high growth start-ups in other industries, where we believe we can add value." ${ }^{50}$ Ein wesentliches Investmentkriterium ist die Darstellbarkeit einer internationalen bzw. globalen Marktnische und einer damit verbundenen Marktmacht. Fondskapital ist nach einem Funds-in-Funds-Prinzip konzipiert, wobei die privaten Venture Capital-Gesellschaften in nicht-börsennotierte Unternehmen mit hohem Wachstumspotential investieren. Aktuell ist Fondskapital in 20 Fonds investiert, die zusammen in insgesamt 150 Unternehmen investiert sind. Vaekstauktion bietet Kreditgarantien bis zu $75 \%$ des Kreditausfallvolumens an.

Betrachtet man das aktuelle Portfolio von Vaekstfonden, so zeigt sich ein Mix an Branchen unterschiedlicher Technologieintensität: Life Sciences, IT, Cleantech, Leisure and Gaming, Design, Trade and Services, Industry (Manufacturing), Technology Components. Dabei beinhalten die Portfolios von Life Science, IT und Industry (Manufacturing) die größte Anzahl an Unternehmensbeteiligungen. Eher mittleren Technologiegehalt dürften insbesondere Unternehmen aus der Branche „Industry“ haben. Hier hat Vaekstfonden beispielsweise auch in Möbelhersteller investiert. Es sei angemerkt, dass die Performance der dänischen Industrie

\footnotetext{
${ }^{50}$ http://www.vaekstfonden.dk/For \%20virksomheder/Vaekstkapital.aspx [20.3.2011].
} 
weitgehend von Branchen niedriger bis mittlerer Technologieintensität bestimmt wird. Auch Industriedesign gilt als eine wichtige Komponente im dänischen Produktionsmodell (e.g. Bang\&Olufsen) (Lindgaard et.al. 2008).

\section{Israel}

\section{Tnufa - Technological Incubators - Nofar}

Diese Programme sind, wie Abbildung 15 zeigt, die drei Programme der israelischen FTIPolitik zur Förderung von Gründungsprojekten in der Pre-Seed- und Seed-Phase. Alle drei Programme werden vom Office of the Chief Scientist operativ umgesetzt. Das Office of the Chief Scientist ist eine 1984 gegründete Institution, die für alle FTI-Programme bezüglich industrieller F\&E zuständig ist (Abbildung 64). Das Office of the Chief Scientist ist angegliedert beim Ministry of Industry, Trade and Labor und hat eine zentrale Position in der israelischen FTI-Politik inne.

Abbildung 64: F\&E-Förderprogramme der israelischen FTI-Politik

\section{Government Support Programs}

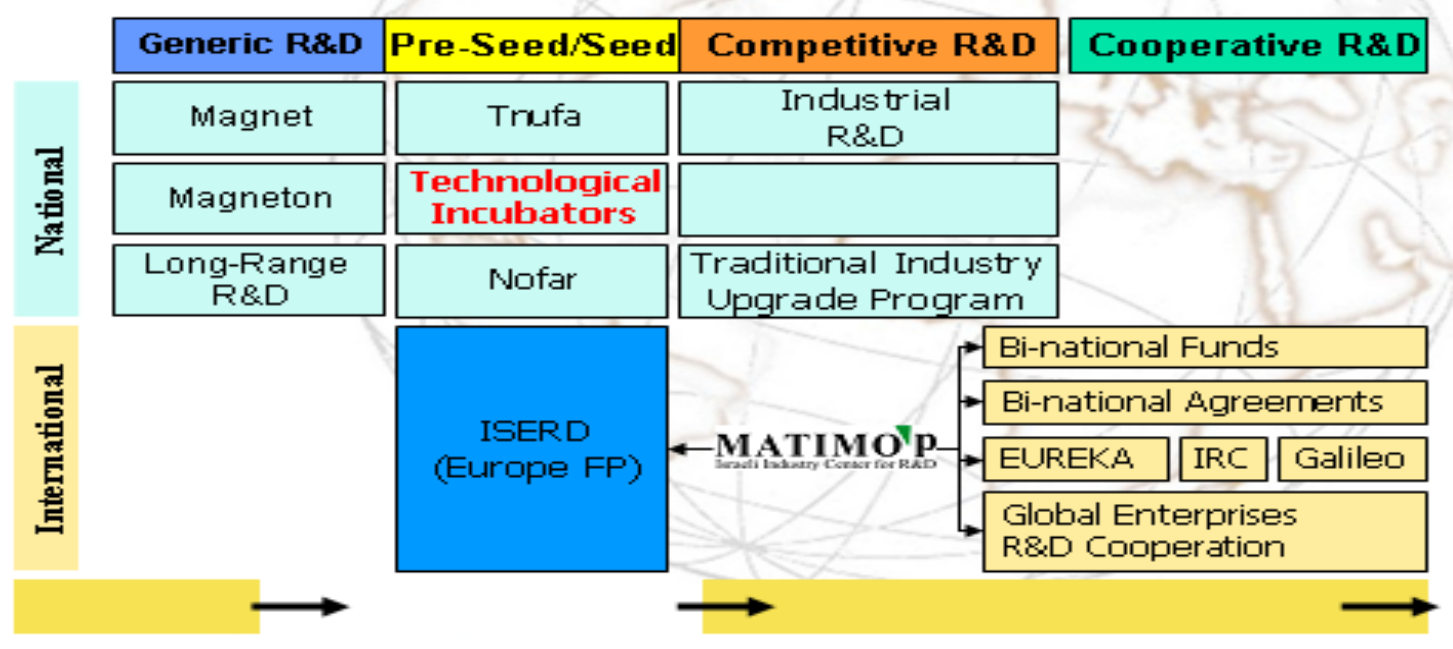

Quelle: Vortrag Yossi Smoler, März 2011

Das Incubator Program wurde 1991 gegründet und ist das bedeutsamste Programm zur Unterstützung von Hochtechnologiegründungen. Seitdem wurden 26 Inkubatoren gegründet, von denen mittlerweile 22 privatisiert sind. Die Betreiber der Inkubatoren agieren als privatwirtschaftliche Shareholder mit Profit-Kalkül. Von den erstmals 1993 gegründeten Unternehmen konnten $61 \%$ eine Folgefinanzierung erreichen; $40 \%$ sind noch am Markt. Das jährliche Budget für dieses Programm beträgt ca. 50 Mio. USD. Die Projekte der Entrepreneure müssen zuerst von einem der Inkubatoren akzeptiert werden. Danach kommt es zu einer Begutachtung durch das Office of the Chief Scientist. Teil der Evaluierung sind Besuche von ExpertInnen vor Ort um u.a. zu ermitteln, ob das Projekt bezogen auf den globalen Markt innovativ ist. Das Office of the Chief Scientist gewährt Zuschüsse bis zu 500 Tsd. USD innerhalb von 2 Jahren oder bis zu 750 Tsd. USD innerhalb von 3 Jahren. Von diesem Kapital wird $85 \%$ durch das Office of the Chief Scientist finanziert, der Rest durch das Management des Inkubators. Das Programm fördert generell alle Technologiefelder, es gibt jedoch die Mög- 
lichkeit, dass sich die Inkubatoren auf bestimmte Technologien wie etwa IKT oder Biotechnologie spezialisieren. Diese Option haben einige Inkubatoren auch wahrgenommen. Die sich daraus ergebende technologische Spezialisierung der in Inkubatoren angesiedelten Unternehmen ist in Abbildung 65 dargestellt.

Abbildung 65: Technologische Spezialisierung der mit Juli 2010 durch das Incubator Program geförderten Unternehmen

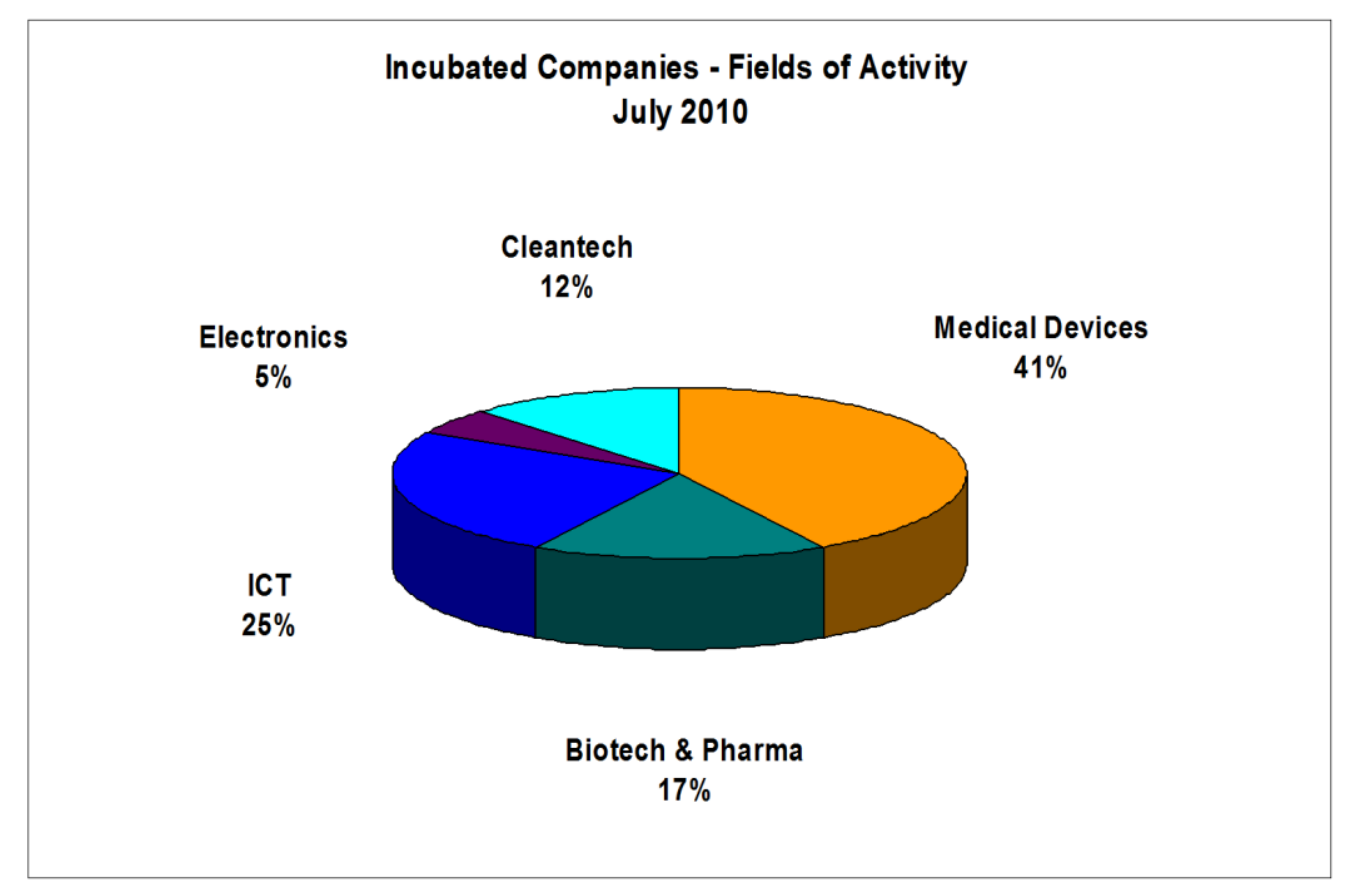

Daten: Vortrag Yossi Smoler, März 2011, eigene Darstellung

Pro Jahr gibt es in den Inkubatoren insgesamt ca. 75-85 Gründungen. Die Rückzahlung der Fördergelder erfolgt durch eine Zahlung in der Höhe von $3 \%$ des Umsatzes. Abbildung 66 zeigt die Entwicklung der kumulierten staatlichen und privaten Investitionen in Inkubatorunternehmen sowie deren Verhältnis zueinander. Daraus wird deutlich, dass das Programm Technological Incubators zunehmende Leverage-Effekte erzielen konnte: Wurden bis 1997 noch weniger als 1 USD private Investitionen pro 1 USD staatliche Investition investiert, beträgt dieses Verhältnis 2009 1: 5,2. 
Abbildung 66: Öffentliche und private Venture Capital-Investition in Hochtechnologiegründunge in Inkubatoren

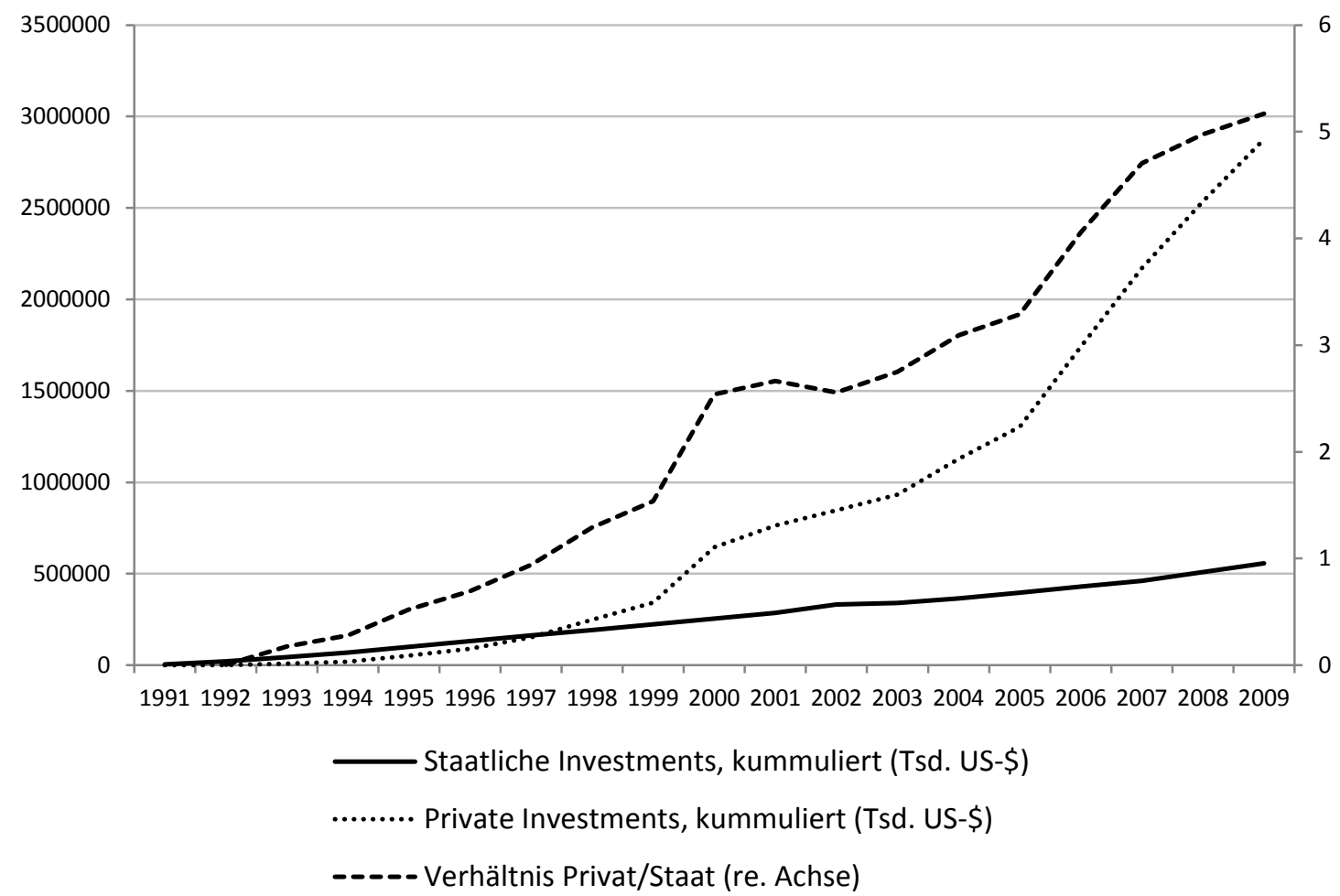

Daten: Vortrag Yossi Smoler, März 2011, eigene Darstellung

Das Programm Tnufa ist eine Finanzierungsunterstützung für Pre-Seed Projekte mit hohem Innovationsgehalt. Das Ziel ist es, den GründerInnen eine Unterstützung zu bieten, um die nächste Finanzierungsrunde am Venture Capital-Markt oder die Integration in einen multinationalen Konzern zu erreichen. Die Finanzierung beträgt max. 60.000 USD. Finanziert werden Patentanmeldungen, Prototypentwicklung, Machbarkeitsstudien sowie die Teilnahme an internationalen Messen. Die Fördernachfrager müssen ihr technisches und betriebswirtschaftliches Können darstellen und die Produktidee wird auf ihre Marktfähigkeit hin überprüft. Jedes Jahr werden ca. 120 Projekte realisiert, von denen etwa $20 \%$ die nächste Finanzierungsrunde erreichen.

Nofar adressiert die Lücke zwischen Grundlagen- und angewandter Forschung. Das Programm soll insbesondere die Verwertung biotechnologischer Forschung unterstützen.

\section{B In Israel}

2B in Israel (2B) wird vom Ministry of Immigrant Absorption zusammen mit der Israel Small and Medium Enterprise Authority betrieben. Im Kern geht es bei diesem Programm um die Förderung von Immigrant Entrepreneurship, das eine wichtige Rolle in Israel spielt. Neben einer Homepage, die erste Informationen anbietet, stellt 2B vor allem Beratungsleistungen durch Coaches zur Verfügung. Ein komplementäres Programm namens IBC (Israel Business Connection) unterstützt die Entrepreneure bei der Suche nach Finanzierungsmöglichkeiten und BranchenexpertInnen.

\subsubsection{Bewertung und Policy Learning}


Welche Schlussfolgerungen lassen sich aus dem internationalen Vergleich ziehen? Im Folgenden sollen jene Aspekte der gründungsbezogenen Rahmenbedingungen und Instrumente erörtert werden, die für Österreich im Allgemeinen und die aws im Besonderen mögliche Hinweise auf Good Practice-Beispiele geben. Von einer vertieften Auseinandersetzung mit diesen Aspekten könnten positive Policy Learning-Effekte erwartet werden.

- Ein Vergleich der innovationsökonomischen Rahmenbedingungen in Österreich ergibt insbesondere beim Humankapital Mängel im Vergleich zu den Vergleichsländern. Damit im Zusammenhang steht die Diskussion, ob die geringen Venture Capital-Investitionen in Österreich eine Folge mangelnder Nachfrage oder mangelnden Angebots sind. Welche Marktseite wirkt limitierend? Die diesbezügliche Datenlage lässt für Österreich keine eindeutigen Schlüsse zu. Eine Evaluierung des deutschen High-Tech Gründerfonds kommt demgegenüber zu einer relativ deutlichen Aussage: „Auch bei einer stärkeren Fokussierung der Akquisitionstätigkeit auf Gründungen mit hoher Technologietiefe wird die Zahl der erfolgreichen Beteiligungen in erster Linie von den verfügbaren Beteiligungsmöglichkeiten bestimmt werden“" (Geyer/ Heimer 2010, S.53). Zu ähnlichen Ergebnissen für Europa kommt auch Meyer (2008) auf Basis von ökonometrischen Modellen. Veugelers (2009) betont demgegenüber die Existenz einer weiterhin bestehenden Finanzierungsrestriktionals Wachstumshemmnis für Young Innovative Companies (YIC). Wenngleich also für Österreich auch die Angebotsseite als kürzere Marktseite konditionierend wirken könnte, nicht zuletzt aufgrund eines nach wie vor mangelnden international wettbewerbsfähigen Regulierungsrahmens, ist die relativ ungünstige Humankapitalausstattung ein potentieller Engpassfaktor, der die verfügbare Zahl an hochwertigen Investitionsprojekten systematisch reduziert. Gerade im Bereich Hochtechnologiegründungen ist ingenieur- und naturwissenschaftliches Humankapital entscheidend. Bei diesen beiden Humankapitalfraktionen ist die Performance Österreichs aber eher mittelmäßig (Leo et. al. 2006). Hier könnte man von Israel lernen, dass intensive Anstrengungen zur Ausbildung von IngenieurInnen und NaturwissenschafterInnen bereits sehr früh gesetzt hat und weiterhin massiv in dieses Segment des Bildungssektors investiert. Diese Fragen gilt es bei zukünftigen Strategien zur Förderung von Hochtechnologiegründungen zu beachten.

- Generell ist zu betonen, dass durch singuläre Merkmale der Vergleichsstaaten eine einfache Nachahmung von Programmen oder Regulierungen problematisch ist (Lerner 2009). Einerseits kann die gleiche Maßnahme in einem anderen Innovationssystem unterschiedliche Effekte haben und andererseits ist es typischerweise nur sehr schwer möglich, exakt die gleiche Maßnahme aufzubauen, da Programmmanagement unter anderem auf Tacit Knowldge beruht, welches per definitionem nur unter bestimmten Bedingungen transferierbar ist (Klofsten et.al. 2010). Das ausführlich diskutierte Beispiel Israel zeigt, dass Erfolge von nicht reproduzierbaren Faktoren und deren Zusammenwirken abhängen können. Weiterhin dürften gerade auch im Beispiel Israels zeitliche Koinzidenzen und „Windows of Opportunity“ eine wichtige Rolle gespielt haben.

- Die hier dargestellten Programme zur Förderung von Unternehmensgründungen entsprechen durchwegs der in der Entrepreneurship-Literatur betonten Bedeutung einer Erhöhung der Qualität von Unternehmensgründungen anstatt einer reinen Maximierung der Gründungsfälle pro Zeitperiode. Shane (2009, S.147), einer der führenden Ökonomen in der Disziplin Entrepreneurship, gibt folgenden Hinweis für die Politik: „The general prin- 
ciple is to shift resources from programs that support generic entrepreneurship efforts to those that support high potential businesses. "Shane (2009) weist weiters darauf hin, dass im Gegensatz zur Kritik an solchen Programmen aufgrund von Identifikationsproblemen sehr wohl robuste Variablen existieren, welche die Probleme derart reduzieren, dass der soziale Nutzen spezifischer Gründungsförderungsprogramme die Kosten signifikant übersteigt. In diesem Sinne sind die Programme der aws im Einklang mit den internationalen Vergleichsprogrammen aus wirtschaftswissenschaftlicher Perspektive prinzipiell geeignet die gesellschaftliche Wohlfahrt zu erhöhen. Der Fokus auf rasch wachsende Unternehmen ist insbesondere in den Förderprogrammen des dänischen Venture Capitalfonds Vaekstfonden zu erkennen.

- Der internationale Vergleich zeigt, dass ein breites Spektrum an Instrumenten zur Förderung von Hochtechnologiegründungen existiert. Gleichzeitig ist eine relativ hohe Übereinstimmung zwischen den eingesetzten Instrumenten in den einzelnen Staaten erkennbar. Dies weist auf bereits vorhandene Lernprozesse sowie auf gemeinsame Orientierungspunkte wie etwa auf die OECD Innovationsstrategien hin. Auch die Europäische Union hat in der Vergangenheit zahlreiche Benchmark-Studien in Auftrag gegeben und damit einen potentiellen Beitrag zur Konvergenz im Instrumentenportfolio geleistet. Generell kann festgestellt werden, dass die Programme der aws den hier dargestellten internationalen Vergleichsprogrammen ähnlich sind. Wie in anderen Staaten sind die Programme aufeinander aufbauend organsiert und inhaltlich aufeinander abgestimmt.

- Ähnlich wie in Österreich existiert auch in Dänemark eine Kooperation zwischen Inkubatoren und staatlichen Venture Capital-Institutionen. In Österreich betrifft dies die Zusammenarbeit zwischen der aws und den AplusB-Zentren; in Dänemark handelt es sich um die Innovation Incubators und den öffentlichen Venture Capital-Fonds Vaekstfonden. Allerdings tritt in Dänemark - wie auch beim israelischen Incubator Program - der Innovation Incubator selbst als eigenständiger staatlicher Venture Capital-Investor auf, während die AplusB-Zentren explizit keine Beteiligungen eingehen dürfen. Diese Regulierung ist ein Ausfluss von beihilferechtlichen Bedenken, die im Zuge der Erstellung der Programmrichtlinien der AplusB-Zentren geäußert wurden. Ein weiteres Problem der österreichischen AplusB-Zentren im Hinblick auf die Beteiligungsfrage ergibt sich weiterhin durch die multiplen Stakeholder und Shareholder, die direkt oder indirekt Einfluss auf das Zentrum nehmen. Da das beschränkte Investitionsvolumen der dänischen Inkubatoren pro Projekt jedoch derart beschränkt ist, dass nur eine kleine Anzahl an Gründungen damit hinreichend finanziert werden kann, ist eine Folge- oder Ko-Finanzierung von Vaekstfonden der Regelfall. Unternehmen, die Unterstützung im Rahmen des Innovation Incubators-Programms erhalten, sind eine der Hauptquellen für Investitionsprojekte des staatlichen Venture Capital-FondsVaekstfonden. Ungefähr ein Drittel des Investitionsvolumens von Vaekstfonden betrifft den aus dem Incubator Program stammenden Dealflow. Ein Review der dänischen Gründungspolitik durch die OECD kommt zu dem Schluss, dass die Konzentration der Venture Capital-Aktivitäten in Vaekstfonden sowie die komplementären Strukturen mit dem Incubator Program positive Effekte auf die Finanzierungsbedingungen von Unternehmensgründungen haben (OECD 2008). Eine genauere Analyse der Kooperationsperformance zwischen dem Incubator Program und Vaekstfonden könnte Hinweise auf Optimierungsmöglichkeiten hinsichtlich der Kooperation der AplusB- 
Zentren und der aws liefern.

- Sektorale Schwerpunkte in der Förderausrichtung finden sich in den hier vorgestellten Programmen generell keine. Insbesondere die deutschen Programme des High-Tech Gründerfonds und der Bayern Kapital geben keine erwünschten Spezialsierungen vor. Jedoch existiert mit dem Programm Go-Bio ein eigenes sektorspezifisches Förderinstrument für gründungsbereite Forscherteams aus der Biotechnologie. Der dänische Fonds Vaekstfonden ist nicht einmal explizit auf High-Tech spezialisiert, sondern finanziert explizit Unternehmen, die trotz mäßiger Technologieintensität internationales Wachstumspotential aufweisen (,Scalable Business Model“). Auch der deutsche High-Tech Gründerfonds ermöglicht eine Substitution des Technologiekriteriums. In diesem Fall erfolgt die Substitution jedoch nicht durch Wachstumskriterien, sondern durch den Innovationsgrad. Vaekstkapital als eine Division von Vaekstfonden definiert zwar einige Zielbranchen (IT, Medizintechnik, Umwelttechnik und Werkstofftechnik), aber unter dem Vorbehalt einer expliziten Offenheit gegenüber allen anderen Branchen. Israel setzt mit seinem Incubator Program auf die dezentrale Entscheidung der Inkubatoren hinsichtlich möglicher Spezialisierungsstrategien. Von Seiten des Office of the Chief Scientist werden jedenfalls keine Vorgaben abgesehen von Technologieniveau und Exportpotential gemacht. Im Vergleich zu den internationalen Vergleichsprogrammen ist festzustellen, dass die Programme des aws zur Förderung von Gründung und Aufbau von Hochtechnologieunternehmen mit ihrer Fokussierung auf IKT, Physical Sciences und Biotechnologie vordergründig den relativ höchsten Grad an Selektion bzw. technologiefeldbezogenem Targeting im internationalen Vergleich aufweisen. Tatsächlich ist jedoch mit der Kategorie Physical Sciences ein sehr weites Feld definiert, das annähernd die Funktion einer residualen Kategorie übernimmt. Sollten damit Probleme die typischerweise mit Targeting verbunden sind reduziert werden, so ist dennoch zu Fragen, in wie weit die Außendarstellung als Förderer für diese drei Technologiefelder nicht dennoch adverse Selektionseffekte aufgrund von Informationsdefiziten über das de facto existierende Förderangebot auf Seiten potentieller Gründer produziert.

- Im Gegensatz zum High-Tech Gründerfonds sowie zum dänischen Fonds Vaekstfonden existiert bei den aws-Technologieprogrammen keine Möglichkeit seitens der potentiellen Gründer mangelnde Technologietiefe durch einen hohen Innovationsgrad (High-Tech Gründerfonds) oder internationale Wachstumsperspektiven (Vaekstfonden) partiell zu substituieren (periphere Substition). In diesem Punkt scheint daher die größte Übereinstimmung mit dem israelischen Incubator Program zu bestehen. Ob diese Orientierung sinnvoll ist, kann nur im Zusammenhang mit einer globalen Analyse des Innovations- und Fördersystems beurteilt werden. In Bezug auf das Innovationssystem ist im Rahmen der Systemevaluierung die Notwendigkeit einer auf radikalen Innovationen beruhenden „Front Runner"-Strategie festgestellt worden. Radikale Innovationen müssen nach Tichy (2010) nicht notwendigerweise an der Technologiegrenze angesiedelt sein. „Präzise Definitionen lassen sich in der industrieökonomischen Literatur allerdings jedoch kaum finden, doch geht es stets um neue Produkte oder Leistungen, die auf einer neuen Kombination von Technologien und/oder Organisationen aufbauen und - vor allem - die Kundenbedürfnisse besser befriedigen als existierende (...)“ (Tichy 2010, S.53). Demnach wäre eine Substitution von Technologiegrad durch Innovationsgrad durchaus im Einklang mit 
den Schlussfolgerungen der Systemevaluierung. Damit erscheint eine Beurteilung der Zweckmäßigkeit einer ausschließlichen Technologieorientierung primär aus der Perspektive des Fördersystems heraus sinnvoll. Aus Perspektive des Fördersystems ist die Frage zu stellen, welche Förderinstrumente in Österreich für dieses spezifische Gründersegment existiert bzw. ob Unternehmen mit hohem Innovationsgrad und/oder hohem Wachstumspotential überhaupt spezifischen Formen des Marktversagens ausgesetzt sind, wie dies im Fall von Hochtechnologiegründern der Fall ist. Würde tatsächlich eine Förderlücke bestehen, so wäre eine Ausweitung des aws Portfolios trotzdem eher kritisch zu beurteilen, da gerade Hochtechnologiegründungen hohe Kompetenzanforderungen an Personal und Institution stellen, die durch Spezialsierungsgewinne und damit verbundene Lernkurveneffekte effizient erfüllt werden können.

- Das Wissen über eine „optimale“ Politik zur Förderung von qualitativ hochwertigen Gründungen ist noch immer sehr begrenzt (Lerner 2009, Veugelers 2009). Umso wichtiger ist es für die Politik, ihren Instrumenteneinsatz der Qualität wie der Quantität nach in einem Lernprozess ständig weiterzuentwickeln. Eine wichtige Basis hierfür bilden Monitoring Instrumente, die regelmäßig wichtige Informationen über die Entwicklung relevanter Erfolgsgrößen geben. Dänemark („Entrepreneurship Index“) und Deutschland (,HighTech Gründungen in Deutschland") veröffentlichen jährliche Berichte über das Gründungsgeschehen. Gerade wenn in der österreichischen FTI-Strategie explizit ein quantitatives Ziel bezüglich Gründungen genannt wird („,Die Anzahl der wissens- und forschungsintensiven Neugründungen soll bis 2020 um jährlich durchschnittlich $3 \%$ gesteigert werden“ (Republik Österreich 2011)), erscheint ein Andenken ähnlicher Monitoring Instrumente eine möglich Option zur Optimierung des Instrumenteneinsatzes. Darüber hinaus kann ein jährlicher Bericht auch das Agenda-Setting in einem ansonsten oftmals wenig präsenten, aber wichtigen Politikbereich unterstützen.

- Eine Besonderheit der deutschen Programme stellt die intensive Nutzung von Coachs dar. Während die Finanzierungsförderung den „Finance Gap“ bei Hochtechnologiegründern adressiert, zielt das Coaching auf die Schließung des in der einschlägigen Literatur diskutierten „Knowledge Gaps“ ab. „The spin-off companies supposedly lack entrepreneurial knowledge skills to develop their businesses” (Mustar et al. 2010, S.68). Darüber hinaus wird durch die regionale Präsenz der Coaches räumliche Nähe hergestellt, die gerade in ökonomischen Situationen, die durch erhöhte Unsicherheit gekennzeichnet sind, eine wichtige Funktion in ökonomischen Transaktionen einnimmt. Während sich der/die GründerIn beim High-Tech Gründerfonds noch mit Einverständnis des Fonds gegen eine Zusammenarbeit mit einem Coach entscheiden kann, ist dies im Fall der Bayern-Kapital obligatorisch. Bei beiden Fonds ist es Ziel der Maßnahme, dass der Coach eine Beteiligung am Unternehmen erwirbt. Die Unternehmen können sich dabei aus einer Liste akkreditierter Coachs eine für sie passende Persönlichkeit wählen. Die Aufgabe der Coachs liegt jedoch nicht nur in der typsicherweise kaufmännischen Beratung der technisch ausgebildeten GründerInnen, sondern auch in der Akquisition von potentiellen Investitionsprojekten. Die aws hat mit dem Programm „Management auf Zeit“ ebenfalls ein Coaching-Element in ihrem Programmportfolio. Dieses Programm ist jedoch als Unterstützung für krisenhafte Entwicklungen gedacht und verfolgt von daher einen anderen Ansatz als die deutschen Coaching-Programme. Die Evaluierungsergebnisse bezüglich 
des deutschen Modells zeigen jedoch keine eindeutig positiven Ergebnisse, wenngleich die grundsätzliche Zweckmäßigkeit des Coaching-Konzepts festgehalten wird. Relevante Probleme bestehen im Matching zwischen der Nachfrage der Gründer nach Unterstützungsleistungen und den Angeboten der Coaches.

- Israel hat mit dem Programm „2B In Israel“ sowie weiteren Maßnahmen zahlreiche Initiativen zur Förderung von Immigrant Entrepreneurship gesetzt. Auch wissenschaftliche Arbeiten zeigen die hohe Bedeutung von ImmigrantInnen bzw. der Diaspora im erfolgreichen Entwicklungsmodell Israels. Ähnliches lässt sich aber auch für andere Länder, wie etwa die USA feststellen. Offenheit gegenüber ImmigrantInnen kann das Angebot an motivierten, gründungswilligen Entrepreneuren signifikant erhöhen. Oftmals sind mobile Bevölkerungsgruppen positiv selektiert und weisen eine höhere unternehmerische Orientierung als immobile Bevölkerungsgruppen auf. Hier könnte Österreich, etwa im Zusammenhang mit der Einführung der Rot-Weiß-Rot Card, neue Instrumente andenken. Ein möglicher Vorschlag wäre etwa, die Angebote der aws aktiv der Zielgruppe hochqualifizierte MigrantInnen anzubieten. Dabei gilt es insbesondere sprachliche Barrieren in der Angebotsgestaltung zu berücksichtigen. Die Einrichtung einer eigenen Homepage mit einschlägigen Informationen würde potentiell prohibitiv wirkende Informationskosten reduzieren. Auch die Nutzung von bereits bestehenden Netzwerken mit der österreichischen Diaspora könnte neue impulse für die österreichische GründerInnen-Szene bringen (Kasten 1). Eine Studie von Egeln et al. (2003) über akademische Spinoff-Gründungen in Österreich kommt zu dem Ergebnis, dass $10 \%$ aller Gründer zuvor an einer ausländischen Einrichtung gearbeitet bzw. studiert haben. Wenngleich freilich der Anteil Deutschlands am höchsten ist, so ist dennoch auch der Anteil amerikanischer Herkunftseinrichtungen durchaus beachtlich. Durch die Einführung der Rot-Weiß-Rot Card sowie aufgrund weiterer, in den letzten Jahren getroffenen Maßnahmen zur Attrahierung ausländischen Humankapitals, könnte sich das Potential an ausländischen Gründern weiter erhöhen (Reiner 2009). 


\subsection{INTERNATIONALE PROGRAMME ZUR FÖRDERUNG VON IPR- AKTIVITÄTEN}

Die geringere Nutzung geistiger Eigentumsrechte durch KMUs kann als Marktversagen interpretiert werden (Blind et al. 2009). Diese Erkenntnis führte in zahlreichen Staaten zur Implementierung politischer Maßnahmen zur Unterstützung von KMUs in der Nutzung des IPRSystems. In diesem Kapitel werden die Förderprogramme in Tabelle 45 dargestellt und anschließend bewertet sowie hinsichtlich möglicher Schlussfolgerungen für das Programmportfolio der aws analysiert.

Tabelle 45: Vergleichsprogramme Patentverwertung

\begin{tabular}{|c|c|c|}
\hline Staat/ Region & Institution & Programm/ Instrument \\
\hline \multirow{4}{*}{ Deutschland } & $\begin{array}{l}\text { Deutsches Patent- und Marken- } \\
\text { amt }\end{array}$ & Patentinformationszentren \\
\hline & SIGNO & KMU-Patentaktion \\
\hline & \multirow{2}{*}{$\begin{array}{l}\text { Bundesministerium für Wirtschaft } \\
\text { und Technologie }\end{array}$} & InnovationMarket \\
\hline & & Erfinderfachauskunft \\
\hline Bayern & Bayrische Patentalianz & $\begin{array}{l}\text { Evaluierung der Erfindung, } \\
\text { Schutzrechtliche Sicherung, Ver- } \\
\text { marktung, Monitoring, Technolo- } \\
\text { gieangebote }\end{array}$ \\
\hline \multirow{3}{*}{ Dänemark } & Nordic Patent Institute & Business Services \\
\hline & $\begin{array}{l}\text { Samian Underwriting Agency } \\
\text { (UK) }\end{array}$ & \multirow{2}{*}{ Patent Enforcer } \\
\hline & $\begin{array}{l}\text { Danish Patent and Trademark } \\
\text { Office }\end{array}$ & \\
\hline \multirow[b]{2}{*}{ Frankreich } & Public Policy & \multirow{2}{*}{$\begin{array}{l}\text { France Brevets } \\
\text { European Patent Fund }\end{array}$} \\
\hline & $\begin{array}{l}\text { Caisse des Depots et Consigna- } \\
\text { tions }\end{array}$ & \\
\hline
\end{tabular}

\subsubsection{Darstellung der Programme}

\section{Deutschland}

Eine Evaluierung der deutschen Fördermaßnahmen zur Nutzung von geistigen Eigentumsrechten durch KMUs kam zu folgenden Ergebnissen (Blind et al. 2009):

1. Die KMUs weisen ein Informationsdefizit bezüglich der verfügbaren Förderprogramme auf.

2. Die Fördermaßnahmen sind qualitativ und quantitativ auf das Instrument des Patentschutzes konzentriert.

3. Die Programme sind mehrheitlich für die frühen Phasen des Patentmanagements d.h. konkret der Patentrecherche und der Patentanmeldung, konzipiert.

Die EvaluatorInnen schlagen insbesondere eine Orientierung der Maßnahmen am umfassenderen Konzept des IP-Managements vor, bei dem es um die optimale Kombination formeller und informeller Schutzrechte im Kontext einer bestimmten Geschäftsstrategie geht. Bei die- 
sem Konzept stellt das Patent nur eines neben anderen Instrumenten dar.

Wie in anderen Politikbereichen Deutschlands existiert auch in der FTI-Politik eine Kombination aus Maßnahmen des Bundes und der Länder. Diese föderale Organisation trifft auch bei Förderungsinstrumenten von IPR-Aktivitäten zu. Während unter SIGNO Programme auf Bundesebene angeboten werden, stellen die Patentinformationszentren und die Bayrische Patentallianz regionale Programme dar.

\section{SIGNO - KMU-Patentaktion - InnovationMarket - Erfinderfachauskunft}

SIGNO fungiert seit 2008 als Dachmarke für eine Reihe von Maßnahmen zur zielgruppendifferenzierten Förderung von IPR-Aktivitäten von Hochschulen, Unternehmen (insbesondere KMUs) und Erfinderpersonen (Abbildung 67). Die einzelnen Programme existieren jedoch i.d.R. bereits seit 2002. Das Bundesministerium für Wirtschaft und Technologie fungiert als Trägerorganisation; das Projektmanagement ist beim Institut der Deutschen Wirtschaft in Köln angesiedelt. Die operativen Aktivitäten werden durch ein bundesweites Netzwerk von 31 SIGNO-Partnern umgesetzt. Diese Partnerinstitutionen stellen einen heterogenen Mix dar und umfassen z.B. ErfinderInnen- und Patentinformationszentren, GründerInnen- und Technologiezentren, Transfereinrichtungen, Hochschuleinrichtungen oder UnternehmensberaterInnen.

Abbildung 67: Die Förderschienen des SIGNO-Programms

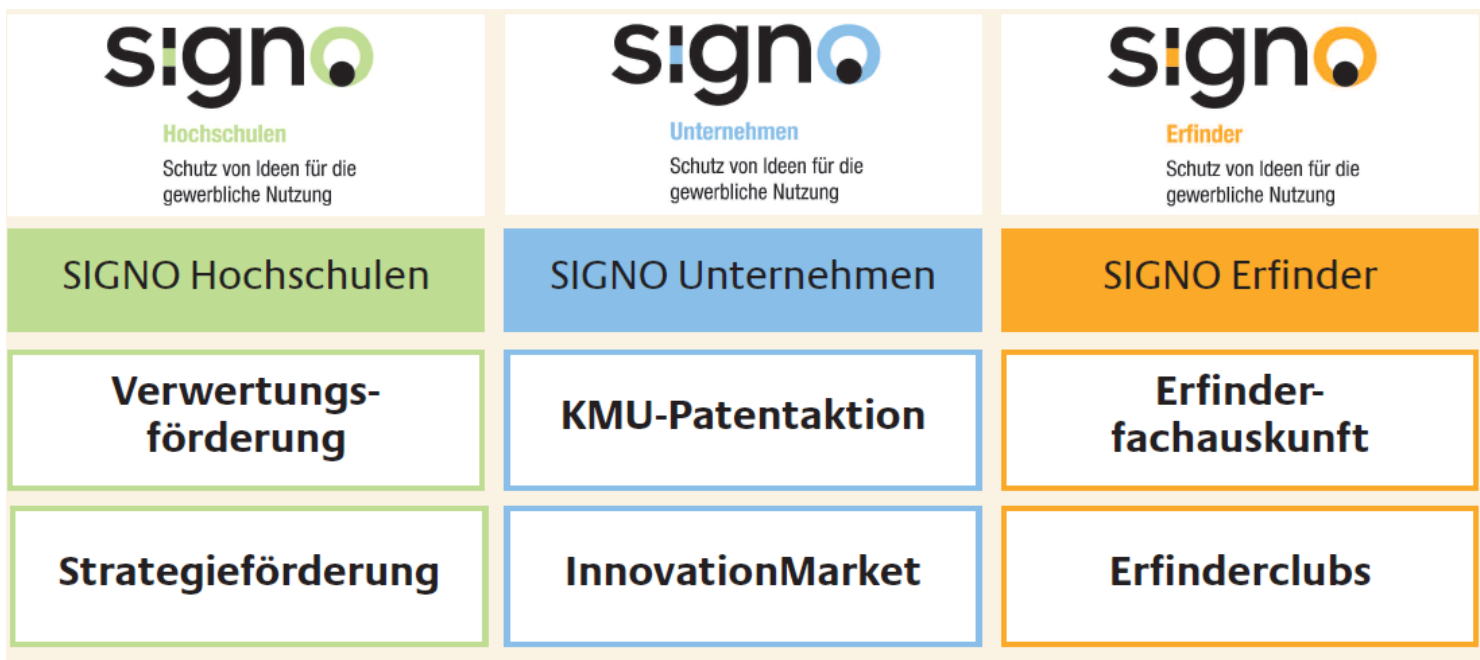

\section{Innovationsbörse / Öffentlichkeitsarbeit / Messen}

Quelle: Bundesministerium für Wirtschaft und Technologie 2010

Das wichtigste Teilprogramm von SIGNO zur Unterstützung von KMUs ist die KMUPatentaktion. Damit sollen ,kleine und mittlere Unternehmen und Existenzgründer der gewerblichen Wirtschaft, der freien Berufe und der Landwirtschaft bei der erstmaligen Sicherung ihrer Ergebnisse aus der Forschung und Entwicklung (FuE) durch gewerbliche Schutzrechte und bei deren Nutzung unterstützt und angeleitet werden" (BMWT 2010, S.15). Ein wesentliches Ziel besteht darin, dass die MaßnahmenteilnehmerInnen jene Kompetenzen erwerben, welche sie zu einem selbständigen Umgang mit Patentangelegenheiten befähigen. Damit wendet sich die Patentaktion explizit an jene KMUs, die noch keine Erfahrung mit Patenten haben oder deren letzte Patentanmeldung mehr als 5 Jahre zurückliegt. 
Die Ziele im Einzelnen sind:

1. Abbau von Hemmnissen gegenüber dem Patentwesen

2. Optimierung des Innovationsmanagements

3. Bessere Nutzung von Patentinformationen

4. Steigerung der Anzahl qualifizierter Patentanmeldungen

5. Sensibilisierung für die ökonomische Relevanz und Verwertbarkeit von Erfindungen

Die Abstimmung der konkreten Förderaktivitäten erfolgt über eine enge Zusammenarbeit der Antragsteller mit dem jeweiligen SIGNO-Partner. Gefördert werden Maßnahmen in fünf Teilpaketen, deren Abfolge dem innerbetrieblichen Leistungsprozess bei Entwicklungsaktivitäten entspricht (in Klammer das Fördervolumen):

1. Recherchen zum Stand der Technik (Zuschuss über $50 \%$ der Kosten, bis max. 800 EUR)

2. Kosten-Nutzen-Analyse (Zuschuss: $50 \%$ der Kosten, bis max. 800 EUR)

3. Patent- oder Gebrauchsmusteranmeldung in Deutschland (Zuschuss über $50 \%$ der Kosten, max. 2.100 EUR)

4. Vorbereitung für die Verwertung einer Erfindung (Zuschuss über $50 \%$ der Kosten, max. 1.600 EUR)

5. Patent- oder Gebrauchsmusteranmeldung für das Ausland (Zuschuss über $50 \%$ der Kosten, max. 2.700 EUR)

Die Auszahlung der Förderungen erfolgt stufenweise je nach dem Erreichen definierter Meilensteine. Die durchschnittliche Fördersumme je Unternehmen liegt bei etwa 4.000 EUR. Die bereits erwähnte Evaluierung von 2009 kam zu dem Schluss, dass der Mehrwert des Programms KMU-Patentaktion v.a. darin liegt, dass KMUs einen „One Stop Shop“ für Unterstützungsmaßnahmen von IPR-Aktivitäten angeboten bekommen. Die Anbietung komplementärer Dienstleistungen schafft darüber hinaus Synergieeffekte und kann insgesamt als „deutlich erkennbarer generischer Erfolgsfaktor für IPR-Services und -Programme“ (Blind et. al. 2009) bewertet werden. Weitere vom Ministerium genannte Erfolgsgrößen betreffen eine Erteilungsquote von $80 \%$ für SIGNO-geförderte Patentanmeldungen, eine deutlich überdurchschnittliche Quote. Weiterhin wird auch ein Lerneffekt dargestellt, der sich darin zeigt, dass beinahe $50 \%$ der MaßnahmenteilnehmerInnen nach Ablauf der Förderung bereits ein weiteres Schutzrecht angemeldet hatten.

Das zweite Programm unter dem Dach SIGNO-Unternehmen ist die Verwertungsaktion InnovationMarket. Im Kern geht es dabei um einen virtuellen Handelsplatz für Technologien. Hintergrund der Einrichtung dieses Handelsplatzes ist die Erkenntnis, dass nicht jede Innovation im innovierenden Unternehmen optimal umgesetzt werden kann. Zielgruppe dieser Aktion sind junge Technologieunternehmen sowie GründerInnen und expandierende Unternehmen auf der Suche nach Finanzierungsmöglichkeiten; Unternehmen, universitäre und außeruniversitäre Forschungseinrichtungen und ErfinderInnen, die Technologieverwertungsmöglichkeiten suchen; Beteiligungsgesellschaften, Banken und Unternehmen, die Technologien nachfragen.

Die Förderung des Programms InnovationMarket umfasst die Kosten für die Erstellung von Inseraten, die als standardisierte Summaries die Angebotsseite auf der Handelsplatt-

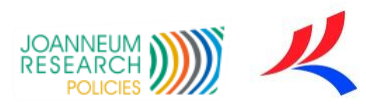


form abbilden. Der Zuschuss beträgt $30 \%$ der Kosten aber max. 800 EUR. Während die Basisdaten für das Inserat der/die InserentIn zur Verfügung stellt, unternimmt der SIGNOPartner weitere Recherchen, die das Inserat einschlägig ergänzen. Vor Veröffentlichung erfolgt eine Prüfung durch einen zweiten SIGNO-Partner. Darüber hinaus kann die Expertise für eigene Verwertungsaktivitäten genutzt werden. Die Verwertungsquote der Technologieangebote liegt bei etwa $14 \%$, wobei auch Großunternehmen als Nachfrager auftreten (BMWT 2010).

Wenngleich das Programm Erfinderfachauskunft nicht explizit und ausschließlich für Unternehmen konzipiert ist, sondern einen weiteren Adressatenkreis hat, ist dieses Programm auch für KMUs von Relevanz. Der inhaltliche Kern des Programms besteht aus einer kostenlosen, bis zu vierstündigen Erstauskunft durch SIGNO-Partner zu ökonomischen und technischen Aspekten sowie Kooperationen. Die wirtschaftlichen Aspekte betreffen Fragen der Verwertung und Finanzierung.

\section{Patentinformationszentren}

In Deutschland gibt es 23 regionale Patentinformationszentren, die als Ansprechpartner für Fragen des gewerblichen Rechtsschutzes fungieren. Diese Zentren sind in der „Arbeitsgemeinschaft Deutsche Patentinformationszentren“ zusammengeschlossen. Sie verfügen einerseits über eine gemeinsame Homepage ${ }^{51}$ sind aber andererseits äußerst heterogen in Bezug auf Trägerschaft, Leistungsangebot und Preisgestaltung. Träger sind u.a. Universitäten, Industrie- und Handelskammern oder die Länder. Beispielhaft sei hier das Patentinformationszentrum in Baden-Württemberg erwähnt, dass als Wirtschaftsförderungsmaßnahme des Landes alle Dienste gratis anbietet.

Die Dienstleistungen der Zentren umfassen in der Regel Informationen zu Anmeldeverfahren für gewerbliche Schutzrechte; Recherchen und Überwachungen, wobei sowohl Eigenrecherchen als auch Auftragsrecherchen möglich sind; Erfinderberatung mit kostenloser Erstberatung, Schulungen und Seminare sowie Annahme von deutschen, Europäischen und internationalen Patentanmeldungen. Weitere Unterstützungsmaßnahmen betreffen strategische Patentberatung, Patentdatenmanagement, F\&E-Projektbetreuung, Normenberatung und Förderberatung.

Als Erfolgsfaktoren der Patentinformationszentren gelten nach Ergebnissen der Evaluation die Qualifikation der MitarbeiterInnen im Umgang mit Patentinformation und die KMUspezifischen Zusatzangebote, die zunehmend auch die Patentinformationszentren zu „One Stop Shops“ machen (Blind et al. 2009).

\section{Bayern}

\section{Bayrische Patentallianz}

Die Bayrische Patentallianz (BP) wurde 2007 durch die Vereine Universität Bayern und Hochschule Bayern gegründet. Sie ist die Nachfolgeinstitution von BayernPatent. Die BP fungiert als zentrale Patent und Vermarktungsagentur von 28 bayrischen Universitäten und Hochschulen. Das Service für ErfinderInnen umfasst die Evaluierung von Erfindungen durch

\footnotetext{
${ }^{51} \mathrm{http}: / /$ www.patentinformation.de/
} 
Markt- und Technikrecherche, die schutzrechtliche Sicherung z.B. durch Patente, Vermarktung der Erfindung und Monitoring der Verträge. Dem Unternehmenssektor wird ein zentraler Zugang zum Technologiepool der bayrischen Universitäten und Hochschulen angeboten, die Lizenzierung und Übertragung von Patenten sowie ein unbürokratische Zusammenarbeit.

Die BP kooperiert mit sogenannten ErfinderberaterInnen, die an den Universitäten und Hochschulen arbeiten. Dieser Erfinderberater erstellt zusammen mit dem/der ErfinderIn eine Erfindungsmeldung die an die BP weitergeleitet wird. Diese führt zentral in München für ganz Bayern eine Evaluierung der Erfindung durch.

Gemessen an den Personalbeständen hat die BP zwei Schwerpunkte in den Technologiefeldern Life Sciences und Physical Sciences. Für beide Technologiefelder existiert jeweils eine Arbeitsgruppe für Lizenzmanagement mit 5 bzw. 4 MitarbeiterInnen, wobei 6 von diesen promoviert sind. Eine weitere Gruppe von 10 Personen gehört zur Arbeitsgruppe Patentmanagement und Verträge, von denen ebenfalls 6 promoviert sind. Auf der Homepage wirbt die BP mit einem bestehenden Netzwerk an kooperationsbereiten Unternehmen unterschiedlicher Branchen und Technologien, das durch mehrere Jahre aktiver Vermarktungsaktivitäten aufgebaut werden konnte.

\section{Dänemark}

Die Aktivitäten von öffentlichen Forschungseinrichtungen betreffend IPRs werden in Dänemark in einem jährlichen Monitoring erfasst, dem „Public Research Commersialisation Survey“. Dabei werden nicht nur Patente und Lizenzen, sondern etwa auch universitäre Spin-off Gründungen erfasst.

\section{Nordic Patent Institute}

Das Nordic Patent Institute (NPI) wurde 2008 als Kooperationsplattform mit eigenständiger Rechtspersönlichkeit zwischen dem dänischen, norwegischen und isländischen Patentamt gegründet. Diese Kooperation wird von den beteiligten Staaten als ein Ausweg aus der ,patent system crisis“ verstanden. Darunter wird die Überlastung des Patentsystems durch zunehmende Patentnachfrage verstanden (Danish Patent and Trademark Office 2008). Deshalb besteht eines der wichtigsten Ziele des NPI in der raschen und fristgerechten Erledigung von Rechercheaufträgen.

Darüber hinaus soll vor allem KMUs ein unbürokratischer Zugang zum internationalen Patentwesen ermöglicht werden. Durch die Kooperation wurden hinreichende Ressourcen gepoolt, um als „International Searching Authority“ im PCT System“ anerkannt zu werden. Dadurch wird es für Unternehmen aus diesen Staaten möglich, PCT-bezogene Aktivitäten in der Landessprache zu verhandeln sowie face-to-face Kontakt mit den Patentämtern zu halten. Sie erhalten damit eine Alternative zur Nutzung des EPO oder des schwedischen PCTOffices.

Diese Zusammenarbeit verlangte insbesondere in der Gründungsphase ein hohes $\mathrm{Ma}$ an Harmonisierung und Kooperationsarbeit. Es mussten Übereinstimmungen in grundlegenden Fragen gefunden werden, wie etwa, was man unter Neuheit und Patentierbarkeit versteht. Dazu wurde z.B. ein Peer View-Verfahren eingeleitet, in welchem jedes Suchprotokoll durch eine/n MitarbeiterIn aus einem anderen Kooperationsstaat begutachtet wird. 
Die bislang neue Intensität der Kooperation wird von beteiligten Institutionen und Beobachtern als vorbildhaft für weiterreichende, zukünftige Europäische und globale Kooperationsform im Patentwesen angesehen. Damit könnte sich diese Kooperation als Vorbild etwa für das Modell eines Europäischen Patents eignen, wenngleich die soziale und kulturelle Nähe der Kooperationsstaaten diese Zusammenarbeit vereinfacht hat und von daher keiner einfachen Wiederholung zugänglich sein dürfte.

\section{Patent Enforcer}

Patent Enforcer (PE) ist ein Versicherungsprodukt zur Versicherung von Kosten, die im Zusammenhang mit Patentstreitigkeiten entstehen (European Commission 2009). Damit adressiert dieses Programm ein Marktversagen, welches zur adversen Selektion und Verdrängung von KMUs am Patentmarkt führt. Konkret fehlen KMUs regelhaft die notwendigen Ressourcen, um Patentstreitigkeiten zu führen, was bei Antizipation dieser prohibitiven Durchsetzungskosten zur Nichtanmeldung von Patenten führt. Die Piraterie-Problematik führt zu einer weiter wachsenden Kluft zwischen Großunternehmen und KMUs in der Patentnutzung. Damit kann ein leistbares Versicherungsprodukt für KMUs in den genannten Fällen eine wichtige Maßnahme zur Reduktion von Marktversagen darstellen.

Das Programm wurde 2007 in Dänemark gestartet. Während das Programm auf privatwirtschaftlicher Basis von der englischen Versicherungsgesellschaft Samian Underwriting Agency betrieben wird, wurde der Markteintritt ebenso wie weitere flankierende Maßnahmen in enger Kooperation mit dem dänischen Patentamt (Danish Patent and Trademark Office, DKPTO) durchgeführt. Die Initiative ging dabei vom DKPTO aus, das private Versicherungsunternehmen mit den notwendigen Informationen und Insiderwissen zum Patentwesen versorgte. Anschließend daran setzte das DKPTO Awarenessmaßnahmen, um die angebotenen Versicherungsprodukte unter den potentiellen Kunden, d.h. ForscherInnen und Unternehmen, bekannt zu machen. Diese Zusammenarbeit zwischen privaten Anbietern und staatlichem Patentamt muss als kritischer Erfolgsfaktor angesehen werden, da ein Versicherungsmarkt auf eine kritische Größe angewiesen ist, um eine hinreichende Risikostreuung und eine für KMUs adäquate Preisgestaltung zu erreichen.

Das PE-Programm ist eine freiwillige Versicherungsoption, die jährlich zu erneuern ist. Die Zielgruppe sind KMUs mit einem Umsatz bis zu 32,5 Mio. EUR. Die jährlichen Kosten liegen etwa zwischen 1.200 EUR und 32.000 EUR, wobei die durchschnittlichen Kosten nahe beim unteren Extremwert liegen.

\section{Frankreich}

\section{France Brevets und der European Patent Fund}

France Brevets ist ein (Patent-)Fonds, der im Jahr 2010 als Public-Private Partnership gegründet wurde und als „IP aggregating mechanism“ fungiert (OECD 2010). Dieser Fonds soll eine spezifische Lücke im Innovationssystem schließen, die eine Folge rezenter, strukturelle Veränderungen im Innovationsprozess ist. In Anlehnung an das Konzept der „Open Innovation“ basieren neue Technologien zunehmend nicht mehr auf einer Invention sondern auf einem Bündel von Erfindungen und den damit verbundenen Patenten. Die einzelnen ErfinderInnen in Form von Unternehmen, WissenschafterInnen, etc. sind jedoch dispers im Innovati- 
onssystem verteilt. Damit entsteht die Notwendigkeit der Bündelung dieser verstreuten Patente zu verwertbaren Technologiegruppen, womit teilweise beträchtliche Transaktionskosten verbunden sein können. Diese Aktivität kann durchaus mit der Sortimentsbildung als einer der Kernfunktionen des Einzelhandels vergleichen werden. Auch hier werden die individuellen Transaktionskosten durch die Delegation der Sortimenterstellung an einen spezialisierten Akteur durch Nutzung von Spezialisierungsvorteilen und Economis of Scale reduziert.

Gerade KMUs haben aufgrund dieser Veränderungen zunehmende Probleme, ihre Technologie extern zu verwerten, da sich ein einzelnes Patent ohne ergänzende Patente zunehmend als problematisch erweist. Um diesen Flaschenhals im Innovationssystem zu schließen, kauft der Fonds France Brevets Lizenzen auf Patente, bündelt diese zu verwertbaren Technologiegruppen und verkauft Lizenzen auf diese Technologiegruppen bzw. „Patent Clusters“. Durch den Ankauf von öffentlichen und privaten Lizenzen kommt es gleichzeitig zu einem Matching des Outputs öffentlicher Forschung und Unternehmensforschung. Damit basiert das Geschäftsmodell des Fonds im Kern auf der geschickten Kombination einer möglichst großen Zahl an Lizenzen bzw. Patenten. Zweifelsohne stellt dies an die Qualifikationen von MitarbeiterInnen hohe Ansprüche. Die Dotierung des Fonds beläuft sich insgesamt auf 100 Mio. EUR, wobei jeweils die Hälfte von staatlicher Seite und privater Seite stammt. Der Unternehmenspartner, die Bank „Caisse de Depôts“, ist für das operative Management des Fonds zuständig.

Frankreich setzt in Anlehnung an das Modell von France Brevets ebenfalls Schritte auf Europäischer Ebene zur Gründung eines Europäischen Patentfonds (Proposal to establish a European patent fund 2011). Als Begründung wird unter anderem auf die inferiore Performance Europäischer Wissensflüsse durch den Austausch von IPRs relativ zu den USA verwiesen. Zwei einschlägige Initiativen wurden diesbezüglich bereits gesetzt: Erstens wurde von der Europäischen Kommission in der ersten Hälfte des Jahres 2010 eine Studie ausgeschrieben, deren Ziel die Darstellung der Möglichkeiten zur Schaffung eines Europäischen Finanzmarktes für IPRs ist. Zweitens existiert seit 2010 eine Arbeitsgruppe, bestehend aus mehreren Europäischen Banken, zur Erarbeitung der Grundlagen eines Europäischen Patentfonds.

Die OECD (2010) weist in ihrer Innovationsstrategie aber auch auf mögliche Wettbewerbsprobleme hin, die durch das starke Engagement eines Patentfonds in einzelnen Technologiefeldern entstehen können.

\subsubsection{Bewertung und Policy Learning}

Grundsätzlich gelten auch bei den IPR-Programmen ähnliche Schlussfolgerungen wie bei gründungsbezogenen Programmen. Das einschlägige Programmportfolio umfasst ein breites Feld von Maßnahmen mit einem Fokus auf KMUs. Die Programme der aws entsprechen durchwegs den internationalen Standards der hier vorgestellten internationalen Programme. Folgende allgemeine und programmbezogene Schlussfolgerungen können gezogen werden:

- Die Regulierung von IPRs in Österreich weist keine signifikanten Unterschiede zu den Vergleichsstaaten auf. Die Patentanmeldungen im Bereich Hochtechnologie liegen etwa gleichauf mit Deutschland oder Dänemark.

- Substanzielle Verbesserungen und damit sinkende Nutzungskosten des IPR-Systems für Unternehmen verlangen internationale Kooperation und sind von daher nur mittelbar 
durch einzelne Staaten beeinflussbar. Das Beispiel der Gründung des Nordic Patent Institute weist aber auf die Möglichkeit von Zwischenschritten durch regionale Kooperationen hin. Die Bedeutung einer Europäischen Lösung wird auch in der Diskussion um einen Europäischen Patentfonds deutlich. Auch in diesem Bereich sind von einer Integration des Marktes Economies of Scale und in diesem Falle vor allem auch Economies of Scope zu erwarten.

- Die Fokussierung der Programme auf den KMU-Sektor entspricht dem Stand der wirtschaftswissenschaftlichen Forschung über Marktversagen des IPR-Systems im Falle von Anmeldung und vor allem auch Durchsetzung von IPRs durch KMUs. Dieses Marktversagen wurde in den letzten Jahren durch Produktpiraterie, Internationalisierung und überlasteten Patentämtern verschärft. Dies führte zur Einführung zahlreicher Unterstützungsprogramme von KMUs, um die Kosten der Nutzung des IPR-Systems zu reduzieren.

- Die Evaluierung der deutschen einschlägigen Förderlandschaft kam zu dem Ergebnis, dass insbesondere eine breitere Orientierung der Programme am Konzept des IPManagements notwendig wäre. Hierbei geht es explizit um die Berücksichtigung formeller und informeller Schutzmöglichkeiten. Das Programm discover.IP entspricht vom Ansatz her dieser Idee. Möglicherweise könnte jedoch auch hier noch expliziter der Fokus der Beratungsdienstleistung im Sinne des IP-Managements erweitert werden.

- Ausdrücklich positiv zu beurteilen ist das Innovationsschutzprogramm ipp. Dieses Programm wird in der deutschen Evaluierung (Blind et al. 2009) als Vorbild erwähnt, da das deutsche Programmportfolio zu sehr auf die Anmeldung und zu wenig auf die Förderung der Durchsetzung von Schutzrechten durch KMUs fokussiert ist. Ein interessantes Beispiel in diesem Zusammenhang ist das Patent Enforcer-Programm in Dänemark. Dieses auf privatwirtschaftlicher Basis organsierte Versicherungsprodukt ermöglicht KMUs eine relativ günstige Versicherung gegen die Kosten, die im Zuge von Verfahren in Patentverletzungsangelegenheiten entstehen können. 


\section{Allgemeine Schlussfolgerungen und spezifische Emp- fehlungen}

\section{Einleitung}

Im Folgenden werden die Evaluierungsergebnisse zu allgemeinen Schlussfolgerungen und Empfehlungen verdichtet, wobei an geeigneter Stelle jeweils auch die Ergebnisse der internationalen Vergleiche einfließen. Zunächst werden die allgemeinen innovationsökonomischen Rahmenbedingungen, in denen die aws-Technologieprogramme operieren, skizziert. Anschließend wird auf die spezifische Interventionslogik der aws-Technologieprogramme eingegangen. Eine Diskussion hinsichtlich der Einbettung der aws-Technologieprogramme in das österreichische Innovationssystem adressiert die Arbeitsteilung der technologiepolitischen Akteure in Österreich. Die Zielsysteme und internen Synergien werden sowohl in Hinblick auf das Subsegment der aws-Technologieprogramme selbst als auch gegenüber den anderen Instrumenten der aws diskutiert. Daran anschließend werden Schlussfolgerungen und Empfehlungen auf Ebene der Einzelprogramme gegeben. Das Kapitel schließt mit einigen Hinweisen bezüglich eines begleitenden Monitorings als Hilfsmittel für die Portfolio- und Programmsteuerung.

\section{Innovationsökonomische Rahmenbedingungen für die aws-Technologieprogramme}

Die Wirkung von innovationspolitischen Maßnahmen wird maßgeblich von vielfach nur mittelbar politisch beeinflussbaren Parametern des Innovationssystems konditioniert. Dies trifft in erhöhtem Ausmaß für die Aktivitäten einzelner Förderagenturen wie der aws zu. Überspitzt formuliert bewegt sich deren Wirksamkeit zwischen zwei Extremen: Einerseits mag bei ausgezeichneten innovationsökonomischen Rahmenbedingungen auch ein fehlerhaft gestaltetes Instrument seine Wirksamkeit entfalten, andererseits wird das beste Instrument und Programmanagement bei ungünstigen Rahmenbedingungen nur eingeschränkt positive Effekte verursachen. Im Kontinuum zwischen diesen Extremen bewegt sich die operative Tätigkeit von Fördergebern. Demnach sollte bei allem social engineering auf Instrumenten- und Fördererebene bei konstanter innovationssystemischer Umwelt kein radikaler Wandel etwa in der Gründungsdynamik oder im Patentierungsverhalten erwartet werden. Die aws ist eben nur ein Akteur neben etlichen anderen und kann von daher die Wirksamkeit ihrer Förderinstrumente nur unter gegeben Restriktionen optimieren. Es ist keineswegs auszuschließen, dass diese Restriktionen bedeutsamere Hebel für die Förderung einschlägiger innovationspolitischer Ziele darstellen als dies für die Instrumente der aws der Fall ist. Beispielhaft sei hier auf folgende Aktionsfelder verwiesen: entrepreneuership education, Europäisches Patent, Humankapitalentwicklung in quantitativer und qualitativer Hinsicht, Risikokapitalmarktregulierung, Risikokultur („Kultur des Scheiterns“), etc. Diese Aspekte gilt es zu beachten, um zu einer realistischen Einschätzung der Beeinflussbarkeit des Innovationssystems durch die Tätigkeit der aws zu gelangen.

Ein Vergleich der innovationsökonomischen Rahmenbedingungen ergibt für Österreich insbesondere beim Humankapital Mängel gegenüber den Vergleichsländern. Damit im Zusammenhang steht die Diskussion, ob die geringen Venture Capital-Investitionen in 
Österreich eine Folge mangelnder Nachfrage oder mangelnden Angebots sind. Welche Marktseite wirkt limitierend? Die diesbezügliche Datenlage lässt für Österreich keine eindeutigen Schlüsse zu. Eine Evaluierung des deutschen High-tech Gründerfonds kommt demgegenüber zu dem Ergebnis, dass das die Zahl von erfolgsversprechenden Beteiligungen primär eine Funktion der verfügbaren Beteiligungsmöglichkeiten ist. Ähnliche Ergebnisse liefern ökonometrische Studien auch für Europa. Allerdings gibt es auch Studien die weiterhin bestehender Finanzierungsrestriktionen als Wachstumshemmnis für junge innovative Unternehmen identifizieren. Wenngleich also für Österreich auch die Angebotsseite als kürzere Marktseite konditionierend wirken könnte, nicht zuletzt aufgrund eines nach wie vor mangelnden international wettbewerbsfähigen Regulierungsrahmens, ist die relativ ungünstige Humankapitalausstattung ein potenzieller Engpassfaktor, der die verfügbare Zahl an hochwertigen Investitionsprojekten systematisch reduziert. Gerade im Bereich Hochtechnologiegründungen ist ingenieur- und naturwissenschaftliches Humankapital entscheidend. Bei diesen beiden Humankapitalfraktionen ist die Performance Österreichs aber eher mittelmäßig. Hier könnte man von Israel lernen, dass intensive Anstrengungen zur Ausbildung von IngenieurInnen und NaturwissenschafterInnen bereits sehr früh gesetzt hat und weiterhin massiv in dieses Segment des Bildungssektors investiert. Diese Fragen gilt es bei zukünftigen Strategien zur Förderung von Hochtechnologiegründungen zu beachten.

\section{Interventionslogik der aws-Technologieprogramme}

Das Portfolio der Technologieprogramme der aws adressiert spezifische Momente des Marktversagens. Insbesondere im Bereich von Hochtechnologiegründungen wird ein Versagen des Kapitalmarkts bei der Finanzierung riskanter, wissensintensiver Gründungen angenommen. Der Effekt dieses Marktversagen ist eine mangelnde Investitionsbereitschaft privaten Kapitals in frühe Phasen des Unternehmenslebenszyklus. Eine Therapiemöglichkeit dieses Versagens verlangt per definitionem die Substitution privater Risikoaversion durch eine staatliche Bereitschaft zur Risikoübernahme. Die politische Ökonomie der Innovationsförderung bewirkt jedoch zahlreiche Anreize zur Risikominimierung durch konservatives Förderverhalten. Dieses Verhalten läuft jedoch Gefahr, Marktversagen lediglich durch Politikversagen zu ersetzen. Es bedarf daher sowohl eines expliziten Bekenntnisses der Innovationspolitik zur Risikobereitschaft als auch entsprechender Anreize auf Ebene der Fördergeber. Sollte die aws die gleiche Ausfallsquote an Förderfällen im Gründerbereich aufweisen wie private Venture Capital-Unternehmen, so ist dies als möglicher Hinweis auf mangelnde Risikoübernahme zu deuten, wenn die Annahme zutrifft, dass Frühphasenfinanzierungen überdurchschnittlich riskant sind.

Die Wirksamkeit innovationspolitischer Instrumente ist in mehrfacher Hinsicht eine Funktion der Zeit. Erstens ermöglicht erst eine hinreichend lange Programmdauer robuste Resultate hinsichtlich der Effektivität und Effizienz eines Instruments. Zweitens ist auch jedes Monitoring und social engineering nur dann sinnvoll, wenn die prospektive Programmdauer über einige wenige Jahre hinausgeht. Drittens ist damit zu rechnen, dass der Start eines Programms Fixkosten verursacht und sinkende Durchschnittskosten eine zunehmende Effizienz des Programms verursacht. Viertens kann angenommen werden, dass Lernkurveneffekte ebenfalls in diese Richtung wirken. Fünftens werden durch zunehmende Programmdauer die Awareness des Programms gesteigert und Informationsasymmetrien sowohl zwischen Fördergebern und 
Fördernehmern als auch zwischen unterschiedlichen Förderinstitutionen reduziert. Zusammengefasst ergeben diese Punkte ein klares Argument für hinreichend lange Programmperioden. In diesem Sinne empfiehlt die Evaluierung auch die Beibehaltung des bestehenden Instrumentenportfolios der aws. Damit die Instrumente optimal eingesetzt werden können, ist neben einer zeitlichen Kontinuität auch eine Verstetigung der finanziellen und personellen Ressourcenausstattung der Programme zu beachten.

Die österreichische FTI-Förderlandschaft ist durch komplexe Vielfalt von Förderinstrumenten gekennzeichnet. Auch das Programmportfolio der Technologieprogramme der aws hat mittlerweile eine kritische Größe erreicht. Eine Einführung zusätzlicher Programme ist von daher negativ zu beurteilen. Neben der Bedeutung der Übersichtlichkeit des Instrumentenportfolios für Fördernehmer und andere, komplementäre Fördereinrichtungen ist insbesondere auch auf zunehmende Agenturkosten zur Kontrolle eigennutzorientierten Verhaltens der Agenten aufgrund wachsender Principal-Agent-Probleme hinzuweisen. Weiterhin wird die Schaffung und Aufrechterhaltung von Synergieeffekten bei zunehmender Anzahl an Programmen ebenfalls ressourcenintensiver und das aktive Management von Komplementaritäten ein aktiv zu gestaltendes Asset. Eine Vervielfachung von Programmen führt bei gleichbleibenden Ressourcen zur Gefahr mangelnder kritischer Massen sowie Ineffizienzen durch niedrige Fallzahlen und damit fehlende economies of scale und Lernkurveneffekte. Last but not least ist eine zeitliche und inhaltliche Kontinuität in der Programmgestaltung auch ein relevanter Standortfaktor im internationalen Innovationswettbewerb sowie ein Unsicherheit reduzierender Faktor für innovative Unternehmen.

Sektorale Schwerpunkte in der Förderausrichtung finden sich in den hier vorgestellten Programmen generell keine. Insbesondere die deutschen Programme des High-Tech Gründerfonds und der Bayern Kapital geben keine erwünschten Spezialsierungen vor. Jedoch existiert mit dem Programm Go-Bio ein eigenes sektorspezifisches Förderinstrument für gründungsbereite Forscherteams aus der Biotechnologie. Der dänische Fonds Vaekstfonden ist nicht einmal explizit auf High-tech spezialisiert, sondern finanziert explizit Unternehmen, die trotz mäßiger Technologieintensität internationales Wachstumspotenzial aufweisen (,Scalable Business Model“). Auch der deutsche High-tech Gründerfonds ermöglicht eine Substitution des Technologiekriteriums. In diesem Fall erfolgt die Substitution jedoch nicht durch Wachstumskriterien, sondern durch den Innovationsgrad. Vaekstkapital als eine Division von Vaekstfonden definiert zwar einige Zielbranchen (IT, Medizintechnik, Umwelttechnik und Werkstofftechnik), aber unter dem Vorbehalt einer expliziten Offenheit gegenüber allen anderen Branchen. Israel setzt mit seinem Incubator Program auf die dezentrale Entscheidung der Inkubatoren hinsichtlich möglicher Spezialisierungsstrategien. Von Seiten des Office of the Chief Scientist werden jedenfalls keine Vorgaben abgesehen von Technologieniveau und Exportpotential gemacht. Im Vergleich zu den internationalen Vergleichsprogrammen ist festzustellen, dass die Programme des aws zur Förderung von Gründung und Aufbau von Hochtechnologieunternehmen mit ihrer Fokussierung auf IKT, Physical Sciences und Biotechnologie vordergründig den relativ höchsten Grad an Selektion bzw. technologiefeldbezogenem Targeting im internationalen Vergleich aufweisen. Tatsächlich ist jedoch mit der Kategorie Physical Sciences ein sehr weites Feld definiert, das annähernd die Funktion einer residualen Kategorie übernimmt. Sollten damit Probleme die typischerweise mit Targeting verbunden sind reduziert werden, so ist dennoch zu Fragen, in wie weit die Außendarstellung 
als Förderer für diese drei Technologiefelder nicht dennoch adverse Selektionseffekte aufgrund von Informationsdefiziten über das de facto existierende Förderangebot auf Seiten potenzieller Gründer produziert.

\section{Einbettung der aws-Technologieprogramme im österreichischen Innovationssystem}

Das Zusammenspiel auf Bundesebene zwischen den verschiedenen an die Technologieprogramme der aws ,anschließenden“ Förderungen der FFG scheint gut zu funktionieren, ist allerdings wenig formalisiert. Es handelt sich dabei vorrangig um die Abstimmung mit Förderungen der FFG im Bereich Basisprogramme, vor allem die Basisförderung. Die Abstimmung erfolgt unter den für die verschiedenen Anträge zuständigen Sachbearbeitern zwar wenig formalisiert (jour fix im Bereich Life sciences), aber doch nach Auskunft der Beteiligten konsequent. Es kann angenommen werden, dass diese Abstimmung stark personenabhängig ist. Im Sinne einer verantwortungsvollen Mittelverwendung sollte dieser Austausch allerdings für obligatorisch erklärt werden. Gleichzeitig sollte die Flexibilität im Abstimmungsprozedere aufrechterhalten werden.

Die Abstimmung und Zusammenarbeit in Bezug auf die AplusB Zentren ist differenziert zu betrachten und tangiert auch die Ebene der Regionen. Die Zusammenarbeit in Bezug auf die konkrete Unterstützung der GründerInnen erfolgt dem Konzept des Förderungsprogramms AplusB des BMVIT / der FFG nicht vorrangig mit dem Programmmanagement der FFG sondern mit den AplusB Zentren selbst. Diese sind je nach Universität und regionalen Gegebenheiten recht unterschiedlich aufgestellt und kooperieren, um ihre GründerInnen zu betreuen, in unterschiedlicher Art und Intensität mit verschiedensten Einrichtungen, z. B. auch mit der aws. Manche dieser Zentren verstehen sich aber auch als regionale Knotenpunkte und Ansprechstellen auch für Förderungen auf Bundesebene. Ähnlich wie in Österreich existiert auch in Dänemark eine Kooperation zwischen Inkubatoren und staatlichen Venture Capital-Institutionen. In Österreich betrifft dies die Zusammenarbeit zwischen der aws und den AplusB-Zentren; in Dänemark handelt es sich um die Innovation Incubators und den öffentlichen Venture Capital-Fonds Vaekstfonden. Allerdings tritt in Dänemark - wie auch beim israelischen Incubator Program - der Innovation Incubator selbst als eigenständiger staatlicher Venture Capital-Investor auf, während die AplusB-Zentren explizit keine Beteiligungen eingehen dürfen. Diese Regulierung ist ein Ausfluss von beihilferechtlichen Bedenken, die im Zuge der Erstellung der Programmrichtlinien der AplusB-Zentren geäußert wurden. Ein weiteres Problem der österreichischen AplusB-Zentren im Hinblick auf die Beteiligungsfrage ergibt sich weiterhin durch die multiplen Stakeholder und Shareholder, die direkt oder indirekt Einfluss auf das Zentrum nehmen. Da das Investitionsvolumen der dänischen Inkubatoren pro Projekt jedoch derart beschränkt ist, dass nur eine kleine Anzahl an Gründungen damit hinreichend finanziert werden kann, ist eine Folge- oder Ko-Finanzierung von Vaekstfonden der Regelfall. Unternehmen, die Unterstützung im Rahmen des Innovation IncubatorsProgramms erhalten, sind eine der Hauptquellen für Investitionsprojekte des staatlichen Venture Capital-FondsVaekstfonden. Ungefähr ein Drittel des Investitionsvolumens von Vaekstfonden betrifft den aus dem Incubator Program stammenden Dealflow. Ein Review der dänischen Gründungspolitik durch die OECD kommt zu dem Schluss, dass die Konzentration der Venture Capital-Aktivitäten in Vaekstfonden sowie die komplementären Strukturen mit dem Incubator Program positive Effekte auf die Finanzierungsbedingungen von Unternehmens-
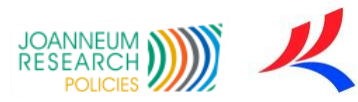
gründungen haben. Eine genauere Analyse der Kooperationsperformance zwischen dem Incubator Program und Vaekstfonden könnte Hinweise auf Optimierungsmöglichkeiten hinsichtlich der Kooperation der AplusB-Zentren und der aws liefern.

In Bezug auf die Positionierung der aws Technologieprogramme im Verhältnis zu den Bundesländern ergibt sich ein vielfältiges Bild. Es zeigt sich, dass die Förderungslandschaft auf Landesebene heterogen und ausdifferenziert ist. Die Recherche der verschiedenen Förderungsmöglichkeiten in den Bundesländern ergab, dass es dort z. B. auch FrühphasenAngebote, Beratung zu Schutzrechts-Fragen, Business-Angel Initiativen etc. gibt. Formalisiert wurde z.B. die Kooperation beim Staatspreis Innovation. In Bezug auf die Wirksamkeit der aws als Bundesagentur wurde auf deren Abnehmen mit zunehmender Distanz verwiesen und auf die Relevanz einer Präsenz vor Ort, wofür sich die aws auch stärker regionaler Partner (wie z. B. AplusB Zentren) bedienen könnte. Manche Regionen orientieren sich in Richtung anderer Zentren als nach Wien (z. B: Biotech-Bereich in Tirol nach München). Es konnte im Rahmen dieser Evaluierung das Verhältnis der Angebote Bund - Bundesländer jedoch nicht vollständig durchleuchtet werden, es sei jedoch auf die verschiedenen Diskussionslinien und Argumente zum Thema „Förderalismus in der Innovationspolitik“ hingewiesen. Dabei ist die österreichische Situation im Vergleich zu Deutschland zusätzlich durch die Kleinheit subnationaler Jurisdiktionen gekennzeichnet. Jedenfalls sind die spezifischen Vor- und Nachteile zentraler und dezentraler institutioneller Arrangements zu berücksichtigen und einzelne Bereiche auf deren Zweckmäßigkeit hinsichtlich dieser Aufgabenverteilung zu überprüfen. Hier besteht zweifelsohne weiterer Forschungsbedarf. Schließlich ist darauf zu verweisen, dass die aws keinen Einfluss auf das Agieren der Akteure auf Landesebene hat (siehe auch die diesbezüglichen Schlussfolgerungen der Zwischenevaluierung der aws-Technologieprogramme aus dem Jahr 2006 (Jörg et al. 2006)).

\section{Zielsysteme und interne Synergien}

Auch wenn aufgrund der Vielzahl an Einzelzielen der jeweiligen aws-Technologieprogramme eine Überfrachtung des Zielkatalogs konstatiert werden muss, zeigt eine Bündelung dieser Einzelziele nach übergeordneten ,Zielcluster“, dass das Zielsystem insgesamt übersichtlich und den adressierten Problemlagen des österreichischen Innovationssystems zweckdienlich ist. Die Überfrachtung des Zielkatalogs ist wohl z.T. „historisch“ entstanden (Definition zusätzlicher Ziele bei Aufbau neuer Programme ohne Durchforstung der bereits bestehenden Ziele) und lässt sich durch Streichen redundanter Ziele leicht vereinfachen. Im Rahmen dieser Evaluierung wurde hierfür ein Vorschlag erarbeitet.

Die Inanspruchnahme der aws-Technologieprogramme durch die Nutzer entspricht etwa der Förderungslogik und zeigt keine Hinweise auf unerwünschte Effekte. Die Analyse zeigt weiters erhebliche Synergien zwischen den Instrumenten (und zwar sowohl innerhalb des Systems der aws-Technologieprogramme einerseits als auch der Synergien mit dem sonstigen Instrumentenangebot der aws außerhalb der Technologieprogramme andererseits). Es finden sich zahlreiche Schnittpunkte zwischen den Programmen und weiters sind etliche Einzelprogramme modulartig (im Sinne eines Baukastensystems) miteinander verwoben. Letztlich führt dies dazu, dass ein Dealflow in einem Programm (z.B. pre-seed) wiederum positive Wirkungen auf den Dealflow eines anderen Programms (z.B. seedfinancing) mit sich bringt. Enge Zusammenhänge bestehen konzeptgemäß z. B. zwischen Seed und Preseed sowie zu I2. 
Gerade zwischen Preseed Biotech und Seed sind die Zusammenhänge in der Nutzung besonders deutlich, was mit den über die direkte monetäre Förderung hinausgehenden Maßnahmen im Rahmen von LISA zusammenhängen kann. Auch der Konnex innerhalb der aws zu Förderungen außerhalb der Technologieprogramme zeigt sich, vor allem zu Double Equity. Geringere Querverbindungen bestehen demgegenüber zwischen Impulse und den anderen Technologieprogrammen. Aus der Sicht des Evaluierungsteams sollten aber potenzielle Synergien in dieser Gruppe der Technologieprogramme systematisch unterstützt werden, wobei die spezifische Situation der Kreativwirtschaft zu berücksichtigen ist: Ein Technologieanspruch ist adäquat für diese Zielgruppen zu definieren und der Zielgruppen der Kreativwirtschaft zu kommunizieren. Denkbar wäre etwa ein Zusammenhang zu I2 (dahingehend zeigen die Daten aus der Systemevaluierung bereits erfolgte Kombinationen), aber auch zu Tecnet oder zu den Angeboten im Bereich Schutzrechte. Die detaillierteren Analysen zu den verschiedenen Programmen der Technologieprogramme haben gezeigt, dass sich in der letzten Zeit Unternehmen der Kreativwirtschaft zunehmend in der Klientel der Angebote zu den Schutzrechten und z. B. bei Tecnet finden. Schutzrechte sind im Bereich der Kreativwirtschaft spezifisch zu diskutieren, dabei könnte eine Kooperation nicht nur von impulse, sondern des IPR-Bereichs der aws mit der cwa (creativwirtschaft austria) und deren creativdepot (www.creativdepot.at) sinnvoll sein.

Die Logik der Abgrenzung der aws-Technologieprogramme gegenüber den anderen Instrumenten der aws ist weniger von der spezifischen Interventionslogik her motiviert als vielmehr eine organisatorische: Die Technologieprogramme bedienen sich - anders als die „herkömmlichen“ Fördermaßnahmen der aws - keiner Bankeninstrumenten. Das führt zum Teil dazu, dass diese Instrumente den Unternehmen nicht oder weniger bekannt sind, da der Vertriebs- und Informationskanal über die Kommerzbanken wegfällt und entsprechend vermehrte Marketing- bzw. Öffentlichkeitsmaßnahmen von Seiten des jeweiligen Programmmanagements getätigt werden müssen. Allerdings wirken die oben erwähnten internen Synergien gleichsam „automatisch“ als Marketingmaßnahme, indem der Dealflow eines Programms auch positive Wirkungen auf jenen anderer Programme zeitigt.

Der Cluster der „Technologieprogramme“ ist dementsprechend eher als Label mit Signal für die Politikebene geeignet und wichtig, aber nicht unbedingt in Richtung Unternehmen / Zielgruppen, da aus deren Perspektive unterschiedlichste Motivbündel aufgegriffen werden. Teile der Zielgruppen (z. B. die Kreativwirtschaft) werden sich mit dem Label „Technologieprogramm“ nicht angesprochen fühlen. Die neue Homepage hat die Kommunikation und Außendarstellung bereits eher an diese Motivbündel angepasst, ähnlich die neuen Folder (z.B. die graphische Umsetzung des Baukastenprinzips beim High-Tech Gründungsfolder).

\section{Schlussfolgerungen und Empfehlungen auf Ebene der Einzelprogramme}

\section{PreSeed/Seedfinancing/MaZ}

Diese Programme werden von Seiten der aws nach außen hin gemäß eines "Baukastenprinzip" vermarktet und stellen in ihrer Gesamtheit als Programmfamilie derzeit das Flaggschiff der hochtechnologieorientierten Gründungsförderung in Österreich dar. Handlungsbedarf ergibt sich im Wesentlichen in der Sicherstellung einer Stetigkeit der Finanzierungsmöglichkeit der Projekte, d.h. in der Vermeidung von Finanzierungslücken in einzelnen Jahren für prinzipiell als positiv zu bewertende Projekte. Zu prüfen wäre eine stärkere Signalisierung der 
prinzipiellen thematischen Offenheit gegenüber der derzeitigen Situation, wo die beiden vorgegebenen Technologiefelder Biotechnologie und IKT mit der Restkategorie Physical Sciencies ergänzt werden.

Im Gegensatz zum High-Tech Gründerfonds sowie zum dänischen Fonds Vaekstfonden existiert bei den aws-Technologieprogrammen zur Förderung von Hochtechnologiegründungen keine Möglichkeit seitens der potenziellen Gründer mangelnde Technologietiefe durch einen hohen Innovationsgrad (High-tech Gründerfonds) oder internationale Wachstumsperspektiven (Vaekstfonden) partiell zu substituieren (periphere Substition). In diesem Punkt scheint daher die größte Übereinstimmung mit dem israelischen Incubator Program zu bestehen. Ob diese Orientierung sinnvoll ist, kann nur im Zusammenhang mit einer breiteren Analyse des Innovations- und Fördersystems beurteilt werden. In Bezug auf das Innovationssystem ist im Rahmen der Systemevaluierung die Notwendigkeit einer auf radikalen Innovationen beruhenden Front Runner-Strategie festgestellt worden. Radikale Innovationen müssen nicht notwendigerweise an der Technologiegrenze angesiedelt sein. Entscheidend ist die Einführung neuer Produkte oder Dienstleistungen als Ergebnis neuer, imitationsresistenter Kombinationen von Technologien bzw. Organisationen, welche Kundenbedürfnisse besser befriedigen als das bestehende Angebot am jeweiligen Markt. Demnach wäre eine Substitution von Technologiegrad durch Innovationsgrad durchaus im Einklang mit den Schlussfolgerungen der Systemevaluierung. Damit erscheint eine Beurteilung der Zweckmäßigkeit einer ausschließlichen Technologieorientierung primär aus der Perspektive des Fördersystems heraus sinnvoll. Aus Perspektive des Fördersystems ist die Frage zu stellen, welche Förderinstrumente in Österreich für dieses spezifische Gründersegment existiert bzw. ob Unternehmen mit hohem Innovationsgrad und/oder hohem Wachstumspotenzial überhaupt spezifischen Formen des Marktversagens ausgesetzt sind, wie dies im Fall von Hochtechnologiegründern der Fall ist. Würde tatsächlich eine Förderlücke bestehen, so wäre eine Ausweitung des awsPortfolios trotzdem eher kritisch zu beurteilen, da gerade Hochtechnologiegründungen hohe Kompetenzanforderungen an Personal und Institution stellen, die durch Spezialisierungsgewinne und damit verbundene Lernkurveneffekte effizient erfüllt werden können.

Eine Besonderheit der deutschen Programme stellt die intensive Nutzung von Coaches dar. Während die Finanzierungsförderung den „Finance Gap“ bei Hochtechnologiegründern adressiert, zielt das Coaching auf die Schließung des in der einschlägigen Literatur diskutierten „Knowledge Gaps“ ab, der insbesondere im Bereich des betriebswirtschaftlichen KnowHows vermutet wird. Darüber hinaus wird durch die regionale Präsenz der Coaches räumliche Nähe hergestellt, die gerade in ökonomischen Situationen, die durch erhöhte Unsicherheit gekennzeichnet sind, eine wichtige Funktion in ökonomischen Transaktionen einnimmt. Während sich der /die GründerIn beim High-tech Gründerfonds noch mit Einverständnis des Fonds gegen eine Zusammenarbeit mit einem Coach entscheiden kann, ist dies im Fall der Bayern-Kapital obligatorisch. Bei beiden Fonds ist es Ziel der Maßnahme, dass der Coach eine Beteiligung am Unternehmen erwirbt. Die Unternehmen können sich dabei aus einer Liste akkreditierter Coachs eine für sie passende Persönlichkeit wählen. Die Aufgabe der Coachs liegt jedoch nicht nur in der typsicherweise kaufmännischen Beratung der technisch ausgebildeten GründerInnen, sondern auch in der Akquisition von potenziellen Investitionsprojekten. Die aws hat mit dem Programm „Management auf Zeit“ ebenfalls ein CoachingElement in ihrem Programmportfolio. Dieses Programm ist jedoch als Unterstützung für kri- 
senhafte Entwicklungen gedacht und verfolgt von daher einen anderen Ansatz als die deutschen Coaching-Programme. Die Evaluierungsergebnisse bezüglich des deutschen Modells zeigen jedoch keine eindeutig positiven Ergebnisse, wenngleich die grundsätzliche Zweckmäßigkeit des Coaching-Konzepts festgehalten wird. Relevante Probleme bestehen im Matching zwischen der Nachfrage der Gründer nach Unterstützungsleistungen und den Angeboten der Coaches.

Israel hat mit dem Programm „2B In Israel“ sowie weiteren Maßnahmen zahlreiche Initiativen zur Förderung von Immigrant Entrepreneurship gesetzt. Auch wissenschaftliche Arbeiten zeigen die hohe Bedeutung von ImmigrantInnen bzw. der Diaspora im erfolgreichen Entwicklungsmodell Israels. Ähnliches lässt sich aber auch für andere Länder, wie etwa die USA feststellen. Offenheit gegenüber ImmigrantInnen kann das Angebot an motivierten, gründungswilligen Entrepreneuren signifikant erhöhen. Oftmals sind mobile Bevölkerungsgruppen positiv selektiert und weisen eine höhere unternehmerische Orientierung als immobile Bevölkerungsgruppen auf. Hier könnte Österreich, etwa im Zusammenhang mit der Einführung der Rot-Weiß-Rot Card, neue Instrumente andenken. Ein möglicher Vorschlag wäre etwa, die Angebote der aws aktiv der Zielgruppe hochqualifizierte MigrantInnen anzubieten. Dabei gilt es insbesondere sprachliche Barrieren in der Angebotsgestaltung zu berücksichtigen. Die Einrichtung einer eigenen Homepage mit einschlägigen Informationen würde potenziell prohibitiv wirkende Informationskosten reduzieren. Auch die Nutzung von bereits bestehenden Netzwerken mit der österreichischen Diaspora könnte neue Impulse für die österreichische GründerInnen-Szene bringen. Wenngleich freilich der Anteil Deutschlands am höchsten ist, so ist dennoch auch der Anteil amerikanischer Herkunftseinrichtungen durchaus beachtlich. Durch die Einführung der Rot-Weiß-Rot Card sowie aufgrund weiterer, in den letzten Jahren getroffenen Maßnahmen zur Attrahierung ausländischen Humankapitals, könnte sich das Potenzial an ausländischen Gründern weiter erhöhen.

impulse

Impulse hat sich mittlerweise als integriertes Programm für die Kreativwirtschaft, das mit den entsprechenden Instrumenten (bzw. Modulen) von der (Vor-)Gründung bis zu bestehenden Unternehmen reicht und zusätzlich durch einschlägige Trainings- bzw. Awarenessmaßnahmen abgerundet wird. Dieses Programm wurde von Seiten der Zielgruppe sehr rasch angenommen, was zu entsprechend hohen Ablehnungsquoten der eingereichten Projekte geführt hat. Mittelfristig kann eine zu hohe Ablehnungsquote (sofern sie nicht aufgrund der geringen Qualität der Projekteinreichungen zustand komme) negative Konsequenzen zeitigen, insofern als das Programm von der potentiellen Zielgruppe gemieden wird, da der Aufwand für die Projekteinreichung den zu erwartenden Nutzen übersteigen würde. Zu prüfen wäre, inwieweit durch eine höhere Dotierung der einzelnen Module dieser hohen Ablehnungsquote - ohne gleichzeitigen Qualitätsverlust der geförderten Projekte - entgegengewirkt werden kann.

\section{LISA}

LISA gilt bis heute als best-practice Förderprogramm, legt es doch mit einer Breite von ausgewählten Fördermaßnahmen (von der allgemeinen/ Gründungsberatung über preseed/ seedfinancing bis zur Schulung/ internationalen Vermarktung) seinen Fokus auf eines der innovativsten Technologiefelder, dem Life Science-Bereich. In der jüngsten Vergangenheit hat LISA 
insbesondere durch die Etablierung der Dachmarke wesentlich zur Nachhaltigkeit und Sichtbarkeit des Unternehmensstandorts Österreich beigetragen. Gerade was diese Etablierung der Dachmarke betrifft, so hat Radauer (2009) in seiner Evaluierung hingewiesen, dass hier durchaus noch Verbesserungspotentiale auszuschöpfen sind. Hierzu zählen u.a. eine weitere Differenzierung zwischen den beiden Technologiebereichen Biotechnologie und Medizintechnik, ein Commitment zur Dachmarke als Corporate Identity von Seiten aller Stakeholder sowie damit einhergehend die Schaffung von Nutzungsregelungen, sowie eine klarere Definition von Rollen zwischen den Kooperationspartnern wie auch zwischen der aws und den Kooperationspartnern und damit einhergehend eine Effizienzsteigerung sämtlicher Entscheidungs- und Kommunikationsprozesse.

Neben der Etablierung der Dachmarke hat auch die zweite große Awareness-Schiene von LISA, der BOB, erheblich dazu beigetragen, sowohl auf nationaler als auch auf internationaler Ebene die Sichtbarkeit von LISA zu stärken. Hat man in den vergangenen Jahren durchaus daran gearbeitet, den BOB weiterzuentwickeln, so soll dies auch in Zukunft mittels verstärkter Einbeziehung wichtiger Stakeholder und Intermediäre (wie z.B. Technologietransferbüros) geschehen.

Sowohl im internationalen Marketing (bei der Dachmarke) als auch beim BOB findet eine immer stärkere Differenzierung zwischen Medizintechnik und Biotechnologie statt. Die Herausforderung, die sich nun angesichts dessen stellt, ist, dieser Differenzierung auch in anderen Programmmaßnahmen (insbesondere in der PR/ Öffentlichkeitsarbeit) Rechnung zu tragen. Damit könnten in Zukunft nicht nur Investoren und Unternehmen, sondern auch Universitäten bzw. deren Technologietransferbüros gezielter angesprochen werden.

$i 2$

Insgesamt ist die Programmentwicklung von i2 als positiv zu bewerten - insbesondere vor dem Hintergrund der globalen Finanz- und Wirtschaftskrise, die zu einem generellen Einbruch des privaten Risikokapitalmarkts im Laufe des Berichtszeitraums gefährt hat. Anzumerken ist, dass die Erwartungen an ein derartiges Programm nicht zu hoch gestellt werden sollten. Vor dem Hintergrund der tristen Lage des VC-Marktes in Österreich ist jedoch ein derartiges Instrument weiterhin - auch wenn seine direkten Wirkungen minimal sind - als eine wichtige Säule zum Aufbau des privaten VC-Marktes in Österreich heranzuziehen.

tecnet

Insgesamt entwickelte sich das Programm gemäß den Zielsetzungen. Der weitere personelle Ausbau von tecnet hat sich bewährt, insbesondere die Spezialisierung der tecnetTechnologieexperten auf jeweils bestimmte Technologie- / Branchenthemen garantiert eine professionelle Bearbeitung der Anfragen bzw. Recherchen. Die zunehmende thematische Heterogenität der Anfragen / Recherchen (z.B. in Richtung Umwelttechnologie i.w.S. aber auch in Richtung Kreativwirtschaft) deutet auf die Notwendigkeit für (thematische) Flexibilität hin. Sollten sich - neben den bestehenden fünf vordefinierten Themenbereiche - weitere Themenfelder (die derzeit unter der „Restkategorie“ Sonstiges gesammelt sind) verdichten und sich eine weiter zunehmende Nachfrage nach Recherchen in der Zielgruppe abzeichnen, wäre also eine Ressourcenaufstockung mit entsprechend spezialisiertem Know-How anzudenken. 
tecma

Insgesamt stellt das Programm tecma aber ein adäquates Instrument zur Nutzung und Verwertung der innovativen Ressourcen von KMUs dar, das durch seine Intervention ein Marktsagen adressiert und die der Zielgruppe inhärente Ressourcen- und Informationsschwäche im Umgang mit dem Patentsystem i.w.S. abzubauen hilft. Durch das Auslaufen von uni:invent kann daher für die nächste Zeit wieder eine Verschiebung in Richtung Betreuung von KMUs / EinzelerfinderInnen erwartet werden bzw. sollte aktiv angestrebt werden.

uni:invent

Der Programmverlauf von uni:invent kann abschließend als durchaus positiv bewertet werden. Im Zuge der Programmlaufzeit ist es gelungen, den Stellenwert von geistigen Eigentumsrechten an den Universitäten zu verankern, einen entsprechenden Bewusstseinswandel zu initialisieren und das IPR-Management zu professionalisieren. Mit dem Auslaufen des Programms im Jahr 2009 stellt sich nun die Frage nach der weiteren Perspektive universitärer IPR-Strategien. Grundsätzlich wird in den Leistungsvereinbarungen mit den Universitäten von Seiten des BMWF festgelegt, dass diese bis spätestens 2012 entsprechende IPRStrategien vorlegen müssen. Die Zukunft betreffende Überlegungen beinhalten u.a. die Etablierung von Wissenstransferzentren an Universitäten, die von externen Fachjurys einschlägig geprüft und gegebenenfalls zertifiziert werden. Das Programm uni:invent hat hierfür einen wegweisenden Entwicklungspfad vorgegeben. Bestand die Rolle der aws bislang in der Programmorganisation und -abwicklung, so steht die aws nun vor der Herausforderung, sich hier neu zu positionieren. Nahe liegend ist, dass sich die aws - mit all ihren über die vergangenen Jahre aufgebauten Ressourcen und Kompetenzen im Bereich IPR - am Markt als Kooperationspartner (Dienstleistungsunternehmen) nicht nur explizit für Unternehmen, sondern auch weiterhin für Universitäten anbietet.

\section{PRIZE}

Insgesamt hat die Entwicklung von PRIZE gezeigt, dass sich ein Förderprogramm für Prototypenentwicklung bzw. Finanzierung von Machbarkeitsstudien im universitären Bereich durchaus bewährt. Die steigende Anzahl an Projekteinreichungen, aber auch die erzielte Anzahl an Kooperations-, Options- und Forschungsverträgen spiegelt das Interesse bzw. den Nutzen eines solchen Programms wider. Darüber hinaus verfügt PRIZE über ein "Alleinstellungsmerkmal": Stehen für Unternehmen (vor allem seitens der FFG) zahlreiche Fördermöglichkeiten für die Herstellung von Prototypen zur Verfügung, so ist PRIZE das einzige Programm im österreichischen Förderportfolio, welches eine Förderung im Bereich der Prototypenentwicklung für universitäre Institutionen anbietet. Bis dato war PRIZE an uni:invent gekoppelt; d.h. es konnten nur Universitäten im Rahmen von PRIZE gefördert werden, die auch am Programm uni:invent teilgenommen haben. Mit dem Auslaufen von uni:invent steht die österreichische FTI-Politik nun vor der Herausforderung, PRIZE als eigenständiges Programm für die Universitäten weiterzuführen und damit im Förderportfolio neu zu positionieren. Die Kompetenzen der aws sollen dabei durchaus auch in Zukunft genutzt werden. 
ipp

Mit dem Innovationsschutzprogramm ipp hat die aws einen weiteren Schritt gesetzt, geistiges Eigentum von Klein- und Mittelunternehmen durch Vermarktung und Durchsetzung von Schutzrechten (bis dato insbesondere in ausgewählten Schwellenländern) zu stärken. Gerade die operative Hilfestellung durch aws-ExpertInnen hat dazu beigetragen, aws-intern länderspezifisches Know-how und weitreichende Kompetenzen aufzubauen. Dieses Know-How soll auch in Zukunft genutzt und weiter ausgebaut werden. Sind bis dato all die Serviceleistungen der aws für Klein- und Mittelunternehmen unentgeltlich, so stellt sich die Frage, ob und inwieweit Unternehmen einen (finanziellen) Beitrag für ihre in Anspruch genommenen Leistungen in Zukunft leisten können. Auch ist anzustreben, dass die Vermarktung und Durchsetzung von Schutzrechten ohne regionale Einschränkung seitens der aws angeboten wird. Die aws - als österreichisches Kompetenzzentrum für Vermarktung und Durchsetzung von Schutzrechten - hat hierzu bereits umfassende Expertise aufgebaut und soll diese weiterhin vor allem dem Unternehmenssektor zur Verfügung stellen.

\section{discover.IP}

Als überaus positiv zu bewerten ist die Evidenz, dass die Initiative discover.IP einen ganzheitlichen Ansatz verfolgt; d.h. dass die Bewertung von Innovationen bzw. Erfindungen umfassend vor dem Hintergrund des unternehmerischen Kontextes erfolgt. Die Evaluierungsergebnisse bestärken hierzu die Intention von discover.IP, dass gerade Klein- und Mittelunternehmen beim Aufbau von Schutzrechten auf externe Expertise und finanzielle Unterstützung angewiesen sind. Weiters bestärkt werden die Ziele von discover.IP, dass solche Initiativen angesichts der Relevanz des geistigen Eigentumsschutzes auch bereits in anderen europäischen Ländern durchgeführt werden. Wird discover.IP u.a. vom Europäischen Patentamt mitfinanziert, so nimmt das Europäische Patentamt auf supra-nationaler Ebene durchaus die Rolle des Multiplikators wahr. Vor diesen Hintergrund gilt es nun auch - unter Einbeziehung internationaler Erfahrungen - discover.IP weiterzuentwickeln.

\section{Internationale Projekte}

Die Teilnahme an EU-Projekten ist eine Begleitmaßnahme. Die aws beteiligt sich an Projekteinreichungen, deren Fokus bislang auf den Bereichen IPR sowie Technologie- und Wissenstransfer gelegen ist. Für die Zukunft ist es überlegenswert, dieses thematische Spannungsfeld weiter aufzumachen und dementsprechend auch die Expertise im Bereich PLM auszubauen. Als potentielle Themenfelder sind in diesem Kontext z.B. zu nennen: Creative Industries, Early Stage-Finanzierung und Open Innovation. Die Entscheidung über die Teilnahme an EU-Projekten ist generell immer nach Maßgabe der unterstützenden Wirkung für die awsFörderprogramme und Beratungsleistungen sowie der zur Verfügung stehenden Ressourcen zu treffen. EU-Projekte dienen vor allem dazu, die Kompetenz der aws-MitarbeiterInnen zu erweitern, aber auch die Reputation der aws als Institution auf supra-nationaler wie auch auf nationaler Ebene zu stärken. 


\section{ProTRANS}

Aufgrund der vorliegenden Daten sowie der aws-eigenen Bewertungs- und Kundenzufriedenheitsanalyse kann dem Programm ein seinen Zielen entsprechender Erfolg zugesprochen werden. Insgesamt ergibt sich also derzeit kein Handlungsbedarf in Bezug auf die Programmgestaltung, zumal auch zu berücksichtigen ist, dass das Programm in seiner jetzigen Ausgestaltung erst zwei operative Jahre hinter sich hat. Anzumerken ist jedoch, dass die geographische Verteilung der geförderten Projekte nach Bundesländern Lücken im Süden (v.a. Steiermark) und im äußersten Westen (Vorarlberg) aufweist. Hier wären entsprechende verstärkte Informationsaktivitäten, etwa in Zusammenarbeit mit den einschlägigen regionalen Akteuren.

\section{Staatspreis Innovation}

In den letzten Jahren hat die aws Bemühungen unternommen, den Staatspreis inhaltlich zu erweitern. Dies ist gelungen, indem ab dem Jahr 2012 (neben dem Econovius) ein weiterer Sonderpreis „VERENA powered by VERBUND“ verliehen wird. Dies bringt mit sich, dass die aws nun vor der Herausforderung steht, diese neue Kategorie - in Kooperation mit den Bundesländern - in den kommenden Jahren nachhaltig zu etablieren. Die aws ist einer solchen Anforderungen durchaus gewachsen, hat sie doch seit der Evaluierung im Jahr 2006 eine gewisse „Neuorientierung“ des Staatspreises nicht nur mitgetragen, sondern die Abwicklung und Organisation auf ein Niveau gehoben, welches das Attribut ,exzellent“ verdient. Gegenüber der Evaluierung 2006 waren es vor allem vier Punkte, deren Abänderung bzw. Weiterentwicklung zu diesem Erfolg führten: (1) Erweiterung des Teilnehmerkreises auf Bundesländerebene, (2) stärkere Gewichtung der Auswahlkriterien auf Innovativität, (3) die Zusammensetzung der Jury aus unabhängigen Fachexperten, sowie (4) die verbesserte PR bzw. Öffentlichkeitsarbeit. Angesichts dieser erfolgreichen Entwicklung gilt es daher als überlegenswert, die bis dato aufgebauten Erfahrungen und Kompetenzen der aws zu nutzen, um künftig auch andere, weitere Staatspreise in der aws abzuwickeln. Die aws würde somit in ihrem Awareness-Portfolio ein Kompetenzzentrum für Staatspreise beinhalten, dessen Aufgabe sowohl die inhaltliche Gesamtkonzeption als auch die Abwicklung mehrerer Staatspreise umfasst. Damit würde der Auftraggeber, nicht nur das Potential an Erfahrung und Know-how der aws optimal ausschöpfen, auch könnten durch eine solche konzentrierte Abwicklung von Staatspreisen Synergieeffekte genutzt werden und daraus Effizienzsteigerungen - sowohl auf Seiten der aws als auch auf Seiten des Auftraggebers - erzielt werden. Über all diese inhaltlichen (Zukunfts-)Themen steht aber allemal die Erfordernis der rechtzeitigen Beauftragung und Budgetbereitstellung an die aws; d.h. dass für all die zukünftigen Ausbauschritte entsprechende finanzielle Rahmenbedingungen gewährt werden müssen. Im Konkreten bedeutet dies, dass - um die inhaltlichen Anforderungen des Auftraggebers erfüllen zu können - die damit verbundenen Budgets zum Start der Umsetzung (in der Regel sechs Monate vor der Verleihung) verbindlich zur Verfügung gestellt werden müssen.

\section{Jugend Innovativ}

Jugend Innovativ konnte im Berichtszeitraum seine Reichweite als wichtigstes jugendorientiertes Motivierungsprogramm weiter ausbauen. Trotz der insgesamt positiven Wirkung lässt sich ein Handlungsbedarf in zwei Richtungen feststellen: Aufgrund der positiven Erfahrungen mit den Sonderpreisen wäre eine weitere thematische Ausweitung (im Idealfall wie- 
derum mit Beteiligung bzw. unter Zusammenarbeit mit privaten Sponsoren aus der Wirtschaft) überlegenswert. Bei einer gezielten Auswahl von neuen Kategorien könnte es durchaus wieder gelingen die Reichweite von Jugend Innovativ neuerlich anzuheben. Denkbare thematische Kategorien wären etwa übergreifende Themenfelder wie z.B. „Wasser“, „Lebensmittel“ oder „Stadt der Zukunft“. Weiterhin problematisch bleibt die große Dominanz des Schultyps HTL. Zwar weist dieser Schultyp unzweifelhaft generische Vorteile in einem technikorientierten Wettbewerb wie Jugend Innovativ, trotzdem sollte eine stärkere Einbindung anderer Schultypen angestrebt werden. Insbesondere der nunmehr sehr geringen Teilnahme von Allgemein Bildenden Höheren Schulen (AHS), die noch zu Beginn der 1990er Jahre die Mehrzahl der Teilnehmer gestellt hatten, sollte gegengesteuert werden. Eine Möglichkeit hierfür bietet wiederum die Definition zusätzlicher Kategorien. Wird die Themengestaltung dieser zusätzlichen Kategorien eher an generischen Wissensgebieten (statt spezifischer Technologiesegmente wie z.B. IKT) ausgerichtet, hat dies eventuell einen für AHS (deren Fächerkanon sich tendenziell eher an die traditionell akademische Gliederung der Wissenschaftsdisziplinen orientiert) motivierenden Effekt.

\section{Monitoring als Hilfsmittel Portfolio- und Programmsteuerung}

2007 bis 2010 verstärkte Bemühungen in Hinblick auf ein programmbegleitendes Monitoring getätigt. Die diesem Monitoring entstammenden Datenquellen waren dann auch ein vorrangiger Input zur hier vorliegenden Evaluierung. Nichtsdestotrotz zeigte sich, dass noch gewisse Problembereiche bezüglich eines systematischen Monitoring vorliegen. Dies betrifft zunächst die interne Organisation der EDV innerhalb der aws, welche durch ungenügende Schnittstellen bzw. Integration der - historisch bedingten unterschiedlichen - Themenbereiche gekennzeichnet ist. Tatsächlich befindet sich die aws derzeit auch in einem aktuell laufenden Umstellungsprozess ihres EDV-Systems. Bei dieser Umstellung sollte auf die Erfordernisse eines laufenden Monitoringprozesses Bedacht genommen werden.

Ein konkretes Problem in diesem Zusammenhang ergibt sich aus dem Charakter einiger Förderprogramme, die die Vorgründungsphase und somit natürliche Personen ansprechen. Nach erfolgter Gründung (und damit dem Wechsel der Rechtspersönlichkeit von einer natürlichen Person hin zur juristischen) ist es im derzeitigen System überaus schwierig Förderhistorien korrekt nachvollziehen zu können. Diese Informationslücke bezüglich der Rückverfolgung (bei Wechsel von Rechtspersönlichkeiten) führt aber letztlich zu einer u.U. unvollständigen Erfassung von Synergien und Schnittstellen zwischen den verschiedenen Programmen und Instrumenten innerhalb der aws. Die Möglichkeit einer derartigen Rückverfolgung aller Förderfälle würde die Analyse des Instrumentennetzwerks insgesamt und somit die Abschätzung der Synergien zwischen den Programmen erheblich erleichtern.

Bezüglich des Monitorings auf der Ebene der Einzelprogramme ist anzumerken, dass eine konzisere Definition von quantitativen Zielerreichungsindikatoren eine einfachere Erfassung des Programmerfolgs ermöglichen könnte. Derzeit sind einige der Zielerreichungsindikatoren nur sehr allgemein bzw. geben keine normativen Sollgrößen vor.

Zusätzlich sollte bei der Erfassung der Förderfälle verstärkt die etablierten Standards der Wirtschaftsstatistik (z.B. Branchengliederung nach ÖNACE) berücksichtigt werden. Anzudenken wäre auch eine Aufstellung der Technologiezugehörigkeit der Projekte nach der IPC (International Patent Classification), z.B. auf dem Aggregationsniveau Sekti- 
on/Klasse. Dies gilt selbstverständlich gilt dies nur für jene Programme, wo prinzipiell die Patentierbarkeit von Projektergebnissen möglich ist bzw. Patente als Fördergegenstand (z.B. im Sinne einer Patentberatung, Patentverwertung etc.) eine wesentliche Rolle spielen. 


\section{Literatur}

Acs, Z. et.al. (2009), The Knowledge Spillover Theory of Entrepreneurship, Small Business Economics, 32, 15-30.

Acs, Z., Szerb, L. (2010), The Global Entrepreneurship and Development Index (GEDI), Paper presented at the DRUID Summer Conference 2010. Online unter: http://www2.druid.dk/conferences/viewpaper.php?id=502261\&cf=43 [3.3.2011].

Aiginger, K. et al. (2006): Mehr Beschäftigung durch Wachstum auf Basis von Innovation und Qualifikation (WIFO Weißbuch). Wien.

aws (2006), Aktualisierung des Anbots: Technologieförderprogramme der aws 2007 - 2009 vom September 2006, Wien.

aws (2007): Leistungsbericht 2007, Wien.

aws (2008): Leistungsbericht 2008, Wien

aws (2009): Leistungsbericht 2009, Wien.

aws (2010), Leistungsbericht LISA 2009, Wien.

aws (2011), Leistungsbericht LISA 2010, Wien.

Blind, K. et.al. (2009), Die volkswirtschaftliche Bedeutung geistigen Eigentums und dessen Schutz mit Fokus auf den Mittelstand. Studie im Auftrag des Bundesministeriums für Wirtschaft und Technologie. Berlin.

BMWF, BMVIT, BMWFJ (2010), Forschungs- und Technologiebericht 2010, Lagebericht gemäß Paragraf 8 (1) Forschungsorganisationsgesetz (FOG) über die aus Bundesmitteln geförderte Forschung, Technologie und Innovation in Österreich, Wien.

BMWFJ (2010), Staatspreis Innovation: Leistungsbericht 2009, Wien.

BMWFJ (ohne Jahr), Staatspreis Innovation: Statuten, abrufbar unter: http://www.staatspreis.at/Satellite.aspx?\&145=162\&143=1490\&8=12.

Bundesministerium für Wirtschaft und Technologie (2010), Mit dem Patent zum Erfolg. Innovationsförderung für Unternehmen. Berlin.

Cohen,N. (2002), The Israel High-Tech Industry - Fifty Years of Excellence. Online unter: http://www.mfa.gov.il $/ \mathrm{mfa} /$ facts $\% 20$ about $\% 20$ israel $/$ science $\% 20$ $\% 20$ technology/the $\% 20$ israel $\% 20$ high-tech $\% 20$ industry $\% 20$ $\% 20$ fifty \%20years \%20of \%20exc [20.3.2011].

Danish Patent and Trademark Office (2008), Status and Perspectives 2008.

De, D. (2005), Entrepreneurship. Gründung und Wachstum von kleinen und mittleren Unternehmen, München: Pearson.

Egeln, J. et.al. (2003), Akademische Spinoff-Gründungen in Österreich. JOANNEUM RESEARCH.

Europäischen Kommission (2010): Grünbuch zur „Erschließung des Potenzials der Kulturund Kreativindustrien“. Brüssel KOM 183/3

European Commission (2009), Making IPR Work for SMEs. Annex b: Best Practice Cases. Online unter:

http://ec.europa.eu/enterprise/newsroom/cf/_getdocument.cfm?doc_id=4055 [3.3.2011].

Gassler, H., Ecker, B., Meyer, S., Schmidmayer, J., Steyer, F. (2010), Patentberatungsstellen in Österreich, Wien.

Geyer, A., Heimer. T. (2010), Evaluierung des High-Tech Gründerfonds. Endbericht. Studie im Auftrag des Bundesministeriums für Wirtschaft und Technologie. Technopolis.

Hölzl, W. (2006), WIFO-Weißbuch: Mehr Beschäftigung auf Basis von Innovation und 
Qualifikation. Teilstudie 20: Neugründung und Entwicklung von Unternehmen. Wien.

Honig, B. et al. (2006), Social Capital and the Linkages of High-Tech Companies to the Military Defense System: Is there a Signalling Mechanism?, Small Business Economics, 27, 419-437.

Janger, J. (2009), Systemevaluierung der österreichischen Forschunghsförderung und finanzierung. Report 1: Rahmenbedingungen. Wien.

Jörg, L., Schibany, A., Nones, B., Gassler, H. (2006): Zwischenevaluierung der awsTechnoloieprogramme. Endbericht im Auftrag des Bundesministeriums für Wirtschaft und Arbeit, Wien.

Klofsten, M. et al. (2010), Transfering Good Practice beyond Organizational Borders: Lessons from Transferring an Entrepreneurship Programme, Regional Studies, 44, 791-799.

Knoll, N. (2020), Interne Evaluierung von ProTRANS \& ERP-Technologieprogramm, Manuskript, Wien

Leo, H. et.al. (2006), WIFO-Weißbuch: Mehr Beschäftigung auf Basis von Innovation und Qualifikation. Teilstudie 8: Forschung und Innovation als Motor des Wachstums. Wien.

Lerner, J. (2009), Boulvards of Broken Dreams: Why Public Efforts to Boost Entreprenership and Venture Capital have failed and what to do about it. Princeton: Princeton University Press.

Lingaard, J. et.al. (2008), An NSI in Transition? Denmark. In: Edquist, C., Hommen, L. (Hrsg.), Small Country Innovation Systems. Globalization, Change and Policy in Aisa and Europe, Cheltenham: Edward Elgar.

Mayer, S., Fischl, I., Ruhland, S., Sheikh, S. (2009): Governance in der FTI-Politik im Wechselspiel zwischen Ministerien und Agenturen, Teilbericht 3 der Systemevaluierung der österreichischen Forschungsförderung und -finanzierung, Wien, im Auftrag des BMVIT und des BMWA

Mayer, S., Fischl. I., Ruhland, S., Sheikh, S. (2009): Das Angebot der direkten FTIFörderung in Österreich, Teilbericht 5 der Systemevaluierung der österreichischen Forschungsförderung und -finanzierung, Wien, im Auftrag des BMVIT und des BMWA

Mayer, S., Sheikh, S., Streicher, J. (2009) Public RTDI Funding in Austria - the Target Groups' Perspective, Teilbericht 7 der Systemevaluierung der österreichischen Forschungsförderung und -finanzierung, Wien, im Auftrag des BMVIT und des BMWA

Meyer, T. (2008), Venture Capital: Bridge between Idea and Innovation? Deutsche Bank Research, Frankfurt am Main.

Mustar, P. (2010), University Spin-off Firms: Lessons from Ten Years of Experience in Europe, Science and Public Policy, 35, 67-80.

OECD (2008), Entrepreneurship Review of Denmark. Paris.

OECD (2009), Science, Technology and Industry Scoreboard. Paris.

OECD (2010), The OECD Innovation Strategy. Getting a Head Start on Tomorrow. Paris.

Park, W.J., Wagh, S. (2002), Chapter 2: Index of Patent Rights, Economic Freedom of the World: 2002 Annual Report, Fraser Institute, 33-41.

Proposal to esablish a European patent fund. Online unter http://www.era.gv.at/space/11442/directory/21218/doc/22124.html [3.3.2011].

Radauer, A. (2009), Evaluierung der LISA (Life Science Austria ) Dachmarke, technopolis

Wien, i.A. des BMWFJ

Rammer, C. et.al. (2004), Internationale Trends in der Forschungs- und Innovationspolitik. Fällt Deutschland zurück? ZEW Wirtschaftsanalysen, Bd.73, Baden-Baden: Nomos Verlagsgessellschaft.

Rat für Forschung und Technologieentwicklung (2008ff): Einzelempfehlungen. 
http://www.rat-fte.at/einzelempfehlungen.html

Rat für Forschung und Technologieentwicklung (2009): Strategie 2020 - Forschung, Technologie und Innovation für Österreich. Wien.

Reiner, C. (2009), How to Lure the New Argonauts to Austria? Brain Competition Policy in a Small Open European Economy, Mitteilungen der Österreichischen Geographischen Gesellschaft, 151, 53-87.

Rheinisch-Westfälisches Institut für Wirtschaftsforschung (2009), Innovationsbericht 2009.

Ruhland, S., Hölzl, K., Scheichenbauer, H., Berger, M., Gassler, H. (2010): Dienstleistungslandschaft in Österreich, Studie im Auftrag der Österreichischen Forschungsförderungsgesellschaft FFG und des Bundesministeriums für Wirtschaft, Familie und Jugend BMWFJ. Wien.

Schartinger, D., Schibany, A., Gassler, H. (2001): Interactive Relations Between Universities and Firms: Empirical Evidence for Austria, Journal of Technology Transfer, Volume 26, Number 3, 255-268.

Schibany, A., Streicher, G., Ecker, B. (2009), Zwischenevaluierung uni:invent, InTeReg Research Report Nr. 89-2009, Wien.

Senor, D. und Singer, S. (2009), Start-up Nation. The Story of Israel's Economic Miracle. New York: Hachette Book.

Shane, S. (2009), Why encouraging more people to become entrepreneurs is bad public policy, Small Business Economics, 33, 141-149.

Tichy, G. (2010), Front Runner-Strategie: Definition und Umsetzung. In: Plattform Forschungs- und Technologieevaluierung, 34, 48-67.

Veugelers, R. (2009), A Lifeline for Europe's Young Radical Innnovators. Bruegel Policy Brief, 1. 